The Iridium (III)-Catalyzed Direct C(sp $\left.{ }^{2}\right)$ - and C(sp $\left.{ }^{3}\right)-H$ Alkynylation of 2Acylimidazoles with Various Alkynyl Bromides: Understanding the Full Catalytic Cycle

Sanjit K. Mahato and Naoto Chatani*

Department of Applied Chemistry, Faculty of Engineering, Osaka University, Suita, Osaka 565-0871, Japan chatani@chem.eng.osaka-u.ac.jp

1. General Consideration

A. Reagent Information S2

B. Analytical Information

2. Experimental Section

A. General Procedure for Preparation of 2-Acyl Imidazoles

B. Synthesis of Coupling Partners

C. Optimization Studies

$\mathrm{S} 12-\mathrm{S} 20$

D. General Procedure for C-H Alkynylation

S21-S29

3. Mechanistic Studies

S30-S36

A. Deuterium Scrambling Experiments

S30-S33

B. KIE Experiments

C. Intermolecular Competition Experiments

S35

D. TEMPO Trapping Experiments

4. Detection of Ir-intermediates

A. Synthesis of Iridacycle B

S37-S38

B. Stoichiometric Reaction with Iridacycle B

S39-S43

C. Standard reaction in presence of Iridacycle B as a catalyst

D. Ligand Exchange Reaction with Iridacycle B

S44

5. Removal of Directing Group and in situ Amidation

6. X-ray Analysis

S47-S76

A. X-ray analysis of intermediate B (CCDC 1949236)

S47-S51

B. X-ray analysis of intermediate B' (CCDC 1949237)

S52-S57

C. X-ray analysis of intermediate $\mathbf{E}$ (CCDC 1964835)

S58-S66

D. X-ray analysis of compound 3ia (CCDC 1974283)

S67-S71

E. X-ray analysis of compound 6sc' (CCDC 1974284)

8. NMR Spectra 


\section{General Consideration}

A. Reagent Information: All the solvents were purchased from Wako Pure Chemical Industries, Ltd. and were used without further purification. [Cp* $\left.\operatorname{IrCl}_{2}\right]_{2}, \mathrm{NaBAr}_{4}{ }_{4}$ were purchased from Aldrich. Acyl chloride and other salts were purchased from Tokyo Chemical Industry Co., Ltd. Silica gel (SilicaFlash ${ }^{\circledR} \mathrm{F} 60 ; 40-63 \mu \mathrm{m}, 230-400 \mathrm{mesh}$ ) obtained from Silicyle was used for column chromatography.

B. Analytical Information : All reactions were performed in a screw cap reaction tube, unless otherwise stated. ${ }^{1} \mathrm{H}$ NMR (400 MHz) and ${ }^{13} \mathrm{C}$ NMR (100 MHz) spectra were recorded on a JEOL ECS-400 spectrometer in $\mathrm{CDCl}_{3}$ or $\mathrm{CDCl}_{3}$ with tetramethylsilane. Copies of the ${ }^{1} \mathrm{H}$ NMR, ${ }^{13} \mathrm{C}$ NMR can be found in the Supporting Information. All ${ }^{1} \mathrm{H}$ NMR experiments are reported in units, parts per million ( $\mathrm{ppm}$ ), and were measured relative to the signals for residual chloroform $(\delta 7.26 \mathrm{ppm})$ in the deuterated solvent or tetramethylsilane $(\delta 0.00 \mathrm{ppm})$ as the internal standard unless otherwise stated. All ${ }^{13} \mathrm{C}$ NMR spectra were reported in ppm relative to $\mathrm{CDCl}_{3}(\delta 77.16 \mathrm{ppm}) .{ }^{11} \mathrm{~B}$ and ${ }^{19} \mathrm{~F}$ NMR experiments are recorded relative to the signals for boron trifluoride diethyl etherate for ${ }^{11} \mathrm{~B}(\delta=0.0 \mathrm{ppm})$ and ${ }^{19} \mathrm{~F}(\delta=-152.8 \mathrm{ppm})$. Highresolution mass spectra (HR-MS) was a recorded on a JEOL JMS-700 spectrometer. Melting points were recorded using a stanford research systems apparatus. Some of the compounds were purified by LC-908 HPLC (GPC). Copies of the ${ }^{1} \mathrm{H}$ NMR, ${ }^{13} \mathrm{C}$ NMR are given in the Supporting Information. 


\section{Experimental Section}

\section{A. General procedure for the preparation of 2 -acyl imidazole starting material $^{1}$}

Procedure A: ${ }^{1 \mathbf{a}}$ To a solution of an imidazole analogue (SM-1; $10 \mathrm{mmol}$; 1 equiv.) in acetonitrile $(20 \mathrm{~mL})$, the appropriate acid chloride (SM-2; $15 \mathrm{mmol}, 1.5$ equiv.) was added at $0{ }^{\circ} \mathrm{C}$ followed by the addition of $\mathrm{Et}_{3} \mathrm{~N}(2.09 \mathrm{~mL}, 15 \mathrm{mmol}, 1.5$ equiv. $)$ at the same temperature. After the addition was complete, the reaction was stirred for 30 minutes and then allowed to stir overnight at room temperature. When the imidazole was fully consumed (monitored by TLC, $50 \%$ ethyl acetate - hexane), water $(50 \mathrm{~mL})$ was added and the resulting solution extracted with ethyl acetate $(50 \mathrm{~mL} \times 3)$, the extract then washed with saturated $\mathrm{NaHCO}_{3}$ solution (50 $\mathrm{mL} \times 2)$ and brine $(100 \mathrm{~mL})$. The organic fractions were dried over $\mathrm{Na}_{2} \mathrm{SO}_{4}$ and evaporated under reduced pressure to give the crude product which was purified by silica gel column chromatography. The corresponding 2-acyl imidazole was eluted at a concentration of $25-35 \%$ ethyl acetate in hexane.

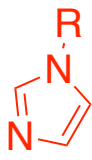

SM-1

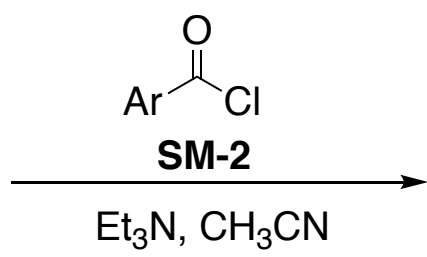

$0{ }^{\circ} \mathrm{C}$ to rt., overnight<smiles>O=C([Al])c1ncc[nH]1</smiles>

1a-s

This procedure was used for the synthesis of the starting materials 1a-s and 1a- $\boldsymbol{d}_{7}$.<smiles>Cc1ccccc1C(=O)c1nccn1C</smiles>

1a<smiles>Cc1ccccc1C(=O)c1nccn1C(C)C</smiles>

$1 b$<smiles>Cc1ccccc1C(=O)c1ncc[nH]1</smiles>

$1 c$<smiles>Cc1ccccc1C(=O)c1nccn1-c1ccccc1</smiles>

1d<smiles>C[M]=Cn1ccnc1C(=O)c1ccccc1C</smiles>

$1 \mathrm{e}$<smiles></smiles>

$1 f$<smiles>Cn1ccnc1C(=O)c1ccccc1</smiles>

$1 \mathrm{~g}$<smiles>Cc1cccc(C(=O)c2nccn2C)c1</smiles>

$1 \mathrm{~h}$<smiles>COc1cccc(C(=O)c2nccn2C)c1</smiles>

$1 i$<smiles>Cn1ccnc1C(=O)c1cccc(C(F)(F)F)c1</smiles>
1j<smiles>Cn1ccnc1C(=O)c1ccccc1C(F)(F)F</smiles>

1n<smiles>Cn1ccnc1C(=O)c1cccc2ccccc12</smiles>

$1 \mathrm{k}$<smiles>Cn1ccnc1C(=O)c1cc(Cl)c(Cl)c[13c]1</smiles><smiles>Cn1cccc1C(=O)c1nccn1C</smiles>
$1 p$<smiles>Cn1ccnc1C(=O)c1ccco1</smiles>

$1 q$

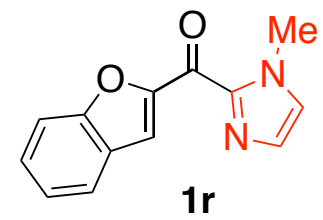

$1 r$

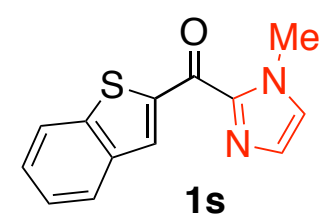<smiles>[2H]c1c([2H])c([2H])c(C([2H])([2H])[2H])c(C(=O)c2nccn2C)c1[2H]</smiles> 


\section{Characterization of the Compounds:}

(1-Methyl-1H-imidazol-2-yl)(o-tolyl)methanone (1a):White crystalline solid; Yield: 76\%;<smiles>Cc1ccccc1C(=O)c1nccn1C</smiles>

1a mp. $90-91{ }^{\circ} \mathrm{C} ; R_{f}=0.39(50 \%$ ethyl acetate-hexane $) . \delta_{\mathrm{H}}\left(400 \mathrm{MHz}, \mathrm{CDCl}_{3}\right)$ $7.63(\mathrm{dd}, J=7.8,1.4 \mathrm{~Hz}, 1 \mathrm{H}), 7.38(\mathrm{td}, J=7.4,1.6 \mathrm{~Hz}, 1 \mathrm{H}), 7.24-7.29$ (m, 2H), $7.20(\mathrm{~d}, J=0.8 \mathrm{~Hz}, 1 \mathrm{H}), 7.11(\mathrm{~d}, J=0.8 \mathrm{~Hz}, 1 \mathrm{H}), 4.13(\mathrm{~s}, 3 \mathrm{H}), 2.41$ $(\mathrm{s}, 3 \mathrm{H}) \mathrm{ppm} . \delta_{\mathrm{C}}\left(100 \mathrm{MHz}, \mathrm{CDCl}_{3}\right) 188.21,143.79,138.00,137.46$, 131.21, 130.89, 130.08, 129.96, 127.15, 125.20, 36.52, 20.32 ppm. HRMS ( $\mathrm{EI}^{+}, \mathrm{m} / \mathrm{z}$ ) calcd for $\mathrm{C}_{12} \mathrm{H}_{12} \mathrm{~N}_{2} \mathrm{O}: 200.0950$, found: 200.0952 .

(1-Isopropyl-1H-imidazol-2-yl)(o-tolyl)methanone (1b): Light yellow liquid; Yield: 83\%;<smiles>Cc1ccccc1C(=O)c1nccn1C(C)C</smiles>

$1 b$ $R_{f}=0.54$ (50\% ethyl acetate-hexane). $\delta_{\mathrm{H}}\left(400 \mathrm{MHz}, \mathrm{CDCl}_{3}\right) 7.56(\mathrm{dd}, J$ $=7.8,1.6 \mathrm{~Hz}, 1 \mathrm{H}), 7.41-7.35(\mathrm{~m}, 1 \mathrm{H}), 7.33(\mathrm{~d}, J=0.8 \mathrm{~Hz}, 1 \mathrm{H}), 7.29-7.23$ $(\mathrm{m}, 2 \mathrm{H}), 7.21(\mathrm{~d}, J=0.4 \mathrm{~Hz}, 1 \mathrm{H}), 5.65-5.58(\mathrm{~m}, 1 \mathrm{H}), 2.39(\mathrm{~s}, 3 \mathrm{H}), 1.59$ $(\mathrm{s}, 3 \mathrm{H}), 1.53(\mathrm{~s}, 3 \mathrm{H}) \mathrm{ppm} . \delta_{\mathrm{C}}\left(100 \mathrm{MHz}, \mathrm{CDCl}_{3}\right) 188.75,143.23,138.87$, $137.21,131.12,130.74,130.48,129.77,125.23,121.22,49.45,23.98$

(2C), 20.31 ppm. HRMS (EI ${ }^{+}, \mathrm{m} / \mathrm{z}$ ) calcd for $\mathrm{C}_{14} \mathrm{H}_{16} \mathrm{~N}_{2} \mathrm{O}: 228.1263$, found: 228.1262.

(1-Benzyl-1H-imidazol-2-yl)(o-tolyl)methanone (1c): Sticky solid; Yield: $60 \% ; \quad R_{f}=0.50$<smiles>Cc1ccccc1C(=O)c1ncc[nH]1</smiles>

$1 \mathrm{c}$ (50\% ethyl acetate-hexane). $\delta_{\mathrm{H}}\left(400 \mathrm{MHz}, \mathrm{CDCl}_{3}\right) 7.58-7.55(\mathrm{~m}, 1 \mathrm{H}), 7.39-$ $7.28(\mathrm{~m}, 4 \mathrm{H}), 7.27-7.21(\mathrm{~m}, 5 \mathrm{H}), 7.14(\mathrm{~d}, J=0.9 \mathrm{~Hz}, 1 \mathrm{H}), 5.74(\mathrm{~s}, 2 \mathrm{H}), 2.33$ $(\mathrm{s}, 3 \mathrm{H}) \mathrm{ppm} . \delta_{\mathrm{C}}\left(100 \mathrm{MHz}, \mathrm{CDCl}_{3}\right) 188.44,143.48,138.29,137.33,136.65$, $131.15,130.87,130.32,129.86,129.05$ (2C), 128.29, 127.78 (2C), 126.07, 125.22, 52.06, 20.24 ppm. HRMS $\left(\mathrm{EI}^{+}, \mathrm{m} / \mathrm{z}\right)$ calcd for $\mathrm{C}_{18} \mathrm{H}_{16} \mathrm{~N}_{2} \mathrm{O}$ : 276.1263, found: 276.1257 .

(1-Phenyl-1H-imidazol-2-yl)(o-tolyl)methanone (1d): Light Yellow solid; 53\%; mp. 77-78<smiles>Cc1ccccc1C(=O)c1nccn1-c1ccccc1</smiles>

$1 d$ ${ }^{\circ} \mathrm{C} ; R_{f}=0.47\left(50 \%\right.$ ethyl acetate-hexane). $\delta_{\mathrm{H}}\left(400 \mathrm{MHz}, \mathrm{CDCl}_{3}\right) 7.73$ (dd, $J=7.6,1.2 \mathrm{~Hz}, 1 \mathrm{H}), 7.51-7.45(\mathrm{~m}, 3 \mathrm{H}), 7.40-7.35(\mathrm{~m}, 3 \mathrm{H}), 7.31(\mathrm{~d}, J=1.2$ $\mathrm{Hz}, 1 \mathrm{H}), 7.30-7.28(\mathrm{~m}, 1 \mathrm{H}), 7.27(\mathrm{~d}, J=1.2 \mathrm{~Hz}, 1 \mathrm{H}), 7.26-7.24(\mathrm{~m}, 1 \mathrm{H})$, $2.41(\mathrm{~s}, 3 \mathrm{H}) \mathrm{ppm} . \delta_{\mathrm{C}}\left(100 \mathrm{MHz}, \mathrm{CDCl}_{3}\right) 186.54,144.05,138.41,138.37$, 137.25, 131.39 (2C), 130.79, 130.17, 129.32 (2C), 128.77, 126.59, 125.60, 125.24 (2C), 20.55 ppm. HRMS ( $\mathrm{EI}^{+}, \mathrm{m} / \mathrm{z}$ ) calcd for $\mathrm{C}_{17} \mathrm{H}_{14} \mathrm{~N}_{2} \mathrm{O}: 262.1106$, found: 262.1103 .

o-Tolyl(1-((2-(trimethylsilyl)ethoxy)methyl)-1H-imidazol-2-yl)methanone (1e): Colorless<smiles>Cc1ccccc1C(=O)c1nccn1S#N</smiles>

$1 e$ thick oil; Yield: $79 \% ; R_{f}=0.59\left(50 \%\right.$ ethyl acetate-hexane). $\delta_{\mathrm{H}}(400 \mathrm{MHz}$, $\left.\mathrm{CDCl}_{3}\right)$ 7.62-7.60 (m, 1H), 7.41-7.37 (m, 2H), 7.28-7.24 (m, 3H), $5.89(\mathrm{~s}$, $2 \mathrm{H}), 3.66-3.62(\mathrm{~m}, 2 \mathrm{H}), 2.40(\mathrm{~s}, 3 \mathrm{H}), 0.98-0.94(\mathrm{~m}, 2 \mathrm{H}),-0.02(\mathrm{~s}, 9 \mathrm{H})$ ppm. $\delta_{\mathrm{C}}\left(100 \mathrm{MHz}, \mathrm{CDCl}_{3}\right) 188.41,143.72,137.99,137.58,131.26$, $131.07,130.51,130.11,125.27,124.85,67.16,20.34,18.06,0.37,-1.29$ (3C) ppm. HRMS (EI, $\mathrm{m} / \mathrm{z}$ ) calcd for $\mathrm{C}_{17} \mathrm{H}_{24} \mathrm{~N}_{2} \mathrm{O}_{2} \mathrm{Si}$ : 316.1607 , found: 316.1605 . 
(2-Fluorophenyl)(1-methyl-1H-imidazol-2-yl)methanone (1f): Off-white solid; Yield: 90.5<smiles></smiles>

$\%$; mp. 105-106 ${ }^{\circ} \mathrm{C} ; R_{f}=0.27$ (50\% ethyl acetate-hexane). $\delta_{\mathrm{H}}(400 \mathrm{MHz}$, $\left.\mathrm{CDCl}_{3}\right)$ 7.76-7.71 (m, 1H), 7.53-7.47 (m, 1H), 7.27-7.22 (m, 2H), 7.18$7.13(\mathrm{~m}, 1 \mathrm{H}), 7.12(\mathrm{~s}, 1 \mathrm{H}), 4.12(\mathrm{~s}, 3 \mathrm{H}) \mathrm{ppm} . \delta_{\mathrm{C}}\left(100 \mathrm{MHz}, \mathrm{CDCl}_{3}\right)$ 182.93, $160.44(\mathrm{~d}, J=252.0 \mathrm{~Hz}), 143.09,133.09$ (d, $J=8.7 \mathrm{~Hz}), 131.12(\mathrm{~d}, J=$ $1.9 \mathrm{~Hz}), 130.10,127.48,127.01$ (d, $J=12.4 \mathrm{~Hz}), 123.77$ (d, $J=3.9 \mathrm{~Hz}), 116.28$ (d, $J=22.1$ $\mathrm{Hz}), 36.25$ ppm. $\delta_{\mathrm{F}}\left(376 \mathrm{MHz}, \mathrm{CDCl}_{3}\right)-112.63 \mathrm{ppm}$. HRMS $\left(\mathrm{EI}^{+}, \mathrm{m} / \mathrm{z}\right)$ calcd for $\mathrm{C}_{11} \mathrm{H}_{9} \mathrm{FN}_{2} \mathrm{O}$ : 204.0699, found: 204.0696 .

(1-Methyl-1H-imidazol-2-yl)(phenyl)methanone (1g): Reddish oil; Yield: 91\%; $R_{f}=0.41$<smiles>Cn1ccnc1C(=O)c1ccccc1</smiles>
(50\% ethyl acetate-hexane). $\delta_{\mathrm{H}}\left(400 \mathrm{MHz}, \mathrm{CDCl}_{3}\right) 8.29-8.25(\mathrm{~m}, 2 \mathrm{H}), 7.60-$ $7.55(\mathrm{~m}, 1 \mathrm{H}), 7.51-7.46(\mathrm{~m}, 2 \mathrm{H}), 7.24(\mathrm{~d}, J=0.9 \mathrm{~Hz}, 1 \mathrm{H}), 7.12(\mathrm{~d}, J=0.4$ $\mathrm{Hz}, 1 \mathrm{H}), 4.09(\mathrm{~s}, 3 \mathrm{H}) \mathrm{ppm} . \delta_{\mathrm{C}}\left(100 \mathrm{MHz}, \mathrm{CDCl}_{3}\right) 184.41,143.30,137.46$, 132.83, 130.91 (2C), 129.47, 128.26 (2C), 126.95, 36.61 ppm. HRMS (CI', $\mathrm{m} / \mathrm{z}$ ) calcd for $\mathrm{C}_{11} \mathrm{H}_{11} \mathrm{~N}_{2} \mathrm{O}^{+}[\mathrm{M}+\mathrm{H}]^{+}:$187.0871, found: 187.0871 .

(1-Methyl-1H-imidazol-2-yl)(m-tolyl)methanone (1h): Yellowish liquid; Yield: 79\%; $R_{f}=$

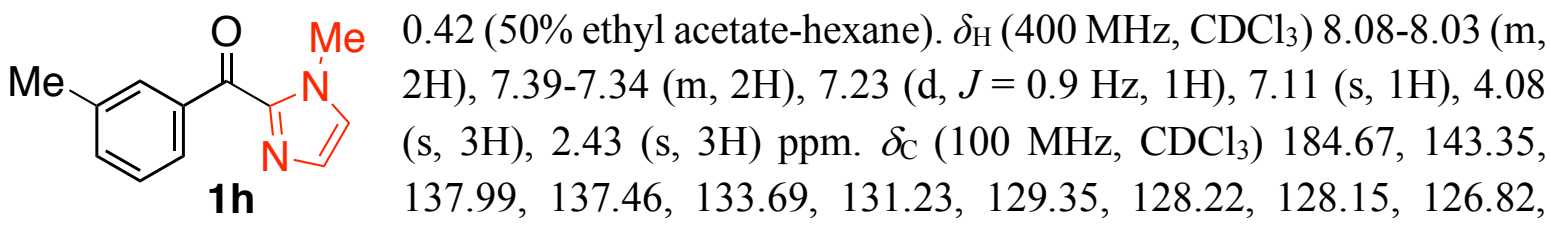
36.54, $21.58 \mathrm{ppm}$. HRMS (EI ${ }^{+}, \mathrm{m} / \mathrm{z}$ ) calcd for $\mathrm{C}_{12} \mathrm{H}_{12} \mathrm{~N}_{2} \mathrm{O}: 200.0950$, found: 200.0947.

(3-Methoxyphenyl)(1-methyl-1H-imidazol-2-yl)methanone (1i): Colorless thick oil; Yield:

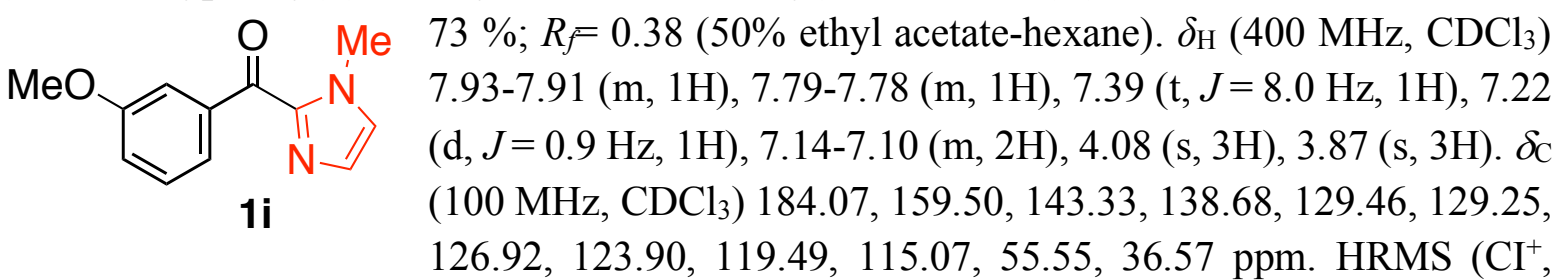
$\mathrm{m} / \mathrm{z}$ ) calcd for $\mathrm{C}_{12} \mathrm{H}_{13} \mathrm{~N}_{2} \mathrm{O}_{2}{ }^{+}[\mathrm{M}+\mathrm{H}]^{+}: 217.0977$, found: 217.0975 .

(1-Methyl-1H-imidazol-2-yl)(3-(trifluoromethyl)phenyl)methanone (1j): Reddish yellow oil; Yield: $92 \% ; R_{f}=0.50$ (50\% ethyl acetate-hexane). $\delta_{\mathrm{H}}(400$<smiles>Cn1ccnc1C(=O)c1cccc(C(F)(F)F)c1</smiles>
$\left.\mathrm{MHz}, \mathrm{CDCl}_{3}\right) 8.58(\mathrm{~s}, 1 \mathrm{H}), 8.54(\mathrm{~d}, J=8 \mathrm{~Hz}, 1 \mathrm{H}), 7.82(\mathrm{~d}, J=7.8$ $\mathrm{Hz}, 1 \mathrm{H}), 7.62$ (t, $J=7.78 \mathrm{~Hz}, 1 \mathrm{H}), 7.26(\mathrm{~s}, 1 \mathrm{H}), 7.14(\mathrm{~s}, 1 \mathrm{H}), 4.11$ $(\mathrm{s}, 3 \mathrm{H}) \mathrm{ppm} . \delta_{\mathrm{C}}\left(100 \mathrm{MHz}, \mathrm{CDCl}_{3}\right) 182.42,142.70,137.92,134.23$, 130.61 (qt, $J=32.6), 129.77,129.01(\mathrm{~d}, J=2.88 \mathrm{~Hz}), 128.70,127.72$ (qt, $J=3.83 \mathrm{~Hz}), 127.46,123.94(\mathrm{qt}, J=270.2 \mathrm{~Hz}), 36.60 \mathrm{ppm} . \delta_{\mathrm{F}}\left(376 \mathrm{MHz}, \mathrm{CDCl}_{3}\right)-62.43$ ppm. HRMS $\left(\mathrm{CI}^{+}, \mathrm{m} / \mathrm{z}\right)$ calcd for $\mathrm{C}_{12} \mathrm{H}_{10} \mathrm{~F}_{3} \mathrm{~N}_{2} \mathrm{O}^{+}[\mathrm{M}+\mathrm{H}]^{+}: 255.0745$, found: 255.0744 . 
(1-Methyl-1H-imidazol-2-yl)(naphthalen-1-yl)methanone (1k): White crystalline solid;<smiles>Cn1ccnc1C(=O)c1cccc2ccccc12</smiles>

$1 \mathrm{k}$ Yield: $69 \%$; mp. $129-130{ }^{\circ} \mathrm{C} ; R_{f}=0.27$ (50\% ethyl acetate-hexane). $\delta_{\mathrm{H}}$ $\left(400 \mathrm{MHz}, \mathrm{CDCl}_{3}\right)$ 8.28-8.26 (m, 1H), 8.01-7.96 (m, 2H), 7.91-7.88 (m, $1 \mathrm{H}), 7.56-7.48(\mathrm{~m}, 3 \mathrm{H}), 7.21(\mathrm{~d}, J=0.9 \mathrm{~Hz}, 1 \mathrm{H}), 7.16(\mathrm{~s}, 1 \mathrm{H}), 4.21(\mathrm{~s}$, $3 \mathrm{H})$ ppm. $\delta_{\mathrm{C}}\left(100 \mathrm{MHz}, \mathrm{CDCl}_{3}\right) 187.17,144.13,135.20,133.95,132.05$, $131.10,129.97,129.93,128.58,127.35$ (2C), 126.27, 125.45, 124.38, $36.68 \mathrm{ppm}$. HRMS $\left(\mathrm{EI}^{+}, \mathrm{m} / \mathrm{z}\right)$ calcd for $\mathrm{C}_{15} \mathrm{H}_{12} \mathrm{~N}_{2} \mathrm{O}: 236.0950$, found: 236.0950 .

(1-Methyl-1H-imidazol-2-yl)(4-(trifluoromethoxy)phenyl)methanone (11): Colorless<smiles>Cn1ccnc1C(=O)c1ccc(OC(F)(F)F)cc1</smiles>
thick oil; Yield: $87 \% ; R_{f}=0.50$ (50\% ethyl acetate-hexane). $\delta_{\mathrm{H}}(400$ $\left.\mathrm{MHz}, \mathrm{CDCl}_{3}\right)$ 8.40-8.35 (m, 2H), 7.33-7.29 (m, 2H), $7.24(\mathrm{~d}, J=$ $0.9 \mathrm{~Hz}, 1 \mathrm{H}), 7.14(\mathrm{~d}, J=0.4 \mathrm{~Hz}, 1 \mathrm{H}), 4.10(\mathrm{~s}, 3 \mathrm{H}) \mathrm{ppm} . \delta_{\mathrm{C}}(100$ $\left.\mathrm{MHz}, \mathrm{CDCl}_{3}\right) 182.47,152.39,142.89,135.65,132.94$ (2C), $129.60,127.27,120.43(\mathrm{qt}, J=256.8 \mathrm{~Hz}), 120.04(2 \mathrm{C}), 36.63 \mathrm{ppm} . \delta_{\mathrm{F}}\left(376 \mathrm{MHz}, \mathrm{CDCl}_{3}\right)-$ $57.21 \mathrm{ppm}$. HRMS $\left(\mathrm{EI}^{+}, \mathrm{m} / \mathrm{z}\right)$ calcd for $\mathrm{C}_{12} \mathrm{H}_{9} \mathrm{~F}_{3} \mathrm{~N}_{2} \mathrm{O}_{2}: 270.0616$, found: 270.0618 .

Methyl 4-(1-methyl-1H-imidazole-2-carbonyl)benzoate (1m): White powder; Yield: 85\%;<smiles>COC(=O)c1ccc(C(=O)c2nccn2C)cc1</smiles>
mp. $149-150{ }^{\circ} \mathrm{C} ; R_{f}=0.46\left(50 \%\right.$ ethyl acetate-hexane). $\delta_{\mathrm{H}}(400$ $\left.\mathrm{MHz}, \mathrm{CDCl}_{3}\right)$ 8.34-8.30 (m, 2H), 8.16-8.12 (m, 2H), $7.26(\mathrm{~d}, J=$ $0.8 \mathrm{~Hz}, 1 \mathrm{H}), 7.15(\mathrm{~s}, 1 \mathrm{H}), 4.11(\mathrm{~s}, 3 \mathrm{H}), 3.96(\mathrm{~s}, 3 \mathrm{H}) \mathrm{ppm} . \delta_{\mathrm{C}}(100$ $\left.\mathrm{MHz}, \mathrm{CDCl}_{3}\right) \quad 183.57, \quad 166.64,143.02,141.09,133.38$, $130.78(2 \mathrm{C}), 129.85,129.40(2 \mathrm{C}), 127.43,52.55,36.73 \mathrm{ppm}$.

HRMS (EI ${ }^{+}, \mathrm{m} / \mathrm{z}$ ) calcd for $\mathrm{C}_{13} \mathrm{H}_{12} \mathrm{~N}_{2} \mathrm{O}_{3}: 244.0848$, found: 244.0846 .

(1-methyl-1H-imidazol-2-yl)(2-(trifluoromethyl)phenyl)methanone (1n): Colorless thick<smiles>Cn1ccnc1C(=O)c1ccccc1C(F)(F)F</smiles>
oil; Yield: $70 \% ; R_{f}=0.27\left(50 \%\right.$ ethyl acetate-hexane). $\delta_{\mathrm{H}}(400 \mathrm{MHz}$, $\left.\mathrm{CDCl}_{3}\right) 7.76-7.73(\mathrm{~m}, 1 \mathrm{H}), 7.65-7.56(\mathrm{~m}, 3 \mathrm{H}), 7.18(\mathrm{~d}, J=0.44 \mathrm{~Hz}, 1 \mathrm{H})$, $7.13(\mathrm{~s}, 1 \mathrm{H}), 4.13(\mathrm{~s}, 3 \mathrm{H}) \mathrm{ppm} . \delta_{\mathrm{C}}\left(100 \mathrm{MHz}, \mathrm{CDCl}_{3}\right)$ 185.64, 142.91, 1n $137.82,131.22,130.39,130.09,129.07,127.96$ (qt, $J=31.6 \mathrm{~Hz}), 127.78$, $126.63(\mathrm{qt}, J=4.79 \mathrm{~Hz}), 123.80(\mathrm{qt}, J=272.2 \mathrm{~Hz}), 36.28 \mathrm{ppm} . \delta_{\mathrm{F}}\left(376 \mathrm{MHz}, \mathrm{CDCl}_{3}\right)-57.73$ ppm. HRMS $\left(\mathrm{CI}^{+}, \mathrm{m} / \mathrm{z}\right)$ calcd for $\mathrm{C}_{12} \mathrm{H}_{10} \mathrm{~F}_{3} \mathrm{~N}_{2} \mathrm{O}^{+}[\mathrm{M}+\mathrm{H}]^{+}: 255.0745$, found: 255.0741 .

(3,4-Dichlorophenyl)(1-methyl-1H-imidazol-2-yl)methanone (10): Yield: 59\%; white<smiles>Cn1ccnc1C(=O)c1ccc(Cl)c(Cl)c1</smiles>
crystalline solid; $\mathrm{mp} .79-80{ }^{\circ} \mathrm{C} ; R_{f}=0.54$ (50\% ethyl acetate-hexane). $\delta_{\mathrm{H}}\left(400 \mathrm{MHz}, \mathrm{CDCl}_{3}\right) 8.46(\mathrm{~d}, J=2.08 \mathrm{~Hz}, 1 \mathrm{H}), 8.20(\mathrm{dd}, J=8.48$, $2.08 \mathrm{~Hz}, 1 \mathrm{H}), 7.56(\mathrm{~d}, J=8.44 \mathrm{~Hz}, 1 \mathrm{H}), 7.24(\mathrm{~d}, J=0.88 \mathrm{~Hz}, 1 \mathrm{H})$, $7.14(\mathrm{~d}, J=0.48 \mathrm{~Hz}, 1 \mathrm{H}), 4.08(\mathrm{~s}, 3 \mathrm{H}) \mathrm{ppm} . \delta_{\mathrm{C}}\left(100 \mathrm{MHz}, \mathrm{CDCl}_{3}\right)$ $181.20,142.51,137.17,136.79,132.79,132.52$, 130.13, 129.99, 129.67, 127.38, 36.61 ppm. HRMS (EI ${ }^{+}, \mathrm{m} / \mathrm{z}$ ) calcd for $\mathrm{C}_{11} \mathrm{H}_{8} \mathrm{Cl}_{2} \mathrm{~N}_{2} \mathrm{O}: 254.0014$, found: 254.0012 . 
(1- Methyl-1H-imidazol-2-yl)(1-methyl-1H-pyrrol-2-yl)methanone (1p): White solid Me O Me (purified by GPC); Yield: 36\%; mp. 102-103 ${ }^{\circ} \mathrm{C} R_{f}=0.25(50 \%$ ethyl N $7.15(\mathrm{~d}, J=0.9 \mathrm{~Hz}, 1 \mathrm{H}), 7.02(\mathrm{~s}, 1 \mathrm{H}), 6.90-6.89(\mathrm{~m}, 1 \mathrm{H}), 6.20-6.18(\mathrm{~m}$, $1 p$ $1 \mathrm{H}), 4.01(\mathrm{~s}, 6 \mathrm{H}) \mathrm{ppm} . \delta_{\mathrm{C}}\left(100 \mathrm{MHz}, \mathrm{CDCl}_{3}\right) 174.30,144.08,132.15$, $130.51,128.67,125.85,124.81,108.88,37.78,36.00$ ppm. HRMS $\left(\mathrm{EI}^{+}\right.$, $\mathrm{m} / \mathrm{z}$ ) calcd for $\mathrm{C}_{10} \mathrm{H}_{11} \mathrm{~N}_{3} \mathrm{O}: 189.0902$, found: 189.0902 .

Furan-2-yl(1-methyl-1H-imidazol-2-yl)methanone (1q): Off-white solid (purified by<smiles>Cn1ccnc1C(=O)c1ccco1</smiles>

$1 q$ GPC); Yield: $47 \%$; mp. $69-70{ }^{\circ} \mathrm{C} . R_{f}=0.32\left(50 \%\right.$ ethyl acetate-hexane). $\delta_{\mathrm{H}}$ $\left(400 \mathrm{MHz}, \mathrm{CDCl}_{3}\right) 8.04(\mathrm{dd}, J=3.68,0.68 \mathrm{~Hz}, 1 \mathrm{H}), 7.73-7.71(\mathrm{~m}, 1 \mathrm{H})$, $7.21(\mathrm{~d}, J=0.88 \mathrm{~Hz}, 1 \mathrm{H}), 7.09(\mathrm{~d}, J=0.44 \mathrm{~Hz}, 1 \mathrm{H}), 6.61-6.59(\mathrm{~m}, 1 \mathrm{H})$, $4.08(\mathrm{~s} 3 \mathrm{H}) \mathrm{ppm} . \delta_{\mathrm{C}}\left(100 \mathrm{MHz}, \mathrm{CDCl}_{3}\right) 170.75,151.26,147.49,142.05$, $129.41,126.86,123.10,112.41,36.31 \mathrm{ppm}$.

HRMS (EI+ ${ }^{+}, \mathrm{z}$ ) calcd for $\mathrm{C}_{9} \mathrm{H}_{8} \mathrm{~N}_{2} \mathrm{O}: 176.0586$, found: 176.0583 .

Benzofuran-2-yl(1-methyl-1H-imidazol-2-yl)methanone (1r): White solid (purified by<smiles>Cn1ccnc1C(=O)c1cc2ccccc2o1</smiles>
GPC); Yield: $73 \%$; mp. $145-146{ }^{\circ} \mathrm{C} . R_{f}=0.39$ (50\% ethyl acetatehexane). $\delta_{\mathrm{H}}\left(400 \mathrm{MHz}, \mathrm{CDCl}_{3}\right) 8.50(\mathrm{~d}, J=1.1 \mathrm{~Hz}, 1 \mathrm{H}), 7.77-7.74(\mathrm{~m}$, $1 \mathrm{H}), 7.65-7.62(\mathrm{~m}, 1 \mathrm{H}), 7.50-7.46(\mathrm{~m}, 1 \mathrm{H}), 7.33-7.29(\mathrm{~m}, 1 \mathrm{H}), 7.26(\mathrm{~s}$. $1 \mathrm{H}), 7.14(\mathrm{~d}, J=0.4 \mathrm{~Hz}, 1 \mathrm{H}), 4.13(\mathrm{~s}, 3 \mathrm{H}) \delta_{\mathrm{C}}\left(100 \mathrm{MHz}, \mathrm{CDCl}_{3}\right)$ $172.35,156.02,151.37,142.35,129.75,128.47,127.73,127.36$, 123.88, 123.74, 119.31, 112.55, 36.51 ppm. HRMS (EI ${ }^{+}, \mathrm{m} / \mathrm{z}$ ) calcd for $\mathrm{C}_{13} \mathrm{H}_{10} \mathrm{~N}_{2} \mathrm{O}_{2}: 226.0742$, found: 226.0739 .

Benzo $[\boldsymbol{b}]$ thiophen-2-yl(1-methyl-1H-imidazol-2-yl)methanone (1s): White powder; Yield:<smiles>Cn1ccnc1C(=O)c1cc2ccccc2s1</smiles>
$75 \%$; mp. $148-149{ }^{\circ} \mathrm{C} ; R_{f}=0.50$ (50\% ethyl acetate-hexane). $\delta_{\mathrm{H}}(400$ $\left.\mathrm{MHz}, \mathrm{CDCl}_{3}\right) 8.89(\mathrm{~d}, J=0.9 \mathrm{~Hz}, 1 \mathrm{H}), 7.96-7.93(\mathrm{~m}, 1 \mathrm{H}), 7.90-7.87$ (m, 1H), 7.48-7.37 (m, 2H), $7.29(\mathrm{~d}, J=0.9 \mathrm{~Hz}, 1 \mathrm{H}), 7.14(\mathrm{~s}, 1 \mathrm{H}), 4.11$ $(\mathrm{s}, 3 \mathrm{H}) \mathrm{ppm} . \quad \delta_{\mathrm{C}}\left(100 \mathrm{MHz}, \mathrm{CDCl}_{3}\right) 176.52,143.52,142.64,141.99$, $139.39,134.01,129.64,127.46,127.41,126.61,124.92,122.80,36.71$ ppm. HRMS (EI ${ }^{+}, \mathrm{m} / \mathrm{z}$ ) calcd for $\mathrm{C}_{13} \mathrm{H}_{10} \mathrm{~N}_{2} \mathrm{OS}$ : 242.0514, found: 242.0515.

(1-Methyl-1H-imidazol-2-yl)(2-(methyl- $\left.d_{3}\right)$ phenyl-3,4,5,6- $\left.d_{4}\right)$ methanone ([D $]_{7}-1$ a): This<smiles>[2H]c1c([2H])c([2H])c(C([2H])([2H])[3H])c(C(=O)c2nccn2C)c1[2H]</smiles>
compound was prepared from the corresponding deuterated acid. The acid was converted to acid chloride by reported method. ${ }^{1 \mathrm{~b}}$ Then, it was followed the above mention general procedure.

White crystalline solid; Yield: $52 \%$; mp. $81-82{ }^{\circ} \mathrm{C} ; R_{f}=0.39$ (50\% ethyl acetate-hexane). $\delta_{\mathrm{H}}\left(400 \mathrm{MHz}, \mathrm{CDCl}_{3}\right) 7.19(\mathrm{~d}, J=0.9 \mathrm{~Hz}, 1 \mathrm{H}), 7.10$ $(\mathrm{s}, 1 \mathrm{H}), 4.12(\mathrm{~s}, 3 \mathrm{H}) \mathrm{ppm} . \delta_{\mathrm{C}}\left(100 \mathrm{MHz}, \mathrm{CDCl}_{3}\right)$ 188.03, 143.63, 
137.79, 137.24, 130.93, 130.45 (m), 129.79, $129.67(\mathrm{~m}), 127.10,124.60$ (t, $J=99.68 \mathrm{~Hz})$, 36.42, 19.39 (m) ppm. HRMS (EI ${ }^{+}, \mathrm{m} / \mathrm{z}$ ) calcd for $\mathrm{C}_{12} \mathrm{H}_{5} \mathrm{D}_{7} \mathrm{~N}_{2} \mathrm{O}$ : 207.1389, found: 207.1386.

Procedure B (via Weinreb amides): The corresponding Weinreb amides were prepared from acids or acid chlorides and reacted with imidazole analogues as per an earlier reported method. ${ }^{2 \mathrm{c}}$

To a stirred solution of imidazole analogue (38.8 mmol, 1.0 equiv.) in dry THF, n-BuLi (1.1 equiv.) was added dropwise at $-78{ }^{\circ} \mathrm{C}$ and the reaction mixture was stirred for 30 minutes at the same temperature and for an additional $1 \mathrm{~h}$ at room temperature. The reaction was then cooled to $-78^{\circ} \mathrm{C}$ and the corresponding Weinreb amides (1.0 equiv.) in anhydrous THF was added dropwise over a period of 30 minutes. After the addition was complete, the solution was stirred for $1 \mathrm{~h}$ at the same temperature, after which, the temperature was increased to room temperature and the solution stirred overnight. After the Weinreb amides were completely consumed, the reaction was quenched by the direct addition of acetic acid (6 equiv.) at room temperature. The resulting solution was extracted with ethyl acetate, washed with saturated $\mathrm{NaHCO}_{3}$ and brine. The organic fraction was dried over $\mathrm{Na}_{2} \mathrm{SO}_{4}$ and evaporated under reduced pressure to give the crude product as an oil which was purified by silica gel column chromatography. The pure compound was eluted at a concentration of $15-20 \%$ ethyl acetate in hexane.

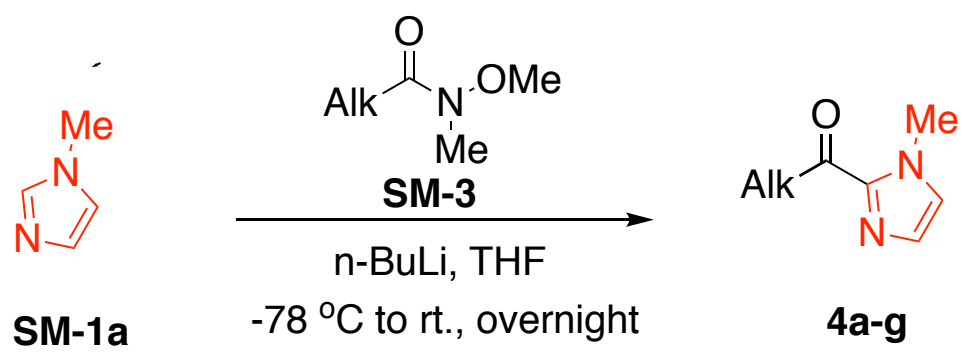

Starting materials 4a-g were prepared following the general procedure B via Weinreb amides.

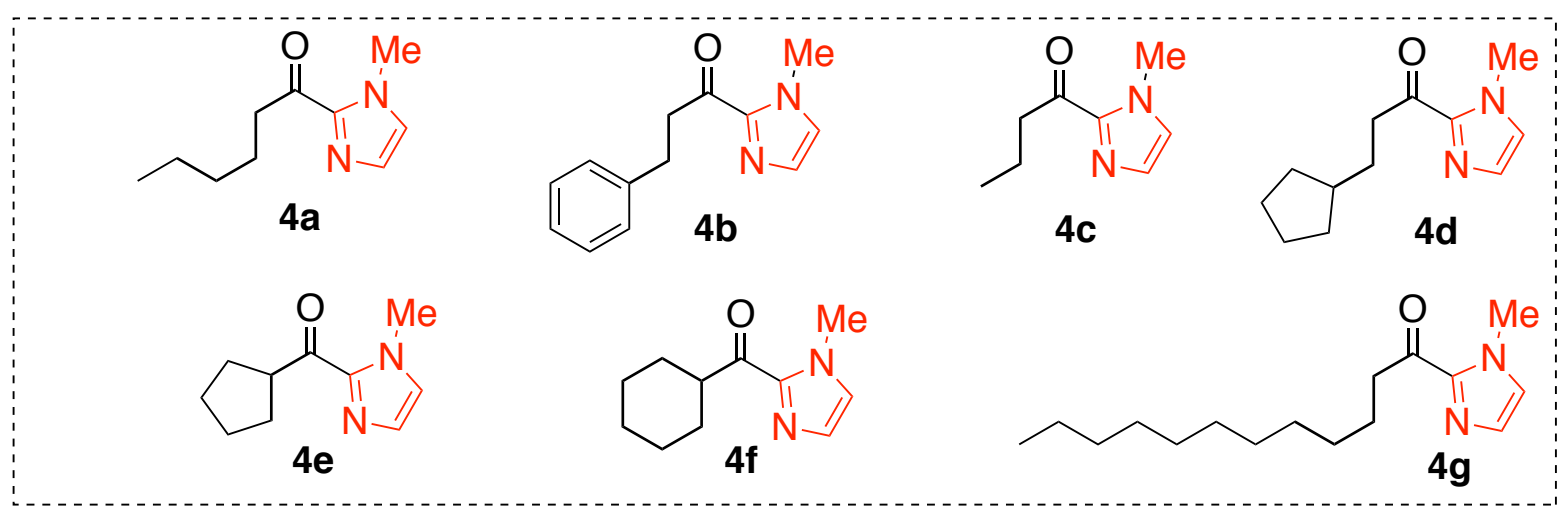

\section{Characterization of the Compounds:}

1-(1-Methyl-1H-imidazol-2-yl)hexan-1-one (4a): Colorless oil; Yield: 73\%; $R_{f}=0.43(50 \%$

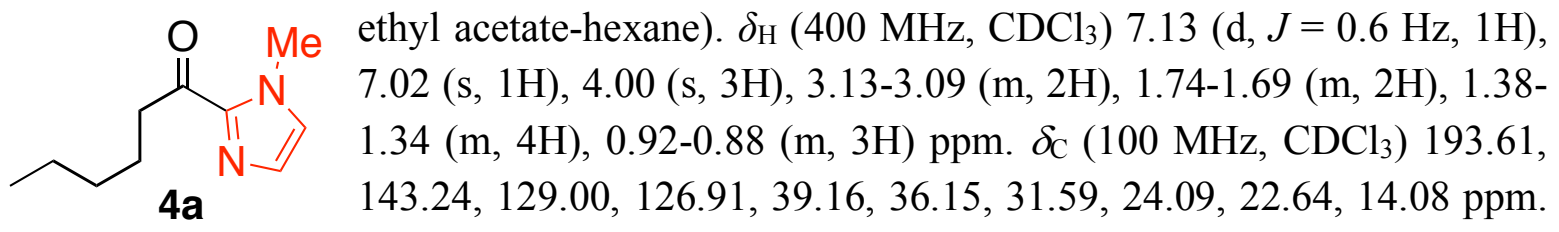
HRMS $\left(\mathrm{EI}^{+}, \mathrm{m} / \mathrm{z}\right)$ calcd for $\mathrm{C}_{10} \mathrm{H}_{16} \mathrm{~N}_{2} \mathrm{O}: 180.1263$, found: 180.1262 . 
1-(1-Methyl-1H-imidazol-2-yl)-3-phenylpropan-1-one (4b): Colorless oil; Yield: 92\%; $R_{f}=$<smiles>Cn1ccnc1C(=O)CCc1ccccc1</smiles>
$0.43\left(50 \%\right.$ ethyl acetate-hexane). $\delta_{\mathrm{H}}\left(400 \mathrm{MHz}, \mathrm{CDCl}_{3}\right) 7.27-7.24(\mathrm{~m}$, $4 \mathrm{H}), 7.22-7.15(\mathrm{~m}, 1 \mathrm{H}), 7.12-7.11(\mathrm{~m}, 1 \mathrm{H}), 7.01(\mathrm{~s}, 1 \mathrm{H}), 3.99(\mathrm{~s}, 3 \mathrm{H})$, $3.50-3.45(\mathrm{~m}, 2 \mathrm{H}), 3.06-3.02(\mathrm{~m}, 2 \mathrm{H}) \mathrm{ppm} . \delta_{\mathrm{C}}\left(100 \mathrm{MHz}, \mathrm{CDCl}_{3}\right)$ $192.13,143.03,141.19,129.15,128.57(2 \mathrm{C}), 128.48(2 \mathrm{C}), 127.02$, 126.10, 40.49, 36.29, 30.03 ppm. HRMS $\left(\mathrm{EI}^{+}, \mathrm{m} / \mathrm{z}\right)$ calcd for $\mathrm{C}_{13} \mathrm{H}_{14} \mathrm{~N}_{2} \mathrm{O}$ :

214.1106, found: 214.1105 .

1-(1-Methyl-1H-imidazol-2-yl)butan-1-one (4c): Colorless oil; Yield: $16 \% ; R_{f}=0.36(50 \%$<smiles>CCCC(=O)c1nccn1C</smiles>
ethyl acetate-hexane). $\delta_{\mathrm{H}}\left(400 \mathrm{MHz}, \mathrm{CDCl}_{3}\right) 7.13(\mathrm{~s}, 1 \mathrm{H}), 7.01(\mathrm{~s}, 1 \mathrm{H}), 4.00$ $(\mathrm{s}, 3 \mathrm{H}), 3.12-3.07(\mathrm{~m}, 2 \mathrm{H}), 1.79-1.68(\mathrm{~m}, 2 \mathrm{H}), 1.02-0.98(\mathrm{~m}, 3 \mathrm{H}) \mathrm{ppm} . \delta_{\mathrm{C}}(100$ $\left.\mathrm{MHz}, \mathrm{CDCl}_{3}\right)$ 193.47, 143.27, 129.02, 126.92, 41.13, 36.36, 17.90, 13.98 ppm. HRMS $\left(\mathrm{EI}^{+}, \mathrm{m} / \mathrm{z}\right)$ calcd for $\mathrm{C}_{8} \mathrm{H}_{12} \mathrm{~N}_{2} \mathrm{O}: 152.0950$, found: 152.0952 .

3-Cyclopentyl-1-(1-methyl-1H-imidazol-2-yl)propan-1-one (4d): Colorless oil; Yield: 93\%;<smiles>Cn1ccnc1C(=O)CCC1CCCC1</smiles>
$R_{f}=0.36$ (50\% ethyl acetate-hexane). $\delta_{\mathrm{H}}\left(400 \mathrm{MHz}, \mathrm{CDCl}_{3}\right) 7.13(\mathrm{~d}, J=$ $0.9 \mathrm{~Hz}, 1 \mathrm{H}), 7.02(\mathrm{~s}, 1 \mathrm{H}), 4.00(\mathrm{~s}, 3 \mathrm{H}), 3.16-3.11(\mathrm{~m}, 2 \mathrm{H}), 1.88-1.46(\mathrm{~m}$, $9 \mathrm{H}), 1.16-1.12(\mathrm{~m}, 2 \mathrm{H}) \mathrm{ppm} . \quad \delta_{\mathrm{C}}\left(100 \mathrm{MHz}, \mathrm{CDCl}_{3}\right)$ 193.71, 143.24, 129.02, 126.92, 39.92, 38.53, 36.37, 32.73 (2C), 30.54, 25.34 (2C) ppm. HRMS (EI, $\mathrm{m} / \mathrm{z}$ ) calcd for $\mathrm{C}_{12} \mathrm{H}_{18} \mathrm{~N}_{2} \mathrm{O}: 206.1419$, found: 206.1422 .

Cyclopentyl(1-methyl-1H-imidazol-2-yl)methanone (4e): Colorless oil; Yield: $89 \%$; $R_{f}=$<smiles>Cn1ccnc1C(=O)C1CCCC1</smiles>

$4 \mathrm{e}$ $0.43\left(50 \%\right.$ ethyl acetate-hexane). $\delta_{\mathrm{H}}\left(400 \mathrm{MHz}, \mathrm{CDCl}_{3}\right) 7.14(\mathrm{~s}, 1 \mathrm{H}), 7.01$ $(\mathrm{s}, 1 \mathrm{H}), 4.13-4.04(\mathrm{~m}, 1 \mathrm{H}), 4.00(\mathrm{~s}, 3 \mathrm{H}), 2.01-1.92(\mathrm{~m}, 2 \mathrm{H}), 1.86-1.61(\mathrm{~m}$, $6 \mathrm{H})$ ppm. $\delta_{\mathrm{C}}\left(100 \mathrm{MHz}, \mathrm{CDCl}_{3}\right)$ 196.13, 143.29, 129.03, 126.97, 47.16, $36.41,30.33(2 \mathrm{C}), 26.43(2 \mathrm{C}) \mathrm{ppm}$. HRMS $\left(\mathrm{EI}^{+}, \mathrm{m} / \mathrm{z}\right)$ calcd for $\mathrm{C}_{10} \mathrm{H}_{14} \mathrm{~N}_{2} \mathrm{O}$ : 178.1106, found: 178.1103 .

Cyclohexyl(1-methyl-1H-imidazol-2-yl)methanone (4f): Colorless oil; Yield: $72 \% ; R_{f}=0.43$<smiles>Cn1ccnc1C(=O)C1CCCCC1</smiles>
$\left(50 \%\right.$ ethyl acetate-hexane). $\delta_{\mathrm{H}}\left(400 \mathrm{MHz}, \mathrm{CDCl}_{3}\right) 7.14(\mathrm{~s}, 1 \mathrm{H}), 7.02(\mathrm{~d}, J$ $=0.4 \mathrm{~Hz}, 1 \mathrm{H}), 3.99(\mathrm{~s}, 3 \mathrm{H}), 3.71-3.65(\mathrm{~m}, 1 \mathrm{H}), 1.96-1.90(\mathrm{~m}, 2 \mathrm{H}), 1.83-$ $1.77(\mathrm{~m}, 2 \mathrm{H}), 1.75-1.70(\mathrm{~m}, 1 \mathrm{H}), 1.50-1.37(\mathrm{~m}, 4 \mathrm{H}), 1.30-1.19(\mathrm{~m}, 1 \mathrm{H})$ ppm. $\delta_{\mathrm{C}}\left(100 \mathrm{MHz}, \mathrm{CDCl}_{3}\right) 196.70,142.66,129.00,127.12,46.10,36.46$, 29.23 (2C), 26.10, 25.82 (2C) ppm. HRMS (EI, $\mathrm{m} / \mathrm{z})$ calcd for $\mathrm{C}_{11} \mathrm{H}_{16} \mathrm{~N}_{2} \mathrm{O}: 192.1263$, found: 192.1262 .

1-(1-Methyl-1H-imidazol-2-yl)dodecan-1-one (4g): Colorless thick oil; Yield: 94\%; $R_{f}=$

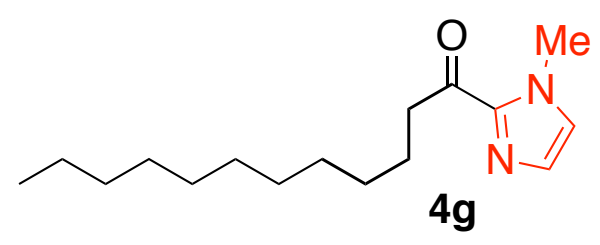
$0.50\left(50 \%\right.$ ethyl acetate-hexane). $\delta_{\mathrm{H}}\left(400 \mathrm{MHz}, \mathrm{CDCl}_{3}\right)$ $7.13(\mathrm{~d}, J=1.2 \mathrm{~Hz}, 1 \mathrm{H}), 7.01(\mathrm{~d}, J=0.4 \mathrm{~Hz}, 1 \mathrm{H}), 4.00$ (s, 3H), 3.13-3.09 (m, 2H), 1.74-1.66 (m, 2H), 1.43-1.25 $(\mathrm{m}, 16 \mathrm{H}), 0.90-0.86(\mathrm{~m}, 3 \mathrm{H}) \mathrm{ppm} . \delta_{\mathrm{C}}\left(100 \mathrm{MHz}, \mathrm{CDCl}_{3}\right)$ $193.62,143.22,128.99,126.91,39.21,36.37,32.05$, 
29.75 (2C), 29.64, 29.59, 29.47, 29.44, 24.82, 22.82, 14.26 ppm. HRMS (EI ${ }^{+}, \mathrm{m} / \mathrm{z}$ ) calcd for $\mathrm{C}_{16} \mathrm{H}_{28} \mathrm{~N}_{2} \mathrm{O}: 264.2202$, found: 264.2200 .

\section{B. Synthesis of coupling partner}

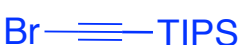

$2 a$

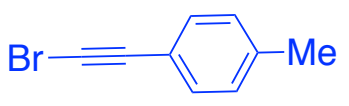

$2 b$

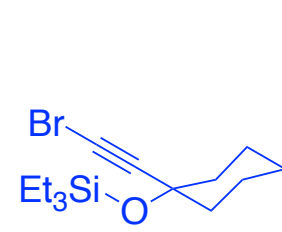

2c

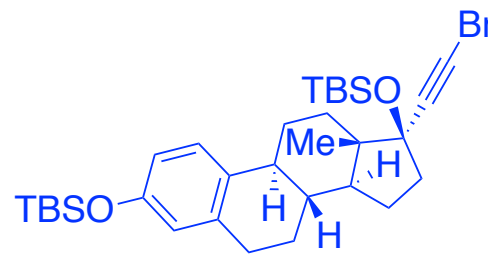

2d

Synthesis of alkynyl bromides (2a and $2 b)$ from alkynes: ${ }^{2}$ To a stirred solution of the alkyne $(15 \mathrm{mmol})$ in acetone $(50 \mathrm{~mL}), \mathrm{N}$-bromosuccinimide $(3.2 \mathrm{gm}, 18 \mathrm{mmol})$ and silver nitrate $(252$ $\mathrm{mg}, 1.5 \mathrm{mmol})$ were added and the reaction mixture was stirred for 3 hours at room temperature. The reaction was then quenched with cold water $(50 \mathrm{~mL})$ and extracted with $\mathrm{n}$ pentane $\left(100 \mathrm{~mL}\right.$ x 3). The combined organic fractions were dried over anhydrous $\mathrm{Na}_{2} \mathrm{SO}_{4}$ and concentrated under reduced pressure. The crude product was purified by silica gel column chromatography ( $0.5 \%$ ethyl acetate in hexane) to give the desired alkynyl bromide.

\section{Characterization of the Compounds:}

(Bromoethynyl)triisopropylsilane (2a): ${ }^{2 a}$ Colorless liquid; Yield: 93\%; $R_{f}=0.92(10 \%$ ethyl $\mathrm{Br}=$-TIPS acetate-hexane). $\delta_{\mathrm{H}}\left(400 \mathrm{MHz}, \mathrm{CDCl}_{3}\right) 1.08-1.05(\mathrm{~m}, 21 \mathrm{H}) \mathrm{ppm} . \delta_{\mathrm{C}}(100$ 2a $\left.\mathrm{MHz}, \mathrm{CDCl}_{3}\right)$ 83.62, 61.86, 18.63, $11.41 \mathrm{ppm}$. The spectroscopic properties of this compound were consistent with previously reported data.

1-(Bromoethynyl)-4-methylbenzene (2b): ${ }^{2 b}$ Light yellowish liquid; Yield: 84\%; $R_{f}=$

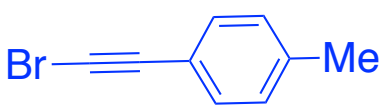

2b $0.81(10 \%$ ethyl acetate-hexane $) . \delta_{\mathrm{H}}\left(400 \mathrm{MHz}, \mathrm{CDCl}_{3}\right) 7.35-7.32(\mathrm{~m}$, $2 \mathrm{H}), 7.12-7.09(\mathrm{~m}, 2 \mathrm{H}), 2.34(\mathrm{~s}, 3 \mathrm{H}) \mathrm{ppm} . \delta_{\mathrm{C}}\left(100 \mathrm{MHz}, \mathrm{CDCl}_{3}\right)$ 139.03, 132.02 (2C), 129.24 (2C), 119.78, 80.29, 48.88, $21.67 \mathrm{ppm}$.

The spectroscopic properties of this compound were consistent with previously reported data.

Synthesis of ((1-(bromoethynyl)cyclohexyl)oxy)triethylsilane $\quad(2 \mathrm{c}) \mathbf{:}^{2 \mathrm{a}, 2 \mathrm{c}} \quad 1-$ Ethynylcyclohexan-1-ol (a) protected by triethyl silane according to reported method and

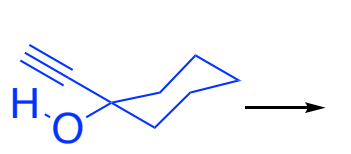

a

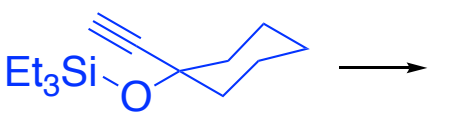

b

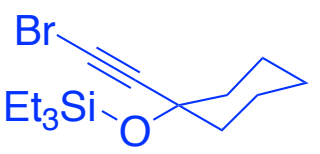

2c characterized by spectral analyses and the spectroscopic data of intermediate (b) is consistent with previously reported data. ${ }^{2 \mathrm{c}}$ Colorless liquid; Yield: $73 \% ; R_{f}=0.8$ (10\% ethyl<smiles>C#CC1(O[Si]C)CCCCC1</smiles>
acetate-hexane; UV inactive, visualization through $\mathrm{I}_{2}$ vapor). $\delta_{\mathrm{H}}(400$ $\left.\mathrm{MHz}, \mathrm{CDCl}_{3}\right) 2.45(\mathrm{~s}, 1 \mathrm{H}), 1.84-1.80(\mathrm{~m}, 2 \mathrm{H}), 1.69-1.42(\mathrm{~m}, 8 \mathrm{H}), 1.05-$ $0.91(\mathrm{~m}, 9 \mathrm{H}), 0.75-0.61(\mathrm{~m}, 6 \mathrm{H}) \delta_{\mathrm{C}}\left(100 \mathrm{MHz}, \mathrm{CDCl}_{3}\right) 88.25,72.74$, 69.20, 41.11(2C), 25.22, 22.89 (2C), 7.03 (3C), 6.19 (3C) ppm. 
The intermediate (b) was brominated as per above mention procedure. Colorless liquid; Yield: $89 \% ; R_{f}=0.8$ ( $10 \%$ ethyl acetate-hexane; slightly UV active, visualization through $\mathrm{I}_{2}$ vapor).

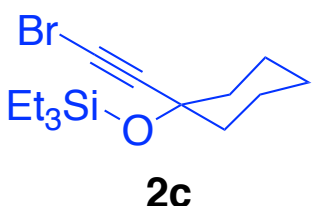

Synthesis $\delta_{\mathrm{H}}\left(400 \mathrm{MHz}, \mathrm{CDCl}_{3}\right) 1.84-1.79(\mathrm{~m}, 2 \mathrm{H}), 1.66-1.44(\mathrm{~m}, 7 \mathrm{H}), 1.30-1.21$ $(\mathrm{m}, 1 \mathrm{H}), 0.99-0.93(\mathrm{~m}, 9 \mathrm{H}), 0.68-0.62(\mathrm{~m}, 6 \mathrm{H}) \mathrm{ppm} . \delta_{\mathrm{C}}(100 \mathrm{MHz}$, $\left.\mathrm{CDCl}_{3}\right)$ 84.45, 70.62, 44.38, 41.20 (2C), 25.35, 23.07 (2C), 7.18 (3C), 6.27(3C) ppm. HRMS $\left(\mathrm{CI}^{+}, \mathrm{m} / \mathrm{z}\right)$ calcd for $\mathrm{C}_{14} \mathrm{H}_{26} \mathrm{BrOSi}^{+}[\mathrm{M}+\mathrm{H}]^{+}$: 317.0936, found: 317.0932 .

\section{$7,8,9,11,12,13,14,15,16,17-d e c a h y d r o-6 H$-cyclopenta[a]phenanthrene-3,17-}

diyl)bis(oxy))bis(tert-butyldimethylsilane) (2d) as reported by $\mathbf{Y u}$ et $a \boldsymbol{l}^{\mathrm{dd}}$ : White solid (purified by GPC). Yield: $32 \%$; mp. $127-128{ }^{\circ} \mathrm{C} ; R_{f}=0.9\left(10 \%\right.$ ethyl acetate-hexane). $\delta_{\mathrm{H}}(400$ $\left.\mathrm{MHz}, \mathrm{CDCl}_{3}\right) 7.13(\mathrm{~d}, J=8.24 \mathrm{~Hz}, 1 \mathrm{H}), 6.61(\mathrm{dd}, J=8.35$,

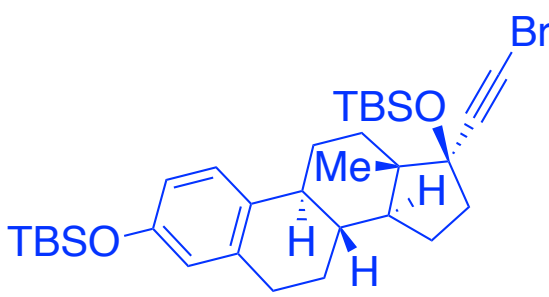

2d $2.63 \mathrm{~Hz}, 1 \mathrm{H}), 6.55(\mathrm{~d}, J=2.76 \mathrm{~Hz}, 1 \mathrm{H}), 2.81-2.78(\mathrm{~m}$, 2H), 2.24- $2.19(\mathrm{~m}, 3 \mathrm{H}), 1.92-1.34(\mathrm{~m}, 10 \mathrm{H}), 0.97(\mathrm{~s}$, 9H), 0.88 (s, 9H), $0.82(\mathrm{~s}, 3 \mathrm{H}), 0.19-0.14(\mathrm{~m}, 12 \mathrm{H}) \mathrm{ppm}$. $\delta_{\mathrm{C}}\left(100 \mathrm{MHz}, \mathrm{CDCl}_{3}\right) 153.22,137.86,133.14,126.17$, $119.88,117.09,84.23,81.84,48.53,48.45,45.53,43.64$, $39.98,39.42,33.03,29.69,27.29,26.37,25.78,25.70$, 23.10, 18.21, 18.16, 13.14, -3.12 (2C), -3.27(3C), -4.39(3C) HRMS $\left(\mathrm{EI}^{+}, \mathrm{m} / \mathrm{z}\right)$ calcd for $\mathrm{C}_{32} \mathrm{H}_{51} \mathrm{BrO}_{2} \mathrm{Si}: 602.2611$, found: 602.2607 . 


\section{Optimization studies}

Optimization for $\mathbf{C}\left(\mathbf{s p}^{2}\right)-\mathrm{H}$ Alkynylation

Table S1. Solvent Optimization

$\mathrm{Br}=-\mathrm{TIPS} 2 \mathrm{2a}$ (1.1 equiv)<smiles>Cc1ccccc1C(=O)c1nccn1C</smiles>

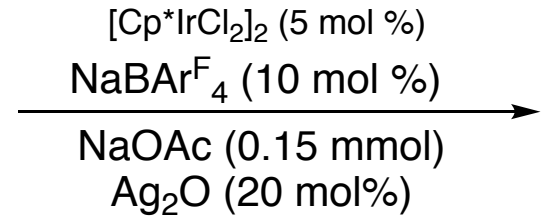

1a $0.1 \mathrm{mmol}$ Solvent $(1 \mathrm{~mL}), 130^{\circ} \mathrm{C}, 17 \mathrm{~h}$<smiles>Cc1cccc(C#CC=S)c1C(=O)c1nccn1C</smiles>

\begin{tabular}{|c|c|c|c|c|}
\hline \multirow{2}{*}{ Entry } & \multirow{2}{*}{ Solvents } & \multirow{2}{*}{ Temperature $\left({ }^{\circ} \mathrm{C}\right)$} & \multicolumn{2}{|c|}{ NMR Yields $(\%)^{2}$} \\
\hline & & & $3 \mathbf{a a}$ & $1 \mathrm{a}$ \\
\hline 1 & DCE & $130(42 \mathrm{~h})$ & $(73)^{b}$ & - \\
\hline 2 & DCE & $130(20 \mathrm{~h})$ & $94(81)^{\mathrm{b}}$ & - \\
\hline 3 & 1,4-Dioxane & 130 & 88 & - \\
\hline 4 & DMF & 130 & - & 86 \\
\hline 5 & Toluene & 130 & 83 & - \\
\hline 6 & Chlorobenzene & 130 & 88.6 & - \\
\hline 7 & Acetonitrile & 100 & - & 92.9 \\
\hline 8 & NMP & 130 & - & 70.4 \\
\hline 9 & DMSO & 130 & - & 94.2 \\
\hline 10 & HFIP & 130 & 60 & - \\
\hline 11 & Acetic acid & 130 & 17.3 & 66.3 \\
\hline 12 & 4-Methyloxane & 130 & 89 & 9 \\
\hline 13 & DCE & 100 & 98 & - \\
\hline
\end{tabular}

${ }^{a}$ Yields are calculated based on ${ }^{1} \mathrm{H}$ NMR spectroscopy using 1,1,2,2tertachloroethane as an internal standard. ${ }^{\mathrm{b}}$ Isolated yield.

Table S2. Catalyst Loading

$\mathrm{Br}=$ TIPS 2a (1.1 equiv)<smiles>Cc1ccccc1C(=O)c1nccn1C</smiles>

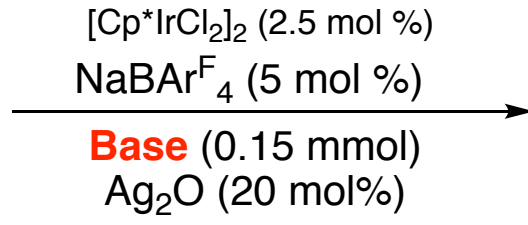

1a $0.1 \mathrm{mmol}$

DCE (1mL), $130^{\circ} \mathrm{C}, 17 \mathrm{~h}$<smiles>Cc1cccc(C#CC(C)C)c1C(=O)c1nccn1C</smiles>

\begin{tabular}{cccc}
\hline \multirow{2}{*}{ Entry } & Catalyst $(\mathbf{X}$ mol\%) & \multicolumn{2}{c}{ NMR Yields (\%) } \\
& & 3aa & 1a \\
\hline 1 & {$\left[\mathrm{Cp}^{*} \mathrm{IrCl}_{2}\right]_{2}(5)$} & 98 & - \\
$\mathbf{2}$ & {$\left[\mathrm{Cp}^{*} \mathrm{IrCl}_{2}\right]_{2}(\mathbf{2 . 5})$} & $\mathbf{9 6 . 4}$ & - \\
3 & {$\left[\mathrm{Cp}^{*} \mathrm{IrCl}_{2}\right]_{2}(1.25)$} & 88 & 11 \\
\hline
\end{tabular}


Table S3. Base Optimization

$$
\mathrm{Br}=\text { TIPS 2a (1.1 equiv) }
$$<smiles>Cc1ccccc1C(=O)c1nccn1C</smiles>

$$
\begin{gathered}
{\left[\mathrm{Cp}^{*} \mathrm{IrCl}_{2}\right]_{2}(\mathrm{x} \mathrm{mol} \%)} \\
\underset{\mathrm{NaBAr}_{4}(2 \times \mathrm{mol} \%)}{\mathrm{NaOAc}(0.15 \mathrm{mmol})} \\
\mathrm{Ag}_{2} \mathrm{O}(20 \mathrm{~mol} \%)
\end{gathered}
$$

1a $0.1 \mathrm{mmol} \operatorname{DCE}(1 \mathrm{~mL}), 130^{\circ} \mathrm{C}, 17 \mathrm{~h}$

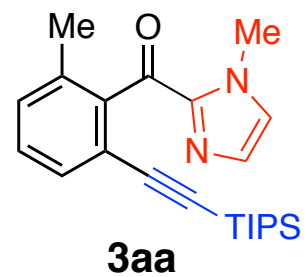

\begin{tabular}{cccc}
\hline \multirow{2}{*}{ Entry } & Base & \multicolumn{2}{c}{ NMR Yields (\%) } \\
& & 3aa & 1a \\
\hline 1 & $\mathrm{NaOAc}$ & 94 & - \\
2 & $\mathrm{LiOAc}$ & Decompose & Decompose \\
$\mathbf{3}$ & $\mathrm{K}_{2} \mathrm{CO}_{3}$ & 99 & - \\
4 & $\mathrm{Na}_{2} \mathrm{CO}_{3}$ & 99 & - \\
5 & $\mathrm{KOAc}$ & 83 & - \\
6 & ${ }^{\mathrm{BuOK}}$ & 47 & 50.6 \\
\hline
\end{tabular}

Table S4. Effect of Base Loading

$$
\mathrm{Br}=-\mathrm{TIPS} 2 \mathrm{2a} \text { (1.1 equiv) }
$$

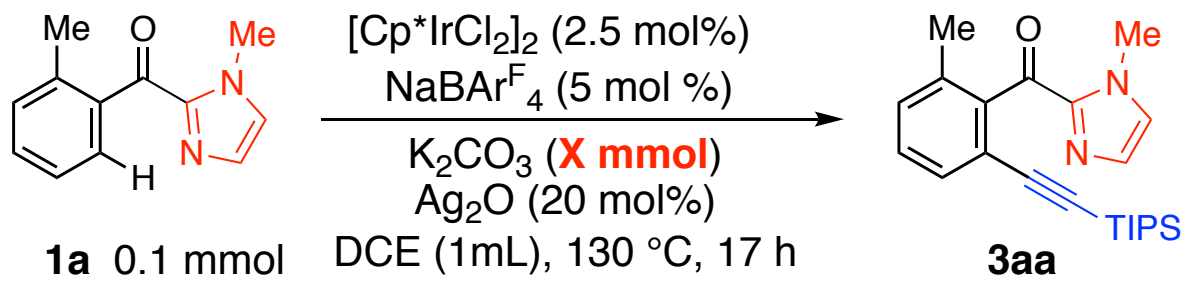

\begin{tabular}{cccc}
\hline \multirow{2}{*}{ Entry } & Base Loading (X mmol) & \multicolumn{2}{c}{ NMR Yields (\%) } \\
& & 3aa & 1a \\
\hline $\mathbf{1}$ & $\mathbf{0 . 1 5}$ & $\mathbf{9 9}$ & - \\
2 & 0.10 & 83 & - \\
3 & 0.05 & 80 & Traces \\
4 & 0.20 & 98 & - \\
\hline
\end{tabular}


Table S5. Co-catalyst Optimization

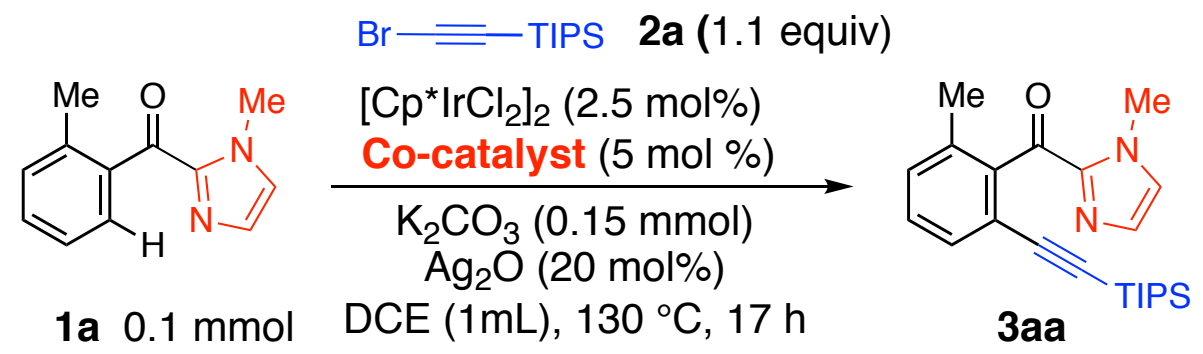

\begin{tabular}{cccc}
\hline \multirow{2}{*}{ Entry } & Co-catalyst (5 mol\%) & \multicolumn{3}{c}{ NMR Yields (\%) } \\
\hline $\mathbf{1}$ & $\mathbf{N a B A r}_{4}$ & $\mathbf{3 a a}$ & $\mathbf{1 a}$ \\
2 & $\mathrm{NaBF}_{4}$ & $\mathbf{9 9}$ & - \\
3 & $\mathrm{AgSbF}_{6}$ & 15 & 91 \\
4 & $\mathrm{NaOTf}_{5}$ & 18 & 85 \\
5 & $\mathrm{AgBF}_{4}$ & 11 & 71 \\
\hline
\end{tabular}

Table S6. Additive Optimization

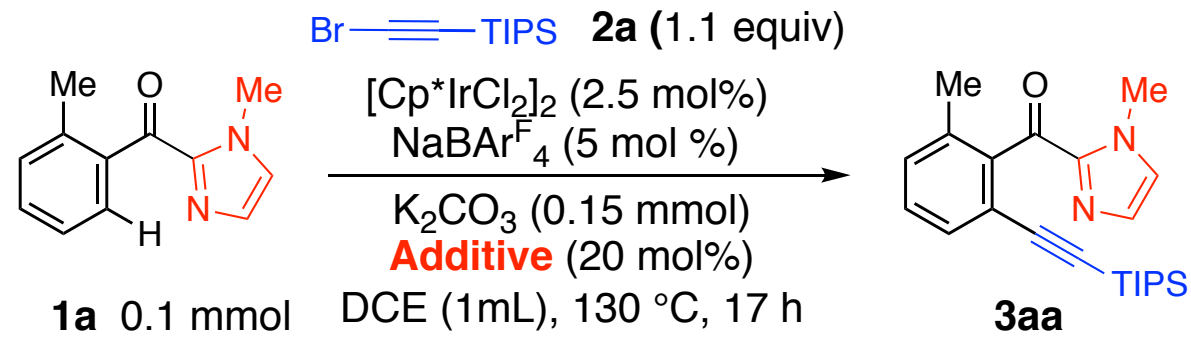

\begin{tabular}{cccc}
\hline \multirow{2}{*}{ Entry } & Additive (20 mol\%) & \multicolumn{2}{c}{ NMR Yields (\%) } \\
& & $\mathbf{3 a a}$ & $\mathbf{1 a}$ \\
\hline 1 & $\mathrm{Ag}_{2} \mathrm{O}$ & 99 & - \\
2 & $\mathrm{Ag}_{2} \mathrm{CO}_{3}$ & 87 & 7 \\
$\mathbf{3}$ & $\mathbf{A g O A c}$ & $\mathbf{1 0 0}$ & - \\
4 & $\mathrm{Cu}(\mathrm{OAc})_{2}$ & 94 & - \\
5 & $\mathrm{CuO}$ & 70 & 13 \\
6 & $\mathrm{Cu}_{2} \mathrm{O}$ & 44 & 28 \\
7 & $\mathrm{AgOTs}$ & - & 98 \\
8 & $\mathrm{AgOCOCF}_{3}$ & 93 & traces \\
\hline
\end{tabular}


Table S7. Effect of Additive Loading

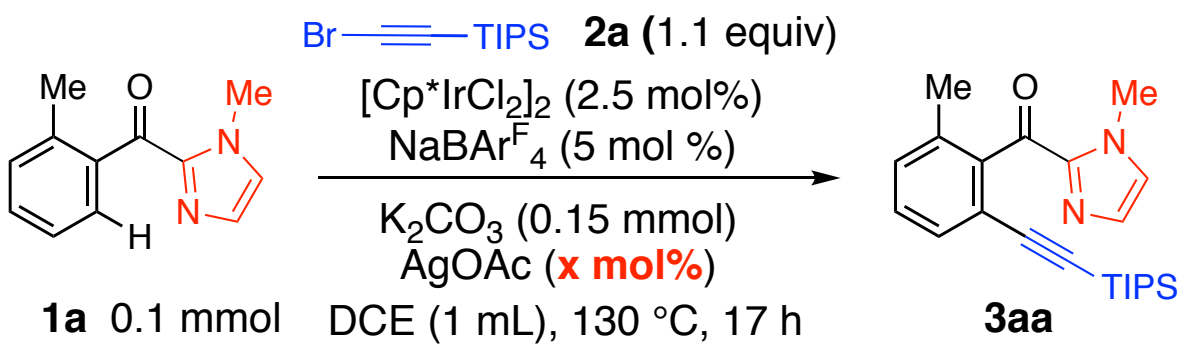

\begin{tabular}{cccc}
\hline \multirow{2}{*}{ Entry } & AgOAc Loading (X mol\%) & \multicolumn{2}{c}{ NMR Yields (\%) } \\
& & $\mathbf{3 a a}$ & $\mathbf{1 a}$ \\
\hline 1 & 20 & 100 & - \\
2 & 10 & 100 & - \\
$\mathbf{3}$ & $\mathbf{5}$ & $\mathbf{1 0 0}$ & - \\
4 & - & 68 & 32 \\
\hline
\end{tabular}

Table S8. Effect of Temperature

\begin{tabular}{|c|c|c|c|}
\hline \multicolumn{4}{|c|}{$\mathrm{Br}=-\mathrm{TIPS} \quad 2 \mathrm{a}$ (1.1 equiv) } \\
\hline $\mathrm{Me} \stackrel{\mathrm{O}}{\mathrm{Me}}$ & $\begin{array}{c}{\left[\mathrm{Cp}^{*} \operatorname{IrCl}_{2}\right]_{2}(2.5 \mathrm{~mol} \%)} \\
\mathrm{NaBAr}_{4}(5 \mathrm{~mol} \%)\end{array}$ & \multirow[b]{3}{*}{$3 a$} & \\
\hline & $\begin{array}{c}\mathrm{K}_{2} \mathrm{CO}_{3}(0.15 \mathrm{mmol}) \\
\mathrm{AgOAc}(5 \mathrm{~mol} \%)\end{array}$ & & \\
\hline $1 \mathrm{a} 0.1 \mathrm{mmol}$ & $\mathrm{DCE}(1 \mathrm{~mL}), \mathrm{X}^{\circ} \mathrm{C}, 17 \mathrm{~h}$ & & \\
\hline \multirow{2}{*}{ Entry } & \multirow{2}{*}{ Temperature $\left({ }^{\circ} \mathrm{C}\right)$} & \multicolumn{2}{|c|}{ NMR Yields (\%) } \\
\hline & & 3aa & $1 \mathbf{a}$ \\
\hline 1 & 25 & 10 & 88 \\
\hline 2 & 100 & 100 & - \\
\hline 3 & 90 & 100 & - \\
\hline 4 & 80 & 100 & - \\
\hline 5 & 70 & 100 & - \\
\hline 6 & 60 & 100 & - \\
\hline 7 & 50 & 94 & 5 \\
\hline
\end{tabular}


Table S9. Effect of Stereoelectronic Modification in Imidazole N-amine $\mathrm{Br}=-\mathrm{TIPS} 2 \mathrm{a}$ (1.1 equiv)

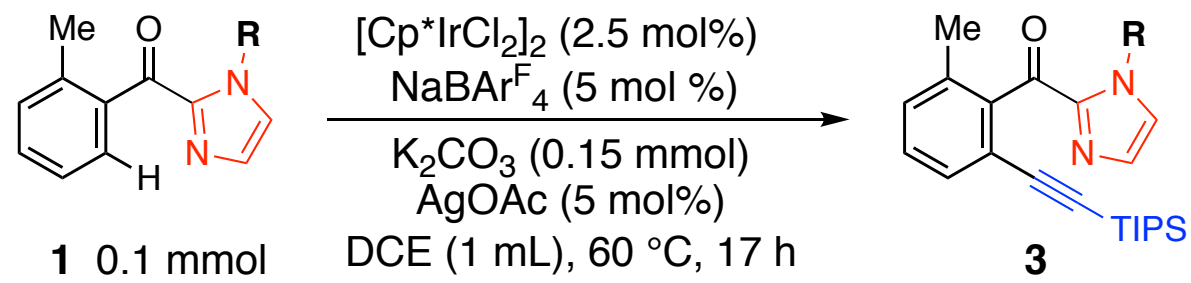

\begin{tabular}{cccc}
\hline \multirow{2}{*}{ Entry } & $\mathbf{R}$ & \multicolumn{2}{c}{ Isolated Yields (\%) } \\
& & $\mathbf{3}$ & $\mathbf{1}$ \\
\hline $\mathbf{1}$ & Me & $\mathbf{9 7}$ & - \\
2 & $\mathrm{Ph}$ & 27 & 62 \\
3 & $\mathrm{i} P$ & 84 & - \\
4 & $\mathrm{Br}$ & 88 & - \\
5 & $\mathrm{BEM}$ & 92 & - \\
\hline
\end{tabular}

Table S10. Optimization Summary of $s p^{2}$ C-H Alkynylation<smiles>Cc1ccccc1C(=O)c1nccn1C</smiles>
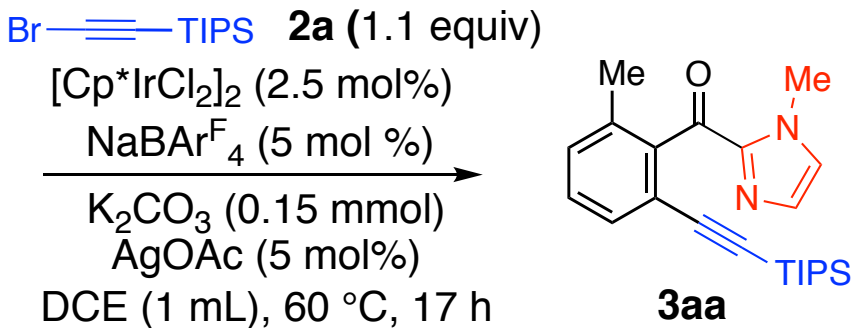

1a $0.1 \mathrm{mmol}$

$$
\text { DCE }(1 \mathrm{~mL}), 60^{\circ} \mathrm{C}, 17 \mathrm{~h}
$$

\begin{tabular}{|c|c|c|c|}
\hline \multirow{2}{*}{ Entry } & \multirow{2}{*}{ Deviations from the standard conditions } & \multicolumn{2}{|c|}{ NMR Yields (\%) } \\
\hline & & 3aa & 1a \\
\hline 1 & None & $100(97 ;$ Isolated $)$ & - \\
\hline 2 & Without $\left[\mathrm{Cp}^{*} \mathrm{IrCl}_{2}\right]_{2}$ & - & 98 \\
\hline 3 & Without $\mathrm{K}_{2} \mathrm{CO}_{3}$ & 5.5 & 83 \\
\hline 4 & Without $\mathrm{NaBAr}_{4}$ & - & 91 \\
\hline 5 & $\mathrm{AgSbF}_{6}$ instead of $\mathrm{NaBAr}_{4}{ }_{4}$ & 15 & 84 \\
\hline 6 & NaOTf instead of $\mathrm{NaBAr}_{4}{ }_{4}$ & 18 & 71 \\
\hline 7 & $\mathrm{LiOAc}$ instead of $\mathrm{K}_{2} \mathrm{CO}_{3}$ & - & - \\
\hline 8 & ${ }^{t} \mathrm{BuOK}$ instead of $\mathrm{K}_{2} \mathrm{CO}_{3}$ & 47 & 51 \\
\hline 9 & Without AgOAc & 68 & 32 \\
\hline 10 & AgOTf instead of AgOAc & - & 98 \\
\hline 11 & $\mathrm{CuO}$ instead of $\mathrm{AgOAc}$ & 70 & 13 \\
\hline 12 & Toluene instead of DCE @ $130^{\circ} \mathrm{C}$ & 83 & - \\
\hline 13 & 1,4-Dioxane instead of DCE @ $130{ }^{\circ} \mathrm{C}$ & 88 & - \\
\hline 14 & Acetonitrile instead of DCE @ $130{ }^{\circ} \mathrm{C}$ & - & 93 \\
\hline 15 & TIPS-acetylene instead of $\mathbf{2 a}$ & - & 98 \\
\hline
\end{tabular}




\section{C(sp $\left.{ }^{3}\right)-H$ Alkynylation Optimization.}

Table S11. Base Optimization for $s p^{3} \mathrm{C}-\mathrm{H}$ functionalization<smiles>CCCCC(=O)c1nccn1C</smiles>

$4 a(0.1 \mathrm{mmol})$

$$
\mathrm{Br}-\mathrm{TIPS} 2 \mathrm{2a}(1.1 \text { equiv.) }
$$$$
\mathrm{NaBAr}_{4}(5 \mathrm{~mol} \%)
$$

Base $(0.15 \mathrm{mmol})$

AgOAc (5 mol\%) DCE $(1 \mathrm{~mL}), 120^{\circ} \mathrm{C}, 17 \mathrm{~h}$

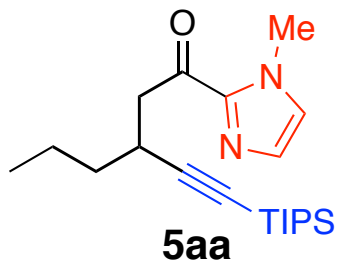

\begin{tabular}{cccc}
\hline \multirow{2}{*}{ Entry } & \multirow{2}{*}{ Base } & \multicolumn{2}{c}{ YNMR yields (\%) } \\
& & $\mathbf{5 a a}$ & $\mathbf{4 a}$ \\
\hline 1 & $\mathrm{NaOAc}$ & 19.6 & 31 \\
$\mathbf{2}$ & $\mathbf{L i O A c}$ & $\mathbf{4 3}(\mathbf{3 9})^{\mathbf{b}}$ & $\mathbf{2 4 . 3}$ \\
3 & $\mathrm{~K}_{2} \mathrm{CO}_{3}$ & 16 & 70 \\
4 & $\mathrm{KOAc}$ & 25 & 41 \\
5 & ${ }^{\mathrm{B}} \mathrm{BuOK}$ & 19 & 36 \\
6 & $\mathrm{CF}_{3} \mathrm{SO}_{3} \mathrm{~K}$ & - & -
\end{tabular}

${ }^{a}$ Yields are calculated based on ${ }^{1} \mathrm{H}$ NMR spectroscopy using 1,1,2,2tertachloroethane as an internal standard. ${ }^{b}$ Isolated yield.

Table S12. Effect of Base Loading<smiles>CCCCC(=O)c1nccn1C</smiles>

4a $(0.1 \mathrm{mmol})$ $\mathrm{Br}=-\mathrm{TIPS} 2 \mathrm{2a}$ (1.1 equiv.) $\left[\mathrm{Cp}^{\star} \mathrm{IrCl}_{2}\right]_{2}(2.5 \mathrm{~mol} \%)$ $\underset{\mathrm{LiOAc}(\mathrm{X} \mathrm{mmol})}{\stackrel{\mathrm{NaBAr}_{4}}{\longrightarrow}(5 \mathrm{~mol} \%)}$ $\mathrm{AgOAc}(5 \mathrm{~mol} \%)$ DCE $(1 \mathrm{~mL}), 120^{\circ} \mathrm{C}, 17 \mathrm{~h}$

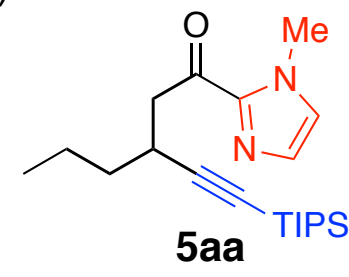

NMR Yields (\%)

\begin{tabular}{cccc} 
Entry & LiOAc Loading (mmol) & 5aa & 4a \\
\hline 1 & 0.15 & $43(39)$ & 24.3 \\
2 & 0.20 & 44 & 37 \\
3 & 0.25 & 45 & 32 \\
$\mathbf{4}$ & $\mathbf{0 . 3 0}$ & $\mathbf{4 9}$ & $\mathbf{2 9}$ \\
\hline
\end{tabular}


Table S13. Coupling Partner Loading<smiles>CCCCC(=O)c1nccn1C</smiles>

4a $(0.1 \mathrm{mmol})$ $\mathrm{Br}=-\mathrm{TIPS} 2 \mathrm{a}$ (X equiv.) $\left[\mathrm{Cp}{ }^{*} \mathrm{IrCl}_{2}\right]_{2}(2.5 \mathrm{~mol} \%)$ $\underset{\operatorname{LiOAc}(0.30 \mathrm{mmol})}{\stackrel{\mathrm{NaBAr}_{4}(5 \mathrm{~mol} \%)}{\longrightarrow}}$ AgOAc (5 mol\%) DCE $(1 \mathrm{~mL}), 120^{\circ} \mathrm{C}, 17 \mathrm{~h}$

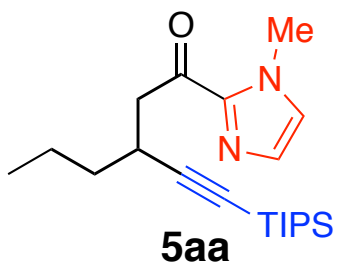

\begin{tabular}{cccc}
\hline \multirow{2}{*}{ Entry } & Coupling Partner Loading & \multicolumn{2}{c}{ NMR Yields (\%) } \\
\hline 1 & 1.1 equiv. & 5aa & 4a \\
\hline 2 & 1.5 equiv. & 49 & 29 \\
$\mathbf{3}$ & $\mathbf{2}$ equiv. & 45 & 26 \\
4 & 3 equiv. & $\mathbf{4 9}$ & $\mathbf{1 2}$ \\
5 & 4 equiv. & 45 & 4 \\
\hline
\end{tabular}

Table S14. Additive Loading<smiles>CCCCC(=O)c1nccn1C</smiles>

$4 a(0.1 \mathrm{mmol})$ $\mathrm{Br}=\mathrm{TIPS} \quad 2 \mathrm{a}$ (2 equiv.) $\left[\mathrm{Cp}^{\star} \mathrm{IrCl}_{2}\right]_{2}(2.5 \mathrm{~mol} \%)$ $\underset{\text { LiOAc }(0.30 \mathrm{mmol})}{\stackrel{\mathrm{NaBAr}_{4}(5 \mathrm{~mol} \%)}{\longrightarrow}}$ AgOAc (X mol\%) DCE (1mL), $120^{\circ} \mathrm{C}, 17 \mathrm{~h}$

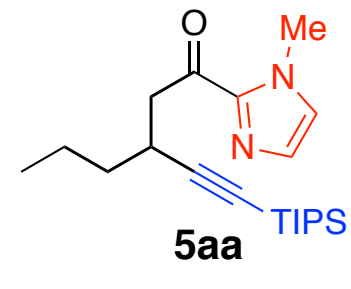

\begin{tabular}{cccc}
\hline \multirow{2}{*}{ Entry } & AgOAc (X mol\%) & \multicolumn{2}{c}{ NMR Yields (\%) } \\
& & $\mathbf{5 a a}$ & $\mathbf{4 a}$ \\
\hline 1 & 5 & 49 & 12 \\
$\mathbf{2}$ & $\mathbf{1 0}$ & $\mathbf{5 0}$ & $\mathbf{1 3}$ \\
3 & 20 & 44 & 13 \\
4 & 50 & 41 & 13 \\
5 & 75 & 43 & 23 \\
6 & 100 & 37 & 10 \\
\hline
\end{tabular}


Table S15. Temperature Optimization (Doubling the catalyst and co-catalyst loading)

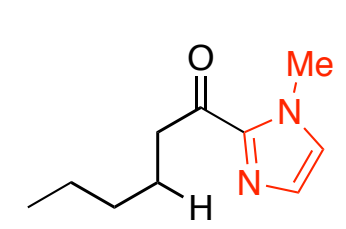

4 a $(0.1 \mathrm{mmol})$

$$
\mathrm{Br}=\text { TIPS 2a (2 equiv.) }
$$

$$
\underset{\mathrm{LiOAc}(0.30 \mathrm{mmol})}{\stackrel{\left[\mathrm{Cp}^{*} \mathrm{IrCl}_{2}\right]_{2}(5 \mathrm{~mol} \%)}{\underset{\mathrm{NaBAr}}{4}(10 \mathrm{~mol} \%)}}
$$

AgOAc (10 mol\%)

DCE $(1 \mathrm{~mL})$, Temp $\left({ }^{\circ} \mathrm{C}\right)$, Time $(\mathrm{h})$

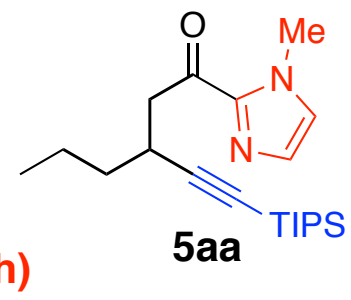

NMR Yield (\%)

\begin{tabular}{ccccc} 
Entry & Temperature $\left({ }^{\mathbf{0}} \mathbf{C}\right)$ & Time $(\mathbf{h})$ & $\mathbf{5 a a}$ & $\mathbf{4 a}$ \\
\hline 1 & 80 & 24 & 37 & 26 \\
2 & 100 & 24 & 56 & 22.5 \\
3 & 120 & 24 & 52 & 15 \\
4 & 140 & 24 & 34 & 22 \\
$\mathbf{5}$ & $\mathbf{1 0 0}$ & $\mathbf{3 6}$ & $\mathbf{7 3}(\mathbf{6 9 . 4})^{\mathbf{b}}$ & $\mathbf{1 8}(\mathbf{1 4})^{\mathbf{b}}$ \\
6 & 100 & 48 & 58 & 16
\end{tabular}

a Yields were calculated based on ${ }^{1} \mathrm{H}$ NMR spectroscopy data using 1,1,2,2tertachloroethane as an internal standard. ${ }^{b}$ Isolated yield. 
Table S16. Effect of Coupling Partner

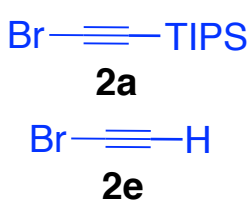

TIPS-EBX

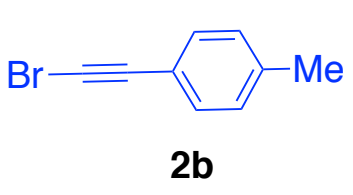

2b

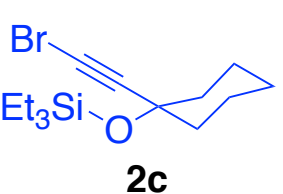

2c

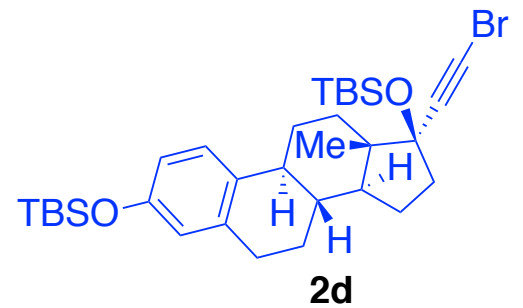

$2 f$<smiles>Cc1ccccc1C(=O)c1nccn1C</smiles>

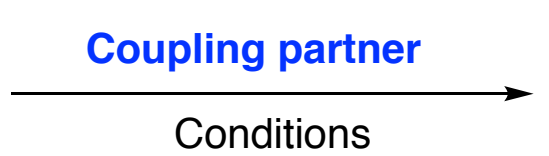

1a $0.2 \mathrm{mmol}$<smiles>[R]C#Cc1cccc(O)c1C(=O)c1nccn1C</smiles>

\begin{tabular}{|c|c|c|c|c|}
\hline \multirow{2}{*}{ Entry } & \multirow{2}{*}{ Coupling partner } & \multirow{2}{*}{ Conditions } & \multicolumn{2}{|c|}{ Yield $(\%)^{\mathrm{a}}$} \\
\hline & & & 6 & 1a \\
\hline 1 & $2 a$ & Table S9 (Entry 1) & $100(97)^{b}$ & - \\
\hline 2 & $2 b$ & - do- & Traces & 89 \\
\hline 3 & $2 b$ & Table S15 (Entry 5) & 10 & 77 \\
\hline 4 & $2 \mathbf{b}$ & Table S15 (Entry 5) ${ }^{\mathrm{c}}$ & $53(46)^{b}$ & 25 \\
\hline 5 & $2 \mathrm{c}$ & Table S9 (Entry 1) @ $100^{\circ} \mathrm{C}$ & 30 & 76.3 \\
\hline 6 & $2 \mathrm{c}$ & Table S15 (Entry 5) ${ }^{\mathrm{c}}$ & 50 & 20 \\
\hline 7 & $2 c$ & Table S15 (Entry 5) & $91.5(89.3)^{b}$ & 5 \\
\hline 8 & 2d & Table S15 (Entry 5) & $(44)^{b}$ & - \\
\hline 9 & $2 \mathrm{e}$ & Table S10 (Entry 1) & - & 98 \\
\hline 10 & $2 \mathrm{e}$ & Table S15 (Entry 5) ${ }^{\mathrm{d}}$ & - & 98 \\
\hline 11 & $2 \mathrm{f}$ & Table S10 (Entry 1) & - & 92 \\
\hline
\end{tabular}




\section{General Procedure for Ir(III)-Catalyzed C-H Alkynylation of 2-Acyl Imidazoles}

To an oven dried screw capped reaction tube $(5 \mathrm{~mL})$, 2-acylimidazole $(0.2 \mathrm{mmol}), \mathrm{K}_{2} \mathrm{CO}_{3}(0.3$ mmol), $\mathrm{NaBAr}_{4}(0.01 \mathrm{mmol}), \mathrm{AgOAc}(0.01 \mathrm{mmol}),\left[\mathrm{Cp}^{*} \mathrm{IrCl}_{2}\right]_{2}(0.005 \mathrm{mmol})$, alkynyl bromide $(0.22 \mathrm{mmol})$ and DCE $(1.0 \mathrm{~mL})$ were added. The tube then was sealed and placed in a preheated heating-block at $60{ }^{\circ} \mathrm{C}$, stirred for 17 hours and then allowed to cool to room temperature. The reaction mixture was filtered through a celite-pad with ethyl acetate and concentrated under reduced pressure. The crude residue was purified by silica (230-400 mesh) column chromatography. The alkynylated product (a thick oil) was eluted at a concentration of $5-15 \%$ ethyl acetate-hexane.

It is noteworthy that this process is applicable for $\mathrm{sp}^{2} \mathrm{C}-\mathrm{H}$ alkynylation (1a-o). The $\mathrm{sp}^{2} \mathrm{C}-\mathrm{H}$ alkynylation of heterocycles analogues (1p-s) involved the conditions stated in Table S15 (entry 5).

(1-Methyl-1H-imidazol-2-yl)(2-methyl-6-((triisopropylsilyl)ethynyl)phenyl)methanone<smiles>Cc1cccc(C#C[AsH2+])c1C(=O)c1nccn1C</smiles>
(3aa): White solid; Yield: 97\% (73.7 mg); mp. 110-111 ${ }^{\circ} \mathrm{C} . R_{f}=0.54(50 \%$ ethyl acetate-hexane). $\delta_{\mathrm{H}}\left(400 \mathrm{MHz}, \mathrm{CDCl}_{3}\right) 7.38-7.35(\mathrm{~m}, 1 \mathrm{H}), 7.25$ (t, $J$ $=7.6 \mathrm{~Hz}, 1 \mathrm{H}), 7.20-7.17(\mathrm{~m}, 1 \mathrm{H}), 7.16(\mathrm{~d}, J=0.8 \mathrm{~Hz}, 1 \mathrm{H}), 7.05(\mathrm{~d}, J=$ $0.8 \mathrm{~Hz}, 1 \mathrm{H}), 4.12(\mathrm{~s}, 3 \mathrm{H}), 2.23(\mathrm{~s}, 3 \mathrm{H}), 0.98-0.90(\mathrm{~m}, 21 \mathrm{H}) \mathrm{ppm} . \delta_{\mathrm{C}}(100$ $\left.\mathrm{MHz}, \mathrm{CDCl}_{3}\right) 189.09,143.52,142.35,134.66,130.79,130.46,130.39$, 128.91, 127.11, 120.86, 104.82, 94.97, 36.25, 19.41, 18.60 (6C), 11.22(3C) ppm. HRMS (EI ${ }^{+}$, $\mathrm{m} / \mathrm{z}$ ) calcd for $\mathrm{C}_{23} \mathrm{H}_{32} \mathrm{~N}_{2} \mathrm{OSi}$ : 380.2284 , found: 380.2279 .

\section{(1-Isopropyl-1H-imidazol-2-yl)(2-methyl-6-}

((triisopropylsilyl)ethynyl)phenyl)methanone (3ba): White powder; Yield: 84\% (68.2 mg);

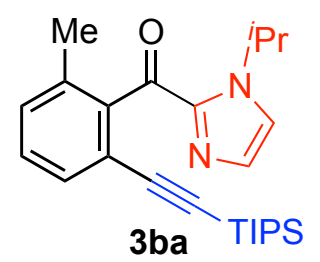
mp. $144-145{ }^{\circ}$ C. $R_{f}=0.57$ (84\% ethyl acetate-hexane). $\delta_{\mathrm{H}}(400 \mathrm{MHz}$, $\left.\mathrm{CDCl}_{3}\right) 7.39-7.36(\mathrm{~m}, 1 \mathrm{H}), 7.29(\mathrm{~d}, J=0.8 \mathrm{~Hz}, 1 \mathrm{H}), 7.24(\mathrm{t}, J=7.6 \mathrm{~Hz}$, $1 \mathrm{H}), 7.20(\mathrm{~d}, J=0.8 \mathrm{~Hz}, 1 \mathrm{H}), 7.19-7.16(\mathrm{~m}, 1 \mathrm{H}), 5.74-5.63(\mathrm{~m}, 1 \mathrm{H}), 2.19$ $(\mathrm{s}, 3 \mathrm{H}), 1.53(\mathrm{~s}, 3 \mathrm{H}), 1.52(\mathrm{~s}, 3 \mathrm{H}), 0.95-0.94(\mathrm{~m}, 21 \mathrm{H}) \mathrm{ppm} . \delta_{\mathrm{C}}(100 \mathrm{MHz}$, $\left.\mathrm{CDCl}_{3}\right)$ 188.96, 143.00, 142.69, 134.39, 131.28, 130.52, 130.35, 128.70, 121.35, 120.66, 104.97, 94.43, 49.56, 23.78, 19.38 (2C), 18.62 (6C), 11.23 (3C) ppm. HRMS $\left(\mathrm{EI}^{+}, \mathrm{m} / \mathrm{z}\right.$ ) calcd for $\mathrm{C}_{25} \mathrm{H}_{36} \mathrm{~N}_{2} \mathrm{OSi}$ : 408.2597, found: 408.2593 .

\section{(1-Benzyl-1H-imidazol-2-yl)(2-methyl-6-((triisopropylsilyl)ethynyl)phenyl)methanone}<smiles>Cc1cccc(C#CC(C)(F)F)c1C(=O)c1nccn1Cc1ccccc1</smiles>
(3ca): White solid; Yield: $86 \%(78.4 \mathrm{mg})$; mp. $144-145^{\circ} \mathrm{C} . R_{f}=0.61(50 \%$ ethyl acetate-hexane). $\delta_{\mathrm{H}}\left(400 \mathrm{MHz}, \mathrm{CDCl}_{3}\right) 7.39-7.31(\mathrm{~m}, 4 \mathrm{H}), 7.30-7.22$ (m, 3H), 7. 19 (d, $J=0.8,1 \mathrm{H}), 7.18-7.14(\mathrm{~m}, 1 \mathrm{H}), 7.07$ (d, $J=0.8 \mathrm{~Hz}$, $1 \mathrm{H}), 5.72$ (brs, 2H), $2.21(\mathrm{~s}, 3 \mathrm{H}), 1.00-0.94(\mathrm{~m}, 21 \mathrm{H}) \mathrm{ppm} . \delta_{\mathrm{C}}(100 \mathrm{MHz}$, $\left.\mathrm{CDCl}_{3}\right)$ 189.06, 143.01, 142.52, 136.42, 134.54, 131.21, 130.40, 130.36, 129.03 (2C), 128.88, 128.33, 128.05 (2C), 125.98, 120.77, 104.84, 94.66, 51.96, 19.25, 18.65 (6C), 11.25 (3C) ppm. HRMS (EI, $\mathrm{m} / \mathrm{z}$ ) calcd for $\mathrm{C}_{29} \mathrm{H}_{36} \mathrm{~N}_{2} \mathrm{OSi}$ : 456.2597, found: 456.2592 .

\section{(2-Methyl-6-((triisopropylsilyl)ethynyl)phenyl)(1-phenyl-1H-imidazol-2-yl)methanone}<smiles>Cc1cccc(C#CC(C)(C)C)c1C(=O)c1nccn1-c1ccccc1</smiles>

(3da): Off-white solid; Yield: 27\% (23.9 mg); mp. 82-83 ${ }^{\circ} \mathrm{C} ; R_{f}=0.63$ (50\% ethyl acetate-hexane). $\delta_{\mathrm{H}}\left(400 \mathrm{MHz}, \mathrm{CDCl}_{3}\right) 7.50-7.43(\mathrm{~m}, 5 \mathrm{H})$, 7.41-7.37 (m, 1H), $7.29(\mathrm{~d}, J=0.8 \mathrm{~Hz}, 1 \mathrm{H}), 7.28-7.23(\mathrm{~m}, 1 \mathrm{H}), 7.22(\mathrm{~d}, J$ $=1.2 \mathrm{~Hz}, 1 \mathrm{H}), 7.20-7.17(\mathrm{~m}, 1 \mathrm{H}), 2.24(\mathrm{~s}, 3 \mathrm{H}), 1.01-0.93(\mathrm{~m}, 21 \mathrm{H}) \mathrm{ppm}$. $\delta_{\mathrm{C}}\left(100 \mathrm{MHz}, \mathrm{CDCl}_{3}\right) 187.18,143.52,141.44,138.24,135.35,131.19$, 
$131.15,130.63,129.16,129.12$ (2C), 128.76, 126.91, 125.75 (2C), 121.30, 105.44, 95.39, 19.53, 18.71 (6C), 11.31 (3C) ppm. HRMS (EI ${ }^{+}, \mathrm{m} / \mathrm{z}$ ) calcd for $\mathrm{C}_{28} \mathrm{H}_{34} \mathrm{~N}_{2} \mathrm{OSi}$ : 442.2440, found: 442.2435 .

(2-Methyl-6-((triisopropylsilyl)ethynyl)phenyl)(1-((2-(trimethylsilyl)ethoxy)methyl)-1Himidazol-2-yl)methanone (3ea): Colorless sticky; Yield: $92 \%(91.3 \mathrm{mg}) ; R_{f}=0.68(50 \%$ ethyl

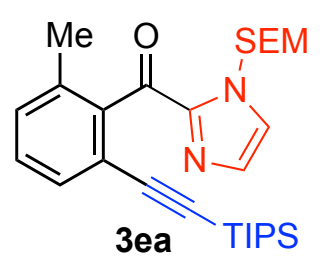
acetate-hexane). $\delta_{\mathrm{H}}\left(400 \mathrm{MHz}, \mathrm{CDCl}_{3}\right) 7.39-7.36(\mathrm{~m}, 1 \mathrm{H}), 7.33(\mathrm{~d}, J=$ $1.2 \mathrm{~Hz}, 1 \mathrm{H}), 7.28-7.24(\mathrm{~m}, 1 \mathrm{H}), 7.22(\mathrm{~d}, J=1.2 \mathrm{~Hz}, 1 \mathrm{H}), 7.20-7.17$ (m, 1H), 5. 90 (brs, 2H), 3.72-3.67 (m, 2H), $2.21(\mathrm{~s}, 3 \mathrm{H}), 1.01-0.92(\mathrm{~m}, 23 \mathrm{H})$, $0.02-0.00(\mathrm{~m}, 9 \mathrm{H}) \delta_{\mathrm{C}}\left(100 \mathrm{MHz}, \mathrm{CDCl}_{3}\right) 189.25,143.09,142.38,134.48$, $131.42,130.40,130.34,128.97,124.87,120.80,104.70,94.78,76.97$, 67.22, 19.37, 18.58, 18.13 (6C), 11.19 (3C), -1.31 (3C) ppm. HRMS (EI $\left.{ }^{+}, \mathrm{m} / \mathrm{z}\right)$ calcd for $\mathrm{C}_{28} \mathrm{H}_{44} \mathrm{~N}_{2} \mathrm{OSi}_{2}$ : 496.2941, found: 496.2947.

(2-Fluoro-6-((triisopropylsilyl)ethynyl)phenyl)(1-methyl-1H-imidazol-2-yl)methanone (3fa): Pale yellow solid; Yield: $97 \%$ (74.5 mg); mp. 95-96 ${ }^{\circ} \mathrm{C} . R_{f}=0.57$ (50\% ethyl acetate-<smiles>Cn1ccnc1C(=O)c1c(F)cccc1C#C[AsH2-]</smileshexane). $\delta_{\mathrm{H}}\left(400 \mathrm{MHz}, \mathrm{CDCl}_{3}\right)$ 7.37-7.32 (m, 2H), $7.19(\mathrm{~s}, 1 \mathrm{H}), 7.14-7.09$ $(\mathrm{m}, 1 \mathrm{H}), 7.08(\mathrm{~s}, 1 \mathrm{H}), 4.11(\mathrm{~s}, 3 \mathrm{H}), 1.01-0.92(\mathrm{~m}, 21 \mathrm{H}) \mathrm{ppm} . \delta_{\mathrm{C}}(100 \mathrm{MHz}$, $\left.\mathrm{CDCl}_{3}\right) 183.12,158.86(\mathrm{~d}, J=246.3 \mathrm{~Hz}), 143.20,131.01,130.71(\mathrm{~d}, J=$ $9.58 \mathrm{~Hz}), 130.54,128.75(\mathrm{~d}, J=2.87 \mathrm{~Hz}), 127.58,123.26(\mathrm{~d}, J=4.79 \mathrm{~Hz})$, $116.14(\mathrm{~d}, J=22.04 \mathrm{~Hz}), 103.04(\mathrm{~d}, J=2.87 \mathrm{~Hz}), 96.34,36.19,18.54(6 \mathrm{C})$, $11.15(3 \mathrm{C}) \mathrm{ppm} . \quad \delta_{\mathrm{F}}\left(376 \mathrm{MHz}, \mathrm{CDCl}_{3}\right)-115.63 \mathrm{ppm}$. HRMS $\left(\mathrm{EI}^{+}, \mathrm{m} / \mathrm{z}\right)$ calcd for $\mathrm{C}_{22} \mathrm{H}_{29} \mathrm{FN}_{2} \mathrm{OSi}$ : 384.2033, found: 384.2027 .

\section{(1-Methyl-1H-imidazol-2-yl)(2-((triisopropylsilyl)ethynyl)phenyl)methanone}

(3ga):<smiles></smiles>
Light yellow solid; Yield: $74 \%$ (54.1 mg); mp. $98-99{ }^{\circ} \mathrm{C} . R_{f}=0.46(50 \%$ ethyl acetate-hexane). $\delta_{\mathrm{H}}\left(400 \mathrm{MHz}, \mathrm{CDCl}_{3}\right) 7.58-7.54(\mathrm{~m}, 2 \mathrm{H}), 7.44-7.36$ $(\mathrm{m}, 2 \mathrm{H}), 7.18(\mathrm{~d}, \mathrm{~J}=0.8 \mathrm{~Hz}, 1 \mathrm{H}), 7.06(\mathrm{~s}, 1 \mathrm{H}), 4.09(\mathrm{~s}, 3 \mathrm{H}), 1.04-0.94(\mathrm{~m}$, $21 \mathrm{H})$ ppm. $\delta_{\mathrm{C}}\left(100 \mathrm{MHz}, \mathrm{CDCl}_{3}\right) 186.92,143.48,141.97,133.65,130.43$, $130.13,128.14,128.07,127.05,122.10,104.71,95.64,36.25,18.68(6 \mathrm{C})$,

11.34 (3C) ppm. HRMS $\left(\mathrm{CI}^{+}, \mathrm{m} / \mathrm{z}\right)$ calcd for $\mathrm{C}_{22} \mathrm{H}_{31} \mathrm{~N}_{2} \mathrm{OSi}^{+}[\mathrm{M}+\mathrm{H}]^{+}: 367.2206$, found: 267.2212.

\section{(2,6-Bis((triisopropylsilyl)ethynyl)phenyl)(1-methyl-1H-imidazol-2-yl)methanone}

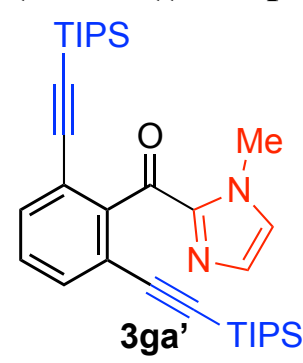

(3ga'): Yellow solid; Yield: 7\% (7.7 mg); mp. 148-149 ${ }^{\circ}$ C. $R_{f}=0.93(50 \%$ ethyl acetate-hexane). $\delta_{\mathrm{H}}\left(400 \mathrm{MHz}, \mathrm{CDCl}_{3}\right) 7.52-7.42(\mathrm{~m}, 2 \mathrm{H}), 7.31-7.27$ $(\mathrm{m}, 1 \mathrm{H}), 7.15(\mathrm{~s}, 1 \mathrm{H}), 7.01(\mathrm{~s}, 1 \mathrm{H}), 4.13(\mathrm{~s}, 3 \mathrm{H}), 1.02-0.92(\mathrm{~m}, 42 \mathrm{H}) \mathrm{ppm}$. $\delta_{\mathrm{C}}\left(100 \mathrm{MHz}, \mathrm{CDCl}_{3}\right) 186.67,145.69,142.90,132.23$ (2C), 130.76, $128.52,126.74,120.90(2 \mathrm{C}), 103.65(2 \mathrm{C}), 95.34$ (2C), 36.92, 18.41 (12C), 11.08 (6C) ppm. HRMS $\left(\mathrm{EI}^{+}, \mathrm{m} / \mathrm{z}\right.$ ) calcd for $\mathrm{C}_{33} \mathrm{H}_{50} \mathrm{~N}_{2} \mathrm{OSi}_{2}: 546.3462$, found: 546.3461 .

(1-Methyl-1H-imidazol-2-yl)(5-methyl-2-((triisopropylsilyl)ethynyl)phenyl)methanone<smiles>Cc1ccc(C#C[AsH2])c(C(=O)c2nccn2C)c1</smiles>

(3ha): Off-white solid; Yield: 93\% (70.4 mg); mp. 68-69 ${ }^{\circ} \mathrm{C} ; R_{f}=$ $0.57\left(50 \%\right.$ ethyl acetate-hexane). $\delta_{\mathrm{H}}\left(400 \mathrm{MHz}, \mathrm{CDCl}_{3}\right) 7.45(\mathrm{~d}, J=$ $8.0 \mathrm{~Hz}, 1 \mathrm{H}), 7.33(\mathrm{~s}, 1 \mathrm{H}), 7.21(\mathrm{dd}, J=8.00,1.21 \mathrm{H}), 7.17(\mathrm{~s}, 1 \mathrm{H})$, $7.05(\mathrm{~s}, 1 \mathrm{H}) 4.08(\mathrm{~s}, 3 \mathrm{H}), 2.38(\mathrm{~s}, 3 \mathrm{H}), 1.02-0.94(\mathrm{~m}, 21 \mathrm{H}) \mathrm{ppm} . \delta_{\mathrm{C}}$ $\left(100 \mathrm{MHz}, \mathrm{CDCl}_{3}\right)$ 187.38, 143.58, 142.05, 138.41, 133.50, 130.94, 
130.46, 128.45, 126.94, 118.14, 104.87, 94.59, 36.18, 21.58, 18.68 (6C), 11.34 (3C) ppm. HRMS (EI ${ }^{+}, \mathrm{m} / \mathrm{z}$ ) calcd for $\mathrm{C}_{23} \mathrm{H}_{32} \mathrm{~N}_{2} \mathrm{OSi}: 380.2284$, found: 380.2277 .

(5-Methoxy-2-((triisopropylsilyl)ethynyl)phenyl)(1-methyl-1H-imidazol-2-yl)methanone<smiles></smiles>

(3ia): Colorless crystalline solid; Yield: $82 \%$ (64.6 mg); mp. 102$103{ }^{\circ} \mathrm{C} . R_{f}=0.46(50 \%$ ethyl acetate-hexane $) . \delta_{\mathrm{H}}\left(400 \mathrm{MHz}, \mathrm{CDCl}_{3}\right)$ $7.48(\mathrm{~d}, J=8.4 \mathrm{~Hz}, 1 \mathrm{H}), 7.18(\mathrm{~d}, J=0.8 \mathrm{~Hz}, 1 \mathrm{H}), 7.06-7.05(\mathrm{~m}, 2 \mathrm{H})$, 6.96-6.92 (m, 1H), $4.08(\mathrm{~s}, 3 \mathrm{H}), 3.82(\mathrm{~s}, 3 \mathrm{H}), 1.00-0.93(\mathrm{~m}, 21 \mathrm{H})$ ppm. $\delta_{\mathrm{C}}\left(100 \mathrm{MHz}, \mathrm{CDCl}_{3}\right) 186.89,159.34,143.51,143.40,135.04$, $130.58,127.07,116.39,114.40,113.12,104.69,93.63,55.63,36.20,18.69$ (6C), 11.37 (3C) ppm. HRMS (EI, $\mathrm{m} / \mathrm{z}$ ) calcd for $\mathrm{C}_{23} \mathrm{H}_{32} \mathrm{~N}_{2} \mathrm{O}_{2} \mathrm{Si}$ : 396.2233, found: 396.2227.

(3-Methoxy-2,6-bis((triisopropylsilyl)ethynyl)phenyl)(1-methyl-1H-imidazol-2-

yl)methanone (3ia'): White crystalline solid; Yield: 5\% (5.7 mg); mp. 202-203 ${ }^{\circ} \mathrm{C} . R_{f}=0.71$<smiles>COc1ccc(C#CC(F)(F)F)c(C(=O)c2nccn2C)c1C#C[In]</smiles>
(50\% ethyl acetate-hexane). $\delta_{\mathrm{H}}\left(400 \mathrm{MHz}, \mathrm{CDCl}_{3}\right) 7.43(\mathrm{~d}, J=8.8$ $\mathrm{Hz}, 1 \mathrm{H}), 7.14(\mathrm{~d}, J=0.4 \mathrm{~Hz}, 1 \mathrm{H}), 6.99(\mathrm{~d}, J=0.4 \mathrm{~Hz}, 1 \mathrm{H}), 6.83$ (d, $J=8.4 \mathrm{~Hz}, 1 \mathrm{H}), 4.08(\mathrm{~s}, 3 \mathrm{H}), 3.87$ (s, 3H), 0.99-0.91 (m, 42H) ppm. $\delta_{\mathrm{C}}\left(100 \mathrm{MHz}, \mathrm{CDCl}_{3}\right)$ 186.40, 160.62, 147.58, 142.97, 133.80, $130.90,126.78,113.10,111.29,110.88,103.90,99.91,99.82$, 93.11, 56.28, 36.03, 18.59 (12C), 11.29 (6C) ppm. HRMS (EI ${ }^{+}$, $\mathrm{m} / \mathrm{z}$ ) calcd for $\mathrm{C}_{34} \mathrm{H}_{52} \mathrm{~N}_{2} \mathrm{O}_{2} \mathrm{Si}_{2}: 576.3567$, found: 576.3566 .

(1-Methyl-1H-imidazol-2-yl)(5-(trifluoromethyl)-2-((triisopropylsilyl)ethynyl)phenyl)<smiles>Cn1ccnc1C(=O)c1cc(C(F)(F)F)ccc1C#[In]</smiles>

methanone (3ja): Colorless thick oil; Yield: $98 \%(84.7 \mathrm{mg}) ; R_{f}=$ $0.64\left(50 \%\right.$ ethyl acetate-hexane) $\delta_{\mathrm{H}}\left(400 \mathrm{MHz}, \mathrm{CDCl}_{3}\right) 7.80-7.79(\mathrm{~m}$, $1 \mathrm{H}), 7.66-7.65(\mathrm{~m}, 2 \mathrm{H}), 7.20$ (d, $J=0.92 \mathrm{~Hz}, 1 \mathrm{H}), 7.0$ (d, $J=0.48$ $\mathrm{Hz}, 1 \mathrm{H}), 4.11(\mathrm{~s}, 3 \mathrm{H}), 1.03-0.95(\mathrm{~m}, 21 \mathrm{H}) \mathrm{ppm} . \delta_{\mathrm{C}}(100 \mathrm{MHz}$, $\mathrm{CDCl}_{3}$ ) $185.39,142.88,142.48,133.83,130.81,129.84$ (qt, $J=33.54$ $\mathrm{Hz}), 127.55,126.63$ (qt, $J=3.83 \mathrm{~Hz}), 125.57,125.02$ (qt, $J=7.66 \mathrm{~Hz}), 123.67$ (qt, $J=271.2$ $\mathrm{Hz}), 103.22,98.96,36.21,18.53(6 \mathrm{C}), 11.15(3 \mathrm{C}) \mathrm{ppm} . \delta_{\mathrm{F}}\left(376 \mathrm{MHz}, \mathrm{CDCl}_{3}\right)-62.65 \mathrm{ppm}$. HRMS (EI ${ }^{+}, \mathrm{m} / \mathrm{z}$ ) calcd for $\mathrm{C}_{23} \mathrm{H}_{29} \mathrm{~F}_{3} \mathrm{~N}_{2} \mathrm{OSi}$ : 434.2001, found: 434.1999.

(1-Methyl-1H-imidazol-2-yl)(2-((triisopropylsilyl)ethynyl)naphthalen-1-yl)methanone<smiles>CC#Cc1ccc2ccccc2c1C(=O)c1nccn1C</smiles>

(3ka): Off-white solid; Yield: $71 \%$ (59.5 mg); mp. 121-122 ${ }^{\circ} \mathrm{C} . \quad R_{f}=$ $0.57\left(50 \%\right.$ ethyl acetate-hexane). $\delta_{\mathrm{H}}\left(400 \mathrm{MHz}, \mathrm{CDCl}_{3}\right) 7.85-7.81(\mathrm{~m}$, 2H), 7.68-7.65(m, 1H), 7.56 (d, $J=8.4 \mathrm{~Hz}, 1 \mathrm{H}), 7.49-7.41(\mathrm{~m}, 2 \mathrm{H}), 7.13$ $(\mathrm{d}, J=0.8 \mathrm{~Hz}, 1 \mathrm{H}), 7.08(\mathrm{~s}, 1 \mathrm{H}), 4.21(\mathrm{~s}, 3 \mathrm{H}), 1.02-0.93(\mathrm{~m}, 21 \mathrm{H}) \mathrm{ppm}$. $\delta_{\mathrm{C}}\left(100 \mathrm{MHz}, \mathrm{CDCl}_{3}\right) 188.64,144.08,140.79,133.08,131.02,130.18$, $129.26,128.98,128.36,127.35,127.23,126.88,125.22,118.87,105.16$, 96.53, 36.36, 18.65 (6C), 11.31(3C) ppm. HRMS (EI ${ }^{+}, \mathrm{m} / \mathrm{z}$ ) calcd for $\mathrm{C}_{26} \mathrm{H}_{32} \mathrm{~N}_{2} \mathrm{OSi}$ : 416.2284, found: 416.2276. 
(1-Methyl-1H-imidazol-2-yl)(4-(trifluoromethoxy)-2-((triisopropylsilyl)ethynyl)phenyl)-<smiles>Cn1ccnc1C(=O)c1ccc(OC(F)(F)F)cc1C#C[In]</smilesmethanone (3la): Yellowish sticky; Yield: $72 \%(64.8 \mathrm{mg}) ; R_{f}=$ 0.57 (50\% ethyl acetate-hexane). $\delta_{\mathrm{H}}\left(400 \mathrm{MHz}, \mathrm{CDCl}_{3}\right) 7.63$ (d, $J$ $=8.8 \mathrm{~Hz}, 1 \mathrm{H}), 7.38-7.37(\mathrm{~m}, 1 \mathrm{H}), 7.26-7.22(\mathrm{~m}, 1 \mathrm{H}), 7.20(\mathrm{~d}, J=$ $0.8 \mathrm{~Hz}, 1 \mathrm{H}), 7.09(\mathrm{~s}, 1 \mathrm{H}), 4.10(\mathrm{~s}, 3 \mathrm{H}), 1.05-0.95(\mathrm{~m}, 21 \mathrm{H}) \mathrm{ppm}$. $\delta_{\mathrm{C}}\left(100 \mathrm{MHz}, \mathrm{CDCl}_{3}\right) 185.33,150.07,143.04,140.32,130.57$, $130.14,127.43,125.51,124.20,120.42$ (qt, $J=257.77 \mathrm{~Hz}$ ), 120.33, 103.01, 97.69, 36.30, 18.59 (6C), $11.20(3 \mathrm{C}) \mathrm{ppm}$. $\delta_{\mathrm{F}}\left(376 \mathrm{MHz}, \mathrm{CDCl}_{3}\right)-57.47 \mathrm{ppm}$. HRMS $\left(\mathrm{EI}^{+}, \mathrm{m} / \mathrm{z}\right)$ calcd for $\mathrm{C}_{23} \mathrm{H}_{29} \mathrm{~F}_{3} \mathrm{~N}_{2} \mathrm{O}_{2} \mathrm{Si}: 450.1950$, found: 450.1943 .

Methyl 4-(1-methyl-1H-imidazole-2-carbonyl)-3-((triisopropylsilyl)ethynyl)benzoate<smiles>COC(=O)c1ccc(C(=O)c2nccn2C)c(C#C[AsH2])c1</smiles>
(3ma): Off-white solid; Yield: 65\% (54.8 mg); mp. 104-105 ${ }^{\circ} \mathrm{C}$. $R_{f}=0.43$ (50\% ethyl acetate-hexane). $\delta_{\mathrm{H}}\left(400 \mathrm{MHz}, \mathrm{CDCl}_{3}\right) 8.21-$ $8.19(\mathrm{~m}, 1 \mathrm{H}), 8.04(\mathrm{dd}, J=8.02,1.62 \mathrm{~Hz}, 1 \mathrm{H}), 7.59(\mathrm{dd}, J=8.00$, $0.48 \mathrm{~Hz}, 1 \mathrm{H}), 7.19(\mathrm{~d}, J=0.68 \mathrm{~Hz}, 1 \mathrm{H}), 7.09(\mathrm{~s}, 1 \mathrm{H}), 4.11(\mathrm{~s}, 3 \mathrm{H})$, $3.95(\mathrm{~s}, 3 \mathrm{H}), 1.03-0.96(\mathrm{~m}, 21 \mathrm{H})$ ppm. $\delta_{\mathrm{C}}\left(100 \mathrm{MHz}, \mathrm{CDCl}_{3}\right)$ $186.08,165.90,145.70,142.92,134.37,131.39,130.64,128.88,127.83,127.28,122.15$, 103.44, 96.67, 52.40, 36.10, 18.49(6C), 11.14(3C) ppm. HRMS $\left(\mathrm{EI}^{+}, \mathrm{m} / \mathrm{z}\right)$ calcd for $\mathrm{C}_{24} \mathrm{H}_{32} \mathrm{~N}_{2} \mathrm{O}_{3} \mathrm{Si}$ : 424.2182 , found: 424.2175 .

(1-Methyl-1H-imidazol-2-yl)(2-(trifluoromethyl)-6-((triisopropylsilyl)ethynyl)phenyl) methanone (3na): Pale yellow solid; Yield: $81 \%$ (70.4 mg); mp. $72-73{ }^{\circ} \mathrm{C} . R_{f}=0.64(50 \%$<smiles>Cn1ccnc1C(=O)c1c(C(F)(F)F)cccc1C(F)(F)F</smiles>
ethyl acetate-hexane). $\delta_{\mathrm{H}}\left(400 \mathrm{MHz}, \mathrm{CDCl}_{3}\right) 7.72-7.64(\mathrm{~m}, 2 \mathrm{H}), 7.51-7.47$ $(\mathrm{m}, 1 \mathrm{H}), 7.16(\mathrm{~s}, 1 \mathrm{H}), 7.06(\mathrm{~s}, 1 \mathrm{H}), 4.11(\mathrm{~s}, 3 \mathrm{H}), 0.99-0.90(\mathrm{~m}, 21 \mathrm{H}) \mathrm{ppm}$. $\delta_{\mathrm{C}}\left(100 \mathrm{MHz}, \mathrm{CDCl}_{3}\right) 185.37,143.08,140.70,136.20,130.99,129.14$, 127.73 (qt, $J=30.0 \mathrm{~Hz}), 127.24,125.98,123.56$ (qt, $J=273.11 \mathrm{~Hz}$ ), $122.77,102.92,97.53,36.14,18.52(6 \mathrm{C}), 11.12(3 \mathrm{C}) \mathrm{ppm} . \delta_{\mathrm{F}}(376 \mathrm{MHz}$, $\left.\mathrm{CDCl}_{3}\right)-58.37$ ppm. HRMS (EI, $\mathrm{m} / \mathrm{z}$ ) calcd for $\mathrm{C}_{23} \mathrm{H}_{29} \mathrm{~N}_{2} \mathrm{OSi}$ : 434.2001, found: 434.2000 .

(4,5-dichloro-2-((triisopropylsilyl)ethynyl)phenyl)(1-methyl-1H-imidazol-2-

yl)methanone (3oa): Colorless sticky (purified by GPC); Yield: 38\% (32.6 mg); $R_{f}=0.64$<smiles></smiles>

(50\% ethyl acetate-hexane). $\delta_{\mathrm{H}}\left(400 \mathrm{MHz}, \mathrm{CDCl}_{3}\right) 7.65(\mathrm{~s}, 1 \mathrm{H}), 7.63$ $(\mathrm{s}, 1 \mathrm{H}), 7.20(\mathrm{~s}, 1 \mathrm{H}), 7.10(\mathrm{~s}, 1 \mathrm{H}), 4.08(\mathrm{~s}, 3 \mathrm{H}), 0.99-0.93(\mathrm{~m}, 21 \mathrm{H})$ ppm. $\delta_{\mathrm{C}}\left(100 \mathrm{MHz}, \mathrm{CDCl}_{3}\right) 184.21,142.80,141.19,134.89,134.49$, $132.62,130.81,130.14,127.63,121.92,102.33,98.18,36.30,18.62$ (6C), 11.21 (3C) ppm. HRMS (EI $\left.{ }^{+}, \mathrm{m} / \mathrm{z}\right)$ calcd for $\mathrm{C}_{22} \mathrm{H}_{28} \mathrm{Cl}_{2} \mathrm{~N}_{2} \mathrm{OSi}$ : 434.1348, found: 434.1346 .

(1-Methyl-1H-imidazol-2-yl)(1-methyl-3-((triisopropylsilyl)ethynyl)-1H-pyrrol-2-

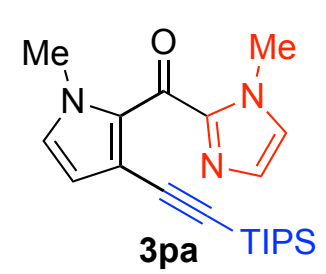

yl)methanone (3pa): Brownish sticky (purified by GPC); Yield: $23 \%$ $(16.9 \mathrm{mg}) ; R_{f}=0.39\left(50 \%\right.$ ethyl acetate-hexane). $\delta_{\mathrm{H}}\left(400 \mathrm{MHz}, \mathrm{CDCl}_{3}\right)$ $7.14(\mathrm{~s}, 1 \mathrm{H}), 7.00(\mathrm{~s}, 1 \mathrm{H}), 6.74(\mathrm{~d}, J=2.4 \mathrm{~Hz}, 1 \mathrm{H}), 6.31(\mathrm{~d}, J=2.4 \mathrm{~Hz}$, $1 \mathrm{H}), 3.93(\mathrm{~s}, 3 \mathrm{H}), 3.82(\mathrm{~s}, 3 \mathrm{H}), 1.04-0.88(\mathrm{~m}, 21 \mathrm{H}) \mathrm{ppm} . \delta_{\mathrm{C}}(100 \mathrm{MHz}$, $\left.\mathrm{CDCl}_{3}\right) 176.63,144.48,133.32,129.70,129.19,125.76,115.02,114.57$, 100.87, 93.65, 36.62, 34.82, 18.69 (6C), 11.36(3C) ppm. HRMS (EI $\left.{ }^{+}, \mathrm{m} / \mathrm{z}\right)$ calcd for $\mathrm{C}_{21} \mathrm{H}_{31} \mathrm{~N}_{3} \mathrm{OSi}$ : 369.2236, found: 369.2232 . 
(1-Methyl-1H-imidazol-2-yl)(3-((triisopropylsilyl)ethynyl)furan-2-yl)methanone (3qa):

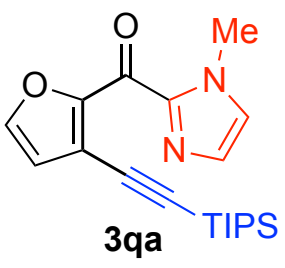

Light brownish sticky; Yield: $76 \%(54 \mathrm{mg}) ; \mathrm{mp} .81-82{ }^{\circ} \mathrm{C} ; R_{f}=0.39(50 \%$ ethyl acetate-hexane). $\delta_{\mathrm{H}}\left(400 \mathrm{MHz}, \mathrm{CDCl}_{3}\right) 7.62(\mathrm{~d}, J=1.6 \mathrm{~Hz}, 1 \mathrm{H}), 7.27$ $(\mathrm{d}, J=0.8 \mathrm{~Hz}, 1 \mathrm{H}), 7.08(\mathrm{~d}, J=0.4 \mathrm{~Hz}, 1 \mathrm{H}), 6.62(\mathrm{~d}, J=1.6 \mathrm{~Hz}, 1 \mathrm{H}), 4.05$ $(\mathrm{s}, 3 \mathrm{H}), 1.13-1.10(\mathrm{~m}, 21 \mathrm{H}) \mathrm{ppm} . \delta_{\mathrm{C}}\left(100 \mathrm{MHz}, \mathrm{CDCl}_{3}\right)$ 171.06, 153.14, $145.87,142.25,129.97,126.83,116.71,116.49,99.89,97.81,36.15,18.75$ (6C), 11.41 (3C) ppm. HRMS (EI+ ${ }^{+}, \mathrm{m} / \mathrm{z}$ ) calcd for $\mathrm{C}_{20} \mathrm{H}_{28} \mathrm{~N}_{2} \mathrm{O}_{2} \mathrm{Si}$ : 356.1920, found: 356.1916 . (1-Methyl-1H-imidazol-2-yl)(3-((triisopropylsilyl)ethynyl)benzofuran-2-yl)methanone (3ra): Brownish sticky; Yield: $66 \%(53.6 \mathrm{mg}) ; R_{f}=0.29$ (50\% ethyl acetate-hexane). $\delta_{\mathrm{H}}(400$

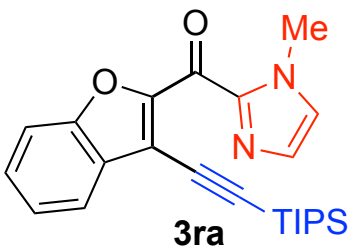

$\left.\mathrm{MHz} \mathrm{CDCl}_{3}\right) 7.77-7.75(\mathrm{~m}, 1 \mathrm{H}), 7.62(\mathrm{~d}, J=8.4 \mathrm{~Hz}, 1 \mathrm{H}), 7.52-7.46$ (m, 1H), 7.38-7.33 (m, 1H), $7.28(\mathrm{~d}, J=0.8 \mathrm{~Hz}, 1 \mathrm{H}), 7.12(\mathrm{~s}, 1 \mathrm{H}), 4.06$ $(\mathrm{s}, 3 \mathrm{H}), 1.14-1.13(\mathrm{~m}, 21 \mathrm{H}) \mathrm{ppm} . \delta_{\mathrm{C}}\left(100 \mathrm{MHz}, \mathrm{CDCl}_{3}\right)$ 173.03, $154.84,153.19,142.52,130.39,128.96,128.60,127.17,124.18$, $122.12,112.80,112.09,102.63,96.39,35.95,18.75(6 \mathrm{C}), 11.33(3 \mathrm{C})$ ppm. HRMS (EI, $\mathrm{m} / \mathrm{z}$ ) calcd for $\mathrm{C}_{24} \mathrm{H}_{30} \mathrm{~N}_{2} \mathrm{O}_{2} \mathrm{Si}$ : 406.2077, found: 406.2073.

\section{(1-Methyl-1H-imidazol-2-yl)(3-((triisopropylsilyl)ethynyl)benzo[b]thiophen-2-}

yl)methanone (3sa): Light yellow solid; Yield: $83 \%(69.7 \mathrm{mg}) ; \mathrm{mp} .76-77{ }^{\circ} \mathrm{C}, R_{f}=0.68(50 \%$

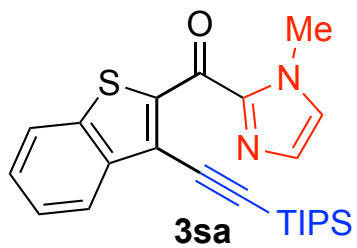
ethyl acetate-hexane). $\delta_{\mathrm{H}}\left(400 \mathrm{MHz}, \mathrm{CDCl}_{3}\right) 8.11-8.08(\mathrm{~m}, 1 \mathrm{H}), 7.85-$ $7.82(\mathrm{~m}, 1 \mathrm{H}), 7.51-7.44(\mathrm{~m}, 2 \mathrm{H}), 7.25(\mathrm{~d}, J=0.92 \mathrm{~Hz}, 1 \mathrm{H}), 7.11(\mathrm{~s}$, $1 \mathrm{H}), 4.11(\mathrm{~s}, 3 \mathrm{H}), 1.25-1.14(\mathrm{~m}, 21 \mathrm{H}) \mathrm{ppm} . \delta_{\mathrm{C}}\left(100 \mathrm{MHz}, \mathrm{CDCl}_{3}\right)$ $175.50,142.96,142.17,140.23,139.59,129.21,127.60,127.27$, $125.13,124.85,124.30,122.40,101.00,100.54,36.28,18.74$ (6C), 11.42 (3C) ppm. HRMS (EI, $\mathrm{m} / \mathrm{z}$ ) calcd for $\mathrm{C}_{24} \mathrm{H}_{30} \mathrm{~N}_{2} \mathrm{OSSi}$ : 422.1848 , found: 422.1847 .

The $\mathrm{sp}^{3} \mathrm{C}-\mathrm{H}$ alkynylation (4a-g) were followed the conditions mentioned in Table S15 (entry 5) while 2 a coupling partner was used.

1-(1-Methyl-1H-imidazol-2-yl)-3-((triisopropylsilyl)ethynyl)hexan-1-one (5aa): Colorless<smiles>CCCC(C#C[PbH])CC(=O)c1nccn1C</smiles>
liquid; Yield: 69\% (49.7 mg); $R_{f}=0.67$ (50\% ethyl acetate-hexane). $\delta_{\mathrm{H}}$ $\left(400 \mathrm{MHz} \mathrm{CDCl}_{3}\right) 7.13(\mathrm{~d}, J=0.8 \mathrm{~Hz}, 1 \mathrm{H}), 7.01(\mathrm{~d}, J=0.8 \mathrm{~Hz}, 1 \mathrm{H})$, $3.99(\mathrm{~s}, 3 \mathrm{H}), 3.38-3.32(\mathrm{~m}, 1 \mathrm{H}), 3.24-3.09(\mathrm{~m}, 2 \mathrm{H}), 1.67-1.48(\mathrm{~m}, 4 \mathrm{H})$, 1.03-0.90 (m, 24H) ppm. $\delta_{\mathrm{C}}\left(100 \mathrm{MHz}, \mathrm{CDCl}_{3}\right) 190.97,143.33,129.21$, $127.05,111.18,81.38,44.62,37.24,36.27,28.60,20.56,18.70$ (6C), 13.96, 11.37 (3C) ppm. HRMS (EI ${ }^{+}, \mathrm{m} / \mathrm{z}$ ) calcd for $\mathrm{C}_{21} \mathrm{H}_{36} \mathrm{~N}_{2} \mathrm{OSi}$ : 360.2597 , found: 360.2596 .

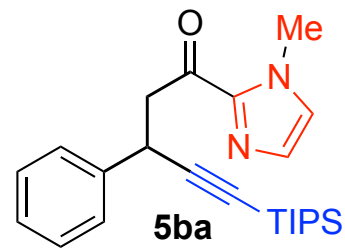
Colorless liquid; Yield: $83 \%$ (65.1mg); $R_{f}=0.61$ (50\% ethyl acetatehexane). $\delta_{\mathrm{H}}\left(400 \mathrm{MHz}, \mathrm{CDCl}_{3}\right)$ 7.47-7.44 (m, 2H), 7.32-7.27 (m, 2H), 7.23-7.18 (m, 1H), $7.12(\mathrm{~d}, J=0.8 \mathrm{~Hz}, 1 \mathrm{H}), 7.00(\mathrm{~s}, 1 \mathrm{H}), 4.49-4.45(\mathrm{~m}$, $1 \mathrm{H}), 3.97(\mathrm{~s}, 3 \mathrm{H}), 3.73-3.66(\mathrm{~m}, 1 \mathrm{H}), 3.50-3.44(\mathrm{~m}, 1 \mathrm{H}), 1.03-0.96(\mathrm{~m}$, $21 \mathrm{H}) \mathrm{ppm} . \delta_{\mathrm{C}}\left(100 \mathrm{MHz}, \mathrm{CDCl}_{3}\right) 189.74,143.08,141.20,129.31$, 128.58 (2C), 127.79 (2C), 127.13, 126.95, 109.08, 83.52, 47.89, 36.26, 34.56, 18.70 (6C), 11.33 (3C) ppm. HRMS (EI, $\mathrm{m} / \mathrm{z}$ ) calcd for $\mathrm{C}_{24} \mathrm{H}_{34} \mathrm{~N}_{2} \mathrm{OSi}$ : 394.2440, found: 394.2444 . 


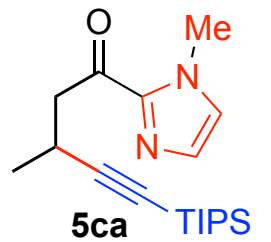

Colorless liquid (purified by GPC); Yield: 41\% (27.1 mg); $R_{f}=0.57(50 \%$ ethyl acetate-hexane). $\delta_{\mathrm{H}}\left(400 \mathrm{MHz}, \mathrm{CDCl}_{3}\right) 7.13(\mathrm{~d}, J=0.8 \mathrm{~Hz}, 1 \mathrm{H}), 7.02$ $(\mathrm{s}, 1 \mathrm{H}), 3.99$ (s, 3H), 3.39-3.31 (m, 1H), 3.25-3.17 (m, 2H), $1.26(\mathrm{~d}, J=6.44$ $\mathrm{Hz}, 3 \mathrm{H}), 1.04-0.95(\mathrm{~m}, 21 \mathrm{H}) \mathrm{ppm} . \delta_{\mathrm{C}}\left(100 \mathrm{MHz}, \mathrm{CDCl}_{3}\right) 190.58,143.03$, $129.03,126.96,112.22,80.15,45.85,36.16,23.11,21.12,18.53$ (6C), 11.13 (3C) ppm. HRMS $\left(\mathrm{EI}^{+}, \mathrm{m} / \mathrm{z}\right)$ calcd for $\mathrm{C}_{19} \mathrm{H}_{32} \mathrm{~N}_{2} \mathrm{OSi}$ : 332.2284, found: 332.2281 .

3-Cyclopentyl-1-(1-methyl-1H-imidazol-2-yl)-5-(triisopropylsilyl)pent-4-yn-1-one (5da):

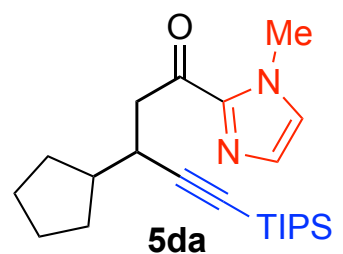

Colorless liquid; Yield: 55\% (42.2 mg); $R_{f}=0.64$ (50\% ethyl acetatehexane). $\delta_{\mathrm{H}}\left(400 \mathrm{MHz}, \mathrm{CDCl}_{3}\right) 7.13(\mathrm{~s}, 1 \mathrm{H}), 7.01(\mathrm{~s}, 1 \mathrm{H}), 3.98(\mathrm{~s}, 3 \mathrm{H})$, 3.42-3.35 (m, 1H), 3.21-3.15 (m, 2H), 2.05-1.43 (m, 9H), 1.06-0.91 (m, $21 \mathrm{H}) \mathrm{ppm} . \delta_{\mathrm{C}}\left(100 \mathrm{MHz}, \mathrm{CDCl}_{3}\right) 191.10,143.49,129.19,127.05$, $109.94,81.63,43.92,43.46,36.33,33.61,31.18,29.06,25.90,25.80$, 18.70 (6C), 11.33 (3C) ppm. HRMS (EI, $\mathrm{m} / \mathrm{z}$ ) calcd for $\mathrm{C}_{23} \mathrm{H}_{38} \mathrm{~N}_{2} \mathrm{OSi}$ : 386.2753, found: 386.2747.

\section{cis-(1-Methyl-1H-imidazol-2-yl)(2-((triisopropylsilyl)ethynyl)cyclopentyl)methanone}

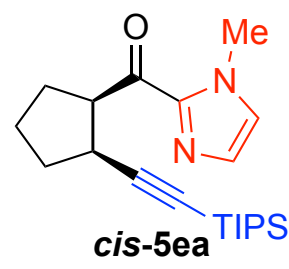

(cis-5ea): Isolated yield 51\% (5:1; cis-trans mixture based on NMR); Colorless liquid (purified by GPC followed by column chromatography); Yield: $32 \%$ ( $22.7 \mathrm{mg}) ; R_{f}=0.57$ (50\% ethyl acetate-hexane). $\delta_{\mathrm{H}}(400 \mathrm{MHz}$, $\left.\mathrm{CDCl}_{3}\right) 7.14(\mathrm{~s}, 1 \mathrm{H}), 7.02(\mathrm{~d}, J=0.8 \mathrm{~Hz}, 1 \mathrm{H}), 4.23$ (ddd, $J=7.2,7.6,8.0$ $\mathrm{Hz}, 1 \mathrm{H}), 3.99(\mathrm{~s}, 3 \mathrm{H}), 3.18(\mathrm{ddd}, J=7.6,7.6,7.2 \mathrm{~Hz}, 1 \mathrm{H}), 2.30-2.10(\mathrm{~m}$, $2 \mathrm{H}), 1.87-1.68(\mathrm{~m}, 4 \mathrm{H}), 1.06-0.91(\mathrm{~m}, 21 \mathrm{H}) \mathrm{ppm} . \delta_{\mathrm{C}}\left(100 \mathrm{MHz}, \mathrm{CDCl}_{3}\right)$ 194.18, 143.15, 129.37, 127.19, 111.68, 80.44, 54.56, 36.37, 34.48, 34.11, 31.68, 25.12, 18.69 (6C), 11.30 (3C) ppm. HRMS (EI ${ }^{+}, \mathrm{m} / \mathrm{z}$ ) calcd for $\mathrm{C}_{21} \mathrm{H}_{34} \mathrm{~N}_{2} \mathrm{OSi}$ : 358.2440, found: 358.2434 . The stereochemistry was confirmed by NOESY experiments.

cis-(1-Methyl-1H-imidazol-2-yl)(2-((triisopropylsilyl)ethynyl)cyclohexyl)methanone (cis5fa): Isolated yield 63\% (10:3.5; cis-trans mixture based on NMR); Colorless liquid (purified<smiles>Cn1ccnc1C(=O)[C@@H]1CCCC[C@@H]1C#CCS</smiles>
by GPC followed by column chromatography); Yield: 47\% (35 mg); $R_{f}=$ $0.71\left(50 \%\right.$ ethyl acetate-hexane). $\delta_{\mathrm{H}}\left(400 \mathrm{MHz}, \mathrm{CDCl}_{3}\right) 7.13(\mathrm{~s}, 1 \mathrm{H}), 7.00$ (s, 1H), 3.98 (s, 3H), 3.92 (ddd, $J=5.6,11.2,3.2 \mathrm{~Hz}, 1 \mathrm{H}), 2.71$ (ddd, $J=$ 4.0, 11.6, $3.6 \mathrm{~Hz}, 1 \mathrm{H}), 2.06-1.93(\mathrm{~m}, 2 \mathrm{H}), 1.75-1.74(\mathrm{~m}, 2 \mathrm{H}), 1.54-1.30$ $(\mathrm{m}, 4 \mathrm{H}), 0.90-0.82(\mathrm{~m}, 21 \mathrm{H}) \mathrm{ppm} . \delta_{\mathrm{C}}\left(100 \mathrm{MHz}, \mathrm{CDCl}_{3}\right)$ 195.31, 143.11, $129.24,127.25,111.34,80.32,50.76,36.39,32.44,32.33,29.73,25.38,25.15,18.52$ (3C), 18.49 (3C), 11.16 (3C) ppm. HRMS $\left(\mathrm{EI}^{+}, \mathrm{m} / \mathrm{z}\right.$ ) calcd for $\mathrm{C}_{22} \mathrm{H}_{36} \mathrm{~N}_{2} \mathrm{OSi}$ : 372.2597, found: 372.2601. The stereochemistry was confirmed by NOESY experiments.

1-(1-Methyl-1H-imidazol-2-yl)-3-((triisopropylsilyl)ethynyl)dodecan-1-one

(5ga):

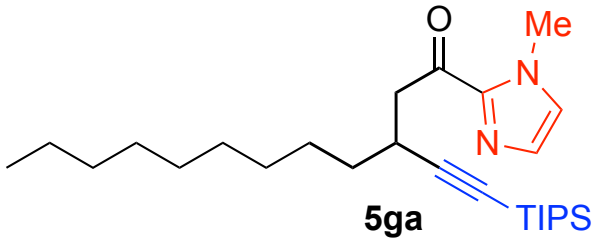

Colorless oily liquid; Yield: $75 \%$ (66.6 mg); $R_{f}=0.64$ (50\% ethyl acetate-hexane). $\delta_{\mathrm{H}}\left(400 \mathrm{MHz}, \mathrm{CDCl}_{3}\right) 7.13$ $(\mathrm{s}, 1 \mathrm{H}), 7.01(\mathrm{~s}, 1 \mathrm{H}), 3.99(\mathrm{~s}, 3 \mathrm{H}), 3.38-3.32(\mathrm{~m}, 1 \mathrm{H})$, 3.24-3.09 (m, 2H), 1.67-1. $13(\mathrm{~m}, 16 \mathrm{H}), 1.06-0.91(\mathrm{~m}$, $21 \mathrm{H}), 0.89-0.85(\mathrm{~m}, 3 \mathrm{H}) \mathrm{ppm} . \delta_{\mathrm{C}}\left(100 \mathrm{MHz}, \mathrm{CDCl}_{3}\right)$ $190.95,143.23$, 129.16, 127.07, 111.16, 81.38, 44.59, 36.33, 35.03, 32.05, 29.71, 29.66, 29.49, 
29.42, 28.79, 27. 30, 22.84, 18.71 (6C), 14.29, 11.33(3C) ppm. HRMS (EI, $\mathrm{m} / \mathrm{z})$ calcd for $\mathrm{C}_{27} \mathrm{H}_{48} \mathrm{~N}_{2} \mathrm{OSi}$ : 444.3536 , found: 444.3533 .

The optimized conditions for $\mathrm{C}-\mathrm{H}$ alkynylation with coupling partner $\mathbf{2 b}, \mathbf{2 c}$ and $\mathbf{2 d}$ were mention in Table 16 (entry 4, 7 and 8 respectively) and followed herein.

(1-Methyl-1H-imidazol-2-yl)(2-methyl-6-(p-tolylethynyl)phenyl)methanone (6ab): Light

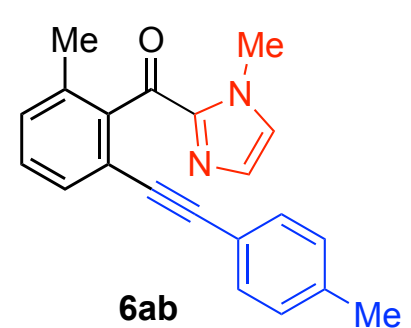

yellow solid (purified by GPC); Yield: $46 \%$ (28.6 mg); mp. 151-152 ${ }^{\circ} \mathrm{C} . \quad R_{f}=0.42\left(50 \%\right.$ ethyl acetate-hexane). $\delta_{\mathrm{H}}\left(400 \mathrm{MHz}, \mathrm{CDCl}_{3}\right)$ 7.40-7.38 (m, 1H), $7.30(\mathrm{t}, J=7.6 \mathrm{~Hz}, 1 \mathrm{H}), 7.24-7.21(\mathrm{~m}, 1 \mathrm{H}), 7.18$ $(\mathrm{d}, J=0.8 \mathrm{~Hz}, 1 \mathrm{H}), 7.09-7.03(\mathrm{~m}, 5 \mathrm{H}) 4.11(\mathrm{~s}, 3 \mathrm{H}), 2.32(\mathrm{~s}, 3 \mathrm{H})$, $2.31(\mathrm{~s}, 3 \mathrm{H}) \mathrm{ppm} . \delta_{\mathrm{C}}\left(100 \mathrm{MHz}, \mathrm{CDCl}_{3}\right) 189.24,144.16,142.01$, $138.55,135.17,131.43(2 \mathrm{C}), 130.59,130.49,129.48,129.22$, 128.09 (2C), 126.85, 121.17, 120.11, 93.76, 86.81, 36.04, 21.60, 19.54 ppm. HRMS (EI $\left.{ }^{+}, \mathrm{m} / \mathrm{z}\right)$ calcd for $\mathrm{C}_{21} \mathrm{H}_{18} \mathrm{~N}_{2} \mathrm{O}: 314.1419$, found: 414.1421 .

(1-Methyl-1H-imidazol-2-yl)(2-(p-tolylethynyl)-5-(trifluoromethyl)phenyl)methanone

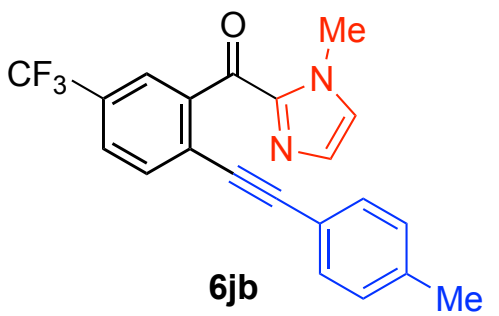

(6jb): Off-white solid (purified by GPC); Yield: 38\% (27.7 $\mathrm{mg}$ ); mp. $121-122{ }^{\circ} \mathrm{C} . \quad R_{f}=0.50$ (50\% ethyl acetate-hexane). $\delta_{\mathrm{H}}\left(400 \mathrm{MHz}, \mathrm{CDCl}_{3}\right) 7.96(\mathrm{t}, J=0.4 \mathrm{~Hz}, 1 \mathrm{H}), 7.71(\mathrm{~d}, J=1.2$ $\mathrm{Hz}, 2 \mathrm{H}), 7.23-7.17(\mathrm{~m}, 3 \mathrm{H}), 7.14(\mathrm{~d}, J=0.4 \mathrm{~Hz}, 1 \mathrm{H}), 7.12-7.09$ $(\mathrm{m}, 2 \mathrm{H}) 4.13(\mathrm{~s}, 3 \mathrm{H}), 2.34(\mathrm{~s}, 3 \mathrm{H}) \mathrm{ppm} . \delta_{\mathrm{C}}\left(100 \mathrm{MHz}, \mathrm{CDCl}_{3}\right)$ $185.05,143.20,141.31,139.34,133.30,131.73$ (2C), 130.57, 129.46 (qt, $J=33.6 \mathrm{~Hz}$ ), 129.21 (2C), 127.39, 127.20 (qt, $J=2.9 \mathrm{~Hz}), 126.35,126.31,123.75$ (qt, $J=271.1 \mathrm{~Hz}), 119.42,96.74,85.93,36.29,21.70 \mathrm{ppm} . \delta_{\mathrm{F}}\left(376 \mathrm{MHz}, \mathrm{CDCl}_{3}\right)-62.49 \mathrm{ppm}$. HRMS (EI ${ }^{+}, \mathrm{m} / \mathrm{z}$ ) calcd for $\mathrm{C}_{21} \mathrm{H}_{15} \mathrm{~F}_{3} \mathrm{~N}_{2} \mathrm{O}: 368.1136$, found: 368.1133 .

(1-Methyl-1H-imidazol-2-yl)(2-methyl-6-((1-

((triethylsilyl)oxy)cyclohexyl)ethynyl)phenyl)methanone (6ac): Colorless thick oil; Yield:

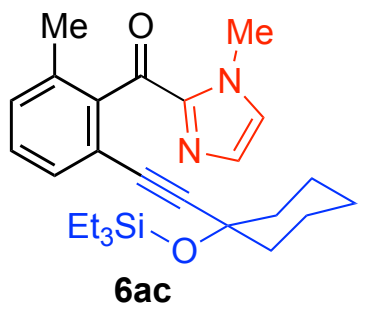
$89 \%(77.3 \mathrm{mg}) ; R_{f}=0.54\left(50 \%\right.$ ethyl acetate-hexane). $\delta_{\mathrm{H}}(400 \mathrm{MHz}$, $\left.\mathrm{CDCl}_{3}\right)$ 7.32-7.25 (m, 2H), 7.21-7.19 (m, 1H), $7.18(\mathrm{~d}, J=0.8 \mathrm{~Hz}, 1 \mathrm{H})$, $7.08(\mathrm{~s}, 1 \mathrm{H}) 4.14(\mathrm{~s}, 3 \mathrm{H}), 2.24(\mathrm{~s}, 3 \mathrm{H}), 1.67-1.06(\mathrm{~m}, 10 \mathrm{H}), 0.93-0.88$ $(\mathrm{m}, 9 \mathrm{H}), 0.63-0.56(\mathrm{~m}, 6 \mathrm{H}) \mathrm{ppm} . \delta_{\mathrm{C}}\left(100 \mathrm{MHz}, \mathrm{CDCl}_{3}\right)$ 189.19, $143.57,142.14,134.77,130.87,130.34,129.60,129.06,127.06$, $120.59,97.31,83.34,69.85,41.40$ (2C), 36.21, 25.40, 23.07 (2C), 19.45, 7.20 (3C), 6.20 (3C) ppm. HRMS ( $\left.\mathrm{EI}^{+}, \mathrm{m} / \mathrm{z}\right)$ calcd for $\mathrm{C}_{26} \mathrm{H}_{36} \mathrm{~N}_{2} \mathrm{O}_{2} \mathrm{Si}$ : 436.2546, found: 436.2546 .

(5-Methoxy-2-((1-((triethylsilyl)oxy)cyclohexyl)ethynyl)phenyl)(1-methyl-1H-imidazol-

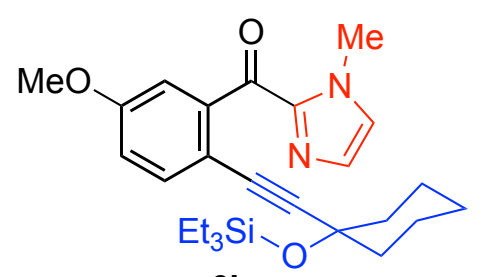

6ic

2-yl)methanone (6ic): Colorless sticky (purified by GPC); Yield: 47\% (42.5 mg); $R_{f}=0.46$ (50\% ethyl acetate-hexane). $\delta_{\mathrm{H}}$ $\left(400 \mathrm{MHz}, \mathrm{CDCl}_{3}\right) 7.41(\mathrm{~d}, J=8.4 \mathrm{~Hz}, 1 \mathrm{H}), 7.19(\mathrm{~d}, J=1.2 \mathrm{~Hz}$, $1 \mathrm{H}), 7.09(\mathrm{~d}, J=0.4 \mathrm{~Hz}, 1 \mathrm{H}), 7.07(\mathrm{~d}, J=2.8 \mathrm{~Hz}, 1 \mathrm{H}), 6.96(\mathrm{dd}$, $J=8.8,2.8 \mathrm{~Hz}, 1 \mathrm{H}), 4.10(\mathrm{~s}, 3 \mathrm{H}), 3.82(\mathrm{~s}, 3 \mathrm{H}), 1.68-1.12(\mathrm{~m}$, $10 \mathrm{H}), 0.92-0.84(\mathrm{~m}, 9 \mathrm{H}), 0.63-0.57(\mathrm{~m}, 6 \mathrm{H}) \mathrm{ppm} . \delta_{\mathrm{C}}(100 \mathrm{MHz}$, $\left.\mathrm{CDCl}_{3}\right)$ 186.82, 159.05, 143.33, 143.05, 134.08, 130.56, 127.04, 116.54, 114.03, 113.39, 96.46, 82.84, 69.86, 55.58, 41.39 (2C), 36.22, 25.45, 23.06 (2C), 7.20 (3C), 6.23 (3C) ppm. HRMS $\left(\mathrm{EI}^{+}, \mathrm{m} / \mathrm{z}\right.$ ) calcd for $\mathrm{C}_{26} \mathrm{H}_{36} \mathrm{~N}_{2} \mathrm{O}_{3} \mathrm{Si}: 452.2495$, found: 452.2490 . 
(1-Methyl-1H-imidazol-2-yl)(2-((1-((triethylsilyl)oxy)cyclohexyl)ethynyl)-5-

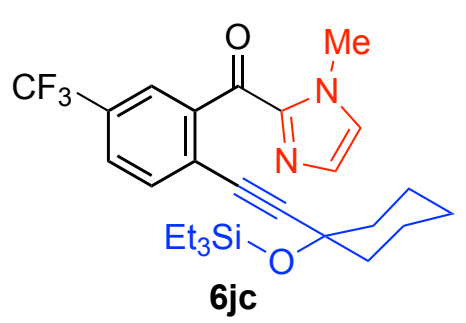

(trifluoromethyl)phenyl)methanone (6jc): Colorless thick oil (purified by GPC); Yield: $40 \%$ (39.1 mg); $R_{f}=0.64$ (50\% ethyl acetate-hexane). $\delta_{\mathrm{H}}\left(400 \mathrm{MHz}, \mathrm{CDCl}_{3}\right) 7.83-7.82(\mathrm{~m}, 1 \mathrm{H}), 7.70-$ $7.66(\mathrm{~m}, 1 \mathrm{H}), 7.61-7.58(\mathrm{~m}, 1 \mathrm{H}), 7.22(\mathrm{~d}, J=0.8 \mathrm{~Hz}, 1 \mathrm{H}), 7.13$ $(\mathrm{s}, 1 \mathrm{H}), 4.13(\mathrm{~s}, 3 \mathrm{H}), 1.72-1.25(\mathrm{~m}, 10 \mathrm{H}), 0.95-0.88(\mathrm{~m}, 9 \mathrm{H})$, $0.67-0.58(\mathrm{~m}, 6 \mathrm{H}) \mathrm{ppm} . \delta_{\mathrm{C}}\left(100 \mathrm{MHz}, \mathrm{CDCl}_{3}\right) 185.31,142.89$, $142.05,133.03,130.82,129.68$ (qt, $J=132.58 \mathrm{~Hz}), 127.77,127.55,126.92,125.48,123.70$ (qt, $J=271.2 \mathrm{~Hz}$ ), 100.74, 81.99, 69.90, 41.18 (2C), 36.28, 25.32, 22.99 (2C), 7.13 (3C), 6.22 (3C) ppm. $\delta_{\mathrm{F}}\left(376 \mathrm{MHz}, \mathrm{CDCl}_{3}\right)-62.69 \mathrm{ppm}$. HRMS $\left(\mathrm{EI}^{+}, \mathrm{m} / \mathrm{z}\right)$ calcd for $\mathrm{C}_{26} \mathrm{H}_{33} \mathrm{~F}_{3} \mathrm{~N}_{2} \mathrm{O}_{2} \mathrm{Si}$ : 490.2263, found: 490.2259 .

(2-Fluoro-6-((1-((triethylsilyl)oxy)cyclohexyl)ethynyl)phenyl)(1-methyl-1H-imidazol-2-

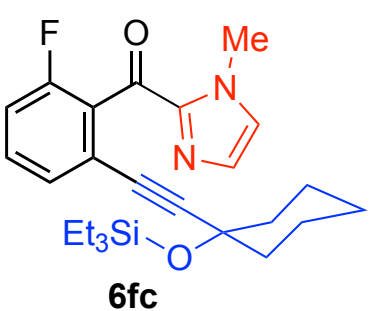

yl)methanone (6fe): Colorless oil; Yield: $61 \%$ (53.4 mg); $R_{f}=0.57$ (50\% ethyl acetate-hexane). $\delta_{\mathrm{H}}\left(400 \mathrm{MHz}, \mathrm{CDCl}_{3}\right) 7.39-7.33(\mathrm{~m}$, $1 \mathrm{H}), 7.28-7.25(\mathrm{~m}, 1 \mathrm{H}), 7.21(\mathrm{~s}, 1 \mathrm{H}), 7.13-7.08(\mathrm{~m}, 2 \mathrm{H}), 4.13(\mathrm{~s}, 3 \mathrm{H})$, $1.72-1.11(\mathrm{~m}, 10 \mathrm{H}), 0.93-0.88(\mathrm{~m}, 9 \mathrm{H}), 0.63-0.56(\mathrm{~m}, 6 \mathrm{H}) \mathrm{ppm} . \delta_{\mathrm{C}}$ $\left(100 \mathrm{MHz}, \mathrm{CDCl}_{3}\right) 183.17,159.02(\mathrm{~d}, J=246.3 \mathrm{~Hz}), 143.26,131.05$ (2C), $130.92(\mathrm{~d}, J=9.6 \mathrm{~Hz}), 130.28(\mathrm{~d}, J=19.2 \mathrm{~Hz}), 128.00$ (d, $J=$ $2.9 \mathrm{~Hz}), 127.57$ (2C), 123.09 (d, $J=5.8 \mathrm{~Hz}), 115.98$ (d, $J=22.0 \mathrm{~Hz}), 98.45,81.86,69.92$, 41.28, 36.22, 25.36, 23.07, $7.16(3 \mathrm{C}), 6.19(3 \mathrm{C}) \mathrm{ppm} . \delta_{\mathrm{F}}\left(376 \mathrm{MHz}, \mathrm{CDCl}_{3}\right)-115.26 \mathrm{ppm}$. HRMS (EI, $\mathrm{m} / \mathrm{z})$ calcd for $\mathrm{C}_{25} \mathrm{H}_{33} \mathrm{FN}_{2} \mathrm{O}_{2} \mathrm{Si}: 440.6344$, found: 440.2297.

\section{(1-Methyl-1H-imidazol-2-yl)(3-((1-}

((triethylsilyl)oxy)cyclohexyl)ethynyl)benzo[b]thiophen-2-yl)methanone (6sc): Colorless

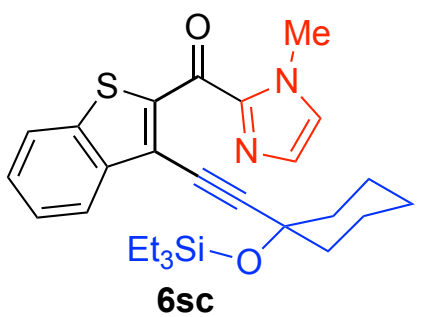
thick oil; Yield: $43 \%(40.7 \mathrm{mg}) ; R_{f}=0.68(50 \%$ ethyl acetatehexane). $\delta_{\mathrm{H}}\left(400 \mathrm{MHz}, \mathrm{CDCl}_{3}\right)$ 8.09-8.06 $(\mathrm{m}, 1 \mathrm{H}), 7.86-7.83(\mathrm{~m}$, $1 \mathrm{H}), 7.51-7.43(\mathrm{~m}, 2 \mathrm{H}), 7.26(\mathrm{~s}, 1 \mathrm{H}), 7.12(\mathrm{~d}, J=0.4 \mathrm{~Hz}, 1 \mathrm{H}), 4.12$ $(\mathrm{s}, 3 \mathrm{H}), 2.09-2.05(\mathrm{~m}, 2 \mathrm{H}), 1.80-1.20(\mathrm{~m}, 8 \mathrm{H}), 0.98-0.94(\mathrm{~m}, 9 \mathrm{H})$, 0.75-0.68 (m, 6H) ppm. $\delta_{\mathrm{C}}\left(100 \mathrm{MHz}, \mathrm{CDCl}_{3}\right) 175.62,143.09$, $142.29,139.56,139.51,129.29,127.75,127.45,125.16,124.95$, 124.14, 122.60, 102.55, 79.57, 70.40, 41.55 (2C), 36.50, 25.56, 23.19 (2C), 7.27 (3C), 6.33 (3C) ppm. HRMS ( $\mathrm{EI}^{+}, \mathrm{m} / \mathrm{z}$ ) calcd for $\mathrm{C}_{27} \mathrm{H}_{34} \mathrm{~N}_{2} \mathrm{O}_{2} \mathrm{SSi}$ : 478.2110, found: 478.2105.

\section{2-(2-(1-Methyl-1H-imidazole-2-carbonyl)benzo[b]thiophen-3-yl)-1-(1-}

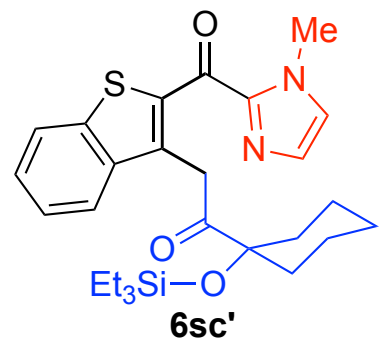

((triethylsilyl)oxy)cyclohexyl)ethan-1-one (6sc'): Off-white solid; Yield: $17 \%(16.8 \mathrm{mg})$; $\mathrm{mp} .132-133{ }^{\circ} \mathrm{C} . R_{f}=0.64(50 \%$ ethyl acetatehexane). $\delta_{\mathrm{H}}\left(400 \mathrm{MHz}, \mathrm{CDCl}_{3}\right) 7.87(\mathrm{~d}, J=8.0 \mathrm{~Hz}, 1 \mathrm{H}), 7.71(\mathrm{~d}, J=$ $8.0 \mathrm{~Hz}, 1 \mathrm{H}), 7.47-7.43(\mathrm{~m}, 1 \mathrm{H}), 7.40-7.35(\mathrm{~m}, 1 \mathrm{H}), 7.25(\mathrm{~d}, J=0.8$ $\mathrm{Hz}, 1 \mathrm{H}), 7.08(\mathrm{~s}, 1 \mathrm{H}), 4.79$ (s, 2H), $4.04(\mathrm{~s}, 3 \mathrm{H}), 2.20-2.14(\mathrm{~m}, 2 \mathrm{H})$, $1.75-1.41(\mathrm{~m}, 8 \mathrm{H}), 1.03-0.99(\mathrm{~m}, 9 \mathrm{H}), 0.74-0.68(\mathrm{~m}, 6 \mathrm{H}) \mathrm{ppm} . \delta_{\mathrm{C}}(100$ $\left.\mathrm{MHz}, \mathrm{CDCl}_{3}\right)$ 208.39, 177.75, 143.52, 143.33, 139.37, 139.18, $134.20,128.98,127.41,127.24,124.53,123.38,122.80,81.53,36.65,36.55,36.19$ (2C), 25.55, 
22.68 (2C), 7.44 (3C), 7.07 (3C) ppm. HRMS ( $\mathrm{EI}^{+}, \mathrm{m} / \mathrm{z}$ ) calcd for $\mathrm{C}_{27} \mathrm{H}_{36} \mathrm{~N}_{2} \mathrm{O}_{3} \mathrm{SSi}$ : 496.2216, found: 496.2207.

(1-Methyl-1H-imidazol-2-yl)(2-((1-((triethylsilyl)oxy)cyclohexyl)ethynyl)cyclohex-1-en-<smiles>CCOC1(C#CC2=C(C(=O)c3nccn3C)CCCC2)CCCCC1</smiles>

7

1-yl)methanone (7): Colorless liquid (purified by GPC); Yield: 44\% (37.5 mg); $R_{f}=0.64$ (50\% ethyl acetate-hexane). $\delta_{\mathrm{H}}(400 \mathrm{MHz}$, $\left.\mathrm{CDCl}_{3}\right) 7.19(\mathrm{~d}, J=0.8 \mathrm{~Hz}, 1 \mathrm{H}), 7.02(\mathrm{~d}, J=0.8 \mathrm{~Hz}, 1 \mathrm{H}), 4.00(\mathrm{~s}, 3 \mathrm{H})$, 2.48-2.44 (m, 2H), 2.32-2.25(m, 2H), 1.76-1.72 (m, 4H), 1.65-1.15 $(\mathrm{m}, 10 \mathrm{H}), 0.91-0.86(\mathrm{~m}, 9 \mathrm{H}), 0.60-0.54(\mathrm{~m}, 6 \mathrm{H}) \mathrm{ppm} . \delta_{\mathrm{C}}(100 \mathrm{MHz}$, $\left.\mathrm{CDCl}_{3}\right) 189.93,143.71,143.10,130.15,126.65,121.69,98.14,84.34$, 69.79, 41.45 (2C), 36.04, 30.06, 27.15, 25.41, 23.00 (2C), 21.87, 21.59, 7.20 (3C), 6.21 (3C) ppm. HRMS (EI, $\mathrm{m} / \mathrm{z}$ ) calcd for $\mathrm{C}_{25} \mathrm{H}_{38} \mathrm{~N}_{2} \mathrm{O}_{2} \mathrm{Si}$ : 426.2703, found: 426.2696.

(2-(((8R,9S,13S,14S,17S)-3,17-Bis((tert-butyldimethylsilyl)oxy)-13-methyl-

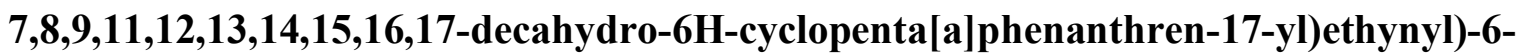
methylphenyl)(1-methyl-1H-imidazol-2-yl)methanone (6ad): Yellowish sticky (purified by

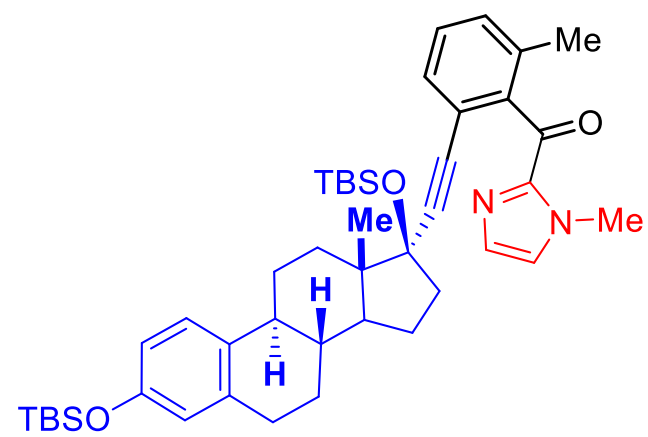
GPC); Yield: 44\% (63.5 mg); $R_{f}=0.57$ (50\% ethyl acetate-hexane). $\delta_{\mathrm{H}}\left(400 \mathrm{MHz}, \mathrm{CDCl}_{3}\right)$ 7.37-7.34 (m, $1 \mathrm{H}), 7.30-7.26(\mathrm{~m}, 1 \mathrm{H}), 7.20-7.17(\mathrm{~m}, 1 \mathrm{H}), 7.15(\mathrm{~d}, J$ $=0.8 \mathrm{~Hz}, 1 \mathrm{H}), 7.09(\mathrm{~d}, J=8.8 \mathrm{~Hz}, 1 \mathrm{H}), 7.03(\mathrm{~s}, 1 \mathrm{H})$, 6.63-6.60 (m, 1H), $6.54(\mathrm{~d}, J=2.4 \mathrm{~Hz}, 1 \mathrm{H}), 4.14(\mathrm{~s}$, $3 \mathrm{H}), 2.80-2.69(\mathrm{~m}, 2 \mathrm{H}), 2.20(\mathrm{~s}, 3 \mathrm{H}), 2.05-1.15(\mathrm{~m}$, $13 \mathrm{H}), 0.98(\mathrm{~s}, 9 \mathrm{H}), 0.84(\mathrm{~s}, 9 \mathrm{H}), 0.77(\mathrm{~s}, 3 \mathrm{H}), 0.19(\mathrm{~s}$, $6 \mathrm{H}), 0.09(\mathrm{~s}, 6 \mathrm{H}) \mathrm{ppm}$.

$\delta_{\mathrm{C}}\left(100 \mathrm{MHz}, \mathrm{CDCl}_{3}\right) 189.11,153.31,143.49,141.86$, $138.03,134,70,133.30,130.87,130.25,130.13,129.08,127.13,126.16,120.64,120.05$, $117.21,97.35,84.67,80.96,48.51,48.36,43.51,40.63,39.48,36.34,32.85,29.84,27.32$, 26.69, 25.94 (3C), 25.84 (3C), 23.16, 19.39, 18.30 (2C), 13.32, -3.04 (2C), -4.24 (2C) ppm. HRMS $\left(\mathrm{EI}^{+}, \mathrm{m} / \mathrm{z}\right.$ ) calcd for $\mathrm{C}_{44} \mathrm{H}_{62} \mathrm{~N}_{2} \mathrm{O}_{3} \mathrm{Si}_{2}: 722.4299$, found: 722.4309 . 


\section{Mechanistic Studies}

\section{A. Deuterium Scrambling Experiments}

(a) Without coupling partner<smiles>Cc1ccccc1C(=O)c1nccn1C</smiles>

$\left[\mathrm{Cp}^{*} \mathrm{IrCl}_{2}\right]_{2}(2.5 \mathrm{~mol} \%)$

$\underset{\mathrm{K}_{2} \mathrm{CO}_{3}(1.5 \text { eq. })}{\mathrm{NaBAr}_{4}(5 \mathrm{~mol} \%)}$

AgOAc (5 mol\%)

$1 \mathrm{a}(0.2 \mathrm{mmol})$

$\mathrm{DCE} / \mathrm{D}_{2} \mathrm{O}(0.9 / 0.1 \mathrm{~mL}), 60^{\circ} \mathrm{C}, 17 \mathrm{~h}$

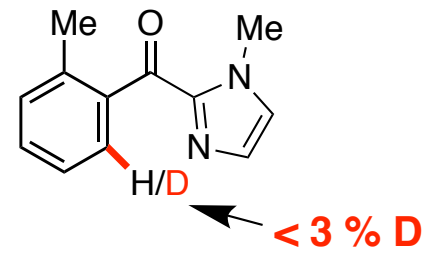

$[D]_{n}-1 a ; 95 \%$

In a screw-cap reaction tube, compound $1 \mathrm{a}(40 \mathrm{mg}, 0.2 \mathrm{mmol}),\left[\mathrm{Cp}^{*} \mathrm{IrCl}_{2}\right]_{2}(4 \mathrm{mg}, 2.5 \mathrm{~mol} \%)$, $\mathrm{K}_{2} \mathrm{CO}_{3}(41.4 \mathrm{mg}, 0.3 \mathrm{mmol}), \mathrm{NaBAr}_{4}(8.8 \mathrm{mg}, 5 \mathrm{~mol} \%)$, AgOAc (2 mg, $\left.5 \mathrm{~mol} \%\right)$, DCE (0.9 $\mathrm{mL})$ and $\mathrm{D}_{2} \mathrm{O}(0.1 \mathrm{~mL})$ were placed under $\mathrm{N}_{2}$ and were then stirred at $60{ }^{\circ} \mathrm{C}$ for $17 \mathrm{~h}$. The reaction mixture was then diluted with $\mathrm{H}_{2} \mathrm{O}(10 \mathrm{~mL})$ and extracted with EtOAc $(3 \times 15 \mathrm{~mL})$. The combined organic layers were dried over $\mathrm{Na}_{2} \mathrm{SO}_{4}$, concentrated under reduced pressure and purified by silica gel column chromatography. The pure compound was eluted at a concentration of $20 \%$ EtOAc-hexane as a white viscous oil [D] $]_{\mathbf{n}}-\mathbf{1 a}(38 \mathrm{mg}, 95 \%) .{ }^{1} \mathrm{H}$ NMR spectroscopy was used to determine the D incorporation (Figure 1).

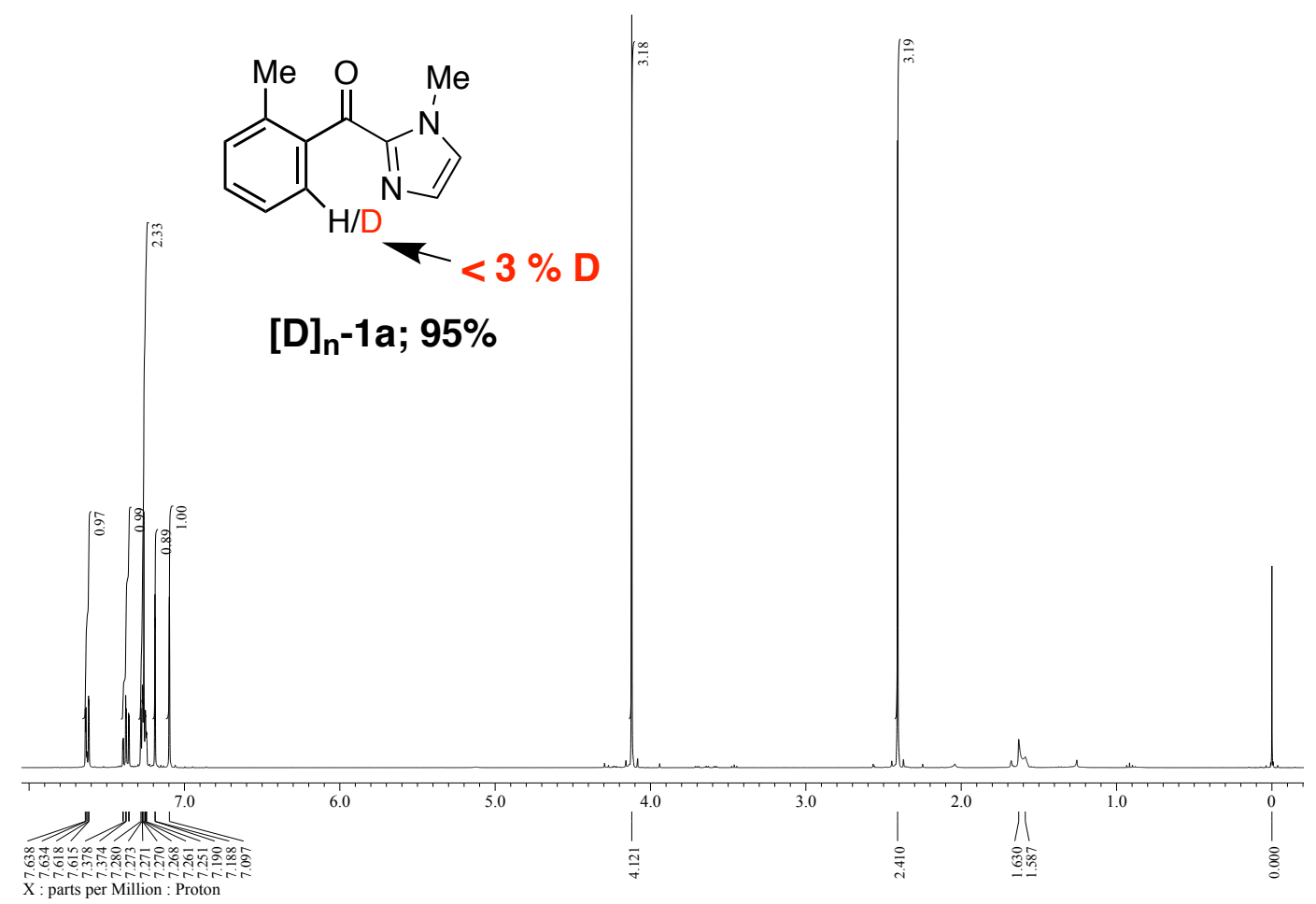

Figure S1: ${ }^{1} \mathrm{H}$ NMR spectrum of $[\mathbf{D}]_{\mathbf{n}}-\mathbf{1 a}\left(400 \mathrm{MHz}, \mathrm{CDCl}_{3}\right)$ for D incorporation study. 
(b) With coupling partner<smiles>Cc1ccccc1C(=O)c1nccn1C</smiles>

$1 \mathrm{a}(0.1 \mathrm{mmol})$

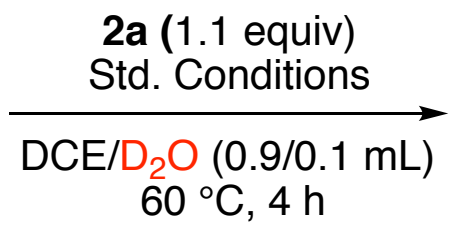

(NIPS

$[D]_{n}-1 a ; 60 \% \quad 3 a a ; 36 \%$

In a screw-cap reaction tube, compound $1 \mathbf{a}(40 \mathrm{mg}, 0.2 \mathrm{mmol})$, TIPS-bromoalkyne 2a $(57.2$ $\mathrm{mg}, 0.22 \mathrm{mmol}),\left[\mathrm{Cp}^{*} \mathrm{IrCl}_{2}\right]_{2}(4 \mathrm{mg}, 2.5 \mathrm{~mol} \%), \mathrm{K}_{2} \mathrm{CO}_{3}(41.4 \mathrm{mg}, 0.3 \mathrm{mmol}), \mathrm{NaBAr}_{4}{ }_{4}(8.8$ $\mathrm{mg}, 5 \mathrm{~mol} \%)$, AgOAc $(2 \mathrm{mg}, 5 \mathrm{~mol} \%)$, DCE $(0.9 \mathrm{~mL})$ and $\mathrm{D}_{2} \mathrm{O}(0.1 \mathrm{~mL})$ were placed under $\mathrm{N}_{2}$ and were then stirred at $60{ }^{\circ} \mathrm{C}$ for $4 \mathrm{~h}$. The reaction mixture was then diluted with $\mathrm{H}_{2} \mathrm{O}(10$ $\mathrm{mL})$ and extracted with EtOAc $(3 \times 15 \mathrm{~mL})$. The combined organic layers were dried with $\mathrm{Na}_{2} \mathrm{SO}_{4}$ and concentrated under reduced pressure and purified by silica gel column chromatography (230-400 mesh). Compound 3aa was eluted at 10\% EtOAc-hexane as a white solid (27 mg, 35.5\%) and compound [D] $\mathbf{n}-1 \mathbf{a}$ at $20 \%$ EtOAc-hexane as a white sticky (22 mg, $55 \%$ ). ${ }^{1} \mathrm{H}-\mathrm{NMR}$ spectroscopy was used to determine the D incorporation (Figure 2).

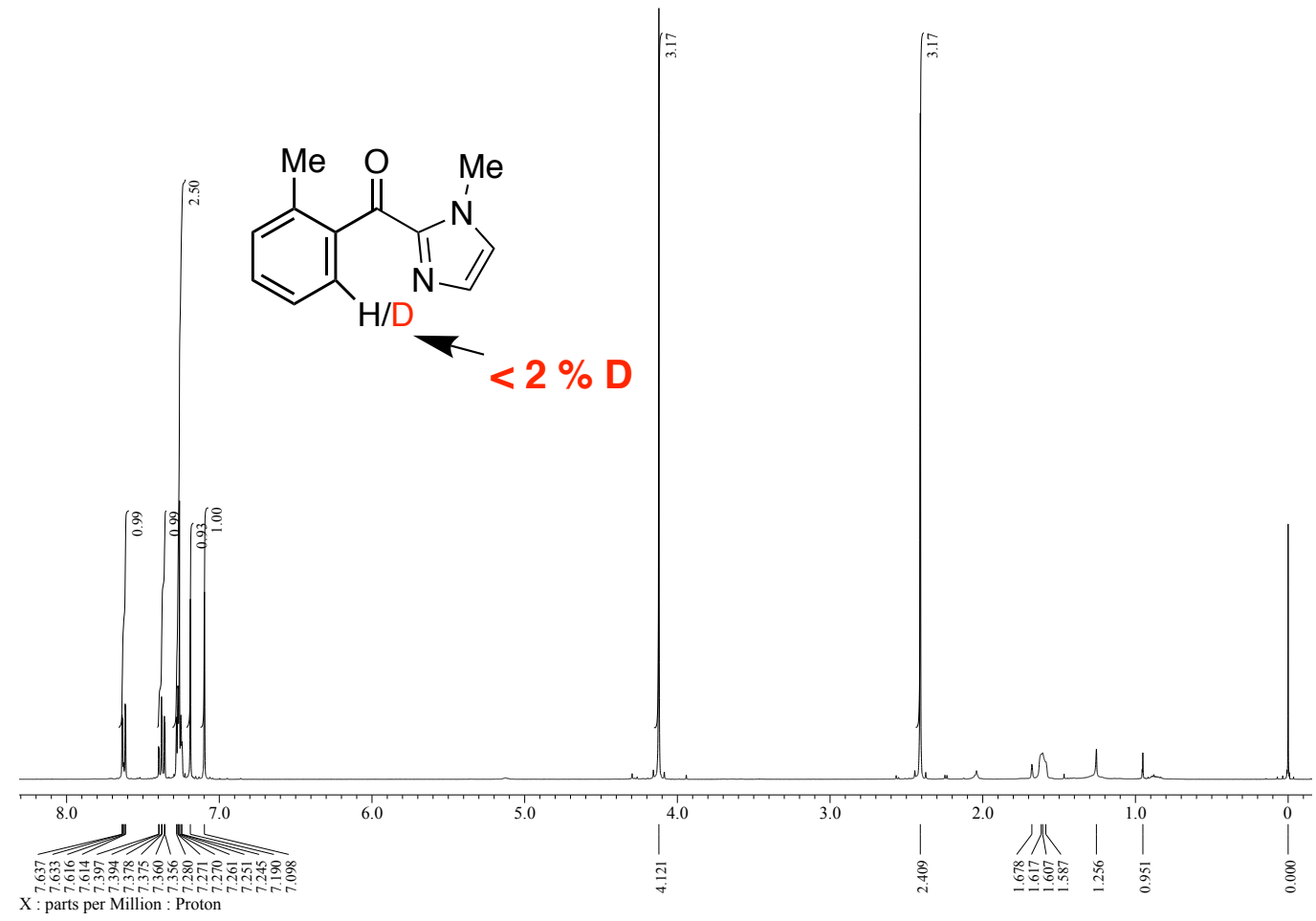

Figure S2: ${ }^{1} \mathrm{H}$ NMR spectrum of $[\mathbf{D}]_{\mathbf{n}}-\mathbf{1 a}\left(400 \mathrm{MHz}, \mathrm{CDCl}_{3}\right)$ for $\mathrm{D}$ incorporation study. 
<smiles></smiles>

3aa; $36 \%$

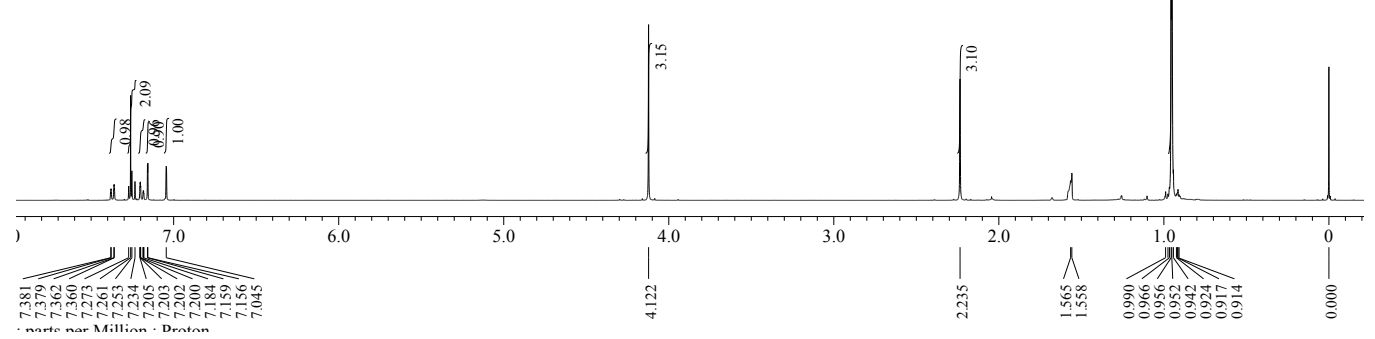

Figure S3: ${ }^{1} \mathrm{H}$ NMR spectrum of compound 3aa (400 $\left.\mathrm{MHz}, \mathrm{CDCl}_{3}\right)$.

(c) $\mathrm{H} / \mathrm{D}$ exchange experiments in absence of $\left[\mathrm{Cp}^{*} \mathrm{IrCl}_{2}\right]_{2}$ catalyst<smiles>Cc1ccccc1C(=O)c1nccn1C</smiles>

1a $(0.2 \mathrm{mmol})$

$$
\begin{gathered}
\mathrm{Br}=-\mathrm{TIPS}_{2} \mathbf{2 a}(1.1 \text { equiv. }) \\
\frac{\mathrm{NaBAr}_{4}(5 \mathrm{~mol} \%)}{\mathrm{K}_{2} \mathrm{CO}_{3}(1.5 \mathrm{eq} .)} \\
\operatorname{AgOAc}(5 \mathrm{~mol} \%)
\end{gathered}
$$$$
\mathrm{DCE} / \mathrm{D}_{2} \mathrm{O}(0.9 / 0.1 \mathrm{~mL}), 60^{\circ} \mathrm{C}, 4 \mathrm{~h}
$$<smiles>Cc1cccc(O)c1C(=O)c1nccn1C</smiles>

$[D]_{n}-1 a ; 98 \%$

In a screw-cap reaction tube, compound $\mathbf{1 a}(40 \mathrm{mg}, 0.2 \mathrm{mmol})$, bromoalkyne $\mathbf{1 b}(57.2 \mathrm{mg}, 0.22$ mmol), $\mathrm{K}_{2} \mathrm{CO}_{3}$ (41.4 mg, $\left.0.15 \mathrm{mmol}\right), \mathrm{NaBAr}_{4}$ ( $8.8 \mathrm{mg}, 5 \mathrm{~mol} \%$ ), $\operatorname{AgOAc}(2 \mathrm{mg}, 5 \mathrm{~mol} \%$ ), DCE $(0.9 \mathrm{~mL})$ and $\mathrm{D}_{2} \mathrm{O}(0.1 \mathrm{~mL})$ were placed under $\mathrm{N}_{2}$ and were then stirred at $60{ }^{\circ} \mathrm{C}$ for $4 \mathrm{~h}$. The reaction mixture was then diluted with $\mathrm{H}_{2} \mathrm{O}(10 \mathrm{~mL})$ and extracted with EtOAc $(3 \times 15$ $\mathrm{mL}$ ). The combined organic layers were dried with $\mathrm{Na}_{2} \mathrm{SO}_{4}$ and concentrated under reduced pressure and purified by silica gel column chromatography (230-400 mesh). The pure compound was eluted at 20\% EtOAc-hexane as a white sticky [D] $]_{\mathbf{n}}-1 \mathbf{a}(39.2 \mathrm{mg}, 98 \%) .{ }^{1} \mathrm{H}-$ NMR spectroscopy was used to determine the D incorporation (Figure S4). 


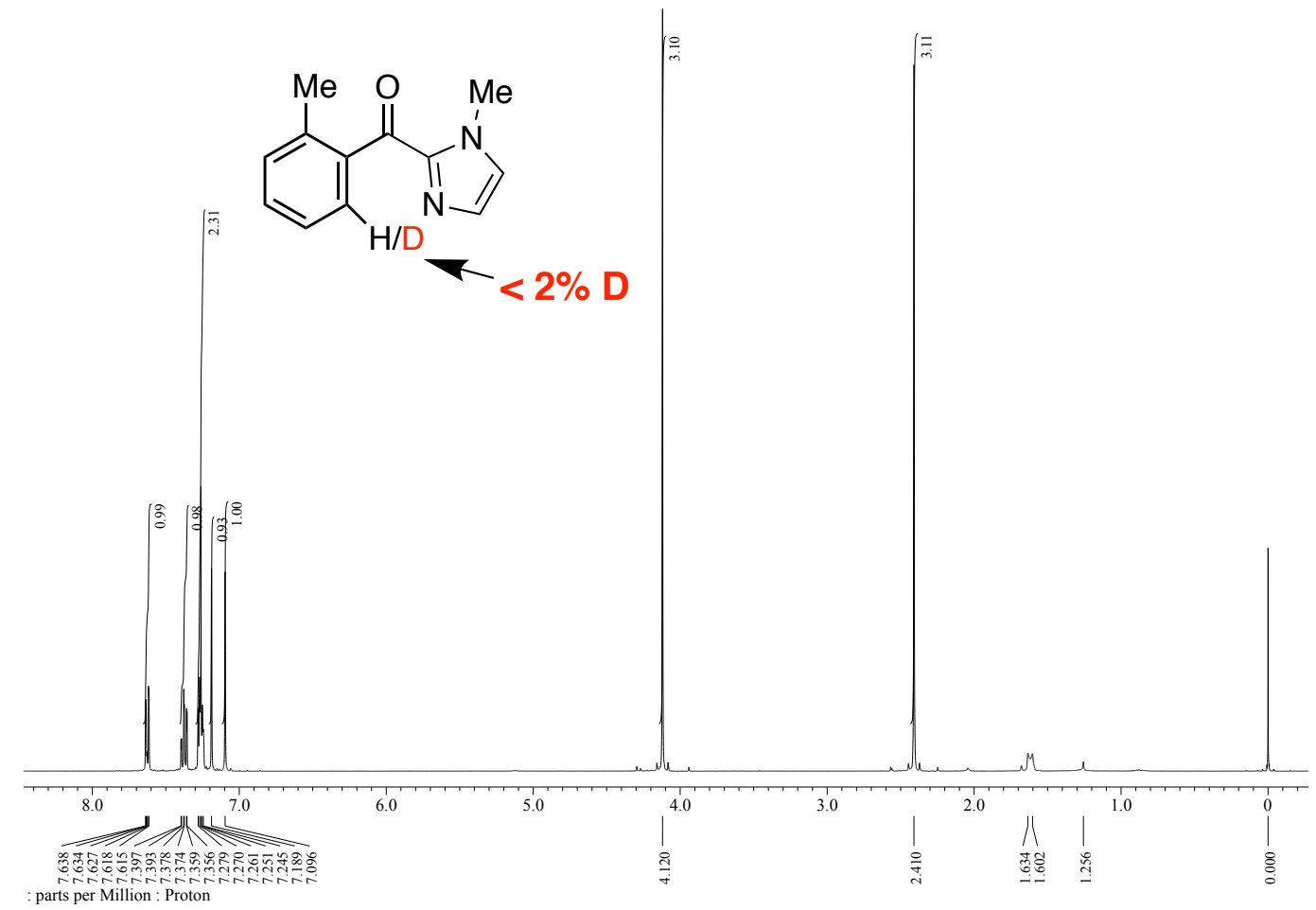

Figure S4: ${ }^{1} \mathrm{H}$ NMR spectrum of $[\mathbf{D}]_{\mathbf{n}}-\mathbf{1 a}\left(400 \mathrm{MHz}, \mathrm{CDCl}_{3}\right)$ for $\mathrm{D}$ incorporation study.

Conclusion: No H/D exchange was observed in the recovered 1a in the three sets of deuterium scrambling experiments, which suggest that $\mathrm{C}-\mathrm{H}$ activation step is irreversible in nature. 


\section{B. KIE experiment}<smiles>[2H]c1c([2H])c([18OH])c(C)c(C(=O)c2nccn2C)c1[2H]</smiles>

1a/[D] $]_{7}-1 \mathrm{a}(0.1 \mathrm{mmol})$

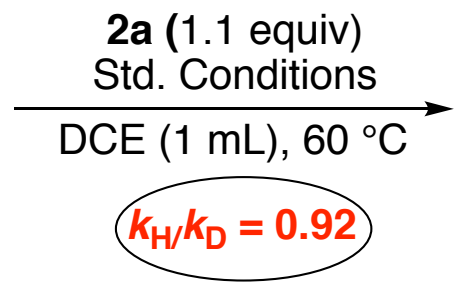<smiles>[2H]c1c([2H])c(C)c(C(=O)c2nccn2C)c(C#C[In])c1[2H]</smiles>

3aa/[D] $]_{7}$-3aa

The KIE was measured by reacting 2-acylimidazole 1a or deuterated 2-acyl imidazole [D] ${ }_{7-1 a}$ and TIPS-bromoalkyne 2a in two independent experiments in NMR tube (1,1,2,2-TCE as an internal standard) under standard reaction conditions. The internal standard doesn't hampered the reaction yields. Crude ${ }^{1} \mathrm{H}$ NMR spectra were recorded over a period of $8 \mathrm{~h}(1 \mathrm{~h}$ interval $)$ to evaluate the yield of the reaction and following table were recorded (Table S17) used to construct a \% Yield vs Time (h) graph (Figure S5).

Table S17. Datasets for KIE

\begin{tabular}{|c|c|c|c|c|c|c|c|c|}
\hline T [h) & $\mathbf{1}$ & $\mathbf{2}$ & $\mathbf{3}$ & $\mathbf{4}$ & $\mathbf{5}$ & $\mathbf{6}$ & $\mathbf{7}$ & $\mathbf{8}$ \\
\hline $\mathbf{3 a a \%}$ & 4.86 & 7.96 & 12.38 & 12.82 & 17.68 & 19.89 & 21.22 & 26.08 \\
\hline$[\mathbf{D}]_{7-3}-3 \mathbf{a a} \%$ & 7.52 & 8.4 & 14.15 & 18.57 & 19.89 & 23.43 & 25.64 & 28.73 \\
\hline
\end{tabular}

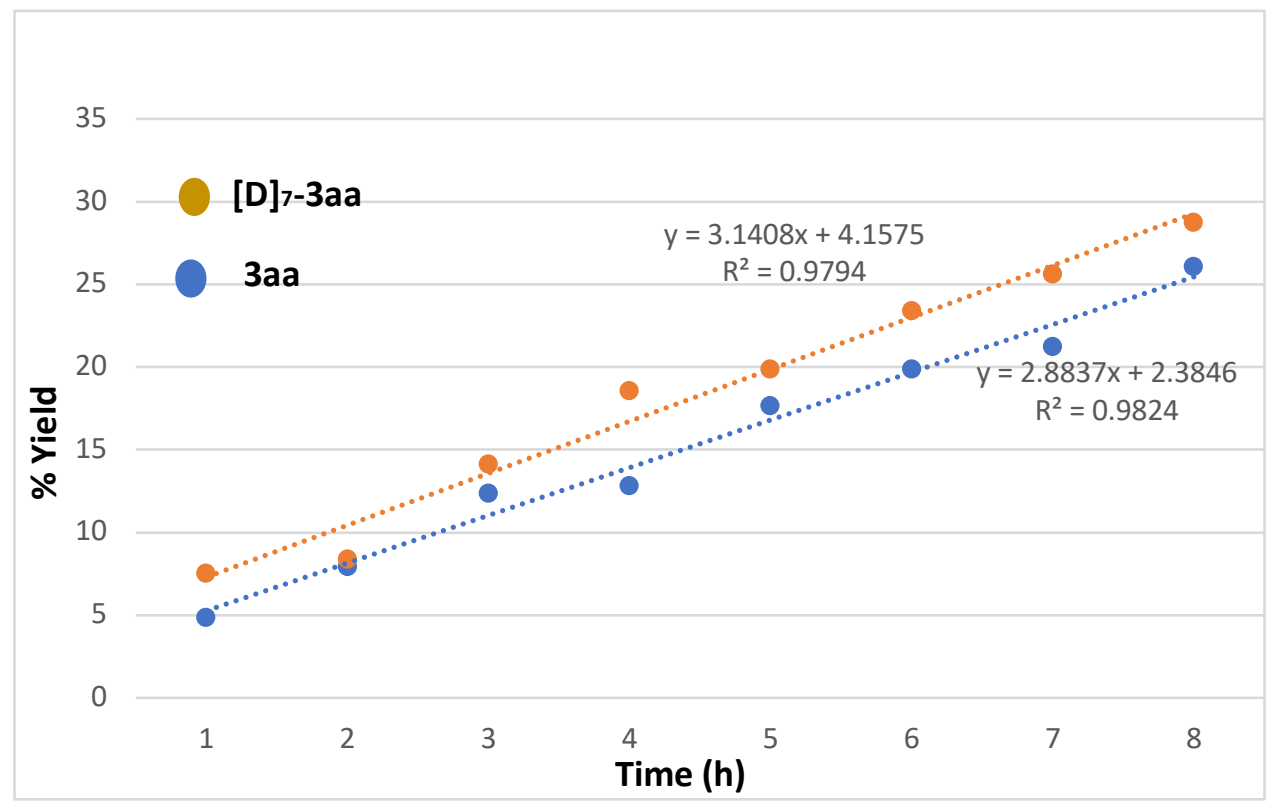

Figure S5: Representative plot of \% yield of product (Y-axis) vs time (h) (X-axis) for the reaction of $\mathbf{1 a}$ or $[\mathbf{D}]_{7}-\mathbf{1 a}$ with TIPS-bromoalkyne. Slopes for the reaction of $\mathbf{1 a}$ and $[\mathbf{D}]_{7}-\mathbf{1 a}$ are $2.8837\left(k_{\mathrm{H}}\right)$ and $3.1408\left(k_{\mathrm{D}}\right)$, respectively. Kinetic isotopic effect $=k_{\mathrm{H}} / k_{\mathrm{D}}=2.8837 / 3.1408$ $=0.92$. 


\section{Intermolecular Competition Experiment}

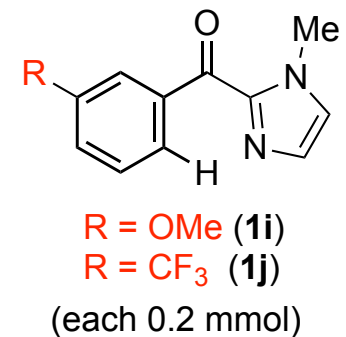

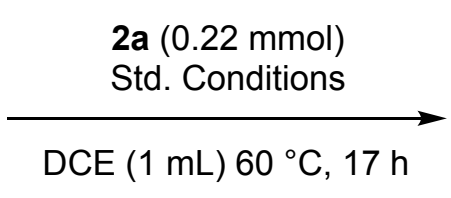

DCE $(1 \mathrm{~mL}) 60^{\circ} \mathrm{C}, 17 \mathrm{~h}$<smiles>[R]c1ccc(C#C[In]S)c(C(=O)c2nccn2C)c1</smiles>

3ja: $: 3 \mathbf{i a}=3.7: 1$

In a screw cap reaction tube, equimolar mixture of compound $\mathbf{1 j}(50.8 \mathrm{mg}, 0.2 \mathrm{mmol})$ and $\mathbf{1 i}$ (43.3 $\mathrm{mg}, 0.2 \mathrm{mmol})$ were reacted with TIPS-bromoalkyne $2 \mathrm{a}(57.2 \mathrm{mg}, 0.22 \mathrm{mmol})$ in the presence of $\left[\mathrm{Cp}^{*} \mathrm{IrCl}_{2}\right]_{2}(2.5 \mathrm{~mol} \%), \mathrm{K}_{2} \mathrm{CO}_{3}(41.5 \mathrm{mg}, 0.3 \mathrm{mmol}), \mathrm{NaBAr}_{4}(8.8 \mathrm{mg}, 5 \mathrm{~mol} \%)$, AgOAc ( $2 \mathrm{mg}, 5 \mathrm{~mol} \%)$ and DCE (I mL) were placed under $\mathrm{N}_{2}$ and were then stirred at $60{ }^{\circ} \mathrm{C}$ for $17 \mathrm{~h}$. The reaction mixture was cooled down and filtered through celite pad with ethyl acetate. The organic fraction was concentrated under reduce pressure and the mixture of $\mathbf{3} \mathbf{j a}$ and 3ia were analyzed by ${ }^{1} \mathrm{H}$ NMR spectroscopy $(\mathbf{3} \mathbf{j a}: 3 \mathbf{i a}=\mathbf{3 . 7 : 1})$.

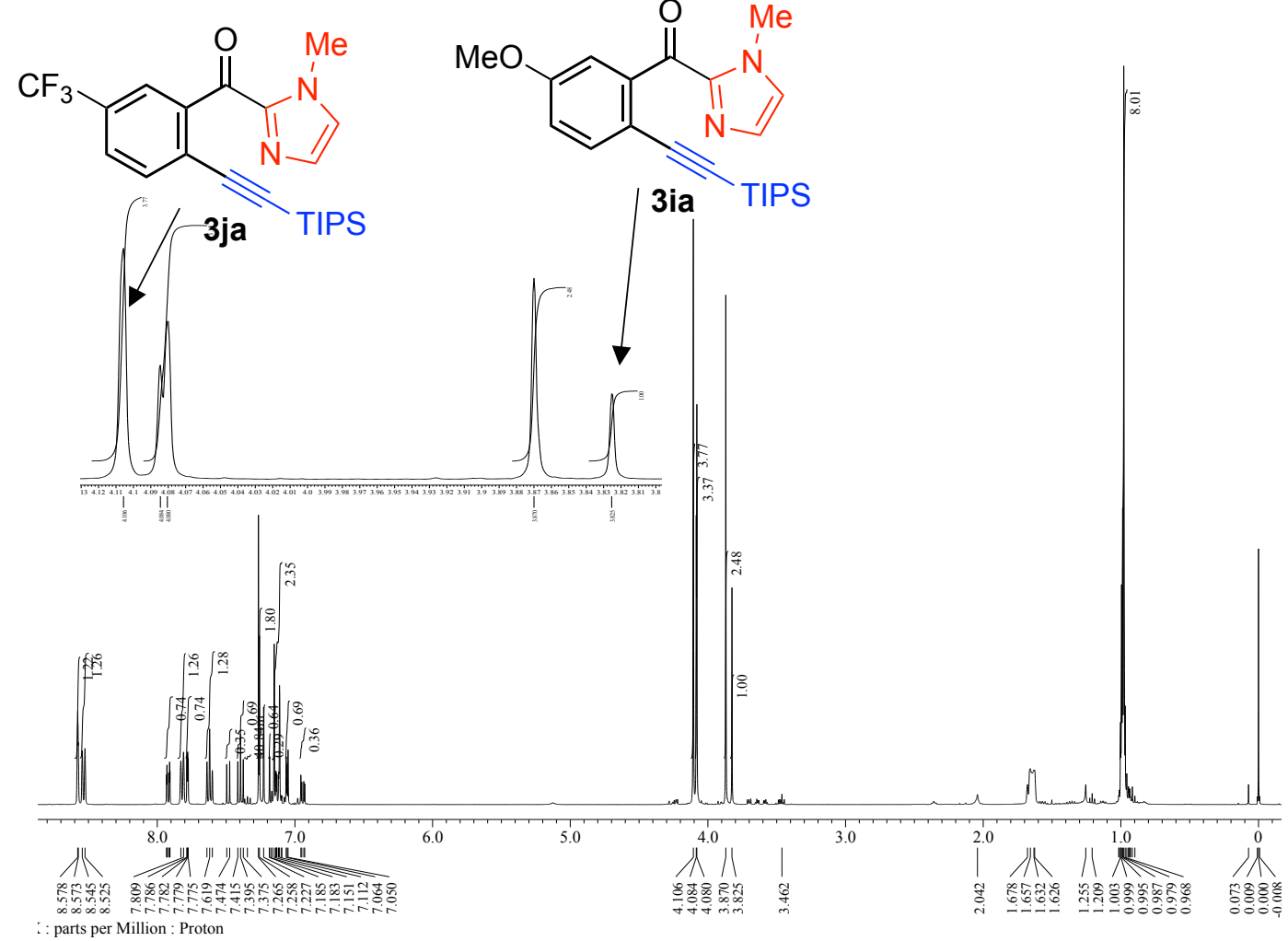

Figure S6: ${ }^{1} \mathrm{H}$ NMR spectrum of $\mathbf{3 j a}$ and $\mathbf{3 i a}$ crude reaction mixture $\left(400 \mathrm{MHz}, \mathrm{CDCl}_{3}\right)$.

Conclusion: Intermolecular competitive experiments reflect the biasness of the $\mathrm{C}-\mathrm{H}$ alkynylation reaction where the electron deficient $\mathbf{1 j}$ is preferred to the electron rich $\mathbf{1 i}$. 


\section{TEMPO-Trapping Experiments}<smiles>Cc1ccccc1C(=O)c1nccn1C</smiles>

1a $(0.2 \mathrm{mmol})$

\author{
2a (1.1 equiv) \\ Std. Conditions \\ DCE $(1 \mathrm{~mL}) 60^{\circ} \mathrm{C}, 17 \mathrm{~h}$
}<smiles>Cc1cccc(C#CC(F)F)c1C(=O)c1nccn1C</smiles>

- : 3aa; $97 \%$

TEMPO (1 eq.): 3aa; $96 \%$ TEMPO (3 eq.): 3aa; 51\%, 1a; $40 \%$

\section{Low concentration of TEMPO:}

In a screw capped reaction tube, compound $1 \mathrm{a}(40 \mathrm{mg}, 0.2 \mathrm{mmol})$ was reacted with TIPSbromoalkyne $2 \mathrm{a}(57.2 \mathrm{mg}, 0.22 \mathrm{mmol})$ in the presence of $\left[\mathrm{Cp}^{*} \mathrm{IrCl}_{2}\right]_{2}(2.5 \mathrm{~mol} \%), \mathrm{K}_{2} \mathrm{CO}_{3}(41.5$ $\mathrm{mg}, 0.3 \mathrm{mmol}), \mathrm{NaBAr}_{4}{ }_{4}(8.8 \mathrm{mg}, 5 \mathrm{~mol} \%), \operatorname{AgOAc}(2 \mathrm{mg}, 5 \mathrm{~mol} \%)$, TEMPO (31.3 mg, 02 mmol) and DCE $(1 \mathrm{~mL})$ by stirring under a $\mathrm{N}_{2}$ atmosphere at $60{ }^{\circ} \mathrm{C}$ for $17 \mathrm{~h}$. The reaction mixture was cooled to room temperature, and filtered through a celite pad which was then washed with ethyl acetate. The organic layers were concentrated under a vacuum and the crude residue was purified by silica gel column chromatography. A colourless sticky solid 3aa was isolated (73 mg, yield: 96\%).

\section{High concentration of TEMPO:}

Similar experiment was executed as describe above with 3 fold increase of TEMPO loading. The product 3aa (38.5 mg, 51\%) and starting materials $1 \mathbf{a}(19.2 \mathrm{mg}, 40 \%)$ were recovered.

Conclusion: Above two sets of experiments suggested that the reaction is not following radial pathway. 


\section{Detection of Ir-intermediates}

\section{A. Synthesis of Iridacycle B}<smiles>Cc1ccccc1C(=O)c1nccn1C</smiles>

$1 \mathrm{a}(0.2 \mathrm{mmol})$

$$
\begin{gathered}
\underset{\left.\mathrm{Cp}^{*} \mathrm{ICl}_{2}\right]_{2}(0.11 \mathrm{mmol})}{\mathrm{K}_{2} \mathrm{CO}_{3}(0.3 \mathrm{mmol})} \\
\underset{\mathrm{AgOAC}(0.2 \mathrm{mmol})}{\mathrm{ACE}(5 \mathrm{~mL}), 140^{\circ} \mathrm{C}, 24 \mathrm{~h}}
\end{gathered}
$$

In a screw cap reaction tube, compound $1 \mathrm{a}(40 \mathrm{mg}, 0.2 \mathrm{mmol})$ were heated with $\mathrm{K}_{2} \mathrm{CO}_{3}(41.5$ $\mathrm{mg}, 0.3 \mathrm{mmol}), \mathrm{AgOAc}(33.4 \mathrm{mg}, 0.2 \mathrm{mmol})$ and [Cp* $\left.\mathrm{IrCl}_{2}\right]_{2}(88 \mathrm{mg}, 0.11 \mathrm{mmol}$ in DCE (5 $\mathrm{mL}$ ) for $24 \mathrm{~h}$ at $60^{\circ} \mathrm{C}$. Then reaction mixture was cooled down and filtered through celite pad with ethyl acetate. The organic layers were concentrated under vacuum and the crude residue was purified by silica gel column chromatography. The Iridacycle B was eluted at $50 \%$ ethyl acetate-hexane as a red solid which was recrystallized in $\mathrm{CHCl}_{3}$. The product was confirmed by NMR, HRMS and X-ray crystallography.

Red crystalline solid; (104 mg, yield: $92.5 \%)$; mp. $253-254{ }^{\circ} \mathrm{C} ; R_{f}=0.15$ (50\% ethyl acetatehexane). $\delta_{\mathrm{H}}\left(400 \mathrm{MHz}, \mathrm{CDCl}_{3}\right) 7-75-7.73(\mathrm{~m}, 1 \mathrm{H}), 7.23(\mathrm{~d}, J=1.4 \mathrm{~Hz}, 1 \mathrm{H}), 7.03(\mathrm{t}, J=7.6 \mathrm{~Hz}$, $1 \mathrm{H}), 6.97(\mathrm{~d}, J=1.4 \mathrm{~Hz}, 1 \mathrm{H}), 3.98(\mathrm{~s}, 3 \mathrm{H}), 2.51(\mathrm{~s}, 3 \mathrm{H}), 1.43(\mathrm{~s}, 15 \mathrm{H}) \mathrm{ppm} . \delta_{\mathrm{C}}(100 \mathrm{MHz}$, $\left.\mathrm{CDCl}_{3}\right) 185.90,158.81,142.89,140.30,139.38,138.03,131.30,130.19,126.69,125.22,88.34$ (5C), 36.05, 23.16, 8.65 (5C) ppm. HRMS ( $\left.\mathrm{EI}^{+}, \mathrm{m} / \mathrm{z}\right)$ calcd for $\mathrm{C}_{22} \mathrm{H}_{26} \mathrm{ClN}_{2} \mathrm{OIr}: 562.1363$, found: 562.1353 .

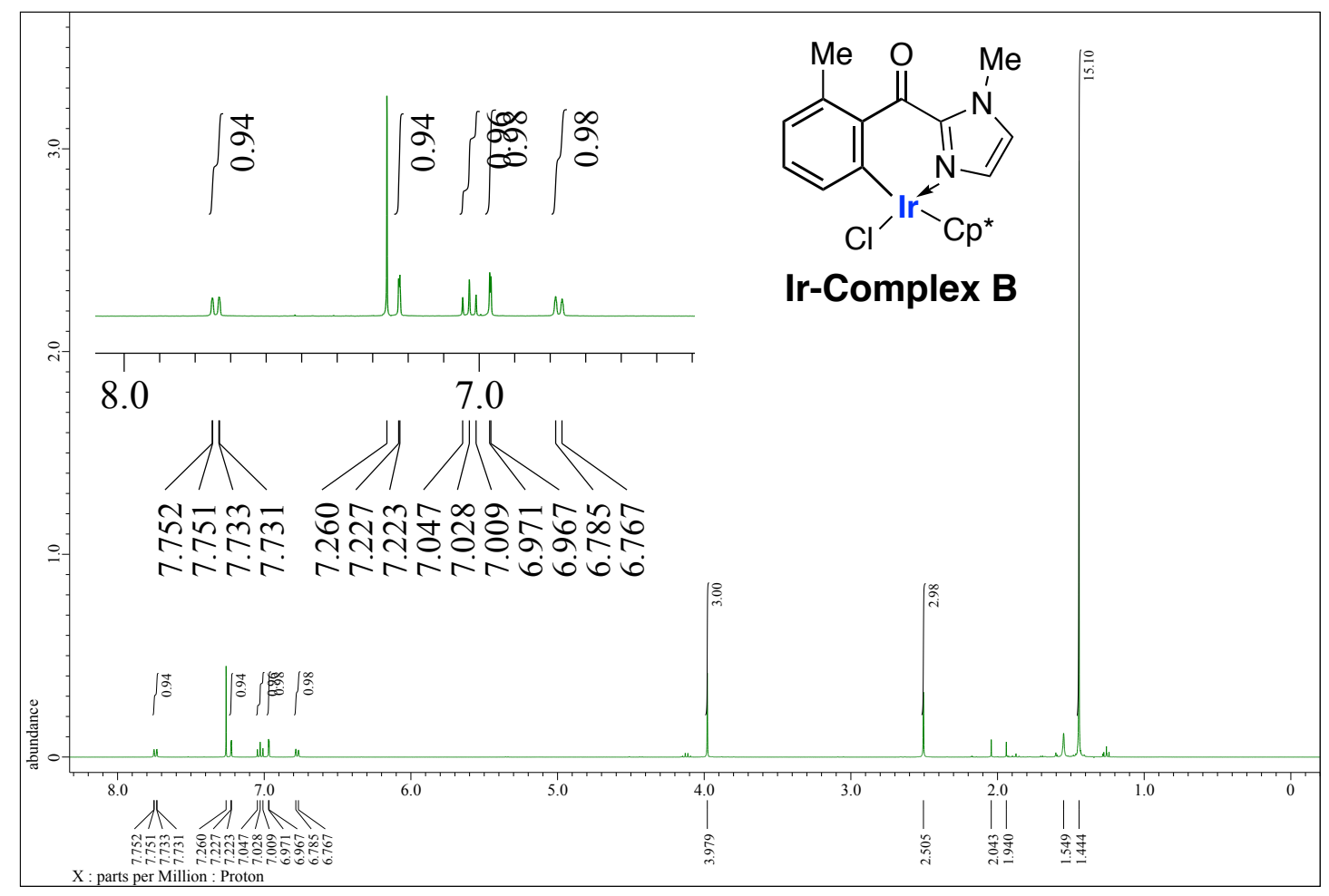

Figure S7: ${ }^{1} \mathrm{H}$ NMR spectrum of iridacycle intermediate $\mathbf{B}\left(400 \mathrm{MHz}, \mathrm{CDCl}_{3}\right)$. 


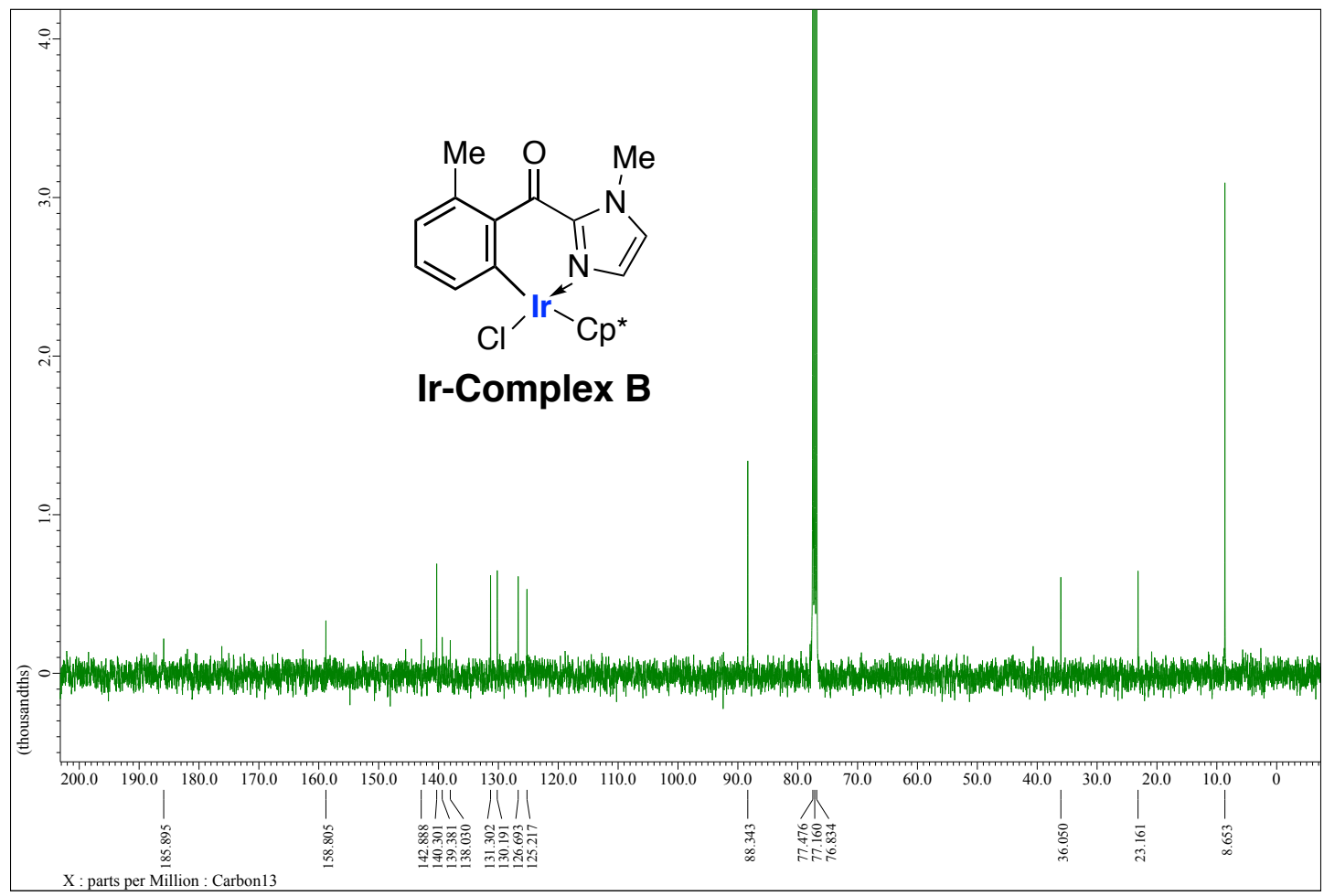

Figure S8: ${ }^{13} \mathrm{C}$ NMR spectrum of iridacycle intermediate $\mathbf{B}\left(100 \mathrm{MHz}, \mathrm{CDCl}_{3}\right)$.

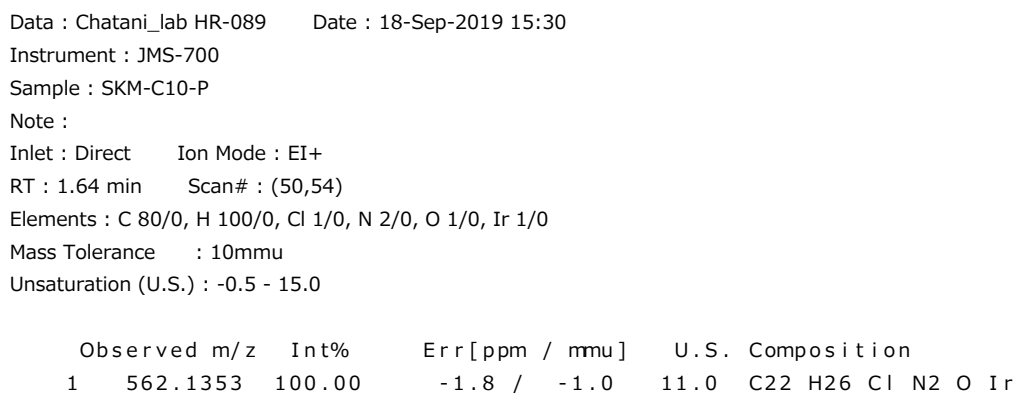

Figure S9: HRMS $\left(\mathrm{EI}^{+}, m / z\right)$ of iridacycle intermediate $\mathbf{B}$. 


\section{B. Stoichiometric Reaction of 2a with Iridacycle B}<smiles></smiles>

Ir-Complex B (0.1 mmol)

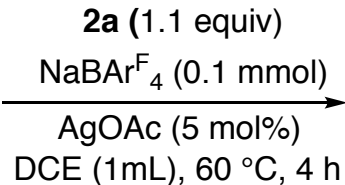

(1.5 equiv.): $3 a a ; 48 \%$, 1a; $14 \%$

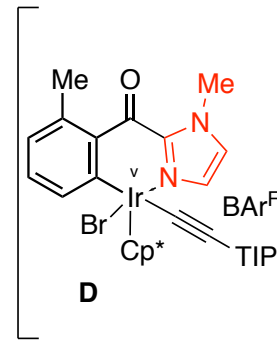

$D: E=1: 9$

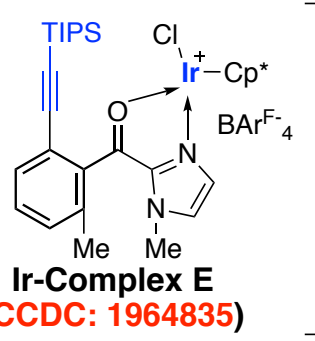

Detected
FAB-MS

C. Reaction in the absence of $\mathrm{K}_{2} \mathbf{C O}_{3}$ : In a screw capped reaction tube, iridacycle $\mathbf{B}$ (56 $\mathrm{mg}$, $0.1 \mathrm{mmol})$ was reacted with TIPS-bromoalkyne $2 \mathrm{a}(28.6 \mathrm{mg}, 0.11 \mathrm{mmol})$ in the presence of $\mathrm{NaBAr}_{4} \mathrm{~F}_{4}(88 \mathrm{mg}, 0.1 \mathrm{mmol}), \operatorname{AgOAc}(0.8 \mathrm{mg}, 5 \mathrm{~mol} \%)$ and DCE $(0.5 \mathrm{~mL})$ under a $\mathrm{N}_{2}$ atmosphere with stirring at $60{ }^{\circ} \mathrm{C}$ for $4 \mathrm{~h}$. The reaction mixture was allowed to cool to room temperature, filtered through a celite pad which was then washed with ethyl acetate. The organic layers were concentrated under a vacuum to give a sticky red solid. The formation of the desired product 3aa was analyzed by ${ }^{1} \mathrm{H}$ NMR spectroscopy (5.5\% NMR yield). The residue was washed several times with $10 \%$ ethyl acetate-hexane to remove non-polar impurities and dissolved in $80 \% \mathrm{CHCl}_{3}$-diethylether followed by evaporation to afford a mixture of the iridacycles $\mathbf{D}$ and $\mathbf{E}$ in a 1:9 ratio $\left(\mathrm{FAB}^{+}\right.$, Figure S10; NMR, Figure S14). The formation of intermediate $\mathbf{D}$ and $\mathbf{E}$ was confirmed by NMR and FAB mass analyses. Moreover, the structure of intermediate $\mathbf{E}$ was a confirmed by X-ray analyses.

Note: HRMS analyses showed that the cationic part of the complex was observed in $\mathrm{FAB}^{+}$ mode whereas the anionic part $\left(\mathrm{BAr}_{4}^{-}\right)$was observed in $\mathrm{FAB}^{-}$mode.

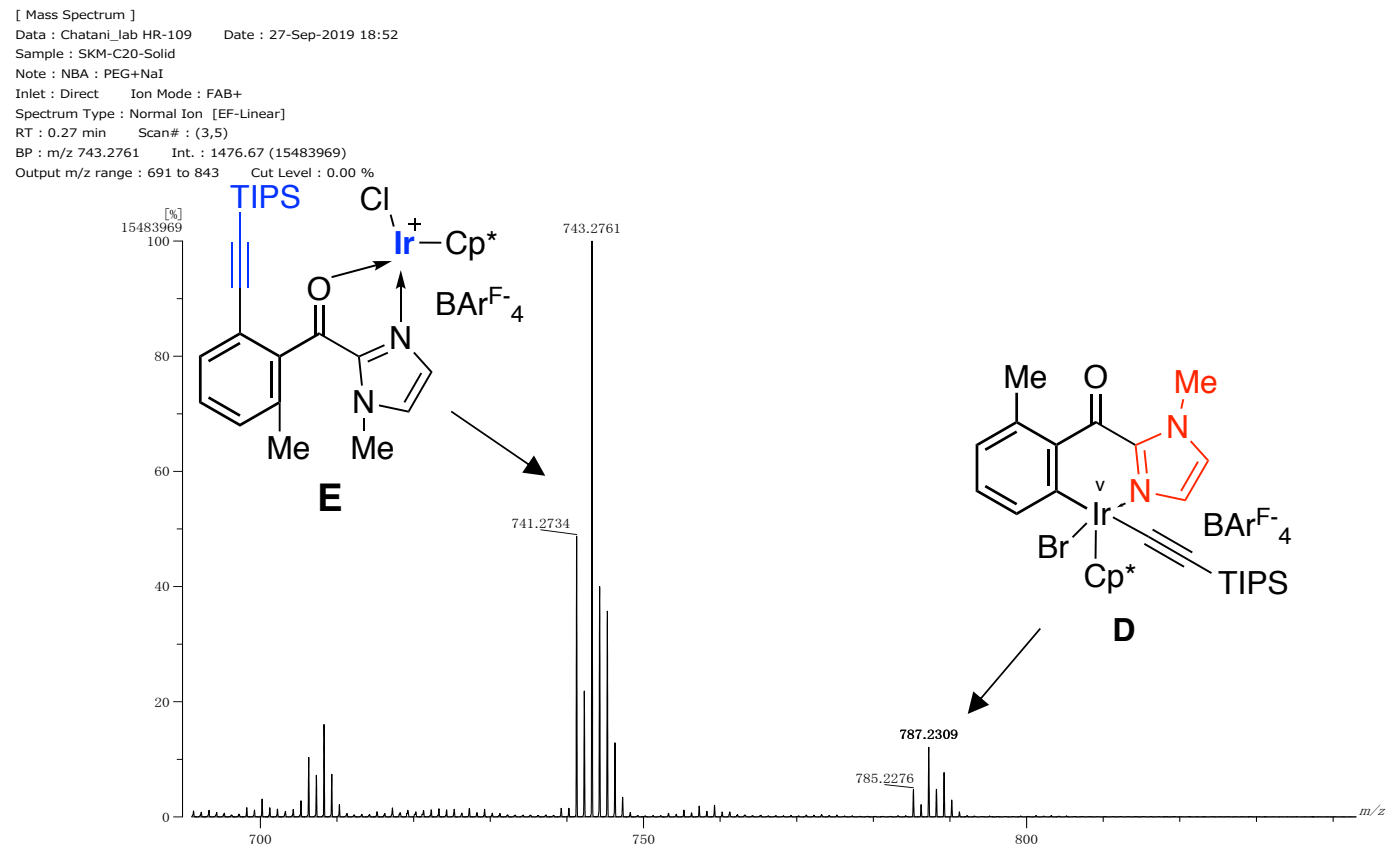

Figure S10: HRMS $\left(\mathrm{FAB}^{+}, \mathrm{m} / \mathrm{z}\right)$ of intermediate $\mathbf{D}$ and $\mathbf{E}$ mixture. 
HRMS $\left(\mathrm{FAB}^{+}, \mathrm{m} / \mathrm{z}\right.$ ) calcd for $\mathrm{C}_{33} \mathrm{H}_{47} \mathrm{ClN}_{2} \mathrm{OSi}^{191} \mathrm{Ir}$ : 741.2752 and $\mathrm{C}_{33} \mathrm{H}_{47} \mathrm{ClN}_{2} \mathrm{OSi}^{193} \mathrm{Ir}$ : 743.2775, found: 741.2734 and 743.2761 respectively (Cationic part of intermediate $\mathbf{E}$ ).

For $\mathrm{C}_{33} \mathrm{H}_{47} \mathrm{BrN}_{2} \mathrm{OSi}^{191} \mathrm{Ir}$ : 785.2247 and $\mathrm{C}_{33} \mathrm{H}_{47} \mathrm{BrN}_{2} \mathrm{OSi}^{193} \mathrm{Ir}$ : 787.2270, found 785.2276 and 787.2309 respectively (Cationic part of intermediate $\mathbf{D}$ ).

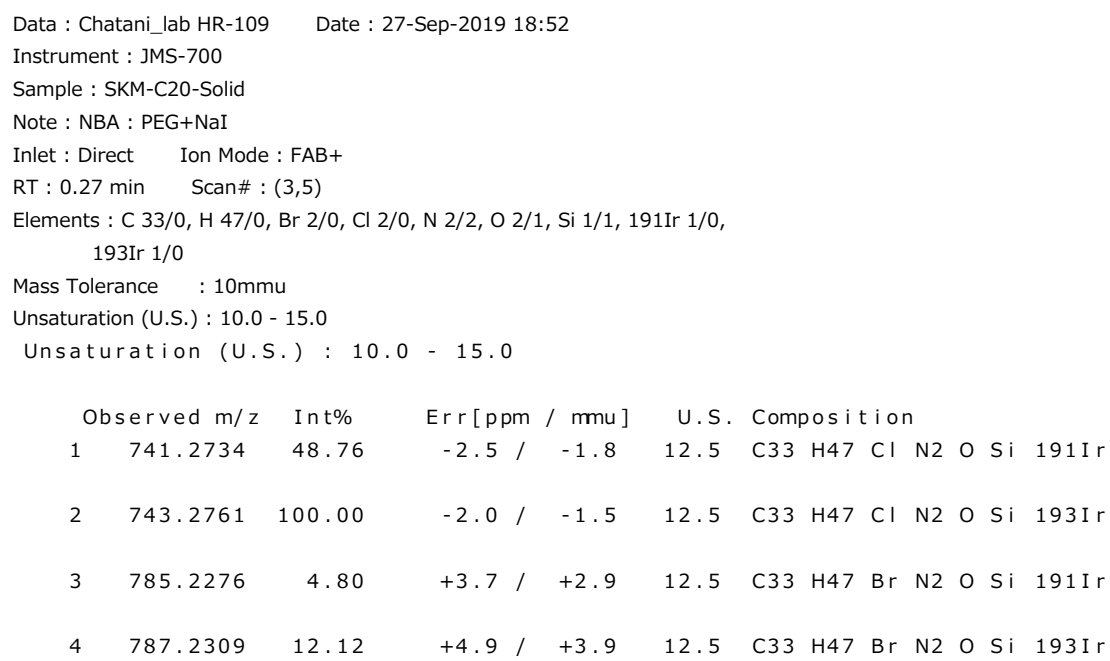

Figure S11: HRMS $\left(\mathrm{FAB}^{+}, \mathrm{m} / \mathrm{z}\right)$ data for intermediate $\mathbf{D}$ and $\mathbf{E}$.

HRMS (FAB $-\mathrm{m} / \mathrm{z}$ ) calcd for $\mathrm{C}_{32} \mathrm{H}_{12}{ }^{10} \mathrm{BF}_{24}$ : 862.0691and $\mathrm{C}_{32} \mathrm{H}_{12}{ }^{11} \mathrm{BF}_{24}$ : 863.0654, found: $862.0670(24.46 \%)$ and $863.0628(100 \%)$.

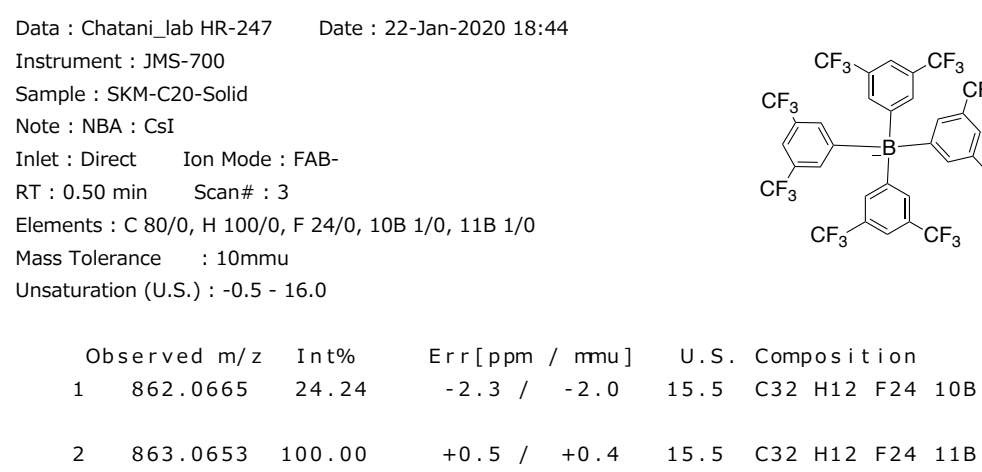

Figure S12: HRMS $\left(\mathrm{FAB}^{-}, \mathrm{m} / \mathrm{z}\right)$ data for intermediate $\mathbf{D}$ and $\mathbf{E}$ mixture. 


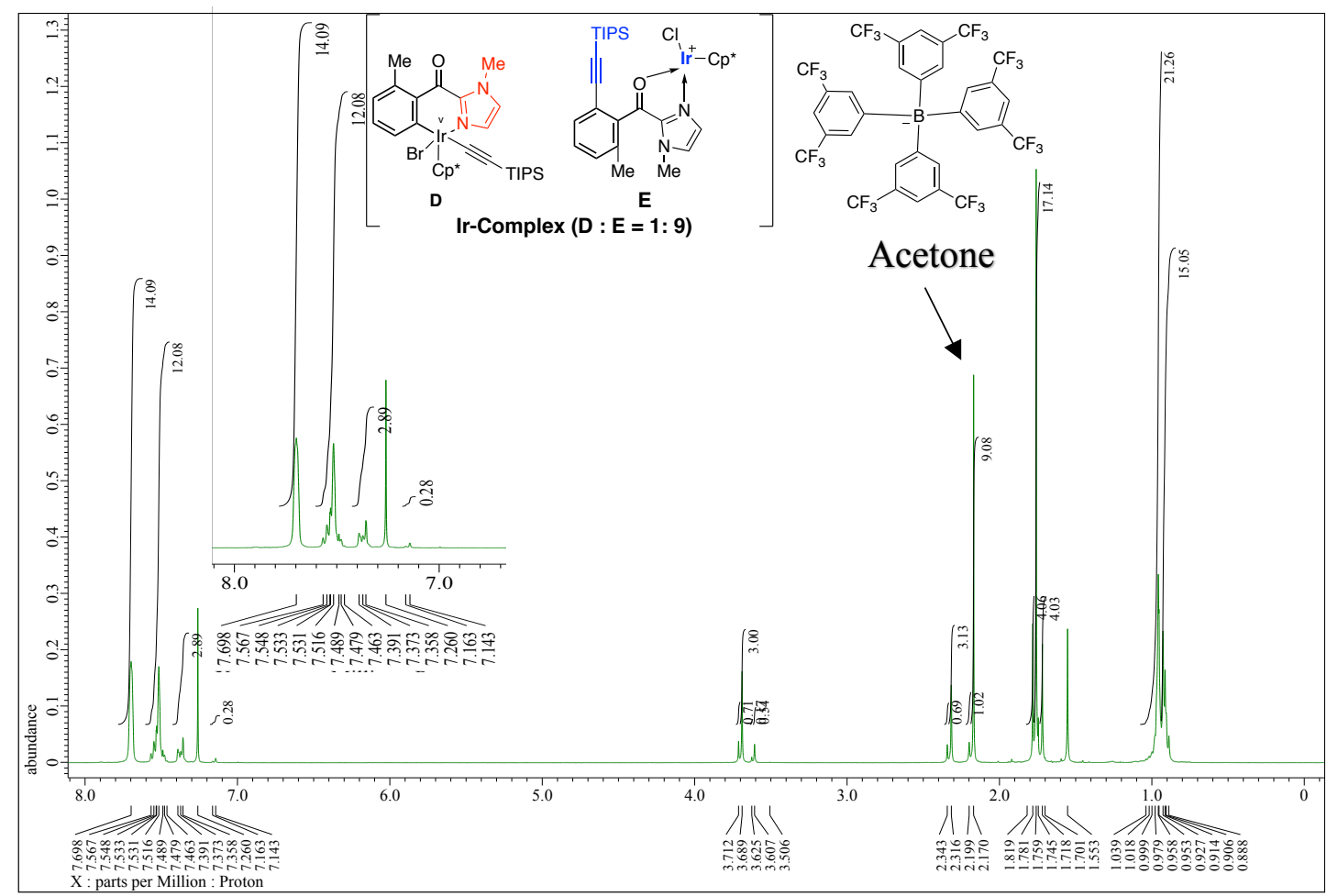

Figure S13: ${ }^{1} \mathrm{H}$ NMR spectrum of intermediate $\mathbf{D}$ and $\mathbf{E}$ mixture (400 $\left.\mathrm{MHz}, \mathrm{CDCl}_{3}\right)$.

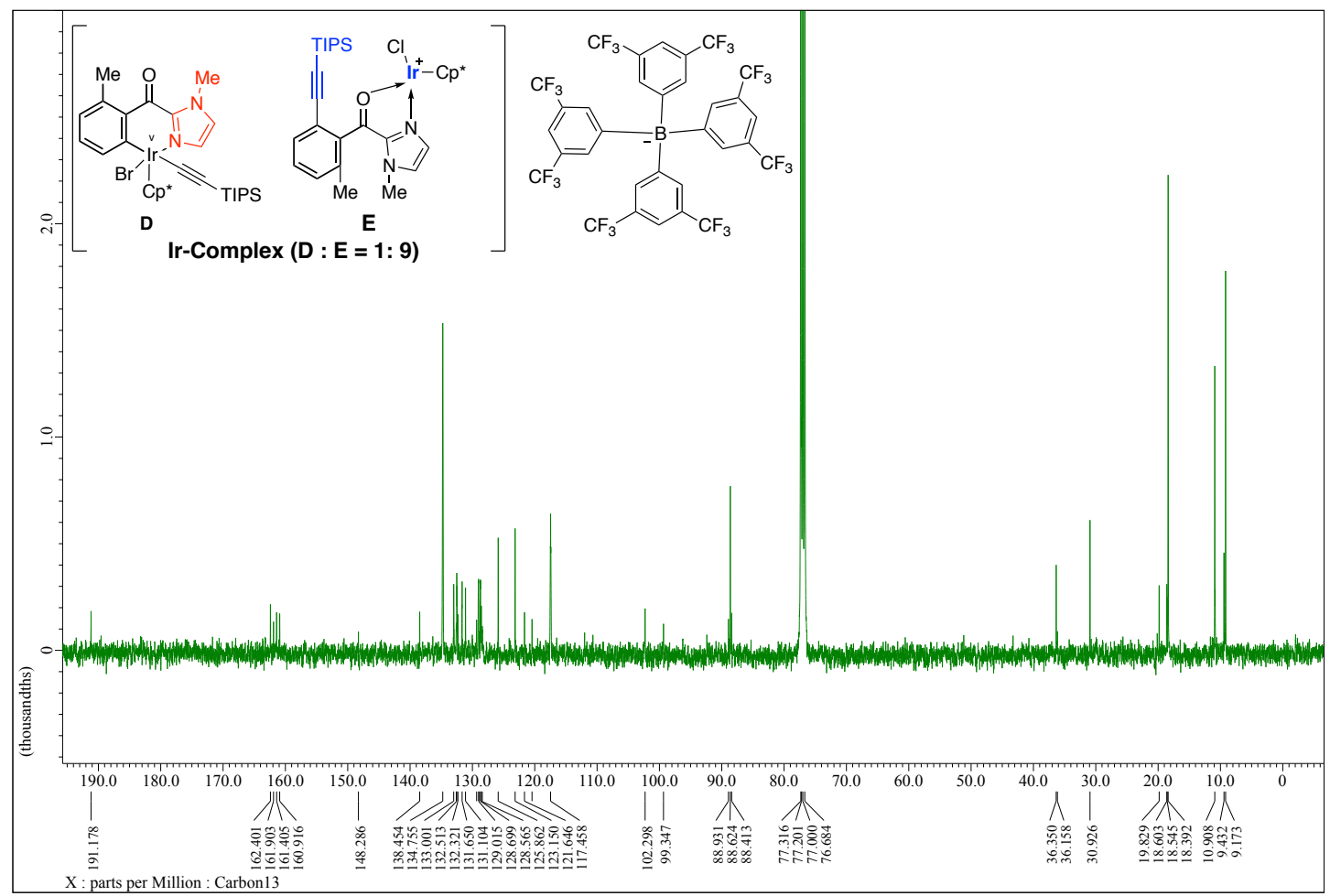

Figure S14. ${ }^{13} \mathrm{C}$ NMR spectrum of intermediate $\mathbf{D}$ and $\mathbf{E}$ mixture $\left(100 \mathrm{MHz}, \mathrm{CDCl}_{3}\right)$. 


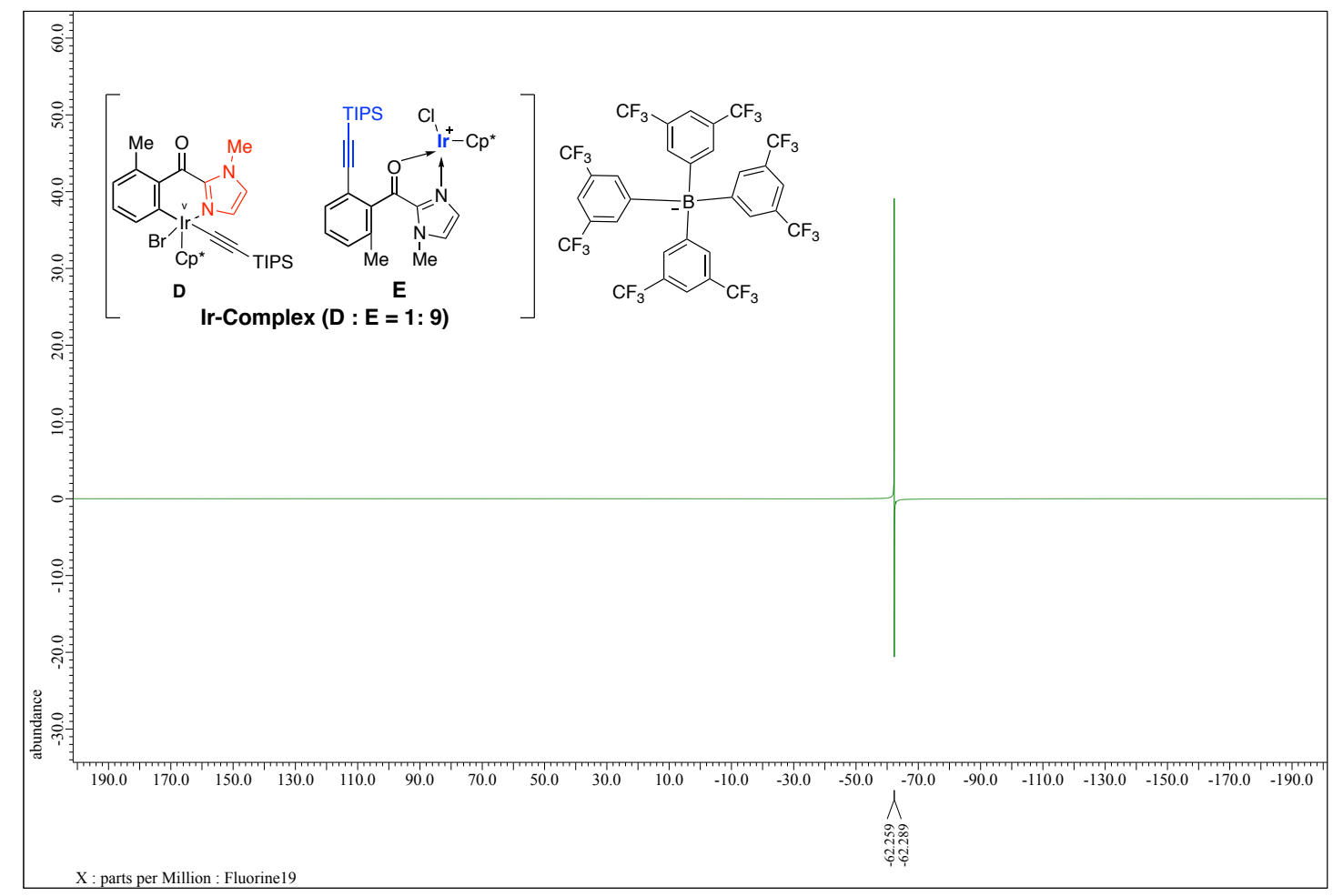

Figure S15: ${ }^{19} \mathrm{~F}$ NMR spectrum of intermediate $\mathbf{D}$ and $\mathbf{E}$ mixture $\left(376 \mathrm{MHz}, \mathrm{CDCl}_{3}\right)$.

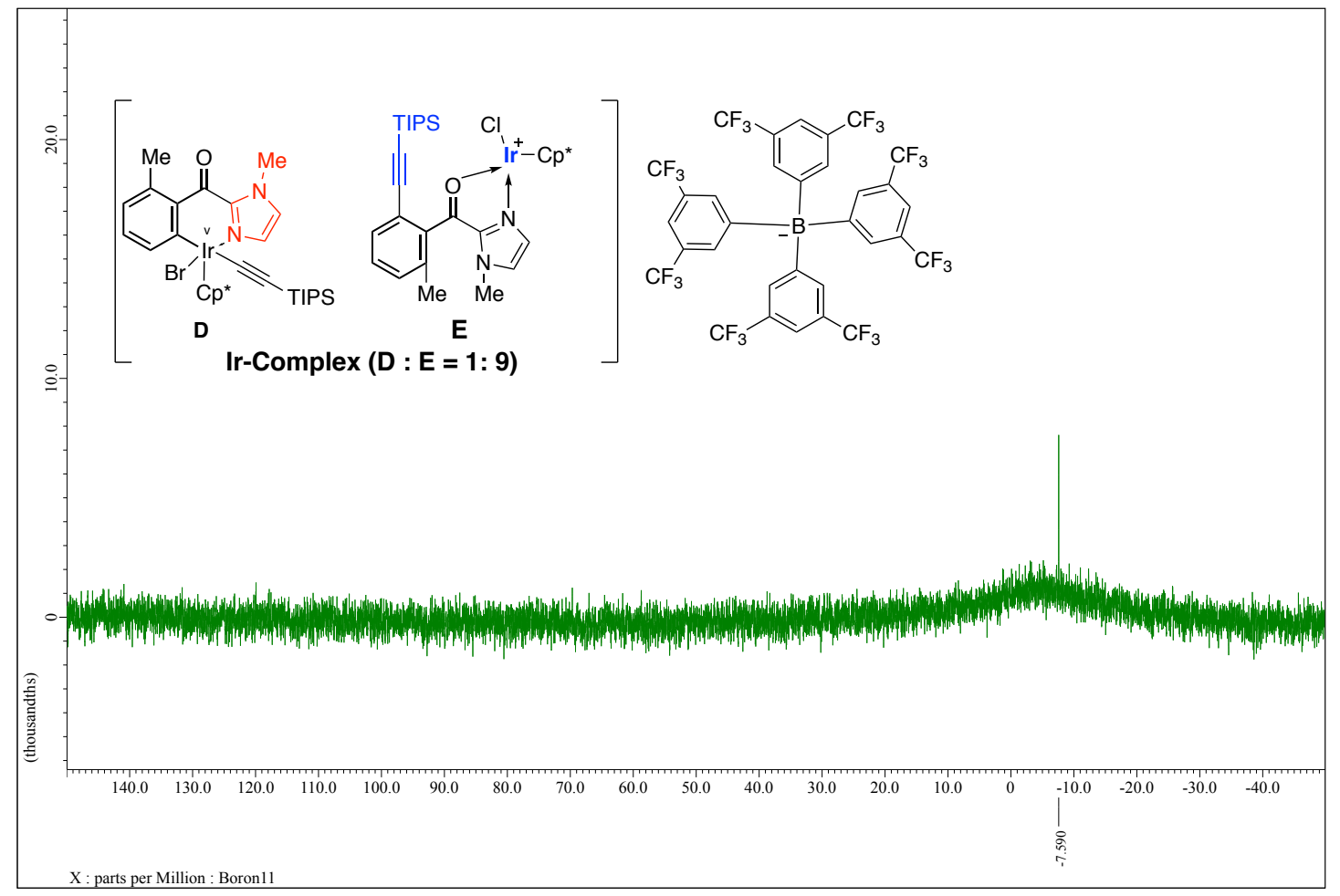

Figure S16: ${ }^{11} \mathrm{~B}$ NMR spectrum of intermediate $\mathbf{D}$ and $\mathbf{E}$ mixture $\left(128 \mathrm{MHz}, \mathrm{CDCl}_{3}\right)$. 
Reaction in the presence of $\mathbf{K}_{2} \mathbf{C O}_{3}$ : An experiment similar to that described above was carried out with the addition of $\mathrm{K}_{2} \mathrm{CO}_{3}(0.15 \mathrm{mmol})$. The products $3 \mathrm{aa}(48 \%$; NMR yield) and 1a $\left(14 \%\right.$; NMR yield) that were formed in the reaction were detected by ${ }^{1} \mathrm{H}$ NMR spectroscopy.

Conclusions: The above two sets of experiments confirm that iridium (V) intermediate (D) are formed. $\mathrm{K}_{3} \mathrm{CO}_{3}$ is essential for this reaction, in that it serves to regenerate the catalyst which followed the CMD pathway. Otherwise the reaction would have stopped after the formation of complex $\mathbf{E}$ at the reductive elimination step.

\section{Standard reaction in presence of Iridacycle B as a catalyst}<smiles>Cc1ccccc1C(=O)c1nccn1C</smiles>

1a $(0.2 \mathrm{mmol})$

\author{
$2 \mathbf{a}$ (1.1 equiv) \\ $\mathrm{K}_{2} \mathrm{CO}_{3}$ (1.5 equiv.) \\ $\mathrm{NaBAr}_{4}(2.5 \mathrm{~mol} \%)$ \\ $\operatorname{AgOAc}(2.5 \mathrm{~mol} \%)$ \\ DCE $(1 \mathrm{~mL}), 60^{\circ} \mathrm{C}, 4 \mathrm{~h}$
}

Ir-Complex B (2.5 mol \%)<smiles>Cc1cccc(C#C[In])c1C(=O)c1nccn1C</smiles>

3aa; 25\%, 1a; $67 \%$

In a screw cap reaction tube, compound 1a $(20 \mathrm{mg}, 0.1 \mathrm{mmol})$ were reacted with TIPSbromoalkyne 2a $(28.6 \mathrm{mg}, 0.11 \mathrm{mmol})$ in the presence of Iridacycle $\mathbf{B}(1.4 \mathrm{mg}, 2.5 \mathrm{~mol} \%)$, $\mathrm{K}_{2} \mathrm{CO}_{3}(20.7 \mathrm{mg}, 0.15 \mathrm{mmol}), \mathrm{NaBAr}_{4}{ }_{4}(2.22 \mathrm{mg}, 2.5 \mathrm{~mol} \%)$, AgOAc (0.4 mg, $2.5 \mathrm{~mol} \%$ ) and DCE (I mL) were placed under $\mathrm{N}_{2}$. Then the reaction was stirred at $60^{\circ} \mathrm{C}$ for $4 \mathrm{~h}$. The reaction mixture was cooled down and filtered through celite pad with ethyl acetate. The organic fraction was concentrated under reduce pressure and the crude were analyzed by ${ }^{1} \mathrm{H}$ NMR spectroscopy which indicated the formation of desired product 3aa $(25 \%)$ and unreacted starting materials 1a (67\%).

A standard reaction with $5 \mathrm{~mol} \%$ of iridacycle $\mathbf{B}$ was also performed which afforded the quantitative yield of product (3aa; 100\% NMR yield)<smiles>Cc1ccccc1C(=O)c1nccn1C</smiles>

1 a $(0.2 \mathrm{mmol})$
$2 \mathbf{a}$ (1.1 equiv) $\mathrm{K}_{2} \mathrm{CO}_{3}$ (1.5 equiv.) $\underset{\mathrm{AgOAc}(5 \mathrm{~mol} \%)}{\stackrel{\mathrm{NaBAr}_{4}(5 \mathrm{~mol} \%)}{\longrightarrow}}$ Ir-Complex B (5 mol \%)<smiles></smiles>

3aa; $100 \%$

Conclusion: The above result also supported the formation of intermediate $\mathrm{B}$ in this catalytic cycle 


\section{E. Ligand exchange reaction of Iridacycle $B$ with $\operatorname{NaBAr}^{\mathrm{F}}$.}<smiles></smiles>

Ir-Complex B (0.05 mmol)<smiles></smiles>

Ir-Complex C $49 \%$

In a screw cap reaction tube, Ir-Complex B (28 mg, $0.05 \mathrm{mmol}), \mathrm{NaBAr}^{\mathrm{F}} 4(48.7 \mathrm{mg}, 0.055$ $\mathrm{mmol}$ ) and DCE (I $\mathrm{mL}$ ) were placed under $\mathrm{N}_{2}$. Then the reaction was stirred at room temperature for $1 \mathrm{~h}$. The reaction mixture extracted with ethyl acetate and the solvent was remove under vacuum to get a reddish solid (49\%). The product was detected by FAB mass analyses.

Reddish solid; (49\%); $R_{f}=0.00$ ( $100 \%$ ethyl acetate).

HRMS analyses showed that the cationic part of the complex was observed in $\mathrm{FAB}^{+}$mode whereas the anionic part was observed in $\mathrm{FAB}^{-}$mode.

HRMS (FAB ${ }^{+}, \mathrm{m} / \mathrm{z}$ ) calcd for $\mathrm{C}_{22} \mathrm{H}_{26} \mathrm{~N}_{2} \mathrm{O}^{191} \mathrm{Ir}: 525.1651$ and $\mathrm{C}_{22} \mathrm{H}_{26} \mathrm{~N}_{2} \mathrm{O}^{193} \mathrm{Ir}: 527.1674$, found: 525.1648 .

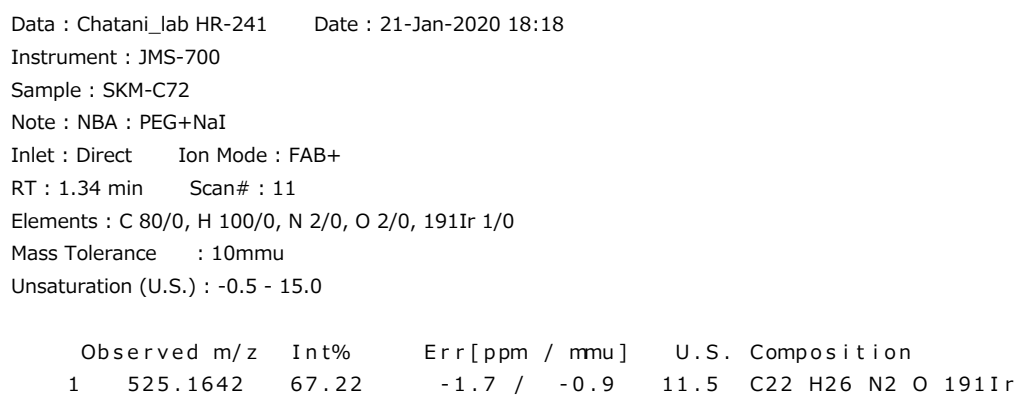

Figure S17: HRMS $\left(\mathrm{FAB}^{+}, \mathrm{m} / \mathrm{z}\right)$ data for intermediate $\mathbf{C}$.

HRMS (FAB- m/z) calcd for $\mathrm{C}_{32} \mathrm{H}_{12}{ }^{10} \mathrm{BF}_{24}$ : 862.0691and $\mathrm{C}_{32} \mathrm{H}_{12}{ }^{11} \mathrm{BF}_{24}$ : 863.0654, found: $862.0670(24.46 \%)$ and $863.0628(100 \%)$.

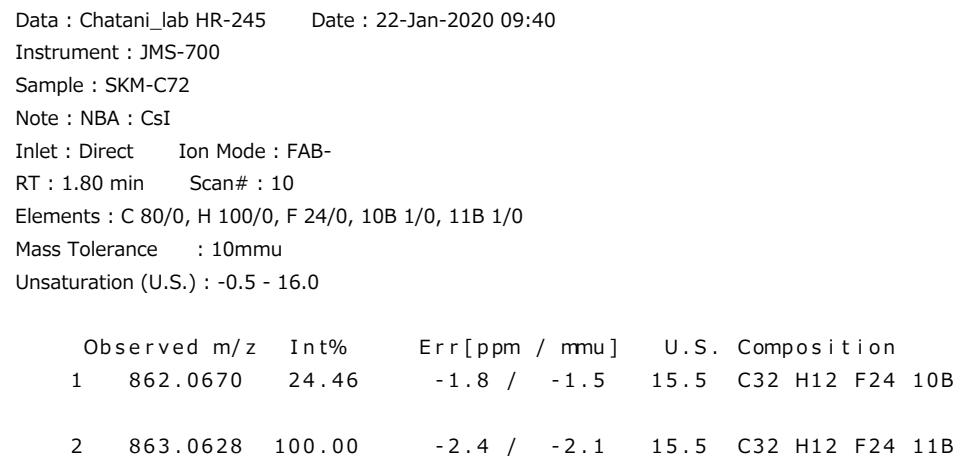

Figure S18: HRMS (FAB- $\left.{ }^{-} \mathrm{m} / \mathrm{z}\right)$ data for intermediate $\mathbf{C}$. 


\section{Removal of Directing group and in-situ Amidation Reaction ${ }^{3}$}

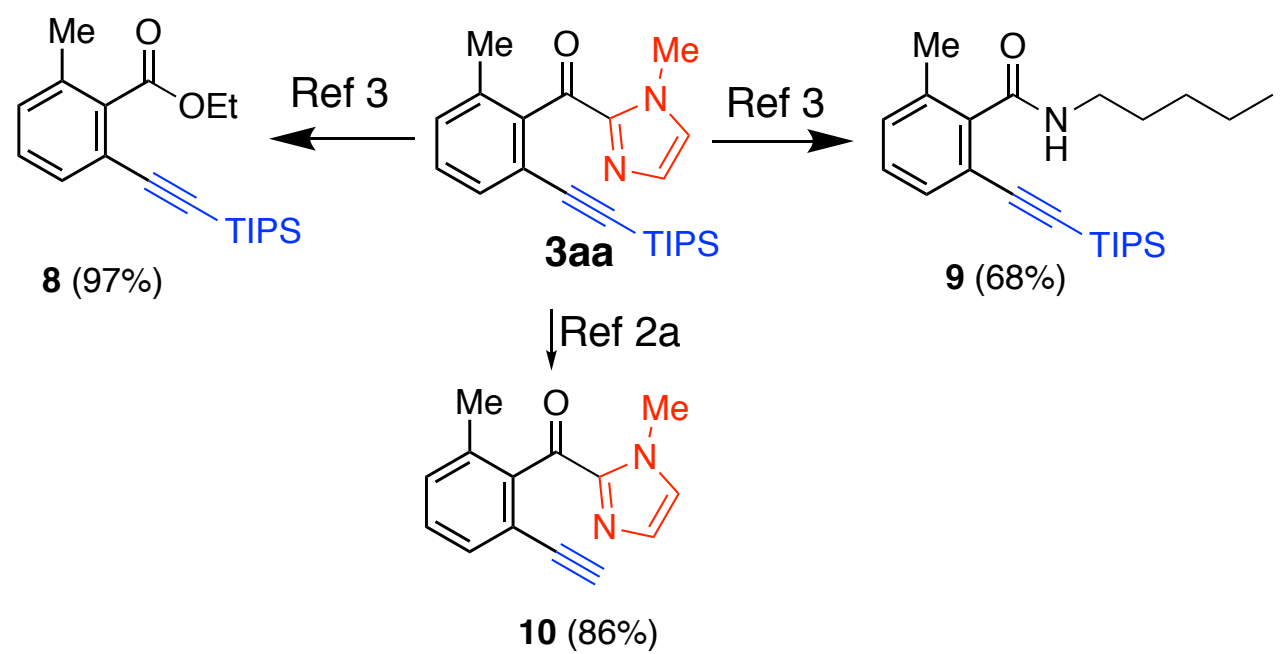

Synthesis of ester 8: The imidazole directing group was easily removed by following the Okamoto protocol. ${ }^{3 a}$ This protocol was originally followed by Megers ${ }^{3 b}$ but we modified the method slightly to produce a better yield.

In a two neck round bottom flask, $4 \AA \mathrm{MS}(500 \mathrm{mg} ; 100 \mathrm{mg} / 0.1 \mathrm{mmol})$ was heated under a vacuum for 30 minutes and was then cooled down to room temperature under a nitrogen atmosphere and compound 3aa (190 $\mathrm{mg}, 0.5 \mathrm{mmol}$ ) was added, followed by the addition of anhydrous $\mathrm{CH}_{3} \mathrm{CN}(5 \mathrm{~mL})$. The suspension was stirred for $3 \mathrm{~h}$ at room temperature under a $\mathrm{N}_{2}$ atmosphere. Methyl trifluoromethanesulfonate $(60.3 \mu \mathrm{L}, 0.55 \mathrm{mmol})$ was then slowly added at room temperature and the reaction mixture was stirred for $3 \mathrm{~h}$.

After stirring for $3 \mathrm{~h}$, the reaction mixture was cooled to $0{ }^{\circ} \mathrm{C}$ and ethanol $(5 \mathrm{~mL})$ and DBU (82 $\mu \mathrm{L}, 0.55 \mathrm{mmol})$ were added. The reaction was then stirred for an additional $2 \mathrm{~h}$ at $0{ }^{\circ} \mathrm{C}$. The progress of the reaction was monitored by TLC ( $10 \%$ ethyl acetate -hexane). The solvent was evaporated and extracted with ethyl acetate, washed with brine and dried over $\mathrm{Na}_{2} \mathrm{SO}_{4}$. The organic fraction was evaporated to give the product $\mathbf{8}$ in pure form.

Ethyl 2-methyl-6-((triisopropylsilyl)ethynyl)benzoate (8): Colorless oil; Yield: 97\% (167<smiles>CCOC(=O)c1c(C)cccc1C#CC=S</smiles>
$\mathrm{mg}) ; R_{f}=0.86\left(50 \%\right.$ ethyl acetate-hexane). $\delta_{\mathrm{H}}\left(400 \mathrm{MHz}, \mathrm{CDCl}_{3}\right) 7.35(\mathrm{~d}$, $J=7.6 \mathrm{~Hz}, 1 \mathrm{H}), 7.22(\mathrm{t}, J=7.6 \mathrm{~Hz}, 1 \mathrm{H}), 7.16-7.13(\mathrm{~m}, 1 \mathrm{H}), 4.37$ (qt, $J=$ $7.2 \mathrm{~Hz}, 2 \mathrm{H}), 2.32(\mathrm{~s}, 3 \mathrm{H}), 1.38(\mathrm{t}, J=7.2 \mathrm{~Hz}, 3 \mathrm{H}), 1.11(\mathrm{~s}, 21 \mathrm{H}) \mathrm{ppm} . \delta_{\mathrm{C}}$ $\left(100 \mathrm{MHz}, \mathrm{CDCl}_{3}\right) 168.81,136.89,134.94,130.57,130.25,129.08$, $120.92,104.25,94.36,61.51,19.51,18.75$ (6C), 14.30, 11.39 (3C) ppm.

HRMS (EI, $\mathrm{m} / \mathrm{z}$ ) calcd for $\mathrm{C}_{21} \mathrm{H}_{32} \mathrm{O}_{2} \mathrm{Si}: 344.2172$, found: 344.2171 .

Synthesis of amide 9 (In-situ amidation): A similar reaction was executed as mentioned above for synthesis of ester 8. But here only amyl amine $(288 \mu \mathrm{L}, 2.5 \mathrm{mmol})$ were added instead of EtOH and DBU at $0{ }^{\circ} \mathrm{C}$. Then reaction was stirred for overnight at room temperature. After completion the reaction, water was added and extracted with ethyl acetate, washed with brine and dried over $\mathrm{Na}_{2} \mathrm{SO}_{4}$. The organic fraction was evaporated under vacuum to get the crude product which was purified by silica get column chromatography. The pure compound 9 was eluted at $15 \%$ ethyl acetate - hexane as a white solid. 
2-Methyl-N-pentyl-6-((triisopropylsilyl)ethynyl)benzamide (9): White solid; Yield: 68\%<smiles>CCCCCNC(=O)c1c(C)cccc1C#CC(F)F</smiles>

(131 mg); mp. $104-105{ }^{\circ} \mathrm{C} ; R_{f}=0.4$ (50\% ethyl acetate-hexane). $\delta_{\mathrm{H}}$ $\left(400 \mathrm{MHz}, \mathrm{CDCl}_{3}\right) 7.23(\mathrm{~d}, J=7.6,1.4 \mathrm{~Hz}, 1 \mathrm{H}), 7.21-7.13(\mathrm{~m}, 2 \mathrm{H})$, $5.76(\mathrm{t}, J=5.2 \mathrm{~Hz}, 1 \mathrm{H}), 3.44-3.38(\mathrm{~m}, 2 \mathrm{H}), 2.34(\mathrm{~s}, 3 \mathrm{H}), 1.63-1.55$ (m, 2H), 1.40-1.30 (m, 4H), 1.15-1.05 (m, 21H), 0.92-0.89 (m, 3H) ppm. $\delta_{\mathrm{C}}\left(100 \mathrm{MHz}, \mathrm{CDCl}_{3}\right) 168.66,139.89,135.62,130.70$, $130.53,128.67,120.60,104.56,94.43,40.08,29.35,29.29,22.52,19.50,18.79$ (6C), 14.15, 11.40 (3C) ppm. HRMS (EI, $\mathrm{m} / \mathrm{z}$ ) calcd for $\mathrm{C}_{24} \mathrm{H}_{39} \mathrm{NOSi}$ : 385.2801, found: 385.2801 .

Synthesis of 10 (TIPS deprotection): To a stirred solution of $1 \mathrm{a}(0.21 \mathrm{mmol})$ in THF $(10 \mathrm{~mL})$, TBAF $(0.22 \mathrm{mmol})$ was added dropwise at $0{ }^{\circ} \mathrm{C}$ and stirred for $2 \mathrm{~h}$ at room temperature. After completion the reaction, water was added and extracted with ethyl acetate, washed with brine and dried over $\mathrm{Na}_{2} \mathrm{SO}_{4}$. The organic fraction was evaporated under vacuum to get the crude product which was purified by silica get column chromatography. The pure compound $\mathbf{1 0}$ was eluted at $18 \%$ ethyl acetate - hexane as a white solid.

(2-Ethynyl-6-methylphenyl)(1-methyl-1H-imidazol-2-yl)methanone $\quad \mathbf{( 1 0}^{\mathbf{2 a}}$ : White<smiles>C#Cc1cccc(OC)c1C(=O)c1nccn1C</smiles>
crystalline solid; Yield: $98 \%(46 \mathrm{mg}) ; \mathrm{mp} .137-138{ }^{\circ} \mathrm{C} ; R_{f}=0.4(50 \%$ ethyl acetate-hexane). $\delta_{\mathrm{H}}\left(400 \mathrm{MHz}, \mathrm{CDCl}_{3}\right) 7.39(\mathrm{~d}, J=7.6 \mathrm{~Hz}, 1 \mathrm{H}), 7.28(\mathrm{t}, J$ $=7.6 \mathrm{~Hz}, 1 \mathrm{H}), 7.26-7.22(\mathrm{~m}, 1 \mathrm{H}), 7.18(\mathrm{~s}, 1 \mathrm{H}), 7.11(\mathrm{~s}, 1 \mathrm{H}), 4.14(\mathrm{~s}, 3 \mathrm{H})$, $2.97(\mathrm{~s}, 1 \mathrm{H}), 2.25(\mathrm{~s}, 3 \mathrm{H}) \mathrm{ppm} . \delta_{\mathrm{C}}\left(100 \mathrm{MHz}, \mathrm{CDCl}_{3}\right) 188.89,143.81$, $142.78,135.00,131.08,130.71,130.36,129.14,127.15,119.51,81.50$, 80.80, 36.16, 19.53 ppm. HRMS (EI $\left.{ }^{+}, \mathrm{m} / \mathrm{z}\right)$ calcd for $\mathrm{C}_{14} \mathrm{H}_{12} \mathrm{~N}_{2} \mathrm{O}: 224.0950$, found: 224.0949 . 


\section{X-Ray Crystallographic Structure Analysis}

A. X-ray analysis of intermediate $\mathrm{B}\left(\mathrm{C}_{22} \mathrm{H}_{26} \mathrm{CIIrN}_{2} \mathrm{O}\right)$ : $\mathrm{CCDC} 1949236$

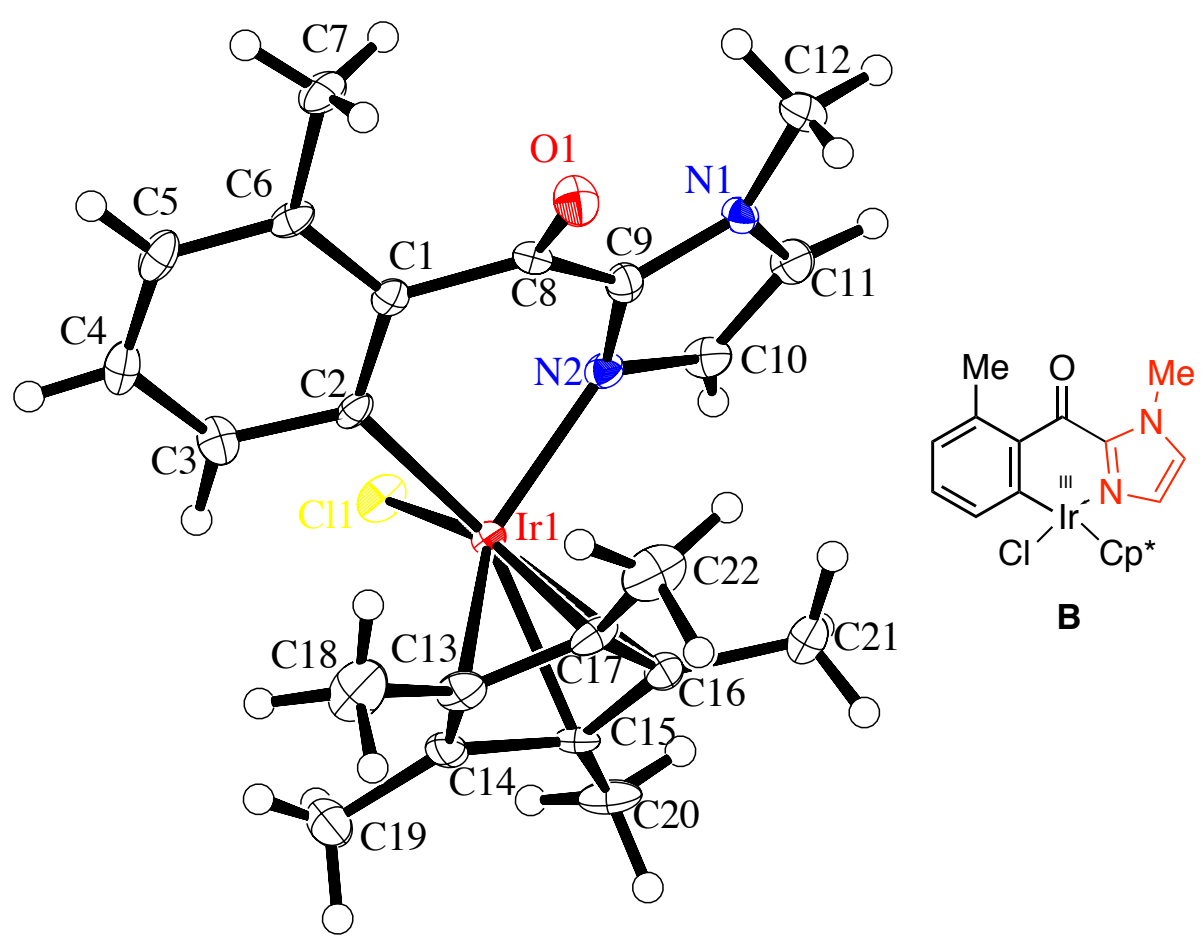

Figure S19: ORTEP diagram of intermediate $\mathbf{B}$. 
CCDC 1949236 contains the supplementary crystallographic data. X-Ray crystallographic structure analysis of intermediate $\mathbf{B}\left(\mathrm{C}_{22} \mathrm{H}_{26} \mathrm{ClIrN}_{2} \mathrm{O}\right)$ was recorded on Rigaku XtaLAB P200 using multi-layer mirror monochromated Mo-K $\alpha$ radiation. The crystal-to-detector distance was $34.00 \mathrm{~mm}$. The data were collected at a temperature of $-150 \pm 1^{\circ} \mathrm{C}$ to a maximum $2 \theta$ value of $61.5^{\circ}$. A total of 13998 reflections were collected, where 5026 were unique (Rint $=0.0455$ ). Data were collected and processed using CrysAlisPro (Rigaku Oxford Diffraction) ${ }^{4}$ and refined by the full matrix least-squares on F2 (Crystals). The structure was solved by direct methods (SIR92) $^{5}$ and expanded using Fourier techniques. The non-hydrogen atoms were refined anisotropically. Hydrogen atoms were refined using the riding model. The final cycle of fullmatrix least-squares refinement ${ }^{6}$ on F2 was based on 5026 observed reflections and 244 variable parameters. The crystal data are given below:

X-ray data of intermediate $\mathbf{B}\left(\mathrm{C}_{23} \mathrm{H}_{32} \mathrm{~N}_{2} \mathrm{O}_{2} \mathrm{Si}\right)$ : CCDC 1949236

Empirical Formula

Formula Weight

Crystal Color, Habit

Crystal Dimensions

Crystal System

Lattice Type

Lattice Parameters

$$
\mathrm{C}_{22} \mathrm{H}_{26} \mathrm{ClIrN}_{2} \mathrm{O}
$$

\subsection{3}

red, prism

$0.700 \times 0.100 \times 0.100 \mathrm{~mm}$

monoclinic

Primitive

$$
\begin{aligned}
& \mathrm{a}=8.0634(2) \AA \\
& \mathrm{b}=19.6046(6) \AA \\
& \mathrm{c}=12.6297(4) \AA \\
& \mathrm{b}=93.722(3) \mathrm{o} \\
& \mathrm{V}=1992.29(10) \AA^{3}
\end{aligned}
$$

Space Group

$\mathrm{P} 21 / \mathrm{n}(\# 14)$

$\mathrm{Z}$ value

4

$\mathrm{D}_{\text {calc }}$

$1.874 \mathrm{~g} / \mathrm{cm}^{3}$

F000

1096.00

$\mathrm{m}(\mathrm{MoK} \alpha)$
$68.686 \mathrm{~cm}^{-1}$ 
Table S18. Bond lengths ( $\AA$ )

$\begin{array}{llllll}\text { atom } & \text { atom } & \text { distance } & \text { atom } & \text { atom } & \text { distance } \\ \mathrm{Ir} 1 & \mathrm{C} 11 & 2.4197(12) & \mathrm{I} 1 & \mathrm{~N} 2 & 2.064(4) \\ \mathrm{Ir} 1 & \mathrm{C} 2 & 2.045(4) & \mathrm{I} 1 & \mathrm{C} 13 & 2.147(5) \\ \mathrm{Ir} 1 & \mathrm{C} 14 & 2.170(4) & \mathrm{I} 11 & \mathrm{C} 15 & 2.259(4) \\ \mathrm{Ir} 1 & \mathrm{C} 16 & 2.237(4) & \mathrm{I} 1 & \mathrm{C} 17 & 2.134(5) \\ \mathrm{O} 1 & \mathrm{C} 8 & 1.229(6) & \mathrm{N} 1 & \mathrm{C} 9 & 1.362(5) \\ \mathrm{N} 1 & \mathrm{C} 11 & 1.367(6) & \mathrm{N} 1 & \mathrm{C} 12 & 1.474(6) \\ \mathrm{N} 2 & \mathrm{C} 9 & 1.333(6) & \mathrm{N} 2 & \mathrm{C} 10 & 1.359(6) \\ \mathrm{C} 1 & \mathrm{C} 2 & 1.422(6) & \mathrm{C} 1 & \mathrm{C} 6 & 1.420(6) \\ \mathrm{C} 1 & \mathrm{C} 8 & 1.488(6) & \mathrm{C} 2 & \mathrm{C} 3 & 1.401(6) \\ \mathrm{C} 3 & \mathrm{C} 4 & 1.373(7) & \mathrm{C} 4 & \mathrm{C} 5 & 1.399(7) \\ \mathrm{C} 5 & \mathrm{C} 6 & 1.387(7) & \mathrm{C} 6 & \mathrm{C} 7 & 1.510(6) \\ \mathrm{C} 8 & \mathrm{C} 9 & 1.476(6) & \mathrm{C} 10 & \mathrm{C} 11 & 1.363(7) \\ \mathrm{C} 13 & \mathrm{C} 14 & 1.430(7) & \mathrm{C} 13 & \mathrm{C} 17 & 1.438(6) \\ \mathrm{C} 13 & \mathrm{C} 18 & 1.510(7) & \mathrm{C} 14 & \mathrm{C} 15 & 1.466(6) \\ \mathrm{C} 14 & \mathrm{C} 19 & 1.489(7) & \mathrm{C} 15 & \mathrm{C} 16 & 1.407(6) \\ \mathrm{C} 15 & \mathrm{C} 20 & 1.491(7) & \mathrm{C} 16 & \mathrm{C} 17 & 1.427(6) \\ \mathrm{C} 16 & \mathrm{C} 21 & 1.502(7) & \mathrm{C} 17 & \mathrm{C} 22 & 1.516(7)\end{array}$

Table S19. Bond lengths involving hydrogens $(\AA)$

$\begin{array}{llllll}\text { atom } & \text { atom } & \text { distance } & \text { atom } & \text { atom } & \text { distance } \\ \text { C3 } & \text { H1 } & 1.001(5) & \mathrm{C} 4 & \mathrm{H} 2 & 0.975(5) \\ \mathrm{C} 5 & \mathrm{H} 3 & 0.910(5) & \mathrm{C} 7 & \mathrm{H} 4 & 0.914(4) \\ \mathrm{C} 7 & \mathrm{H} 5 & 0.911(5) & \mathrm{C} 7 & \mathrm{H} 6 & 0.937(5) \\ \mathrm{C} 10 & \mathrm{H} 7 & 0.741(4) & \mathrm{C} 11 & \mathrm{H} 8 & 0.971(5) \\ \mathrm{C} 12 & \mathrm{H} 9 & 0.980(5) & \mathrm{C} 12 & \mathrm{H} 10 & 0.980(5) \\ \mathrm{C} 12 & \mathrm{H} 11 & 0.980(5) & \mathrm{C} 18 & \mathrm{H} 12 & 0.989(5) \\ \mathrm{C} 18 & \mathrm{H} 13 & 0.994(6) & \mathrm{C} 18 & \mathrm{H} 14 & 1.049(6) \\ \mathrm{C} 19 & \mathrm{H} 15 & 0.888(6) & \mathrm{C} 19 & \mathrm{H} 16 & 0.958(6) \\ \mathrm{C} 19 & \mathrm{H} 17 & 0.961(6) & \mathrm{C} 20 & \mathrm{H} 18 & 0.973(6) \\ \mathrm{C} 20 & \mathrm{H} 19 & 0.984(5) & \mathrm{C} 20 & \mathrm{H} 20 & 0.893(5) \\ \mathrm{C} 21 & \mathrm{H} 21 & 0.981(5) & \mathrm{C} 21 & \mathrm{H} 22 & 0.979(5) \\ \mathrm{C} 21 & \mathrm{H} 23 & 0.980(6) & \mathrm{C} 22 & \mathrm{H} 24 & 0.958(5) \\ \mathrm{C} 22 & \mathrm{H} 25 & 1.081(5) & \mathrm{C} 22 & \mathrm{H} 26 & 1.035(6)\end{array}$

Table S20. Bond angles $\left({ }^{\circ}\right)$

\begin{tabular}{lllllllr} 
atom & atom & atom & angle & atom & atom & atom & \multicolumn{1}{c}{ angle } \\
C11 & Ir1 & N2 & $88.95(10)$ & C11 & Ir1 & C2 & $88.65(13)$ \\
C11 & Ir1 & C13 & $131.94(13)$ & C11 & Ir1 & C14 & $98.67(12)$ \\
C11 & Ir1 & C15 & $97.68(11)$ & C11 & Ir1 & C16. & $126.68(12)$ \\
C11 & Ir1 & C17 & $160.92(12)$ & N2 & Ir1 & C2 & $85.68(15)$
\end{tabular}


Table S20. Bond angles ( ${ }^{\circ}$ ) (Continued)

\begin{tabular}{|c|c|c|c|c|c|c|c|}
\hline atom & atom & atom & angle & atom & atom & atom & angle \\
\hline $\mathrm{N} 2$ & Ir1 & $\mathrm{C} 13$ & $139.10(16)$ & $\mathrm{N} 2$ & Ir 1 & C14. & $156.39(15)$ \\
\hline $\mathrm{N} 2$ & Ir1 & $\mathrm{C} 15$ & $118.45(14)$ & $\mathrm{N} 2$ & Ir 1 & $\mathrm{C} 16$ & $94.04(15)$ \\
\hline N2 & Ir1 & $\mathrm{C} 17$ & $102.05(16)$ & $\mathrm{C} 2$ & Ir 1 & $\mathrm{C} 13$ & $94.15(16)$ \\
\hline $\mathrm{C} 2$ & Ir1 & $\mathrm{C} 14$ & $116.64(16)$ & $\mathrm{C} 2$ & Ir1 & C15. & $155.00(15)$ \\
\hline $\mathrm{C} 2$ & Ir1 & $\mathrm{C} 16$ & $144.67(17)$ & $\mathrm{C} 2$ & Ir 1 & C17. & $107.49(17)$ \\
\hline C13 & Ir1 & $\mathrm{C} 14$ & $38.68(17)$ & $\mathrm{C} 13$ & Ir1 & $\mathrm{C} 15$ & $63.73(16)$ \\
\hline C13 & Ir1 & $\mathrm{C} 16$ & $63.63(16)$ & $\mathrm{C} 13$ & Ir1 & $\mathrm{C} 17$ & $39.25(17)$ \\
\hline C14 & Ir1 & $\mathrm{C} 15$ & $38.58(16)$ & C14 & Ir1 & $\mathrm{C} 16$ & $63.50(16)$ \\
\hline $\mathrm{C} 14$ & Ir1 & $\mathrm{C} 17$ & $65.33(17)$ & $\mathrm{C} 15$ & Ir1 & $\mathrm{C} 16$ & $36.47(16)$ \\
\hline $\mathrm{C} 15$ & Ir1 & $\mathrm{C} 17$ & $63.41(16)$ & $\mathrm{C} 16$ & Ir 1 & $\mathrm{C} 17$ & $38.03(16)$ \\
\hline C9 & N1 & $\mathrm{C} 11$ & $107.6(4)$ & C9 & N1 & $\mathrm{C} 12$ & $128.0(4)$ \\
\hline $\mathrm{C} 11$ & N1 & $\mathrm{C} 12$ & $124.4(4)$ & Ir 1 & $\mathrm{~N} 2$ & C9 & $120.4(3)$ \\
\hline Ir1 & $\mathrm{N} 2$ & $\mathrm{C} 10$ & $126.8(3)$ & C9 & $\mathrm{N} 2$ & $\mathrm{C} 10$ & $107.6(4)$ \\
\hline $\mathrm{C} 2$ & $\mathrm{C} 1$ & C6 & $121.1(4)$ & $\mathrm{C} 2$ & $\mathrm{C} 1$ & $\mathrm{C} 8$ & $120.8(4)$ \\
\hline C6 & $\mathrm{C} 1$ & $\mathrm{C} 8$ & $118.1(4)$ & Ir 1 & $\mathrm{C} 2$ & $\mathrm{C} 1$ & $124.2(3)$ \\
\hline Ir1 & $\mathrm{C} 2$ & $\mathrm{C} 3$ & $118.7(3)$ & $\mathrm{C} 1$ & $\mathrm{C} 2$ & $\mathrm{C} 3$ & $116.9(4)$ \\
\hline $\mathrm{C} 2$ & $\mathrm{C} 3$ & $\mathrm{C} 4$ & $122.7(4)$ & $\mathrm{C} 3$ & $\mathrm{C} 4$ & $\mathrm{C} 5$ & $119.7(4)$ \\
\hline $\mathrm{C} 4$ & $\mathrm{C} 5$ & C6 & $120.8(4)$ & $\mathrm{C} 1$ & C6 & $\mathrm{C} 5$ & $118.8(4)$ \\
\hline $\mathrm{C} 1$ & C6 & $\mathrm{C} 7$ & $123.7(4)$ & $\mathrm{C} 5$ & C6 & $\mathrm{C} 7$ & $117.4(4)$ \\
\hline $\mathrm{O} 1$ & $\mathrm{C} 8$ & $\mathrm{C} 1$ & $123.1(4)$ & $\mathrm{O} 1$ & $\mathrm{C} 8$ & C9 & $118.8(4)$ \\
\hline $\mathrm{C} 1$ & $\mathrm{C} 8$ & C9 & $118.0(4)$ & N1 & C9 & $\mathrm{N} 2$ & $109.2(4)$ \\
\hline N1 & C9 & $\mathrm{C} 8$ & $125.0(4)$ & $\mathrm{N} 2$ & C9 & $\mathrm{C} 8$ & $125.7(4)$ \\
\hline N2 & $\mathrm{C} 10$ & $\mathrm{C} 11$ & $108.8(4)$ & N1 & $\mathrm{C} 11$ & $\mathrm{C} 10$ & $106.8(4)$ \\
\hline Ir1 & $\mathrm{C} 13$ & $\mathrm{C} 14$ & $71.5(3)$ & Ir 1 & $\mathrm{C} 13$ & $\mathrm{C} 17$ & $69.9(3)$ \\
\hline Ir1 & C13 & $\mathrm{C} 18$ & $125.8(3)$ & C14 & C13 & $\mathrm{C} 17$ & $108.2(4)$ \\
\hline $\mathrm{C} 14$ & $\mathrm{C} 13$ & $\mathrm{C} 18$ & $126.2(4)$ & $\mathrm{C} 17$ & $\mathrm{C} 13$ & $\mathrm{C} 18$ & $125.6(4)$ \\
\hline Ir 1 & $\mathrm{C} 14$ & $\mathrm{C} 13$ & $69.8(3)$ & Ir 1 & $\mathrm{C} 14$ & $\mathrm{C} 15$ & $74.0(2)$ \\
\hline Ir 1 & $\mathrm{C} 14$ & C19 & $127.0(3)$ & $\mathrm{C} 13$ & $\mathrm{C} 14$ & $\mathrm{C} 15$ & $107.0(4)$ \\
\hline C13 & C14 & C19 & $127.8(4)$ & C15 & C14 & C19 & $124.8(4)$ \\
\hline Ir 1 & $\mathrm{C} 15$ & $\mathrm{C} 14$ & $67.4(2)$ & Ir 1 & $\mathrm{C} 15$ & $\mathrm{C} 16$ & $70.9(3)$ \\
\hline Ir1 & $\mathrm{C} 15$ & $\mathrm{C} 20$ & 128.1(3) & $\mathrm{C} 14$ & $\mathrm{C} 15$ & $\mathrm{C} 16$ & $107.6(4)$ \\
\hline $\mathrm{C} 14$ & $\mathrm{C} 15$ & $\mathrm{C} 20$ & $125.3(4)$ & $\mathrm{C} 16$ & $\mathrm{C} 15$ & $\mathrm{C} 20$ & $127.0(4)$ \\
\hline Ir1 & $\mathrm{C} 16$ & $\mathrm{C} 15$ & $72.6(2)$ & $\operatorname{Ir} 1$ & C16 & $\mathrm{C} 17$ & $67.1(2)$ \\
\hline Ir1 & $\mathrm{C} 16$ & $\mathrm{C} 21$ & $126.0(3)$ & C15 & $\mathrm{C} 16$ & $\mathrm{C} 17$ & $109.3(4)$ \\
\hline C15 & $\mathrm{C} 16$ & $\mathrm{C} 21$ & $125.0(4)$ & $\mathrm{C} 17$ & C16 & $\mathrm{C} 21$ & $125.7(4)$ \\
\hline Ir1 & $\mathrm{C} 17$ & $\mathrm{C} 13$ & $70.9(3)$ & Ir 1 & $\mathrm{C} 17$ & $\mathrm{C} 16$ & $74.9(3)$ \\
\hline Ir1 & $\mathrm{C} 17$ & $\mathrm{C} 22$ & $127.5(3)$ & C13 & C17 & $\mathrm{C} 16$ & $107.6(4)$ \\
\hline $\mathrm{C} 13$ & $\mathrm{C} 17$ & $\mathrm{C} 22$ & $125.5(4)$ & $\mathrm{C} 16$ & $\mathrm{C} 17$ & $\mathrm{C} 22$ & $126.2(4)$ \\
\hline
\end{tabular}


Table S21. Bond angles involving hydrogens $\left(^{\circ}\right)$

$\begin{array}{llllllll}\text { atom } & \text { atom } & \text { atom } & \text { angle } & \text { atom } & \text { atom } & \text { atom } & \text { angle } \\ \text { C2 } & \text { C3 } & \text { H1 } & 114.6(4) & \text { C4 } & \text { C3 } & \text { H1 } & 122.8(4) \\ \text { C3 } & \text { C4 } & \text { H2 } & 118.6(5) & \text { C5 } & \text { C4 } & \text { H2 } & 121.7(5) \\ \text { C4 } & \text { C5 } & \text { H3 } & 116.5(5) & \text { C6 } & \text { C5 } & \text { H3 } & 122.5(5) \\ \text { C6 } & \text { C7 } & \text { H4 } & 109.2(4) & \text { C6 } & \text { C7 } & \text { H5 } & 115.5(4) \\ \text { C6 } & \text { C7 } & \text { H6 } & 107.8(4) & \text { H4 } & \text { C7 } & \text { H5 } & 107.5(5) \\ \text { H4 } & \text { C7 } & \text { H6 } & 113.1(5) & \text { H5 } & \text { C7 } & \text { H6 } & 103.8(5) \\ \text { N2 } & \text { C10 } & \text { H7 } & 121.6(5) & \text { C11 } & \text { C10 } & \text { H7 } & 129.5(5) \\ \text { N1 } & \text { C11 } & \text { H8 } & 121.7(4) & \text { C10 } & \text { C11 } & \text { H8 } & 131.4(5) \\ \text { N1 } & \text { C12 } & \text { H9 } & 109.5(4) & \text { N1 } & \text { C12 } & \text { H10 } & 109.5(4) \\ \text { N1 } & \text { C12 } & \text { H11 } & 109.5(4) & \text { H9 } & \text { C12 } & \text { H10 } & 109.5(5) \\ \text { H9 } & \text { C12 } & \text { H11 } & 109.5(5) & \text { H10 } & \text { C12 } & \text { H11 } & 109.5(4) \\ \text { C13 } & \text { C18 } & \text { H12 } & 109.4(5) & \text { C13 } & \text { C18 } & \text { H13 } & 107.5(5) \\ \text { C13 } & \text { C18 } & \text { H14 } & 114.5(5) & \text { H12 } & \text { C18 } & \text { H13 } & 110.5(5) \\ \text { H12 } & \text { C18 } & \text { H14 } & 103.5(5) & \text { H13 } & \text { C18 } & \text { H14 } & 111.4(5) \\ \text { C14 } & \text { C19 } & \text { H15 } & 107.4(5) & \text { C14 } & \text { C19 } & \text { H16 } & 111.1(5) \\ \text { C14 } & \text { C19 } & \text { H17 } & 114.3(5) & \text { H15 } & \text { C19 } & \text { H16 } & 111.1(5) \\ \text { H15 } & \text { C19 } & \text { H17 } & 109.0(5) & \text { H16 } & \text { C19 } & \text { H17 } & 103.9(6) \\ \text { C15 } & \text { C20 } & \text { H18 } & 110.6(4) & \text { C15 } & \text { C20 } & \text { H19 } & 113.9(5) \\ \text { C15 } & \text { C20 } & \text { H20 } & 106.3(5) & \text { H18 } & \text { C20 } & \text { H19 } & 107.6(5) \\ \text { H18 } & \text { C20 } & \text { H20 } & 118.1(6) & \text { H19 } & \text { C20 } & \text { H20 } & 100.2(5) \\ \text { C16 } & \text { C21 } & \text { H21 } & 109.4(5) & \text { C16 } & \text { C21 } & \text { H22 } & 109.5(4) \\ \text { C16 } & \text { C21 } & \text { H23 } & 109.4(5) & \text { H21 } & \text { C21 } & \text { H22 } & 109.5(5) \\ \text { H21 } & \text { C21 } & \text { H23 } & 109.4(5) & \text { H22 } & \text { C21 } & \text { H23 } & 109.5(5) \\ \text { C17 } & \text { C22 } & \text { H24 } & 105.9(4) & \text { C17 } & \text { C22 } & \text { H25 } & 107.4(4) \\ \text { C17 } & \text { C22 } & \text { H26 } & 106.7(5) & \text { H24 } & \text { C22 } & \text { H25 } & 103.7(5) \\ \text { H24 } & \text { C22 } & \text { H26 } & 122.4(6) & \text { H25 } & \text { C22 } & \text { H26 } & 109.9(5)\end{array}$


B. X-ray analysis of intermediate B' $\left(\mathrm{C}_{23} \mathrm{H}_{28} \mathrm{CIIrN}{ }_{2} \mathrm{O}\right)$ : CCDC 1949237

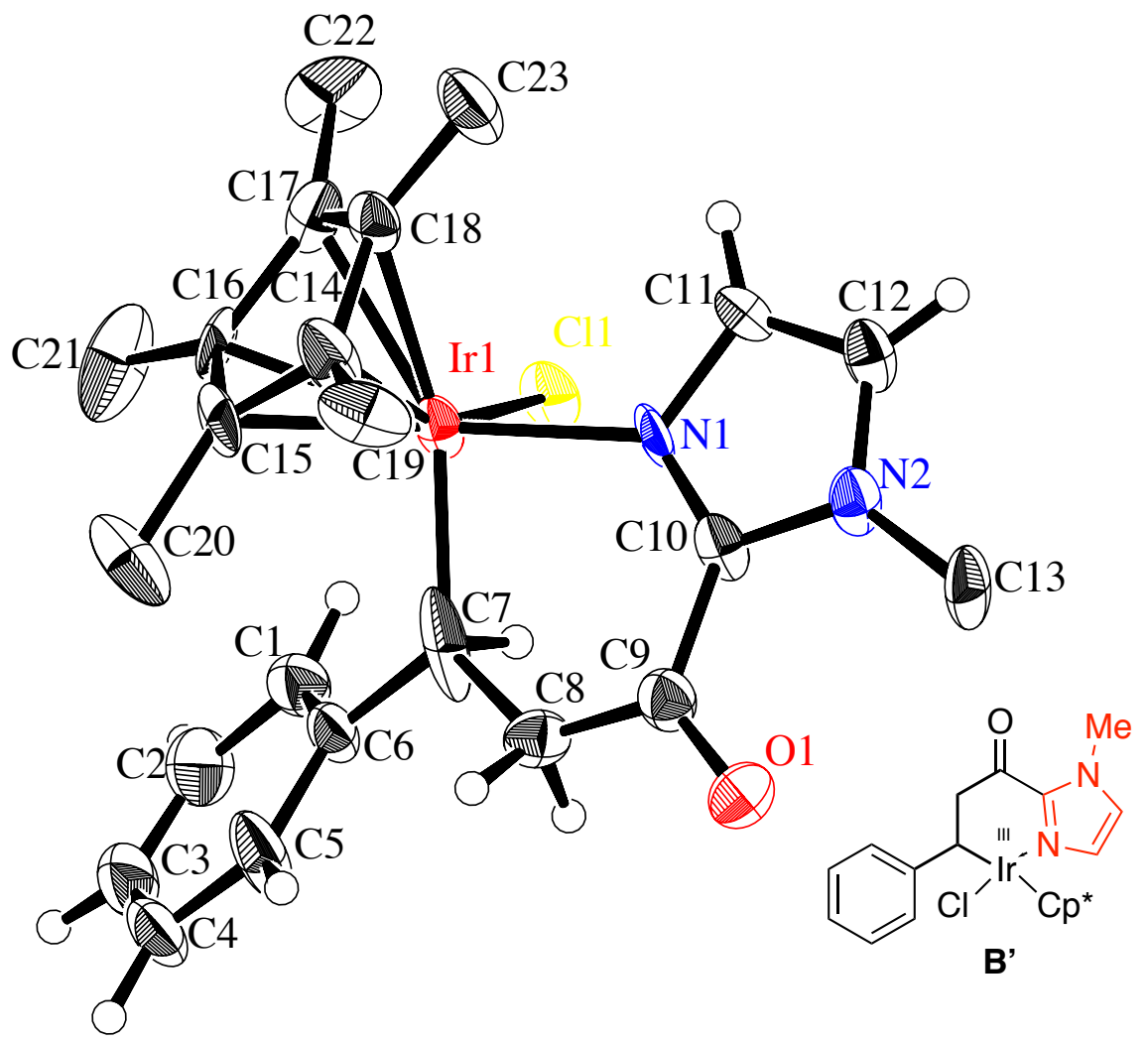

Figure S20: ORTEP diagram of intermediate B'. 
CCDC 1949237 contains the supplementary crystallographic data. X-Ray crystallographic structure analysis of intermediate B' was recorded on Rigaku XtaLAB P200 using multi-layer mirror monochromated $\mathrm{Cu}-\mathrm{K} \alpha$ radiation. The crystal-to-detector distance was $40.00 \mathrm{~mm}$. The data were collected at a temperature of $-150 \pm 1^{\circ} \mathrm{C}$ to a maximum $2 \theta$ value of $149.4^{\circ}$. A total of 34248 reflections were collected, where 8388 were unique $($ Rint $=0.0817$ ). Data were collected and processed using CrysAlisPro (Rigaku Oxford Diffraction) ${ }^{4}$ and refined by the full matrix least-squares on F2 (Crystals). The structure was solved by direct methods (SIR92) ${ }^{5}$ and expanded using Fourier techniques. The non-hydrogen atoms were refined anisotropically. Hydrogen atoms were refined using the riding model. The final cycle of full-matrix leastsquares refinement ${ }^{6}$ on F2 was based on 8388 observed reflections and 500 variable parameters. The crystal data are given below:

\section{Crystal Data for CCDC $1949237\left(\mathrm{C}_{23} \mathrm{H}_{28} \mathrm{ClIrN}_{2} \mathrm{O}\right)$}

Empirical Formula

Formula Weight

Crystal Color, Habit

Crystal Dimensions

Crystal System

Lattice Type

Lattice Parameters

Space Group

$\mathrm{Z}$ value

Dcalc

$\mathrm{F}_{000}$

$\mathrm{m}(\mathrm{CuK} \alpha)$
$\mathrm{C}_{23} \mathrm{H}_{28} \mathrm{ClIrN}_{2} \mathrm{O}$

576.16

orange, prism

$0.200 \times 0.100 \times 0.050 \mathrm{~mm}$

monoclinic

Primitive

$\mathrm{a}=20.6873(11) \AA$

$\mathrm{b}=7.8511(4) \AA$

$\mathrm{c}=26.1279(12) \AA$

$\mathrm{b}=98.161(4)^{\mathrm{O}}$

$\mathrm{V}=4200.7(4) \AA^{3}$

$\mathrm{P} 21 / \mathrm{c}(\# 14)$

8

$1.822 \mathrm{~g} / \mathrm{cm}^{3}$

2256.00

$133.196 \mathrm{~cm}^{-1}$ 
Table S22. Bond lengths ( $)$

\begin{tabular}{|c|c|c|c|c|c|}
\hline atom & atom & distance & atom & atom & distance \\
\hline Ir1 & $\mathrm{Cl1}$ & $2.415(4)$ & Ir 1 & N1 & $2.082(14)$ \\
\hline Ir1 & $\mathrm{C} 7$ & $1.98(3)$ & Ir 1 & $\mathrm{C} 14$ & $2.11(3)$ \\
\hline Ir1 & $\mathrm{C} 15$ & $2.200(19)$ & Ir 1 & $\mathrm{C} 16$ & $2.179(17)$ \\
\hline Ir1 & $\mathrm{C} 17$ & $2.234(19)$ & Ir 1 & $\mathrm{C} 18$ & $2.268(19)$ \\
\hline Ir2 & $\mathrm{Cl} 2$ & $2.408(4)$ & Ir 2 & $\mathrm{~N} 3$ & $2.077(13)$ \\
\hline Ir 2 & $\mathrm{C} 30$ & $2.051(16)$ & $\operatorname{Ir} 2$ & C37 & $2.177(16)$ \\
\hline Ir2 & C38 & $2.247(16)$ & Ir 2 & C39 & $2.194(16)$ \\
\hline Ir 2 & $\mathrm{C} 40$ & $2.13(2)$ & $\operatorname{Ir} 2$ & $\mathrm{C} 41$ & $2.167(17)$ \\
\hline $\mathrm{O} 1$ & C9 & $1.25(2)$ & $\mathrm{O} 2$ & C32 & $1.21(2)$ \\
\hline N1 & $\mathrm{C} 10$ & $1.34(2)$ & N1 & $\mathrm{C} 11$ & $1.38(2)$ \\
\hline N2 & $\mathrm{C} 10$ & $1.38(2)$ & $\mathrm{N} 2$ & $\mathrm{C} 12$ & $1.37(2)$ \\
\hline N2 & $\mathrm{C} 13$ & $1.47(2)$ & $\mathrm{N} 3$ & C33 & $1.33(2)$ \\
\hline N3 & C34 & $1.394(19)$ & N4 & C33 & $1.36(2)$ \\
\hline N4 & C35 & $1.39(2)$ & N4 & C36 & $1.48(2)$ \\
\hline $\mathrm{C} 1$ & $\mathrm{C} 2$ & $1.40(3)$ & $\mathrm{C} 1$ & C6 & $1.41(2)$ \\
\hline $\mathrm{C} 2$ & $\mathrm{C} 3$ & $1.33(3)$ & $\mathrm{C} 3$ & $\mathrm{C} 4$ & $1.37(3)$ \\
\hline $\mathrm{C} 4$ & $\mathrm{C} 5$ & $1.41(3)$ & $\mathrm{C} 5$ & $\mathrm{C} 6$ & $1.39(3)$ \\
\hline C6 & $\mathrm{C} 7$ & $1.53(3)$ & $\mathrm{C} 7$ & $\mathrm{C} 8$ & $1.40(2)$ \\
\hline $\mathrm{C} 8$ & C9 & $1.44(3)$ & C9 & $\mathrm{C} 10$ & $1.48(3)$ \\
\hline $\mathrm{C} 11$ & $\mathrm{C} 12$ & $1.37(2)$ & $\mathrm{C} 14$ & $\mathrm{C} 15$ & $1.42(3)$ \\
\hline $\mathrm{C} 14$ & $\mathrm{C} 18$ & $1.43(3)$ & $\mathrm{C} 14$ & $\mathrm{C} 19$ & $1.54(3)$ \\
\hline $\mathrm{C} 15$ & $\mathrm{C} 16$ & $1.40(2)$ & $\mathrm{C} 15$ & $\mathrm{C} 20$ & $1.54(3)$ \\
\hline $\mathrm{C} 16$ & $\mathrm{C} 17$ & $1.46(3)$ & $\mathrm{C} 16$ & $\mathrm{C} 21$ & $1.50(3)$ \\
\hline $\mathrm{C} 17$ & $\mathrm{C} 18$ & $1.40(2)$ & $\mathrm{C} 17$ & $\mathrm{C} 22$ & $1.51(2)$ \\
\hline C18 & $\mathrm{C} 23$ & $1.52(3)$ & $\mathrm{C} 24$ & $\mathrm{C} 25$ & $1.39(3)$ \\
\hline $\mathrm{C} 24$ & $\mathrm{C} 29$ & $1.41(2)$ & $\mathrm{C} 25$ & $\mathrm{C} 26$ & $1.38(3)$ \\
\hline $\mathrm{C} 26$ & $\mathrm{C} 27$ & $1.40(3)$ & $\mathrm{C} 27$ & $\mathrm{C} 28$ & $1.40(3)$ \\
\hline $\mathrm{C} 28$ & $\mathrm{C} 29$ & $1.39(2)$ & $\mathrm{C} 29$ & C30 & $1.47(2)$ \\
\hline $\mathrm{C} 30$ & C31 & $1.36(2)$ & C31 & C32 & $1.43(3)$ \\
\hline C32 & C33 & $1.52(2)$ & C34 & C35 & $1.36(3)$ \\
\hline C37 & C38 & $1.47(2)$ & C37 & $\mathrm{C} 41$ & $1.45(2)$ \\
\hline $\mathrm{C} 37$ & $\mathrm{C} 42$ & $1.50(2)$ & C38 & C39 & $1.40(2)$ \\
\hline C38 & $\mathrm{C} 43$ & $1.50(2)$ & C39 & $\mathrm{C} 40$ & $1.43(2)$ \\
\hline C39 & $\mathrm{C} 44$ & $1.52(2)$ & $\mathrm{C} 40$ & $\mathrm{C} 41$ & $1.41(2)$ \\
\hline $\mathrm{C} 40$ & $\mathrm{C} 45$ & $1.53(3)$ & $\mathrm{C} 41$ & $\mathrm{C} 46$ & $1.50(2)$ \\
\hline
\end{tabular}

Table S23. Bond lengths involving hydrogens $(\AA)$

$\begin{array}{llllll}\text { atom } & \text { atom } & \text { distance } & \text { atom } & \text { atom } & \text { distance } \\ \text { C1 } & \mathrm{H} 1 & 0.950 & \mathrm{C} 2 & \mathrm{H} 2 & 0.950 \\ \mathrm{C} 3 & \mathrm{H} 3 & 0.950 & \mathrm{C} 4 & \mathrm{H} 4 & 0.950\end{array}$


Table S23. Bond lengths involving hydrogens $(\AA)$ (Continued)

$\begin{array}{llllll}\text { atom } & \text { atom } & \text { distance } & \text { atom } & \text { atom } & \text { distance } \\ \text { C5 } & \text { H5 } & 0.950 & \text { C7 } & \text { H7 } & 1.000 \\ \text { C8 } & \text { H8A } & 0.990 & \text { C8 } & \text { H8B } & 0.990 \\ \text { C11 } & \text { H11 } & 0.950 & \text { C12 } & \text { H12 } & 0.950 \\ \text { C24 } & \text { H24 } & 0.950 & \text { C25 } & \text { H25 } & 0.950 \\ \text { C26 } & \text { H26 } & 0.950 & \text { C27 } & \text { H27 } & 0.950 \\ \text { C28 } & \text { H28 } & 0.950 & \text { C30 } & \text { H30 } & 1.000 \\ \text { C31 } & \text { H31A } & 0.990 & \text { C31 } & \text { H31B } & 0.990 \\ \text { C34 } & \text { H34 } & 0.950 & \text { C35 } & \text { H35 } & 0.950\end{array}$

Table S24. Bond angles $\left({ }^{\circ}\right)$

\begin{tabular}{|c|c|c|c|c|c|c|c|}
\hline atom & atom & atom & angle & atom & atom & atom & angle \\
\hline Cl1 & Ir1 & N1 & $85.2(4)$ & Cl1 & Ir1 & C7 & $93.6(7)$ \\
\hline $\mathrm{Cl1}$ & Ir1 & $\mathrm{C} 14$ & $155.6(6)$ & $\mathrm{Cl1}$ & Ir1 & $\mathrm{C} 15$ & $138.9(5)$ \\
\hline Cl1 & Ir 1 & $\mathrm{C} 16$ & $103.1(5)$ & $\mathrm{Cl1}$ & Ir 1 & $\mathrm{C} 17$ & $93.5(5)$ \\
\hline Cl1 & Ir 1 & $\mathrm{C} 18$ & $118.2(4)$ & N1 & Ir 1 & $\mathrm{C} 7$ & $87.0(7)$ \\
\hline N1 & Ir 1 & $\mathrm{C} 14$ & $100.0(6)$ & N1 & Ir1 & $\mathrm{C} 15$ & $135.4(6)$ \\
\hline N1 & Ir1 & $\mathrm{C} 16$ & $158.6(7)$ & N1 & Ir1 & $\mathrm{C} 17$ & $122.2(7)$ \\
\hline N1 & Ir1 & $\mathrm{C} 18$ & $96.1(6)$ & $\mathrm{C} 7$ & Ir1 & $\mathrm{C} 14$ & $110.3(9)$ \\
\hline $\mathrm{C} 7$ & Ir1 & $\mathrm{C} 15$ & $94.2(8)$ & $\mathrm{C} 7$ & Ir1 & $\mathrm{C} 16$ & $111.8(8)$ \\
\hline $\mathrm{C} 7$ & Ir1 & $\mathrm{C} 17$ & $150.5(7)$ & $\mathrm{C} 7$ & Ir1 & $\mathrm{C} 18$ & $148.1(8)$ \\
\hline C14 & Ir1 & $\mathrm{C} 15$ & $38.3(7)$ & $\mathrm{C} 14$ & Ir1 & $\mathrm{C} 16$ & $64.6(7)$ \\
\hline C14 & Ir1 & $\mathrm{C} 17$ & $63.5(7)$ & $\mathrm{C} 14$ & Ir1 & $\mathrm{C} 18$ & $37.9(7)$ \\
\hline C15 & Ir1 & $\mathrm{C} 16$ & $37.3(7)$ & $\mathrm{C} 15$ & Ir1 & $\mathrm{C} 17$ & $62.5(6)$ \\
\hline C15 & Ir1 & C18 & $61.7(6)$ & C16 & Ir1 & C17 & $38.7(7)$ \\
\hline C16 & Ir1 & $\mathrm{C} 18$ & $62.6(7)$ & $\mathrm{C} 17$ & Ir1 & $\mathrm{C} 18$ & $36.2(6)$ \\
\hline $\mathrm{Cl} 2$ & Ir2 & N3 & $84.3(4)$ & $\mathrm{Cl} 2$ & Ir2 & C30 & $95.2(5)$ \\
\hline $\mathrm{Cl} 2$ & Ir2 & C37 & $114.7(5)$ & $\mathrm{Cl} 2$ & Ir2 & C38 & $92.9(4)$ \\
\hline $\mathrm{Cl} 2$ & Ir2 & C39 & $105.2(4)$ & $\mathrm{Cl} 2$ & Ir2 & $\mathrm{C} 40$ & $142.9(5)$ \\
\hline $\mathrm{Cl} 2$ & Ir2 & C41 & $153.5(5)$ & N3 & Ir2 & C30 & $86.1(6)$ \\
\hline N3 & Ir2 & C37 & $159.1(7)$ & N3 & $\operatorname{Ir} 2$ & C38 & $137.5(6)$ \\
\hline N3 & Ir 2 & C39 & $103.3(6)$ & N3 & Ir2 & $\mathrm{C} 40$ & $95.0(6)$ \\
\hline N3 & Ir 2 & C41 & $121.3(6)$ & $\mathrm{C} 30$ & Ir2 & C37 & $100.1(6)$ \\
\hline $\mathrm{C} 30$ & Ir 2 & C38 & $136.3(6)$ & $\mathrm{C} 30$ & $\operatorname{Ir} 2$ & C39 & $158.2(6)$ \\
\hline $\mathrm{C} 30$ & Ir 2 & $\mathrm{C} 40$ & $121.8(6)$ & $\mathrm{C} 30$ & Ir2 & C41 & $93.6(6)$ \\
\hline $\mathrm{C} 37$ & Ir 2 & C38 & $38.8(6)$ & C37 & Ir2 & C39 & $64.5(6)$ \\
\hline $\mathrm{C} 37$ & Ir 2 & $\mathrm{C} 40$ & $64.7(7)$ & C37 & Ir2 & $\mathrm{C} 41$ & $39.0(7)$ \\
\hline C38 & Ir2 & C39 & $36.8(6)$ & C38 & Ir2 & $\mathrm{C} 40$ & $63.0(6)$ \\
\hline C38 & Ir 2 & $\mathrm{C} 41$ & $64.0(6)$ & C39 & Ir2 & $\mathrm{C} 40$ & $38.8(6)$ \\
\hline C39 & Ir2 & C41 & $64.7(6)$ & $\mathrm{C} 40$ & Ir2 & C41 & $38.3(6)$ \\
\hline $\operatorname{Ir} 1$ & N1 & $\mathrm{C} 10$ & $128.2(12)$ & Ir 1 & N1 & $\mathrm{C} 11$ & $123.3(11)$ \\
\hline $\mathrm{C} 10$ & N1 & $\mathrm{C} 11$ & $107.6(14)$ & $\mathrm{C} 10$ & $\mathrm{~N} 2$ & $\mathrm{C} 12$ & $106.4(13)$ \\
\hline $\mathrm{C} 10$ & $\mathrm{~N} 2$ & $\mathrm{C} 13$ & $128.7(15)$ & $\mathrm{C} 12$ & $\mathrm{~N} 2$ & $\mathrm{C} 13$ & $124.7(15)$ \\
\hline Ir 2 & N3 & C33 & $127.0(10)$ & Ir 2 & N3 & C34 & $125.0(11)$ \\
\hline C33 & N3 & C34 & $106.6(14)$ & C33 & N4 & C35 & $106.6(14)$ \\
\hline
\end{tabular}


Table S24. Bond angles $\left({ }^{\circ}\right)$ (Continued)

\begin{tabular}{|c|c|c|c|c|c|c|c|}
\hline atom & atom & atom & angle & atom & atom & atom & angle \\
\hline C33 & N4 & $\mathrm{C} 36$ & $129.2(14)$ & $\mathrm{C} 35$ & N4 & $\mathrm{C} 36$ & $124.2(14)$ \\
\hline $\mathrm{C} 2$ & $\mathrm{C} 1$ & C6 & $119.3(18)$ & $\mathrm{C} 1$ & $\mathrm{C} 2$ & $\mathrm{C} 3$ & $120.6(19)$ \\
\hline $\mathrm{C} 2$ & $\mathrm{C} 3$ & $\mathrm{C} 4$ & $122(2)$ & $\mathrm{C} 3$ & $\mathrm{C} 4$ & $\mathrm{C} 5$ & $118(2)$ \\
\hline C4 & $\mathrm{C} 5$ & C6 & $120.9(17)$ & $\mathrm{C} 1$ & C6 & $\mathrm{C} 5$ & $118.3(18)$ \\
\hline $\mathrm{C} 1$ & C6 & C7 & $118.7(16)$ & $\mathrm{C} 5$ & C6 & $\mathrm{C} 7$ & $123.0(15)$ \\
\hline Ir1 & C7 & C6 & $121.6(13)$ & Ir1 & C7 & $\mathrm{C} 8$ & $127.3(16)$ \\
\hline C6 & C7 & C8 & 111(2) & C7 & C8 & C9 & $127.3(18)$ \\
\hline $\mathrm{O} 1$ & C9 & $\mathrm{C} 8$ & $121.3(17)$ & $\mathrm{O} 1$ & C9 & $\mathrm{C} 10$ & $119.2(16)$ \\
\hline $\mathrm{C} 8$ & C9 & $\mathrm{C} 10$ & 119.3(14) & N1 & C10 & N2 & $110.1(14)$ \\
\hline N1 & C10 & C9 & $125.1(15)$ & $\mathrm{N} 2$ & $\mathrm{C} 10$ & C9 & $124.6(14)$ \\
\hline N1 & C11 & C12 & $107.5(15)$ & N2 & C12 & $\mathrm{C} 11$ & $108.4(15)$ \\
\hline Ir1 & C14 & C15 & $74.4(13)$ & Ir1 & C14 & $\mathrm{C} 18$ & $77.2(12)$ \\
\hline Ir1 & C14 & C19 & $124.9(16)$ & $\mathrm{C} 15$ & C14 & $\mathrm{C} 18$ & $107.4(15)$ \\
\hline $\mathrm{C} 15$ & C14 & C19 & $123.6(17)$ & $\mathrm{C} 18$ & C14 & C19 & $127.6(19)$ \\
\hline Ir1 & C15 & C14 & $67.2(12)$ & Ir1 & $\mathrm{C} 15$ & $\mathrm{C} 16$ & $70.5(11)$ \\
\hline Ir1 & $\mathrm{C} 15$ & $\mathrm{C} 20$ & $122.7(13)$ & $\mathrm{C} 14$ & $\mathrm{C} 15$ & $\mathrm{C} 16$ & $108.7(16)$ \\
\hline $\mathrm{C} 14$ & $\mathrm{C} 15$ & $\mathrm{C} 20$ & $125.7(16)$ & $\mathrm{C} 16$ & $\mathrm{C} 15$ & $\mathrm{C} 20$ & $125.2(18)$ \\
\hline Ir1 & C16 & $\mathrm{C} 15$ & $72.1(10)$ & Ir1 & C16 & $\mathrm{C} 17$ & $72.7(10)$ \\
\hline Ir1 & C16 & $\mathrm{C} 21$ & $129.1(16)$ & $\mathrm{C} 15$ & C16 & $\mathrm{C} 17$ & $106.7(15)$ \\
\hline $\mathrm{C} 15$ & C16 & $\mathrm{C} 21$ & $129(2)$ & $\mathrm{C} 17$ & C16 & $\mathrm{C} 21$ & $123.4(18)$ \\
\hline Ir1 & C17 & C16 & $68.6(10)$ & Ir1 & $\mathrm{C} 17$ & $\mathrm{C} 18$ & $73.3(11)$ \\
\hline Ir1 & $\mathrm{C} 17$ & $\mathrm{C} 22$ & $127.1(15)$ & $\mathrm{C} 16$ & $\mathrm{C} 17$ & $\mathrm{C} 18$ & $107.7(14)$ \\
\hline $\mathrm{C} 16$ & $\mathrm{C} 17$ & $\mathrm{C} 22$ & $124.8(17)$ & $\mathrm{C} 18$ & $\mathrm{C} 17$ & $\mathrm{C} 22$ & $127.3(18)$ \\
\hline Ir1 & $\mathrm{C} 18$ & C14 & $64.9(13)$ & Ir1 & $\mathrm{C} 18$ & $\mathrm{C} 17$ & $70.6(11)$ \\
\hline Ir1 & $\mathrm{C} 18$ & $\mathrm{C} 23$ & $129.5(14)$ & $\mathrm{C} 14$ & $\mathrm{C} 18$ & $\mathrm{C} 17$ & $108.0(16)$ \\
\hline $\mathrm{C} 14$ & $\mathrm{C} 18$ & $\mathrm{C} 23$ & $125.8(15)$ & $\mathrm{C} 17$ & $\mathrm{C} 18$ & $\mathrm{C} 23$ & $126.2(15)$ \\
\hline $\mathrm{C} 25$ & $\mathrm{C} 24$ & $\mathrm{C} 29$ & $120.1(15)$ & $\mathrm{C} 24$ & $\mathrm{C} 25$ & $\mathrm{C} 26$ & $121.9(18)$ \\
\hline $\mathrm{C} 25$ & $\mathrm{C} 26$ & $\mathrm{C} 27$ & $118(2)$ & $\mathrm{C} 26$ & $\mathrm{C} 27$ & $\mathrm{C} 28$ & $120.1(17)$ \\
\hline $\mathrm{C} 27$ & $\mathrm{C} 28$ & $\mathrm{C} 29$ & $121.0(17)$ & $\mathrm{C} 24$ & $\mathrm{C} 29$ & $\mathrm{C} 28$ & $118.2(16)$ \\
\hline $\mathrm{C} 24$ & $\mathrm{C} 29$ & $\mathrm{C} 30$ & $120.7(14)$ & $\mathrm{C} 28$ & $\mathrm{C} 29$ & $\mathrm{C} 30$ & $121.1(15)$ \\
\hline Ir2 & $\mathrm{C} 30$ & $\mathrm{C} 29$ & $118.0(11)$ & Ir2 & $\mathrm{C} 30$ & $\mathrm{C} 31$ & $124.7(13)$ \\
\hline $\mathrm{C} 29$ & C30 & C31 & $116.8(15)$ & $\mathrm{C} 30$ & C31 & $\mathrm{C} 32$ & $129.3(16)$ \\
\hline $\mathrm{O} 2$ & $\mathrm{C} 32$ & $\mathrm{C} 31$ & $124.6(16)$ & $\mathrm{O} 2$ & $\mathrm{C} 32$ & $\mathrm{C} 33$ & $117.6(16)$ \\
\hline C31 & $\mathrm{C} 32$ & $\mathrm{C} 33$ & $117.6(15)$ & N3 & $\mathrm{C} 33$ & N4 & $111.1(14)$ \\
\hline N3 & C33 & C32 & $124.4(16)$ & N4 & C33 & $\mathrm{C} 32$ & $124.1(16)$ \\
\hline N3 & C34 & $\mathrm{C} 35$ & $108.3(15)$ & N4 & C35 & $\mathrm{C} 34$ & $107.4(14)$ \\
\hline $\operatorname{Ir} 2$ & C37 & $\mathrm{C} 38$ & 73.2(9) & Ir2 & C37 & $\mathrm{C} 41$ & 70.1(9) \\
\hline Ir2 & C37 & $\mathrm{C} 42$ & $132.5(15)$ & $\mathrm{C} 38$ & $\mathrm{C} 37$ & $\mathrm{C} 41$ & $106.5(14)$ \\
\hline $\mathrm{C} 38$ & C37 & $\mathrm{C} 42$ & $125.3(15)$ & $\mathrm{C} 41$ & C37 & $\mathrm{C} 42$ & $126.7(13)$ \\
\hline Ir2 & C38 & C37 & $68.0(9)$ & Ir2 & C38 & C39 & $69.5(9)$ \\
\hline $\operatorname{Ir} 2$ & C38 & $\mathrm{C} 43$ & $123.0(12)$ & $\mathrm{C} 37$ & $\mathrm{C} 38$ & C39 & $108.6(13)$ \\
\hline C37 & C38 & $\mathrm{C} 43$ & $123.8(15)$ & C39 & C38 & $\mathrm{C} 43$ & $127.3(13)$ \\
\hline Ir2 & C39 & $\mathrm{C} 38$ & 73.6(9) & Ir2 & C39 & $\mathrm{C} 40$ & $68.1(10)$ \\
\hline Ir2 & C39 & $\mathrm{C} 44$ & $129.5(11)$ & $\mathrm{C} 38$ & C39 & $\mathrm{C} 40$ & $107.4(13)$ \\
\hline $\mathrm{C} 38$ & C39 & $\mathrm{C} 44$ & $126.5(14)$ & $\mathrm{C} 40$ & C39 & $\mathrm{C} 44$ & $125.7(15)$ \\
\hline Ir2 & $\mathrm{C} 40$ & C39 & $73.2(10)$ & Ir 2 & $\mathrm{C} 40$ & $\mathrm{C} 41$ & $72.4(11)$ \\
\hline Ir2 & $\mathrm{C} 40$ & $\mathrm{C} 45$ & $124.7(14)$ & C39 & $\mathrm{C} 40$ & $\mathrm{C} 41$ & $110.1(15)$ \\
\hline C39 & $\mathrm{C} 40$ & $\mathrm{C} 45$ & $124.5(15)$ & $\mathrm{C} 41$ & $\mathrm{C} 40$ & $\mathrm{C} 45$ & $125.3(15)$ \\
\hline Ir2 & C41 & C37 & $70.9(9)$ & Ir2 & $\mathrm{C} 41$ & $\mathrm{C} 40$ & $69.3(11)$ \\
\hline Ir2 & C41 & $\mathrm{C} 46$ & $127.8(14)$ & $\mathrm{C} 37$ & $\mathrm{C} 41$ & $\mathrm{C} 40$ & $107.2(14)$ \\
\hline C37 & C41 & C46 & $126.8(16)$ & $\mathrm{C} 40$ & C41 & $\mathrm{C} 46$ & $125.9(16)$ \\
\hline
\end{tabular}


Table S25. Bond angles involving hydrogens $\left({ }^{\circ}\right)$

$\begin{array}{llllllll}\text { atom } & \text { atom } & \text { atom } & \text { angle } & \text { atom } & \text { atom } & \text { atom } & \text { angle } \\ \text { C2 } & \text { C1 } & \text { H1 } & 120.3 & \text { C6 } & \text { C1 } & \text { H1 } & 120.3 \\ \text { C1 } & \text { C2 } & \text { H2 } & 119.7 & \text { C3 } & \text { C2 } & \text { H2 } & 119.7 \\ \text { C2 } & \text { C3 } & \text { H3 } & 118.8 & \text { C4 } & \text { C3 } & \text { H3 } & 118.8 \\ \text { C3 } & \text { C4 } & \text { H4 } & 120.9 & \text { C5 } & \text { C4 } & \text { H4 } & 120.9 \\ \text { C4 } & \text { C5 } & \text { H5 } & 119.6 & \text { C6 } & \text { C5 } & \text { H5 } & 119.5 \\ \text { Ir1 } & \text { C7 } & \text { H7 } & 91.9 & \text { C6 } & \text { C7 } & \text { H7 } & 91.9 \\ \text { C8 } & \text { C7 } & \text { H7 } & 91.9 & \text { C7 } & \text { C8 } & \text { H8A } & 105.5 \\ \text { C7 } & \text { C8 } & \text { H8B } & 105.5 & \text { C9 } & \text { C8 } & \text { H8A } & 105.5 \\ \text { C9 } & \text { C8 } & \text { H8B } & 105.5 & \text { H8A } & \text { C8 } & \text { H8B } & 106.1 \\ \text { N1 } & \text { C11 } & \text { H11 } & 126.2 & \text { C12 } & \text { C11 } & \text { H11 } & 126.2 \\ \text { N2 } & \text { C12 } & \text { H12 } & 125.8 & \text { C11 } & \text { C12 } & \text { H12 } & 125.8 \\ \text { C25 } & \text { C24 } & \text { H24 } & 120.0 & \text { C29 } & \text { C24 } & \text { H24 } & 119.9 \\ \text { C24 } & \text { C25 } & \text { H25 } & 119.1 & \text { C26 } & \text { C25 } & \text { H25 } & 119.0 \\ \text { C25 } & \text { C26 } & \text { H26 } & 120.8 & \text { C27 } & \text { C26 } & \text { H26 } & 120.8 \\ \text { C26 } & \text { C27 } & \text { H27 } & 120.0 & \text { C28 } & \text { C27 } & \text { H27 } & 119.9 \\ \text { C27 } & \text { C28 } & \text { H28 } & 119.5 & \text { C29 } & \text { C28 } & \text { H28 } & 119.5 \\ \text { Ir2 } & \text { C30 } & \text { H30 } & 92.2 & \text { C29 } & \text { C30 } & \text { H30 } & 92.2 \\ \text { C31 } & \text { C30 } & \text { H30 } & 92.2 & \text { C30 } & \text { C31 } & \text { H31A } & 105.0 \\ \text { C30 } & \text { C31 } & \text { H31B } & 105.0 & \text { C32 } & \text { C31 } & \text { H31A } & 105.0 \\ \text { C32 } & \text { C31 } & \text { H31B } & 105.0 & \text { H31A } & \text { C31 } & \text { H31B } & 105.8 \\ \text { N3 } & \text { C34 } & \text { H34 } & 125.9 & \text { C35 } & \text { C34 } & \text { H34 } & 125.9 \\ \text { N4 } & \text { C35 } & \text { H35 } & 126.3 & \text { C34 } & \text { C35 } & \text { H35 } & 126.3\end{array}$


C. X-ray analysis of intermediate $\mathrm{E}\left(\mathrm{C}_{65} \mathrm{H}_{59} \mathrm{BCIF}_{24} \mathrm{IrN}_{2} \mathrm{OSi}\right)$ : CCDC 1964835
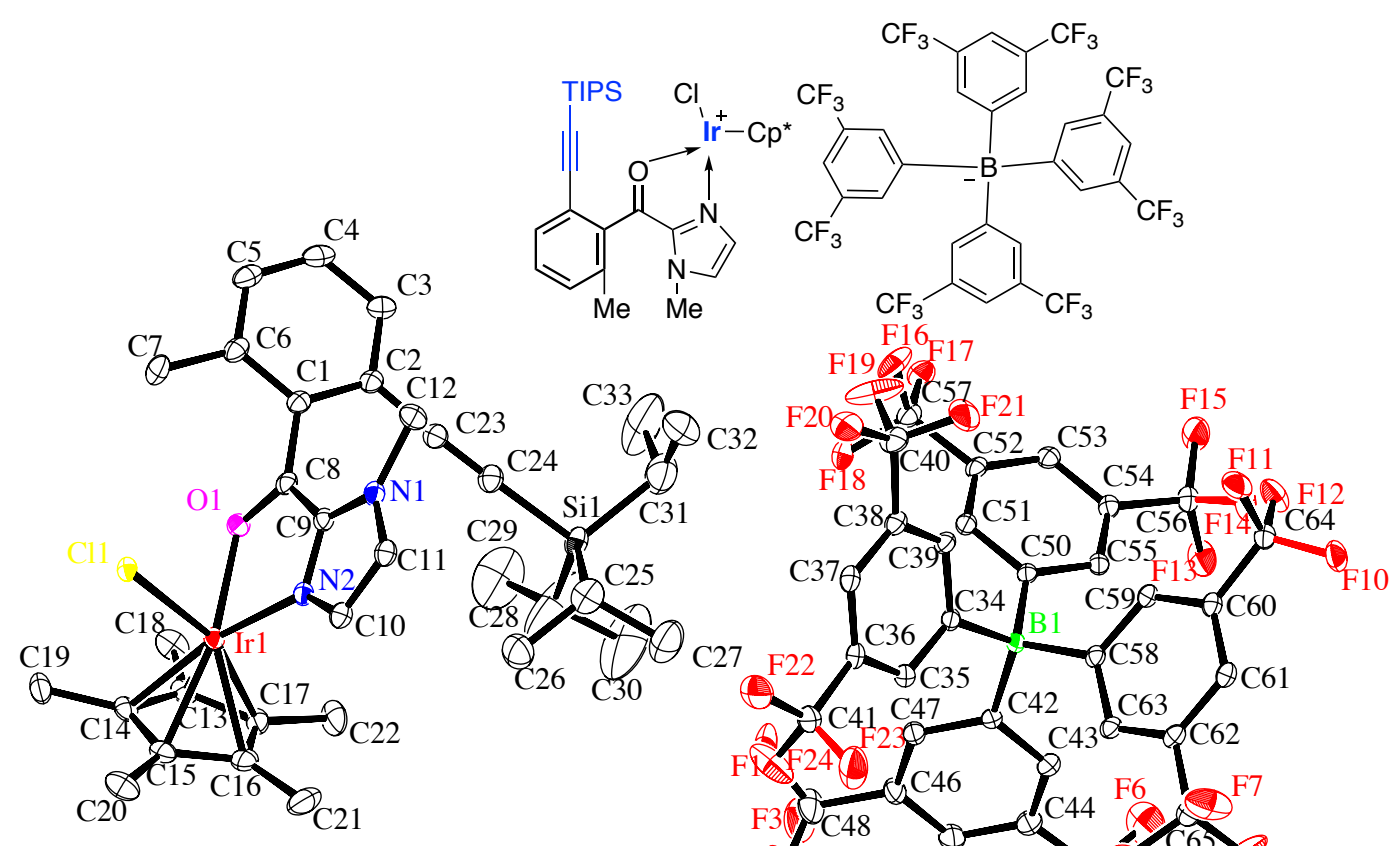

Figure S21: ORTEP diagram of intermediate E. 
CCDC 1964835 contains the supplementary crystallographic data. X-Ray crystallographic structure analysis of intermediate $\mathbf{E}$ was recorded on Rigaku XtaLAB P200 using multi-layer mirror monochromated $\mathrm{Cu}-\mathrm{K} \alpha$ radiation. The crystal-to-detector distance was $64.00 \mathrm{~mm}$. The data were collected at a temperature of $-150 \pm 1^{\circ} \mathrm{C}$ to a maximum $2 \theta$ value of $136.2^{\circ}$. A total of 35550 reflections were collected, where 11978 were unique $(\operatorname{Rint}=0.0323$ ). Data were collected and processed using CrysAlisPro (Rigaku Oxford Diffraction) ${ }^{4}$ and refined by the full matrix least-squares on F2 (Crystals). The structure was solved by direct methods (SIR92) ${ }^{5}$ and expanded using Fourier techniques. The non-hydrogen atoms were refined anisotropically. Hydrogen atoms were refined using the riding model. The final cycle of full-matrix leastsquares refinement ${ }^{6}$ on F2 was based on 11978 observed reflections and 865 variable parameters. The crystal data are given below:

Crystal Data for intermediate E (CCDC 1964835)

Empirical Formula

Formula Weight

Crystal Color, Habit

Crystal Dimensions

Crystal System

Lattice Type

Lattice Parameters

Space Group

$\mathrm{Z}$ value

$\mathrm{D}_{\text {calc }}$

F000

$\mathrm{m}(\mathrm{CuK} \alpha)$
$\mathrm{C}_{65} \mathrm{H}_{59} \mathrm{BClF}_{24} \mathrm{IrN}_{2} \mathrm{OSi}$

1606.72

red, prism

$0.450 \times 0.200 \times 0.150 \mathrm{~mm}$

monoclinic

I-centered

$a=43.3006(8) \AA$

$\mathrm{b}=12.9147(2) \AA$

$c=24.7725(4) \AA$

$\mathrm{b}=103.9972(17)^{\mathrm{o}}$

$\mathrm{V}=13441.8(4) \AA^{3}$

I2/a (\#15)

8

$1.588 \mathrm{~g} / \mathrm{cm}^{3}$

6400.00

$52.797 \mathrm{~cm}^{-1}$ 
Table S26. Bond lengths ( $)$

\begin{tabular}{|c|c|c|c|c|c|}
\hline atom & atom & distance & atom & atom & distance \\
\hline Ir1 & $\mathrm{Cl1}$ & $2.4064(10)$ & $\operatorname{Ir} 1$ & $\mathrm{O} 1$ & $2.144(3)$ \\
\hline Ir1 & $\mathrm{N} 2$ & $2.092(3)$ & $\operatorname{Ir} 1$ & $\mathrm{C} 13$ & $2.159(4)$ \\
\hline Ir1 & $\mathrm{C} 14$ & $2.142(4)$ & Ir1 & $\mathrm{C} 15$ & $2.141(4)$ \\
\hline Ir1 & $\mathrm{C} 16$ & $2.153(5)$ & Ir 1 & $\mathrm{C} 17$ & $2.153(5)$ \\
\hline Si1 & $\mathrm{C} 24$ & $1.841(5)$ & Sil & $\mathrm{C} 25$ & $1.863(7)$ \\
\hline Si1 & $\mathrm{C} 28$ & $1.840(14)$ & Sil & C31 & $1.886(8)$ \\
\hline F1 & $\mathrm{C} 48$ & $1.319(8)$ & $\mathrm{F} 2$ & $\mathrm{C} 48$ & $1.332(9)$ \\
\hline F3 & $\mathrm{C} 48$ & $1.333(8)$ & F4 & $\mathrm{C} 49$ & $1.289(9)$ \\
\hline F5 & $\mathrm{C} 49$ & $1.308(8)$ & F6 & $\mathrm{C} 49$ & $1.307(8)$ \\
\hline F7 & C65 & $1.341(6)$ & F8 & C65 & $1.292(7)$ \\
\hline F9 & C65 & $1.334(5)$ & F10 & C64 & $1.331(5)$ \\
\hline F11 & C64 & $1.348(6)$ & F12 & C64 & $1.338(5)$ \\
\hline F13 & $\mathrm{C} 56$ & $1.337(6)$ & F14 & C56 & $1.329(5)$ \\
\hline F15 & $\mathrm{C} 56$ & $1.325(5)$ & F16 & C57 & $1.336(6)$ \\
\hline F17 & $\mathrm{C} 57$ & $1.347(5)$ & F18 & C57 & $1.341(5)$ \\
\hline F19 & $\mathrm{C} 40$ & $1.318(6)$ & F20 & $\mathrm{C} 40$ & $1.320(6)$ \\
\hline F21 & $\mathrm{C} 40$ & $1.336(5)$ & F22 & $\mathrm{C} 41$ & $1.323(6)$ \\
\hline F23 & $\mathrm{C} 41$ & $1.318(6)$ & F24 & $\mathrm{C} 41$ & $1.318(6)$ \\
\hline $\mathrm{O} 1$ & $\mathrm{C} 8$ & $1.254(5)$ & N1 & C9 & $1.361(5)$ \\
\hline N1 & C11 & $1.362(5)$ & N1 & $\mathrm{C} 12$ & $1.467(5)$ \\
\hline $\mathrm{N} 2$ & C9 & $1.342(5)$ & $\mathrm{N} 2$ & $\mathrm{C} 10$ & $1.350(5)$ \\
\hline $\mathrm{C} 1$ & $\mathrm{C} 2$ & $1.407(5)$ & $\mathrm{C} 1$ & C6 & $1.406(6)$ \\
\hline $\mathrm{C} 1$ & $\mathrm{C} 8$ & $1.482(6)$ & $\mathrm{C} 2$ & $\mathrm{C} 3$ & $1.391(6)$ \\
\hline $\mathrm{C} 2$ & $\mathrm{C} 23$ & $1.441(6)$ & $\mathrm{C} 3$ & $\mathrm{C} 4$ & $1.387(7)$ \\
\hline $\mathrm{C} 4$ & $\mathrm{C} 5$ & $1.379(6)$ & $\mathrm{C} 5$ & C6 & $1.388(6)$ \\
\hline C6 & $\mathrm{C} 7$ & $1.508(6)$ & $\mathrm{C} 8$ & C9 & $1.440(6)$ \\
\hline $\mathrm{C} 10$ & $\mathrm{C} 11$ & $1.372(6)$ & $\mathrm{C} 13$ & $\mathrm{C} 14$ & $1.428(6)$ \\
\hline C13 & $\mathrm{C} 17$ & $1.431(6)$ & $\mathrm{C} 13$ & $\mathrm{C} 18$ & $1.501(7)$ \\
\hline $\mathrm{C} 14$ & $\mathrm{C} 15$ & $1.455(6)$ & $\mathrm{C} 14$ & $\mathrm{C} 19$ & $1.496(6)$ \\
\hline C15 & $\mathrm{C} 16$ & $1.446(6)$ & $\mathrm{C} 15$ & $\mathrm{C} 20$ & $1.503(7)$ \\
\hline $\mathrm{C} 16$ & $\mathrm{C} 17$ & $1.448(7)$ & $\mathrm{C} 16$ & $\mathrm{C} 21$ & $1.485(7)$ \\
\hline $\mathrm{C} 17$ & $\mathrm{C} 22$ & $1.518(6)$ & $\mathrm{C} 23$ & $\mathrm{C} 24$ & $1.206(6)$ \\
\hline $\mathrm{C} 25$ & $\mathrm{C} 26$ & $1.539(9)$ & $\mathrm{C} 25$ & $\mathrm{C} 27$ & $1.536(9)$ \\
\hline $\mathrm{C} 28$ & $\mathrm{C} 29$ & $1.448(18)$ & $\mathrm{C} 28$ & C30 & $1.476(16)$ \\
\hline C31 & $\mathrm{C} 32$ & $1.559(13)$ & C31 & C33 & $1.636(12)$ \\
\hline C34 & $\mathrm{C} 35$ & $1.406(5)$ & C34 & C39 & $1.402(6)$ \\
\hline $\mathrm{C} 34$ & B1 & $1.636(6)$ & $\mathrm{C} 35$ & C36 & $1.393(6)$ \\
\hline $\mathrm{C} 36$ & $\mathrm{C} 37$ & $1.387(6)$ & C36 & $\mathrm{C} 41$ & $1.506(6)$ \\
\hline $\mathrm{C} 37$ & C38 & $1.386(6)$ & C38 & C39 & $1.389(6)$ \\
\hline C38 & $\mathrm{C} 40$ & $1.496(7)$ & $\mathrm{C} 42$ & $\mathrm{C} 43$ & $1.400(6)$ \\
\hline $\mathrm{C} 42$ & $\mathrm{C} 47$ & $1.397(5)$ & $\mathrm{C} 42$ & B1 & $1.634(6)$ \\
\hline
\end{tabular}


Table S26. Bond lengths ( $\AA$ ) (continued)

\begin{tabular}{|c|c|c|c|c|c|}
\hline atom & atom & distance & atom & atom & distance \\
\hline $\mathrm{C} 43$ & $\mathrm{C} 44$ & $1.394(7)$ & $\mathrm{C} 44$ & $\mathrm{C} 45$ & $1.383(6)$ \\
\hline $\mathrm{C} 44$ & C49 & $1.492(7)$ & $\mathrm{C} 45$ & $\mathrm{C} 46$ & $1.391(7)$ \\
\hline $\mathrm{C} 46$ & $\mathrm{C} 47$ & $1.394(7)$ & $\mathrm{C} 46$ & $\mathrm{C} 48$ & $1.497(6)$ \\
\hline $\mathrm{C} 50$ & C51 & $1.405(5)$ & $\mathrm{C} 50$ & C55 & $1.403(5)$ \\
\hline $\mathrm{C} 50$ & B1 & $1.639(6)$ & C51 & $\mathrm{C} 52$ & $1.387(5)$ \\
\hline C52 & C53 & $1.393(5)$ & C52 & $\mathrm{C} 57$ & $1.501(6)$ \\
\hline C53 & C54 & $1.385(5)$ & C54 & C55 & $1.394(5)$ \\
\hline $\mathrm{C} 54$ & C56 & $1.501(5)$ & C58 & C59 & $1.393(5)$ \\
\hline $\mathrm{C} 58$ & C63 & $1.405(5)$ & C58 & B1 & $1.646(5)$ \\
\hline C59 & C60 & $1.401(5)$ & C60 & C61 & $1.384(6)$ \\
\hline C60 & C64 & $1.501(6)$ & C61 & C62 & $1.392(6)$ \\
\hline C62 & C63 & $1.394(5)$ & C62 & C65 & $1.501(6)$ \\
\hline
\end{tabular}

Table S27. Bond lengths involving hydrogens ( $)$

$\begin{array}{llllll}\text { atom } & \text { atom } & \text { distance } & \text { atom } & \text { atom } & \text { distance } \\ \text { C3 } & \text { H3 } & 0.950 & \text { C4 } & \text { H4 } & 0.950 \\ \text { C5 } & \text { H5 } & 0.950 & \text { C7 } & \text { H7A } & 0.980 \\ \text { C7 } & \text { H7B } & 0.980 & \text { C7 } & \text { H7C } & 0.980 \\ \text { C10 } & \text { H10 } & 0.950 & \text { C11 } & \text { H11 } & 0.950 \\ \text { C12 } & \text { H12A } & 0.980 & \text { C12 } & \text { H12B } & 0.980 \\ \text { C12 } & \text { H12C } & 0.980 & \text { C18 } & \text { H18A } & 0.980 \\ \text { C18 } & \text { H18B } & 0.980 & \text { C18 } & \text { H18C } & 0.980 \\ \text { C19 } & \text { H19A } & 0.980 & \text { C19 } & \text { H19B } & 0.980 \\ \text { C19 } & \text { H19C } & 0.980 & \text { C20 } & \text { H20A } & 0.980 \\ \text { C20 } & \text { H20B } & 0.980 & \text { C20 } & \text { H20C } & 0.980 \\ \text { C21 } & \text { H21A } & 0.980 & \text { C21 } & \text { H21B } & 0.980 \\ \text { C21 } & \text { H21C } & 0.980 & \text { C22 } & \text { H22A } & 0.980 \\ \text { C22 } & \text { H22B } & 0.980 & \text { C22 } & \text { H22C } & 0.980 \\ \text { C25 } & \text { H25 } & 1.000 & \text { C26 } & \text { H26A } & 0.980 \\ \text { C26 } & \text { H26B } & 0.980 & \text { C26 } & \text { H26C } & 0.980 \\ \text { C27 } & \text { H27A } & 0.980 & \text { C27 } & \text { H27B } & 0.980 \\ \text { C27 } & \text { H27C } & 0.980 & \text { C28 } & \text { H28 } & 1.000 \\ \text { C29 } & \text { H29A } & 0.980 & \text { C29 } & \text { H29B } & 0.980 \\ \text { C29 } & \text { H29C } & 0.980 & \text { C30 } & \text { H30A } & 0.980 \\ \text { C30 } & \text { H30B } & 0.980 & \text { C30 } & \text { H30C } & 0.980 \\ \text { C31 } & \text { H31 } & 1.000 & \text { C32 } & \text { H32A } & 0.980 \\ \text { C32 } & \text { H32B } & 0.980 & \text { C32 } & \text { H32C } & 0.980 \\ \text { C33 } & \text { H33A } & 0.980 & \text { C33 } & \text { H33B } & 0.980 \\ \text { C33 } & \text { H33C } & 0.980 & \text { H35 } & 0.950\end{array}$


Table S27. Bond lengths involving hydrogens $(\AA)$ (Continued)

$\begin{array}{llllll}\text { atom } & \text { atom } & \text { distance } & \text { atom } & \text { atom } & \text { distance } \\ \text { C37 } & \text { H37 } & 0.950 & \text { C39 } & \text { H39 } & 0.950 \\ \text { C43 } & \text { H43 } & 0.950 & \text { C45 } & \text { H45 } & 0.950 \\ \text { C47 } & \text { H47 } & 0.950 & \text { C51 } & \text { H51 } & 0.950 \\ \text { C53 } & \text { H53 } & 0.950 & \text { C55 } & \text { H55 } & 0.950 \\ \text { C59 } & \text { H59 } & 0.950 & \text { C61 } & \text { H61 } & 0.950 \\ \text { C63 } & \text { H63 } & 0.950 & & & \end{array}$

Table S28. Bond angles $\left({ }^{\circ}\right)$

\begin{tabular}{|c|c|c|c|c|c|c|c|}
\hline atom & atom & atom & angle & atom & atom & atom & angle \\
\hline $\mathrm{Cl1}$ & Ir1 & $\mathrm{O} 1$ & $84.55(8)$ & $\mathrm{Cl1}$ & Ir 1 & $\mathrm{~N} 2$ & $85.57(10)$ \\
\hline Cl1 & Ir 1 & $\mathrm{C} 13$ & $126.26(11)$ & $\mathrm{Cl1}$ & Ir 1 & $\mathrm{C} 14$ & $95.61(12)$ \\
\hline Cl1 & Ir 1 & $\mathrm{C} 15$ & $98.34(12)$ & $\mathrm{Cl1}$ & Ir 1 & C16. & $132.44(13)$ \\
\hline Cl1 & Ir 1 & $\mathrm{C} 17$ & $161.05(11)$ & $\mathrm{O} 1$ & Ir 1 & $\mathrm{~N} 2$ & $75.03(12)$ \\
\hline $\mathrm{O} 1$ & Ir 1 & $\mathrm{C} 13$ & $95.63(14)$ & $\mathrm{O} 1$ & Ir 1 & C14. & $119.20(14)$ \\
\hline $\mathrm{O} 1$ & Ir 1 & $\mathrm{C} 15$ & $158.79(13)$ & $\mathrm{O} 1$ & Ir 1 & C16. & $142.99(15)$ \\
\hline $\mathrm{O} 1$ & Ir 1 & $\mathrm{C} 17$ & $106.16(14)$ & $\mathrm{N} 2$ & Ir 1 & C13. & $146.41(14)$ \\
\hline $\mathrm{N} 2$ & Ir 1 & $\mathrm{C} 14$ & $165.76(15)$ & $\mathrm{N} 2$ & Ir 1 & C15. & $126.05(14)$ \\
\hline $\mathrm{N} 2$ & Ir1 & $\mathrm{C} 16$ & $102.89(14)$ & $\mathrm{N} 2$ & Ir 1 & C17. & $112.04(14)$ \\
\hline C13 & Ir 1 & $\mathrm{C} 14$ & $38.77(16)$ & $\mathrm{C} 13$ & Ir 1 & $\mathrm{C} 15$ & $65.65(17)$ \\
\hline C13 & Ir1 & $\mathrm{C} 16$ & $65.31(17)$ & $\mathrm{C} 13$ & Ir1 & $\mathrm{C} 17$ & $38.75(16)$ \\
\hline C14 & Ir 1 & $\mathrm{C} 15$ & $39.72(16)$ & $\mathrm{C} 14$ & Ir 1 & $\mathrm{C} 16$ & $65.97(16)$ \\
\hline $\mathrm{C} 14$ & Ir1 & $\mathrm{C} 17$ & $65.58(16)$ & $\mathrm{C} 15$ & Ir 1 & $\mathrm{C} 16$ & $39.36(16)$ \\
\hline $\mathrm{C} 15$ & Ir 1 & $\mathrm{C} 17$ & $66.03(17)$ & $\mathrm{C} 16$ & Ir 1 & $\mathrm{C} 17$ & $39.30(18)$ \\
\hline $\mathrm{C} 24$ & Sil & $\mathrm{C} 25$ & $104.3(2)$ & $\mathrm{C} 24$ & Sil & $\mathrm{C} 28$ & $107.4(4)$ \\
\hline $\mathrm{C} 24$ & Sil & C31 & $105.8(3)$ & $\mathrm{C} 25$ & Sil & $\mathrm{C} 28$ & $115.0(5)$ \\
\hline $\mathrm{C} 25$ & Sil & C31 & $110.6(3)$ & $\mathrm{C} 28$ & Sil & C31 & $113.0(5)$ \\
\hline Ir1 & $\mathrm{O} 1$ & $\mathrm{C} 8$ & $117.8(2)$ & C9 & N1 & $\mathrm{C} 11$ & $107.2(3)$ \\
\hline C9 & N1 & $\mathrm{C} 12$ & $128.4(3)$ & $\mathrm{C} 11$ & N1 & $\mathrm{C} 12$ & $124.4(3)$ \\
\hline Ir1 & $\mathrm{N} 2$ & C9 & $114.8(3)$ & Ir 1 & $\mathrm{~N} 2$ & $\mathrm{C} 10$ & $137.0(3)$ \\
\hline C9 & N2 & $\mathrm{C} 10$ & $107.4(3)$ & $\mathrm{C} 2$ & $\mathrm{C} 1$ & C6 & $121.2(4)$ \\
\hline $\mathrm{C} 2$ & $\mathrm{C} 1$ & $\mathrm{C} 8$ & $119.5(4)$ & C6 & $\mathrm{C} 1$ & $\mathrm{C} 8$ & $119.2(3)$ \\
\hline $\mathrm{C} 1$ & $\mathrm{C} 2$ & $\mathrm{C} 3$ & $119.5(4)$ & $\mathrm{C} 1$ & $\mathrm{C} 2$ & $\mathrm{C} 23$ & $121.5(4)$ \\
\hline $\mathrm{C} 3$ & $\mathrm{C} 2$ & $\mathrm{C} 23$ & $118.8(4)$ & $\mathrm{C} 2$ & $\mathrm{C} 3$ & $\mathrm{C} 4$ & $119.1(4)$ \\
\hline $\mathrm{C} 3$ & $\mathrm{C} 4$ & $\mathrm{C} 5$ & $121.0(4)$ & $\mathrm{C} 4$ & $\mathrm{C} 5$ & C6 & $121.7(4)$ \\
\hline $\mathrm{C} 1$ & C6 & $\mathrm{C} 5$ & $117.4(4)$ & $\mathrm{C} 1$ & C6 & $\mathrm{C} 7$ & $122.5(4)$ \\
\hline $\mathrm{C} 5$ & C6 & $\mathrm{C} 7$ & $120.1(4)$ & $\mathrm{O} 1$ & $\mathrm{C} 8$ & $\mathrm{C} 1$ & $118.3(3)$ \\
\hline $\mathrm{O} 1$ & $\mathrm{C} 8$ & C9 & $114.8(4)$ & $\mathrm{C} 1$ & $\mathrm{C} 8$ & C9 & $126.8(3)$ \\
\hline $\mathrm{N} 1$ & C9 & $\mathrm{N} 2$ & $109.5(3)$ & N1 & C9 & $\mathrm{C} 8$ & $133.2(4)$ \\
\hline $\mathrm{N} 2$ & C9 & $\mathrm{C} 8$ & $117.1(3)$ & $\mathrm{N} 2$ & $\mathrm{C} 10$ & $\mathrm{C} 11$ & $108.7(4)$ \\
\hline N1 & $\mathrm{C} 11$ & $\mathrm{C} 10$ & $107.2(4)$ & Ir 1 & $\mathrm{C} 13$ & $\mathrm{C} 14$ & $70.0(2)$ \\
\hline Ir1 & $\mathrm{C} 13$ & $\mathrm{C} 17$ & $70.4(3)$ & $\operatorname{Ir} 1$ & $\mathrm{C} 13$ & $\mathrm{C} 18$ & $122.7(3)$ \\
\hline C14 & $\mathrm{C} 13$ & $\mathrm{C} 17$ & $108.9(4)$ & $\mathrm{C} 14$ & $\mathrm{C} 13$ & C18 & $126.2(4)$ \\
\hline
\end{tabular}


Table S28. Bond angles $\left({ }^{\circ}\right)$ (Continued)

\begin{tabular}{|c|c|c|c|c|c|c|c|}
\hline atom & atom & atom & angle & atom & atom & atom & angle \\
\hline C17 & $\mathrm{C} 13$ & $\mathrm{C} 18$ & $124.8(4)$ & Ir 1 & $\mathrm{C} 14$ & $\mathrm{C} 13$ & $71.3(2)$ \\
\hline Ir 1 & $\mathrm{C} 14$ & $\mathrm{C} 15$ & $70.1(2)$ & Ir 1 & $\mathrm{C} 14$ & C19 & $125.2(3)$ \\
\hline C13 & $\mathrm{C} 14$ & $\mathrm{C} 15$ & $107.9(3)$ & $\mathrm{C} 13$ & $\mathrm{C} 14$ & C19 & $126.6(4)$ \\
\hline $\mathrm{C} 15$ & $\mathrm{C} 14$ & $\mathrm{C} 19$ & $125.5(4)$ & Ir 1 & $\mathrm{C} 15$ & $\mathrm{C} 14$ & $70.2(2)$ \\
\hline Ir1 & $\mathrm{C} 15$ & $\mathrm{C} 16$ & $70.8(2)$ & Ir 1 & $\mathrm{C} 15$ & $\mathrm{C} 20$ & $127.2(3)$ \\
\hline C14 & $\mathrm{C} 15$ & $\mathrm{C} 16$ & $107.4(4)$ & $\mathrm{C} 14$ & $\mathrm{C} 15$ & $\mathrm{C} 20$ & $126.1(4)$ \\
\hline $\mathrm{C} 16$ & $\mathrm{C} 15$ & $\mathrm{C} 20$ & $126.4(4)$ & Ir 1 & $\mathrm{C} 16$ & $\mathrm{C} 15$ & $69.9(3)$ \\
\hline Ir1 & $\mathrm{C} 16$ & $\mathrm{C} 17$ & $70.3(3)$ & Ir 1 & $\mathrm{C} 16$ & $\mathrm{C} 21$ & $125.3(3)$ \\
\hline C15 & $\mathrm{C} 16$ & $\mathrm{C} 17$ & $107.9(4)$ & $\mathrm{C} 15$ & $\mathrm{C} 16$ & $\mathrm{C} 21$ & $124.8(4)$ \\
\hline $\mathrm{C} 17$ & $\mathrm{C} 16$ & $\mathrm{C} 21$ & $127.4(4)$ & Ir 1 & $\mathrm{C} 17$ & $\mathrm{C} 13$ & $70.8(3)$ \\
\hline Ir1 & $\mathrm{C} 17$ & $\mathrm{C} 16$ & $70.4(3)$ & Ir 1 & $\mathrm{C} 17$ & $\mathrm{C} 22$ & $127.6(3)$ \\
\hline C13 & $\mathrm{C} 17$ & $\mathrm{C} 16$ & $107.9(4)$ & $\mathrm{C} 13$ & $\mathrm{C} 17$ & $\mathrm{C} 22$ & $125.8(4)$ \\
\hline $\mathrm{C} 16$ & $\mathrm{C} 17$ & $\mathrm{C} 22$ & $126.2(4)$ & $\mathrm{C} 2$ & $\mathrm{C} 23$ & $\mathrm{C} 24$ & $176.2(4)$ \\
\hline Sil & $\mathrm{C} 24$ & $\mathrm{C} 23$ & $176.0(4)$ & Sil & $\mathrm{C} 25$ & $\mathrm{C} 26$ & $112.6(4)$ \\
\hline Sil & $\mathrm{C} 25$ & $\mathrm{C} 27$ & $112.6(5)$ & $\mathrm{C} 26$ & $\mathrm{C} 25$ & $\mathrm{C} 27$ & $111.2(6)$ \\
\hline Sil & $\mathrm{C} 28$ & $\mathrm{C} 29$ & $121.4(10)$ & Si1 & $\mathrm{C} 28$ & C30 & $114.6(9)$ \\
\hline C29 & $\mathrm{C} 28$ & $\mathrm{C} 30$ & $113.8(12)$ & Sil & C31 & C32 & $111.2(5)$ \\
\hline Sil & C31 & C33 & $106.9(7)$ & $\mathrm{C} 32$ & C31 & $\mathrm{C} 33$ & 119.2(9) \\
\hline C35 & $\mathrm{C} 34$ & C39 & $115.3(4)$ & $\mathrm{C} 35$ & C34 & B1 & $123.0(4)$ \\
\hline C39 & $\mathrm{C} 34$ & B1 & $121.2(3)$ & $\mathrm{C} 34$ & $\mathrm{C} 35$ & C36 & $122.2(4)$ \\
\hline C35 & $\mathrm{C} 36$ & C37 & $121.0(4)$ & $\mathrm{C} 35$ & $\mathrm{C} 36$ & C41 & $118.7(4)$ \\
\hline C37 & $\mathrm{C} 36$ & $\mathrm{C} 41$ & $120.2(4)$ & C36 & C37 & C38 & $117.9(4)$ \\
\hline C37 & C38 & C39 & $121.0(4)$ & C37 & C38 & $\mathrm{C} 40$ & $120.8(4)$ \\
\hline C39 & C38 & $\mathrm{C} 40$ & $118.2(4)$ & $\mathrm{C} 34$ & C39 & C38 & $122.5(4)$ \\
\hline F19 & $\mathrm{C} 40$ & $\mathrm{~F} 20$ & $107.3(4)$ & F19 & $\mathrm{C} 40$ & F21 & $104.3(4)$ \\
\hline F19 & $\mathrm{C} 40$ & $\mathrm{C} 38$ & $112.4(5)$ & $\mathrm{F} 20$ & $\mathrm{C} 40$ & F21 & $105.9(4)$ \\
\hline F20 & $\mathrm{C} 40$ & $\mathrm{C} 38$ & $114.4(4)$ & $\mathrm{F} 21$ & $\mathrm{C} 40$ & C38 & $111.8(4)$ \\
\hline F22 & $\mathrm{C} 41$ & $\mathrm{~F} 23$ & $105.7(4)$ & $\mathrm{F} 22$ & $\mathrm{C} 41$ & F24 & $106.9(4)$ \\
\hline F22 & $\mathrm{C} 41$ & $\mathrm{C} 36$ & $112.8(4)$ & $\mathrm{F} 23$ & $\mathrm{C} 41$ & F24 & $105.1(5)$ \\
\hline F23 & $\mathrm{C} 41$ & $\mathrm{C} 36$ & $112.8(4)$ & $\mathrm{F} 24$ & $\mathrm{C} 41$ & C36 & $113.0(4)$ \\
\hline $\mathrm{C} 43$ & $\mathrm{C} 42$ & $\mathrm{C} 47$ & $115.8(4)$ & $\mathrm{C} 43$ & $\mathrm{C} 42$ & B1 & $121.8(3)$ \\
\hline C47 & $\mathrm{C} 42$ & B1 & $121.7(4)$ & $\mathrm{C} 42$ & $\mathrm{C} 43$ & $\mathrm{C} 44$ & $122.4(3)$ \\
\hline C43 & C44 & $\mathrm{C} 45$ & $120.8(4)$ & C43 & $\mathrm{C} 44$ & C49 & $119.3(4)$ \\
\hline $\mathrm{C} 45$ & $\mathrm{C} 44$ & C49 & $119.9(5)$ & $\mathrm{C} 44$ & $\mathrm{C} 45$ & $\mathrm{C} 46$ & $117.9(5)$ \\
\hline $\mathrm{C} 45$ & $\mathrm{C} 46$ & $\mathrm{C} 47$ & $121.1(4)$ & $\mathrm{C} 45$ & $\mathrm{C} 46$ & $\mathrm{C} 48$ & $118.6(5)$ \\
\hline $\mathrm{C} 47$ & $\mathrm{C} 46$ & $\mathrm{C} 48$ & $120.3(5)$ & $\mathrm{C} 42$ & $\mathrm{C} 47$ & $\mathrm{C} 46$ & $122.0(4)$ \\
\hline $\mathrm{F} 1$ & $\mathrm{C} 48$ & $\mathrm{~F} 2$ & $106.6(6)$ & $\mathrm{F} 1$ & $\mathrm{C} 48$ & F3 & $106.3(6)$ \\
\hline F1 & C48 & $\mathrm{C} 46$ & $112.6(5)$ & $\mathrm{F} 2$ & $\mathrm{C} 48$ & $\mathrm{~F} 3$ & $106.0(5)$ \\
\hline $\mathrm{F} 2$ & $\mathrm{C} 48$ & $\mathrm{C} 46$ & $111.4(5)$ & F3 & $\mathrm{C} 48$ & $\mathrm{C} 46$ & $113.4(5)$ \\
\hline $\mathrm{F} 4$ & C49 & F5 & $106.8(7)$ & $\mathrm{F} 4$ & C49 & F6 & $101.3(5)$ \\
\hline $\mathrm{F} 4$ & $\mathrm{C} 49$ & $\mathrm{C} 44$ & $112.6(6)$ & F5 & C49 & F6 & $107.6(6)$ \\
\hline F5 & C49 & $\mathrm{C} 44$ & $114.1(4)$ & F6 & C49 & $\mathrm{C} 44$ & $113.5(6)$ \\
\hline
\end{tabular}


Table S28. Bond angles $\left(^{\circ}\right)$ (Continued)

$\begin{array}{llllllll}\text { atom } & \text { atom } & \text { atom } & \text { angle } & \text { atom } & \text { atom } & \text { atom } & \text { angle } \\ \text { C51 } & \text { C50 } & \text { C55 } & 115.7(3) & \text { C51 } & \text { C50 } & \text { B1 } & 123.2(3) \\ \text { C55 } & \text { C50 } & \text { B1 } & 121.0(3) & \text { C50 } & \text { C51 } & \text { C52 } & 122.3(3) \\ \text { C51 } & \text { C52 } & \text { C53 } & 121.0(3) & \text { C51 } & \text { C52 } & \text { C57 } & 118.6(3) \\ \text { C53 } & \text { C52 } & \text { C57 } & 120.3(3) & \text { C52 } & \text { C53 } & \text { C54 } & 117.8(4) \\ \text { C53 } & \text { C54 } & \text { C55 } & 121.2(3) & \text { C53 } & \text { C54 } & \text { C56 } & 120.5(3) \\ \text { C55 } & \text { C54 } & \text { C56 } & 118.3(3) & \text { C50 } & \text { C55 } & \text { C54 } & 122.0(3) \\ \text { F13 } & \text { C56 } & \text { F14 } & 105.7(3) & \text { F13 } & \text { C56 } & \text { F15 } & 106.7(4) \\ \text { F13 } & \text { C56 } & \text { C54 } & 111.4(3) & \text { F14 } & \text { C56 } & \text { F15 } & 107.6(3) \\ \text { F14 } & \text { C56 } & \text { C54 } & 112.3(3) & \text { F15 } & \text { C56 } & \text { C54 } & 112.8(3) \\ \text { F16 } & \text { C57 } & \text { F17 } & 106.0(4) & \text { F16 } & \text { C57 } & \text { F18 } & 106.3(3) \\ \text { F16 } & \text { C57 } & \text { C52 } & 112.5(3) & \text { F17 } & \text { C57 } & \text { F18 } & 106.2(3) \\ \text { F17 } & \text { C57 } & \text { C52 } & 112.7(3) & \text { F18 } & \text { C57 } & \text { C52 } & 112.6(3) \\ \text { C59 } & \text { C58 } & \text { C63 } & 115.4(3) & \text { C59 } & \text { C58 } & \text { B1 } & 122.0(3) \\ \text { C63 } & \text { C58 } & \text { B1 } & 122.4(3) & \text { C58 } & \text { C59 } & \text { C60 } & 122.8(4) \\ \text { C59 } & \text { C60 } & \text { C61 } & 120.7(4) & \text { C59 } & \text { C60 } & \text { C64 } & 118.2(3) \\ \text { C61 } & \text { C60 } & \text { C64 } & 121.1(3) & \text { C60 } & \text { C61 } & \text { C62 } & 117.7(3) \\ \text { C61 } & \text { C62 } & \text { C63 } & 121.2(4) & \text { C61 } & \text { C62 } & \text { C65 } & 118.7(3) \\ \text { C63 } & \text { C62 } & \text { C65 } & 120.1(4) & \text { C58 } & \text { C63 } & \text { C62 } & 122.2(3) \\ \text { F10 } & \text { C64 } & \text { F11 } & 106.8(4) & \text { F10 } & \text { C64 } & \text { F12 } & 106.7(3) \\ \text { F10 } & \text { C64 } & \text { C60 } & 113.3(4) & \text { F11 } & \text { C64 } & \text { F12 } & 105.6(4) \\ \text { F11 } & \text { C64 } & \text { C60 } & 111.9(3) & \text { F12 } & \text { C64 } & \text { C60 } & 112.0(4) \\ \text { F7 } & \text { C65 } & \text { F8 } & 107.5(4) & \text { F7 } & \text { C65 } & \text { F9 } & 103.1(4) \\ \text { F7 } & \text { C65 } & \text { C62 } & 111.4(4) & \text { F8 } & \text { C65 } & \text { F9 } & 107.5(5) \\ \text { F8 } & \text { C65 } & \text { C62 } & 113.6(4) & \text { F9 } & \text { C65 } & \text { C62 } & 113.1(3) \\ \text { C34 } & \text { B1 } & \text { C42 } & 113.8(3) & \text { C34 } & \text { B1 } & \text { C50 } & 112.1(3) \\ \text { C34 } & \text { B1 } & \text { C58 } & 103.0(3) & \text { C42 } & \text { B1 } & \text { C50 } & 103.5(3) \\ \text { C42 } & \text { B1 } & \text { C58 } & 113.1(3) & \text { C50 } & \text { B1 } & \text { C58 } & 111.7(3)\end{array}$

Table S29. Bond angles involving hydrogens $\left({ }^{\circ}\right)$

$\begin{array}{llllllll}\text { atom } & \text { atom } & \text { atom } & \text { angle } & \text { atom } & \text { atom } & \text { atom } & \text { angle } \\ \text { C2 } & \text { C3 } & \text { H3 } & 120.4 & \text { C4 } & \text { C3 } & \text { H3 } & 120.4 \\ \text { C3 } & \text { C4 } & \text { H4 } & 119.5 & \text { C5 } & \text { C4 } & \text { H4 } & 119.5 \\ \text { C4 } & \text { C5 } & \text { H5 } & 119.2 & \text { C6 } & \text { C5 } & \text { H5 } & 119.2 \\ \text { C6 } & \text { C7 } & \text { H7A } & 109.5 & \text { C6 } & \text { C7 } & \text { H7B } & 109.5 \\ \text { C6 } & \text { C7 } & \text { H7C } & 109.5 & \text { H7A } & \text { C7 } & \text { H7B } & 109.5 \\ \text { H7A } & \text { C7 } & \text { H7C } & 109.5 & \text { H7B } & \text { C7 } & \text { H7C } & 109.5 \\ \text { N2 } & \text { C10 } & \text { H10 } & 125.7 & \text { C11 } & \text { C10 } & \text { H10 } & 125.7 \\ \text { N1 } & \text { C11 } & \text { H11 } & 126.4 & \text { C10 } & \text { C11 } & \text { H11 } & 126.4 \\ \text { N1 } & \text { C12 } & \text { H12A } & 109.5 & \text { N1 } & \text { C12 } & \text { H12B } & 109.5 \\ \text { N1 } & \text { C12 } & \text { H12C } & 109.5 & \text { H12A } & \text { C12 } & \text { H12B } & 109.5 \\ \text { H12A } & \text { C12 } & \text { H12C } & 109.5 & \text { H12B } & \text { C12 } & \text { H12C } & 109.5 \\ \text { C13 } & \text { C18 } & \text { H18A } & 109.5 & \text { C13 } & \text { C18 } & \text { H18B } & 109.5 \\ \text { C13 } & \text { C18 } & \text { H18C } & 109.5 & \text { H18A } & \text { C18 } & \text { H18B } & 109.5 \\ \text { H18A } & \text { C18 } & \text { H18C } & 109.5 & \text { H18B } & \text { C18 } & \text { H18C } & 109.5\end{array}$


Table S29. Bond angles involving hydrogens ( $\left.{ }^{\circ}\right)$ (Continued)

\begin{tabular}{|c|c|c|c|c|c|c|c|}
\hline atom & atom & atom & angle & atom & atom & atom & angle \\
\hline C14 & C19 & H19A & 109.5 & C14 & C19 & H19B & 109.5 \\
\hline $\mathrm{C} 14$ & C19 & $\mathrm{H} 19 \mathrm{C}$ & 109.5 & H19A & C19 & H19B & 109.5 \\
\hline H19A & C19 & $\mathrm{H} 19 \mathrm{C}$ & 109.5 & H19B & C19 & $\mathrm{H} 19 \mathrm{C}$ & 109.5 \\
\hline C15 & $\mathrm{C} 20$ & $\mathrm{H} 20 \mathrm{~A}$ & 109.5 & $\mathrm{C} 15$ & C20 & $\mathrm{H} 20 \mathrm{~B}$ & 109.5 \\
\hline C15 & $\mathrm{C} 20$ & $\mathrm{H} 20 \mathrm{C}$ & 109.5 & $\mathrm{H} 20 \mathrm{~A}$ & C20 & $\mathrm{H} 20 \mathrm{~B}$ & 109.5 \\
\hline $\mathrm{H} 20 \mathrm{~A}$ & C20 & $\mathrm{H} 20 \mathrm{C}$ & 109.4 & $\mathrm{H} 20 \mathrm{~B}$ & C20 & $\mathrm{H} 20 \mathrm{C}$ & 109.5 \\
\hline C16 & C21 & $\mathrm{H} 21 \mathrm{~A}$ & 109.5 & $\mathrm{C} 16$ & $\mathrm{C} 21$ & $\mathrm{H} 21 \mathrm{~B}$ & 109.5 \\
\hline C16 & C21 & $\mathrm{H} 21 \mathrm{C}$ & 109.5 & $\mathrm{H} 21 \mathrm{~A}$ & $\mathrm{C} 21$ & $\mathrm{H} 21 \mathrm{~B}$ & 109.5 \\
\hline $\mathrm{H} 21 \mathrm{~A}$ & C21 & $\mathrm{H} 21 \mathrm{C}$ & 109.5 & $\mathrm{H} 21 \mathrm{~B}$ & C21 & $\mathrm{H} 21 \mathrm{C}$ & 109.5 \\
\hline C17 & $\mathrm{C} 22$ & $\mathrm{H} 22 \mathrm{~A}$ & 109.5 & $\mathrm{C} 17$ & C22 & $\mathrm{H} 22 \mathrm{~B}$ & 109.5 \\
\hline C17 & $\mathrm{C} 22$ & $\mathrm{H} 22 \mathrm{C}$ & 109.5 & $\mathrm{H} 22 \mathrm{~A}$ & $\mathrm{C} 22$ & $\mathrm{H} 22 \mathrm{~B}$ & 109.5 \\
\hline $\mathrm{H} 22 \mathrm{~A}$ & C22 & $\mathrm{H} 22 \mathrm{C}$ & 109.5 & $\mathrm{H} 22 \mathrm{~B}$ & C22 & $\mathrm{H} 22 \mathrm{C}$ & 109.5 \\
\hline Sil & $\mathrm{C} 25$ & $\mathrm{H} 25$ & 106.7 & C26 & $\mathrm{C} 25$ & $\mathrm{H} 25$ & 106.7 \\
\hline $\mathrm{C} 27$ & $\mathrm{C} 25$ & $\mathrm{H} 25$ & 106.7 & $\mathrm{C} 25$ & C26 & $\mathrm{H} 26 \mathrm{~A}$ & 109.5 \\
\hline $\mathrm{C} 25$ & C26 & $\mathrm{H} 26 \mathrm{~B}$ & 109.5 & $\mathrm{C} 25$ & C26 & $\mathrm{H} 26 \mathrm{C}$ & 109.5 \\
\hline $\mathrm{H} 26 \mathrm{~A}$ & C26 & H26B & 109.5 & $\mathrm{H} 26 \mathrm{~A}$ & C26 & $\mathrm{H} 26 \mathrm{C}$ & 109.5 \\
\hline $\mathrm{H} 26 \mathrm{~B}$ & C26 & $\mathrm{H} 26 \mathrm{C}$ & 109.5 & $\mathrm{C} 25$ & $\mathrm{C} 27$ & $\mathrm{H} 27 \mathrm{~A}$ & 109.5 \\
\hline $\mathrm{C} 25$ & $\mathrm{C} 27$ & $\mathrm{H} 27 \mathrm{~B}$ & 109.5 & $\mathrm{C} 25$ & $\mathrm{C} 27$ & $\mathrm{H} 27 \mathrm{C}$ & 109.5 \\
\hline $\mathrm{H} 27 \mathrm{~A}$ & $\mathrm{C} 27$ & $\mathrm{H} 27 \mathrm{~B}$ & 109.5 & $\mathrm{H} 27 \mathrm{~A}$ & $\mathrm{C} 27$ & $\mathrm{H} 27 \mathrm{C}$ & 109.5 \\
\hline $\mathrm{H} 27 \mathrm{~B}$ & $\mathrm{C} 27$ & $\mathrm{H} 27 \mathrm{C}$ & 109.5 & Sil & C28 & $\mathrm{H} 28$ & 100.7 \\
\hline C29 & C28 & H28 & 100.7 & C30 & C28 & $\mathrm{H} 28$ & 100.7 \\
\hline $\mathrm{C} 28$ & C29 & $\mathrm{H} 29 \mathrm{~A}$ & 109.5 & C28 & C29 & H29B & 109.5 \\
\hline C28. & C29. & $\mathrm{H} 29 \mathrm{C}$. & 109.5 & H29A & C29 & H29B & 109.5 \\
\hline H29A & C29 & $\mathrm{H} 29 \mathrm{C}$ & 109.5 & H29B & C29 & $\mathrm{H} 29 \mathrm{C}$ & 109.5 \\
\hline C28 & C30 & $\mathrm{H} 30 \mathrm{~A}$ & 109.5 & C28 & C30 & H30B & 109.5 \\
\hline C28 & C30 & $\mathrm{H} 30 \mathrm{C}$ & 109.5 & $\mathrm{H} 30 \mathrm{~A}$ & C30 & H30B & 109.5 \\
\hline $\mathrm{H} 30 \mathrm{~A}$ & C30 & $\mathrm{H} 30 \mathrm{C}$ & 109.5 & H30B & C30 & $\mathrm{H} 30 \mathrm{C}$ & 109.5 \\
\hline Si1 & C31 & H31 & 106.2 & $\mathrm{C} 32$ & C31 & H31 & 106.2 \\
\hline C33 & C31 & H31 & 106.2 & C31 & C32 & $\mathrm{H} 32 \mathrm{~A}$ & 109.5 \\
\hline C31 & C32 & H32B & 109.5 & C31 & C32 & $\mathrm{H} 32 \mathrm{C}$ & 109.5 \\
\hline $\mathrm{H} 32 \mathrm{~A}$ & C32 & H32B & 109.5 & H32A & C32 & $\mathrm{H} 32 \mathrm{C}$ & 109.5 \\
\hline H32B & C32 & $\mathrm{H} 32 \mathrm{C}$ & 109.5 & C31 & C33 & $\mathrm{H} 33 \mathrm{~A}$ & 109.5 \\
\hline C31 & C33 & H33B & 109.5 & C31 & C33 & $\mathrm{H} 33 \mathrm{C}$ & 109.5 \\
\hline H33A & C33 & H33B & 109.5 & H33A & C33 & $\mathrm{H} 33 \mathrm{C}$ & 109.5 \\
\hline H33B & C33 & H33C & 109.5 & C34 & C35 & H35 & 118.9 \\
\hline C36 & C35 & H35 & 118.9 & C36 & C37 & H37 & 121.1 \\
\hline C38 & C37 & H37 & 121.1 & C34 & C39 & H39 & 118.7 \\
\hline C38 & C39 & H39 & 118.7 & C42 & C43 & H43 & 118.8 \\
\hline C44 & C43 & H43 & 118.8 & C44 & $\mathrm{C} 45$ & H45 & 121.1 \\
\hline C46 & $\mathrm{C} 45$ & H45 & 121.1 & C42 & $\mathrm{C} 47$ & H47 & 119.0 \\
\hline C46 & C47 & H47 & 119.0 & C50 & C51 & H51 & 118.9 \\
\hline C52 & C51 & H51 & 118.9 & C52 & C53 & H53 & 121.1 \\
\hline C54 & C53 & H53 & 121.1 & C50 & C55 & H55 & 119.0 \\
\hline
\end{tabular}


Table S29. Bond angles involving hydrogens ( $\left.{ }^{\circ}\right)$ (Continued)

$\begin{array}{llllllll}\text { atom } & \text { atom } & \text { atom } & \text { angle } & \text { atom } & \text { atom } & \text { atom } & \text { angle } \\ \text { C54 } & \text { C55 } & \text { H55 } & 119.0 & \text { C58 } & \text { C59 } & \text { H59 } & 118.6 \\ \text { C60 } & \text { C59 } & \text { H59 } & 118.6 & \text { C60 } & \text { C61 } & \text { H61 } & 121.2 \\ \text { C62 } & \text { C61 } & \text { H61 } & 121.2 & \text { C58 } & \text { C63 } & \text { H63 } & 118.9 \\ \text { C62 } & \text { C63 } & \text { H63 } & 118.9 & & & & \end{array}$


D. X-ray analysis of compound 3ia $\left(\mathrm{C}_{23} \mathrm{H}_{32} \mathrm{~N}_{2} \mathrm{O}_{2} \mathrm{Si}\right)$ : $\mathrm{CCDC} 1974283$

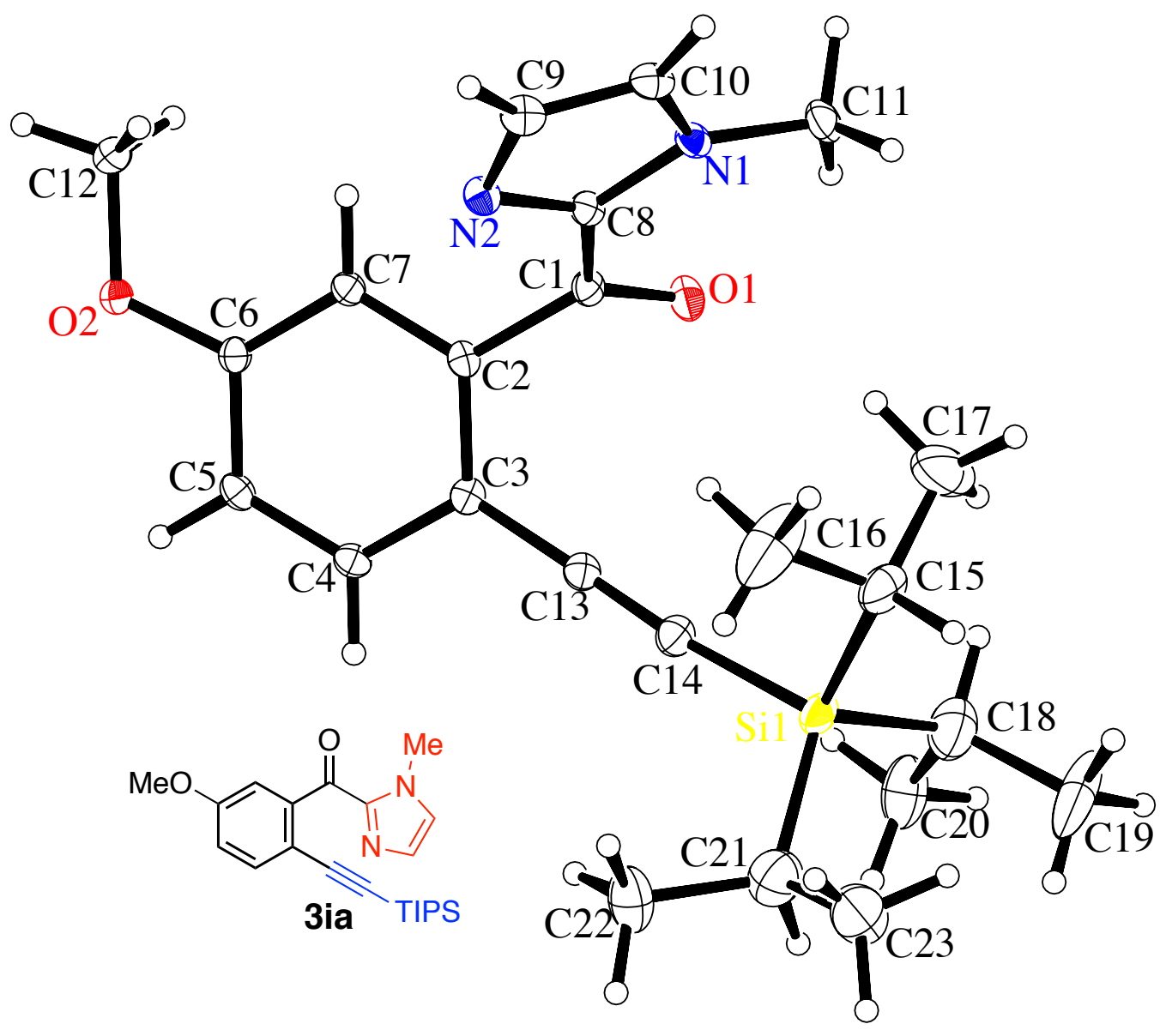

Figure S22: ORTEP diagram of compound 3ia. 
CDC 1974283 contains the supplementary crystallographic data. X-Ray crystallographic structure analysis of compound 3ia $\left(\mathrm{C}_{23} \mathrm{H}_{32} \mathrm{~N}_{2} \mathrm{O}_{2} \mathrm{Si}\right)$ was recorded on Rigaku XtaLAB P200 using multi-layer mirror monochromated $\mathrm{Cu}-\mathrm{K} \alpha$ radiation. The crystal-to-detector distance was $34.00 \mathrm{~mm}$. The data were collected at a temperature of $-150 \pm 1^{\circ} \mathrm{C}$ to a maximum $2 \theta$ value of $152.8^{\circ}$. A total of 15316 reflections were collected, where 4575 were unique $(\operatorname{Rint}=0.0439)$. Data were collected and processed using CrysAlisPro (Rigaku Oxford Diffraction) ${ }^{4}$ and refined by the full matrix least-squares on F2 (Crystals). The structure was solved by direct methods $(\mathrm{SIR} 92)^{5}$ and expanded using Fourier techniques. The non-hydrogen atoms were refined anisotropically. Hydrogen atoms were refined using the riding model. The final cycle of fullmatrix least-squares refinement ${ }^{6}$ on F2 was based on 4575 observed reflections and 253 variable parameters. The crystal data are given below:

\section{X-ray data of compound 3ia $\left(\mathrm{C}_{23} \mathrm{H}_{32} \mathrm{~N}_{2} \mathrm{O}_{2} \mathrm{Si}\right)$ : CCDC 1974283}

Empirical Formula

Formula Weight

Crystal Color, Habit

Crystal Dimensions

Crystal System

Lattice Type

Lattice Parameters

Space Group

$\mathrm{Z}$ value

Dcalc

$\mathrm{F}_{000}$

$\mathrm{m}(\mathrm{CuK} \alpha)$
$\mathrm{C}_{23} \mathrm{H}_{32} \mathrm{~N}_{2} \mathrm{O}_{2} \mathrm{Si}$

396.60

red, prism

$0.500 \times 0.200 \times 0.100 \mathrm{~mm}$

monoclinic

Primitive

$\mathrm{a}=20.4075(4) \AA$

$\mathrm{b}=8.0569(2) \AA$

$\mathrm{c}=14.2212(3) \AA$

$\mathrm{b}=103.892(2)^{\mathrm{O}}$

$\mathrm{V}=2269.87(9) \AA^{3}$

$\mathrm{P} 21 / \mathrm{c}(\# 14)$

4

$1.160 \mathrm{~g} / \mathrm{cm}^{3}$

856.00

$10.604 \mathrm{~cm}^{-1}$ 
Table S30. Bond lengths ( $\AA$ )

$\begin{array}{llllll}\text { atom } & \text { atom } & \text { distance } & \text { atom } & \text { atom } & \text { distance } \\ \text { Si1 } & \text { C14 } & 1.835(3) & \text { Si1 } & \text { C15 } & 1.868(4) \\ \text { Si1 } & \text { C18 } & 1.956(6) & \text { Si1 } & \text { C21 } & 1.841(5) \\ \text { O1 } & \text { C1 } & 1.226(4) & \text { O2 } & \text { C6 } & 1.361(3) \\ \text { O2 } & \text { C12 } & 1.435(4) & \mathrm{N} 1 & \mathrm{C} 8 & 1.370(4) \\ \mathrm{N} 1 & \mathrm{C} 10 & 1.363(4) & \mathrm{N} 1 & \mathrm{C} 11 & 1.461(4) \\ \mathrm{N} 2 & \mathrm{C} 8 & 1.336(4) & \mathrm{N} 2 & \mathrm{C} 9 & 1.367(4) \\ \mathrm{C} 1 & \mathrm{C} 2 & 1.507(4) & \mathrm{C} 1 & \mathrm{C} 8 & 1.462(4) \\ \mathrm{C} 2 & \mathrm{C} 3 & 1.401(4) & \mathrm{C} 2 & \mathrm{C} 7 & 1.393(4) \\ \mathrm{C} 3 & \mathrm{C} 4 & 1.409(4) & \mathrm{C} 3 & \mathrm{C} 13 & 1.434(4) \\ \mathrm{C} 4 & \mathrm{C} 5 & 1.378(4) & \mathrm{C} 5 & \mathrm{C} 6 & 1.399(4) \\ \mathrm{C} 6 & \mathrm{C} 7 & 1.397(4) & \mathrm{C} 9 & \mathrm{C} 10 & 1.364(4) \\ \mathrm{C} 13 & \mathrm{C} 14 & 1.207(4) & \mathrm{C} 15 & \mathrm{C} 16 & 1.559(8) \\ \mathrm{C} 15 & \mathrm{C} 17 & 1.505(9) & \mathrm{C} 18 & \mathrm{C} 19 & 1.546(6) \\ \mathrm{C} 18 & \mathrm{C} 20 & 1.448(7) & \mathrm{C} 21 & \mathrm{C} 22 & 1.527(7) \\ \mathrm{C} 21 & \mathrm{C} 23 & 1.594(8) & & & \end{array}$

Table S31. Bond lengths involving hydrogens $(\AA)$

$\begin{array}{llllll}\text { atom } & \text { atom } & \text { distance } & \text { atom } & \text { atom } & \text { distance } \\ \text { C4 } & \text { H4 } & 0.950 & \text { C5 } & \text { H5 } & 0.950 \\ \text { C7 } & \text { H7 } & 0.950 & \text { C9 } & \text { H9 } & 0.950 \\ \text { C10 } & \text { H10 } & 0.950 & \text { C11 } & \text { H11A } & 0.980 \\ \text { C11 } & \text { H11B } & 0.980 & \text { C11 } & \text { H11C } & 0.980 \\ \text { C12 } & \text { H12A } & 0.980 & \text { C12 } & \text { H12B } & 0.980 \\ \text { C12 } & \text { H12C } & 0.980 & \text { C15 } & \text { H15 } & 1.000 \\ \text { C16 } & \text { H16A } & 0.980 & \text { C16 } & \text { H16B } & 0.980 \\ \text { C16 } & \text { H16C } & 0.980 & \text { C17 } & \text { H17A } & 0.980 \\ \text { C17 } & \text { H17B } & 0.980 & \text { C17 } & \text { H17C } & 0.980 \\ \text { C18 } & \text { H18 } & 1.000 & \text { C19 } & \text { H19A } & 0.980 \\ \text { C19 } & \text { H19B } & 0.980 & \text { C19 } & \text { H19C } & 0.980 \\ \text { C20 } & \text { H20A } & 0.980 & \text { C20 } & \text { H20B } & 0.980 \\ \text { C20 } & \text { H20C } & 0.980 & \text { C21 } & \text { H21 } & 1.000 \\ \text { C22 } & \text { H22A } & 0.980 & \text { C22 } & \text { H22B } & 0.980 \\ \text { C22 } & \text { H22C } & 0.980 & \text { C23 } & \text { H23A } & 0.980 \\ \text { C23 } & \text { H23B } & 0.980 & \text { C23 } & \text { H23C. } & 0.980\end{array}$

Table S32. Bond angles $\left({ }^{\circ}\right)$

$\begin{array}{llllllll}\text { atom } & \text { atom } & \text { atom } & \text { angle } & \text { atom } & \text { atom } & \text { atom } & \text { angle } \\ \text { C14 } & \text { Si1 } & \text { C15 } & 105.27(17) & \text { C14 } & \text { Si1 } & \text { C18. } & 106.04(18) \\ \text { C14 } & \text { Si1 } & \text { C21 } & 110.51(18) & \text { C15 } & \text { Si1 } & \text { C18 } & 106.0(2) \\ \text { C15 } & \text { Si1 } & \text { C21 } & 118.1(2) & \text { C18 } & \text { Si1 } & \text { C21 } & 110.1(2)\end{array}$


Table S32. Bond angles ( $\left.{ }^{\circ}\right)$ (Continued)

\begin{tabular}{|c|c|c|c|c|c|c|c|}
\hline atom & atom & atom & angle & atom & atom & atom & angle \\
\hline C6 & $\mathrm{O} 2$ & $\mathrm{C} 12$ & $116.8(2)$ & $\mathrm{C} 8$ & N1 & $\mathrm{C} 10$ & $106.3(3)$ \\
\hline $\mathrm{C} 8$ & N1 & C11 & $129.3(3)$ & $\mathrm{C} 10$ & N1 & $\mathrm{C} 11$ & $124.2(3)$ \\
\hline $\mathrm{C} 8$ & $\mathrm{~N} 2$ & C9 & $104.8(2)$ & $\mathrm{O} 1$ & $\mathrm{C} 1$ & $\mathrm{C} 2$ & $120.1(3)$ \\
\hline $\mathrm{O} 1$ & $\mathrm{C} 1$ & $\mathrm{C} 8$ & $122.2(2)$ & $\mathrm{C} 2$ & $\mathrm{C} 1$ & $\mathrm{C} 8$ & $117.7(3)$ \\
\hline $\mathrm{C} 1$ & $\mathrm{C} 2$ & $\mathrm{C} 3$ & $119.0(2)$ & $\mathrm{C} 1$ & $\mathrm{C} 2$ & $\mathrm{C} 7$ & $119.9(3)$ \\
\hline $\mathrm{C} 3$ & $\mathrm{C} 2$ & $\mathrm{C} 7$ & $121.0(2)$ & $\mathrm{C} 2$ & $\mathrm{C} 3$ & $\mathrm{C} 4$ & $118.4(3)$ \\
\hline $\mathrm{C} 2$ & $\mathrm{C} 3$ & $\mathrm{C} 13$ & $121.7(2)$ & $\mathrm{C} 4$ & $\mathrm{C} 3$ & $\mathrm{C} 13$ & $119.8(3)$ \\
\hline $\mathrm{C} 3$ & $\mathrm{C} 4$ & $\mathrm{C} 5$ & $120.9(3)$ & $\mathrm{C} 4$ & $\mathrm{C} 5$ & C6 & $120.0(2)$ \\
\hline $\mathrm{O} 2$ & $\mathrm{C} 6$ & $\mathrm{C} 5$ & $115.4(2)$ & $\mathrm{O} 2$ & C6 & $\mathrm{C} 7$ & $124.6(3)$ \\
\hline $\mathrm{C} 5$ & $\mathrm{C} 6$ & $\mathrm{C} 7$ & $120.1(3)$ & $\mathrm{C} 2$ & $\mathrm{C} 7$ & C6 & $119.5(3)$ \\
\hline N1 & $\mathrm{C} 8$ & $\mathrm{~N} 2$ & $111.5(3)$ & N1 & $\mathrm{C} 8$ & $\mathrm{C} 1$ & $124.7(3)$ \\
\hline $\mathrm{N} 2$ & $\mathrm{C} 8$ & $\mathrm{C} 1$ & $123.8(2)$ & $\mathrm{N} 2$ & C9 & $\mathrm{C} 10$ & $110.5(3)$ \\
\hline N1 & $\mathrm{C} 10$ & C9 & $106.8(3)$ & $\mathrm{C} 3$ & $\mathrm{C} 13$ & $\mathrm{C} 14$ & $176.2(3)$ \\
\hline Sil & C14 & $\mathrm{C} 13$ & $170.4(3)$ & Sil & $\mathrm{C} 15$ & $\mathrm{C} 16$ & $110.0(3)$ \\
\hline Sil & $\mathrm{C} 15$ & $\mathrm{C} 17$ & $113.0(4)$ & $\mathrm{C} 16$ & $\mathrm{C} 15$ & $\mathrm{C} 17$ & $110.6(5)$ \\
\hline Sil & C18 & C19 & $109.5(4)$ & Sil & $\mathrm{C} 18$ & $\mathrm{C} 20$ & $113.4(4)$ \\
\hline C19 & $\mathrm{C} 18$ & $\mathrm{C} 20$ & $113.3(5)$ & Sil & $\mathrm{C} 21$ & $\mathrm{C} 22$ & $116.3(4)$ \\
\hline Sil & $\mathrm{C} 21$ & $\mathrm{C} 23$ & $111.5(4)$ & $\mathrm{C} 22$ & $\mathrm{C} 21$ & $\mathrm{C} 23$ & $106.6(4)$ \\
\hline
\end{tabular}

Table S33. Bond angles involving hydrogens $\left({ }^{\circ}\right)$

$\begin{array}{llllllll}\text { atom } & \text { atom } & \text { atom } & \text { angle } & \text { atom } & \text { atom } & \text { atom } & \text { angle } \\ \text { C3 } & \text { C4 } & \text { H4 } & 119.5 & \text { C5 } & \text { C4 } & \text { H4 } & 119.5 \\ \text { C4 } & \text { C5 } & \text { H5 } & 120.0 & \text { C6 } & \text { C5 } & \text { H5 } & 120.0 \\ \text { C2 } & \text { C7 } & \text { H7 } & 120.2 & \text { C6 } & \text { C7 } & \text { H7 } & 120.2 \\ \text { N2 } & \text { C9 } & \text { H9 } & 124.7 & \text { C10 } & \text { C9 } & \text { H9 } & 124.7 \\ \text { N1 } & \text { C10 } & \text { H10 } & 126.6 & \text { C9 } & \text { C10 } & \text { H10 } & 126.6 \\ \text { N1 } & \text { C11 } & \text { H11A } & 109.5 & \text { N1 } & \text { C11 } & \text { H11B } & 109.5 \\ \text { N1 } & \text { C11 } & \text { H11C } & 109.5 & \text { H11A } & \text { C11 } & \text { H11B } & 109.5 \\ \text { H11A } & \text { C11 } & \text { H11C } & 109.5 & \text { H11B } & \text { C11 } & \text { H11C } & 109.5 \\ \text { O2 } & \text { C12 } & \text { H12A } & 109.5 & \text { O2 } & \text { C12 } & \text { H12B } & 109.5 \\ \text { O2 } & \text { C12 } & \text { H12C } & 109.5 & \text { H12A } & \text { C12 } & \text { H12B } & 109.5 \\ \text { H12A } & \text { C12 } & \text { H12C } & 109.5 & \text { H12B } & \text { C12 } & \text { H12C } & 109.5 \\ \text { Si1 } & \text { C15 } & \text { H15 } & 107.7 & \text { C16 } & \text { C15 } & \text { H15 } & 107.7 \\ \text { C17 } & \text { C15 } & \text { H15 } & 107.7 & \text { C15 } & \text { C16 } & \text { H16A } & 109.5 \\ \text { C15 } & \text { C16 } & \text { H16B } & 109.5 & \text { C15 } & \text { C16 } & \text { H16C } & 109.5 \\ \text { H16A } & \text { C16 } & \text { H16B } & 109.5 & \text { H16A } & \text { C16 } & \text { H16C } & 109.5 \\ \text { H16B } & \text { C16 } & \text { H16C } & 109.5 & \text { C15 } & \text { C17 } & \text { H17A } & 109.5 \\ \text { C15 } & \text { C17 } & \text { H17B } & 109.5 & \text { C15 } & \text { C17 } & \text { H17C } & 109.5 \\ \text { H17A } & \text { C17 } & \text { H17B } & 109.5 & \text { H17A } & \text { C17 } & \text { H17C } & 109.5 \\ \text { H17B } & \text { C17 } & \text { H17C } & 109.5 & \text { Si1 } & \text { C18 } & \text { H18 } & 106.7\end{array}$


Table S33. Bond angles involving hydrogens ( $\left.{ }^{\circ}\right)$ (Continued)

$\begin{array}{llllllll}\text { atom } & \text { atom } & \text { atom } & \text { angle } & \text { atom } & \text { atom } & \text { atom } & \text { angle } \\ \text { C19 } & \text { C18 } & \text { H18 } & 106.7 & \text { C20 } & \text { C18 } & \text { H18 } & 106.7 \\ \text { C18 } & \text { C19 } & \text { H19A } & 109.5 & \text { C18 } & \text { C19 } & \text { H19B } & 109.5 \\ \text { C18 } & \text { C19 } & \text { H19C } & 109.5 & \text { H19A } & \text { C19 } & \text { H19B } & 109.5 \\ \text { H19A } & \text { C19 } & \text { H19C } & 109.5 & \text { H19B } & \text { C19 } & \text { H19C } & 109.5 \\ \text { C18 } & \text { C20 } & \text { H20A } & 109.5 & \text { C18 } & \text { C20 } & \text { H20B } & 109.5 \\ \text { C18 } & \text { C20 } & \text { H20C } & 109.5 & \text { H20A } & \text { C20 } & \text { H20B } & 109.5 \\ \text { H20A } & \text { C20 } & \text { H20C } & 109.5 & \text { H20B } & \text { C20 } & \text { H20C } & 109.5 \\ \text { Si1 } & \text { C21 } & \text { H21 } & 107.4 & \text { C22 } & \text { C21 } & \text { H21 } & 107.4 \\ \text { C23 } & \text { C21 } & \text { H21 } & 107.4 & \text { C21 } & \text { C22 } & \text { H22A } & 109.5 \\ \text { C21 } & \text { C22 } & \text { H22B } & 109.5 & \text { C21 } & \text { C22 } & \text { H22C } & 109.5 \\ \text { H22A } & \text { C22 } & \text { H22B } & 109.5 & \text { H22A } & \text { C22 } & \text { H22C } & 109.5 \\ \text { H22B } & \text { C22 } & \text { H22C } & 109.5 & \text { C21 } & \text { C23 } & \text { H23A } & 109.5 \\ \text { C21 } & \text { C23 } & \text { H23B } & 109.5 & \text { C21 } & \text { C23 } & \text { H23C } & 109.5 \\ \text { H23A } & \text { C23 } & \text { H23B } & 109.5 & \text { H23A } & \text { C23 } & \text { H23C } & 109.5 \\ \text { H23B } & \text { C23 } & \text { H23C } & 109.5 & & & & \end{array}$


E. X-ray analysis of compound 6se' $\left(\mathrm{C}_{27} \mathrm{H}_{36} \mathrm{~N}_{2} \mathrm{O}_{3} \mathrm{SSi}\right)$ : $\mathrm{CCDC} 1974284$
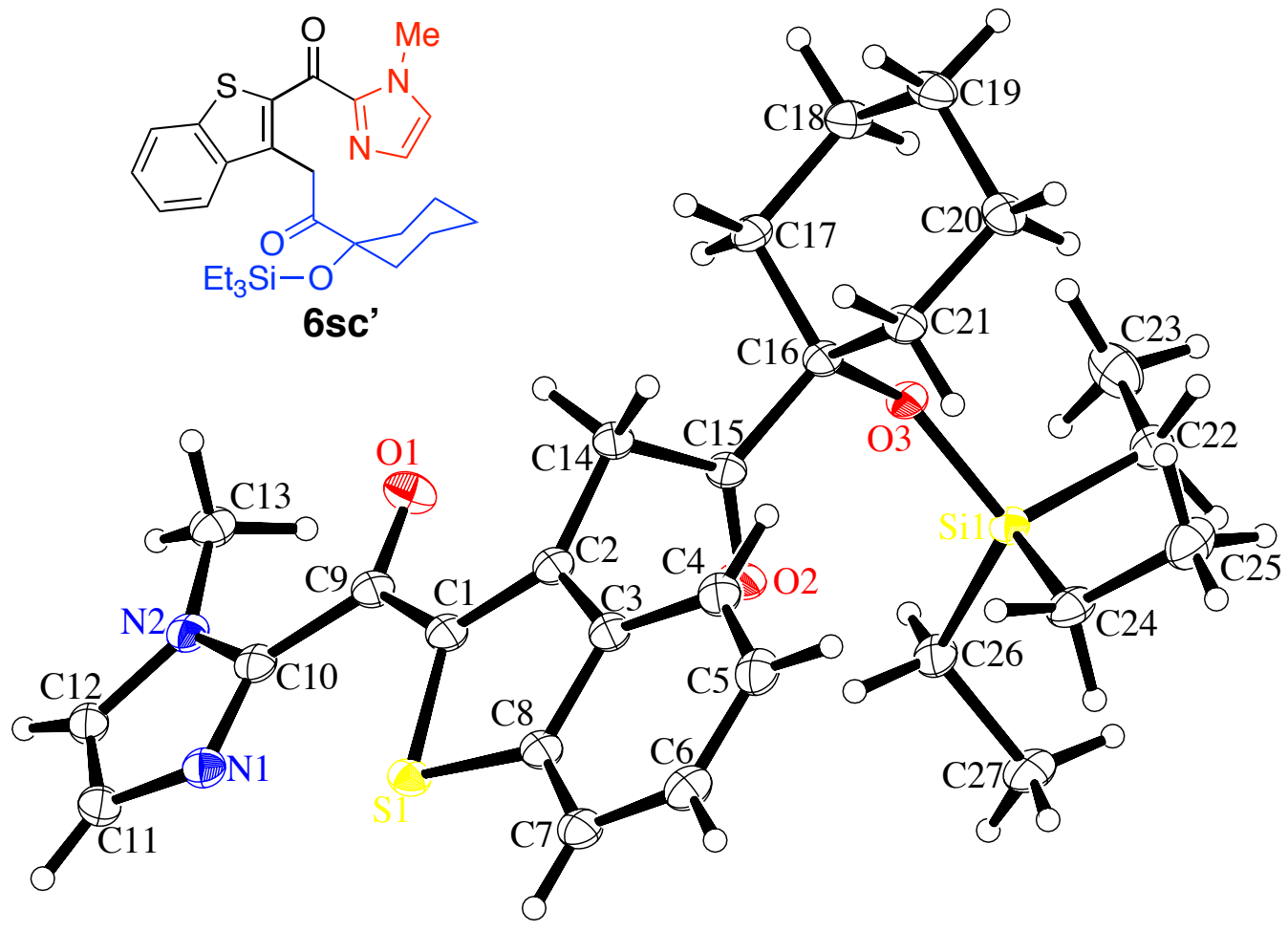

Figure S23: ORTEP diagram of compound 6sc'. 
CCDC 1974284 contains the supplementary crystallographic data. X-Ray crystallographic structure analysis of compound 6sc' $\left(\mathrm{C}_{27} \mathrm{H}_{36} \mathrm{~N}_{2} \mathrm{O}_{3} \mathrm{SSi}\right)$ was recorded on Rigaku XtaLAB P200 using multi-layer mirror monochromated $\mathrm{Cu}-\mathrm{K} \alpha$ radiation. The crystal-to-detector distance was $64.00 \mathrm{~mm}$. The data were collected at a temperature of $-150 \pm 1{ }^{\circ} \mathrm{C}$ to a maximum $2 \theta$ value of $136.2^{\circ}$. A total of 12652 reflections were collected, where 4624 were unique $(\operatorname{Rint}=0.0538)$. Data were collected and processed using CrysAlisPro (Rigaku Oxford Diffraction) ${ }^{4}$ and refined by the full matrix least-squares on F2 (Crystals). The structure was solved by direct methods $(\mathrm{SIR} 92)^{5}$ and expanded using Fourier techniques. The non-hydrogen atoms were refined anisotropically. Hydrogen atoms were refined using the riding model. The final cycle of fullmatrix least-squares refinement ${ }^{6}$ on F2 was based on 4624 observed reflections and 307 variable parameters. The crystal data are given below:

X-ray data of compound 6sc' $\left(\mathrm{C}_{23} \mathrm{H}_{32} \mathrm{~N}_{2} \mathrm{O}_{2} \mathrm{Si}\right)$ : CCDC 1974284

Empirical Formula

Formula Weight

Crystal Color, Habit

Crystal Dimensions

Crystal System

Lattice Type

Lattice Parameters

Space Group

$\mathrm{Z}$ value

$\mathrm{D}_{\text {calc }}$

F000

$\mathrm{m}(\mathrm{CuKa})$
$\mathrm{C}_{27} \mathrm{H}_{36} \mathrm{~N}_{2} \mathrm{O}_{3} \mathrm{SSi}$

496.74

colorless, plate

$0.600 \times 0.400 \times 0.100 \mathrm{~mm}$

triclinic

Primitive

$\mathrm{a}=8.2563(5) \AA$
$\mathrm{b}=8.5661(4) \AA$
$\mathrm{c}=19.6108(11) \AA$
$\mathrm{a}=78.098(4) \mathrm{o}$
$\mathrm{b}=89.653(5)^{\mathrm{o}}$
$\mathrm{g}=71.445(5)^{\mathrm{o}}$
$\mathrm{V}=1283.94(13) \AA^{3}$

P-1 (\#2)

2

$1.285 \mathrm{~g} / \mathrm{cm}^{3}$

532.00

$18.150 \mathrm{~cm}^{-1}$ 
Table S34. Bond lengths ( $\AA$ )

$\begin{array}{llllll}\text { atom } & \text { atom } & \text { distance } & \text { atom } & \text { atom } & \text { distance } \\ \text { S1 } & \text { C1 } & 1.760(3) & \text { S1 } & \text { C8 } & 1.735(3) \\ \text { Si1 } & \text { O3 } & 1.6553(19) & \text { Si1 } & \text { C22 } & 1.875(3) \\ \text { Si1 } & \text { C24 } & 1.874(3) & \text { Si1 } & \text { C26 } & 1.881(3) \\ \text { O1 } & \text { C9 } & 1.227(3) & \text { O2 } & \text { C15 } & 1.212(4) \\ \text { O3 } & \text { C16 } & 1.423(3) & \text { N1 } & \text { C10 } & 1.326(3) \\ \text { N1 } & \text { C11 } & 1.366(4) & \text { N2 } & \text { C10 } & 1.368(3) \\ \text { N2 } & \text { C12 } & 1.363(4) & \mathrm{N} 2 & \mathrm{C} 13 & 1.459(3) \\ \text { C1 } & \text { C2 } & 1.377(4) & \mathrm{C} 1 & \mathrm{C} 9 & 1.483(3) \\ \text { C2 } & \text { C3 } & 1.425(3) & \mathrm{C} 2 & \mathrm{C} 14 & 1.502(4) \\ \mathrm{C} 3 & \mathrm{C} 4 & 1.413(4) & \mathrm{C} 3 & \mathrm{C} 8 & 1.413(4) \\ \mathrm{C} 4 & \mathrm{C} 5 & 1.377(4) & \mathrm{C} 5 & \mathrm{C} 6 & 1.399(4) \\ \mathrm{C} 6 & \mathrm{C} 7 & 1.384(4) & \mathrm{C} 7 & \mathrm{C} 8 & 1.400(3) \\ \mathrm{C} 9 & \mathrm{C} 10 & 1.472(4) & \mathrm{C} 11 & \mathrm{C} 12 & 1.366(3) \\ \mathrm{C} 14 & \mathrm{C} 15 & 1.527(3) & \mathrm{C} 15 & \mathrm{C} 16 & 1.535(3) \\ \mathrm{C} 16 & \mathrm{C} 17 & 1.538(3) & \mathrm{C} 16 & \mathrm{C} 21 & 1.542(4) \\ \mathrm{C} 17 & \mathrm{C} 18 & 1.533(4) & \mathrm{C} 18 & \mathrm{C} 19 & 1.529(4) \\ \mathrm{C} 19 & \mathrm{C} 20 & 1.524(4) & \mathrm{C} 20 & \mathrm{C} 21 & 1.524(4) \\ \mathrm{C} 22 & \mathrm{C} 23 & 1.525(4) & \mathrm{C} 24 & \mathrm{C} 25 & 1.543(5) \\ \mathrm{C} 26 & \mathrm{C} 27 & 1.530(4) & & & \end{array}$

Table S35. Bond lengths involving hydrogens $(\AA)$

$\begin{array}{llllll}\text { atom } & \text { atom } & \text { distance } & \text { atom } & \text { atom } & \text { distance } \\ \text { C4 } & \text { H4 } & 0.950 & \text { C5 } & \text { H5 } & 0.950 \\ \text { C6 } & \text { H6 } & 0.950 & \text { C7 } & \text { H7 } & 0.950 \\ \text { C11 } & \text { H11 } & 0.950 & \text { C12 } & \text { H12 } & 0.950 \\ \text { C13 } & \text { H13A } & 0.980 & \text { C13 } & \text { H13B } & 0.980 \\ \text { C13 } & \text { H13C } & 0.980 & \text { C14 } & \text { H14A } & 0.990 \\ \text { C14 } & \text { H14B } & 0.990 & \text { C17 } & \text { H17A } & 0.990 \\ \text { C17 } & \text { H17B } & 0.990 & \text { C18 } & \text { H18A } & 0.990 \\ \text { C18 } & \text { H18B } & 0.990 & \text { C19 } & \text { H19A } & 0.990 \\ \text { C19 } & \text { H19B } & 0.990 & \text { C20 } & \text { H20A } & 0.990 \\ \text { C20 } & \text { H20B } & 0.990 & \text { C21 } & \text { H21A } & 0.990 \\ \text { C21 } & \text { H21B } & 0.990 & \text { C22 } & \text { H22A } & 0.990 \\ \text { C22 } & \text { H22B } & 0.990 & \text { C23 } & \text { H23A } & 0.980 \\ \text { C23 } & \text { H23B } & 0.980 & \text { C23 } & \text { H23C } & 0.980 \\ \text { C24 } & \text { H24A } & 0.990 & \text { C24 } & \text { H24B } & 0.990 \\ \text { C25 } & \text { H25A } & 0.980 & \text { C25 } & \text { H25B } & 0.980 \\ \text { C25 } & \text { H25C } & 0.980 & \text { C26 } & \text { H26A } & 0.990 \\ \text { C26 } & \text { H26B } & 0.990 & \text { C27 } & \text { H27A } & 0.980 \\ \text { C27 } & \text { H27B } & 0.980 & \text { C27 } & \text { H27C } & 0.980\end{array}$


Table S36. Bond angles $\left({ }^{\circ}\right)$

\begin{tabular}{|c|c|c|c|c|c|c|c|}
\hline atom & atom & atom & angle & atom & atom & atom & angle \\
\hline $\mathrm{C} 1$ & $\mathrm{~S} 1$ & $\mathrm{C} 8$ & $91.26(13)$ & $\mathrm{O} 3$ & Sil & $\mathrm{C} 22$ & $103.21(12)$ \\
\hline $\mathrm{O} 3$ & Sil & $\mathrm{C} 24$ & $114.78(11)$ & $\mathrm{O} 3$ & Sil & $\mathrm{C} 26$. & $111.83(11)$ \\
\hline $\mathrm{C} 22$ & Sil & $\mathrm{C} 24$ & $109.47(12)$ & $\mathrm{C} 22$ & Si1 & C26. & $108.29(13)$ \\
\hline $\mathrm{C} 24$ & Sil & $\mathrm{C} 26$ & $108.96(13)$ & Sil & $\mathrm{O} 3$ & C16. & $135.14(14)$ \\
\hline $\mathrm{C} 10$ & N1 & $\mathrm{C} 11$ & $105.2(2)$ & $\mathrm{C} 10$ & $\mathrm{~N} 2$ & $\mathrm{C} 12$ & $106.1(2)$ \\
\hline $\mathrm{C} 10$ & $\mathrm{~N} 2$ & $\mathrm{C} 13$ & $129.3(2)$ & $\mathrm{C} 12$ & $\mathrm{~N} 2$ & $\mathrm{C} 13$ & $124.5(2)$ \\
\hline S1 & $\mathrm{C} 1$ & $\mathrm{C} 2$ & $112.01(18)$ & S1 & $\mathrm{C} 1$ & C9 & $123.8(2)$ \\
\hline $\mathrm{C} 2$ & $\mathrm{C} 1$ & C9 & $124.1(2)$ & $\mathrm{C} 1$ & $\mathrm{C} 2$ & $\mathrm{C} 3$ & $112.8(2)$ \\
\hline $\mathrm{C} 1$ & $\mathrm{C} 2$ & $\mathrm{C} 14$ & $126.2(2)$ & $\mathrm{C} 3$ & $\mathrm{C} 2$ & $\mathrm{C} 14$ & $121.0(2)$ \\
\hline $\mathrm{C} 2$ & $\mathrm{C} 3$ & $\mathrm{C} 4$ & $129.0(2)$ & $\mathrm{C} 2$ & $\mathrm{C} 3$ & $\mathrm{C} 8$ & $112.4(2)$ \\
\hline $\mathrm{C} 4$ & $\mathrm{C} 3$ & $\mathrm{C} 8$ & $118.6(2)$ & $\mathrm{C} 3$ & $\mathrm{C} 4$ & $\mathrm{C} 5$ & $120.0(3)$ \\
\hline $\mathrm{C} 4$ & $\mathrm{C} 5$ & $\mathrm{C} 6$ & $120.3(3)$ & $\mathrm{C} 5$ & C6 & $\mathrm{C} 7$ & $121.5(2)$ \\
\hline C6 & $\mathrm{C} 7$ & $\mathrm{C} 8$ & $118.2(3)$ & $\mathrm{S} 1$ & $\mathrm{C} 8$ & $\mathrm{C} 3$. & $111.48(18)$ \\
\hline S1 & $\mathrm{C} 8$ & $\mathrm{C} 7$ & $127.1(2)$ & $\mathrm{C} 3$ & $\mathrm{C} 8$ & $\mathrm{C} 7$ & $121.4(3)$ \\
\hline $\mathrm{O} 1$ & C9 & $\mathrm{C} 1$ & $120.6(3)$ & $\mathrm{O} 1$ & C9 & $\mathrm{C} 10$ & $119.4(2)$ \\
\hline $\mathrm{C} 1$ & $\mathrm{C} 9$ & $\mathrm{C} 10$ & $120.0(2)$ & N1 & $\mathrm{C} 10$ & $\mathrm{~N} 2$ & $111.7(2)$ \\
\hline N1 & $\mathrm{C} 10$ & C9 & $125.5(2)$ & $\mathrm{N} 2$ & $\mathrm{C} 10$ & $\mathrm{C} 9$ & $122.7(2)$ \\
\hline N1 & $\mathrm{C} 11$ & $\mathrm{C} 12$ & $110.1(2)$ & $\mathrm{N} 2$ & $\mathrm{C} 12$ & $\mathrm{C} 11$ & $106.8(2)$ \\
\hline $\mathrm{C} 2$ & $\mathrm{C} 14$ & $\mathrm{C} 15$ & $111.2(2)$ & $\mathrm{O} 2$ & C15 & $\mathrm{C} 14$ & $121.0(2)$ \\
\hline $\mathrm{O} 2$ & $\mathrm{C} 15$ & $\mathrm{C} 16$ & $120.5(2)$ & $\mathrm{C} 14$ & $\mathrm{C} 15$ & $\mathrm{C} 16$ & $118.5(2)$ \\
\hline $\mathrm{O} 3$ & $\mathrm{C} 16$ & $\mathrm{C} 15$ & $109.6(2)$ & $\mathrm{O} 3$ & C16 & $\mathrm{C} 17$ & $106.60(16)$ \\
\hline $\mathrm{O} 3$ & $\mathrm{C} 16$ & $\mathrm{C} 21$ & $110.69(19)$ & $\mathrm{C} 15$ & C16 & $\mathrm{C} 17$ & $112.3(2)$ \\
\hline C15 & $\mathrm{C} 16$ & $\mathrm{C} 21$ & $108.49(17)$ & $\mathrm{C} 17$ & C16 & $\mathrm{C} 21$ & $109.2(2)$ \\
\hline C16 & $\mathrm{C} 17$ & $\mathrm{C} 18$ & $111.6(2)$ & $\mathrm{C} 17$ & C18 & C19 & $111.3(2)$ \\
\hline C18 & C19 & $\mathrm{C} 20$ & $111.3(3)$ & C19 & $\mathrm{C} 20$ & $\mathrm{C} 21$ & $110.1(2)$ \\
\hline $\mathrm{C} 16$ & $\mathrm{C} 21$ & $\mathrm{C} 20$ & $111.54(18)$ & Sil & $\mathrm{C} 22$ & $\mathrm{C} 23$ & $115.2(2)$ \\
\hline Sil & $\mathrm{C} 24$ & $\mathrm{C} 25$ & $117.8(2)$ & Si1 & $\mathrm{C} 26$ & $\mathrm{C} 27$ & $113.6(2)$ \\
\hline
\end{tabular}

Table S37. Bond angles involving hydrogens $\left(^{(}\right)$

$\begin{array}{llllllll}\text { atom } & \text { atom } & \text { atom } & \text { angle } & \text { atom } & \text { atom } & \text { atom } & \text { angle } \\ \text { C3 } & \text { C4 } & \text { H4 } & 120.0 & \text { C5 } & \text { C4 } & \text { H4 } & 120.0 \\ \text { C4 } & \text { C5 } & \text { H5 } & 119.8 & \text { C6 } & \text { C5 } & \text { H5 } & 119.8 \\ \text { C5 } & \text { C6 } & \text { H6 } & 119.2 & \text { C7 } & \text { C6 } & \text { H6 } & 119.2 \\ \text { C6 } & \text { C7 } & \text { H7 } & 120.9 & \text { C8 } & \text { C7 } & \text { H7 } & 120.9 \\ \text { N1 } & \text { C11 } & \text { H11 } & 124.9 & \text { C12 } & \text { C11 } & \text { H11 } & 124.9 \\ \text { N2 } & \text { C12 } & \text { H12 } & 126.6 & \text { C11 } & \text { C12 } & \text { H12 } & 126.6 \\ \text { N2 } & \text { C13 } & \text { H13A } & 109.5 & \text { N2 } & \text { C13 } & \text { H13B } & 109.5 \\ \text { N2 } & \text { C13 } & \text { H13C } & 109.5 & \text { H13A } & \text { C13 } & \text { H13B } & 109.5 \\ \text { H13A } & \text { C13 } & \text { H13C } & 109.5 & \text { H13B } & \text { C13 } & \text { H13C } & 109.5\end{array}$


Table S37. Bond angles involving hydrogens $\left({ }^{\circ}\right)$ (Continued)

\begin{tabular}{|c|c|c|c|c|c|c|c|}
\hline atom & atom & atom & angle & atom & atom & atom & angle \\
\hline $\mathrm{C} 2$ & $\mathrm{C} 14$ & H14A & 109.4 & $\mathrm{C} 2$ & C14 & H14B & 109.4 \\
\hline C15 & $\mathrm{C} 14$ & H14A & 109.4 & C15 & $\mathrm{C} 14$ & H14B & 109.4 \\
\hline $\mathrm{H} 14 \mathrm{~A}$ & $\mathrm{C} 14$ & H14B & 108.0 & $\mathrm{C} 16$ & $\mathrm{C} 17$ & H17A & 109.3 \\
\hline C16 & $\mathrm{C} 17$ & H17B & 109.3 & C18 & $\mathrm{C} 17$ & H17A & 109.3 \\
\hline C18 & $\mathrm{C} 17$ & H17B & 109.3 & H17A & $\mathrm{C} 17$ & H17B & 108.0 \\
\hline C17 & C18 & H18A & 109.4 & C17 & C18 & H18B & 109.4 \\
\hline C19 & $\mathrm{C} 18$ & H18A & 109.4 & C19 & $\mathrm{C} 18$ & H18B & 109.4 \\
\hline H18A & C18 & H18B & 108.0 & C18 & C19 & H19A & 109.4 \\
\hline C18 & C19 & H19B & 109.4 & $\mathrm{C} 20$ & C19 & H19A & 109.4 \\
\hline C20 & C19 & H19B & 109.4 & H19A & C19 & H19B & 108.0 \\
\hline C19 & C20 & $\mathrm{H} 20 \mathrm{~A}$ & 109.6 & C19 & C20 & H20B & 109.6 \\
\hline C21 & C20 & $\mathrm{H} 20 \mathrm{~A}$ & 109.6 & $\mathrm{C} 21$ & $\mathrm{C} 20$ & H20B & 109.6 \\
\hline $\mathrm{H} 20 \mathrm{~A}$ & C20 & $\mathrm{H} 20 \mathrm{~B}$ & 108.2 & $\mathrm{C} 16$ & C21 & $\mathrm{H} 21 \mathrm{~A}$ & 109.3 \\
\hline C16 & C21 & $\mathrm{H} 21 \mathrm{~B}$ & 109.3 & $\mathrm{C} 20$ & C21 & $\mathrm{H} 21 \mathrm{~A}$ & 109.3 \\
\hline C20 & $\mathrm{C} 21$ & $\mathrm{H} 21 \mathrm{~B}$ & 109.3 & $\mathrm{H} 21 \mathrm{~A}$ & C21 & $\mathrm{H} 21 \mathrm{~B}$ & 108.0 \\
\hline Sil & $\mathrm{C} 22$ & $\mathrm{H} 22 \mathrm{~A}$ & 108.5 & Sil & $\mathrm{C} 22$ & H22B & 108.5 \\
\hline $\mathrm{C} 23$ & $\mathrm{C} 22$ & $\mathrm{H} 22 \mathrm{~A}$ & 108.5 & $\mathrm{C} 23$ & $\mathrm{C} 22$ & H22B & 108.5 \\
\hline $\mathrm{H} 22 \mathrm{~A}$ & $\mathrm{C} 22$ & $\mathrm{H} 22 \mathrm{~B}$ & 107.5 & $\mathrm{C} 22$ & C23 & $\mathrm{H} 23 \mathrm{~A}$ & 109.5 \\
\hline $\mathrm{C} 22$ & $\mathrm{C} 23$ & $\mathrm{H} 23 \mathrm{~B}$ & 109.5 & $\mathrm{C} 22$ & C23 & $\mathrm{H} 23 \mathrm{C}$ & 109.5 \\
\hline $\mathrm{H} 23 \mathrm{~A}$ & C23 & $\mathrm{H} 23 \mathrm{~B}$ & 109.5 & $\mathrm{H} 23 \mathrm{~A}$ & C23 & $\mathrm{H} 23 \mathrm{C}$ & 109.5 \\
\hline H23B & $\mathrm{C} 23$ & $\mathrm{H} 23 \mathrm{C}$ & 109.5 & Si1 & $\mathrm{C} 24$ & $\mathrm{H} 24 \mathrm{~A}$ & 107.9 \\
\hline Sil & C24 & H24B & 107.9 & $\mathrm{C} 25$ & C24 & $\mathrm{H} 24 \mathrm{~A}$ & 107.9 \\
\hline $\mathrm{C} 25$ & C24 & $\mathrm{H} 24 \mathrm{~B}$ & 107.9 & $\mathrm{H} 24 \mathrm{~A}$ & C24 & H24B & 107.2 \\
\hline C24 & $\mathrm{C} 25$ & $\mathrm{H} 25 \mathrm{~A}$ & 109.5 & $\mathrm{C} 24$ & $\mathrm{C} 25$ & $\mathrm{H} 25 \mathrm{~B}$ & 109.5 \\
\hline $\mathrm{C} 24$ & $\mathrm{C} 25$ & $\mathrm{H} 25 \mathrm{C}$ & 109.5 & $\mathrm{H} 25 \mathrm{~A}$ & $\mathrm{C} 25$ & $\mathrm{H} 25 \mathrm{~B}$ & 109.5 \\
\hline $\mathrm{H} 25 \mathrm{~A}$ & $\mathrm{C} 25$ & $\mathrm{H} 25 \mathrm{C}$ & 109.5 & $\mathrm{H} 25 \mathrm{~B}$ & $\mathrm{C} 25$ & $\mathrm{H} 25 \mathrm{C}$ & 109.5 \\
\hline Si1 & C26 & $\mathrm{H} 26 \mathrm{~A}$ & 108.8 & Si1 & C26 & H26B & 108.8 \\
\hline C27 & C26 & $\mathrm{H} 26 \mathrm{~A}$ & 108.8 & $\mathrm{C} 27$ & C26 & H26B & 108.8 \\
\hline $\mathrm{H} 26 \mathrm{~A}$ & C26 & $\mathrm{H} 26 \mathrm{~B}$ & 107.7 & $\mathrm{C} 26$ & $\mathrm{C} 27$ & $\mathrm{H} 27 \mathrm{~A}$ & 109.5 \\
\hline $\mathrm{C} 26$ & $\mathrm{C} 27$ & $\mathrm{H} 27 \mathrm{~B}$ & 109.5 & C26 & $\mathrm{C} 27$ & $\mathrm{H} 27 \mathrm{C}$ & 109.5 \\
\hline $\mathrm{H} 27 \mathrm{~A}$ & $\mathrm{C} 27$ & $\mathrm{H} 27 \mathrm{~B}$ & 109.5 & H27A & C27 & $\mathrm{H} 27 \mathrm{C}$ & 109.5 \\
\hline H27B & $\mathrm{C} 27$ & $\mathrm{H} 27 \mathrm{C}$ & 109.5 & & & & \\
\hline
\end{tabular}




\section{References}

1. For synthesis of 2-acyl imidazole, see: (a) Ma, J.; Harms, K.; Meggers, E. Enantioselective rhodium/ruthenium photoredox catalysis en route to chiral 1,2aminoalcohols. Chem. Commun., 2016, 52, 10183-10186. (b) Bisht, R.; Hoque, E.; Chattopadhyay, B. Amide Effects in C-H Activation: Noncovalent Interactions with L-Shaped Ligand for meta Borylation of Aromatic Amides. Angew. Chem. Int. Ed. 2018, 57, 15762-15766. (c) Huo, H.; Shen, X.; Wang, C.; Zhang, L.; Röse, P.; Chen, L. -A.; Harms, K.; Marsch, M.; Hilt, G.; Meggers, E. Asymmetric photoredox transition-metal catalysis activated by visible light. Nature 2014, 515, 100-103.

2. For synthesis of coupling partner, see : (a) Porey, S.; Zhang, X.; Bhowmick, A.; Singh, V. K.; Guin, S.; Paton, R. S.; Maiti, D. Alkyne Linchpin Strategy for Drug: Pharmacophore Conjugation: Experimental and Computational Realization of a MetaSelective Inverse Sonogashira Coupling. J. Am. Chem. Soc. 2020, 142, 3762-3774. (b) Pan, R.; Shi, C.; Zhang, D.; Tian, Y.; Guo, S.; Yao, H.; Lin, A. Nickel-Catalyzed Reductive 1,2-Dialkynylation of Alkenes Bearing an 8-Aminoquinoline Directing Group. Org. Lett. 2019, 21, 8915-8920. (c) Li, J.; Peng, J.; Deng, Y.; Ma, C.; Zhang, G.; Bai, Y.; Lai, G. Synthesis of platinum acetylide complexes and their application in curing silicone rubber by hydrosilylation. Appl. Organometal. Chem. 2012, 26, 461-466. (d) Liu, T.; Qiao, J. X.; Poss, M. A.; Yu, J.-Q. Palladium(II)-Catalyzed SiteSelective $\mathrm{C}\left(\mathrm{sp}^{3}\right)-\mathrm{H}$ Alkynylation of Oligopeptides: A Linchpin Approach for Oligopeptide-Drug Conjugation. Angew. Chem. Int. Ed. 2017, 56, $10924-10927$.

3. For removal of directing group, see: (a) Ohta, S.; Hayakawa, S.; Moriwaki, H.; Tsuboi, S.; Okamoto, M. Synthesis of 2-acyl-1-methyl-1H-imidazoles and reactivity of the acyl group. Heterocycles 1985, 23, 1759-1764. (b) Huang, X.; Webster, R. D.; Harms, K.; Meggers, E. Asymmetric Catalysis with Organic Azides and Diazo Compounds Initiated by Photoinduced Electron Transfer. J. Am. Chem. Soc. 2016, 138, 1263612642.

4. CrysAlisPro: Data Collection and Processing Software, Rigaku Corporation (2015). Tokyo 196-8666, Japan.

5. Altomare, A.; Cascarano, G.; Giacovazzo, C.; Guagliardi, A. Completion and refinement of crystal structures with SIR92. J. Appl. Cryst. 1993, 26, 343-350.

6. Least Squares function minimized: (SHELXL97)

$\mathrm{Sw}\left(\mathrm{F}_{\mathrm{O}}{ }^{2}-\mathrm{F}_{\mathrm{c}}^{2}\right)^{2} \quad$ where $\mathrm{w}=$ Least Squares weights. 


\section{NMR spectra}

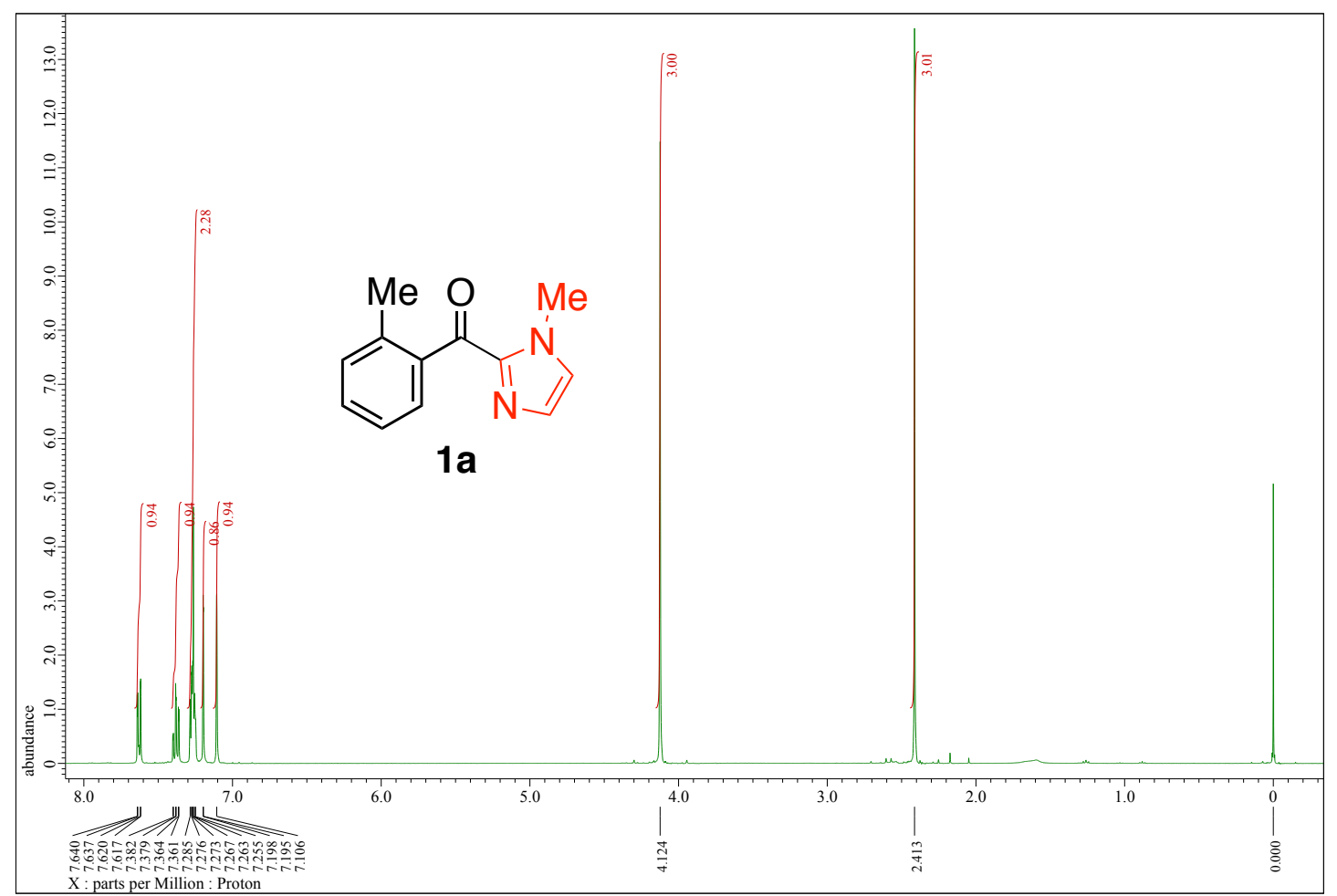

Figure S24: ${ }^{1} \mathrm{H}$ NMR spectrum of compound 1 a $\left(400 \mathrm{MHz}, \mathrm{CDCl}_{3}\right)$.

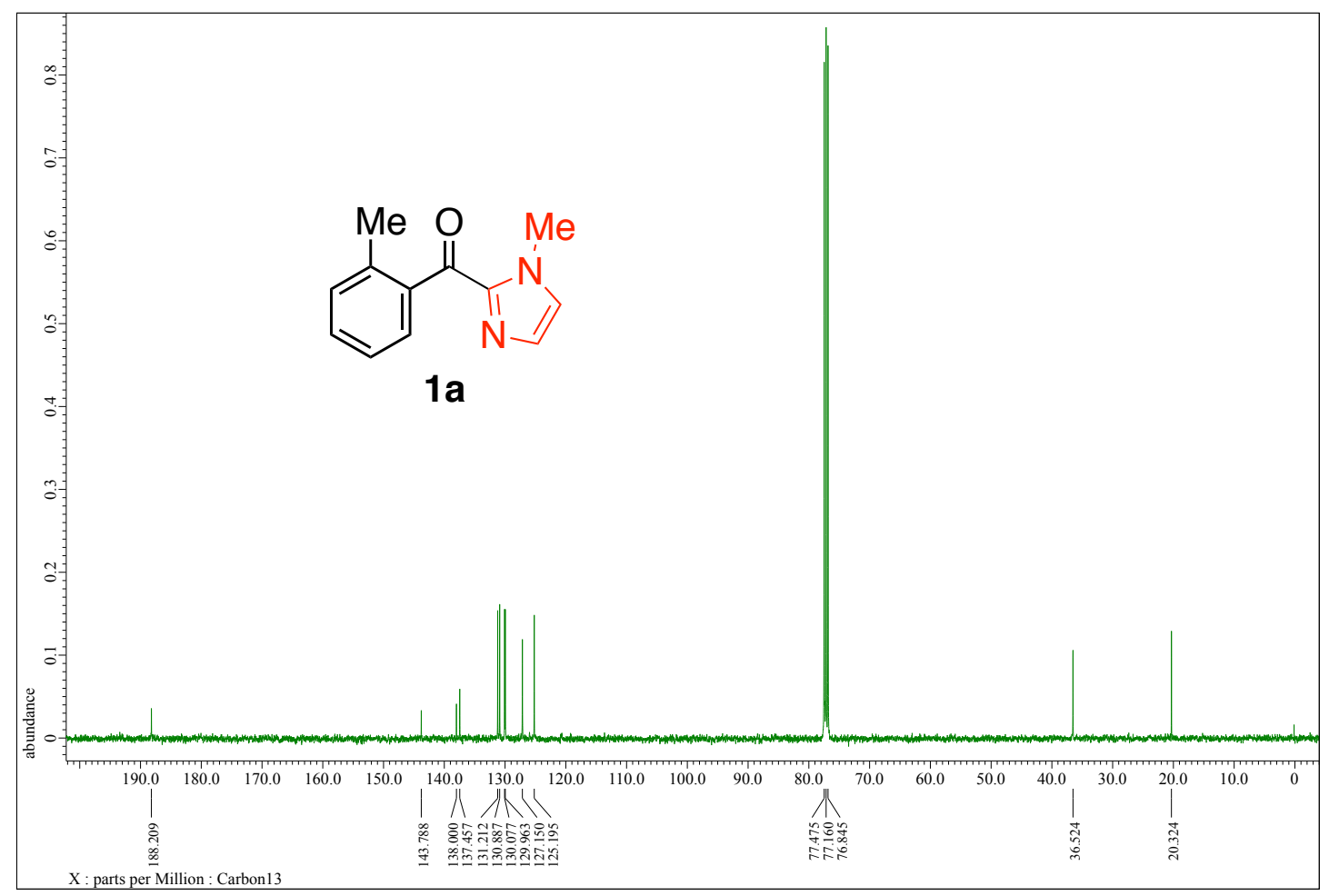

Figure S25. ${ }^{13} \mathrm{C}$ NMR spectrum of compound $1 \mathbf{a}\left(100 \mathrm{MHz}, \mathrm{CDCl}_{3}\right)$. 


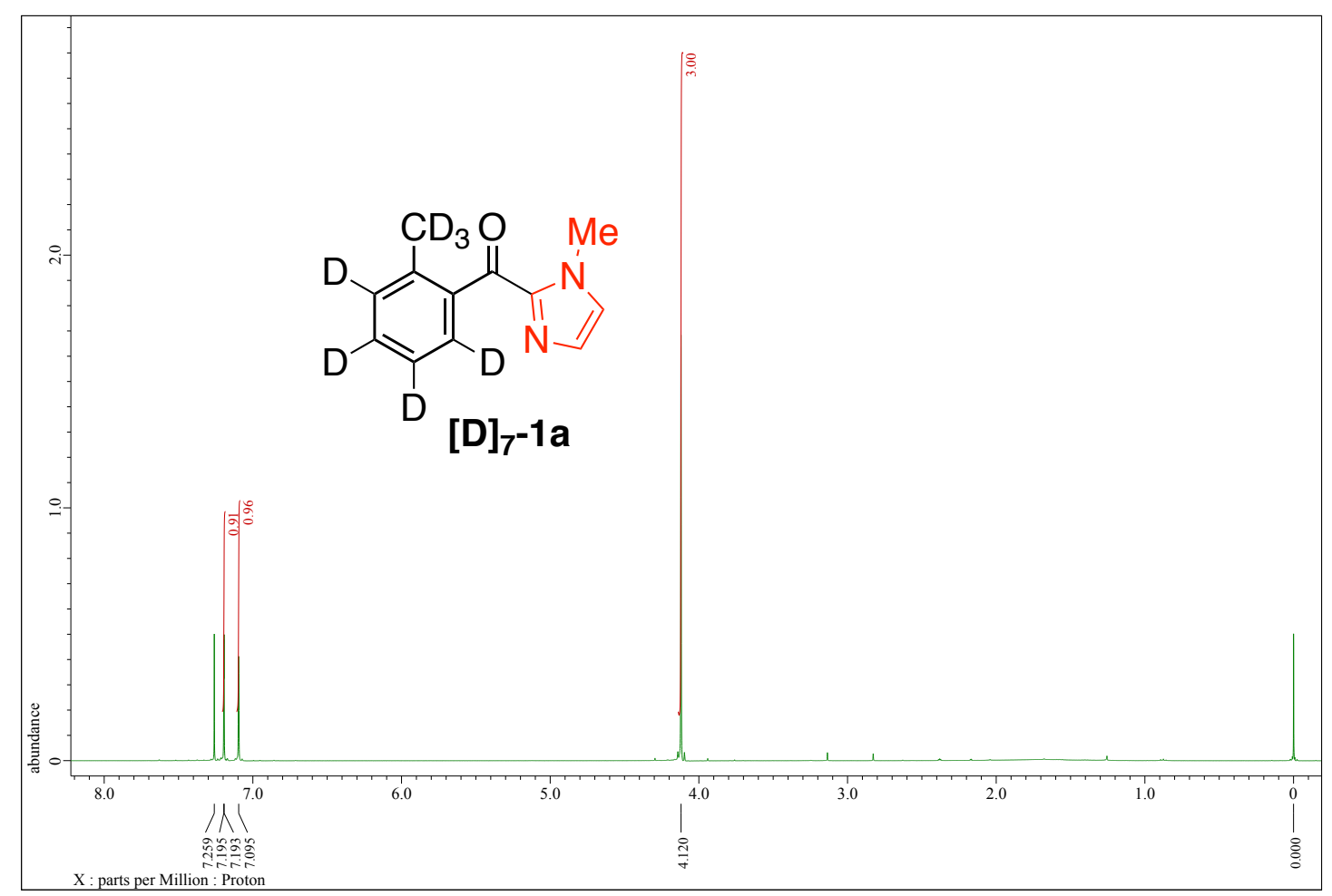

Figure S26: ${ }^{1} \mathrm{H}$ NMR spectrum of compound $[\mathbf{D}]_{7}-1 \mathbf{a}\left(400 \mathrm{MHz}, \mathrm{CDCl}_{3}\right)$.

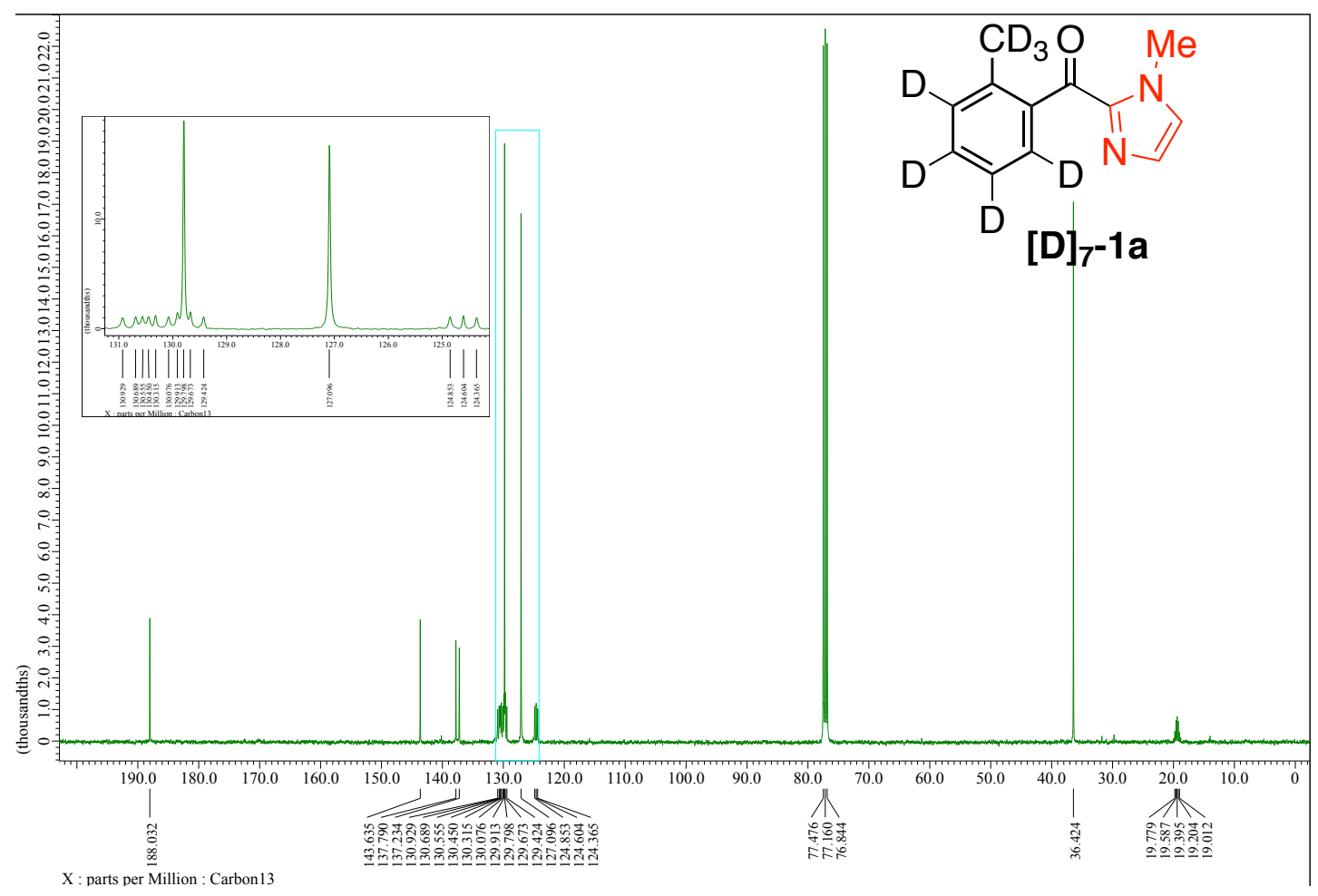

Figure S27: ${ }^{13} \mathrm{C}$ NMR spectrum of compound $[\mathbf{D}]_{7}-\mathbf{1 a}\left(100 \mathrm{MHz}, \mathrm{CDCl}_{3}\right)$. 


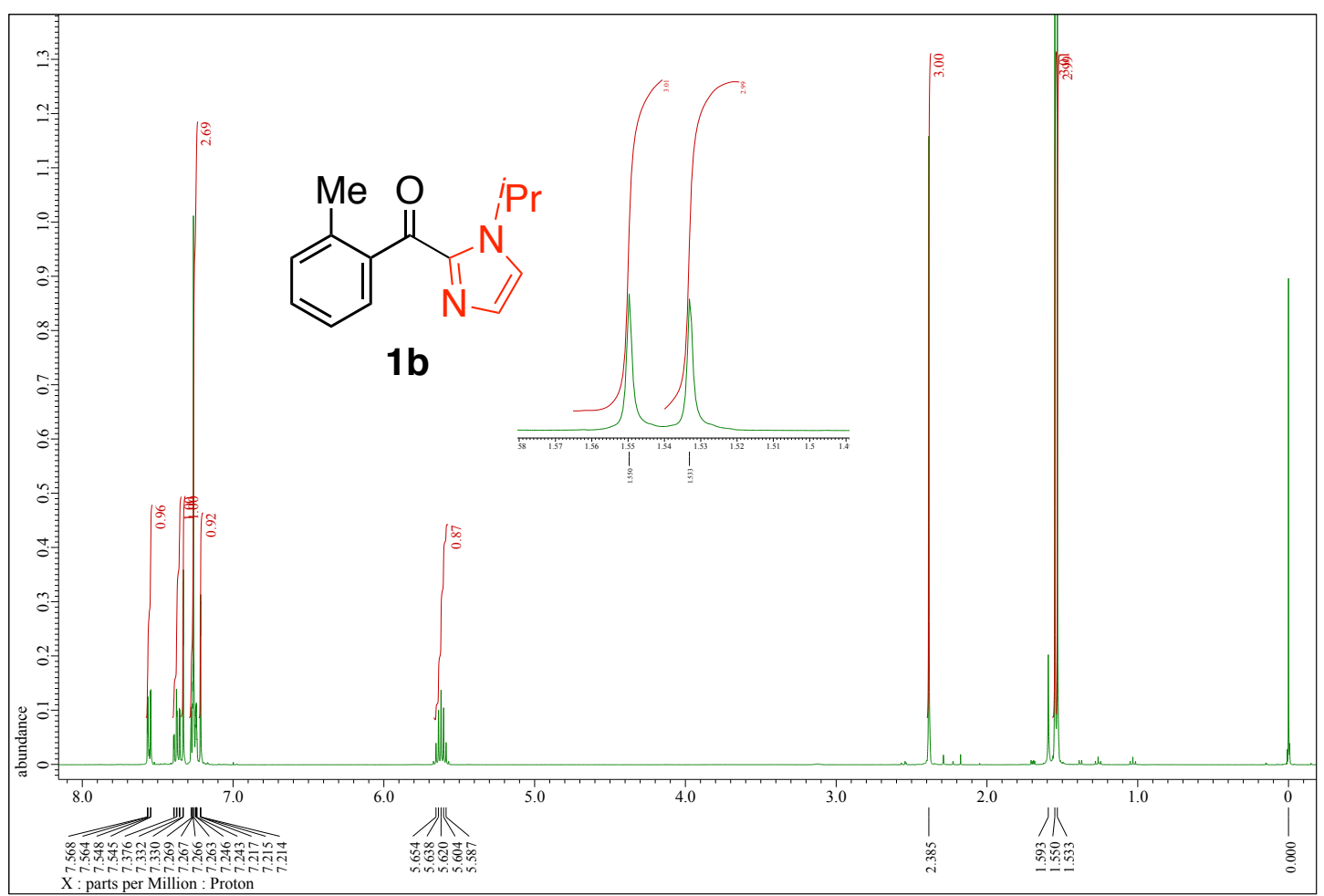

Figure S28: ${ }^{1} \mathrm{H}$ NMR spectrum of compound $1 \mathbf{b}\left(400 \mathrm{MHz}, \mathrm{CDCl}_{3}\right)$.

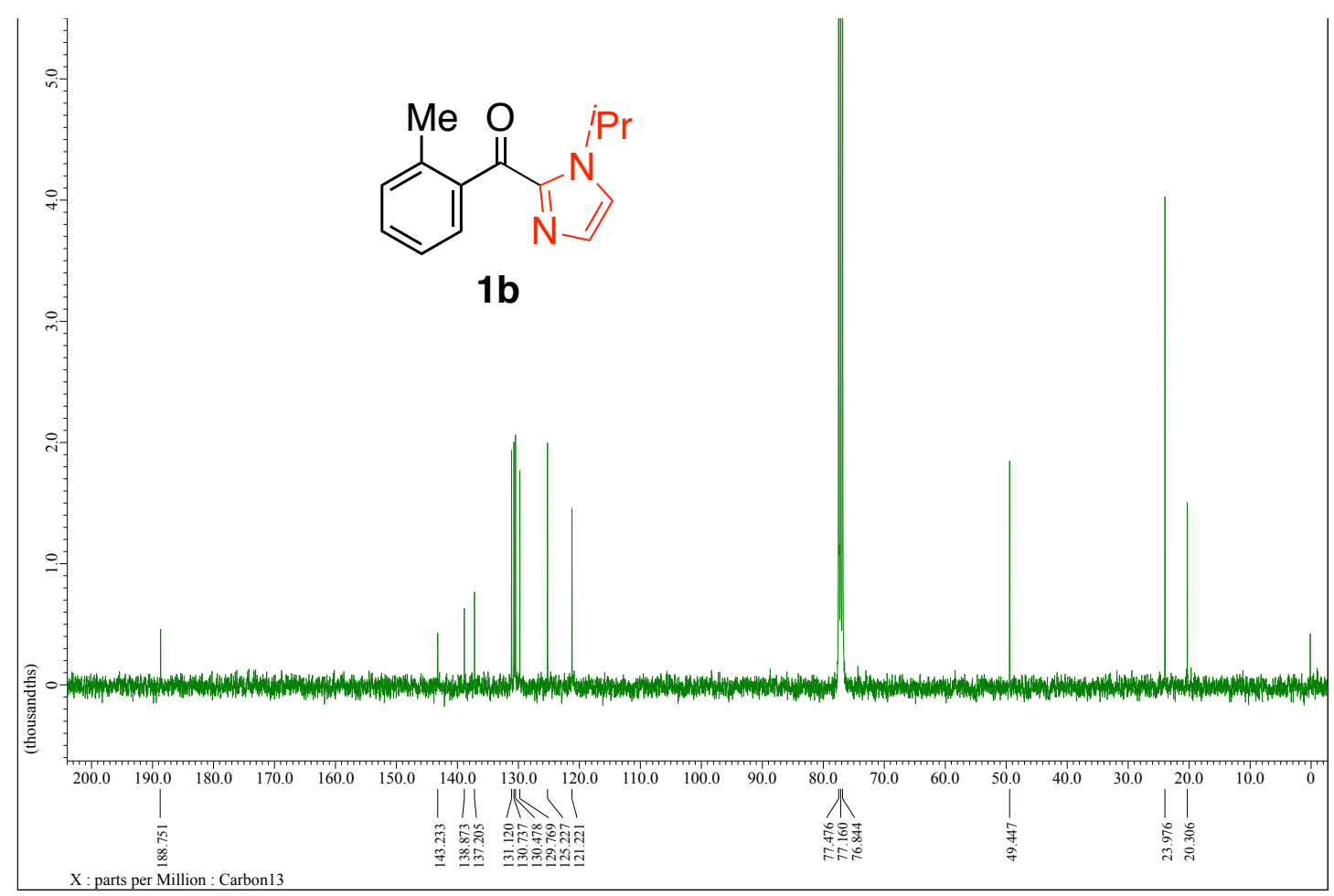

Figure S29: ${ }^{13} \mathrm{C}$ NMR spectrum of compound $\mathbf{1 b}\left(100 \mathrm{MHz}, \mathrm{CDCl}_{3}\right)$. 


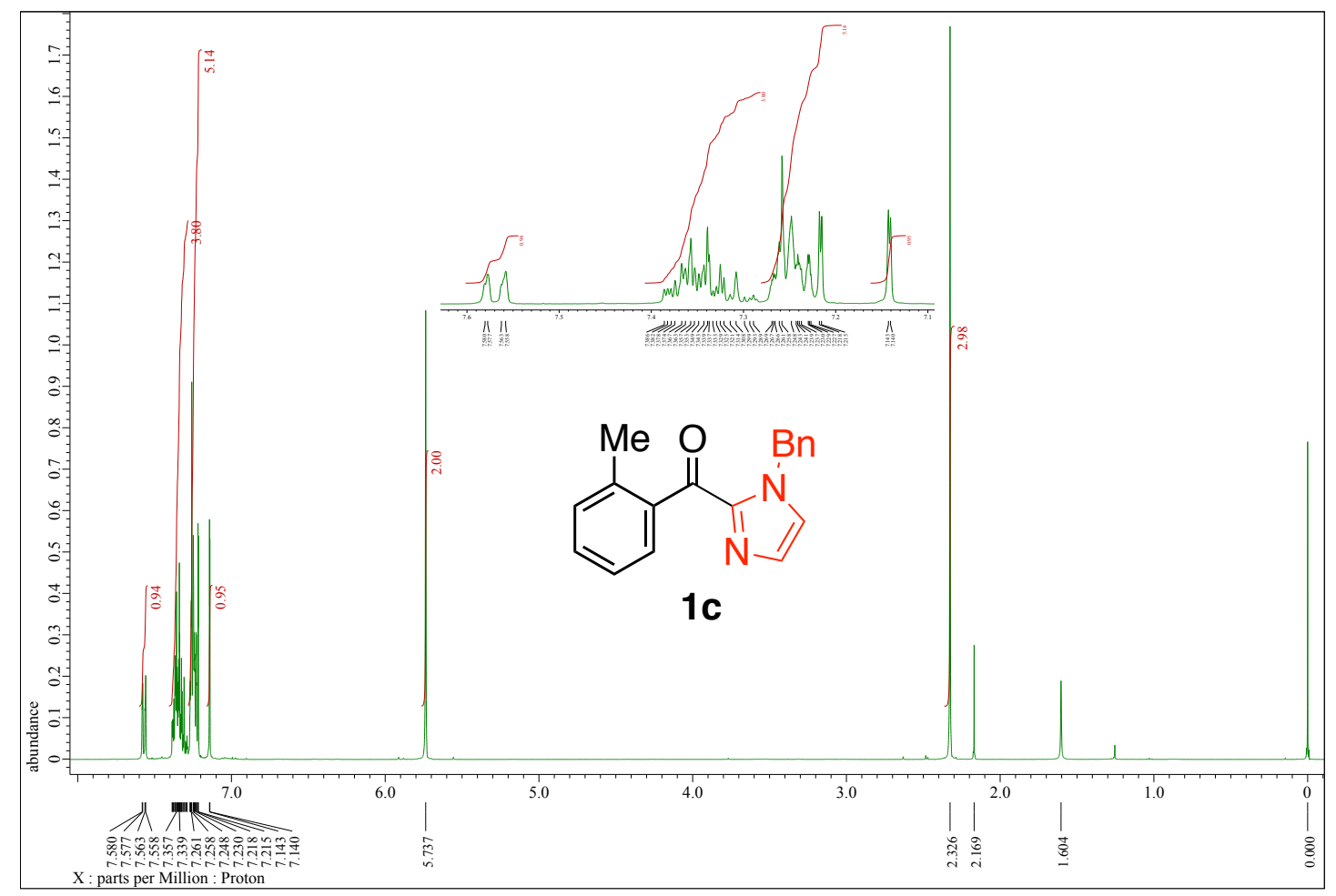

Figure S30: ${ }^{1} \mathrm{H}$ NMR spectrum of compound $1 \mathrm{c}\left(400 \mathrm{MHz}, \mathrm{CDCl}_{3}\right)$.

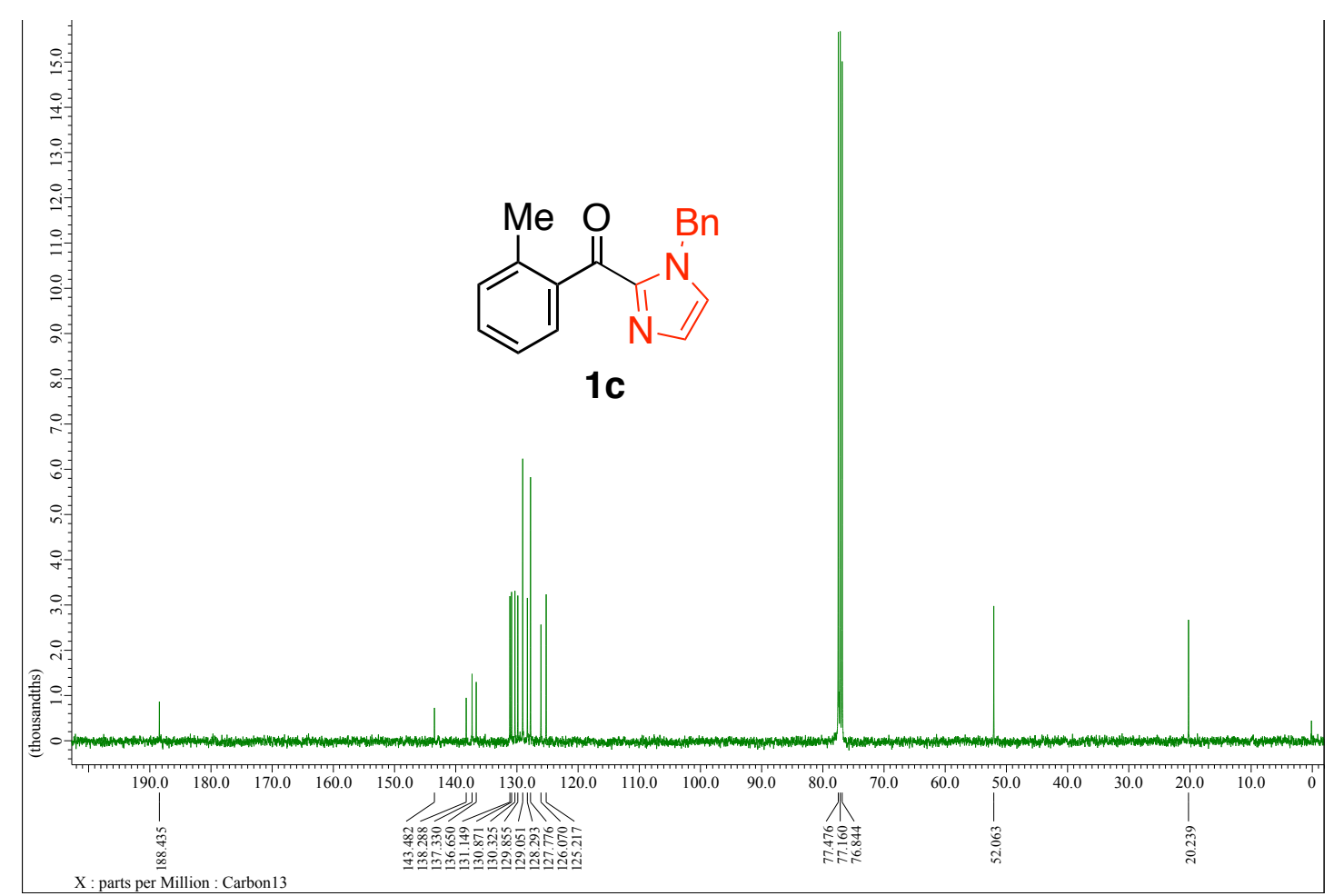

Figure S31: ${ }^{13} \mathrm{C}$ NMR spectrum of compound 1c $\left(100 \mathrm{MHz}, \mathrm{CDCl}_{3}\right)$. 


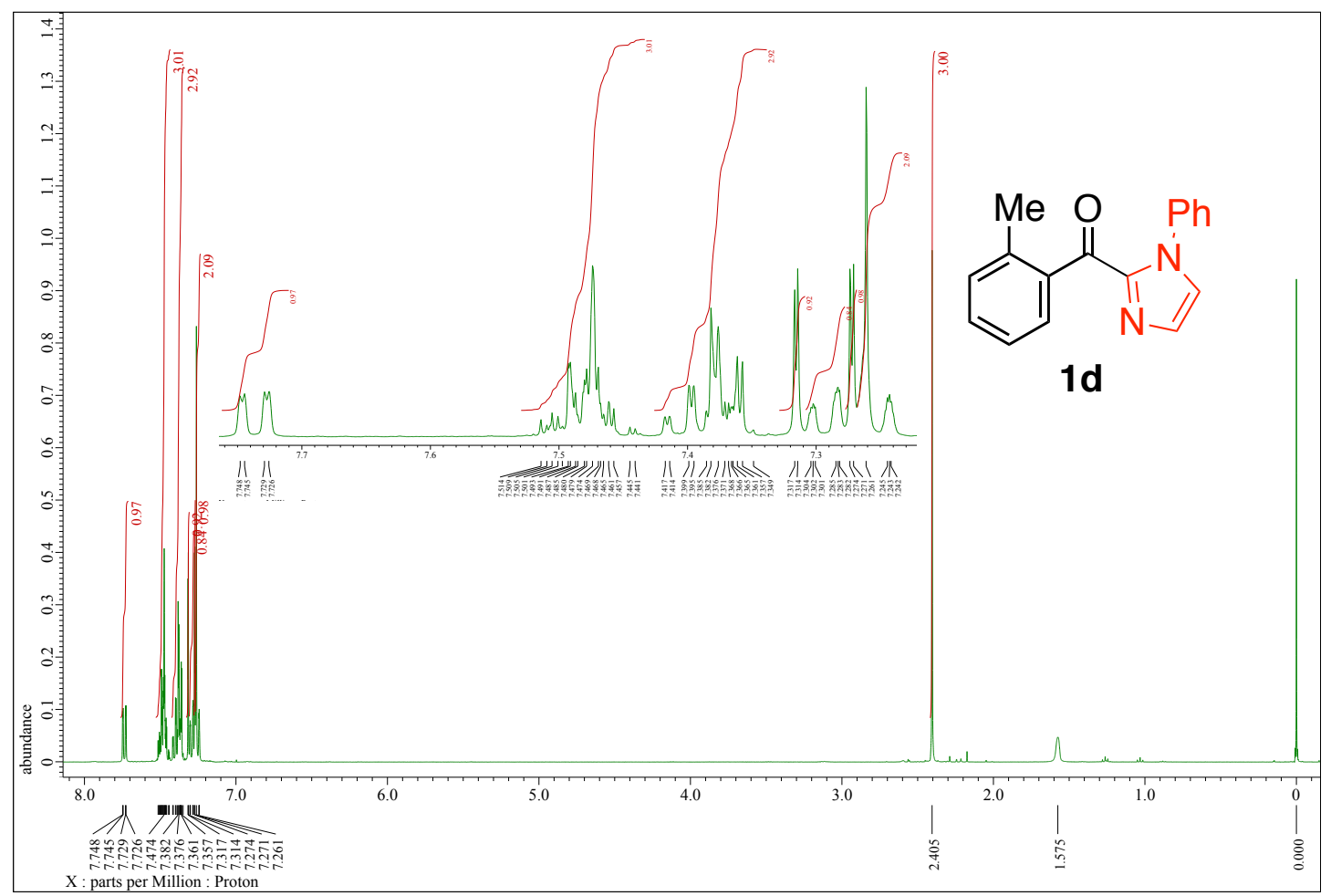

Figure S32: ${ }^{1} \mathrm{H}$ NMR spectrum of compound $1 \mathbf{d}\left(400 \mathrm{MHz}, \mathrm{CDCl}_{3}\right)$.

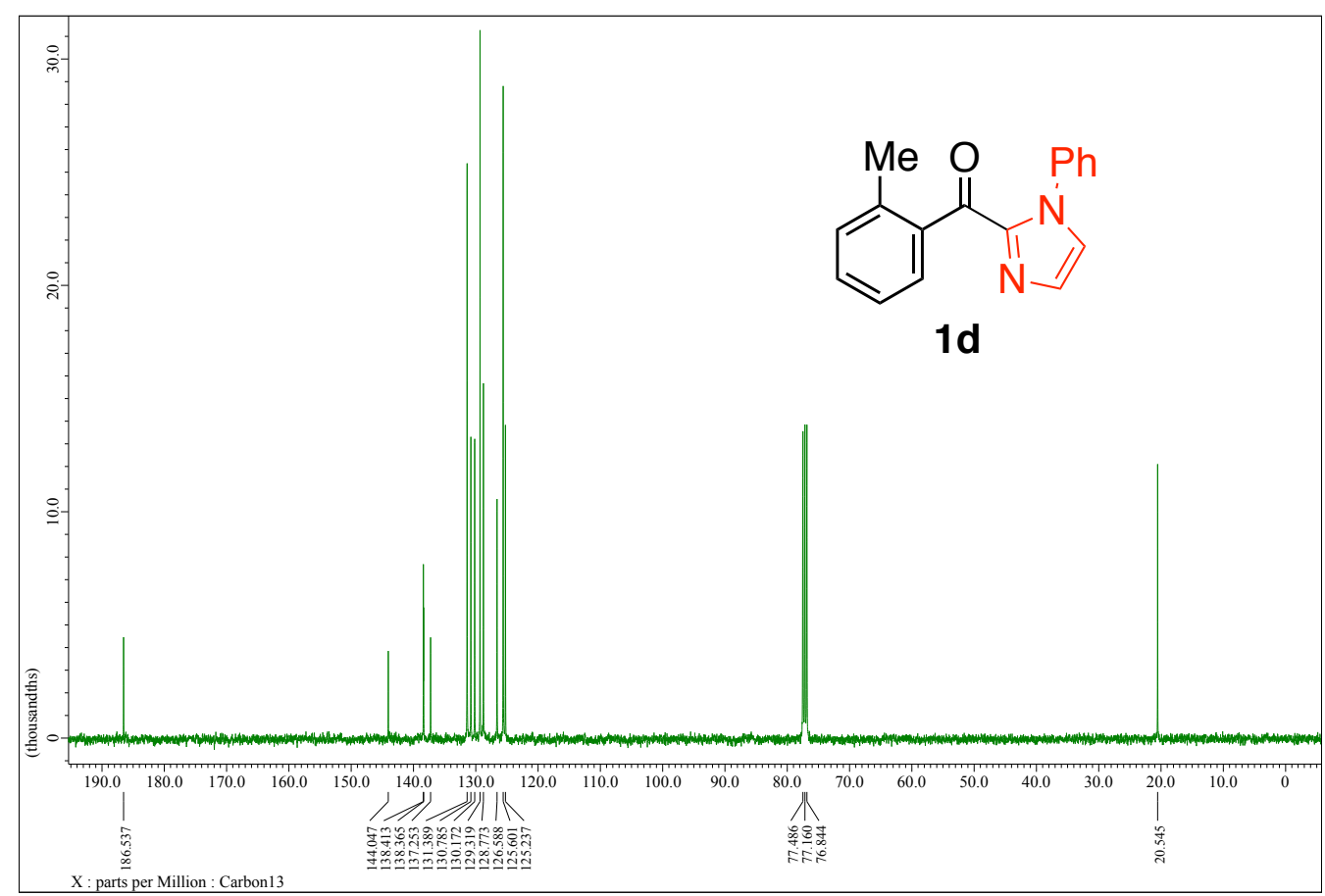

Figure S33: ${ }^{13} \mathrm{C}$ NMR spectrum of compound $1 \mathbf{d}\left(100 \mathrm{MHz}, \mathrm{CDCl}_{3}\right)$. 


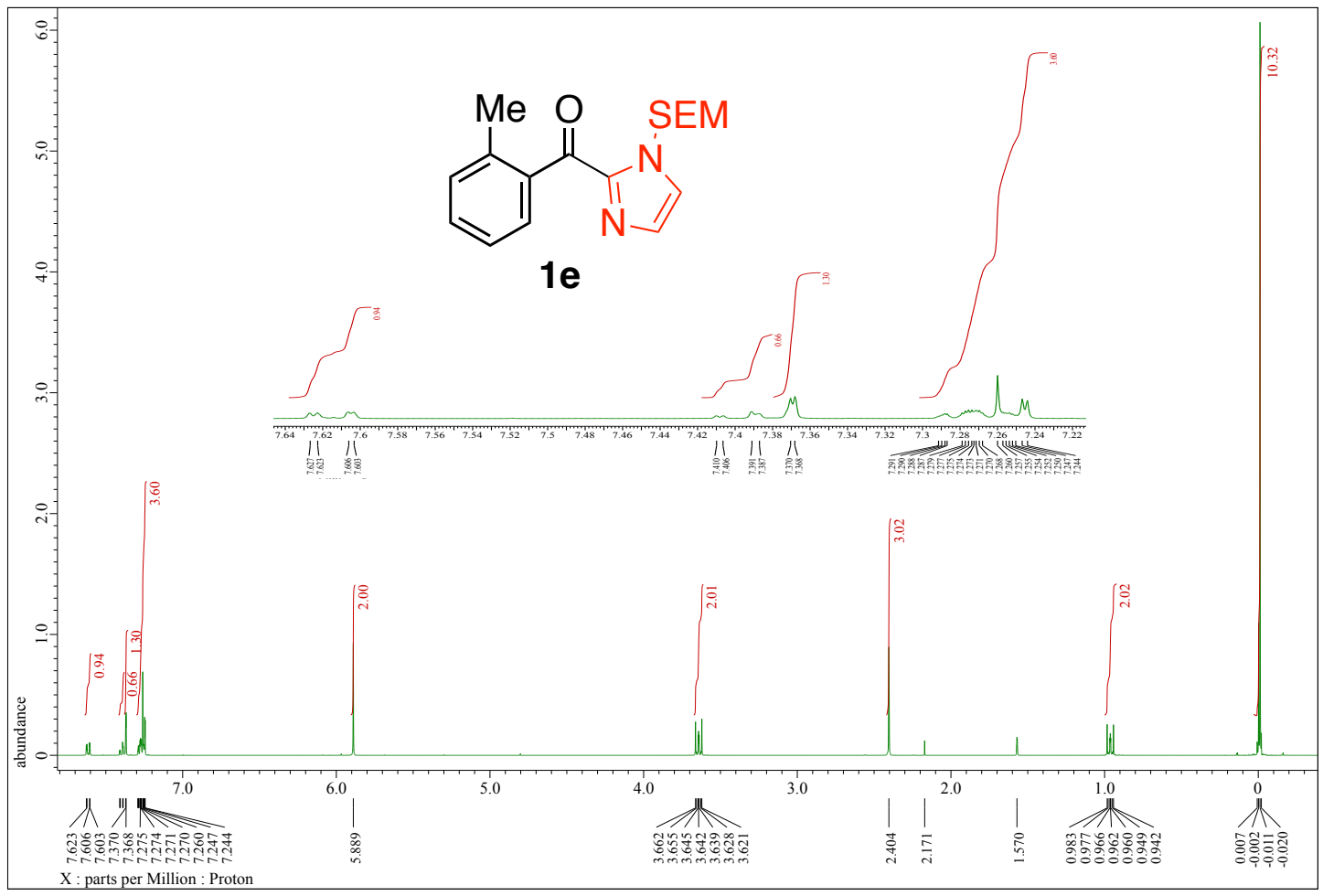

Figure S34: ${ }^{1} \mathrm{H}$ NMR spectrum of compound 1 e $\left(400 \mathrm{MHz}, \mathrm{CDCl}_{3}\right)$.

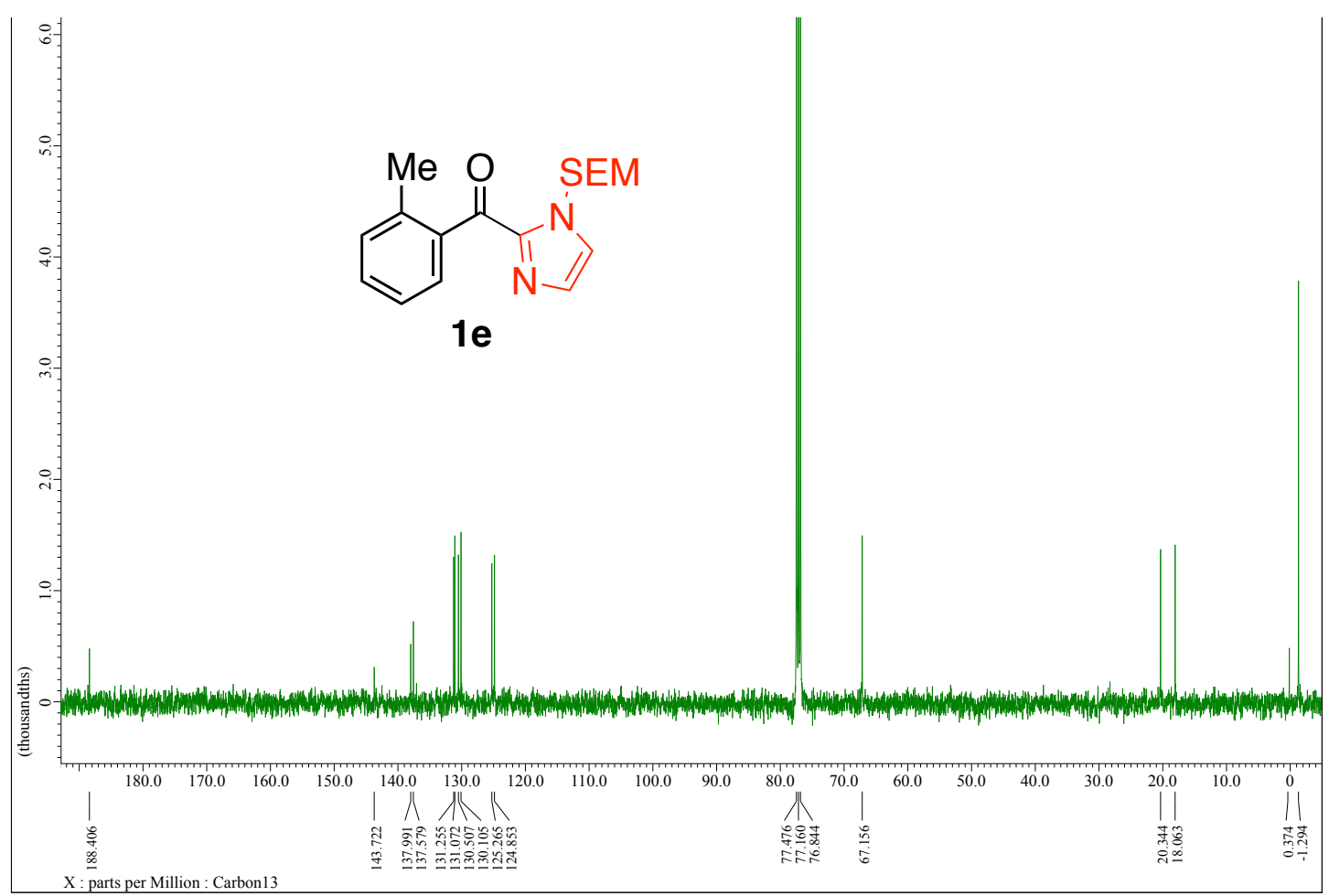

Figure S35: ${ }^{13} \mathrm{C}$ NMR spectrum of compound $1 \mathrm{e}\left(100 \mathrm{MHz}, \mathrm{CDCl}_{3}\right)$. 


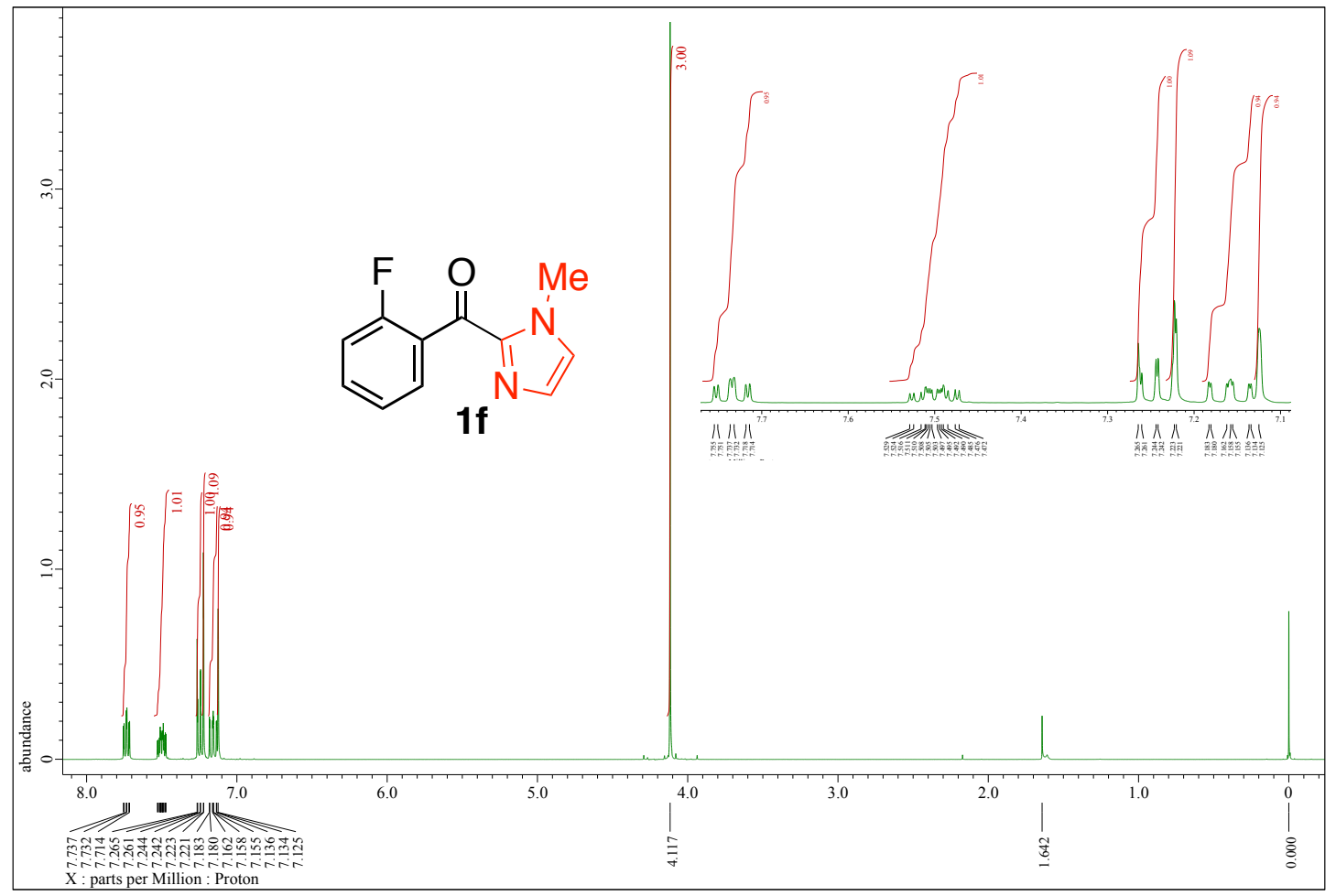

Figure S36: ${ }^{1} \mathrm{H}$ NMR spectrum of compound $1 f\left(400 \mathrm{MHz}, \mathrm{CDCl}_{3}\right)$.



Figure S37: ${ }^{13} \mathrm{C}$ NMR spectrum of compound $1 f\left(100 \mathrm{MHz}, \mathrm{CDCl}_{3}\right)$. 


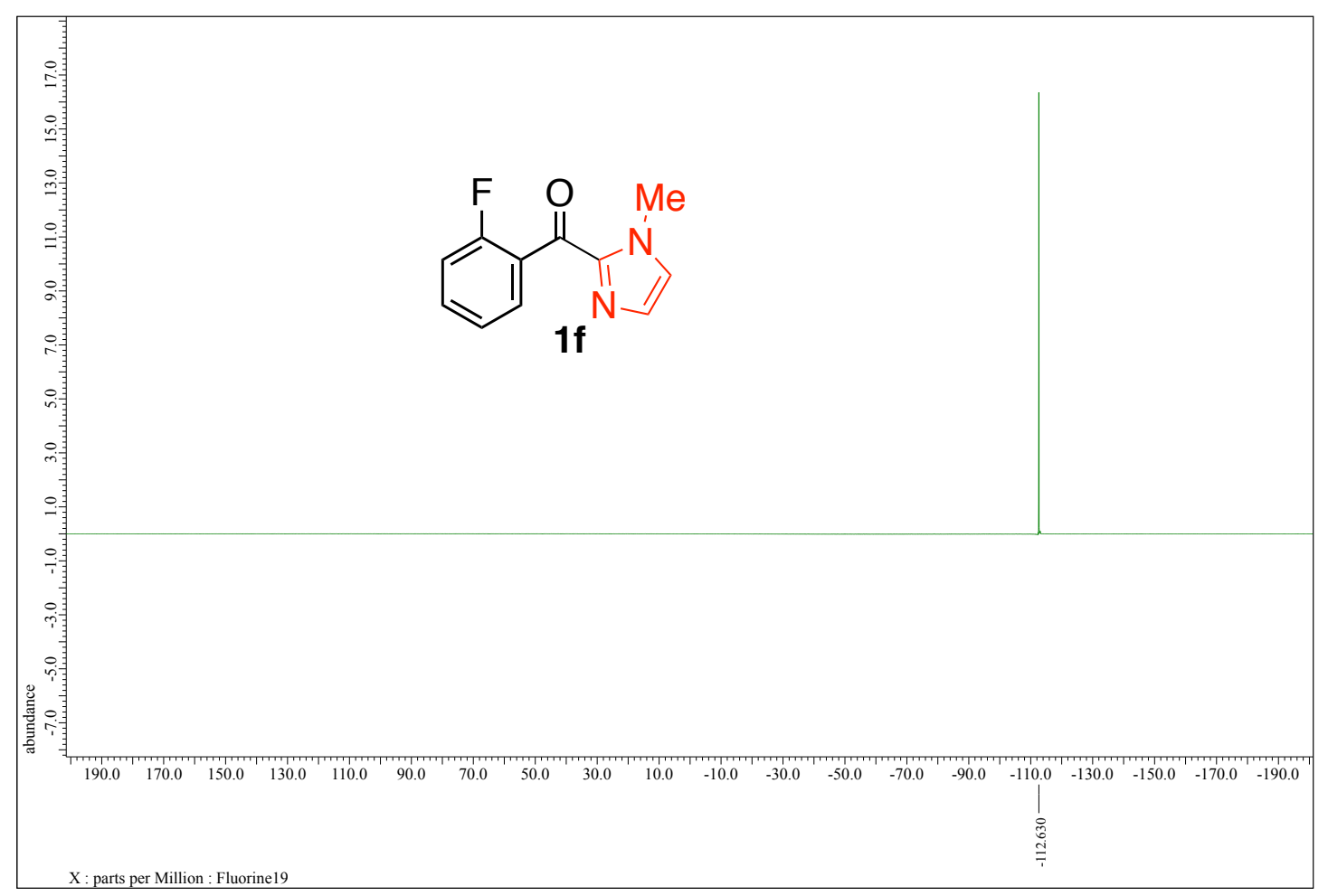

Figure S38: ${ }^{19} \mathrm{~F}$ NMR spectrum of compound 1 (f $\left(376 \mathrm{MHz}, \mathrm{CDCl}_{3}\right)$.

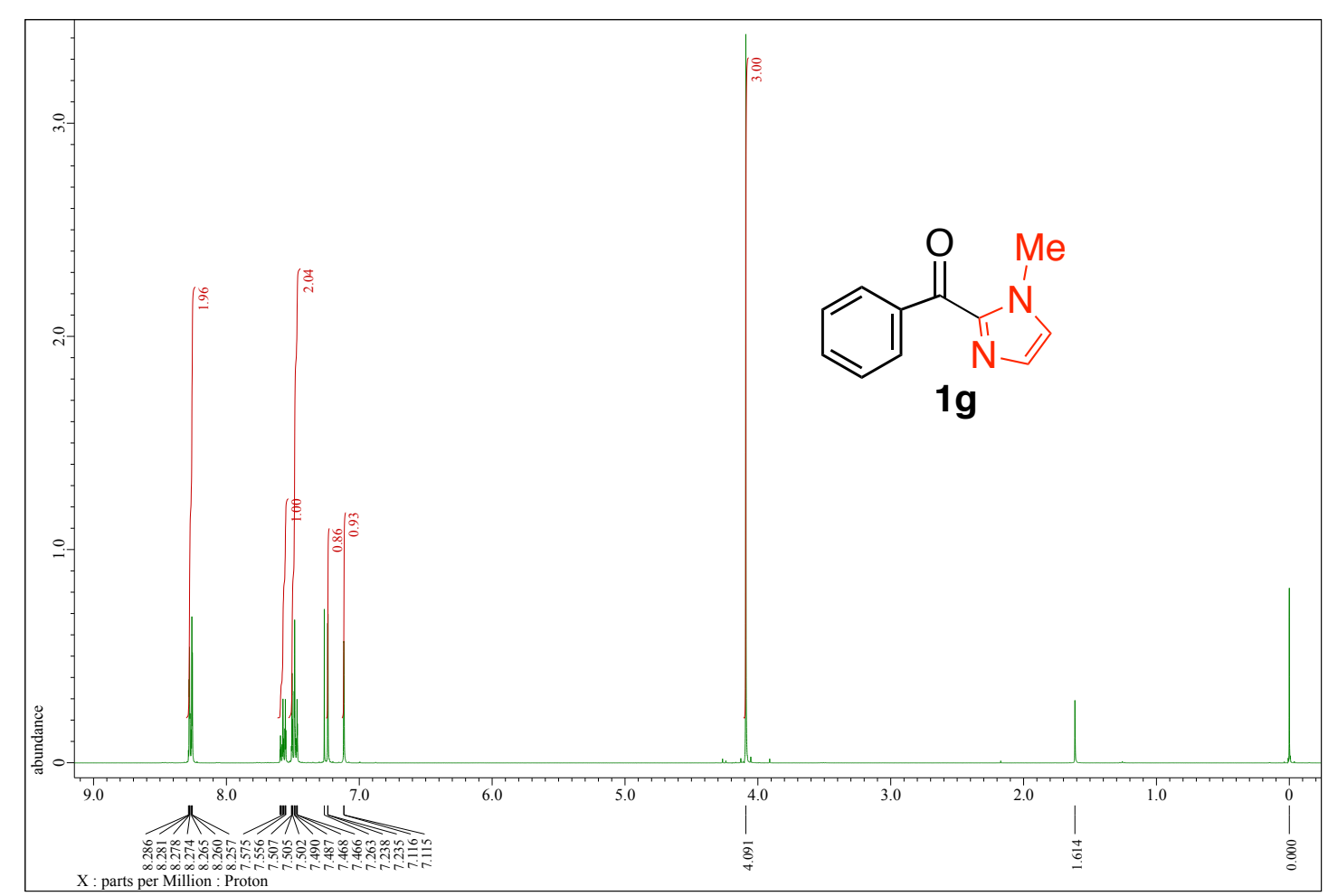

Figure S39: ${ }^{1} \mathrm{H}$ NMR spectrum of compound $1 \mathrm{~g}\left(400 \mathrm{MHz}, \mathrm{CDCl}_{3}\right)$. 


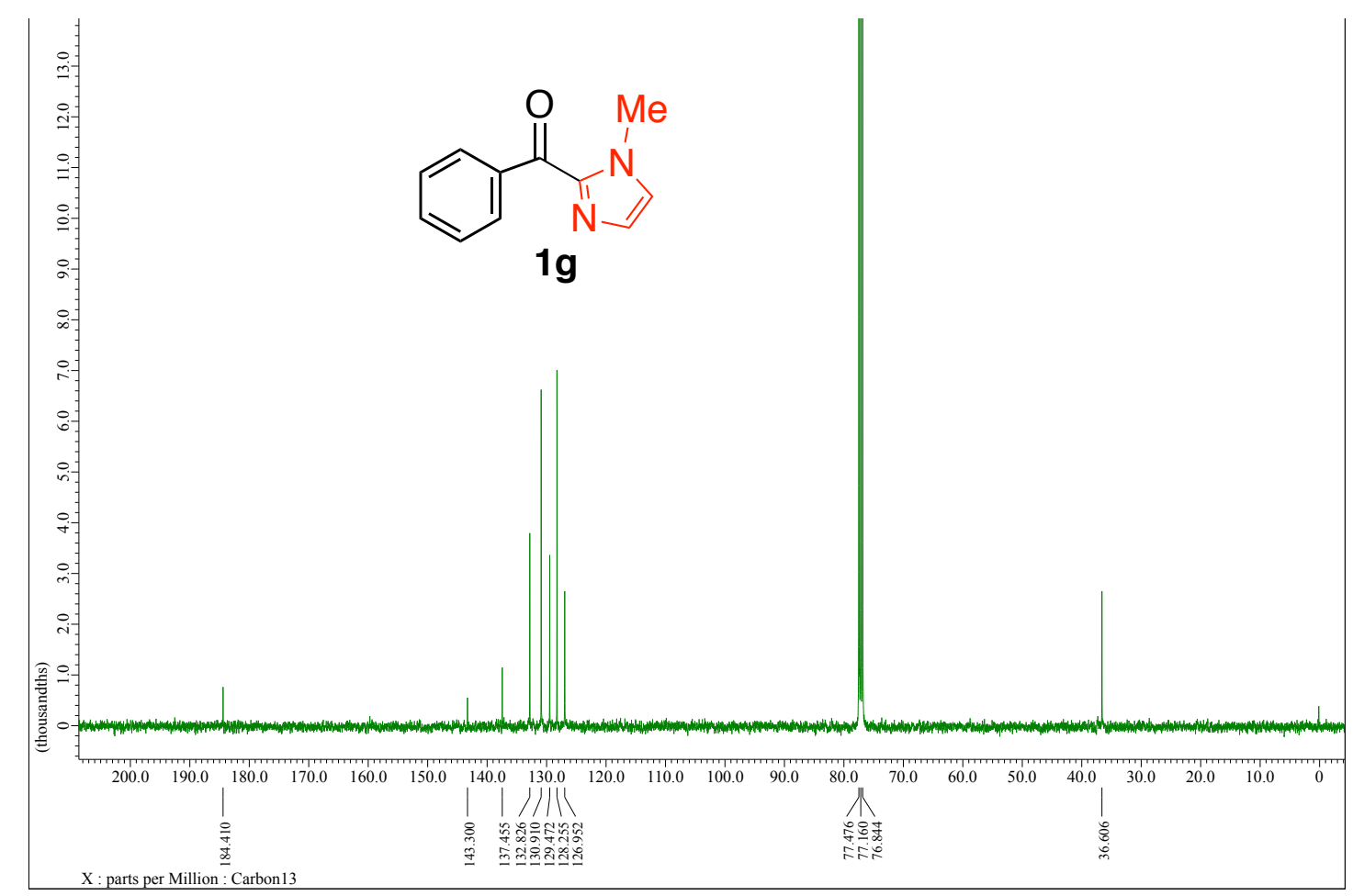

Figure S40: ${ }^{13} \mathrm{C}$ NMR spectrum of compound 1 g $\left(100 \mathrm{MHz}, \mathrm{CDCl}_{3}\right)$.

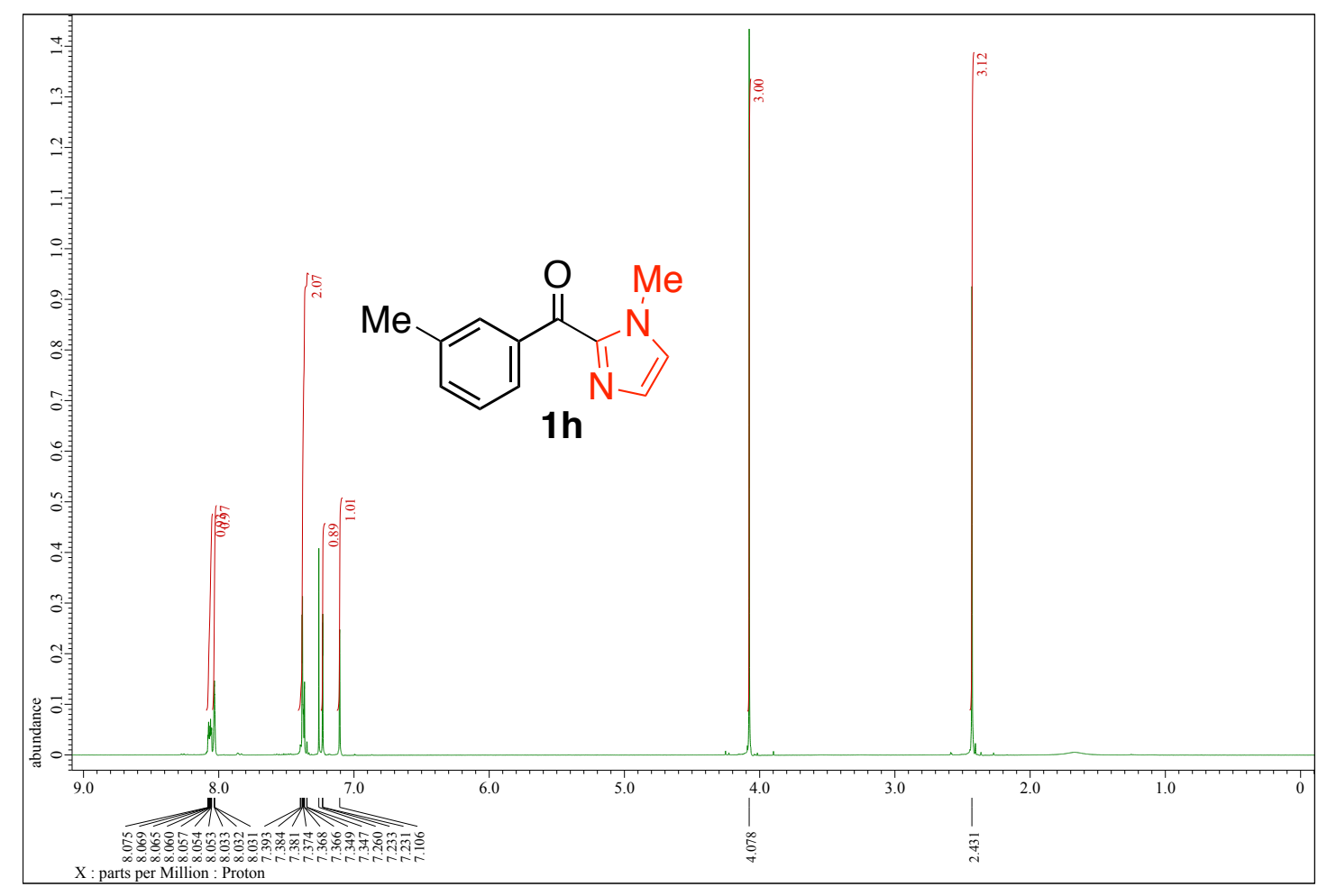

Figure S41: ${ }^{1} \mathrm{H}$ NMR spectrum of compound $\mathbf{1 h}\left(400 \mathrm{MHz}, \mathrm{CDCl}_{3}\right)$. 


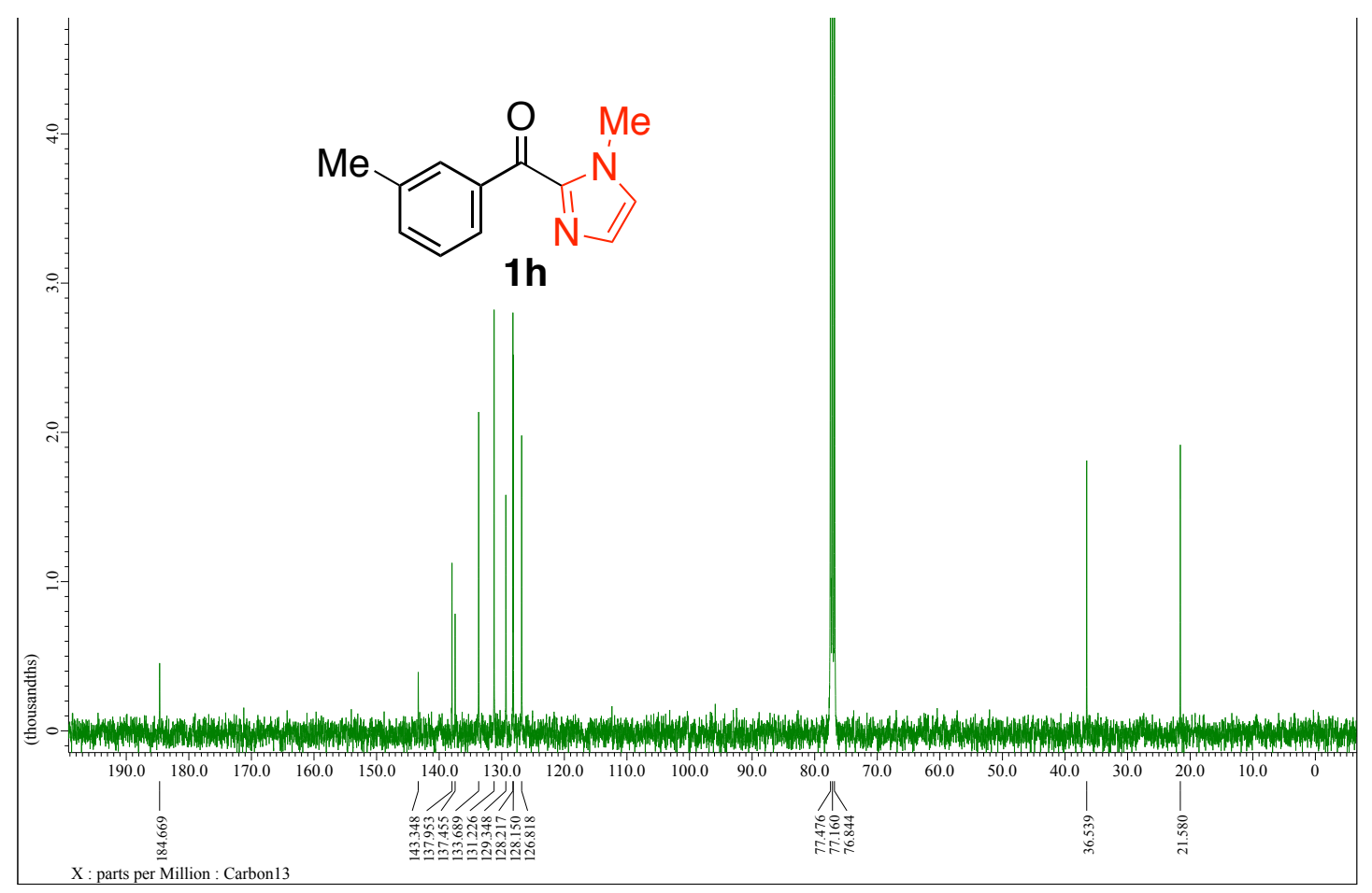

Figure S42: ${ }^{13} \mathrm{C}$ NMR spectrum of compound $\mathbf{1 h}\left(100 \mathrm{MHz}, \mathrm{CDCl}_{3}\right)$.

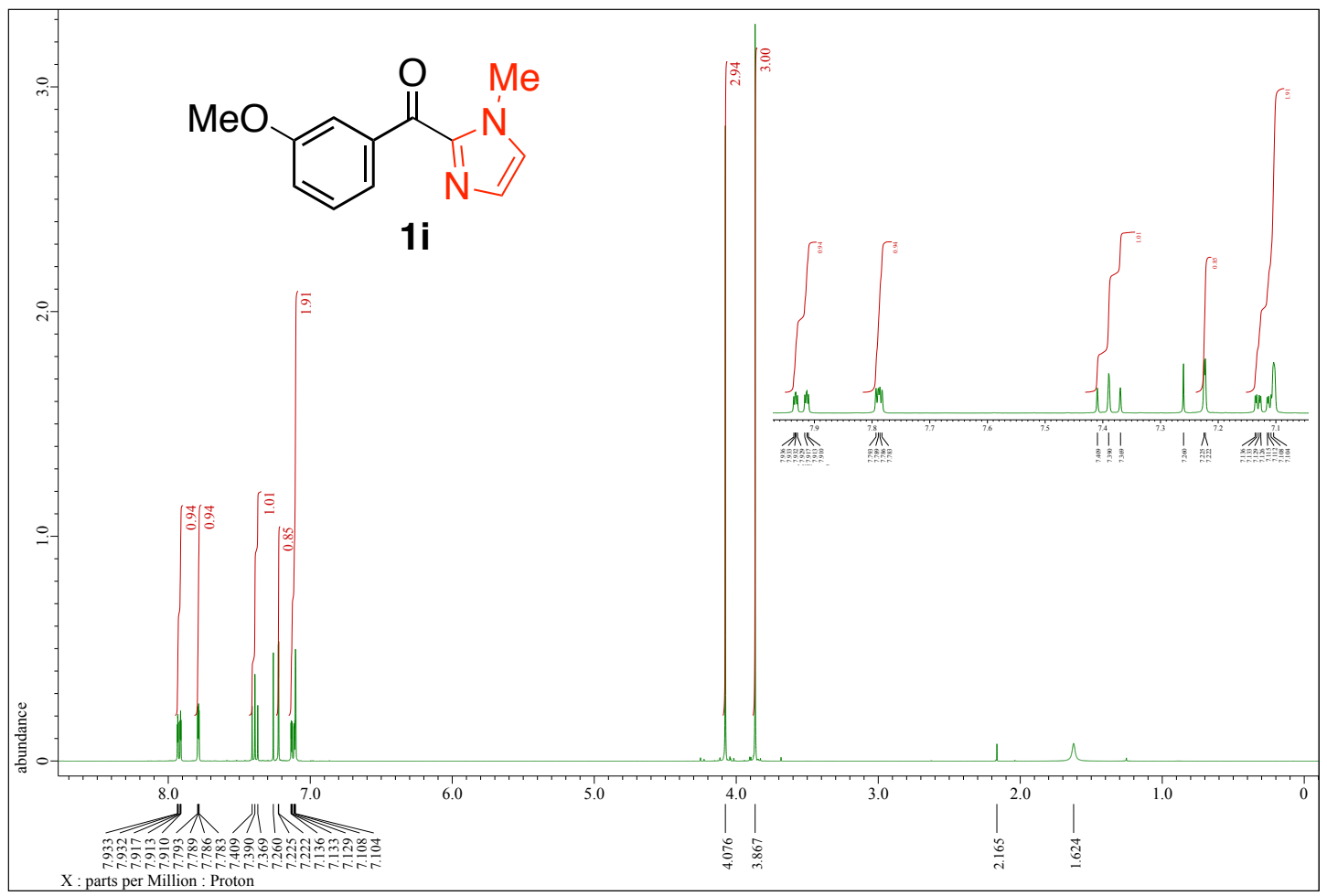

Figure S43: ${ }^{1} \mathrm{H}$ NMR spectrum of compound $1 \mathbf{i}\left(400 \mathrm{MHz}, \mathrm{CDCl}_{3}\right)$. 


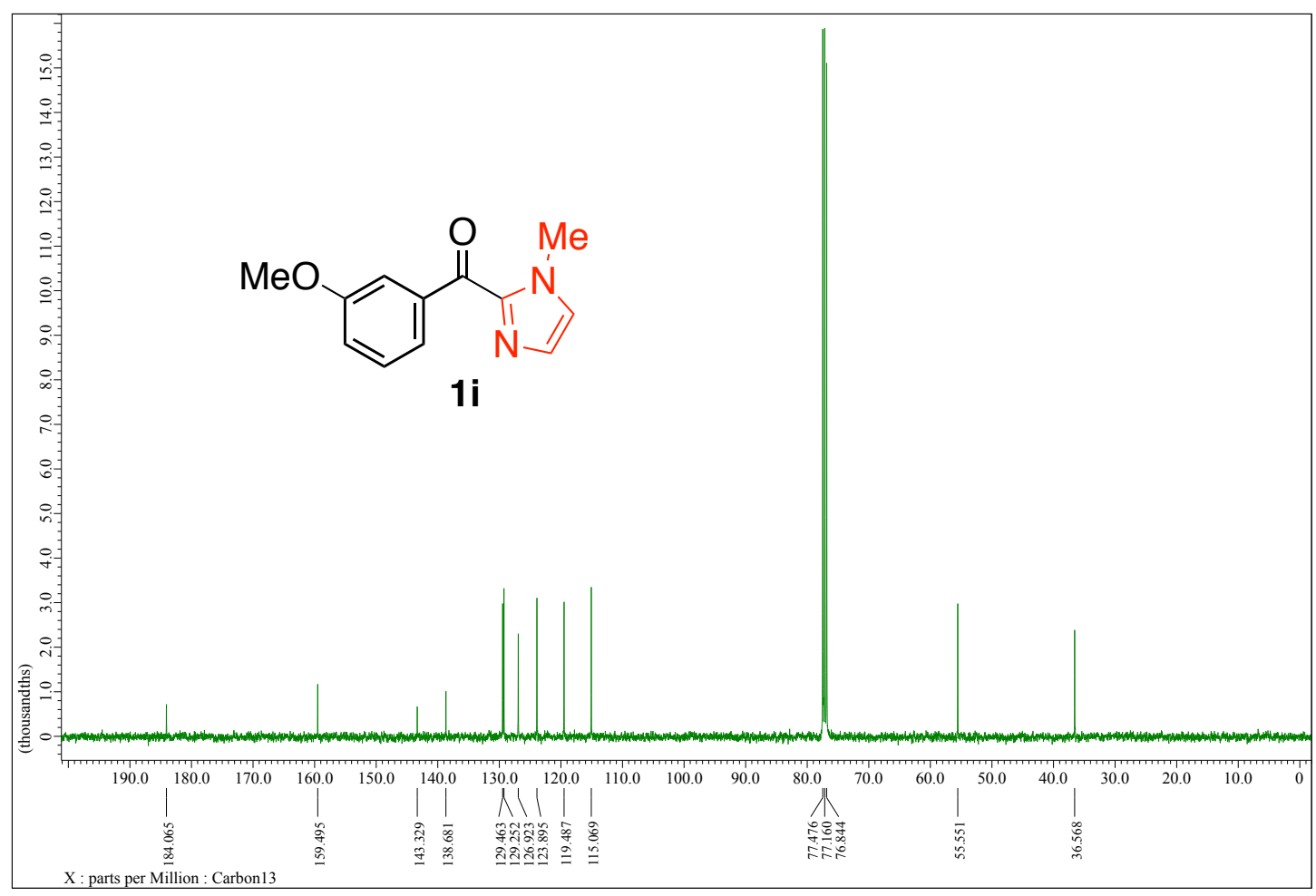

Figure S44: ${ }^{13} \mathrm{C}$ NMR spectrum of compound $1 \mathbf{i}\left(100 \mathrm{MHz}, \mathrm{CDCl}_{3}\right)$.

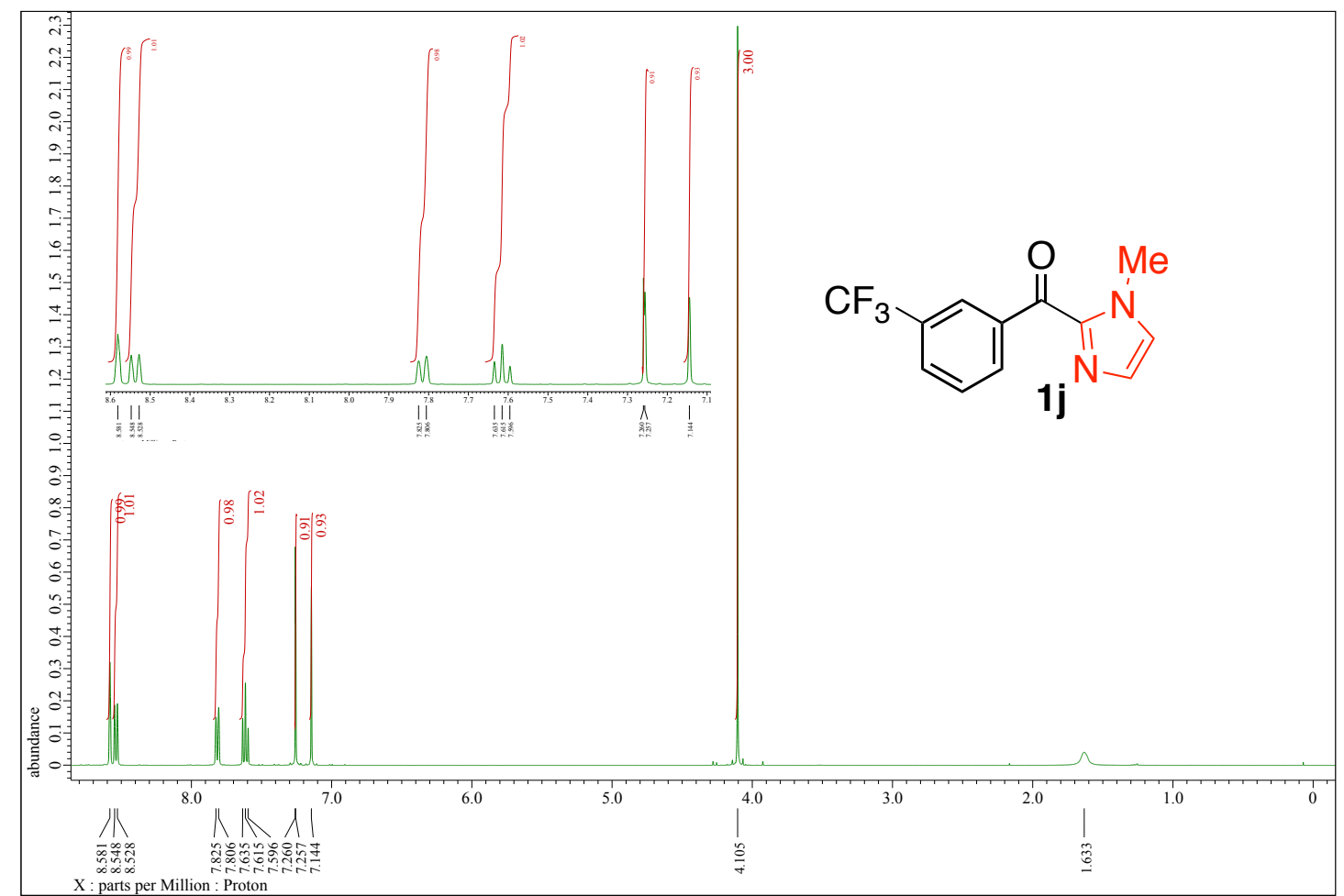

Figure S45: ${ }^{1} \mathrm{H}$ NMR spectrum of compound $\mathbf{1 j}\left(400 \mathrm{MHz}, \mathrm{CDCl}_{3}\right)$. 


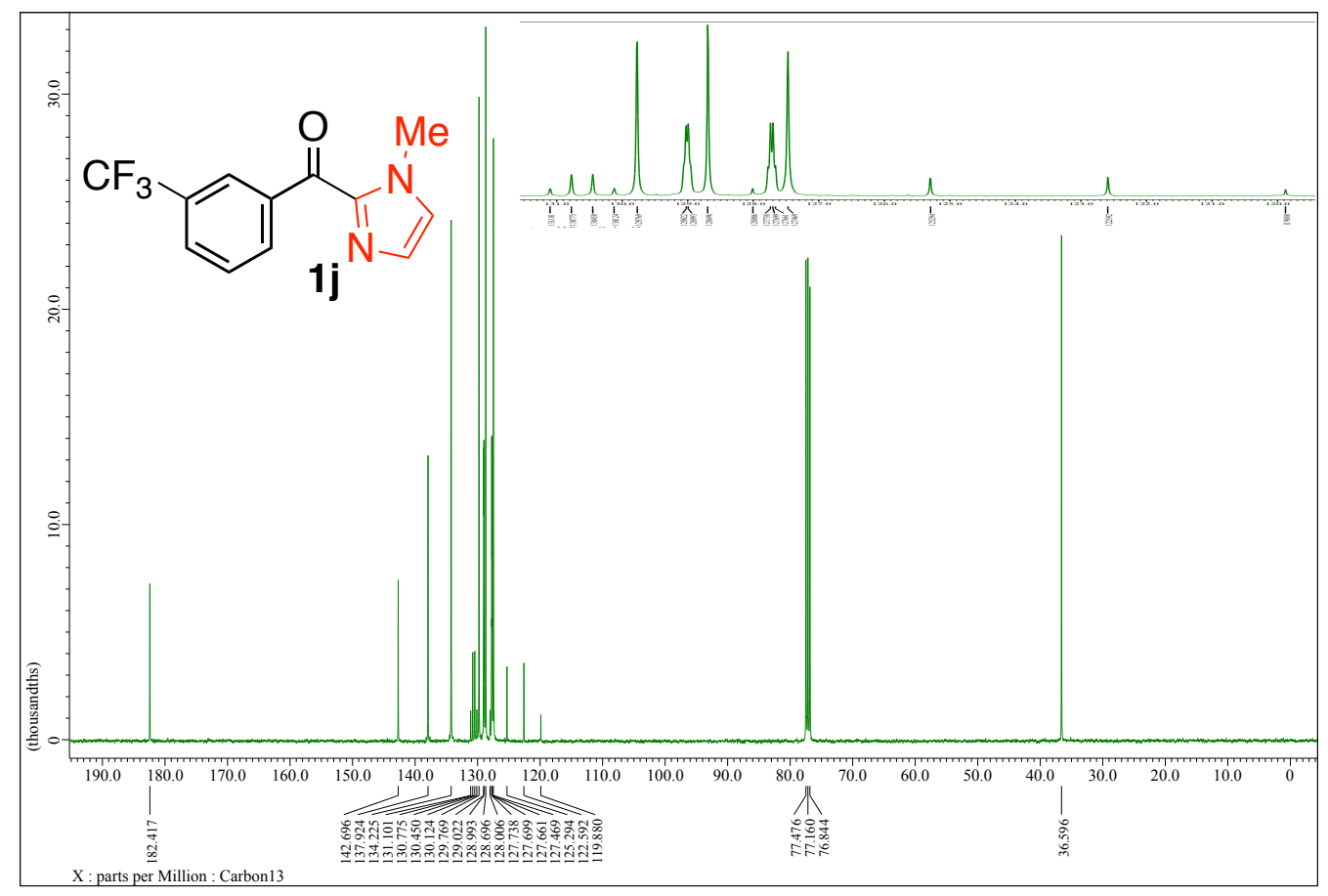

Figure S46: ${ }^{13} \mathrm{C}$ NMR spectrum of compound $\mathbf{1 j}\left(100 \mathrm{MHz}, \mathrm{CDCl}_{3}\right)$.

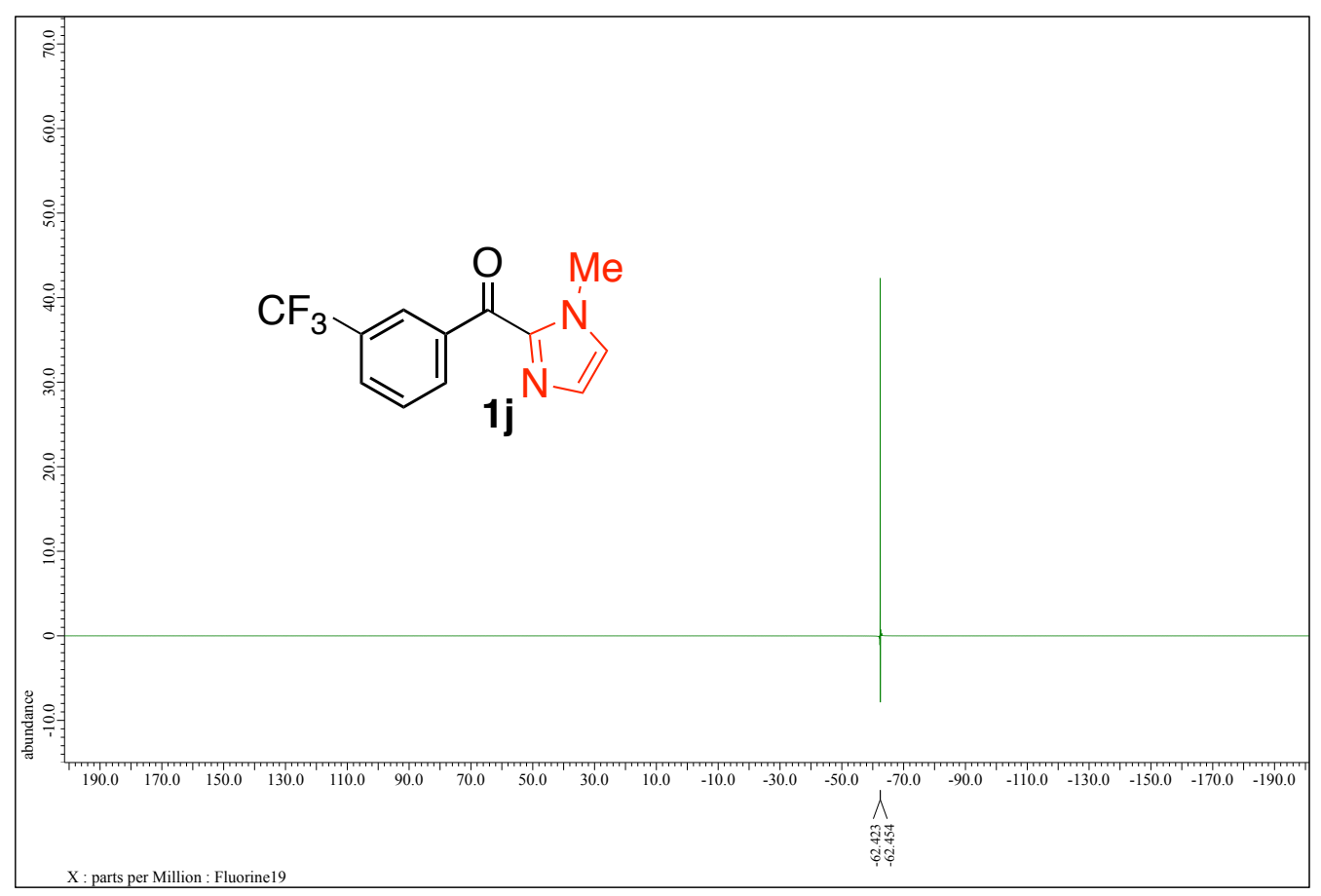

Figure S47: ${ }^{19} \mathrm{~F}$ NMR spectrum of compound $\mathbf{1 j}\left(376 \mathrm{MHz}, \mathrm{CDCl}_{3}\right)$. 


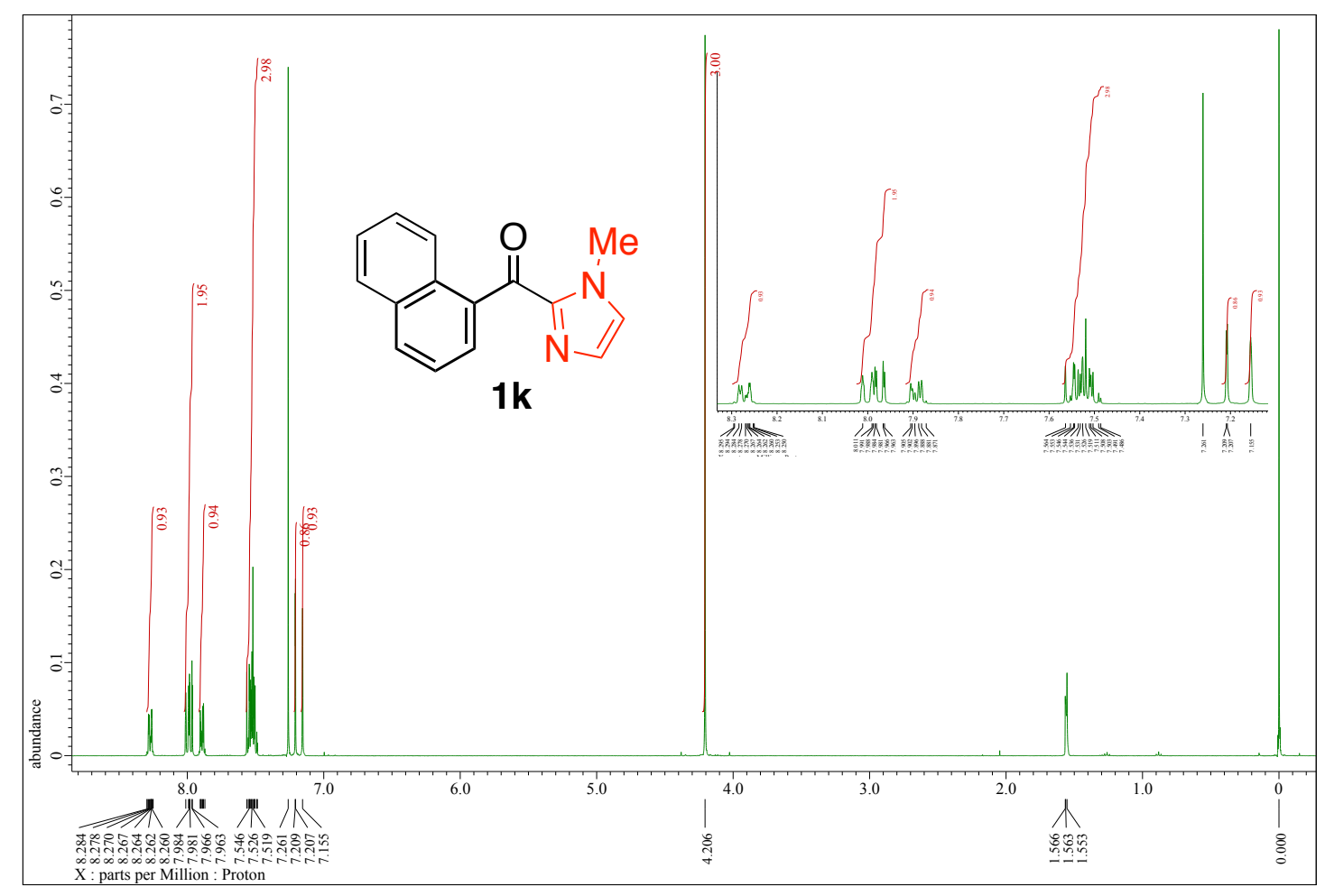

Figure S48: ${ }^{1} \mathrm{H}$ NMR spectrum of compound $1 \mathbf{k}\left(400 \mathrm{MHz}, \mathrm{CDCl}_{3}\right)$.

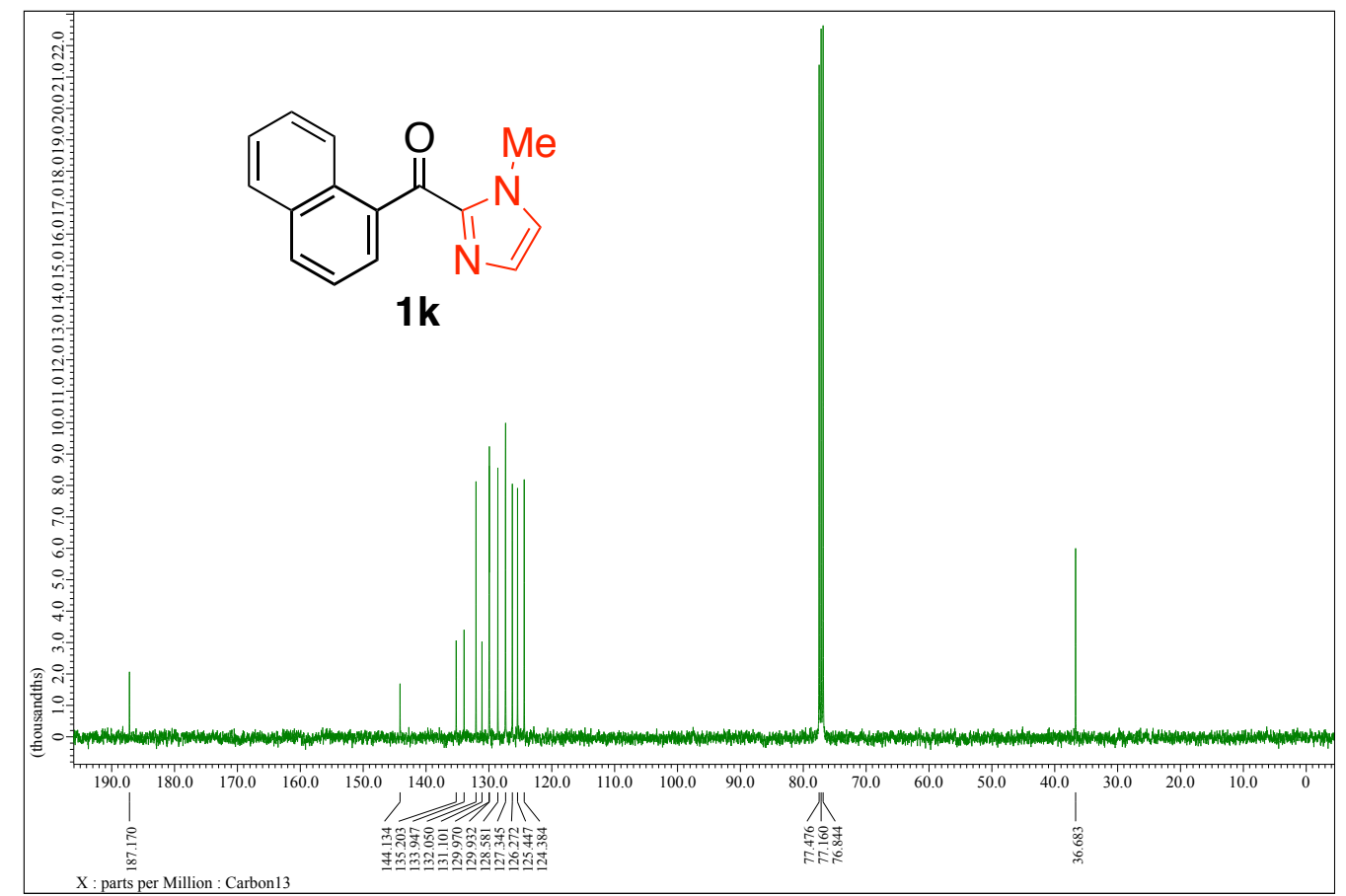

Figure S49: ${ }^{13} \mathrm{C}$ NMR spectrum of compound $1 \mathbf{k}\left(100 \mathrm{MHz}, \mathrm{CDCl}_{3}\right)$. 


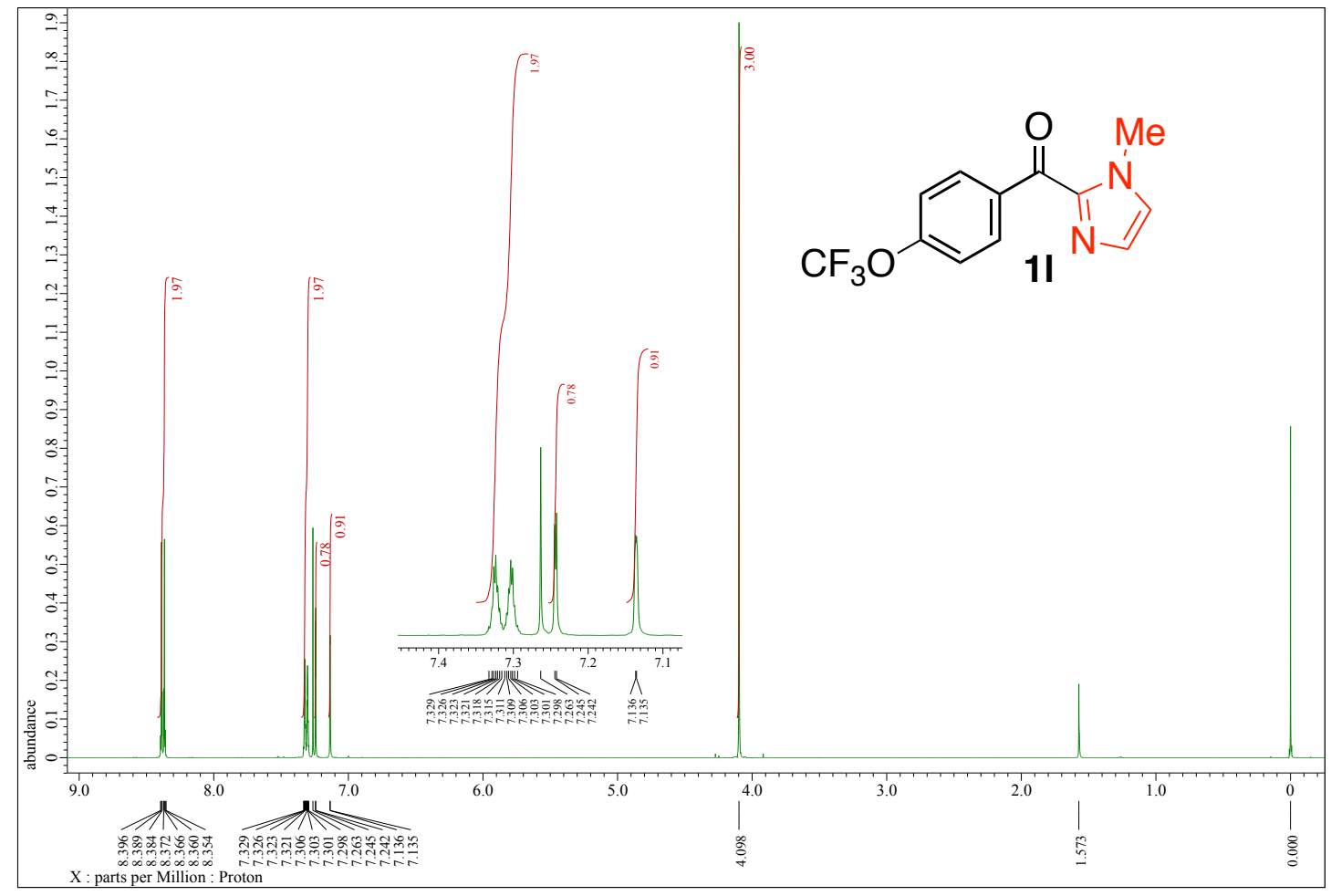

Figure S50: ${ }^{1} \mathrm{H}$ NMR spectrum of compound 11 (400 MHz, $\left.\mathrm{CDCl}_{3}\right)$.

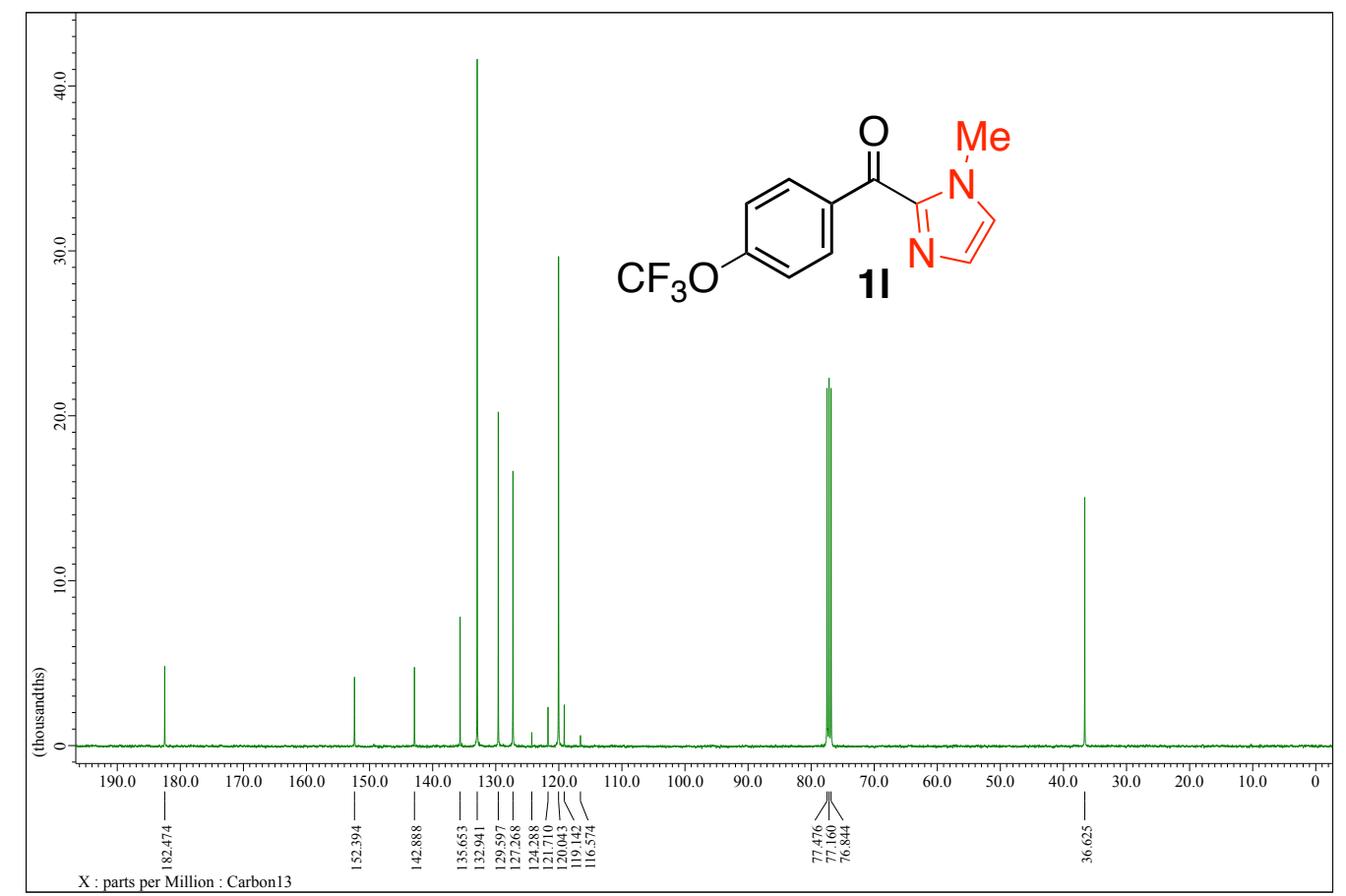

Figure S51: ${ }^{13} \mathrm{C}$ NMR spectrum of compound $11\left(100 \mathrm{MHz}, \mathrm{CDCl}_{3}\right)$. 


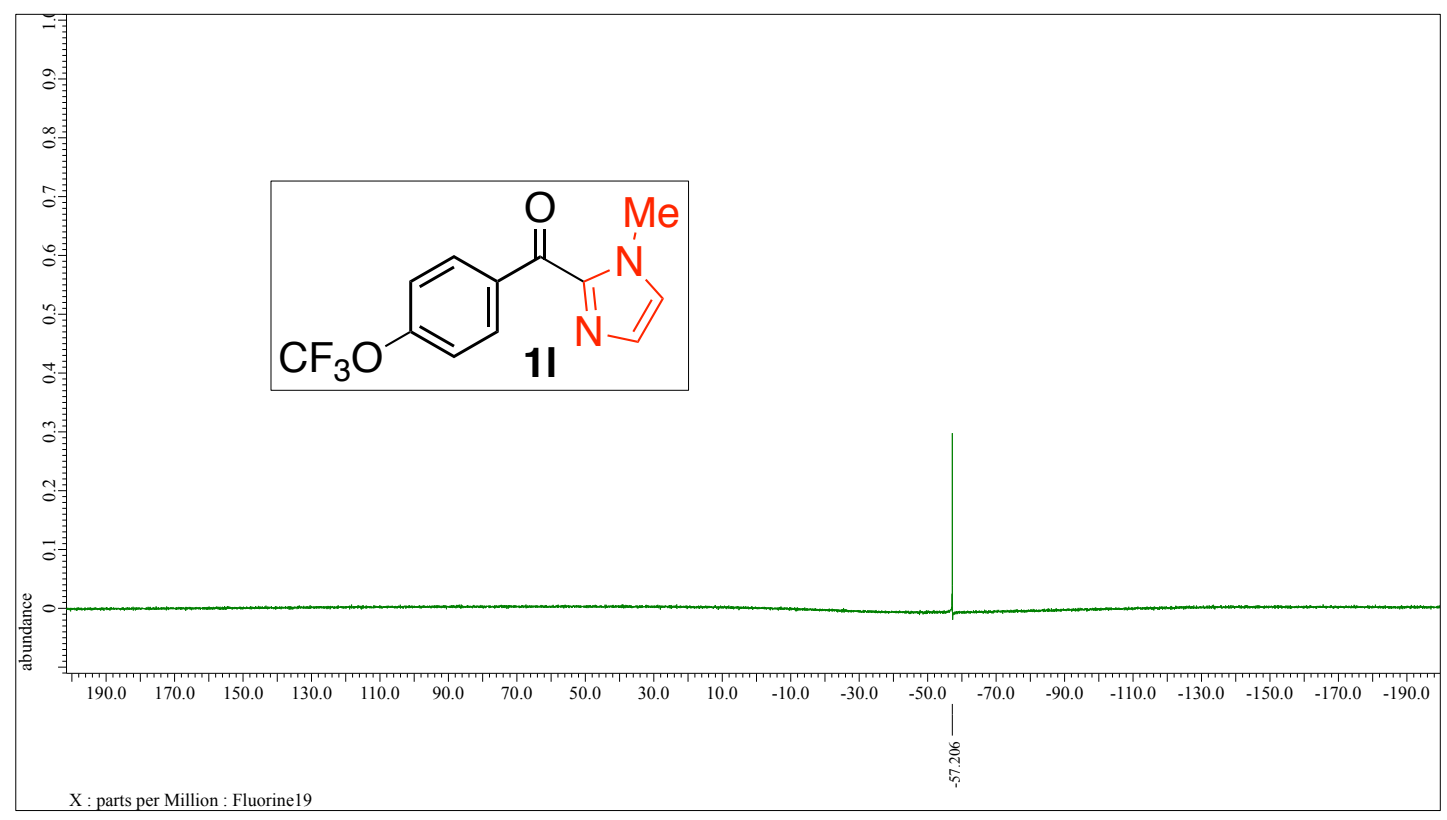

Figure S52: ${ }^{19} \mathrm{~F}$ NMR spectrum of compound 11 (376 $\left.\mathrm{MHz}, \mathrm{CDCl}_{3}\right)$.

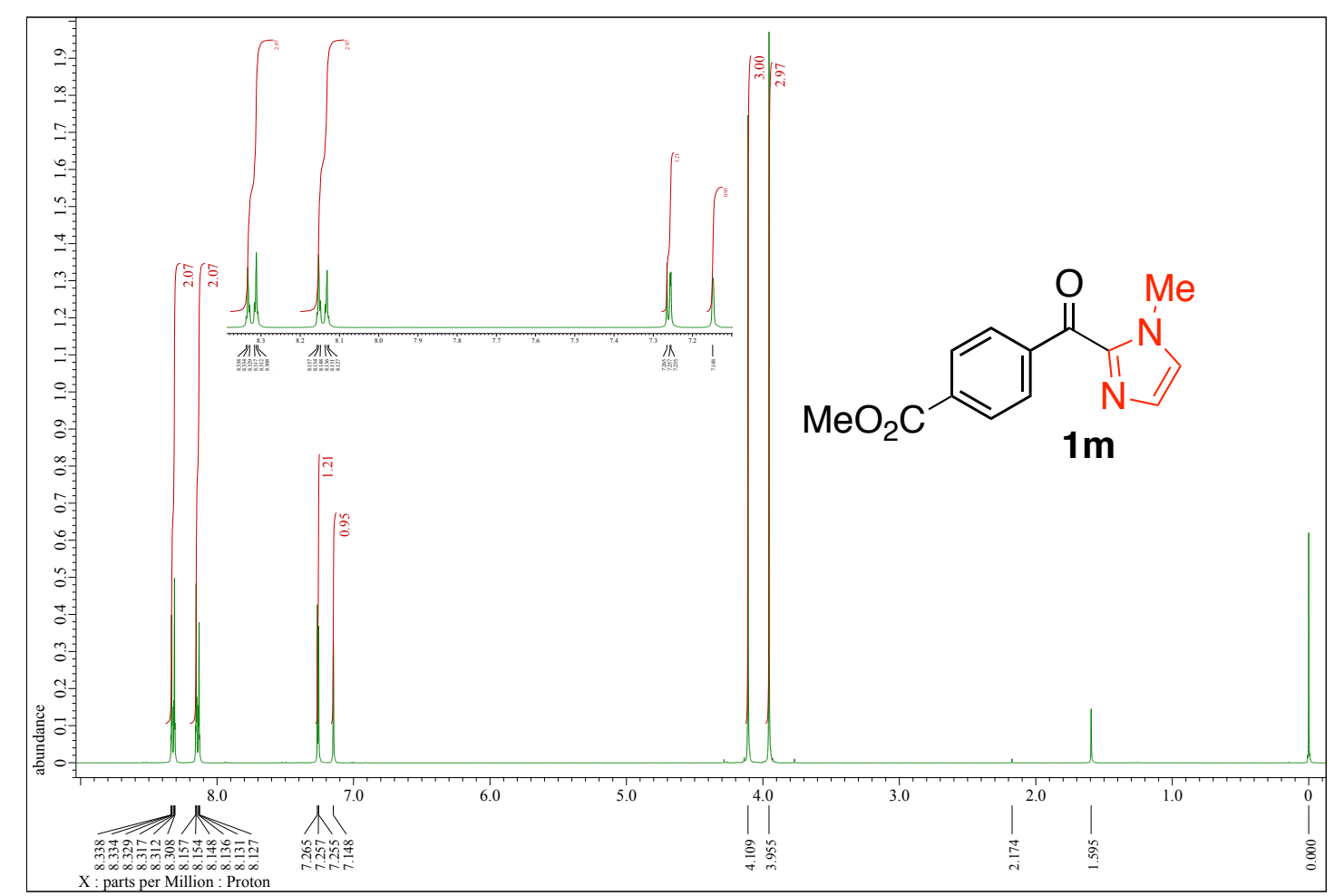

Figure S53: ${ }^{1} \mathrm{H}$ NMR spectrum of compound $1 \mathbf{m}\left(400 \mathrm{MHz}, \mathrm{CDCl}_{3}\right)$. 


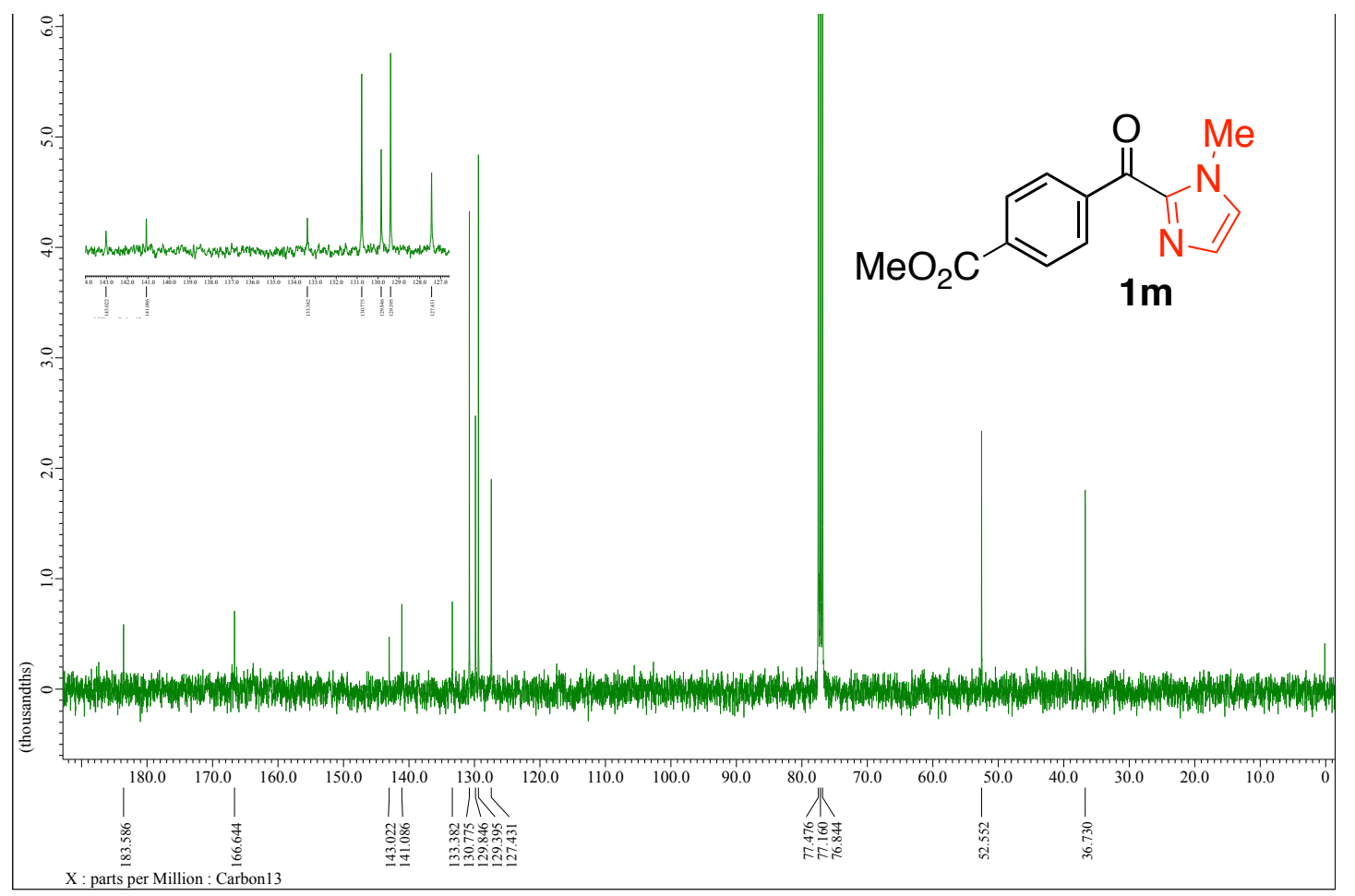

Figure S54: ${ }^{13} \mathrm{C}$ NMR spectrum of compound 1 m $\left(100 \mathrm{MHz}, \mathrm{CDCl}_{3}\right)$.

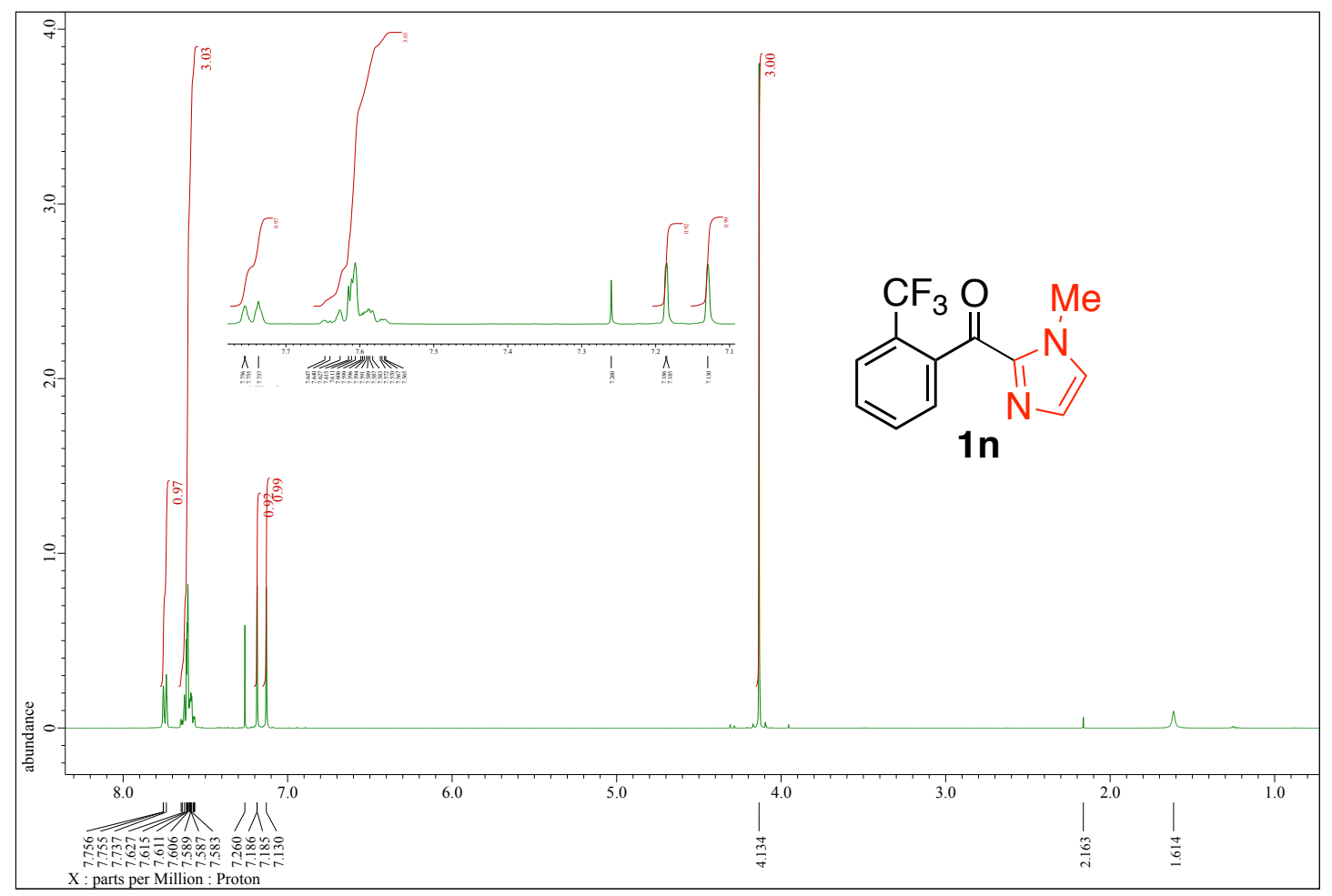

Figure S55: ${ }^{1} \mathrm{H}$ NMR spectrum of compound 1 n $\left(400 \mathrm{MHz}, \mathrm{CDCl}_{3}\right)$. 


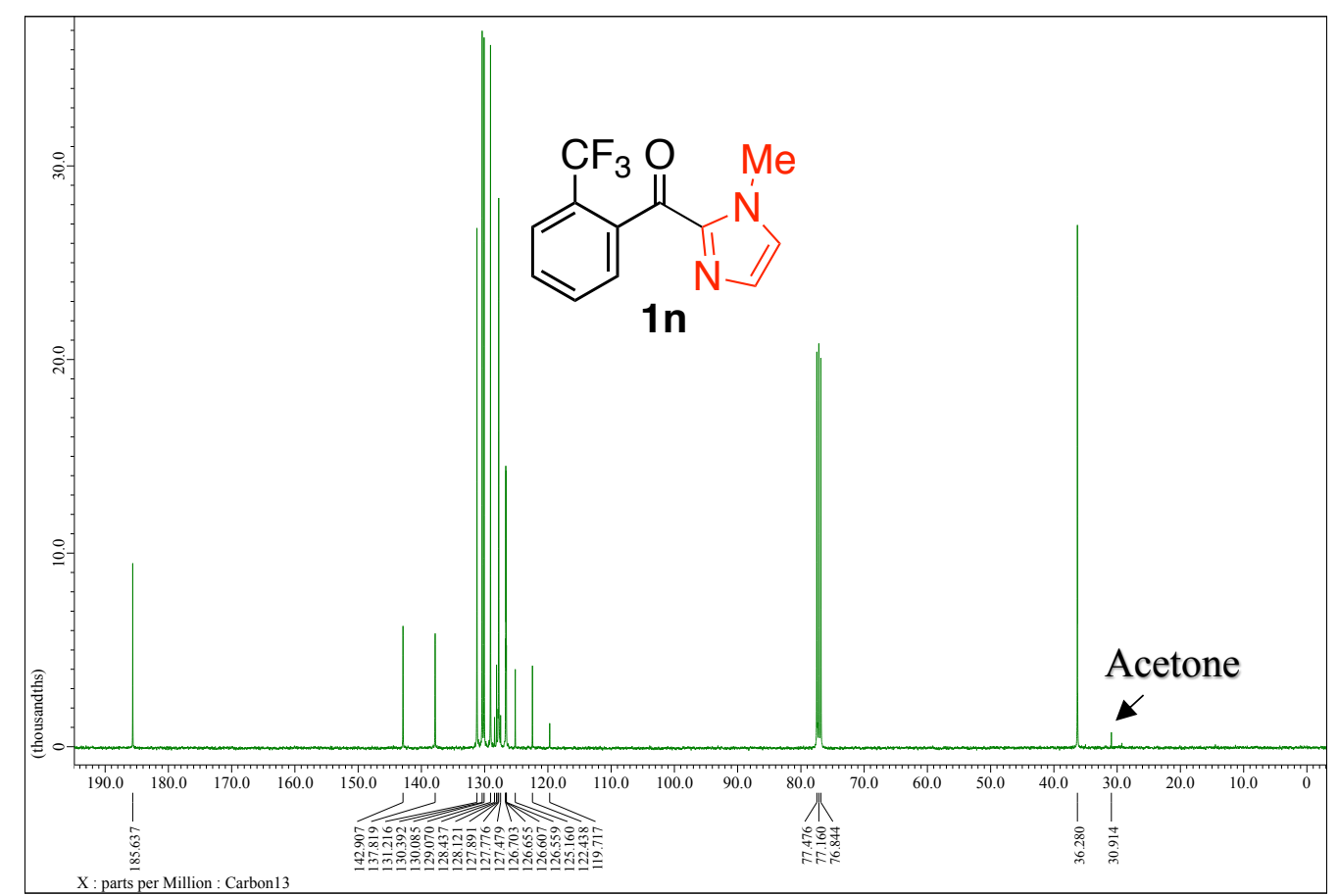

Figure S56: ${ }^{13} \mathrm{C}$ NMR spectrum of compound 1 n $\left(100 \mathrm{MHz}, \mathrm{CDCl}_{3}\right)$.

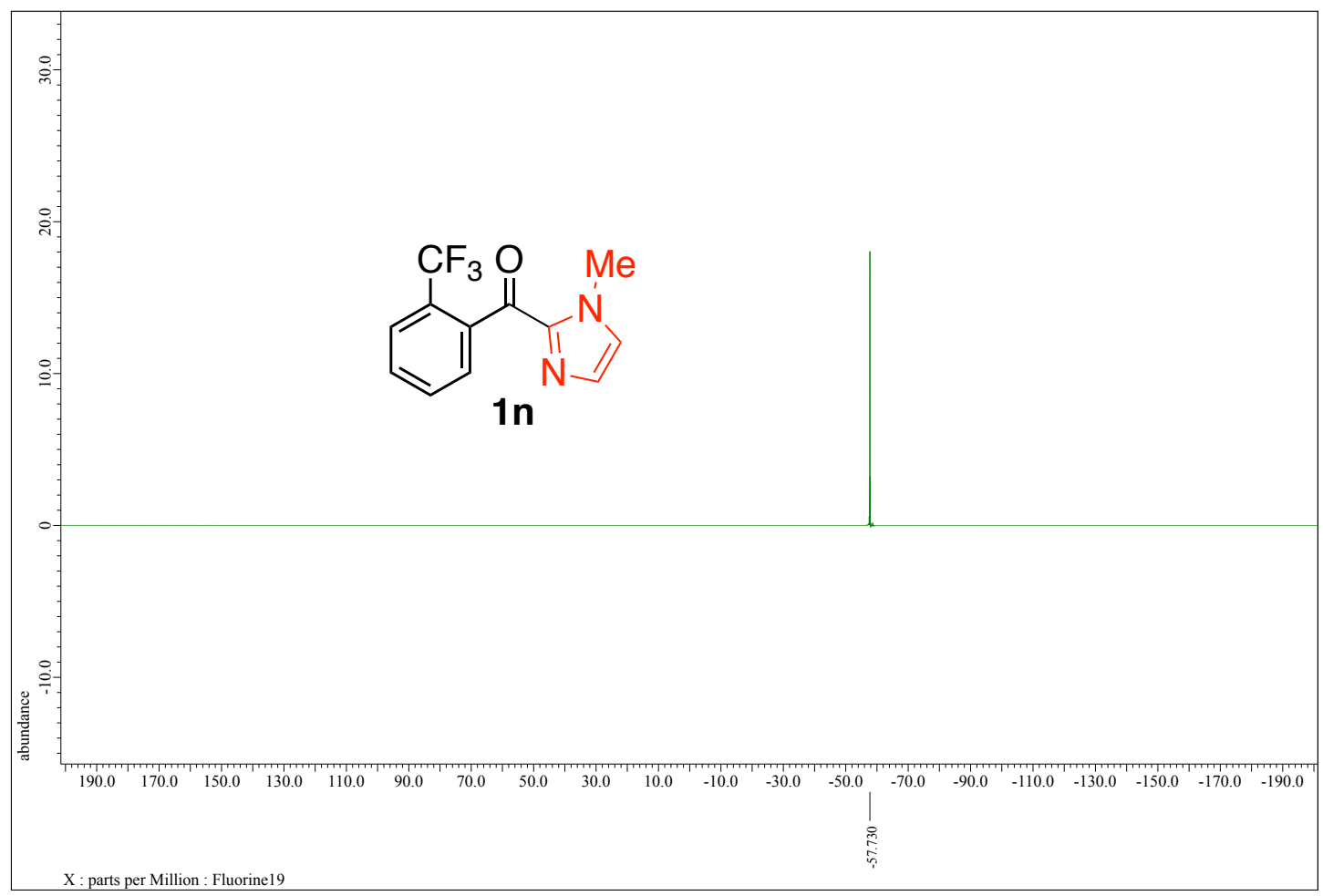

Figure S57: ${ }^{19} \mathrm{~F}$ NMR spectrum of compound 1 n $\left(376 \mathrm{MHz}, \mathrm{CDCl}_{3}\right)$. 


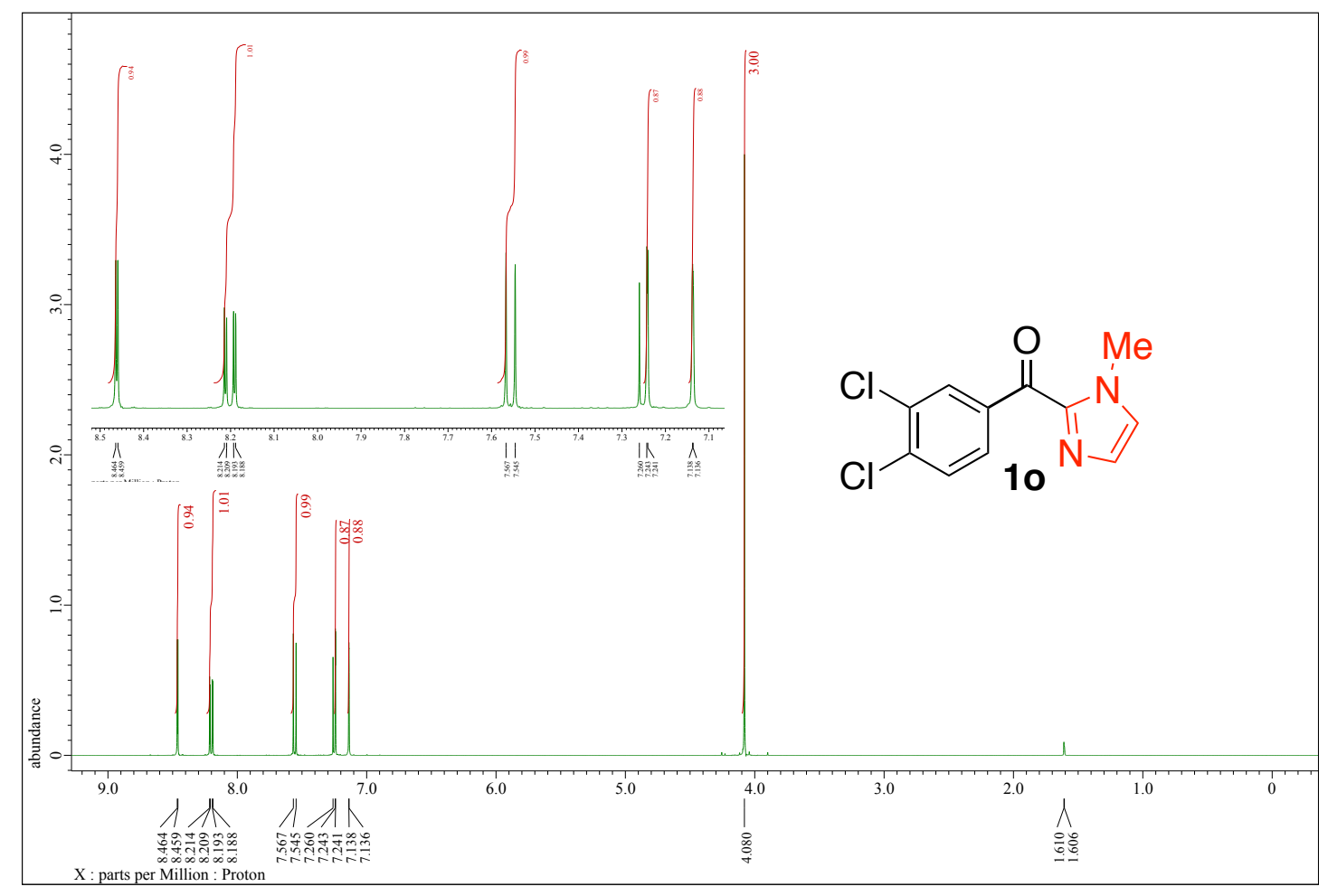

Figure S58: ${ }^{1} \mathrm{H}$ NMR spectrum of compound $10\left(400 \mathrm{MHz}, \mathrm{CDCl}_{3}\right)$.

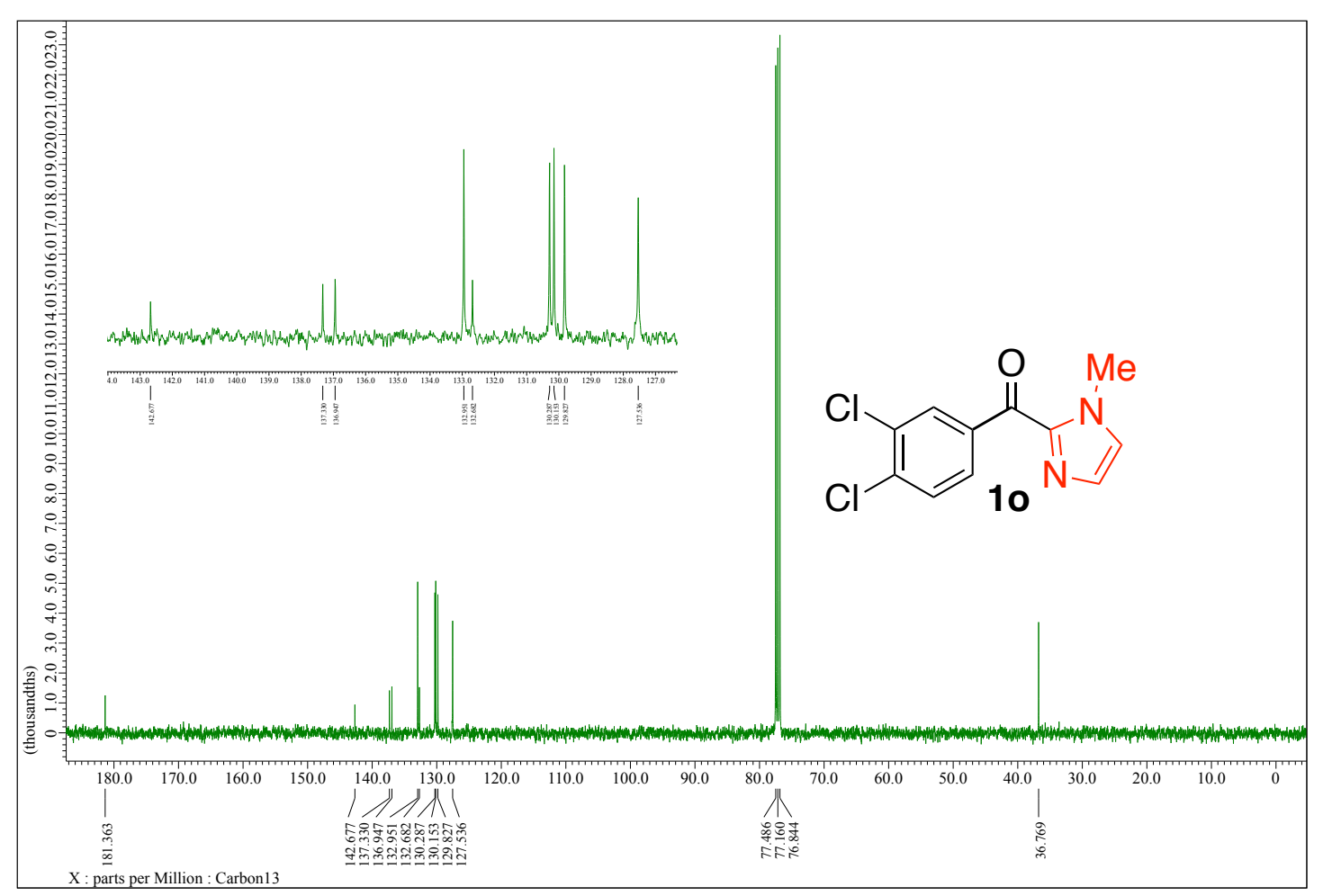

Figure S59: ${ }^{13} \mathrm{C}$ NMR spectrum of compound $10\left(100 \mathrm{MHz}, \mathrm{CDCl}_{3}\right)$. 


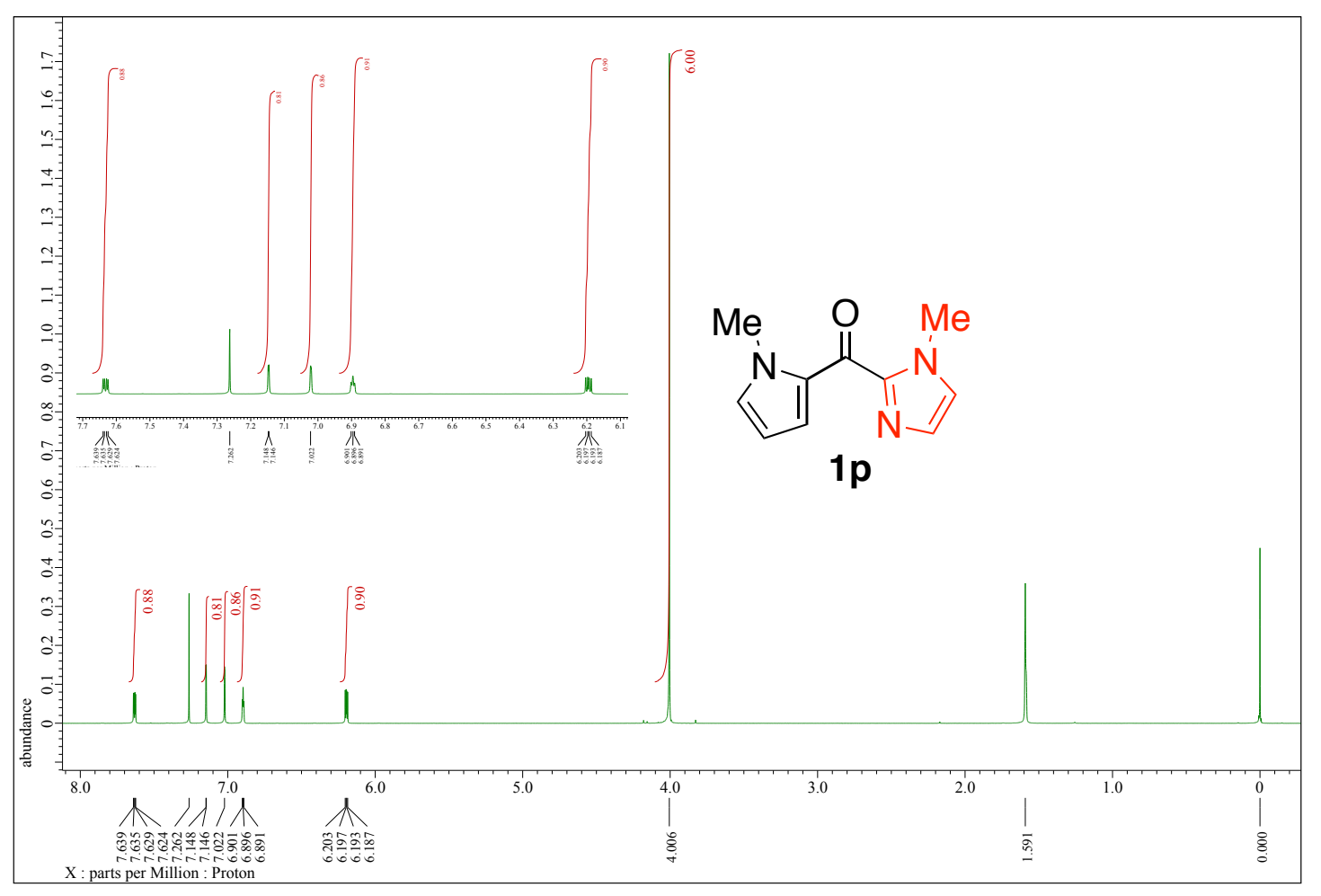

Figure S60: ${ }^{1} \mathrm{H}$ NMR spectrum of compound 1 p $\left(400 \mathrm{MHz}, \mathrm{CDCl}_{3}\right)$.

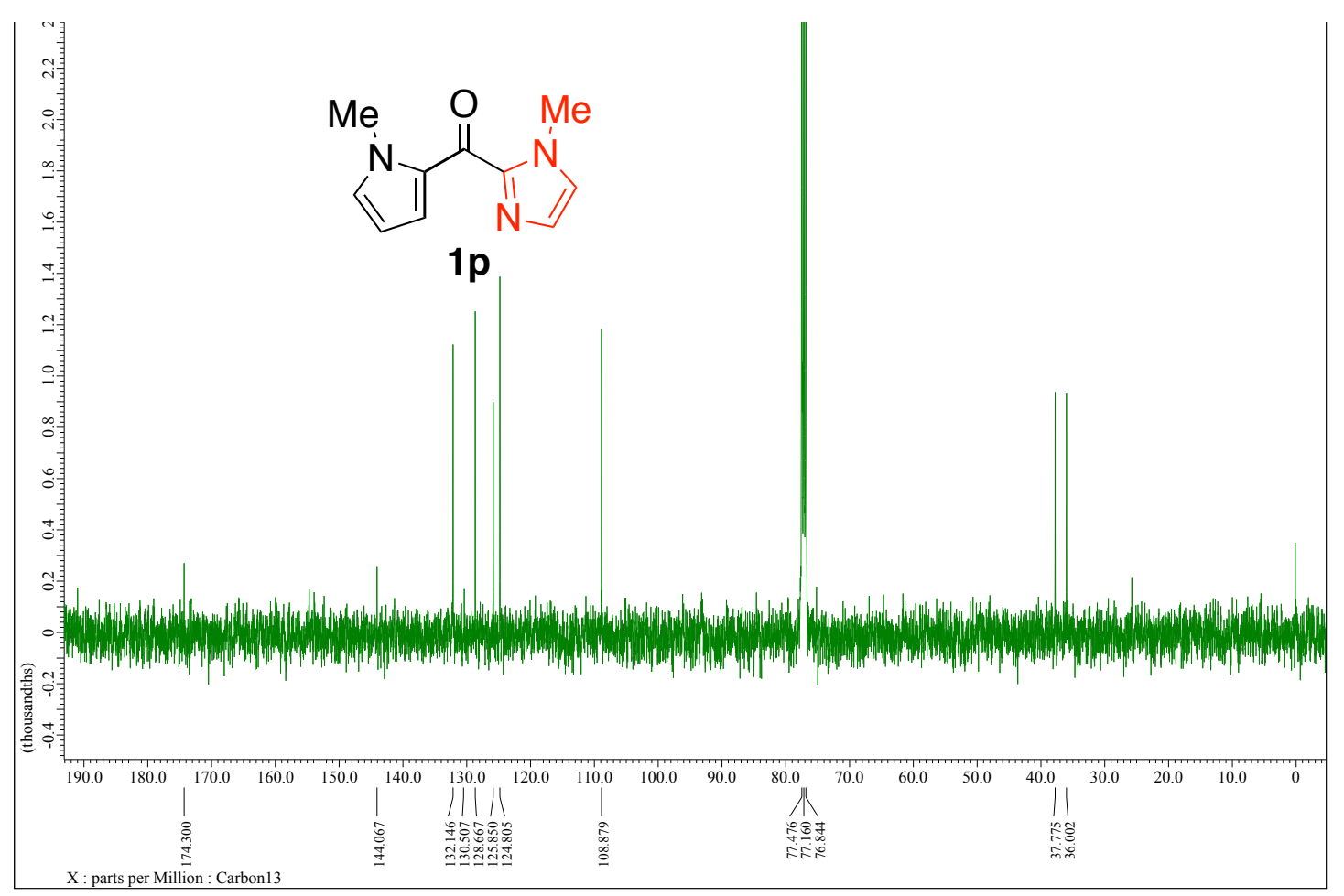

Figure S61: ${ }^{13} \mathrm{C}$ NMR spectrum of compound $1 p\left(100 \mathrm{MHz}, \mathrm{CDCl}_{3}\right)$. 


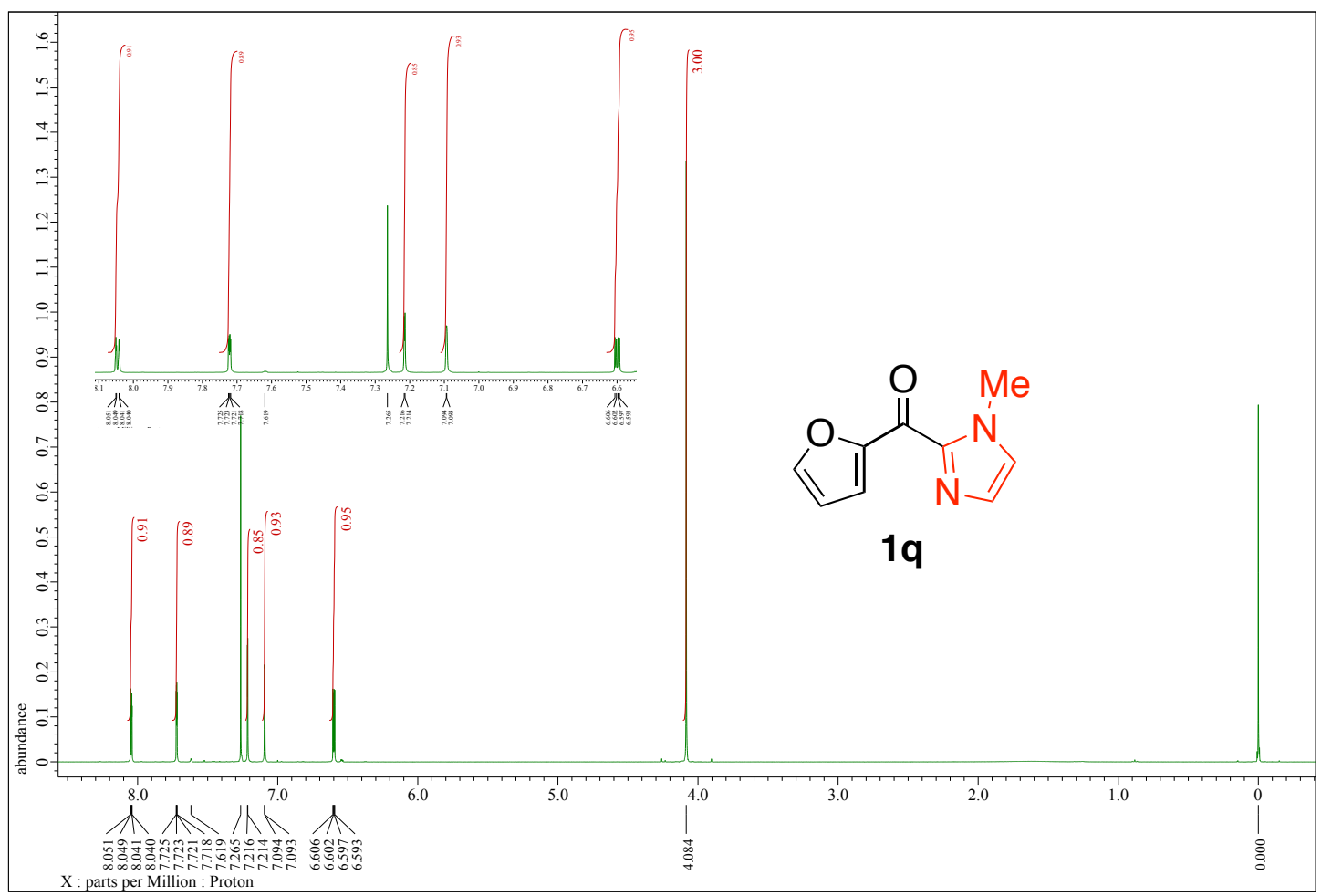

Figure S62: ${ }^{1} \mathrm{H}$ NMR spectrum of compound 1q $\left(400 \mathrm{MHz}, \mathrm{CDCl}_{3}\right)$.

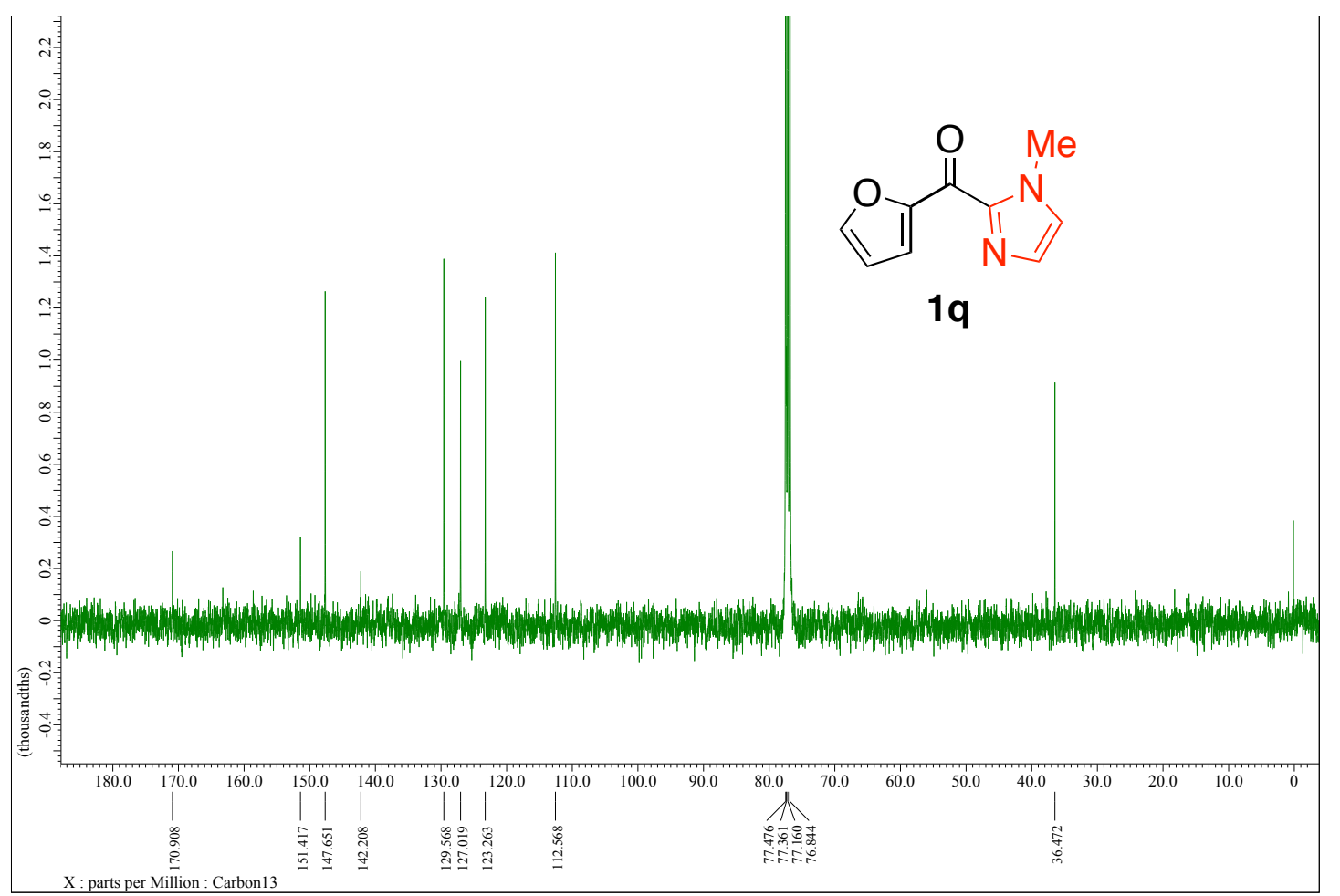

Figure S63: ${ }^{13} \mathrm{C}$ NMR spectrum of compound $1 \mathbf{q}\left(100 \mathrm{MHz}, \mathrm{CDCl}_{3}\right)$. 


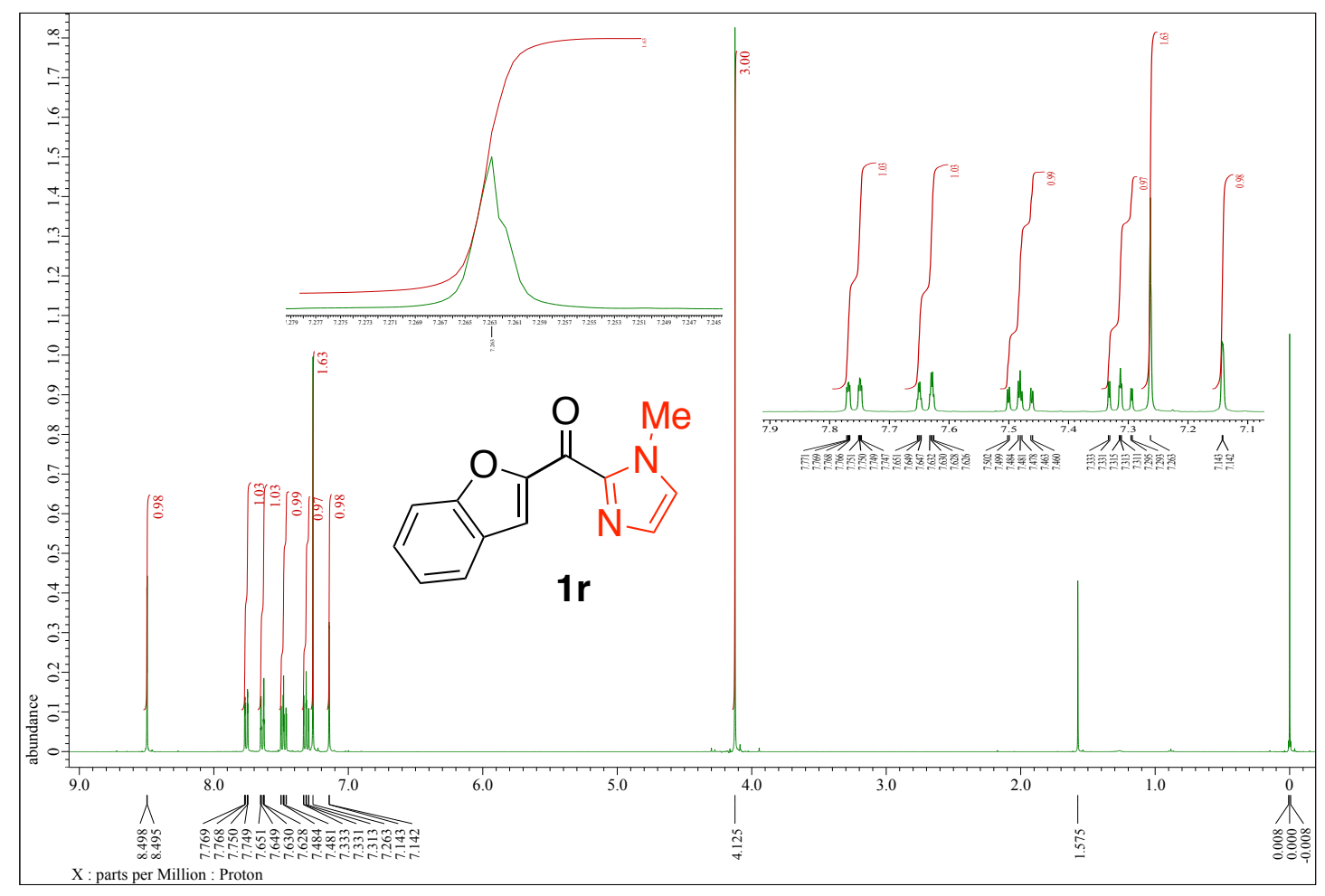

Figure S64: ${ }^{1} \mathrm{H}$ NMR spectrum of compound 1 r $\left(400 \mathrm{MHz}, \mathrm{CDCl}_{3}\right)$.

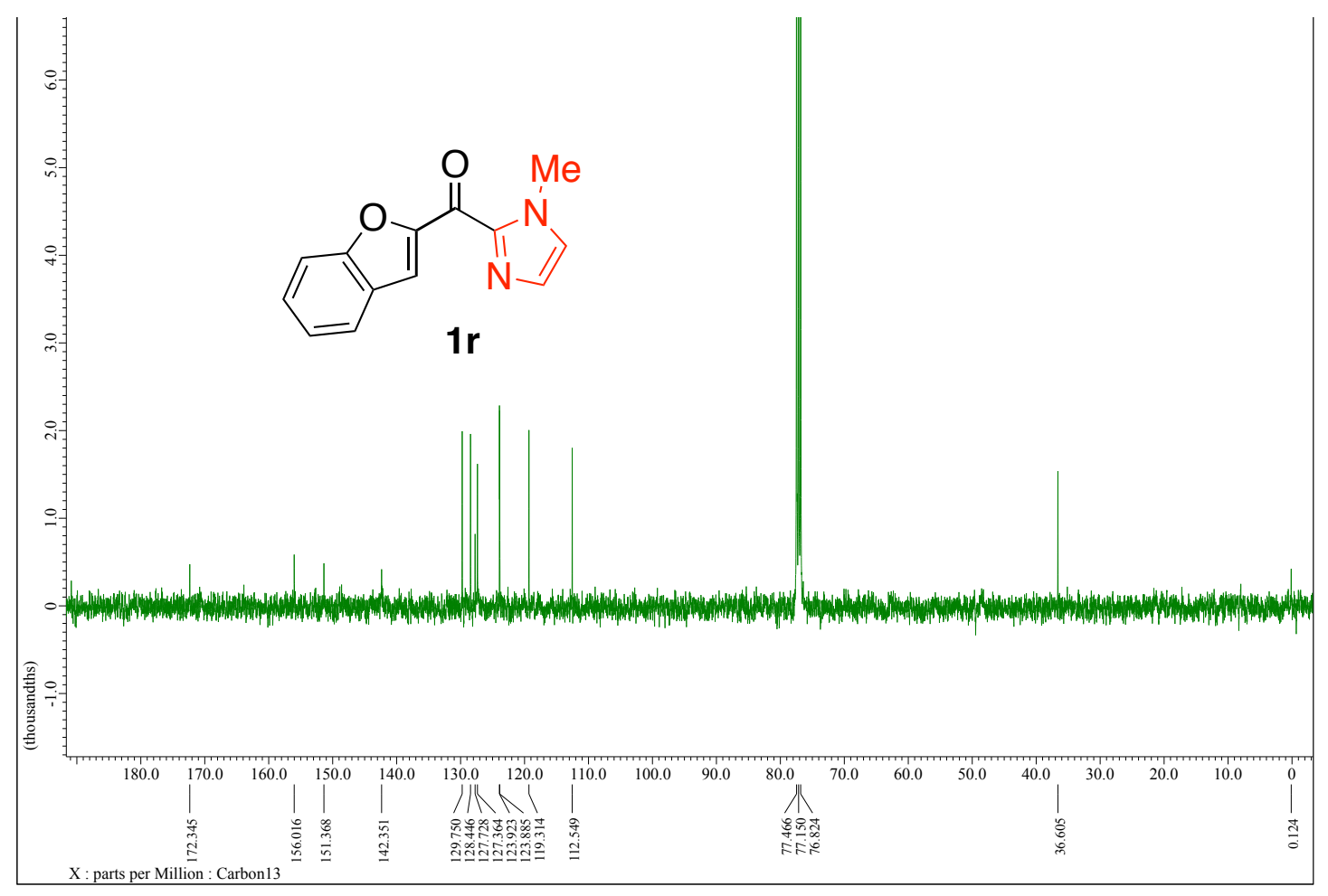

Figure S65: ${ }^{13} \mathrm{C}$ NMR spectrum of compound $1 \mathbf{r}\left(100 \mathrm{MHz}, \mathrm{CDCl}_{3}\right)$. 


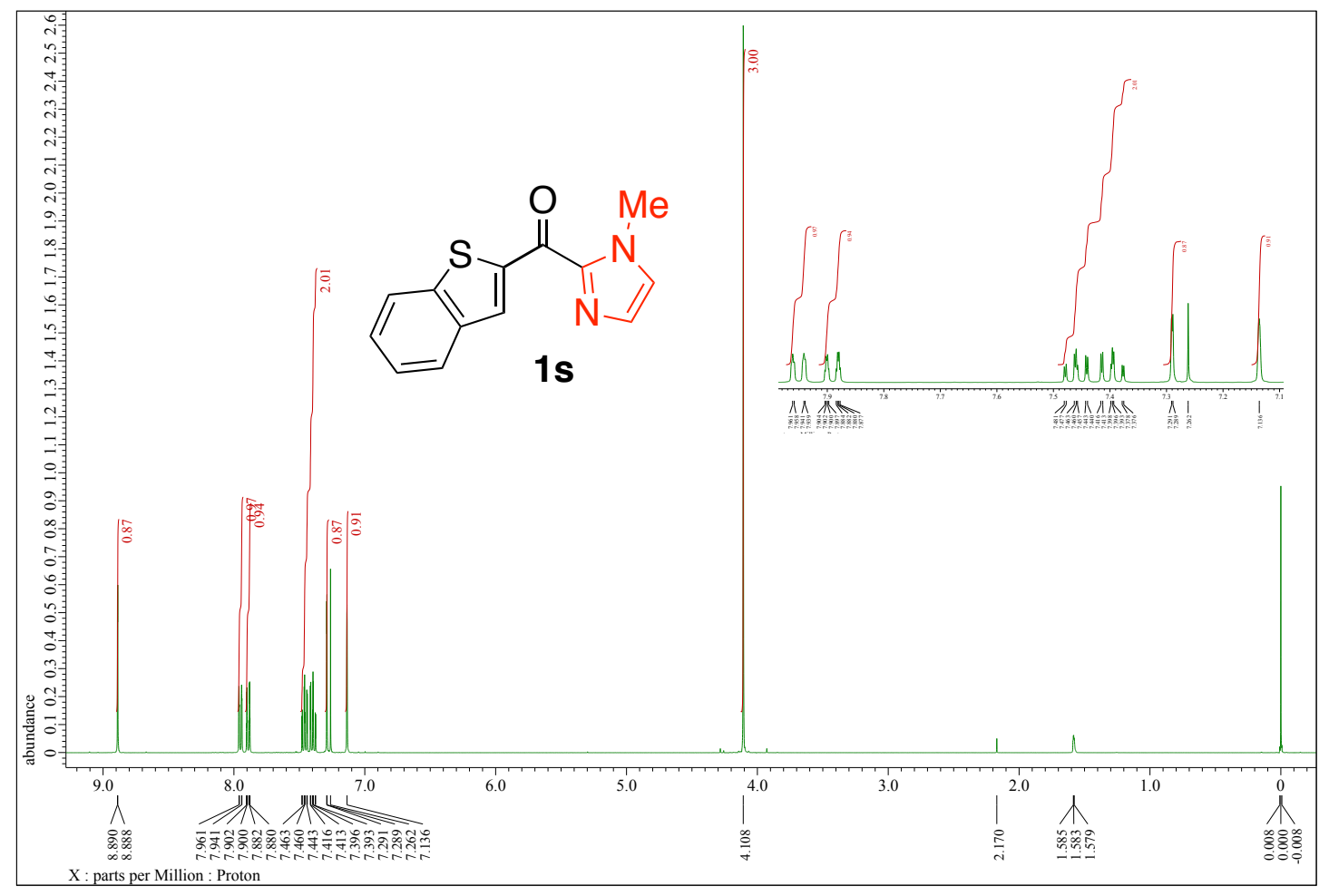

Figure S66: ${ }^{1} \mathrm{H}$ NMR spectrum of compound 1 s $\left(400 \mathrm{MHz}, \mathrm{CDCl}_{3}\right)$.

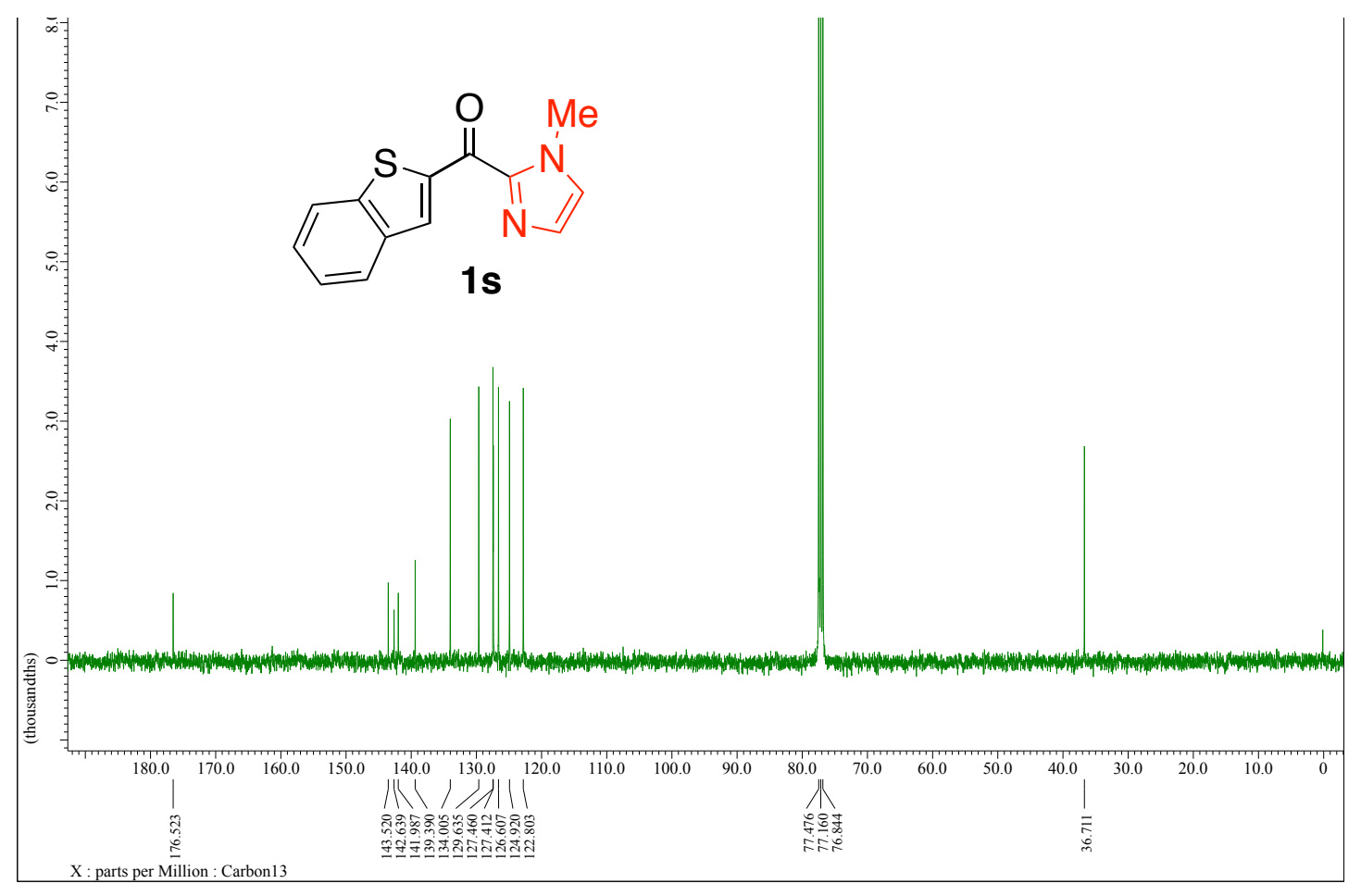

Figure S67: ${ }^{13} \mathrm{C}$ NMR spectrum of compound 1 s $\left(100 \mathrm{MHz}, \mathrm{CDCl}_{3}\right)$. 


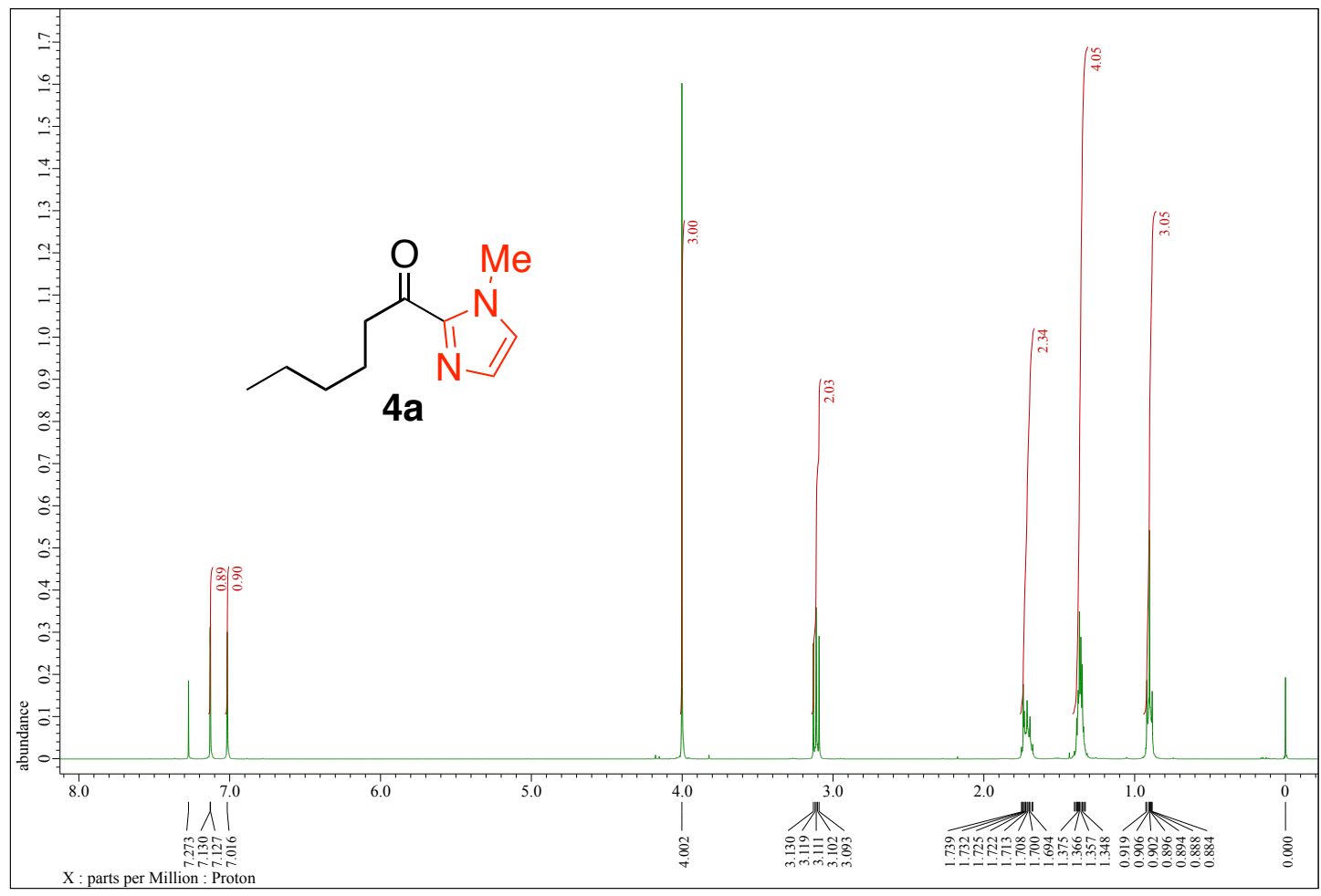

Figure S68: ${ }^{1} \mathrm{H}$ NMR spectrum of compound $4 \mathbf{a}\left(400 \mathrm{MHz}, \mathrm{CDCl}_{3}\right)$.

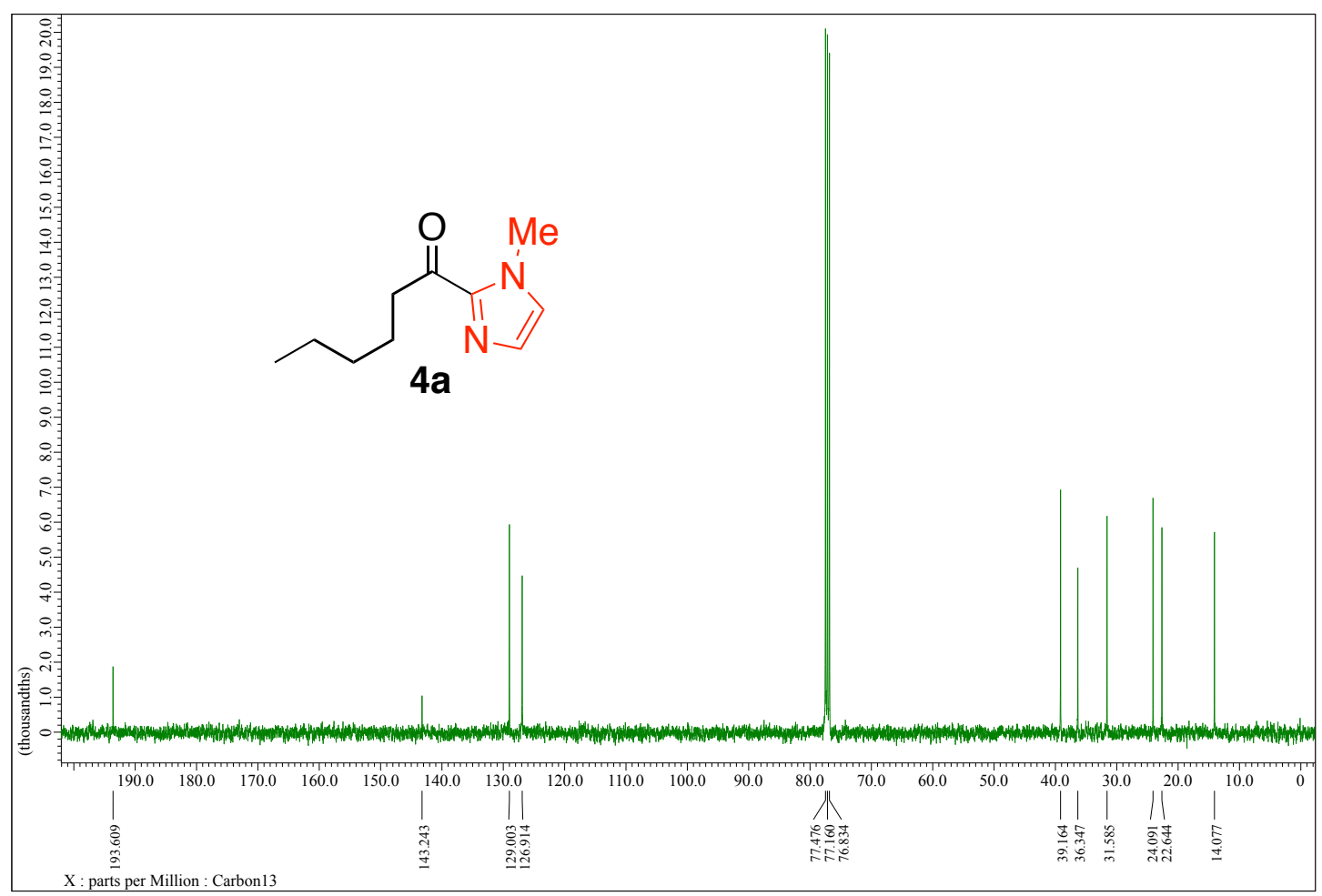

Figure S69: ${ }^{13} \mathrm{C}$ NMR spectrum of compound $4 \mathbf{a}\left(100 \mathrm{MHz}, \mathrm{CDCl}_{3}\right)$. 


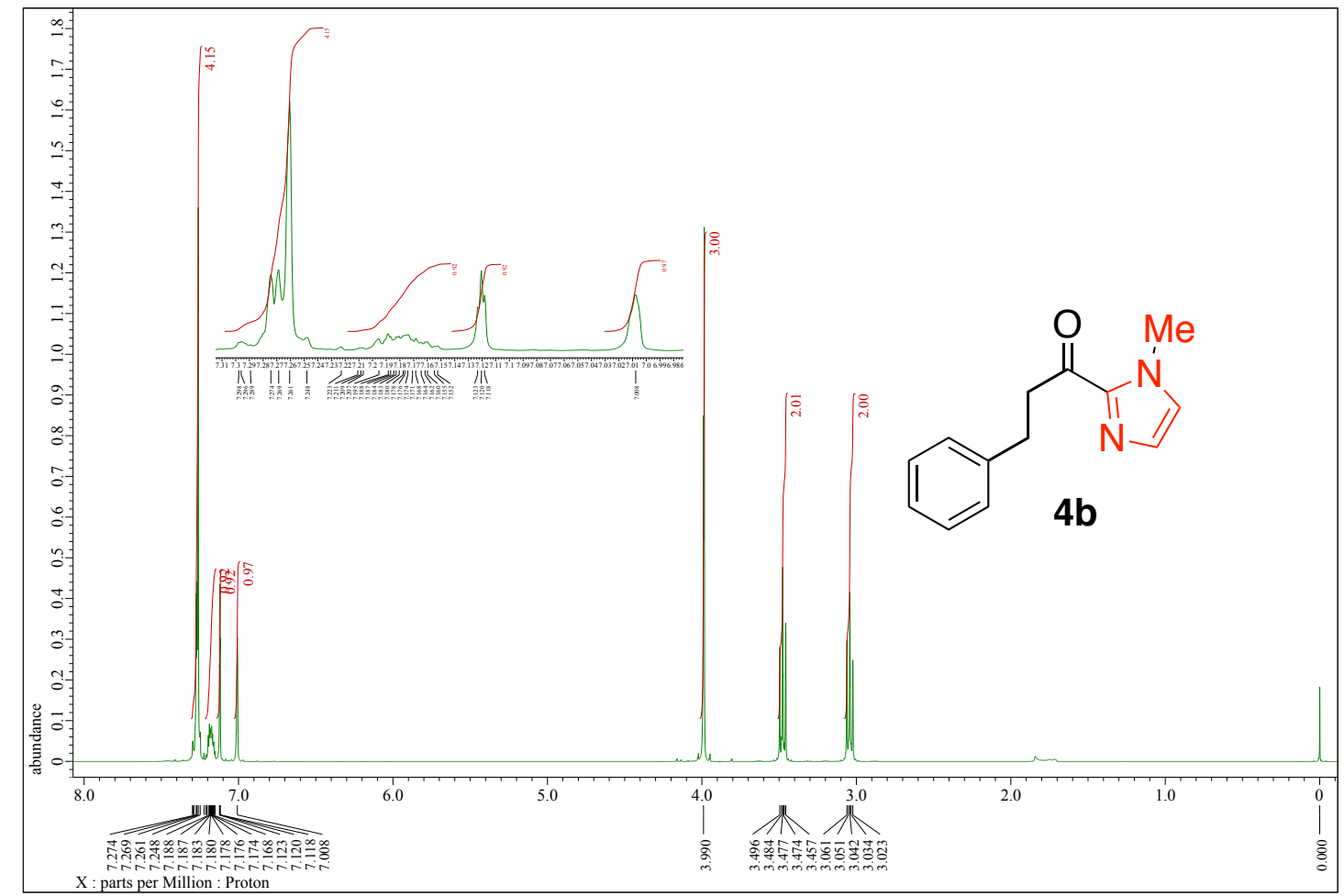

Figure S70: ${ }^{1} \mathrm{H}$ NMR spectrum of compound $\mathbf{4 b}\left(400 \mathrm{MHz}, \mathrm{CDCl}_{3}\right)$.

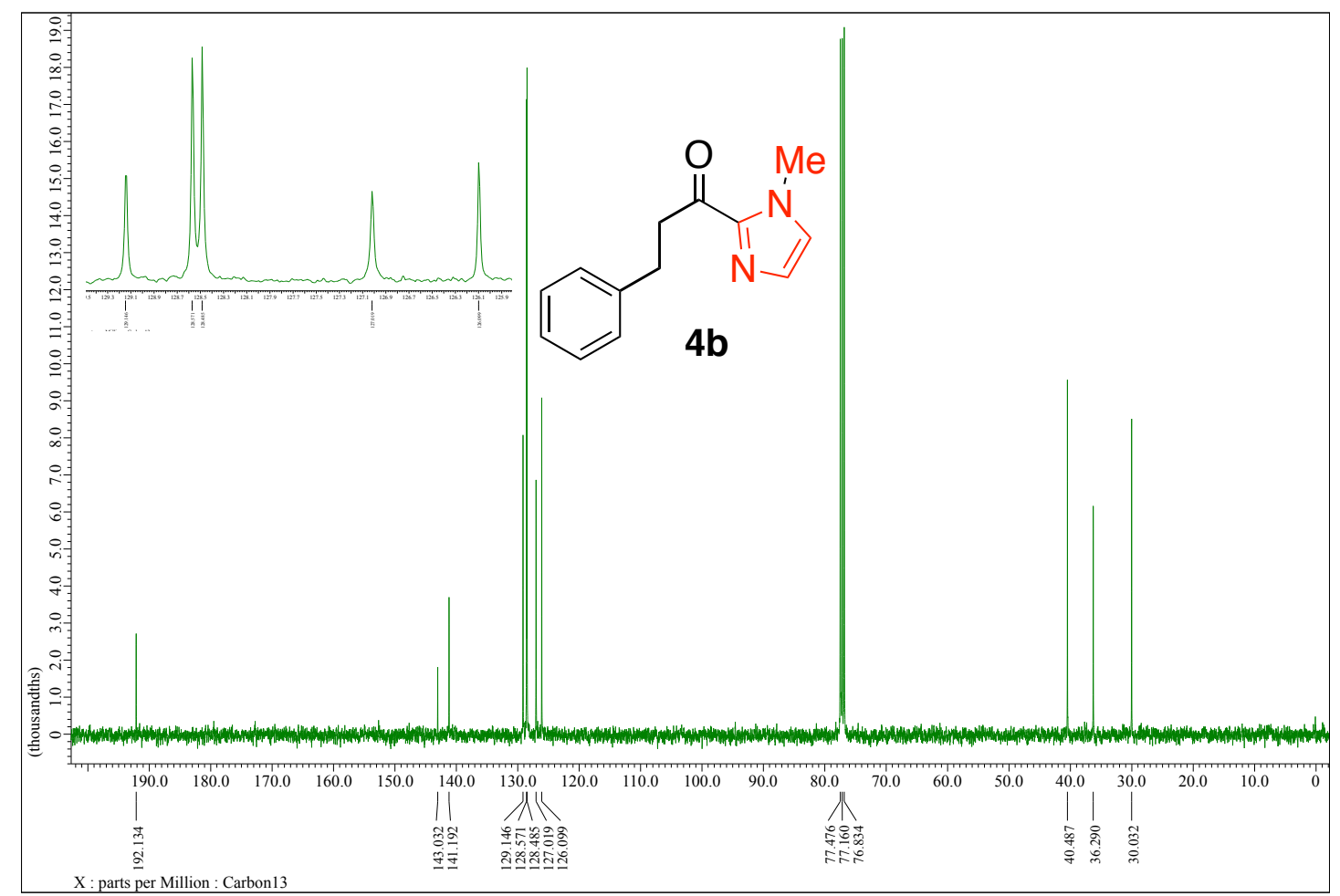

Figure S71: ${ }^{13} \mathrm{C}$ NMR spectrum of compound $\mathbf{4 b}\left(100 \mathrm{MHz}, \mathrm{CDCl}_{3}\right)$. 


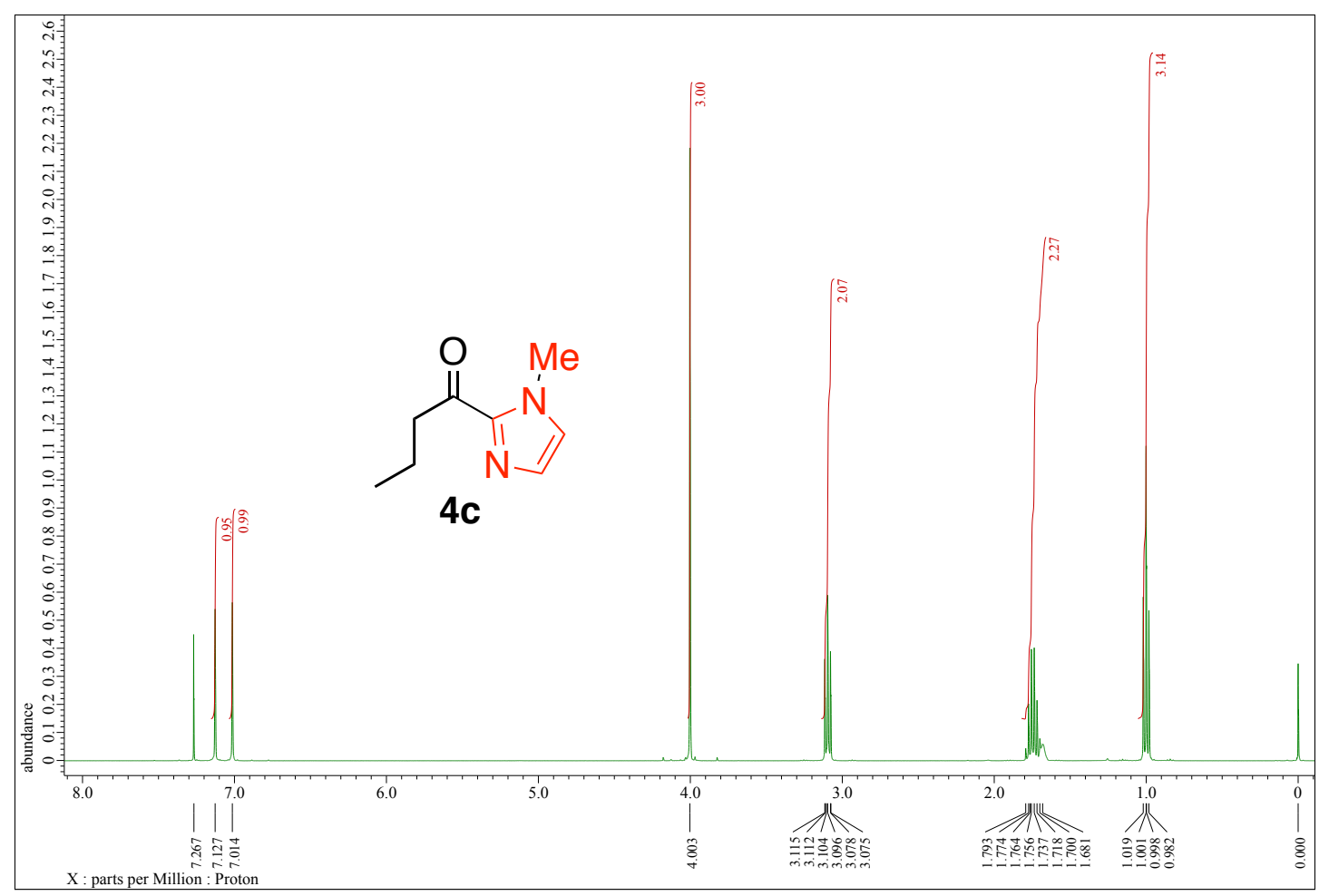

Figure S72: ${ }^{1} \mathrm{H}$ NMR spectrum of compound $4 \mathbf{c}\left(400 \mathrm{MHz}, \mathrm{CDCl}_{3}\right)$.

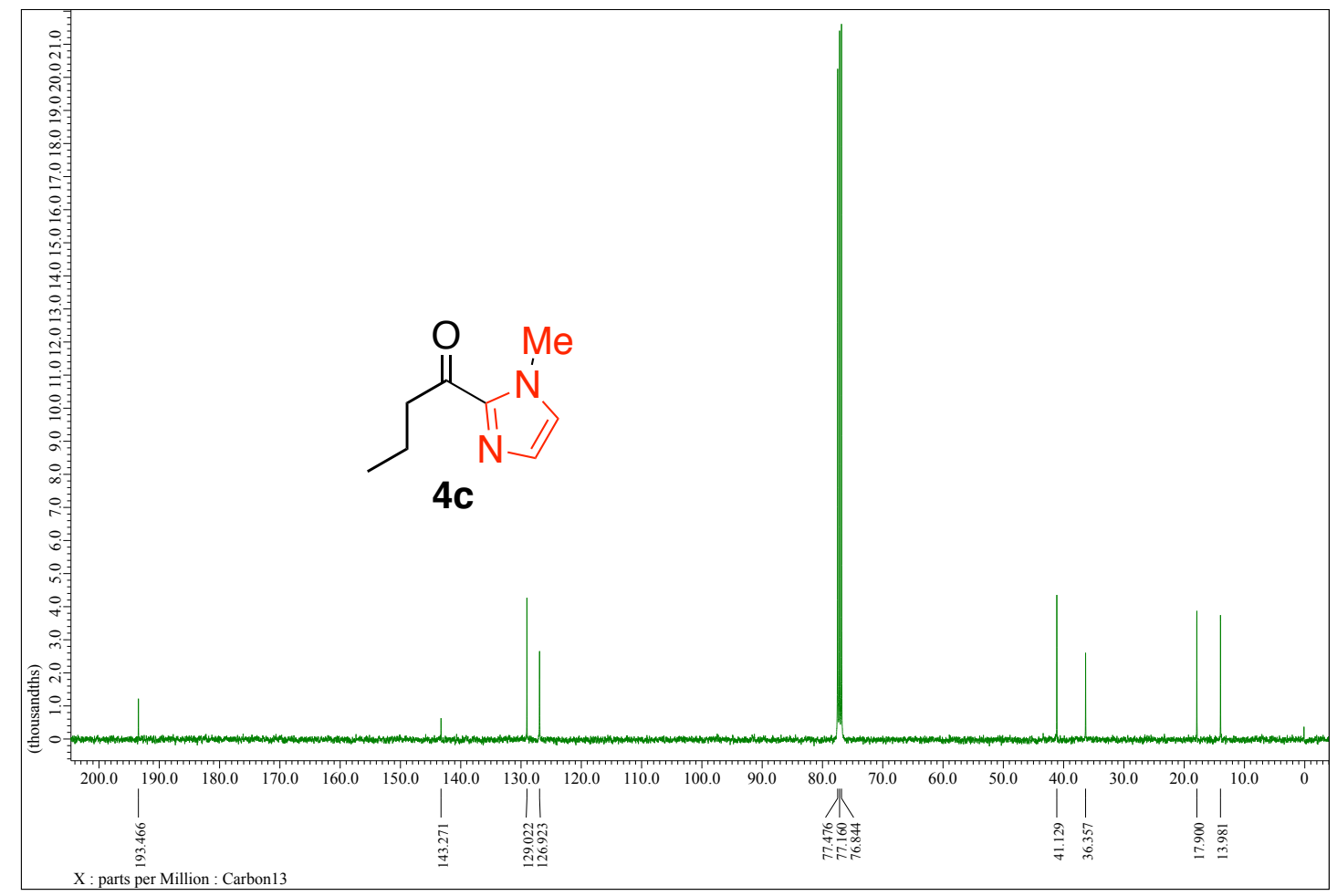

Figure S73: ${ }^{13} \mathrm{C}$ NMR spectrum of compound $4 \mathbf{c}\left(100 \mathrm{MHz}, \mathrm{CDCl}_{3}\right)$. 


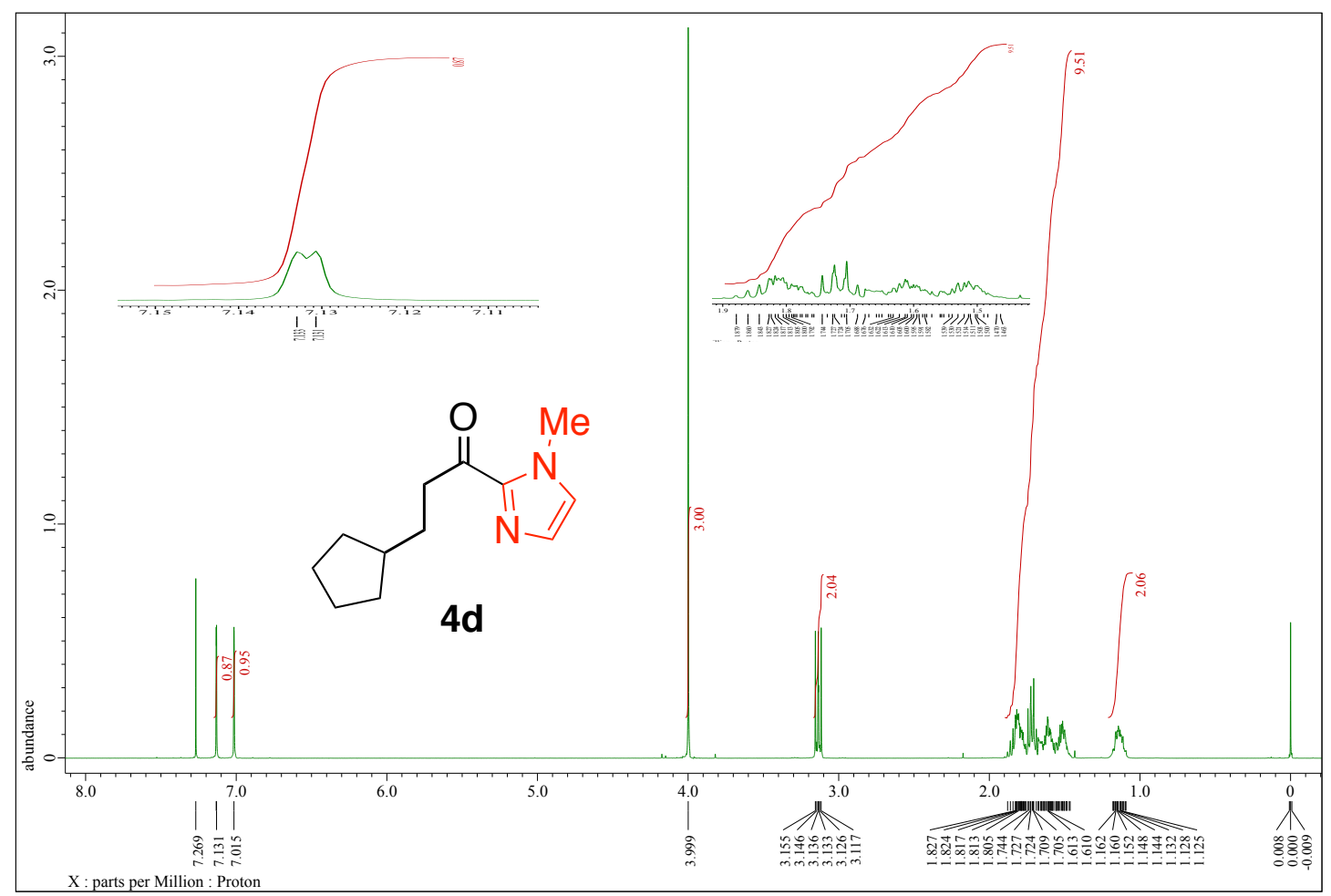

Figure S74: ${ }^{1} \mathrm{H}$ NMR spectrum of compound $4 \mathbf{d}\left(400 \mathrm{MHz}, \mathrm{CDCl}_{3}\right)$.

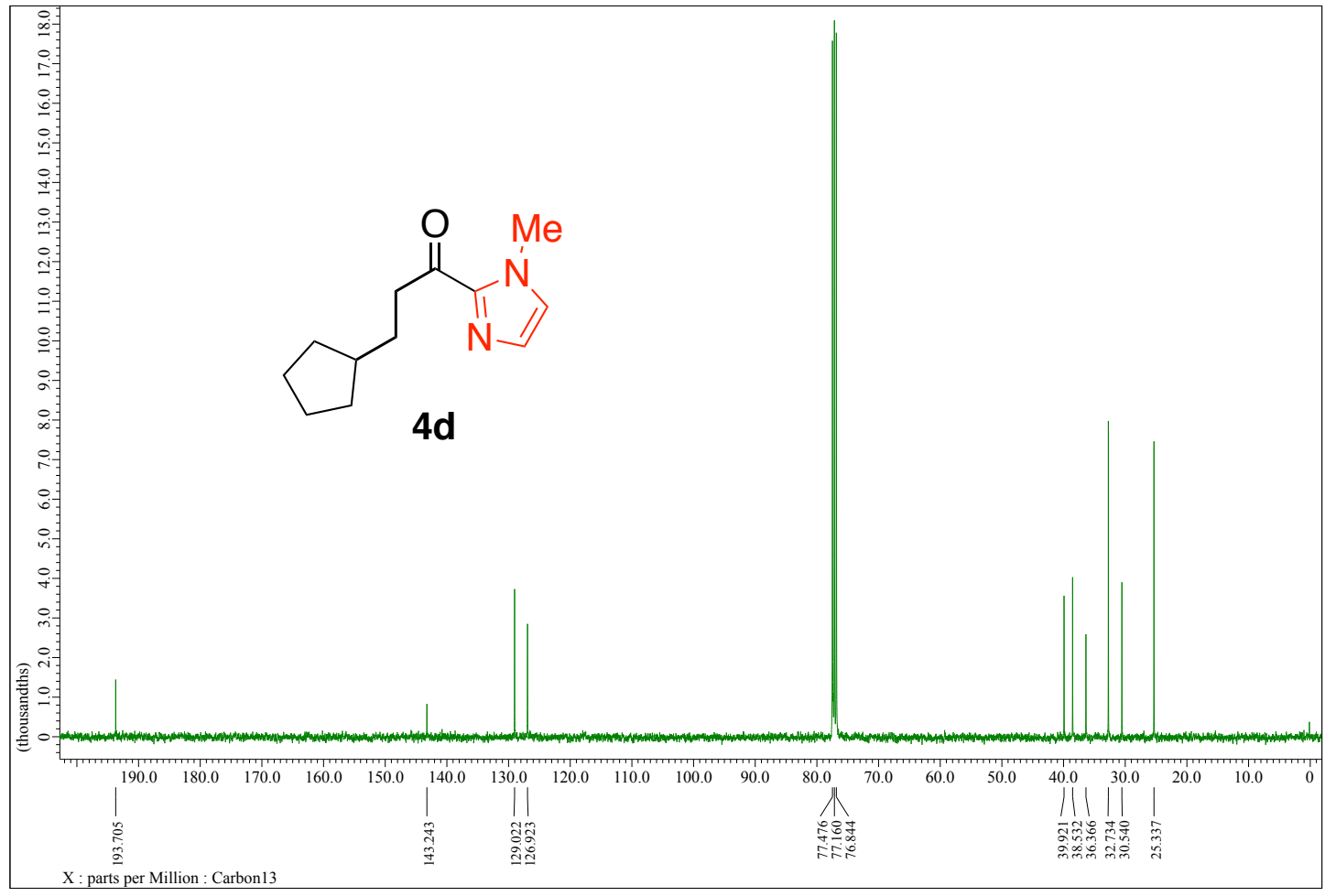

Figure S75: ${ }^{13} \mathrm{C}$ NMR spectrum of compound $4 \mathbf{d}\left(100 \mathrm{MHz}, \mathrm{CDCl}_{3}\right)$. 


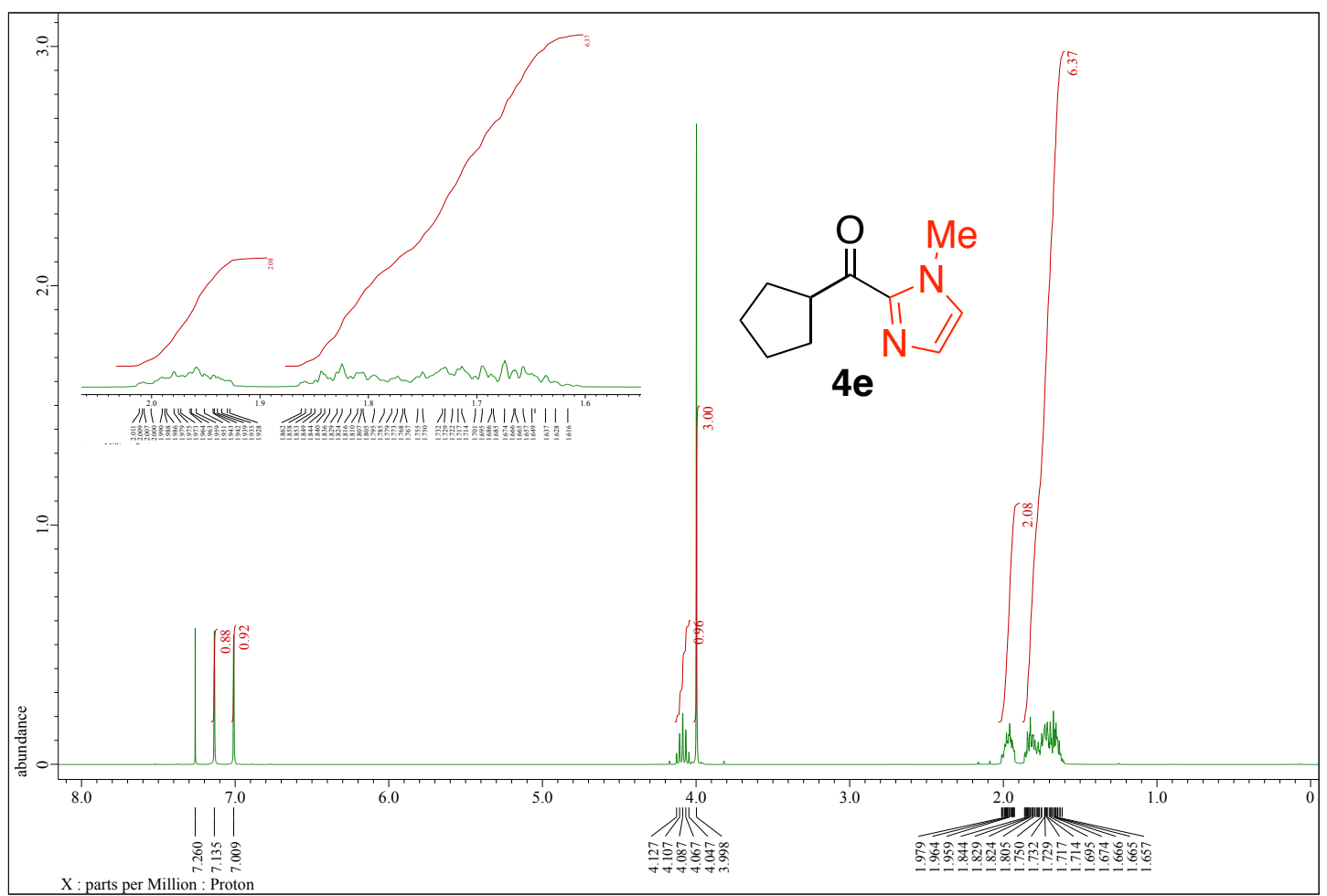

Figure S76: ${ }^{1} \mathrm{H}$ NMR spectrum of compound $4 \mathrm{e}\left(400 \mathrm{MHz}, \mathrm{CDCl}_{3}\right)$.

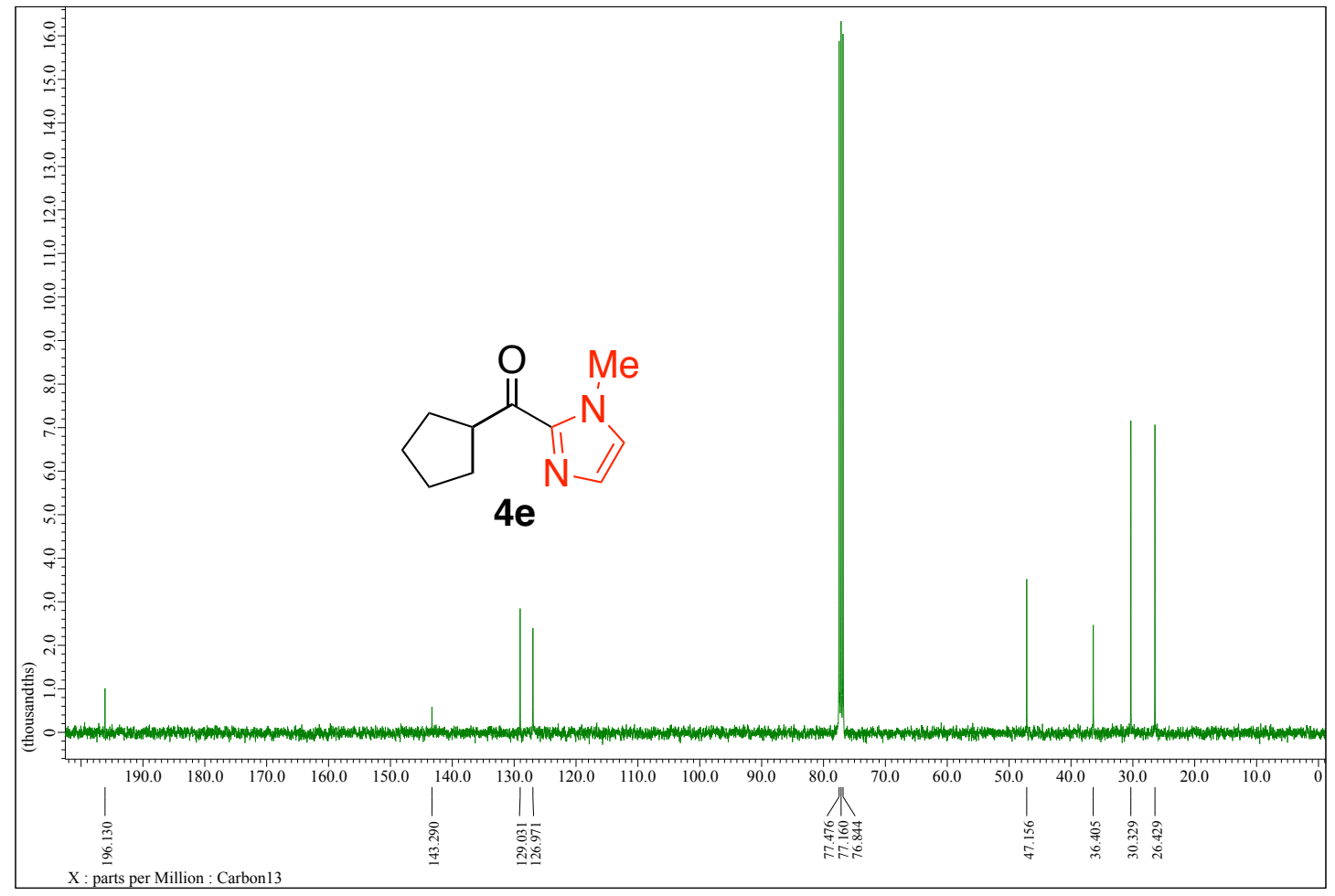

Figure S77: ${ }^{13} \mathrm{C}$ NMR spectrum of compound $4 \mathbf{e}\left(100 \mathrm{MHz}, \mathrm{CDCl}_{3}\right)$. 


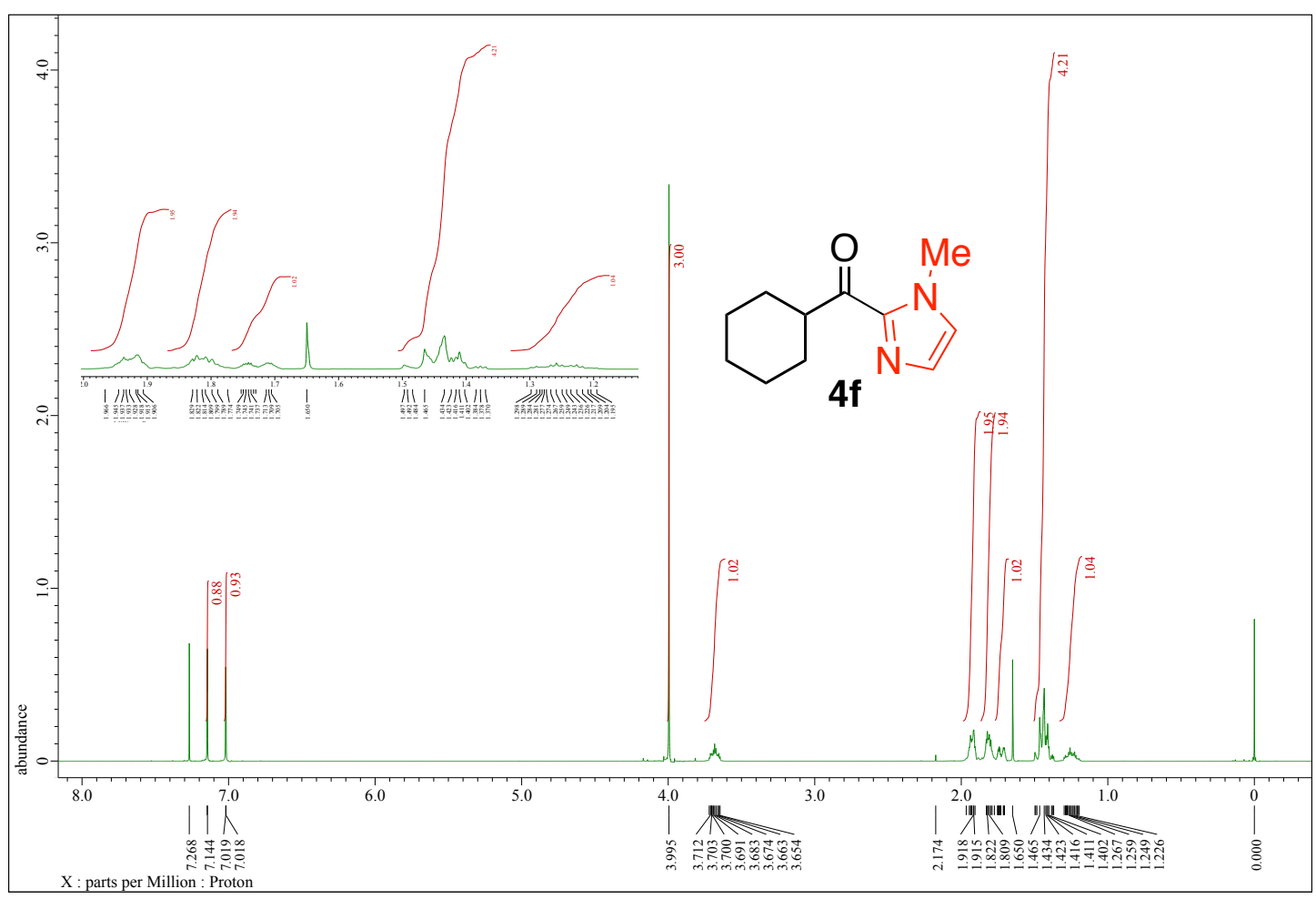

Figure S78: ${ }^{1} \mathrm{H}$ NMR spectrum of compound $4 \mathbf{f}\left(400 \mathrm{MHz}, \mathrm{CDCl}_{3}\right)$.

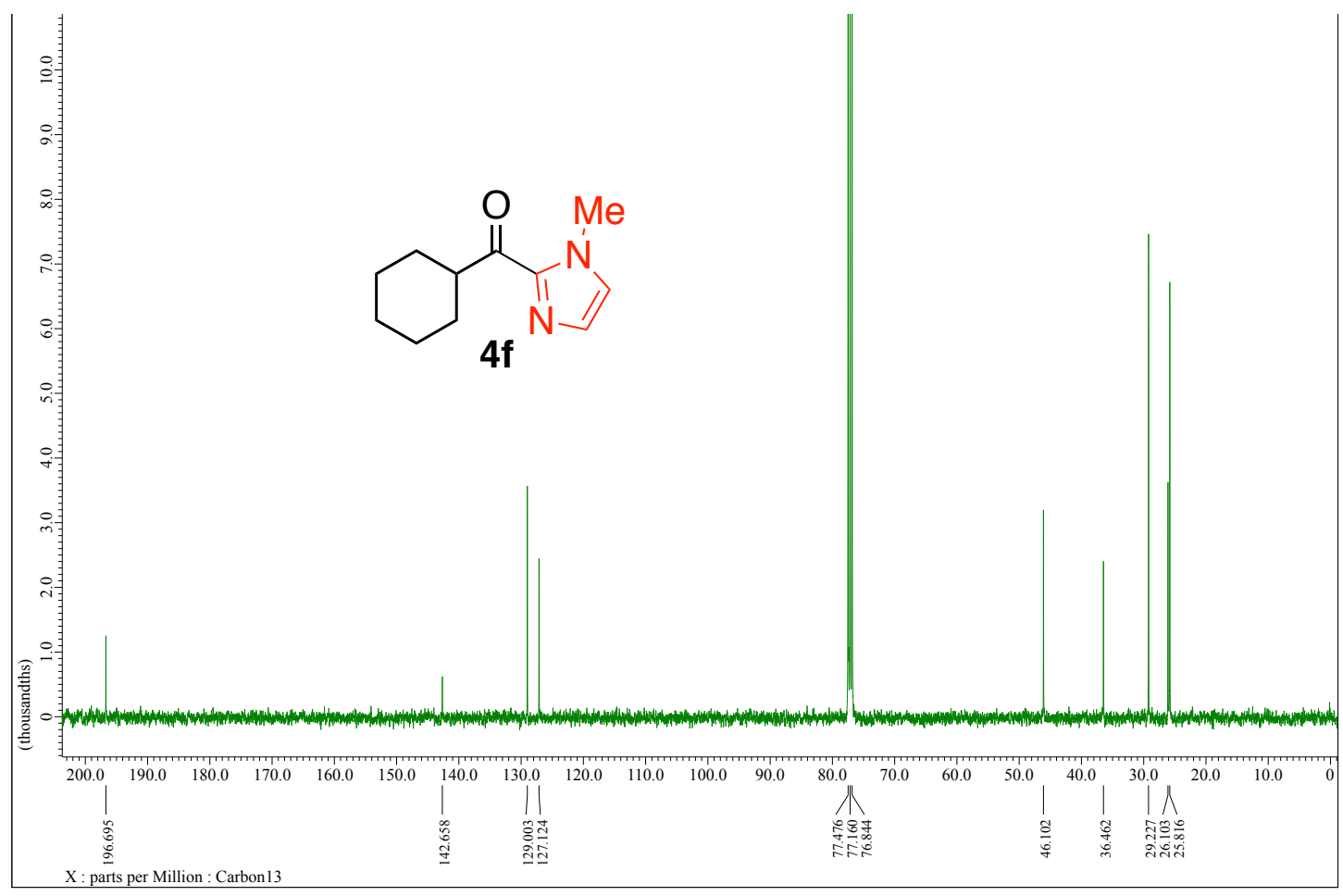

Figure S79: ${ }^{13} \mathrm{C}$ NMR spectrum of compound $\mathbf{4 f}\left(100 \mathrm{MHz}, \mathrm{CDCl}_{3}\right)$. 


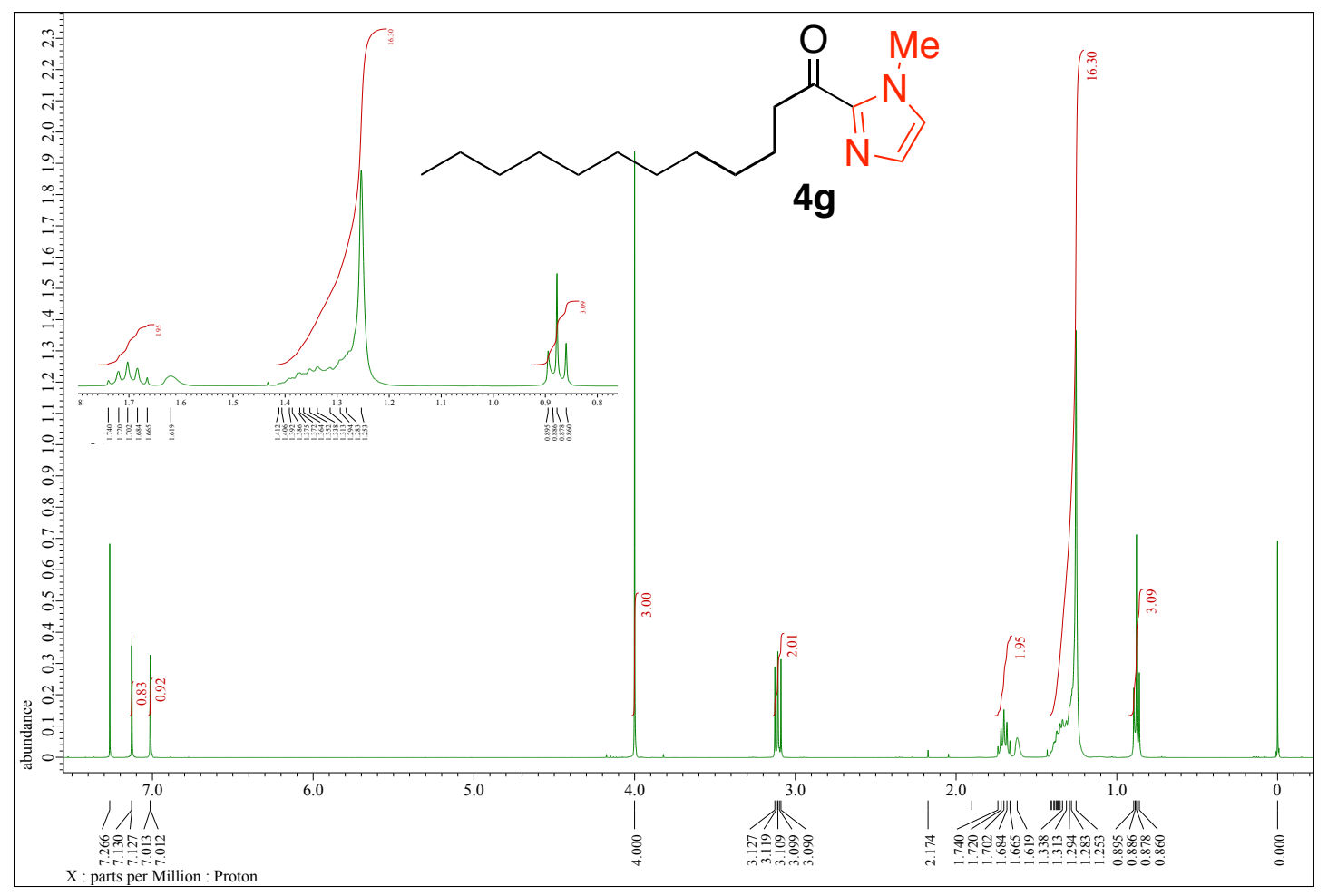

Figure S80: ${ }^{1} \mathrm{H}$ NMR spectrum of compound $\mathbf{4 g}\left(400 \mathrm{MHz}, \mathrm{CDCl}_{3}\right)$.

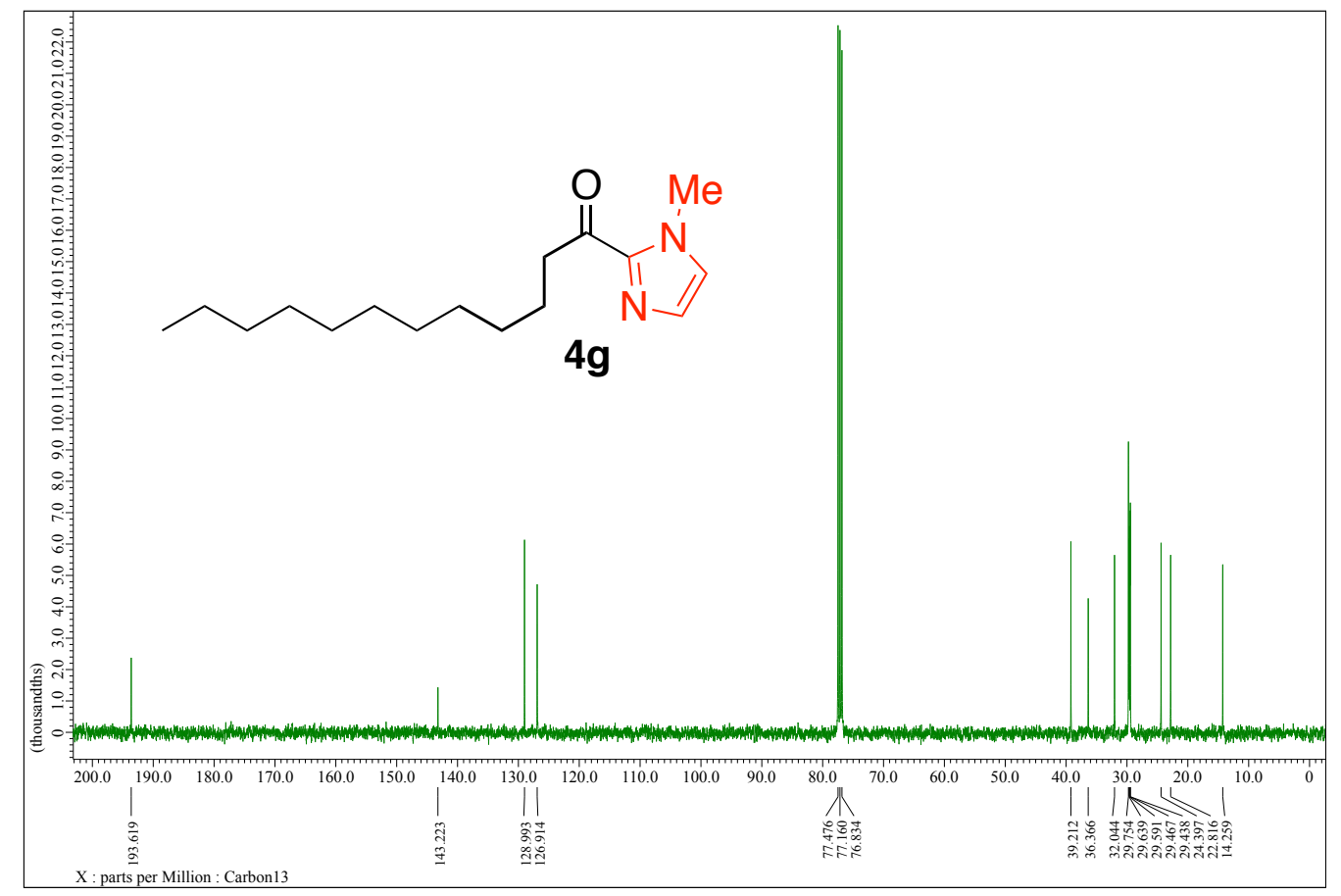

Figure S81: ${ }^{13} \mathrm{C}$ NMR spectrum of compound $4 \mathbf{g}\left(100 \mathrm{MHz}, \mathrm{CDCl}_{3}\right)$. 


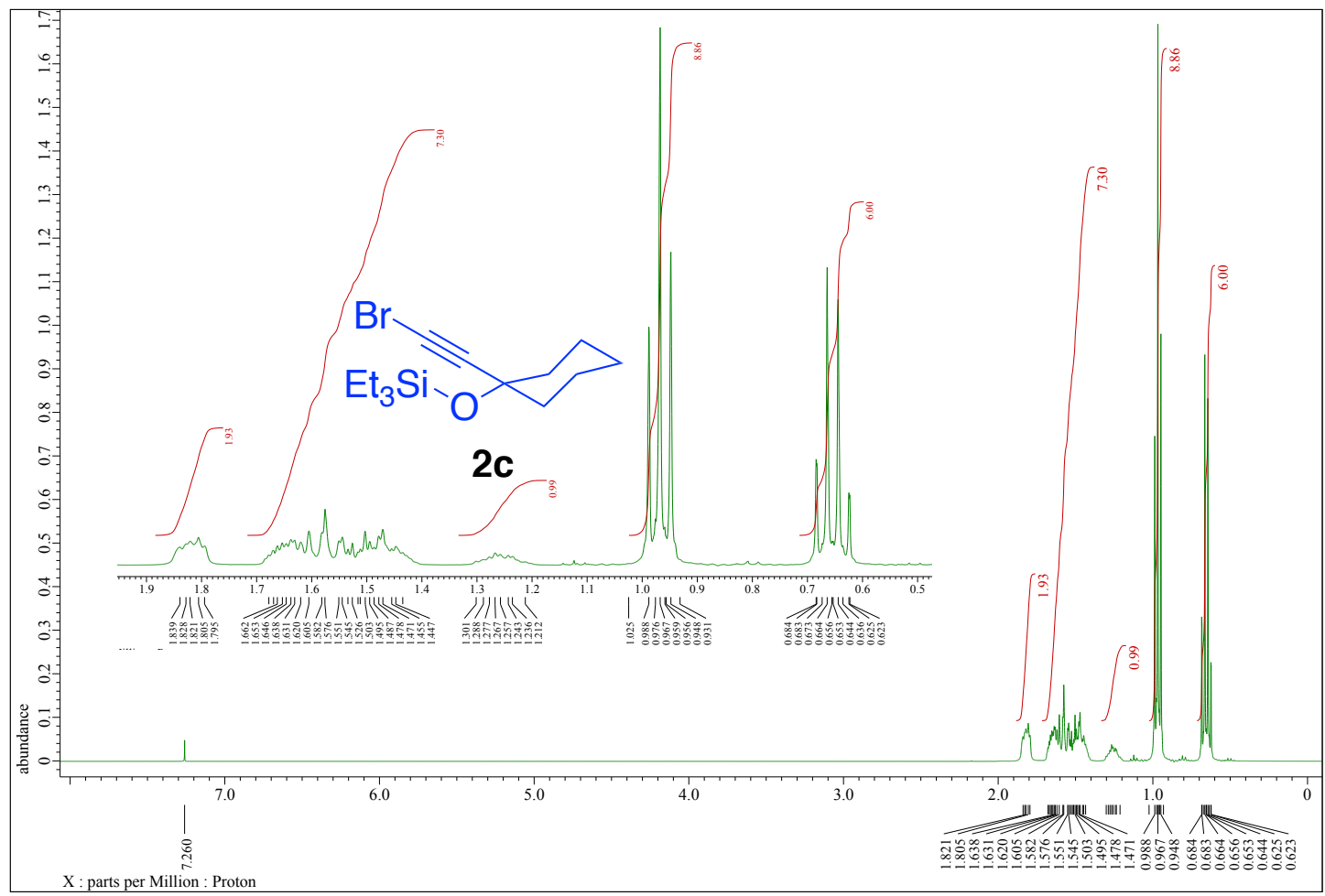

Figure S82: ${ }^{1} \mathrm{H}$ NMR spectrum of compound $2 c\left(400 \mathrm{MHz}, \mathrm{CDCl}_{3}\right)$

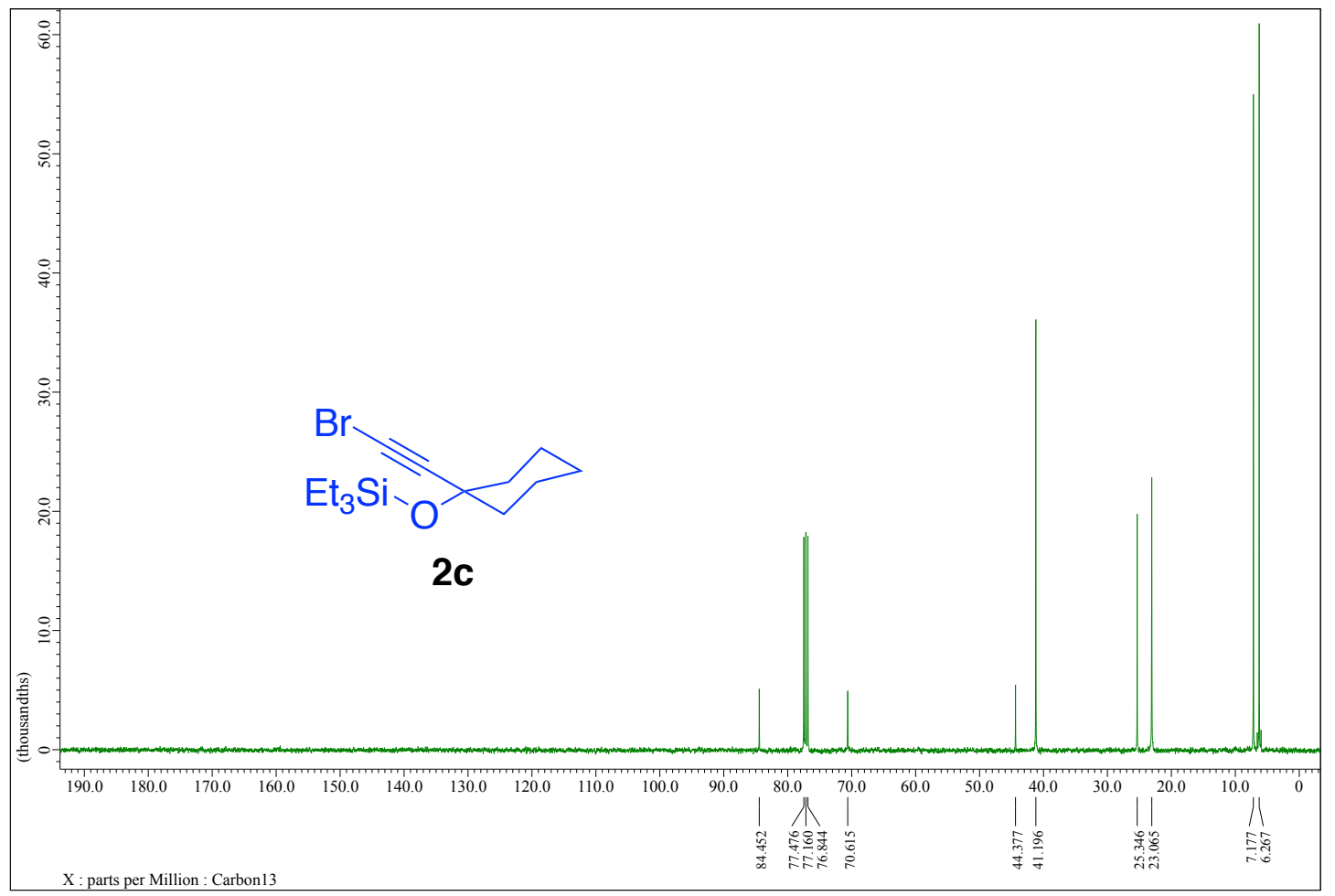

Figure S83: ${ }^{13} \mathrm{C}$ NMR spectrum of compound $2 \mathbf{c}\left(100 \mathrm{MHz}, \mathrm{CDCl}_{3}\right)$. 


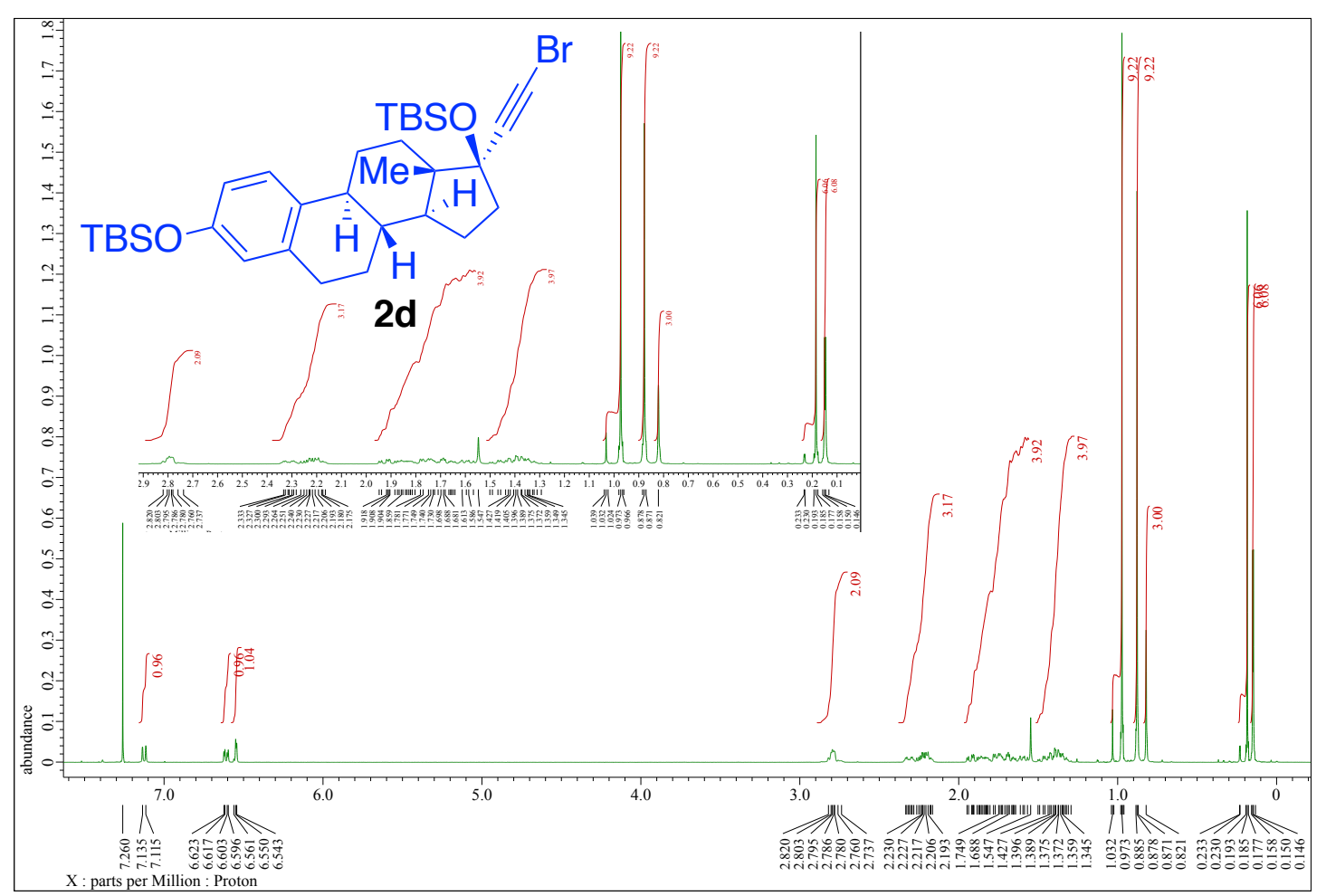

Figure S84: ${ }^{1} \mathrm{H}$ NMR spectrum of compound 2d (400 $\left.\mathrm{MHz}, \mathrm{CDCl}_{3}\right)$

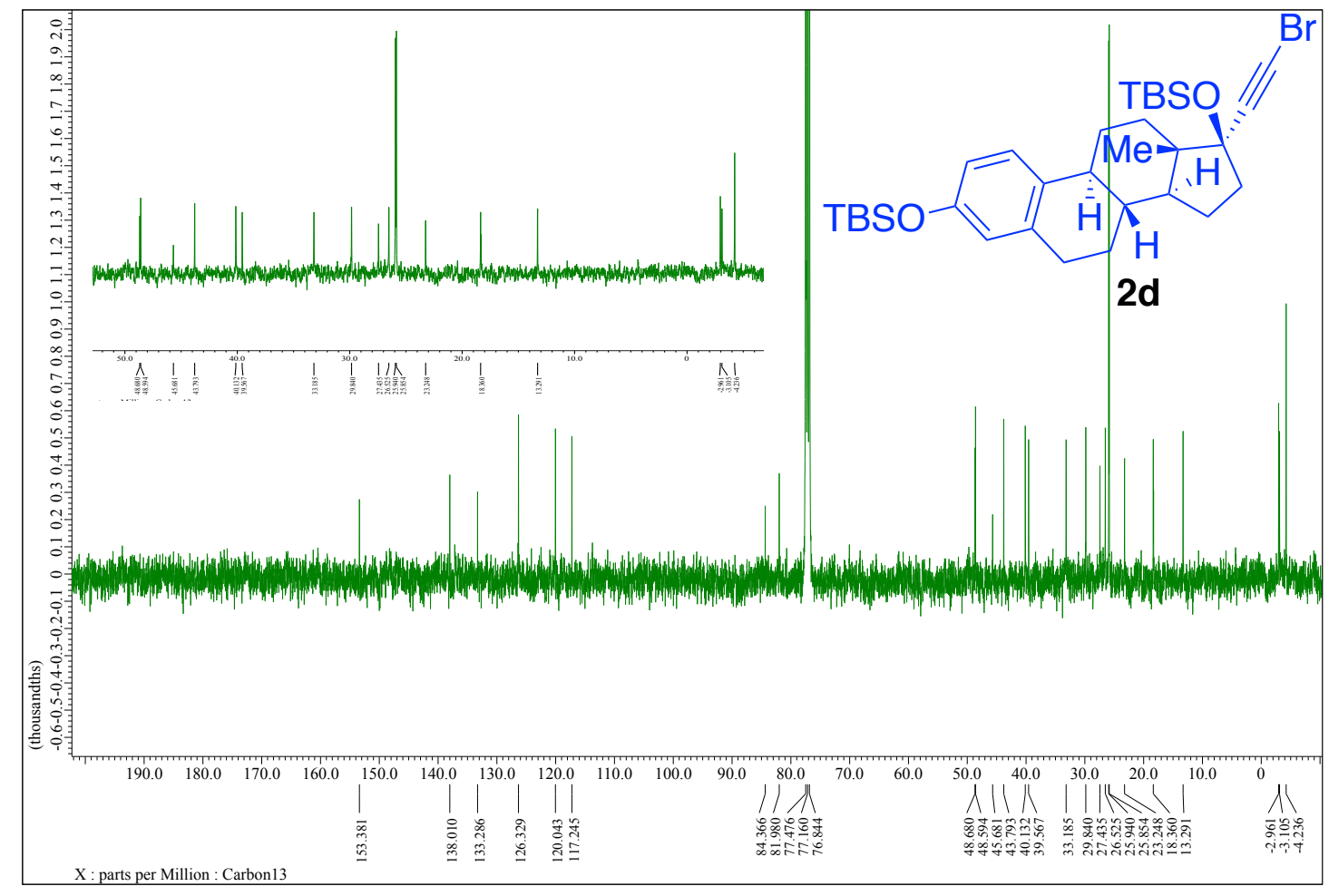

Figure S85: ${ }^{13} \mathrm{C}$ NMR spectrum of compound $2 d\left(100 \mathrm{MHz}, \mathrm{CDCl}_{3}\right)$. 


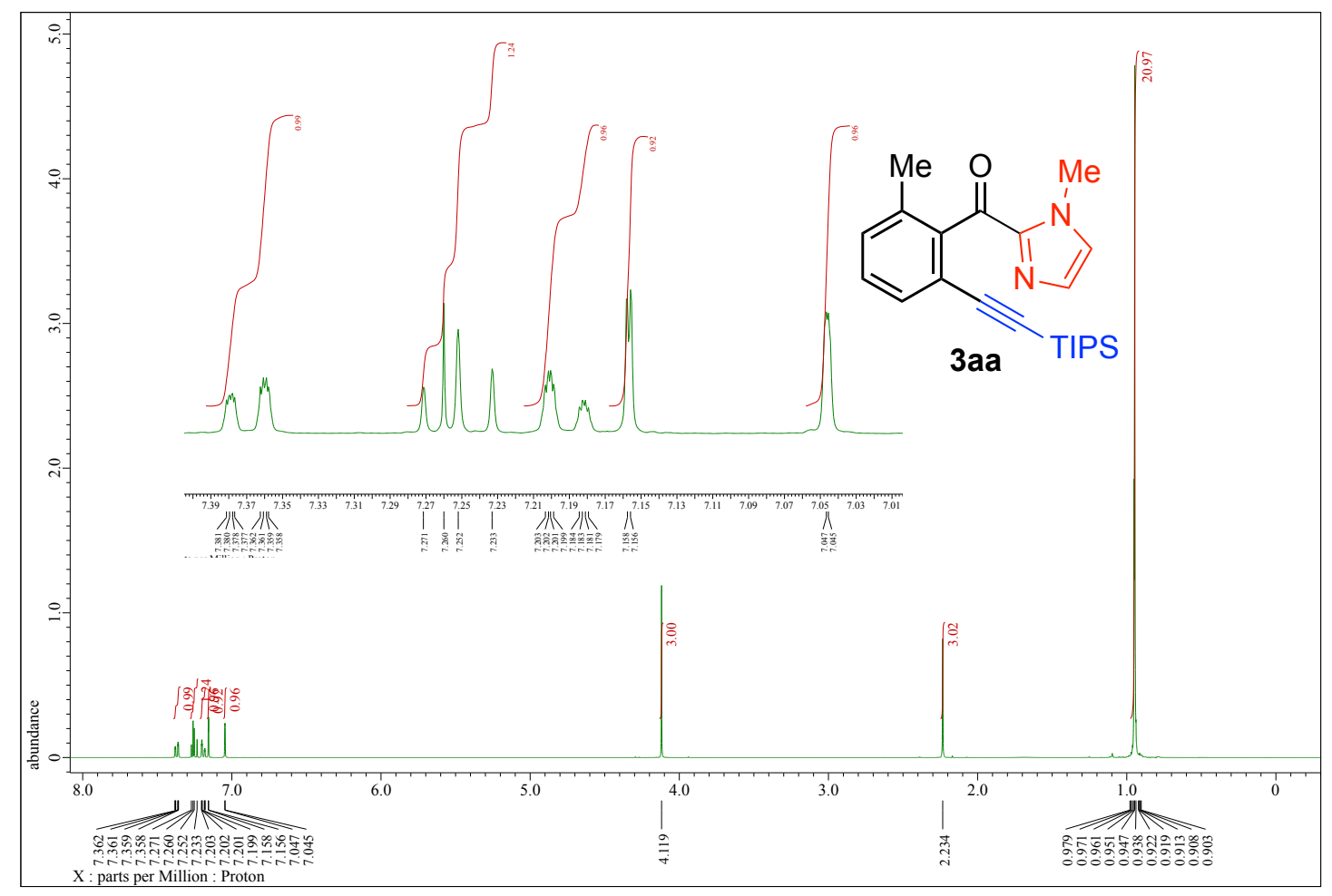

Figure S86: ${ }^{1} \mathrm{H}$ NMR spectrum of compound 3aa (400 $\mathrm{MHz}, \mathrm{CDCl}_{3}$ )

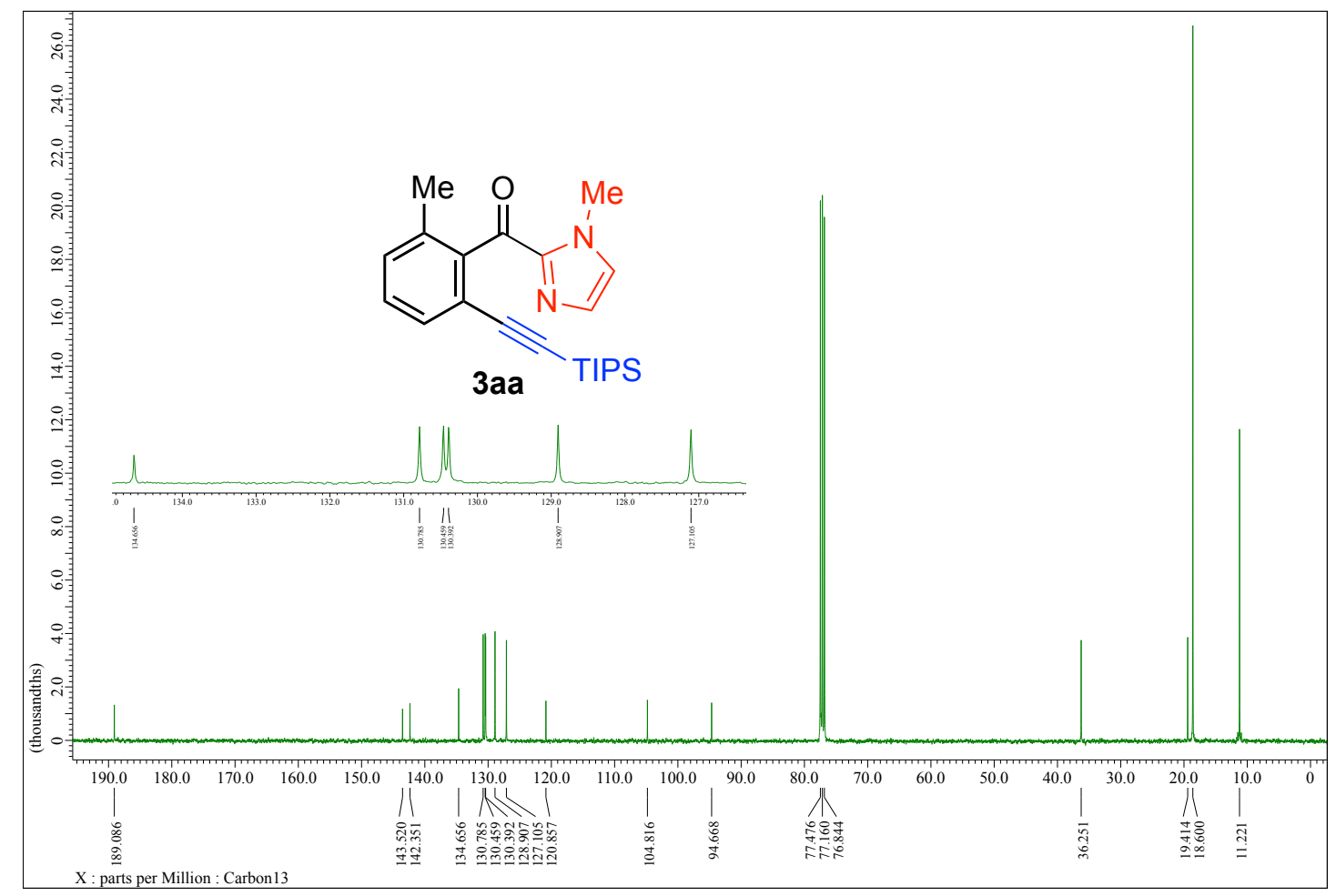

Figure S87: ${ }^{13} \mathrm{C}$ NMR spectrum of compound 3aa $\left(100 \mathrm{MHz}, \mathrm{CDCl}_{3}\right)$. 


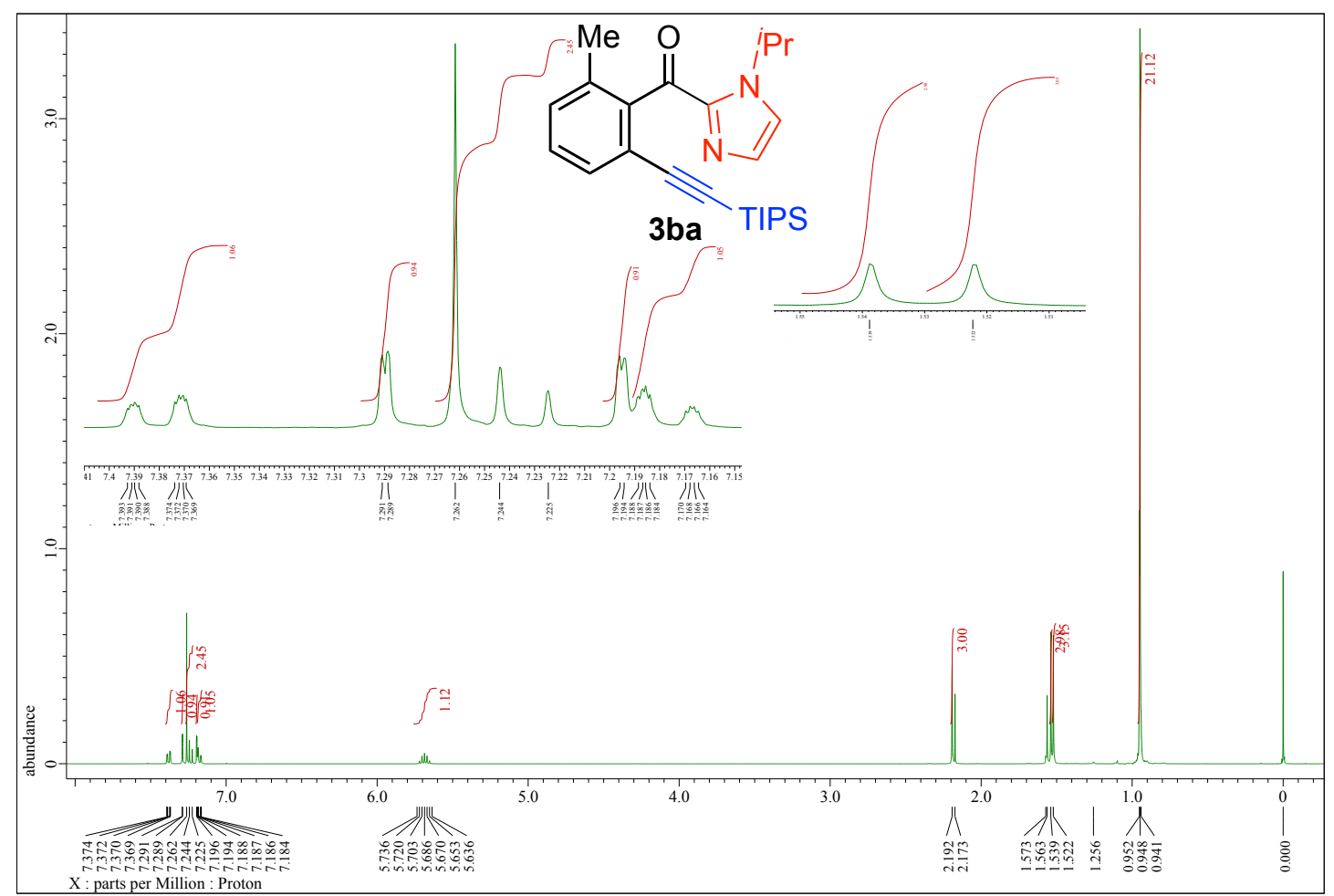

Figure S88: ${ }^{1} \mathrm{H}$ NMR spectrum of compound $\mathbf{3 b a}\left(400 \mathrm{MHz}, \mathrm{CDCl}_{3}\right)$

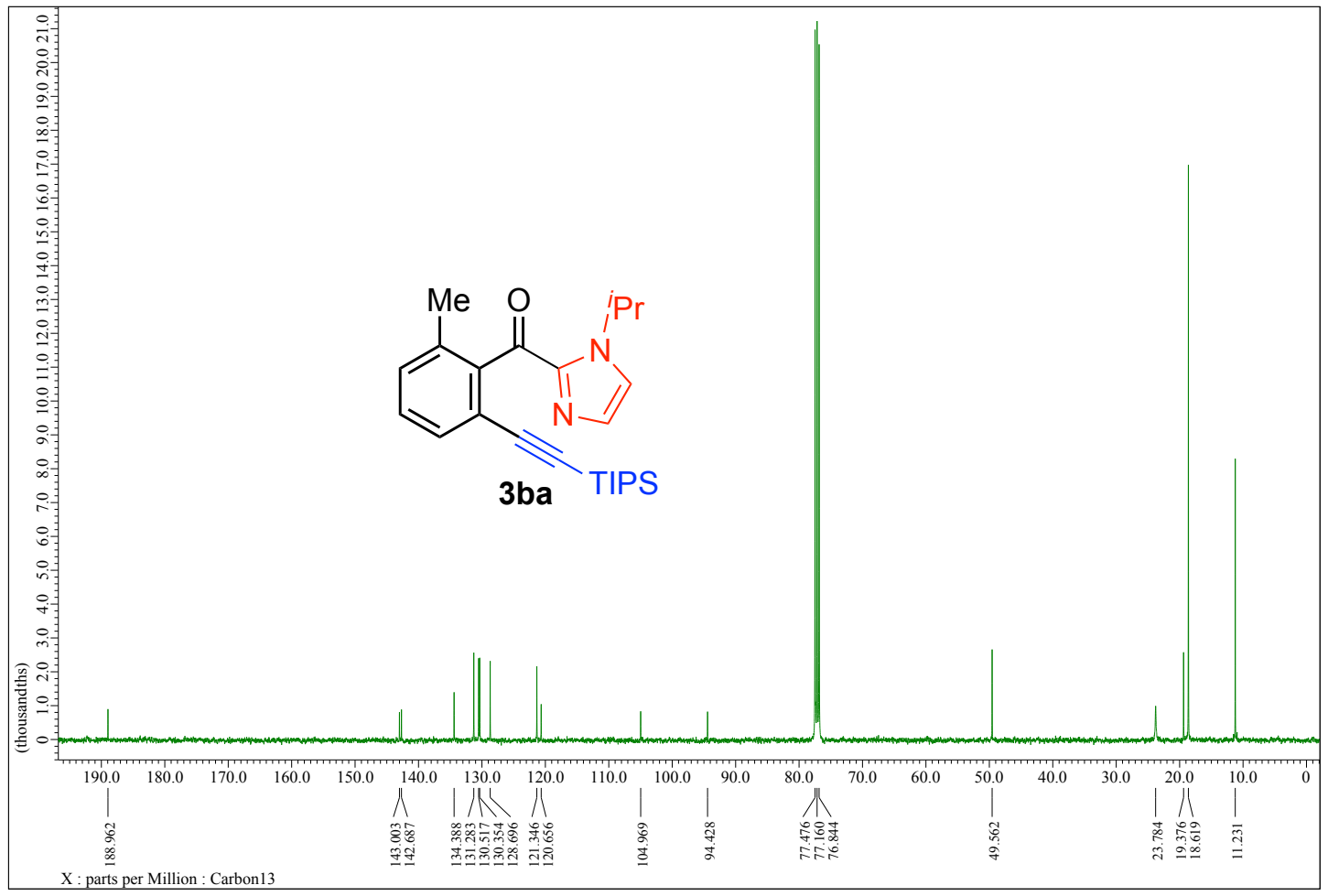

Figure S89: ${ }^{13} \mathrm{C}$ NMR spectrum of compound $3 \mathbf{b a}\left(100 \mathrm{MHz}, \mathrm{CDCl}_{3}\right)$. 


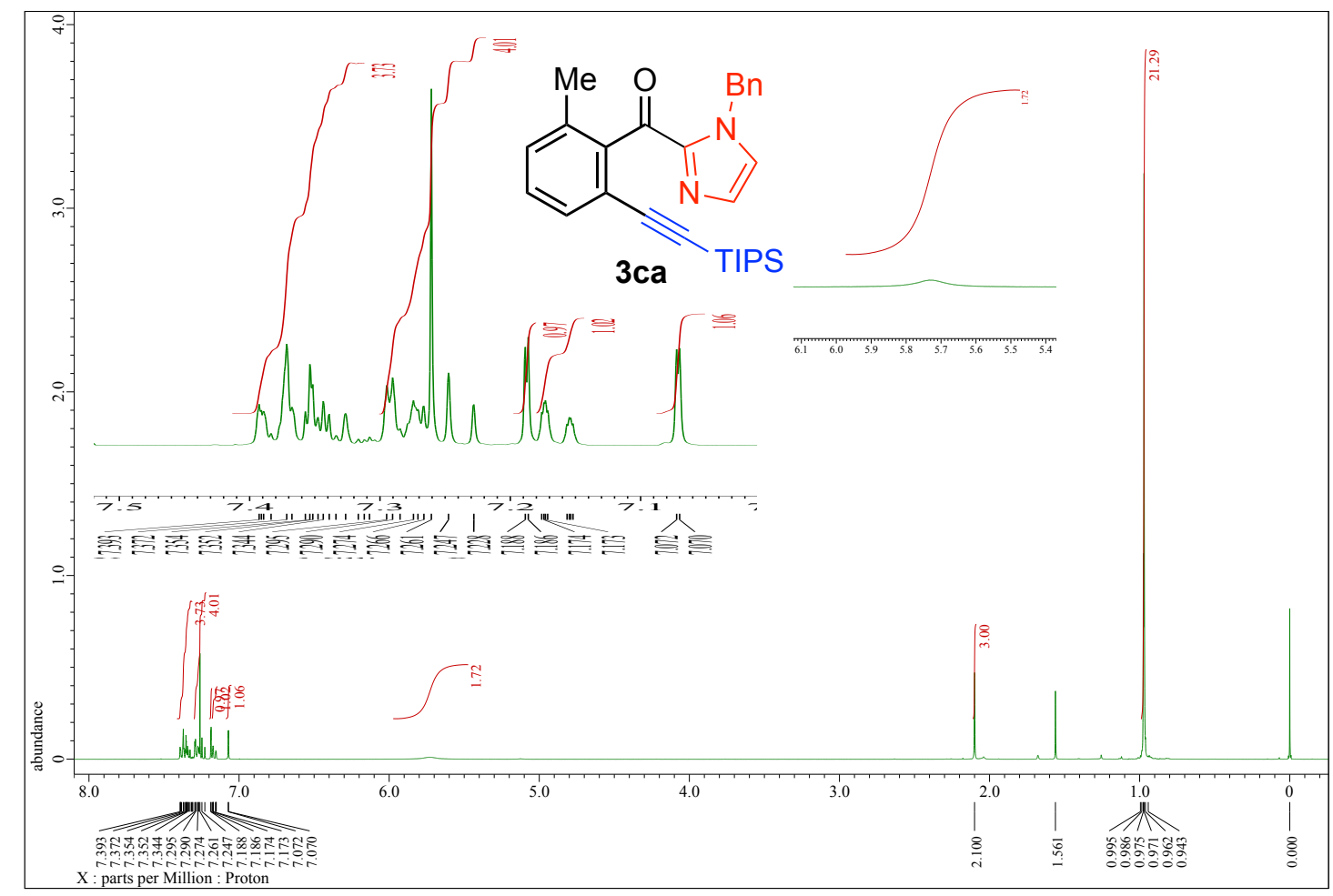

Figure S90: ${ }^{1} \mathrm{H}$ NMR spectrum of compound $3 \mathbf{c a}\left(400 \mathrm{MHz}, \mathrm{CDCl}_{3}\right)$.

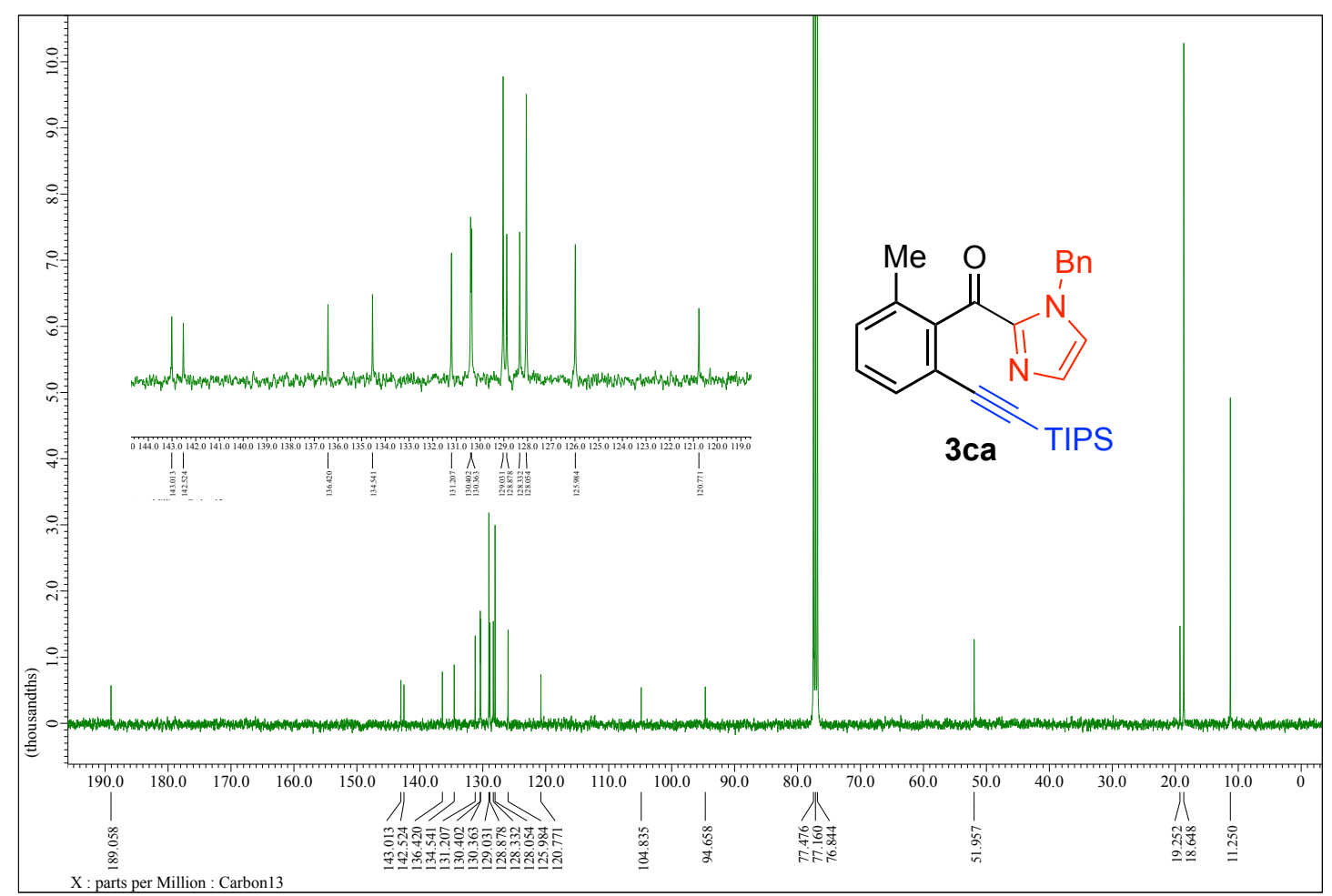

Figure S91: ${ }^{13} \mathrm{C}$ NMR spectrum of compound 3ca $\left(100 \mathrm{MHz}, \mathrm{CDCl}_{3}\right)$. 


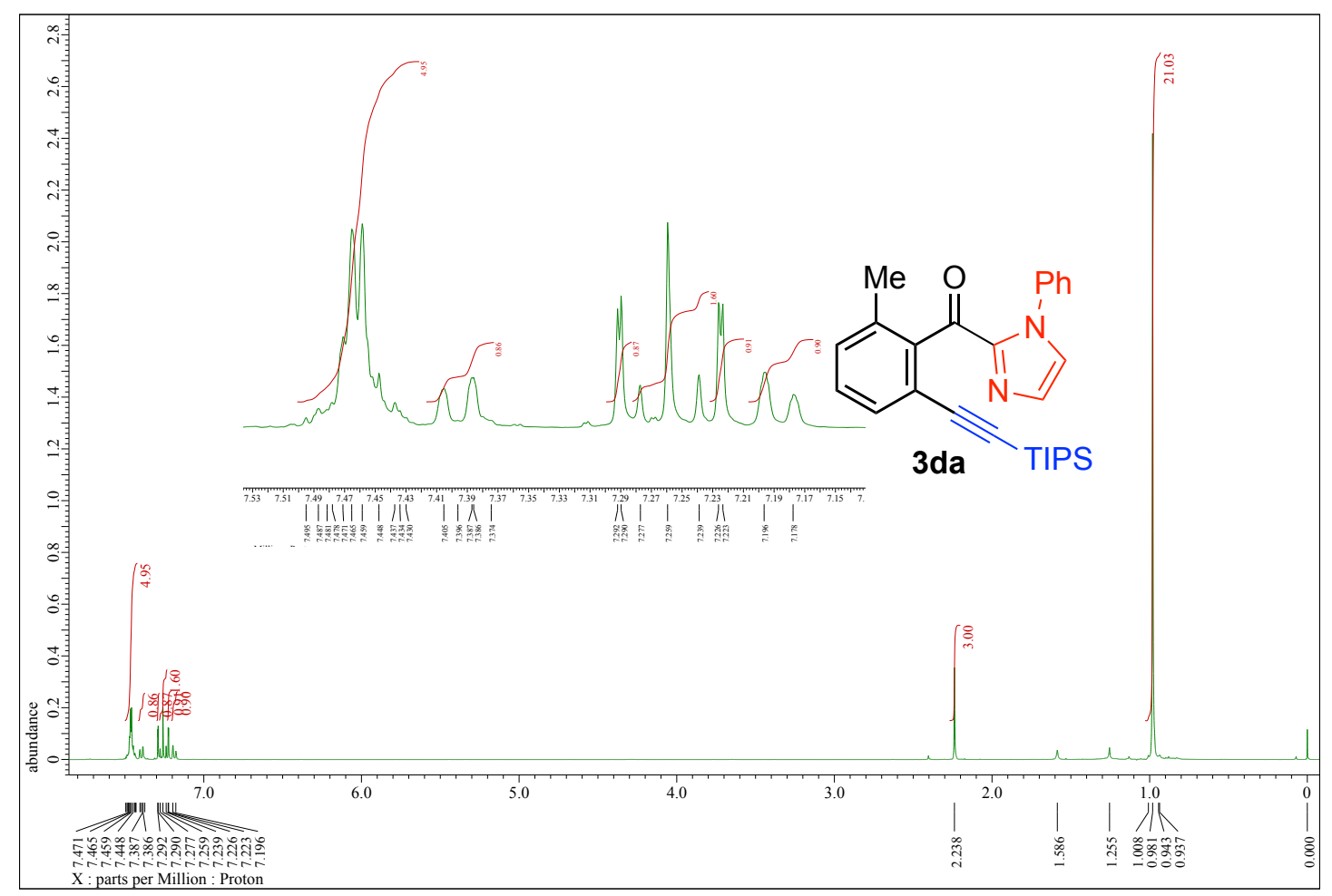

Figure S92: ${ }^{1} \mathrm{H}$ NMR spectrum of compound 3da (400 $\left.\mathrm{MHz}, \mathrm{CDCl}_{3}\right)$.

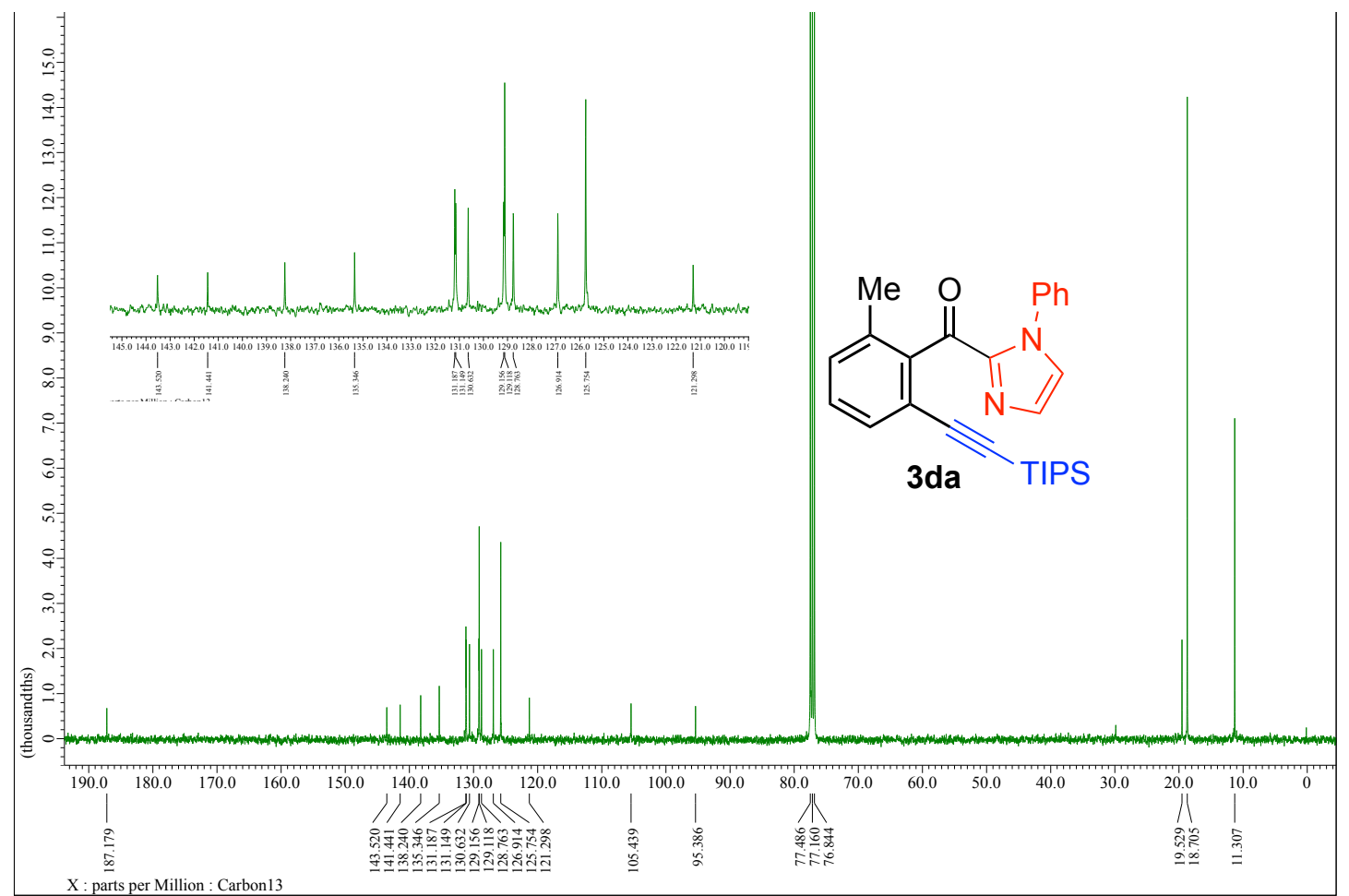

Figure S93: ${ }^{13} \mathrm{C}$ NMR spectrum of compound 3da $\left(100 \mathrm{MHz}, \mathrm{CDCl}_{3}\right)$. 


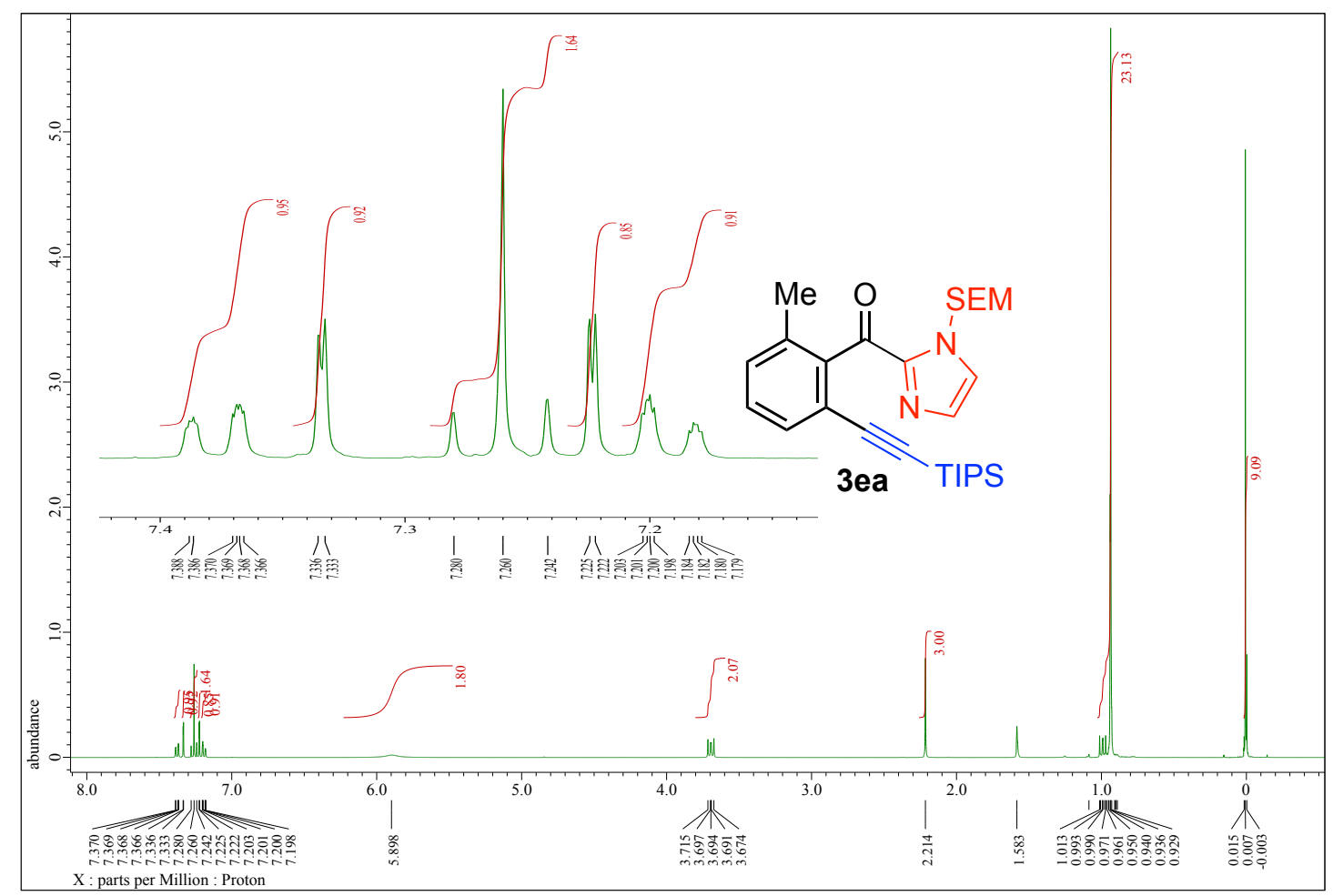

Figure S94: ${ }^{1} \mathrm{H}$ NMR spectrum of compound 3ea $\left(400 \mathrm{MHz}, \mathrm{CDCl}_{3}\right)$.

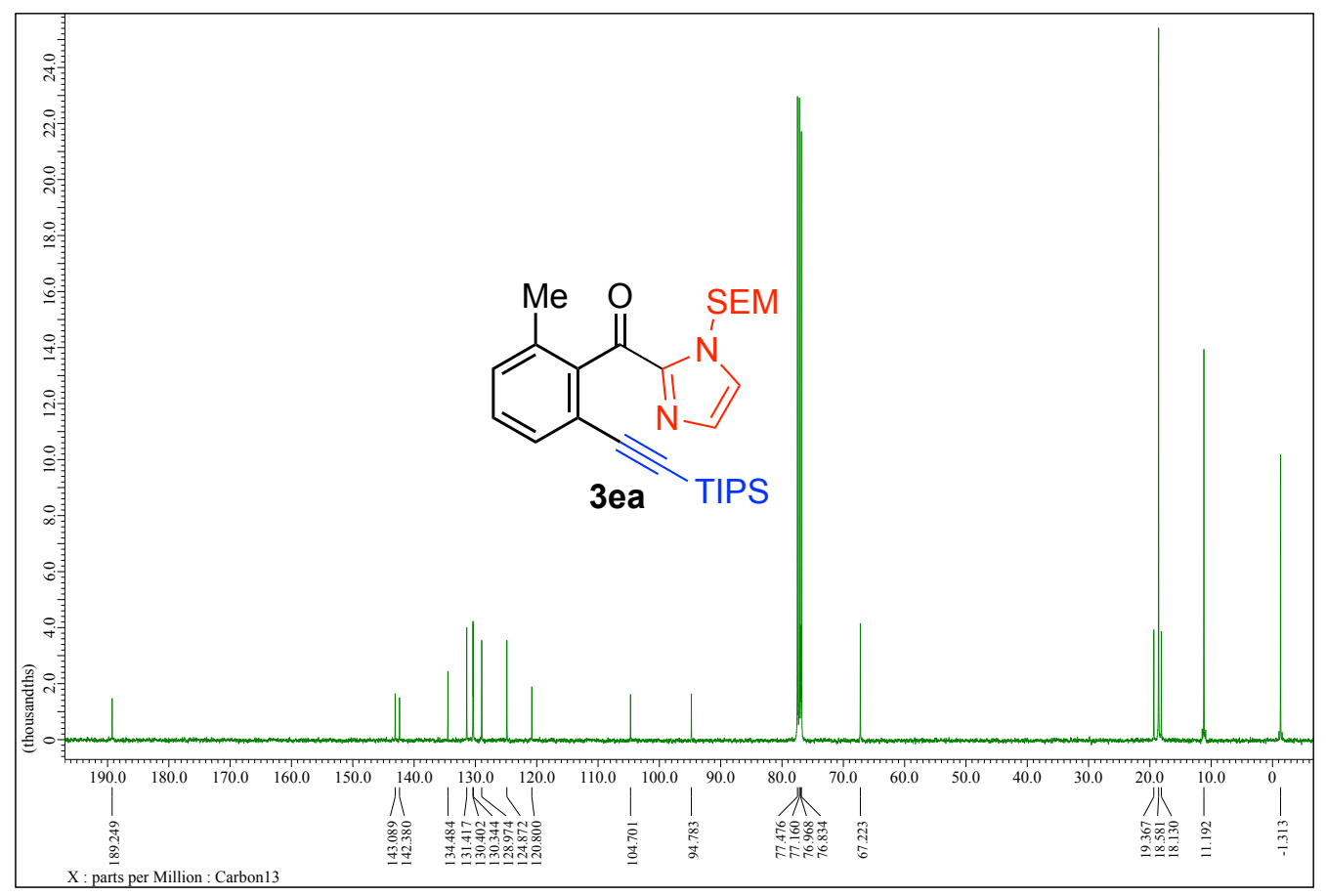

Figure S95: ${ }^{13} \mathrm{C}$ NMR spectrum of compound 3ea $\left(100 \mathrm{MHz}, \mathrm{CDCl}_{3}\right)$. 


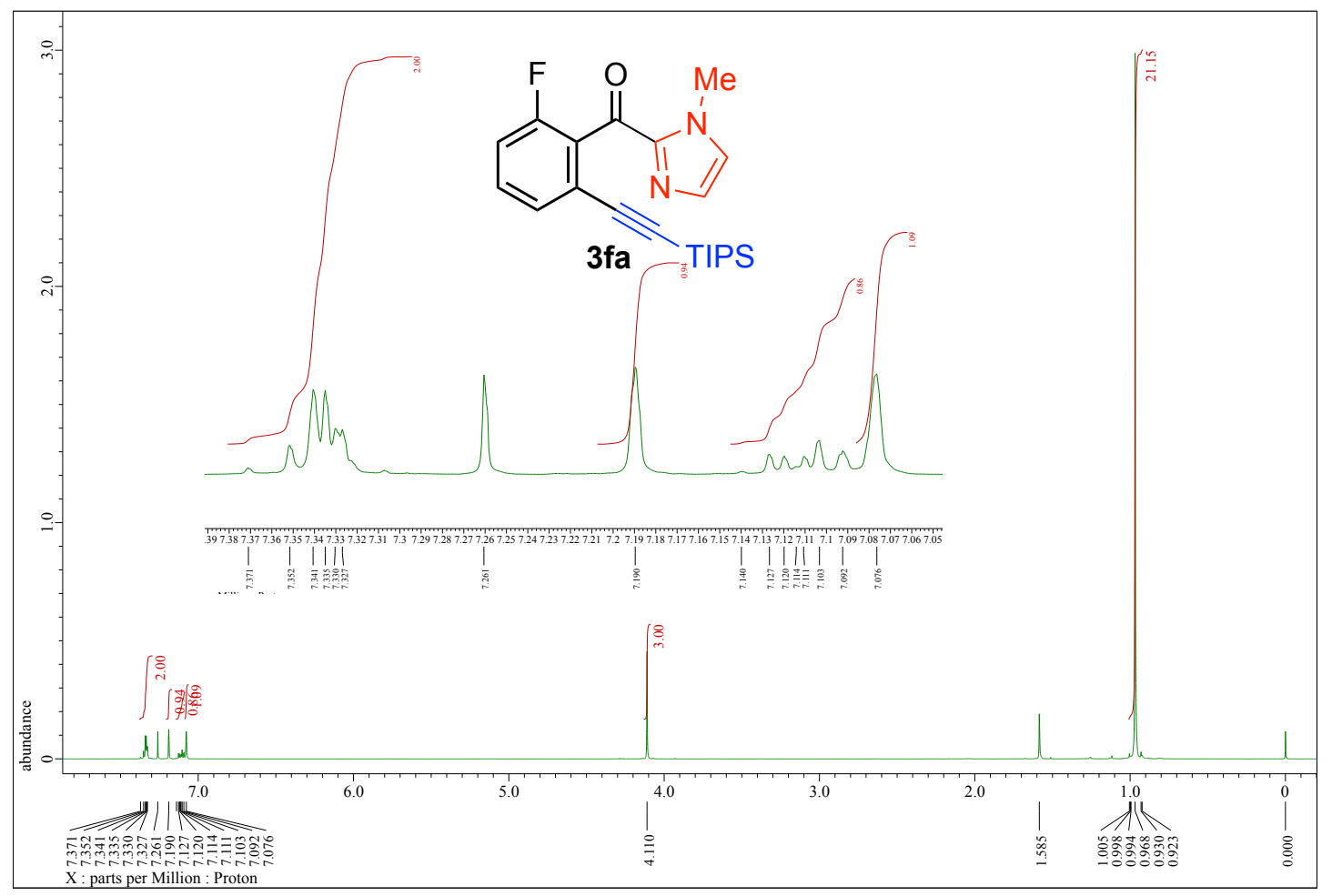

Figure S96: ${ }^{1} \mathrm{H}$ NMR spectrum of compound $\mathbf{3 f a}\left(400 \mathrm{MHz}, \mathrm{CDCl}_{3}\right)$.

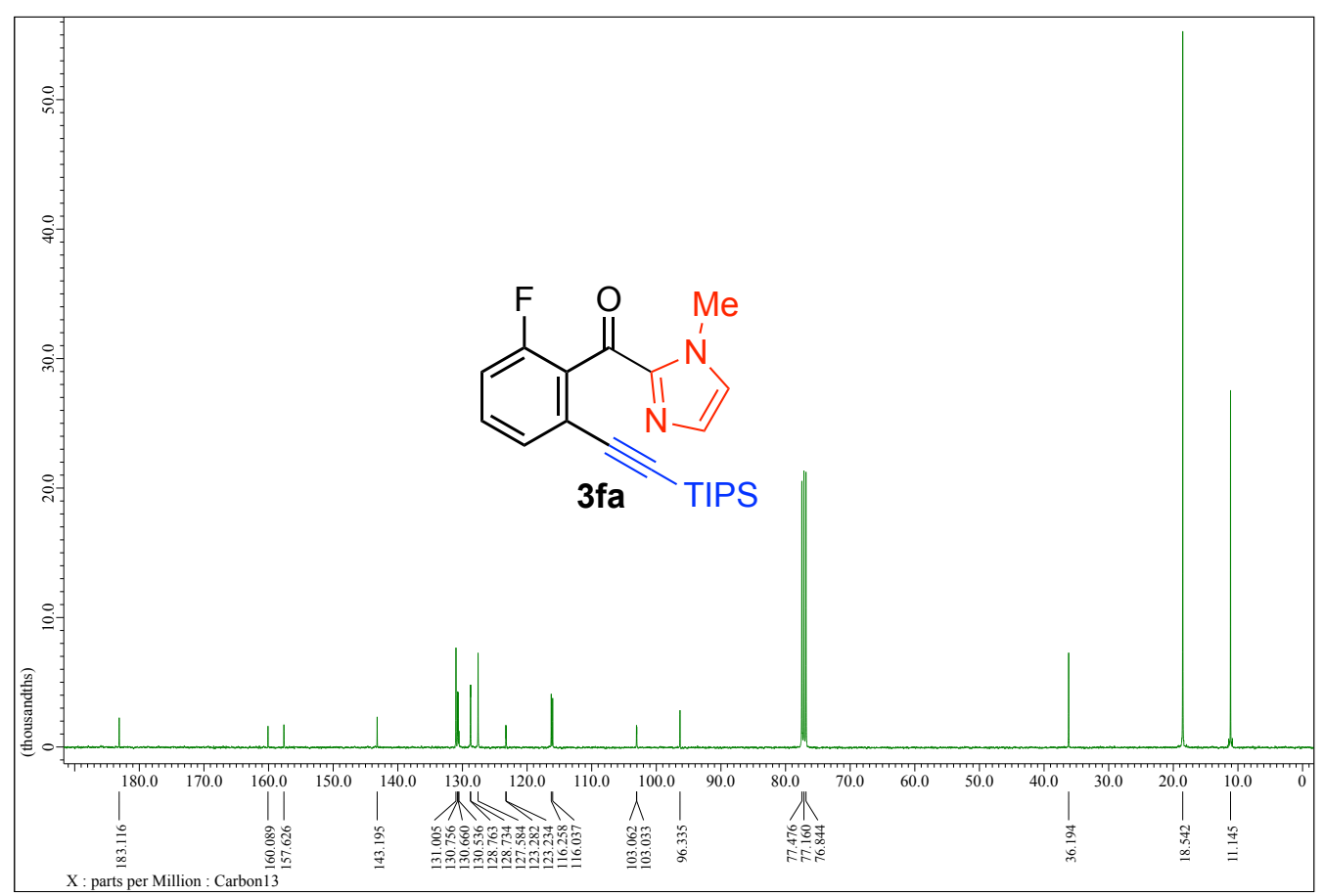

Figure S97: ${ }^{13} \mathrm{C}$ NMR spectrum of compound $3 f a\left(100 \mathrm{MHz}, \mathrm{CDCl}_{3}\right)$. 


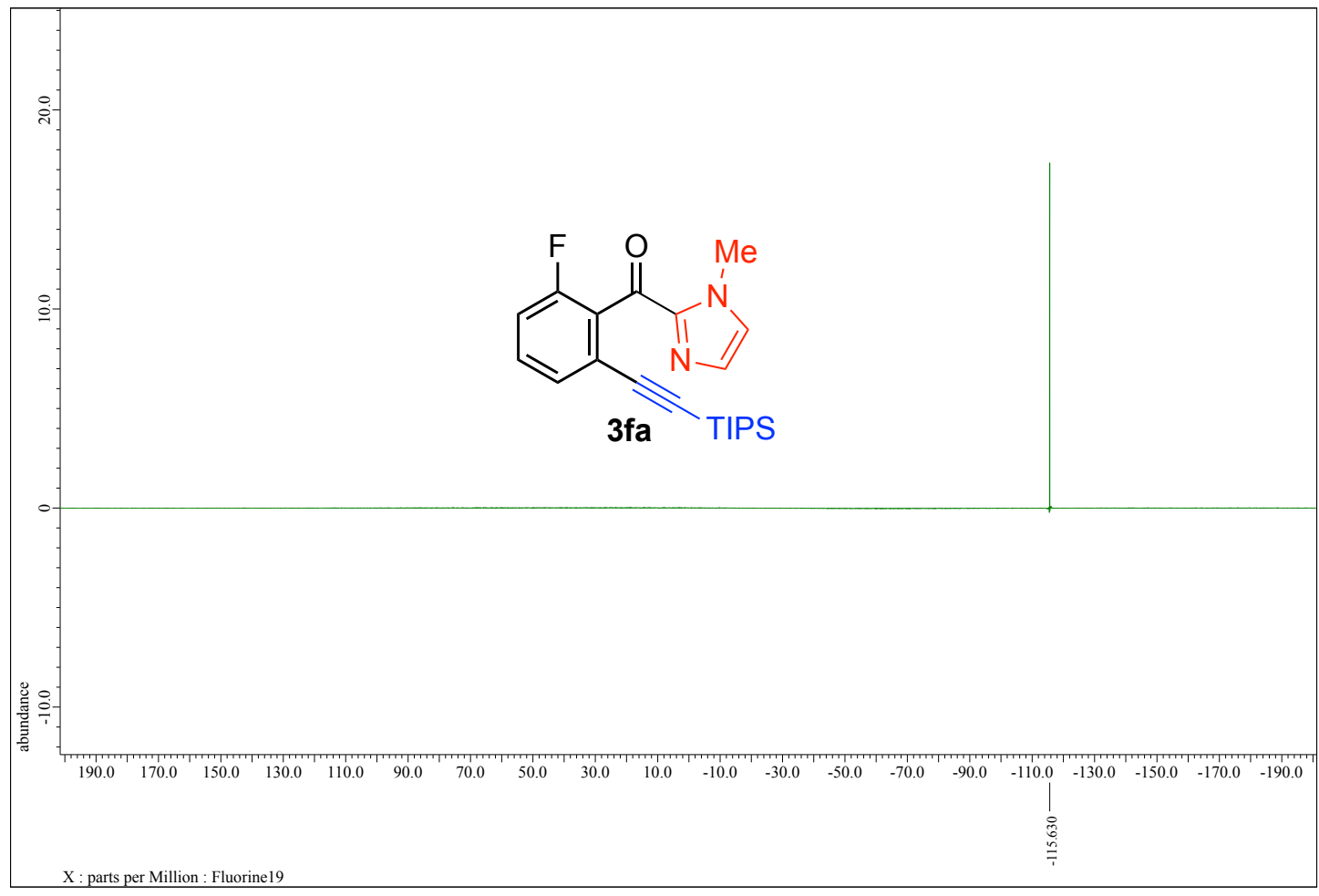

Figure S98: ${ }^{19} \mathrm{~F}$ NMR spectrum of compound $3 f a\left(376 \mathrm{MHz}, \mathrm{CDCl}_{3}\right)$.

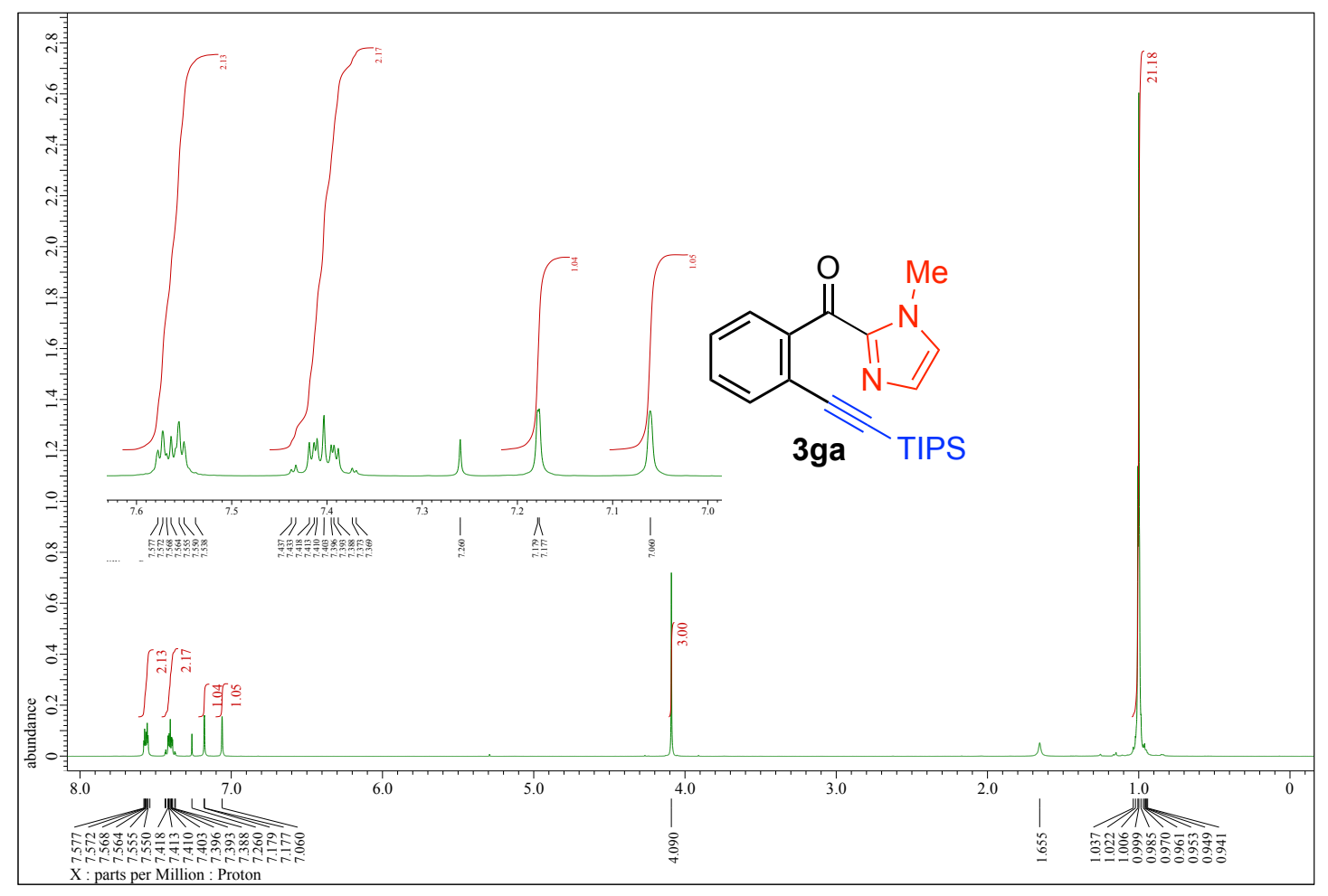

Figure S99: ${ }^{1} \mathrm{H}$ NMR spectrum of compound 3ga (400 $\left.\mathrm{MHz}, \mathrm{CDCl}_{3}\right)$. 


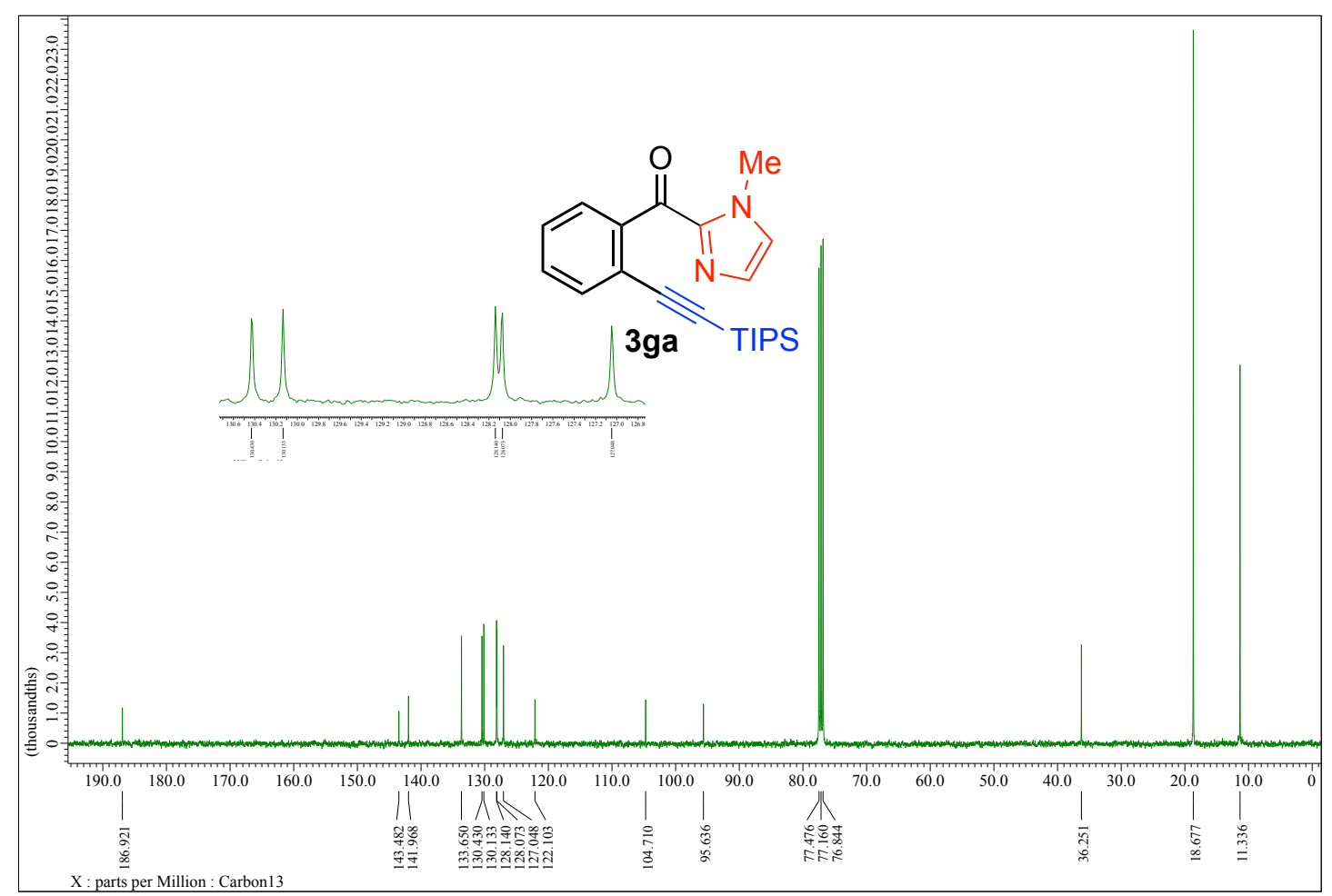

Figure S100: ${ }^{13} \mathrm{C}$ NMR spectrum of compound 3ga (100 $\left.\mathrm{MHz}, \mathrm{CDCl}_{3}\right)$.

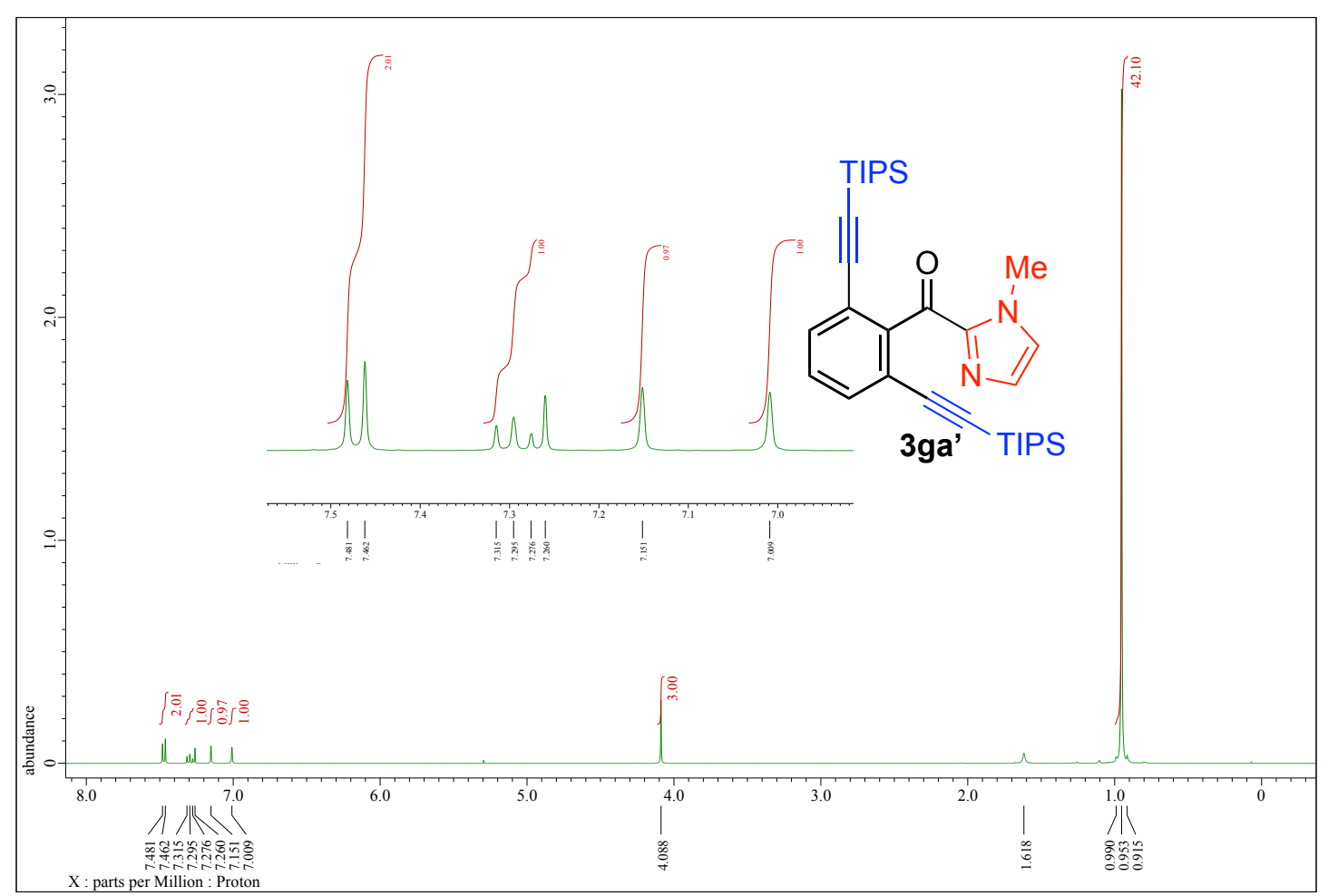

Figure S101: ${ }^{1} \mathrm{H}$ NMR spectrum of compound 3ga' (400 $\mathrm{MHz}, \mathrm{CDCl}_{3}$ ). 


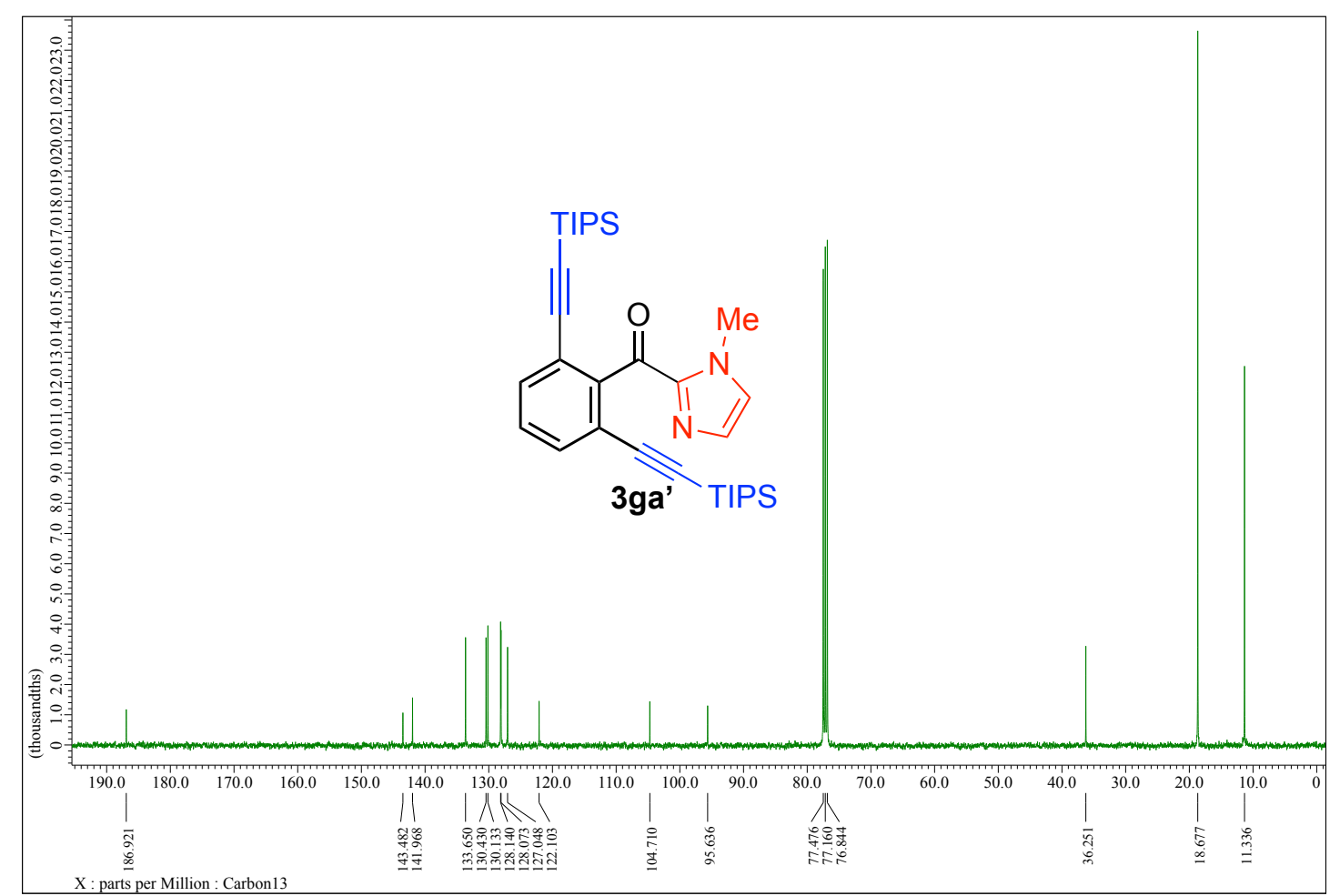

Figure S102: ${ }^{13} \mathrm{C}$ NMR spectrum of compound 3ga' (100 MHz, $\left.\mathrm{CDCl}_{3}\right)$.

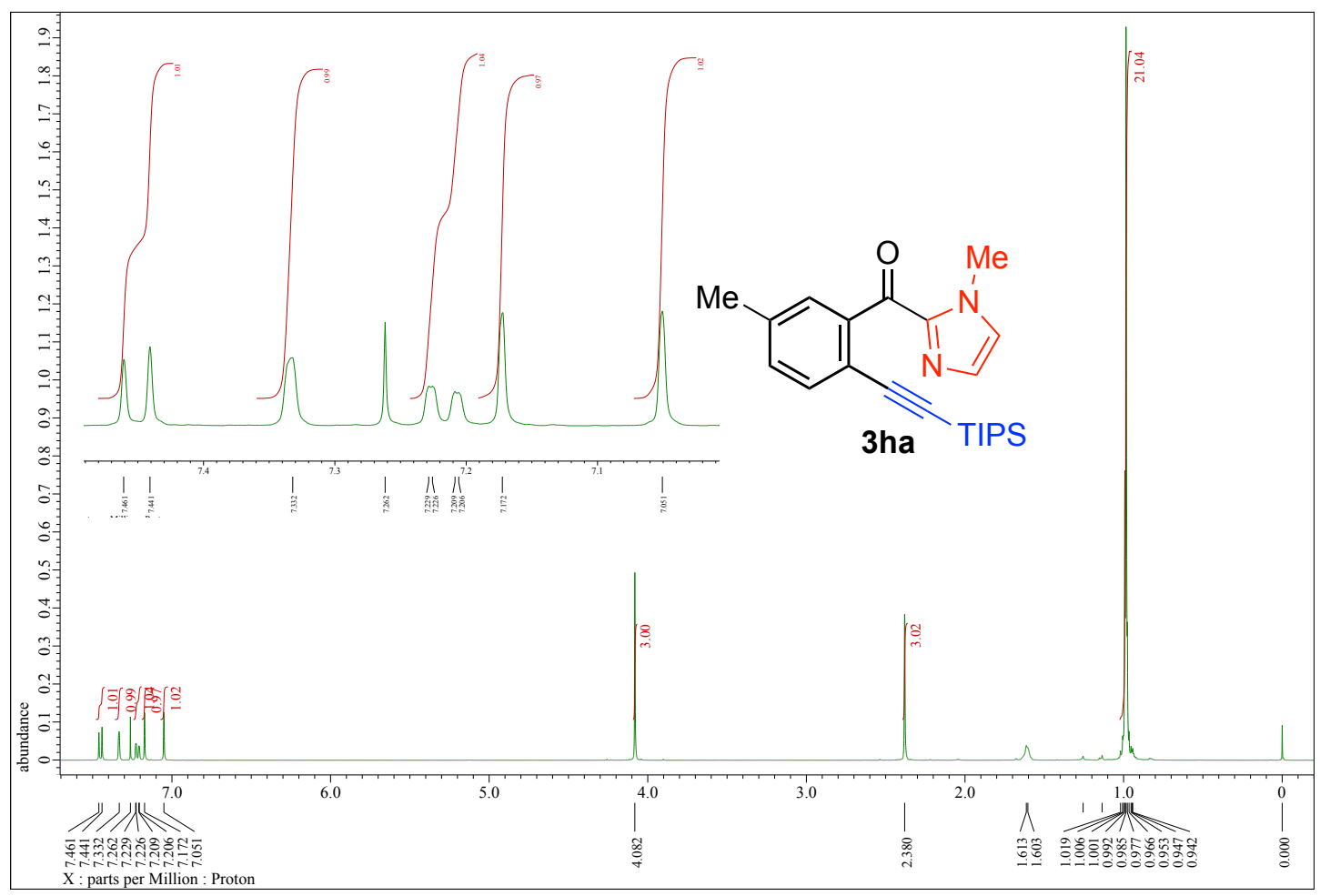

Figure S103: ${ }^{1} \mathrm{H}$ NMR spectrum of compound 3 ha $\left(400 \mathrm{MHz}, \mathrm{CDCl}_{3}\right)$. 


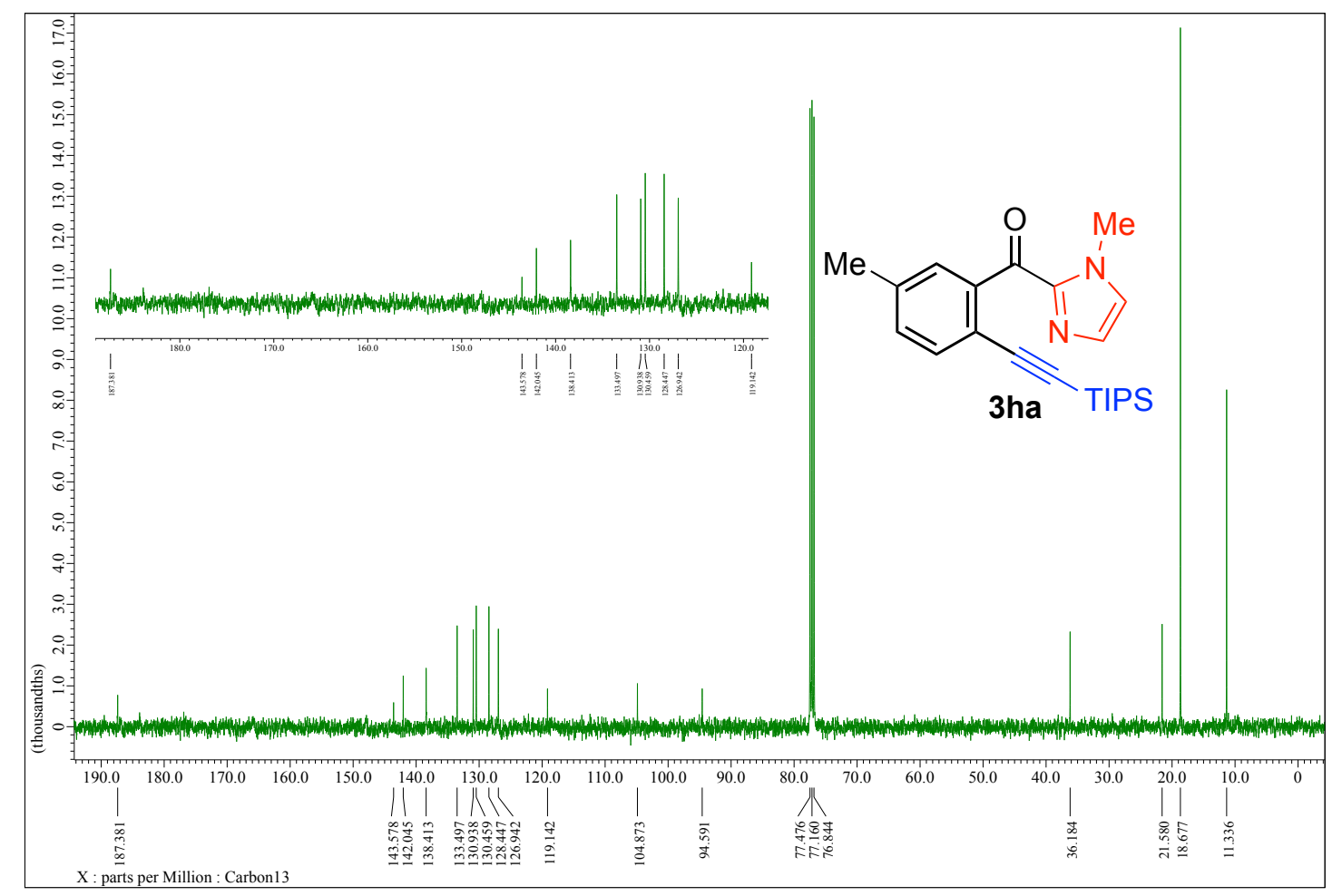

Figure S104: ${ }^{13} \mathrm{C}$ NMR spectrum of compound 3ha $\left(100 \mathrm{MHz}, \mathrm{CDCl}_{3}\right)$.

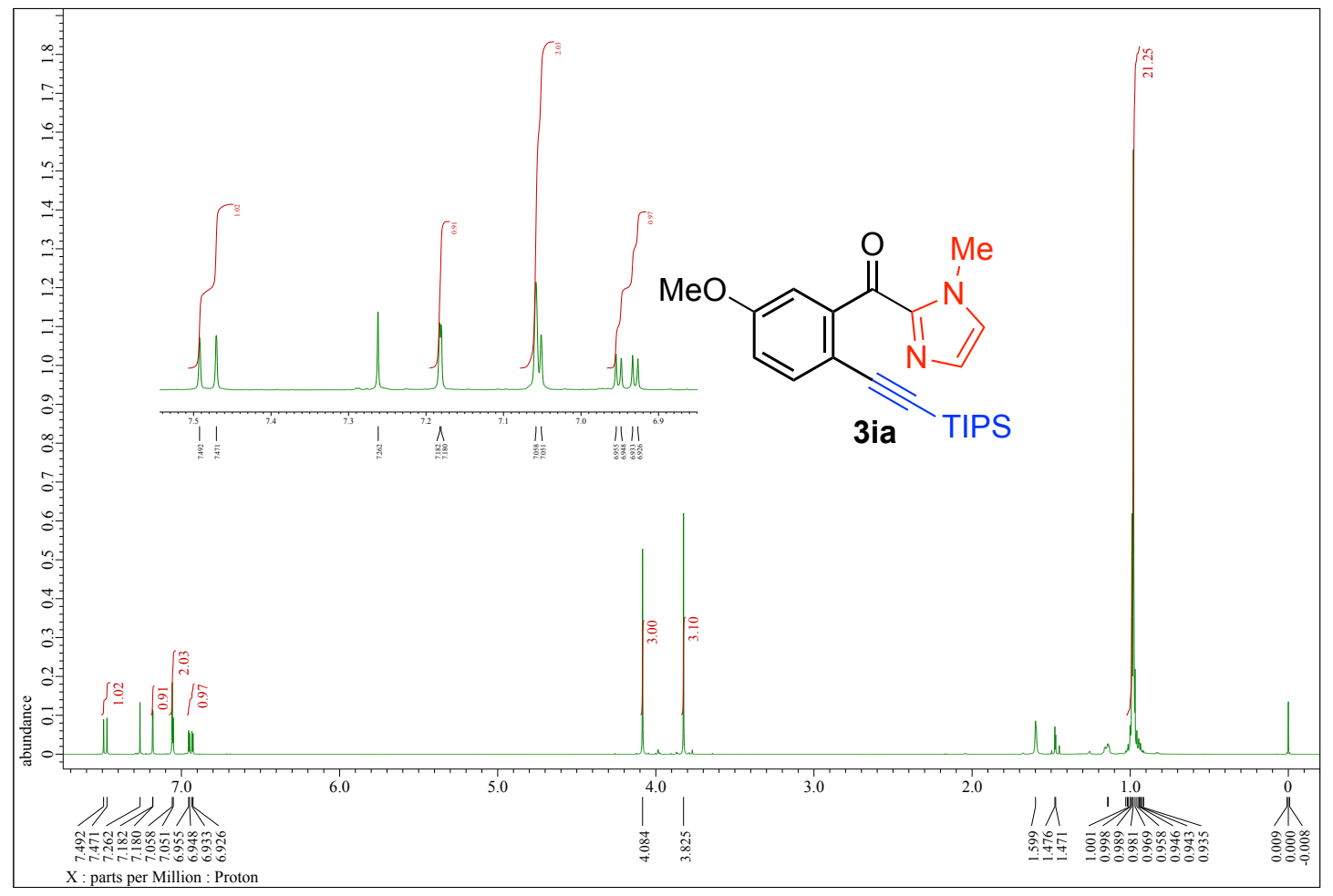

Figure S105: ${ }^{1} \mathrm{H}$ NMR spectrum of compound 3ia (400 $\mathrm{MHz}, \mathrm{CDCl}_{3}$ ). 


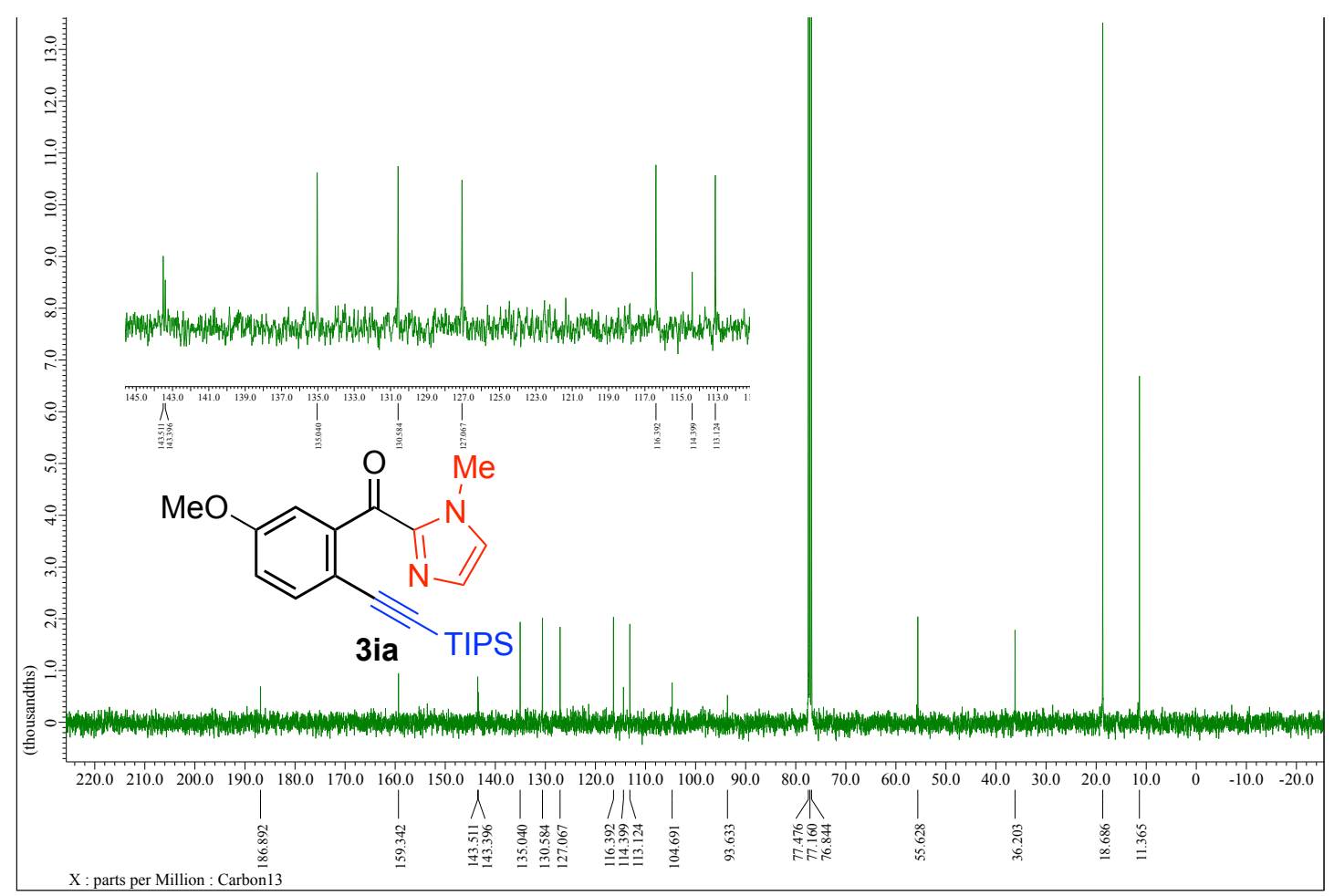

Figure S106: ${ }^{13} \mathrm{C}$ NMR spectrum of compound 3ia $\left(100 \mathrm{MHz}, \mathrm{CDCl}_{3}\right)$.

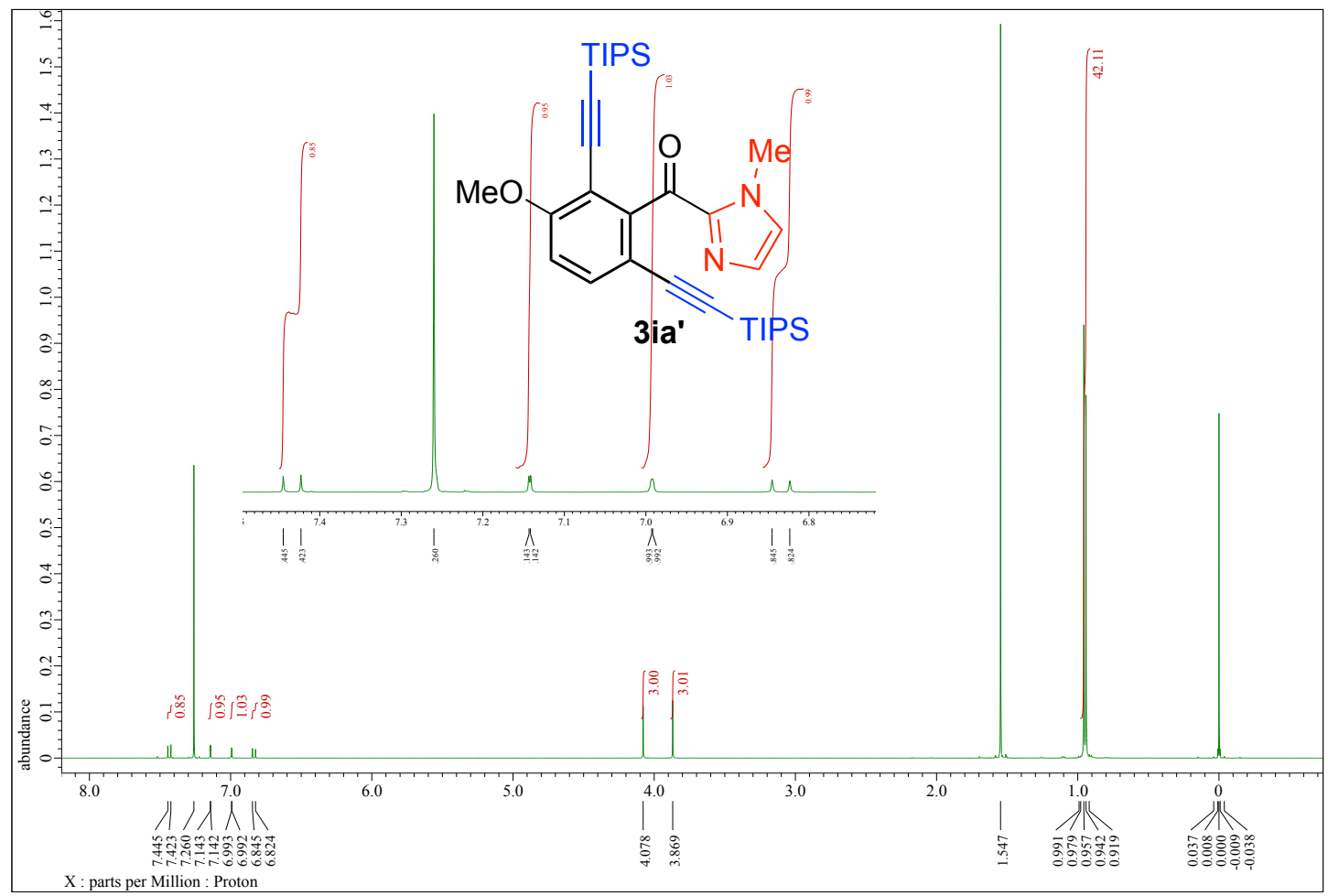

Figure S107: ' $\mathrm{H}$ NMR spectrum of compound 3ia' (400 MHz, $\mathrm{CDCl}_{3}$ ). 


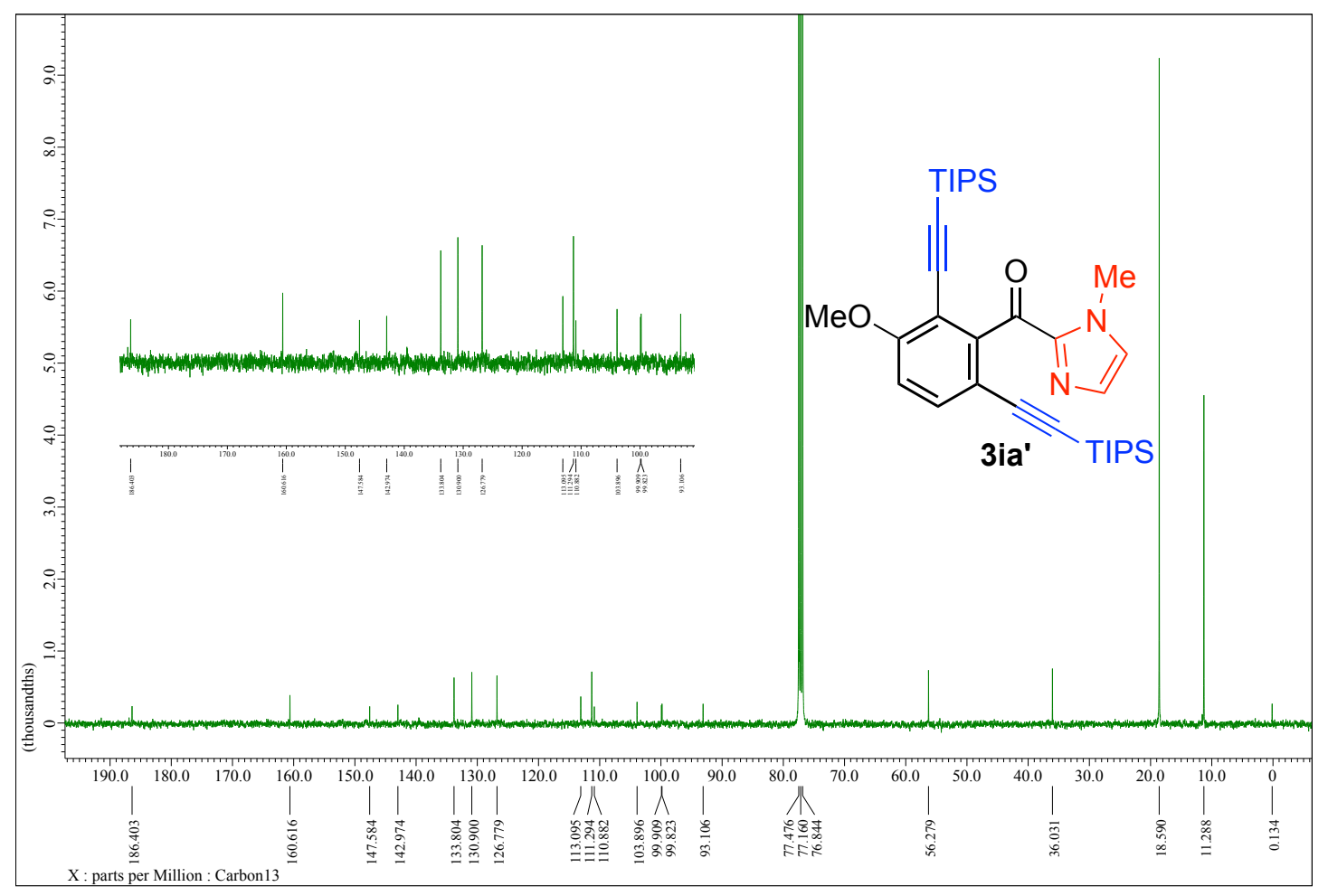

Figure S108: ${ }^{13} \mathrm{C}$ NMR spectrum of compound 3ia' $\left(100 \mathrm{MHz}, \mathrm{CDCl}_{3}\right)$.

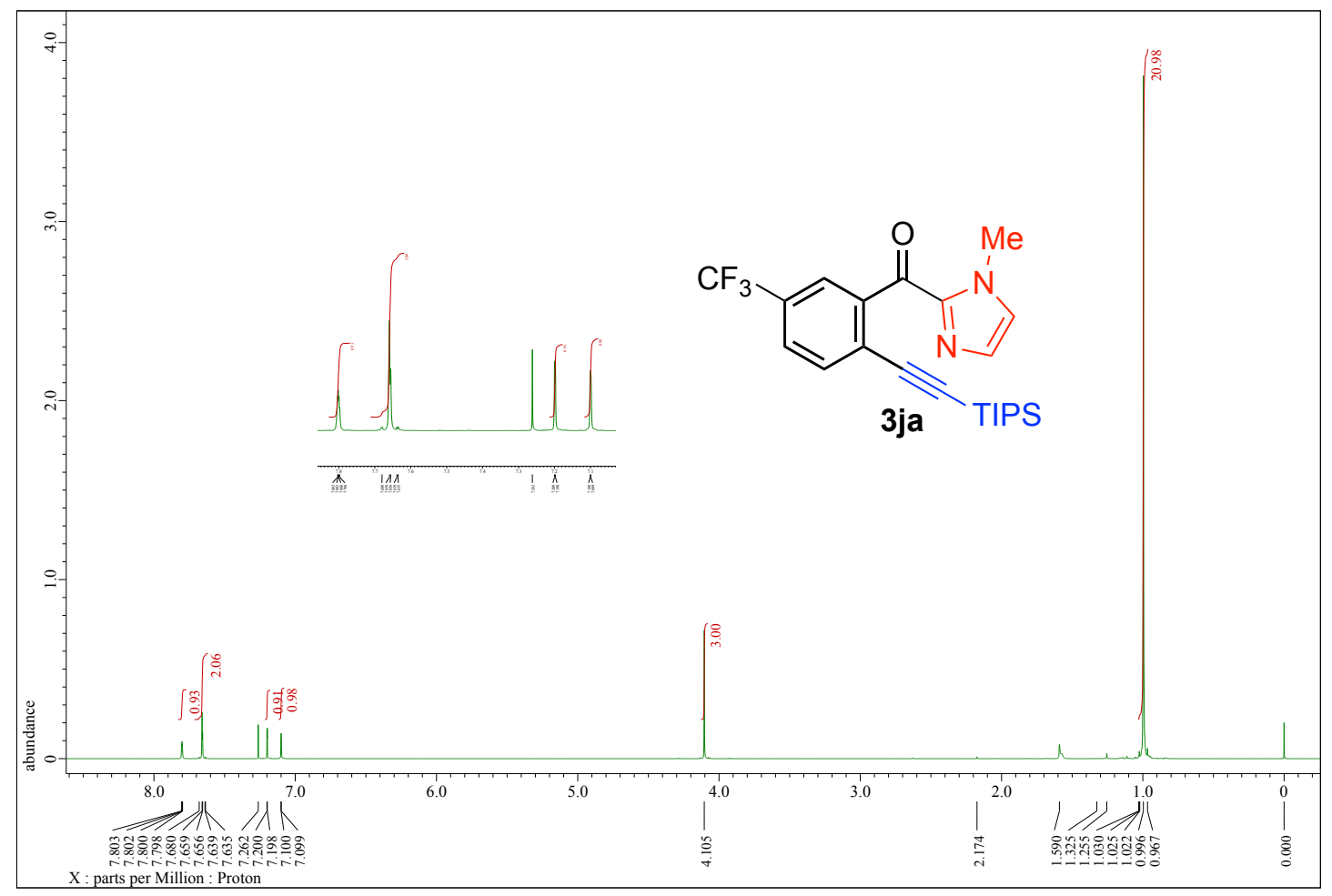

Figure S109: ${ }^{1} \mathrm{H}$ NMR spectrum of compound 3ja (400 MHz, $\mathrm{CDCl}_{3}$ ). 


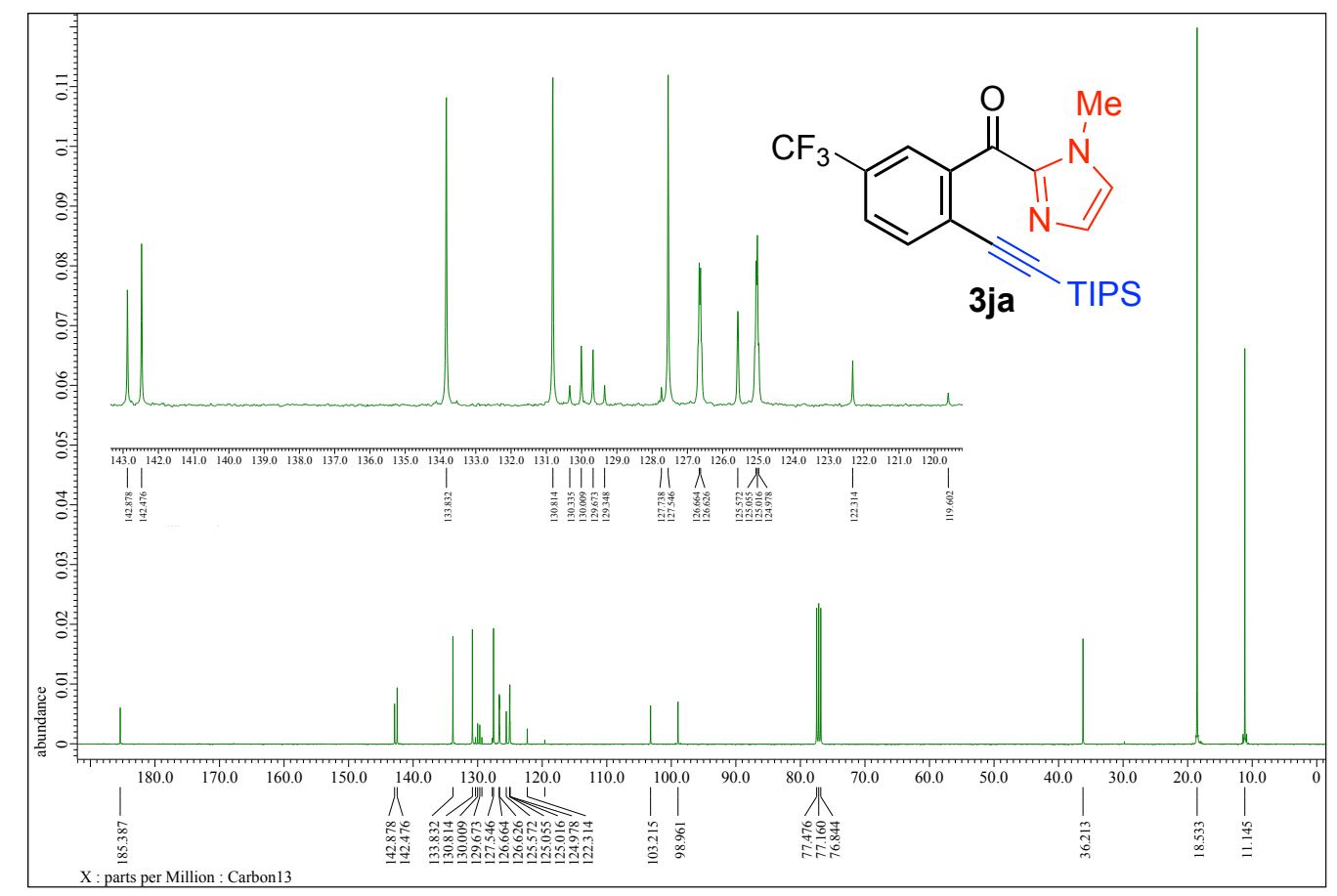

Figure S110: ${ }^{13} \mathrm{C}$ NMR spectrum of compound 3ja (100 $\left.\mathrm{MHz}, \mathrm{CDCl}_{3}\right)$.

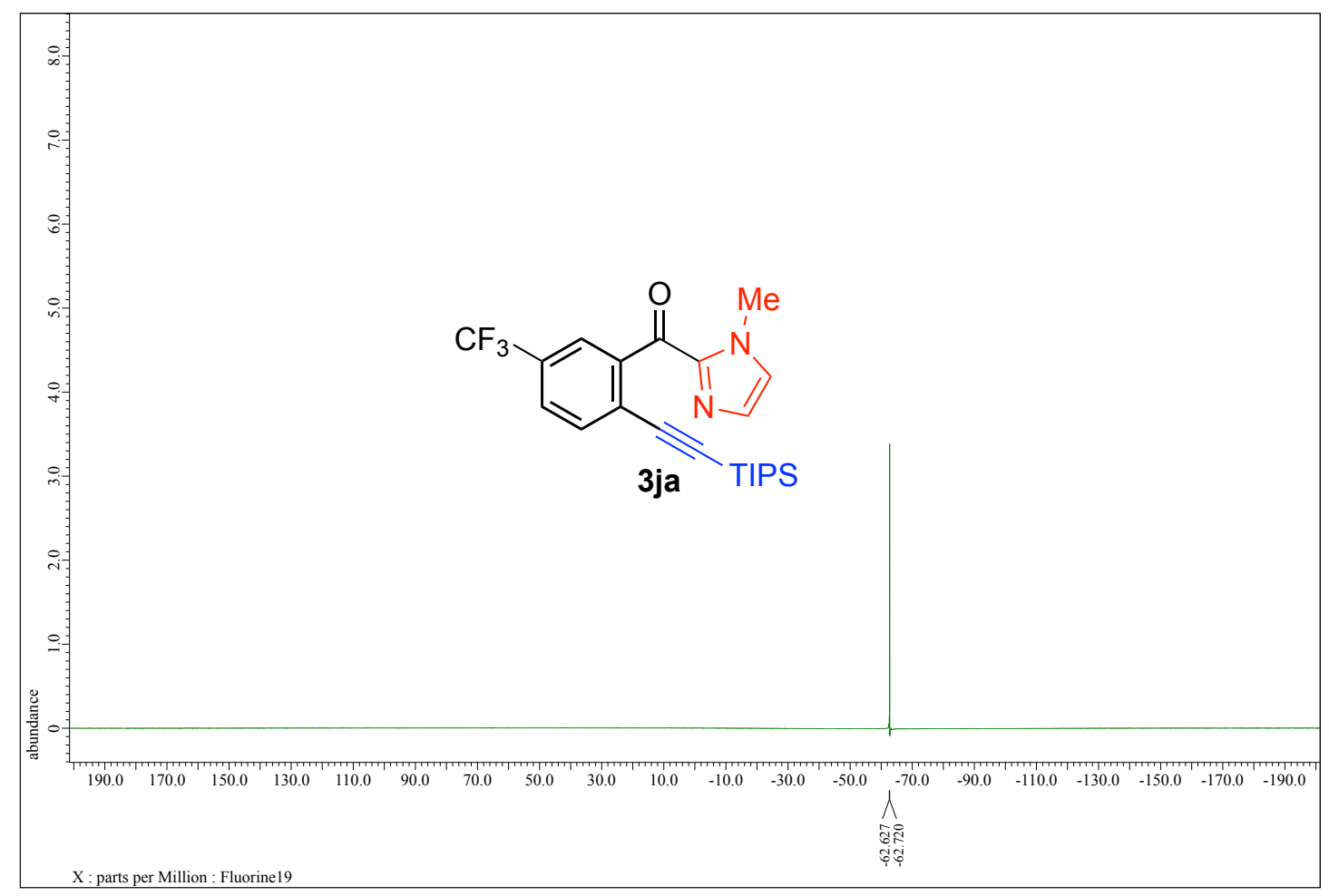

Figure S111: ${ }^{19} \mathrm{~F}$ NMR spectrum of compound $\mathbf{3 j a}\left(376 \mathrm{MHz}, \mathrm{CDCl}_{3}\right)$. 


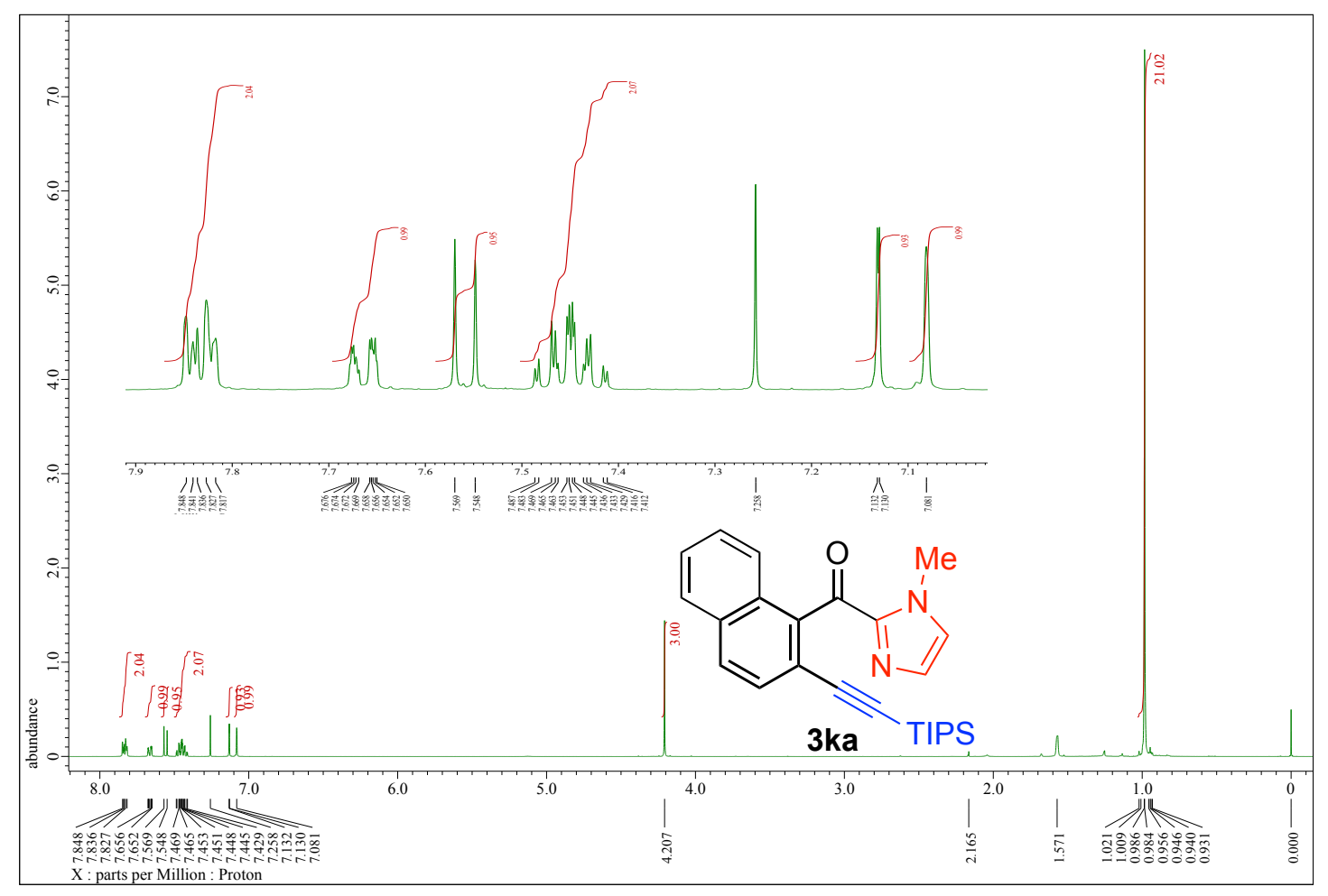

Figure S112: ${ }^{1} \mathrm{H}$ NMR spectrum of compound 3ka (400 $\left.\mathrm{MHz}, \mathrm{CDCl}_{3}\right)$.

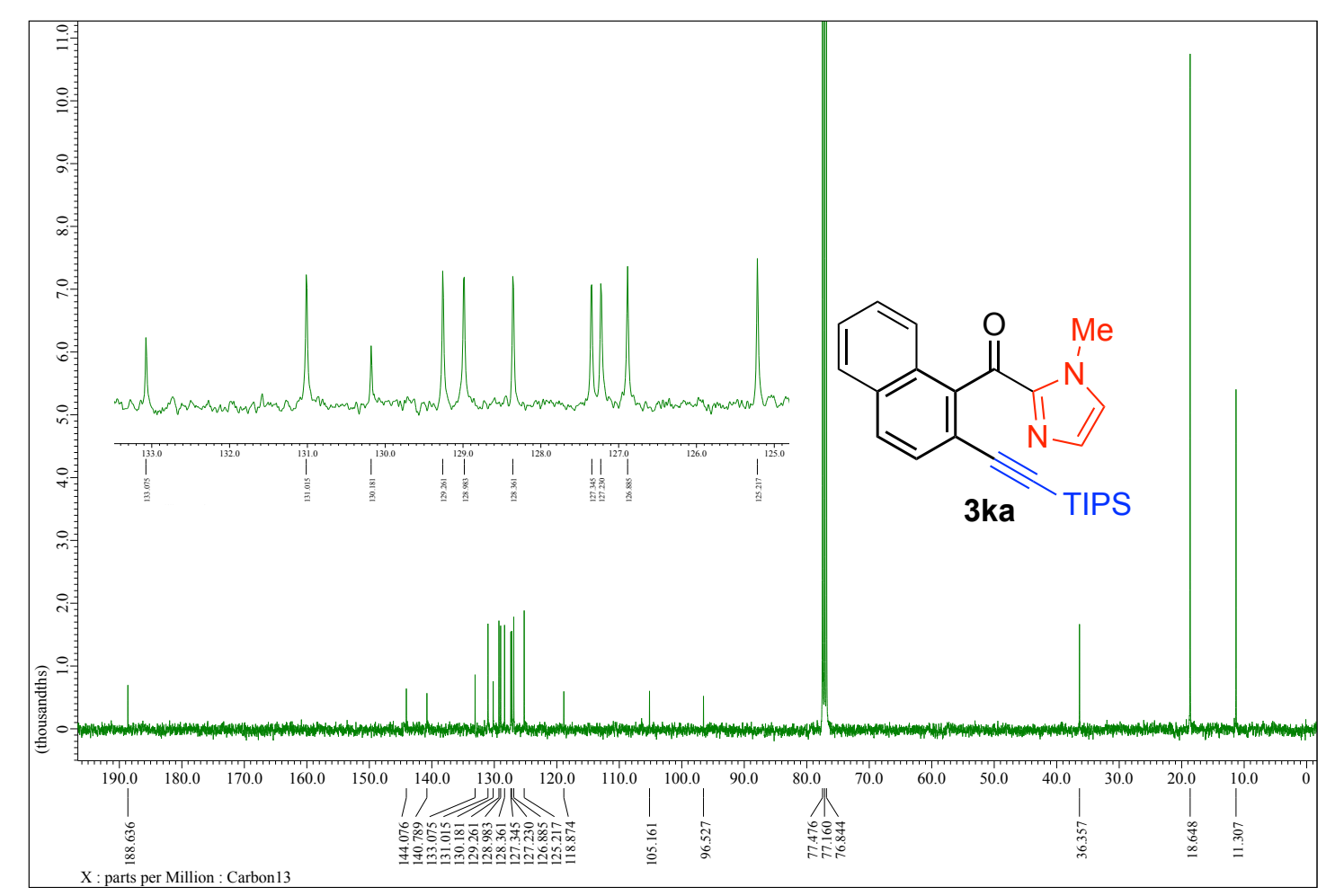

Figure S113: ${ }^{13} \mathrm{C}$ NMR spectrum of compound 3ka (100 $\left.\mathrm{MHz}, \mathrm{CDCl}_{3}\right)$. 


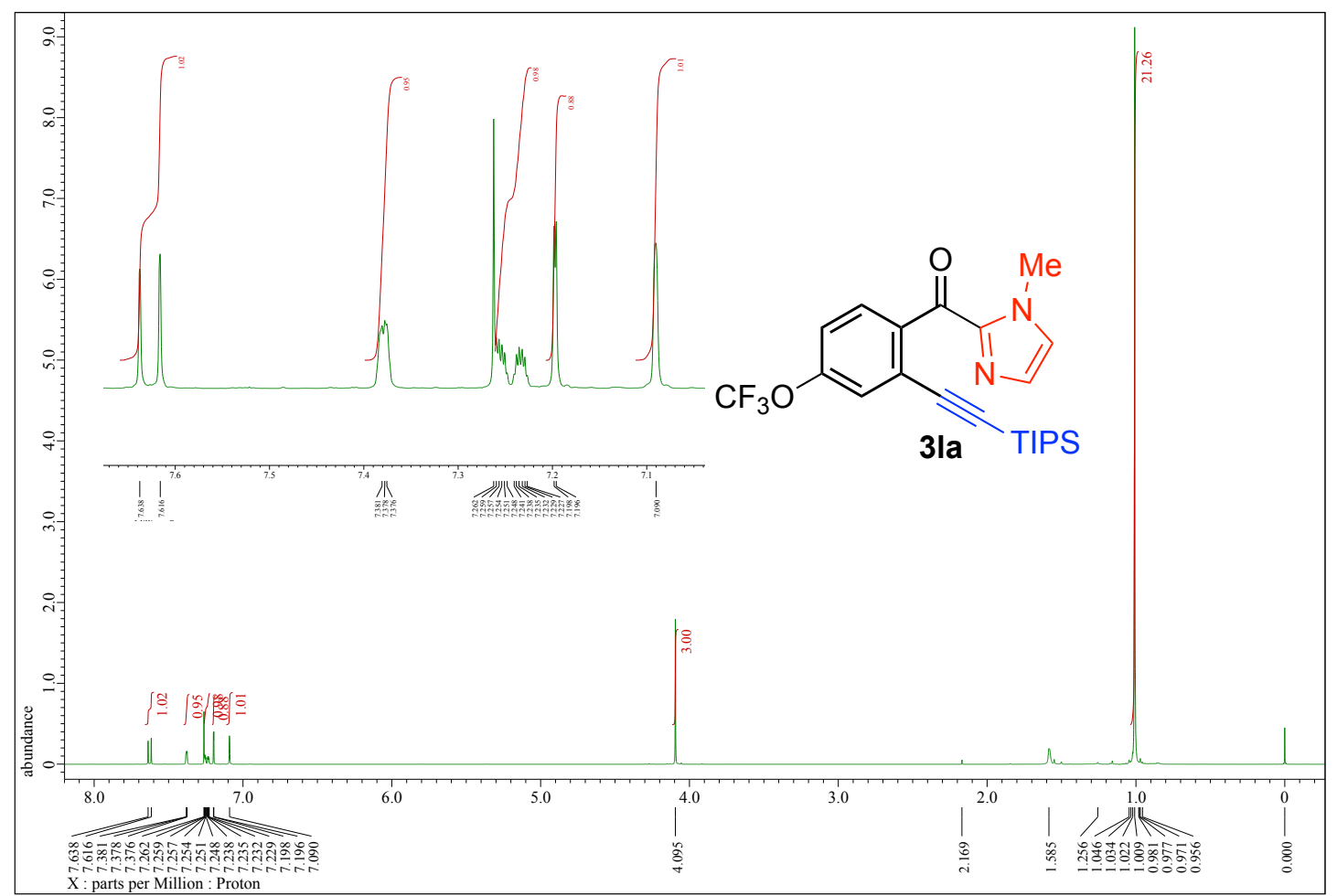

Figure S114: ${ }^{1} \mathrm{H}$ NMR spectrum of compound 3la $\left(400 \mathrm{MHz}, \mathrm{CDCl}_{3}\right)$.

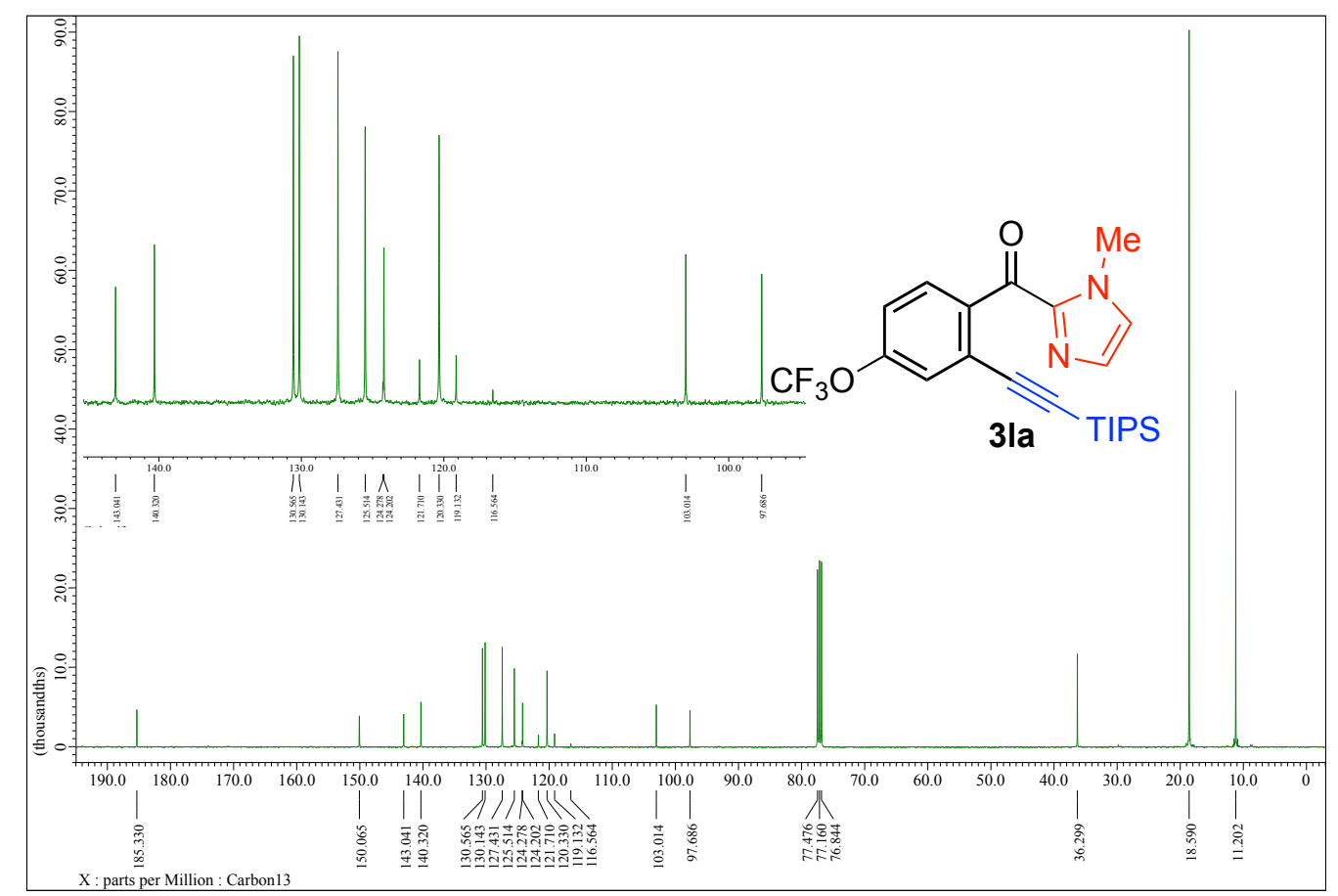

Figure S115: ${ }^{13} \mathrm{C}$ NMR spectrum of compound 3la (100 $\left.\mathrm{MHz}, \mathrm{CDCl}_{3}\right)$. 


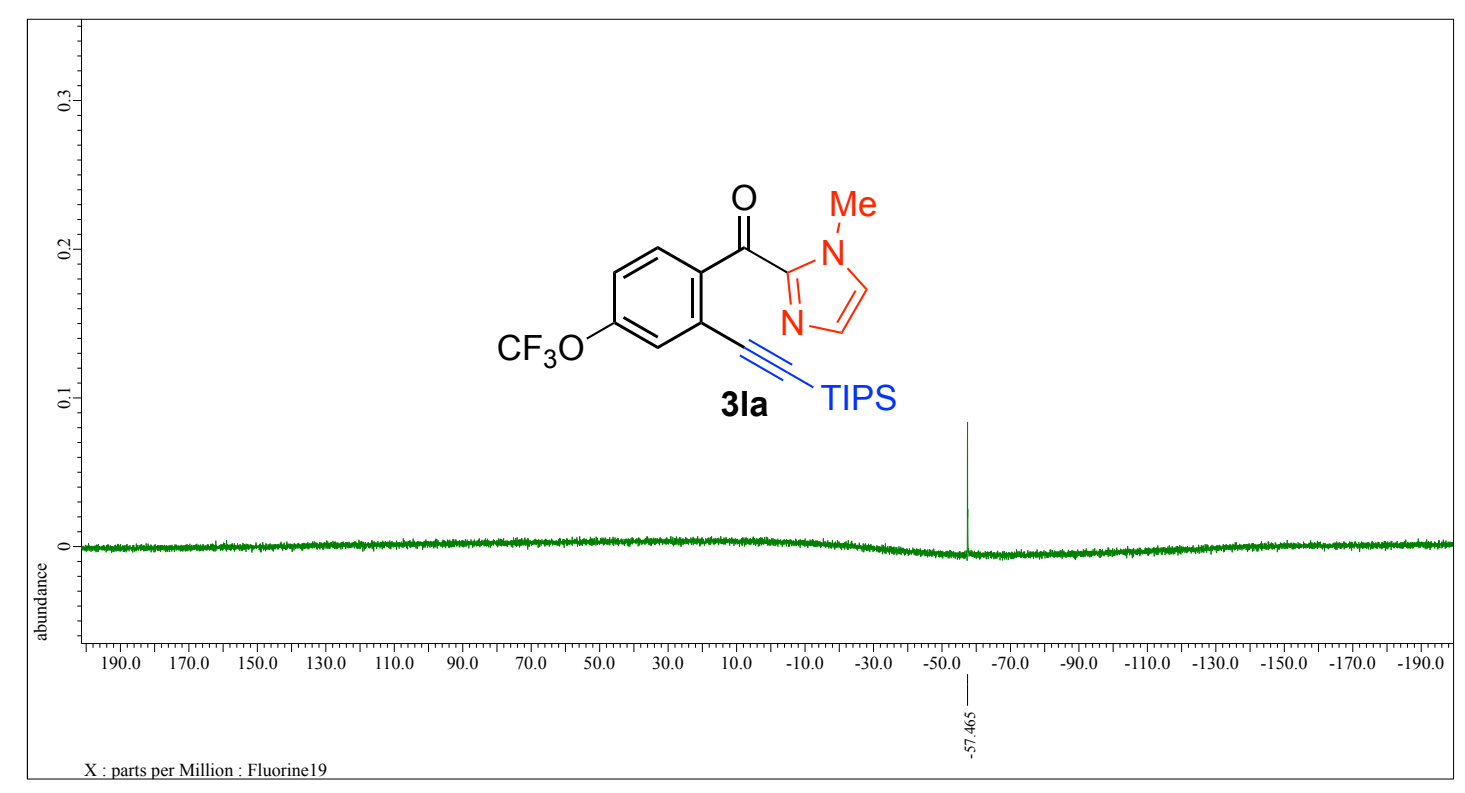

Figure S116: ${ }^{19} \mathrm{~F}$ NMR spectrum of compound 3la (376 $\mathrm{MHz}, \mathrm{CDCl}_{3}$ ).

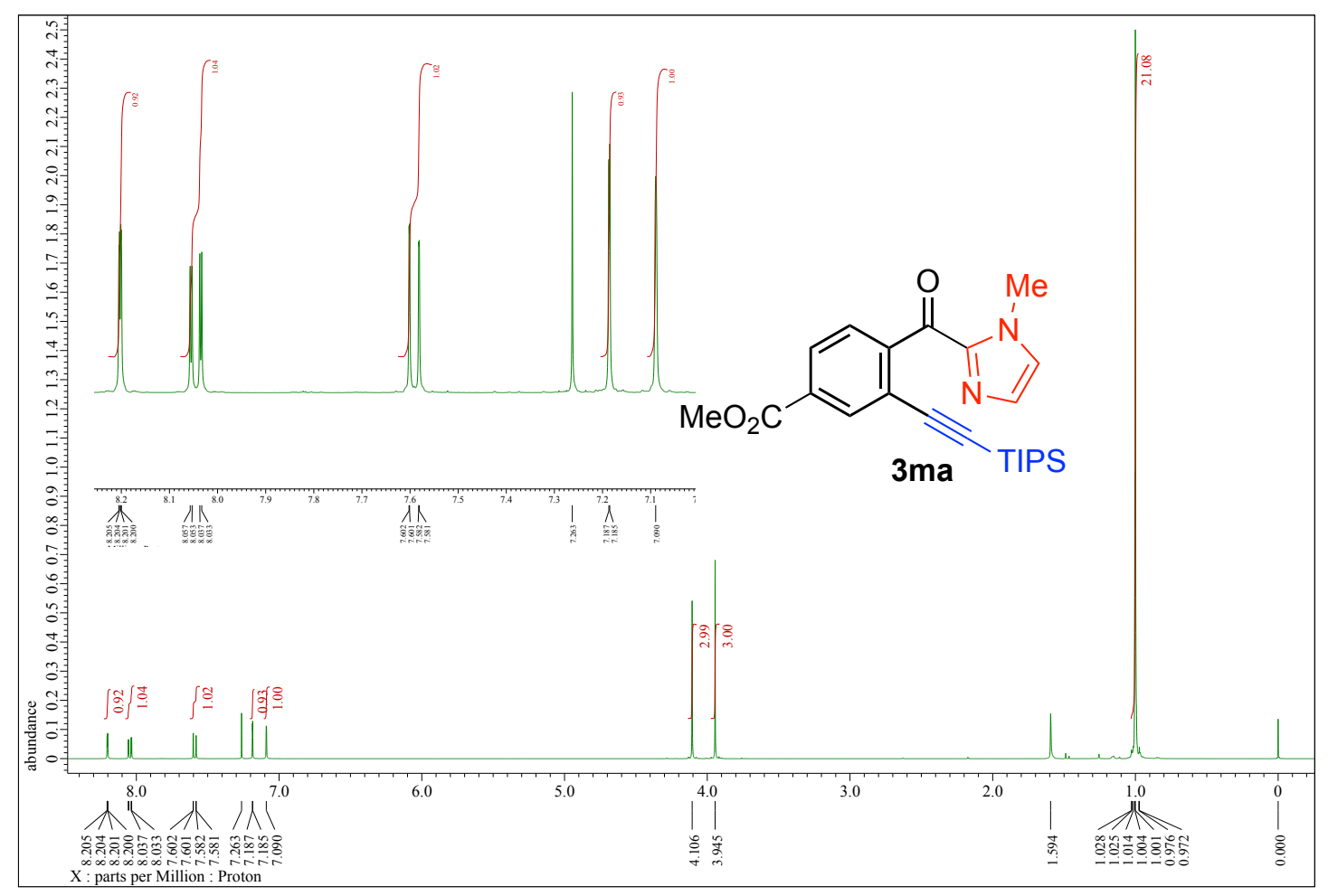

Figure S117: ${ }^{1} \mathrm{H}$ NMR spectrum of compound 3ma (400 $\left.\mathrm{MHz}, \mathrm{CDCl}_{3}\right)$. 


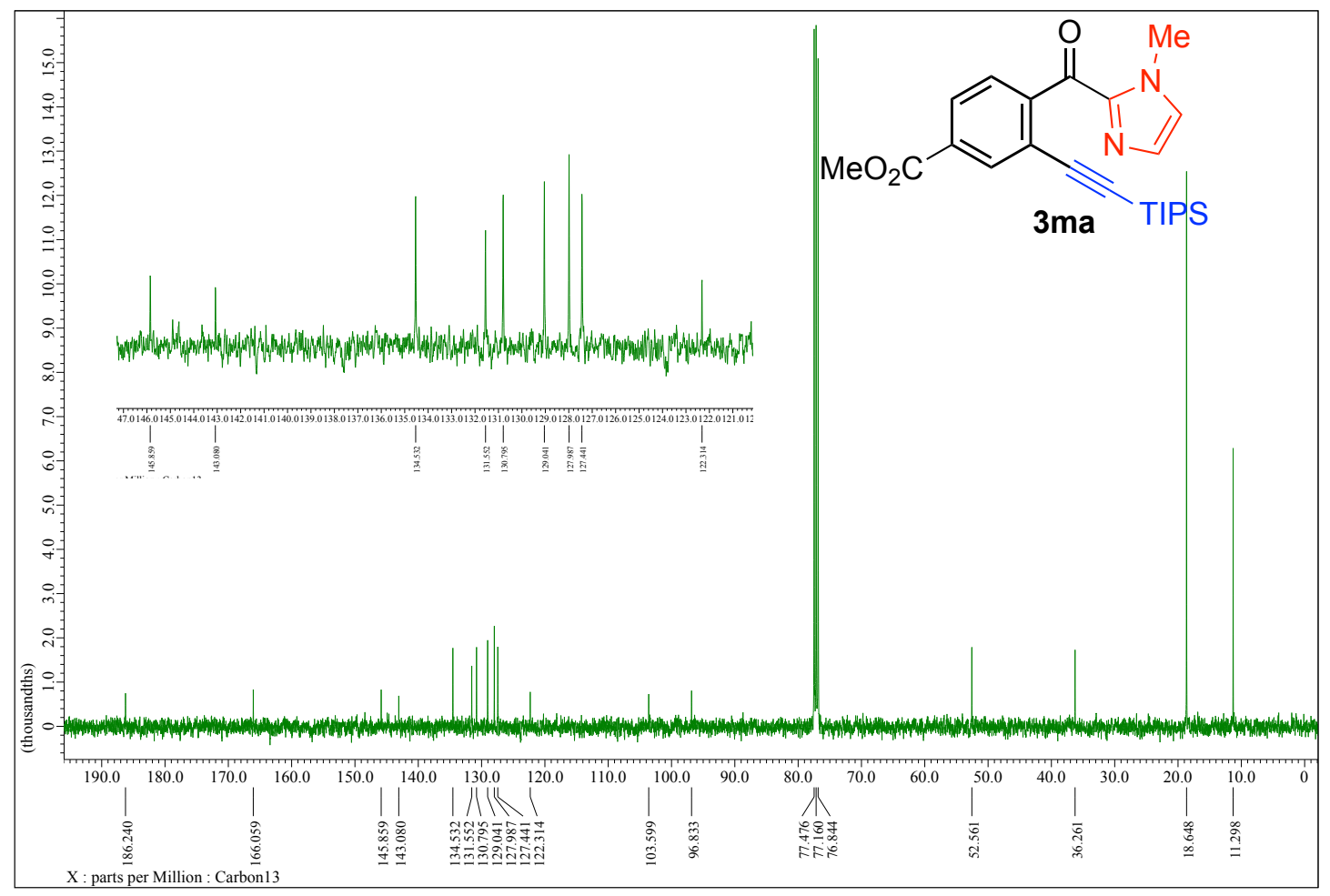

Figure S118: ${ }^{13} \mathrm{C}$ NMR spectrum of compound 3ma $\left(100 \mathrm{MHz}, \mathrm{CDCl}_{3}\right)$.

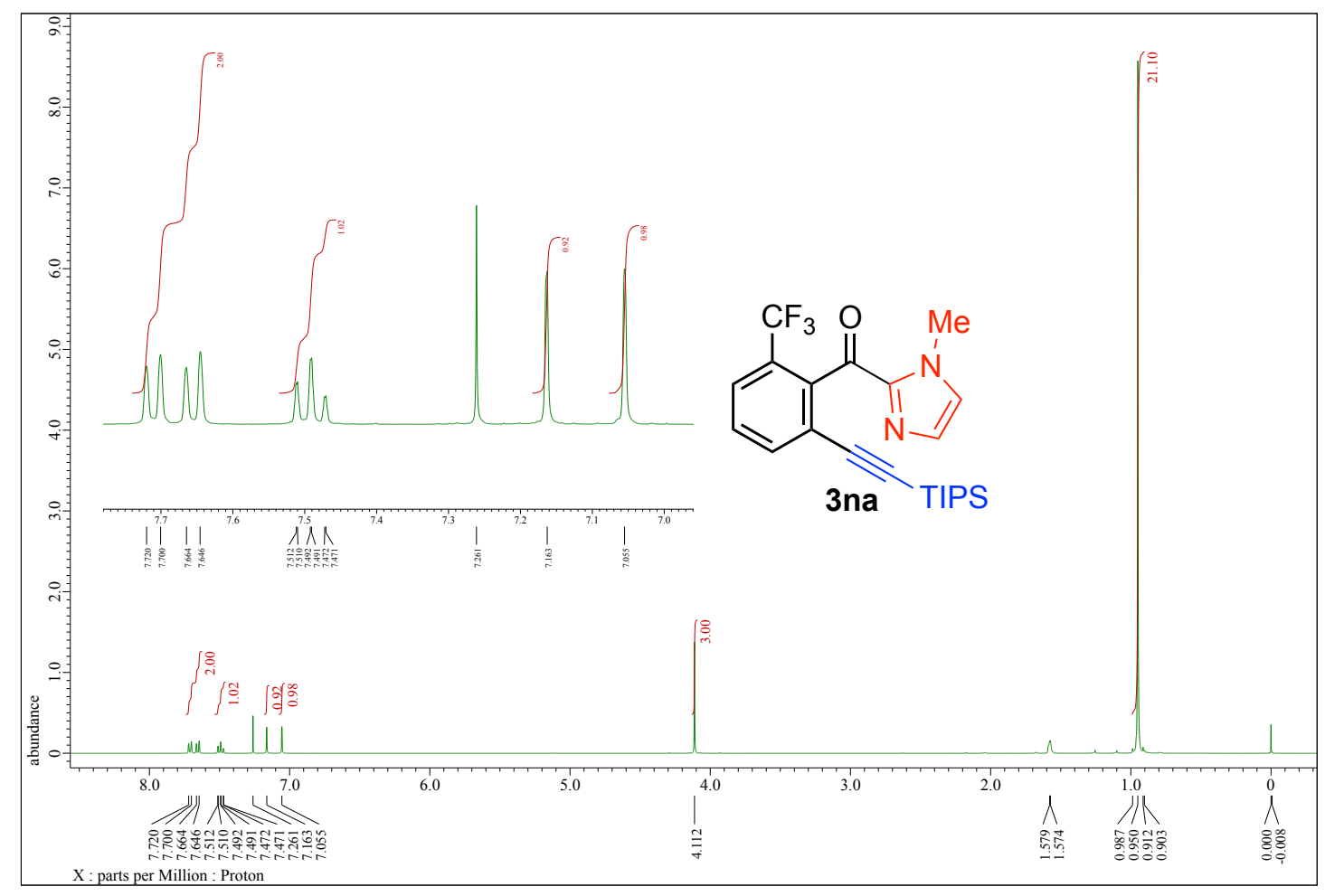

Figure S119: ${ }^{1} \mathrm{H}$ NMR spectrum of compound 3na (400 MHz, $\left.\mathrm{CDCl}_{3}\right)$. 


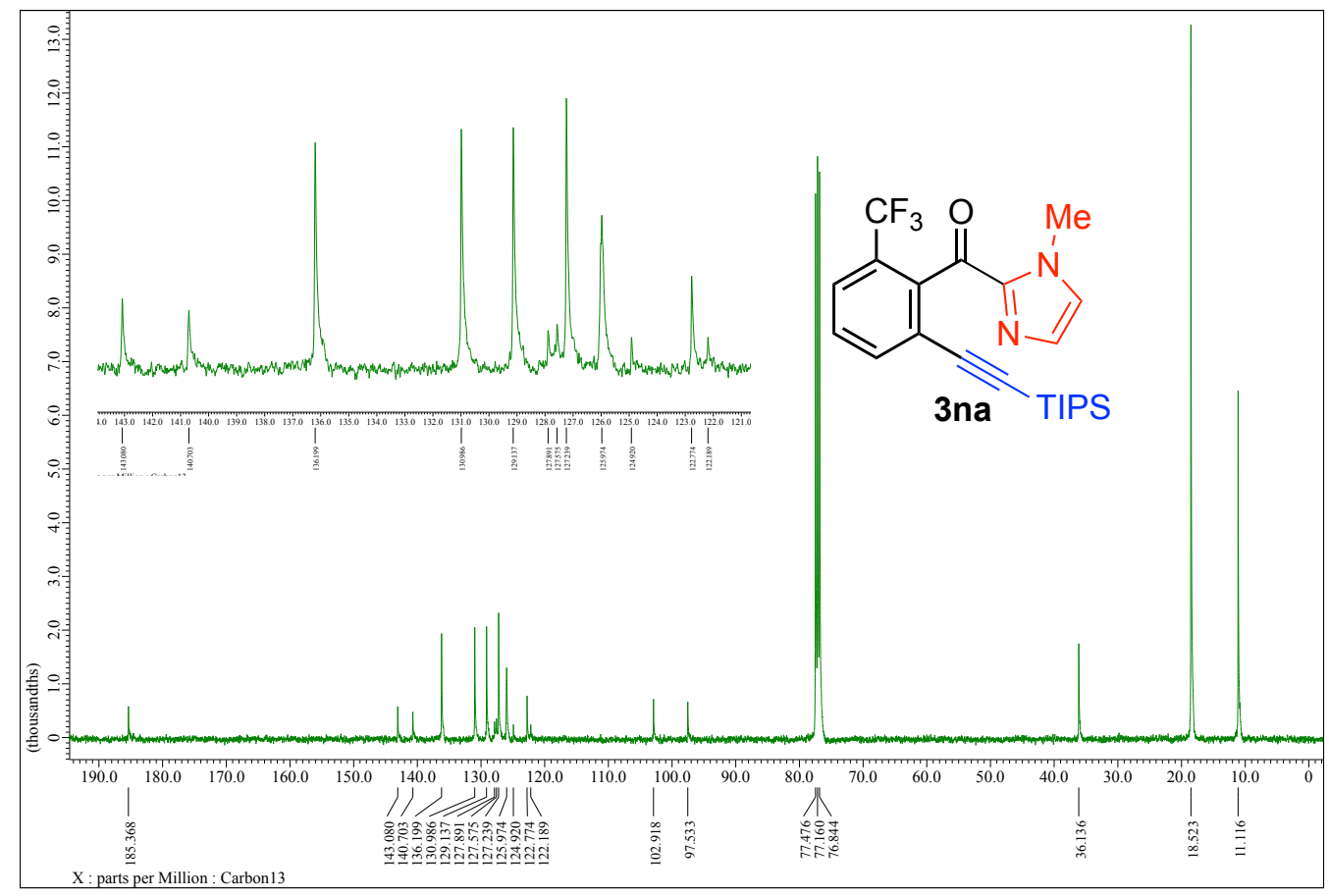

Figure S120: ${ }^{13} \mathrm{C}$ NMR spectrum of compound 3na $\left(100 \mathrm{MHz}, \mathrm{CDCl}_{3}\right)$.

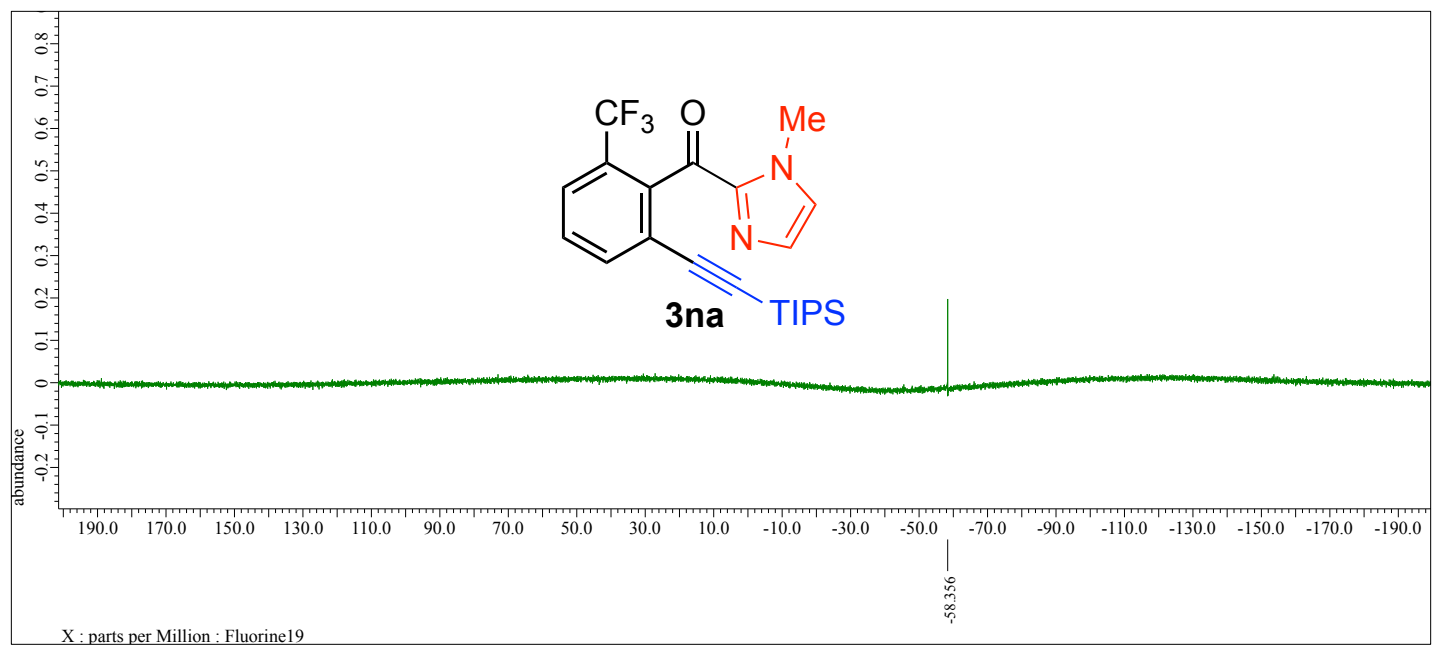

Figure S121: ${ }^{19} \mathrm{~F}$ NMR spectrum of compound 3na (376 $\mathrm{MHz}, \mathrm{CDCl}_{3}$ ). 


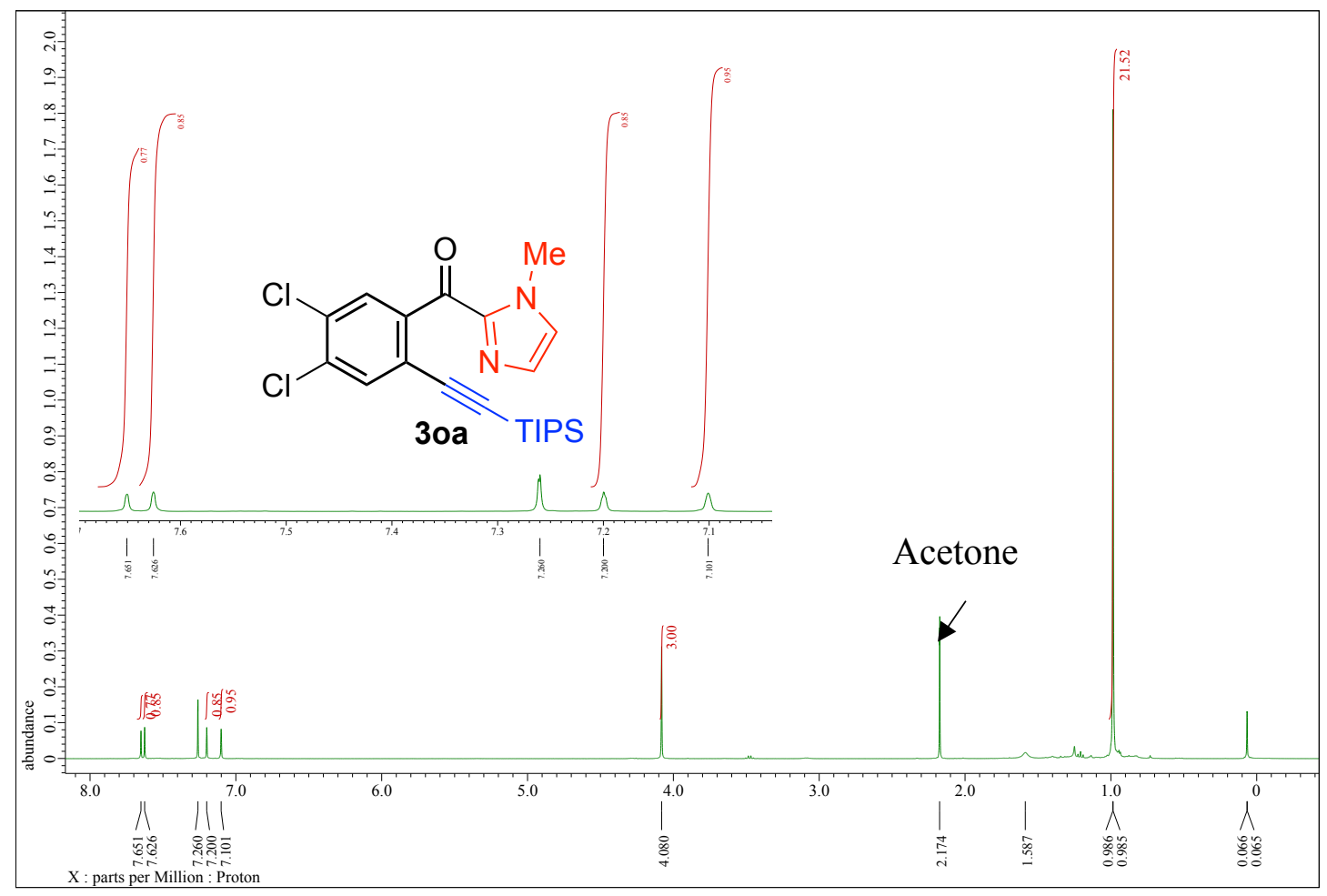

Figure S122: ${ }^{1} \mathrm{H}$ NMR spectrum of compound $30 a\left(400 \mathrm{MHz}, \mathrm{CDCl}_{3}\right)$.

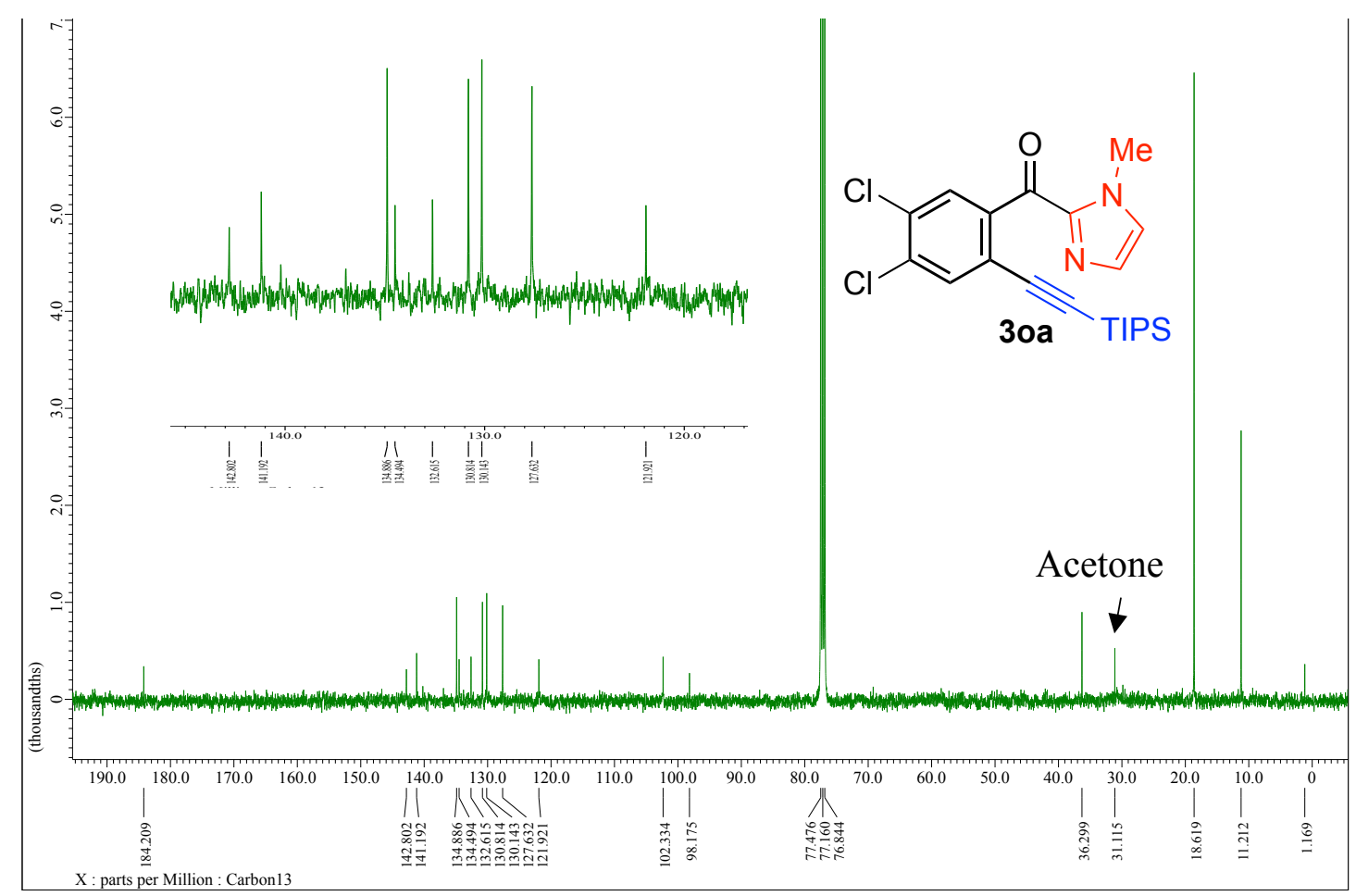

Figure S123: ${ }^{13} \mathrm{C}$ NMR spectrum of compound $30 a\left(100 \mathrm{MHz}, \mathrm{CDCl}_{3}\right)$. 


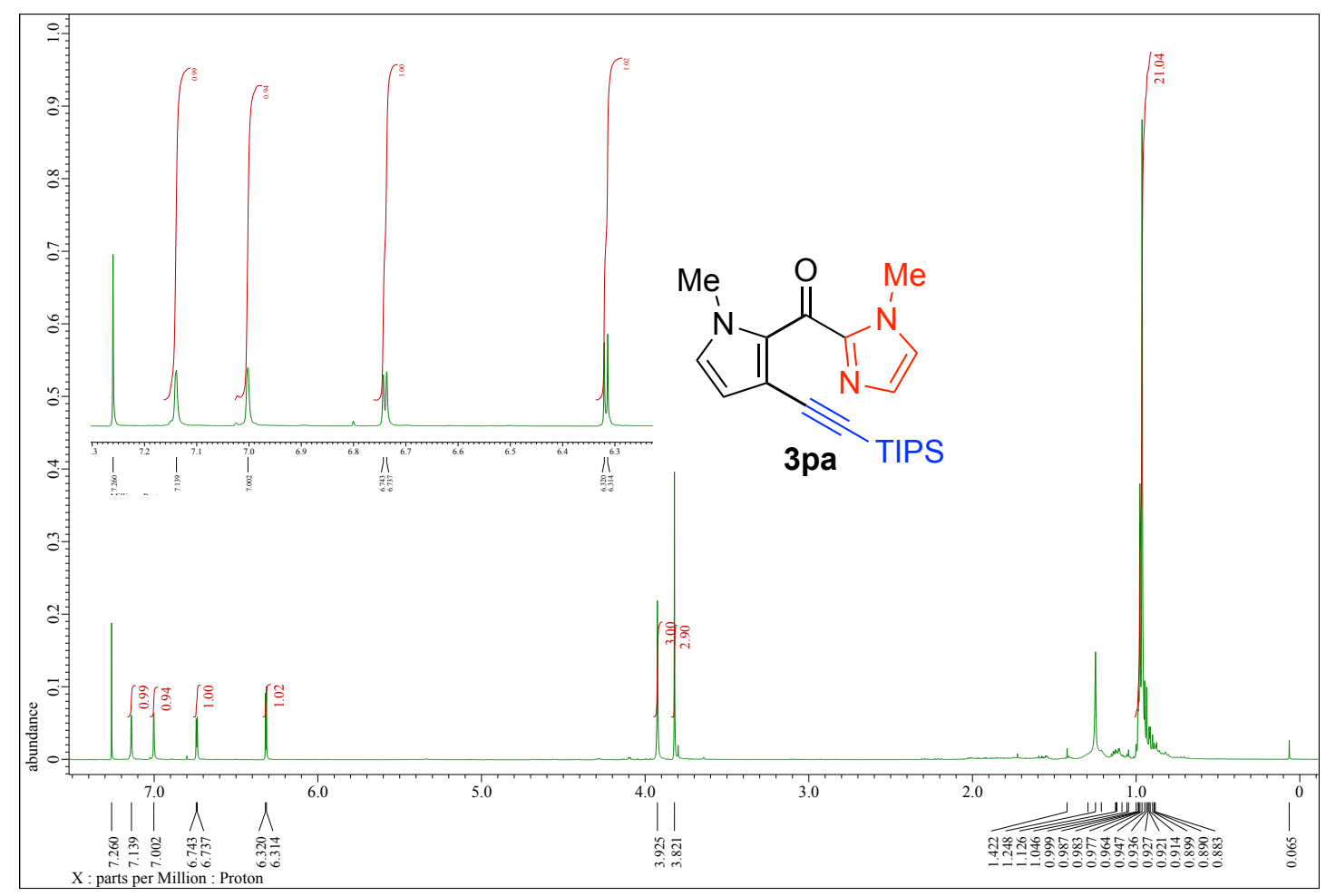

Figure S124: ${ }^{1} \mathrm{H}$ NMR spectrum of compound 3pa (400 $\mathrm{MHz}, \mathrm{CDCl}_{3}$ ).

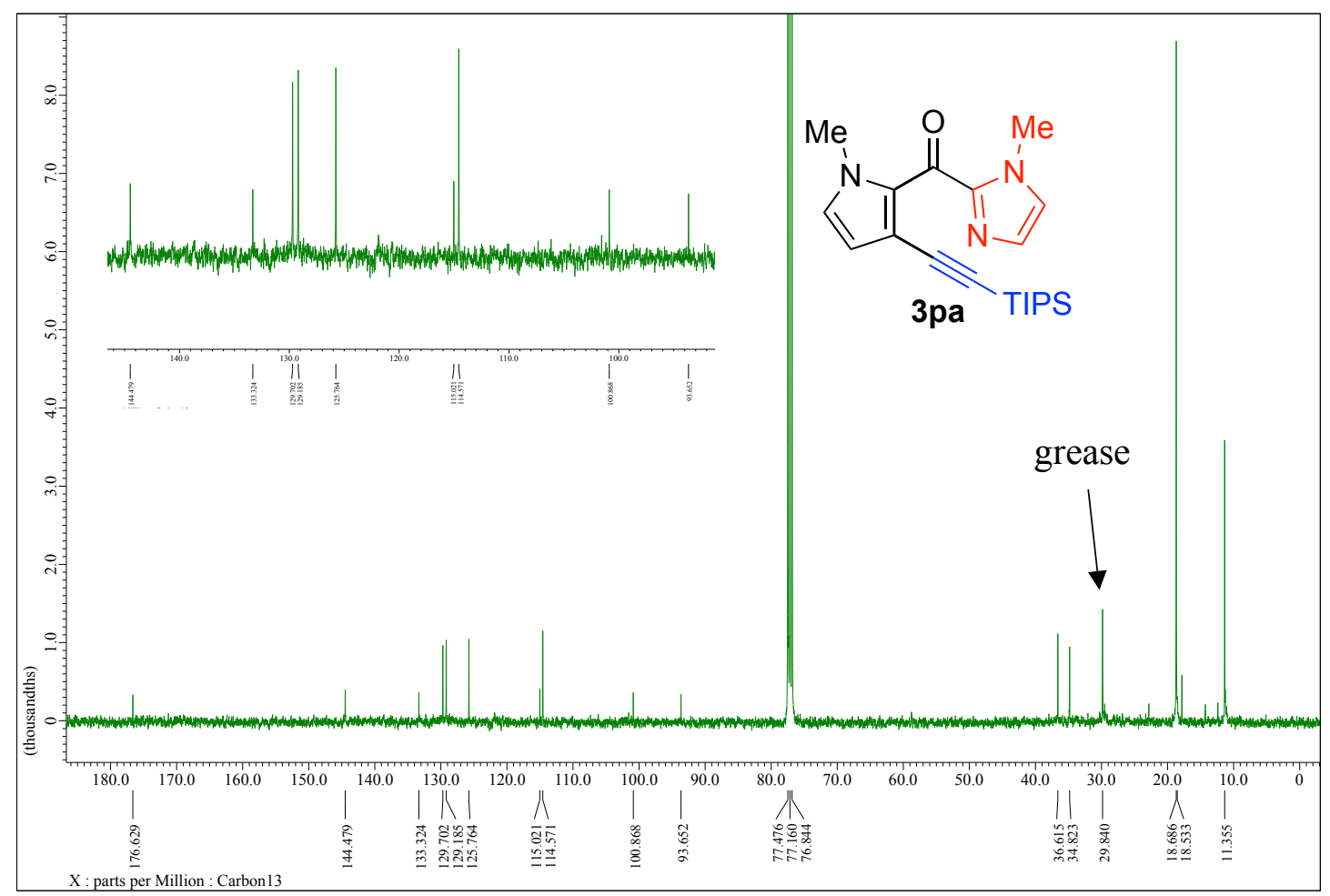

Figure S125: ${ }^{13} \mathrm{C}$ NMR spectrum of compound 3pa $\left(100 \mathrm{MHz}, \mathrm{CDCl}_{3}\right)$. 


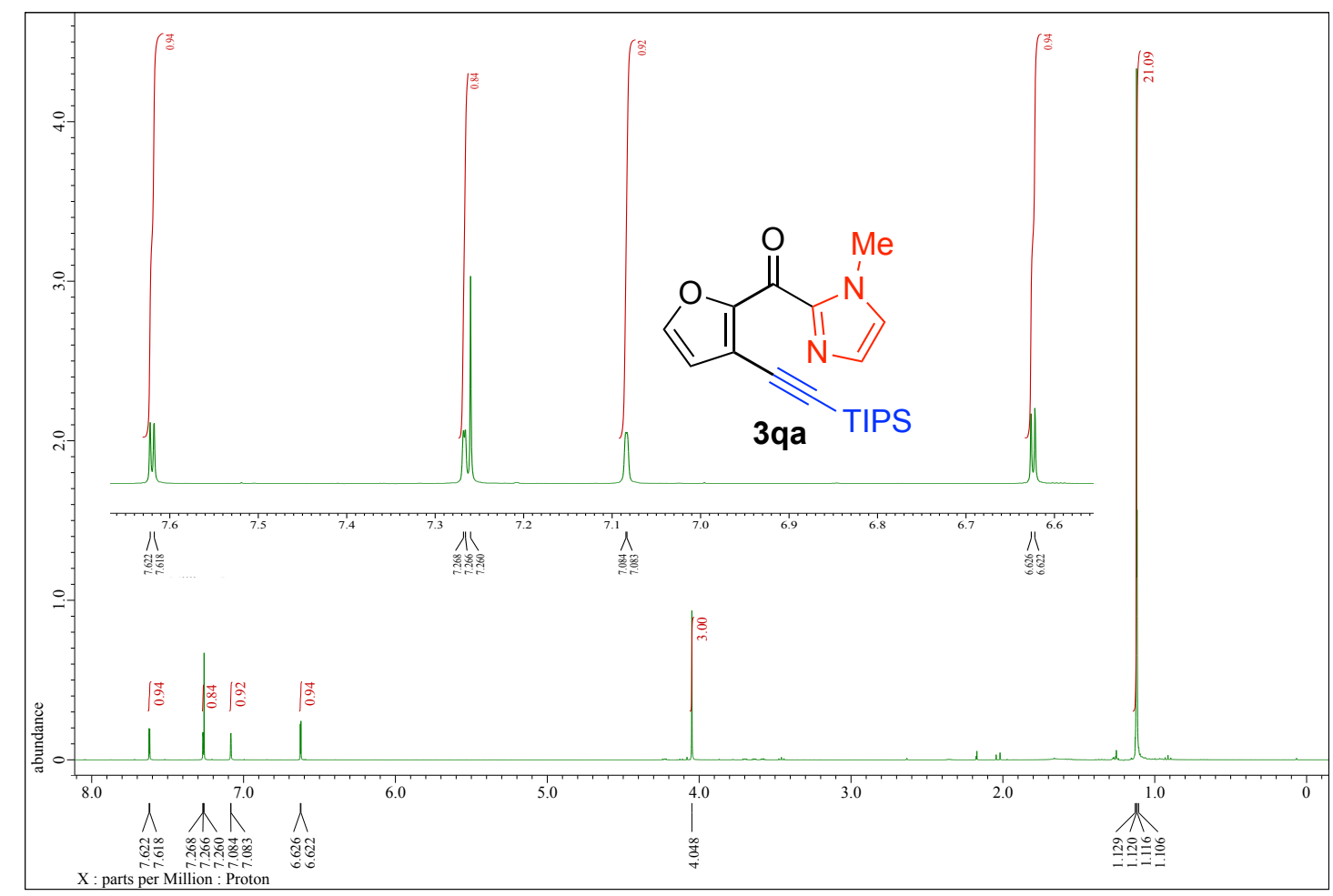

Figure S126: ${ }^{1} \mathrm{H}$ NMR spectrum of compound $3 q a\left(400 \mathrm{MHz}, \mathrm{CDCl}_{3}\right.$ ).

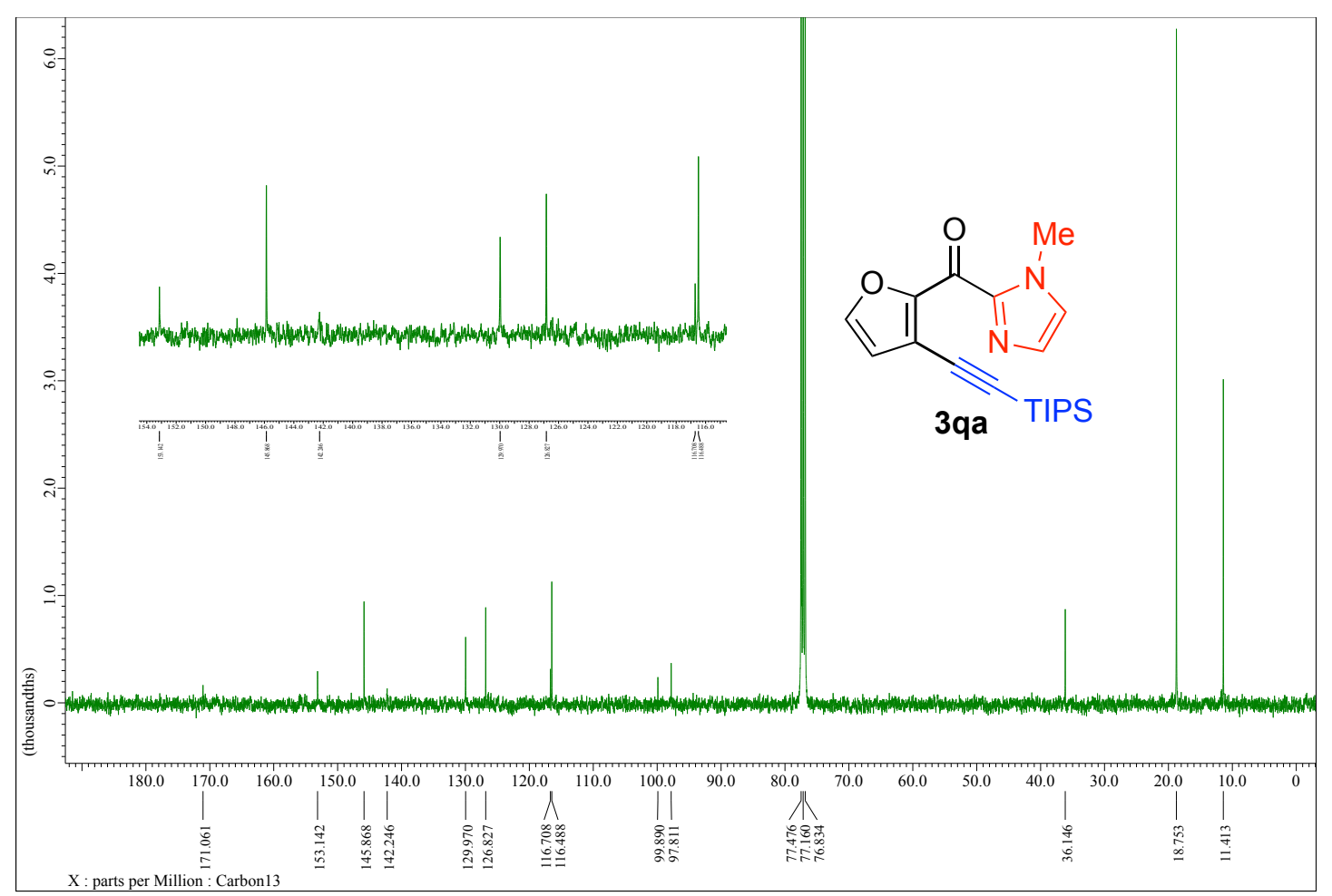

Figure S127: ${ }^{13} \mathrm{C}$ NMR spectrum of compound 3qa (100 $\left.\mathrm{MHz}, \mathrm{CDCl}_{3}\right)$. 


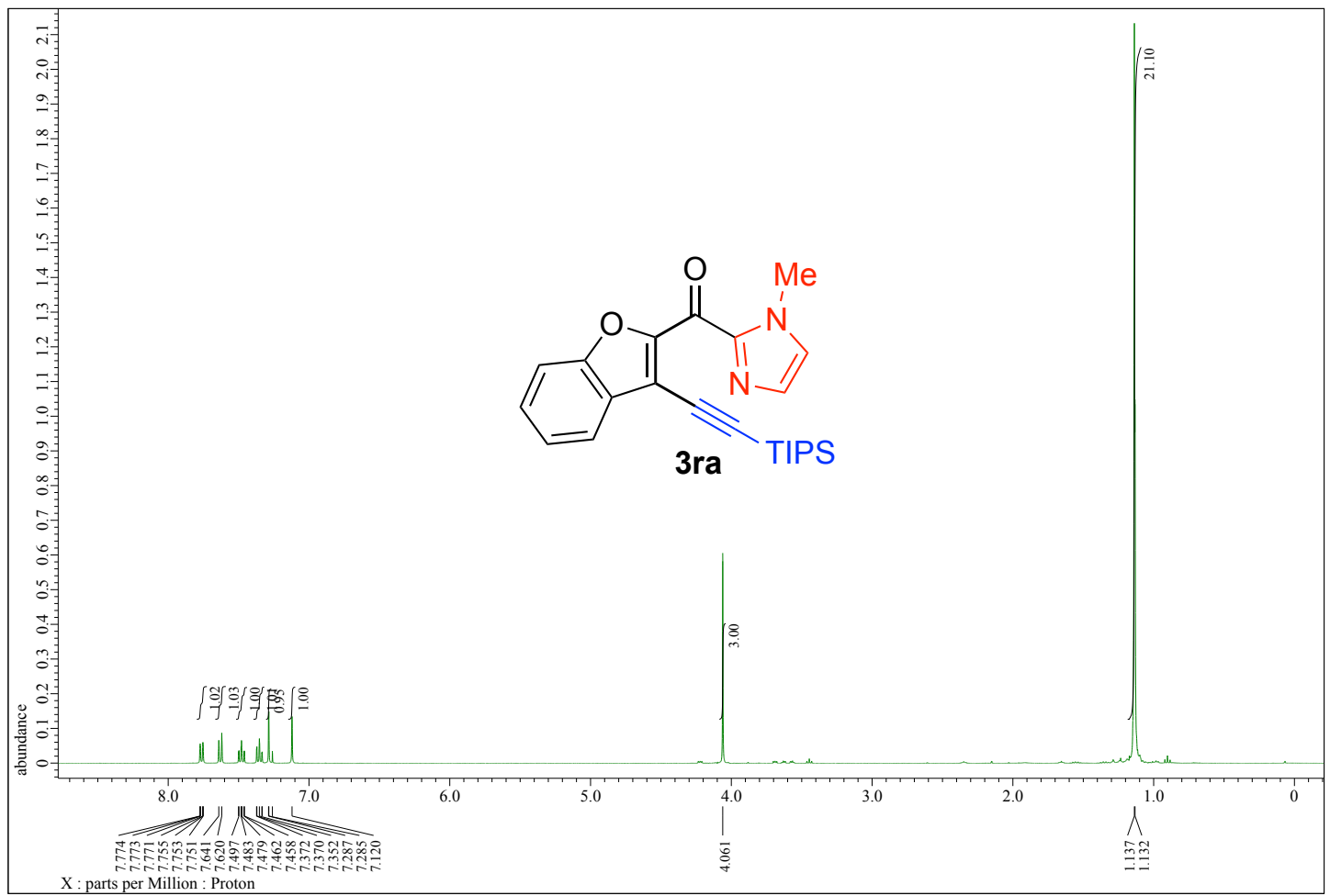

Figure S128: ${ }^{1} \mathrm{H}$ NMR spectrum of compound 3ra $\left(400 \mathrm{MHz}, \mathrm{CDCl}_{3}\right)$.

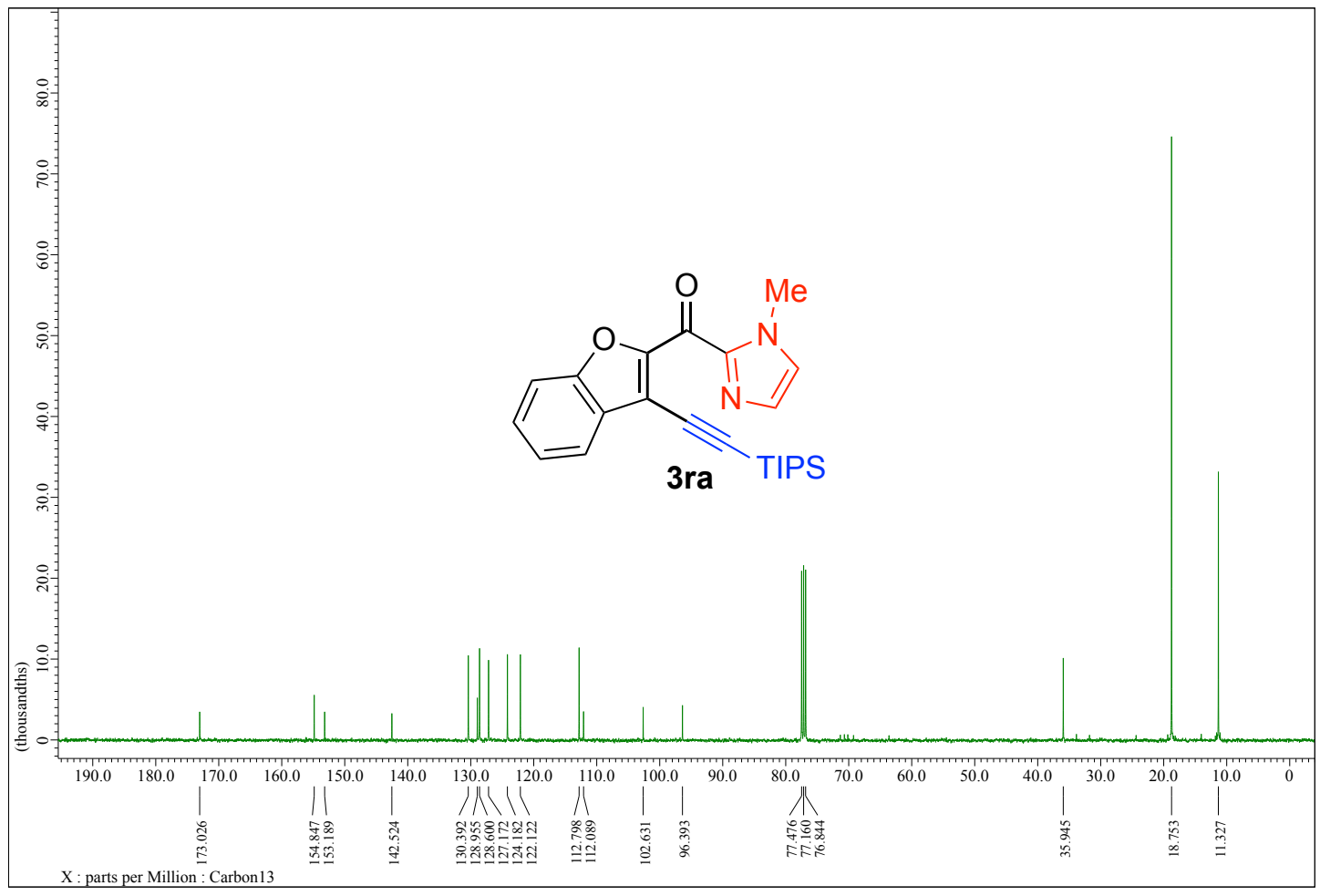

Figure S129: ${ }^{13} \mathrm{C}$ NMR spectrum of compound 3ra (100 $\left.\mathrm{MHz}, \mathrm{CDCl}_{3}\right)$. 


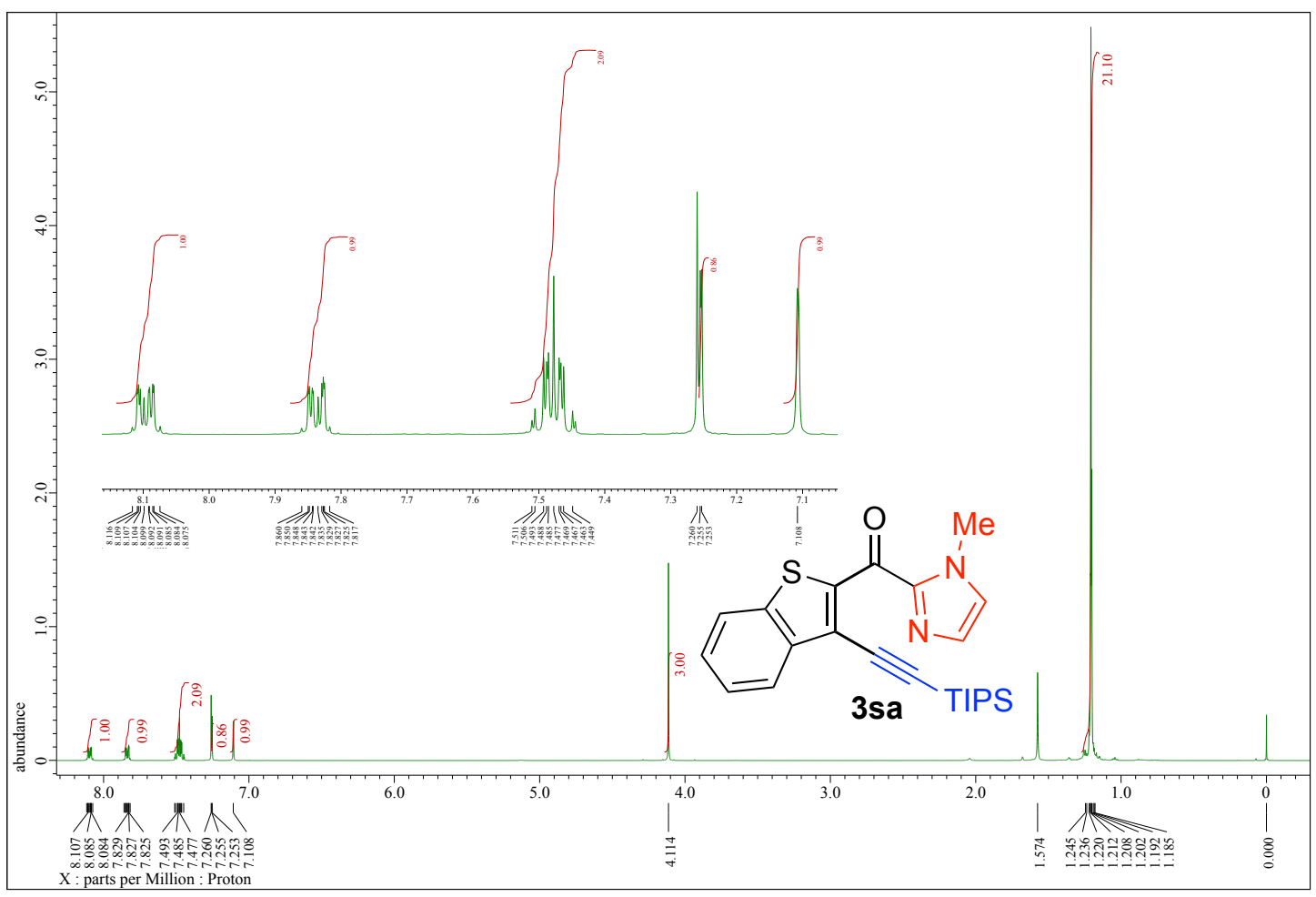

Figure S130: ${ }^{1} \mathrm{H}$ NMR spectrum of compound 3sa (400 MHz, $\left.\mathrm{CDCl}_{3}\right)$.

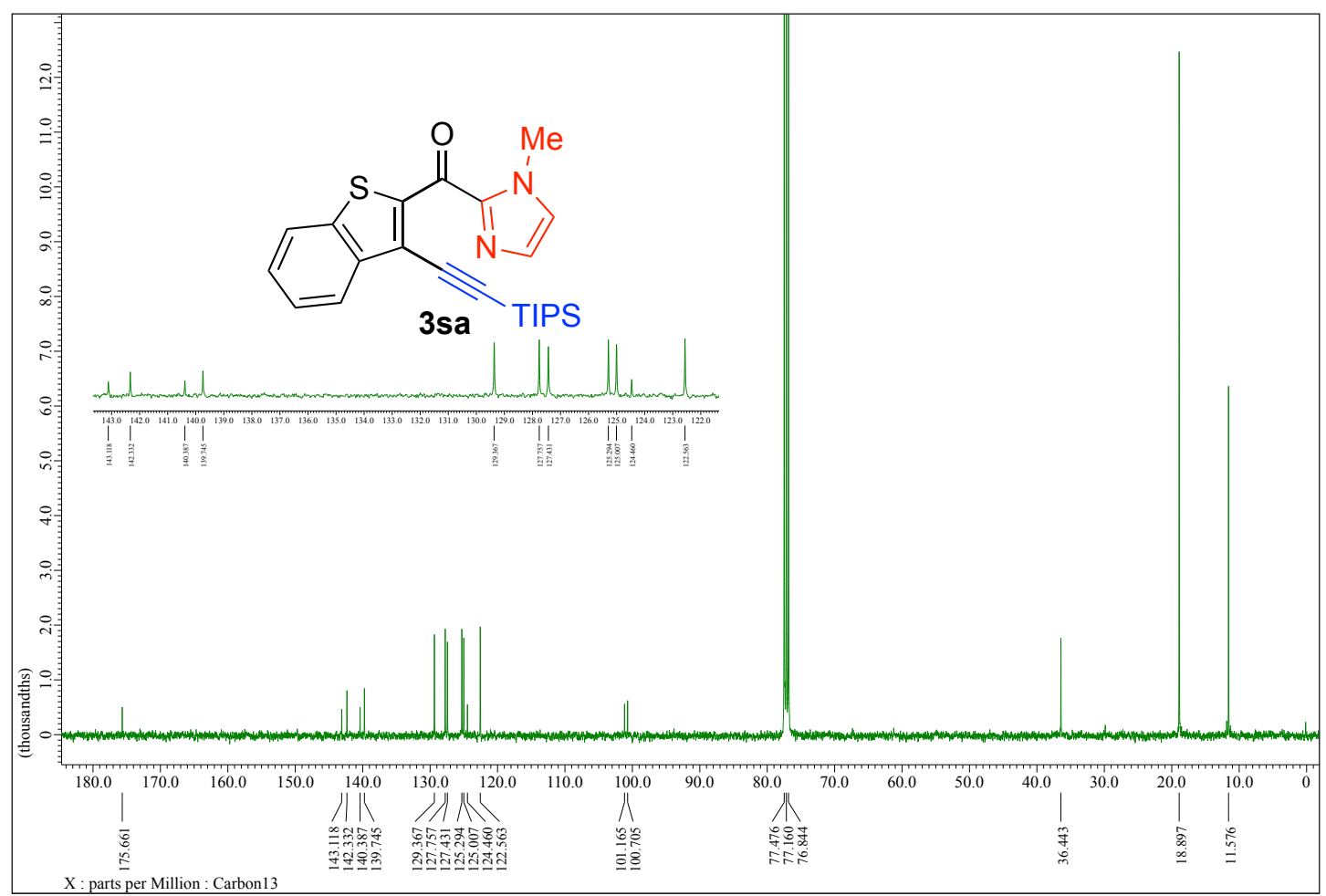

Figure S131: ${ }^{13} \mathrm{C}$ NMR spectrum of compound 3sa (100 $\left.\mathrm{MHz}, \mathrm{CDCl}_{3}\right)$. 


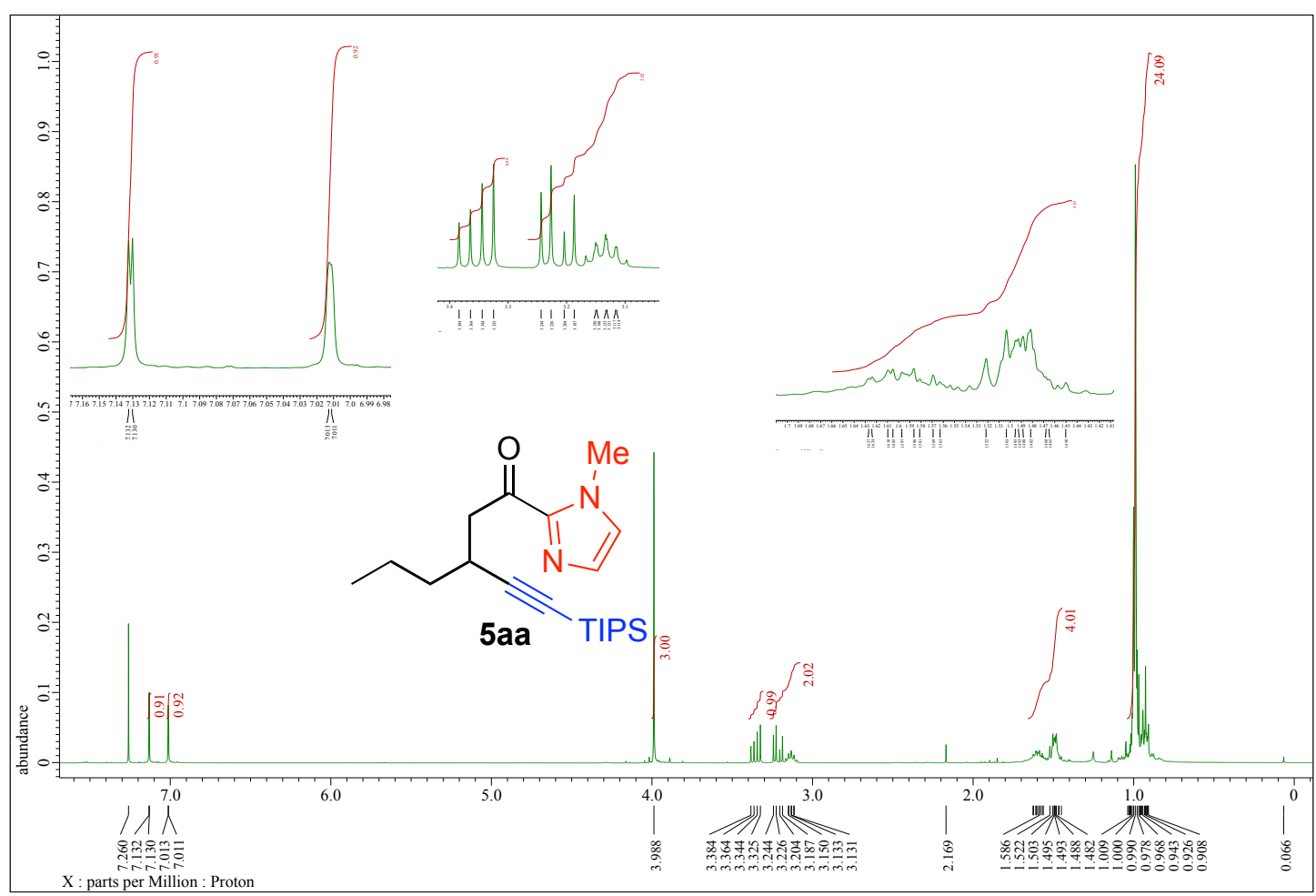

Figure S132: ${ }^{1} \mathrm{H}$ NMR spectrum of compound $\mathbf{5 a a}\left(400 \mathrm{MHz}, \mathrm{CDCl}_{3}\right)$.

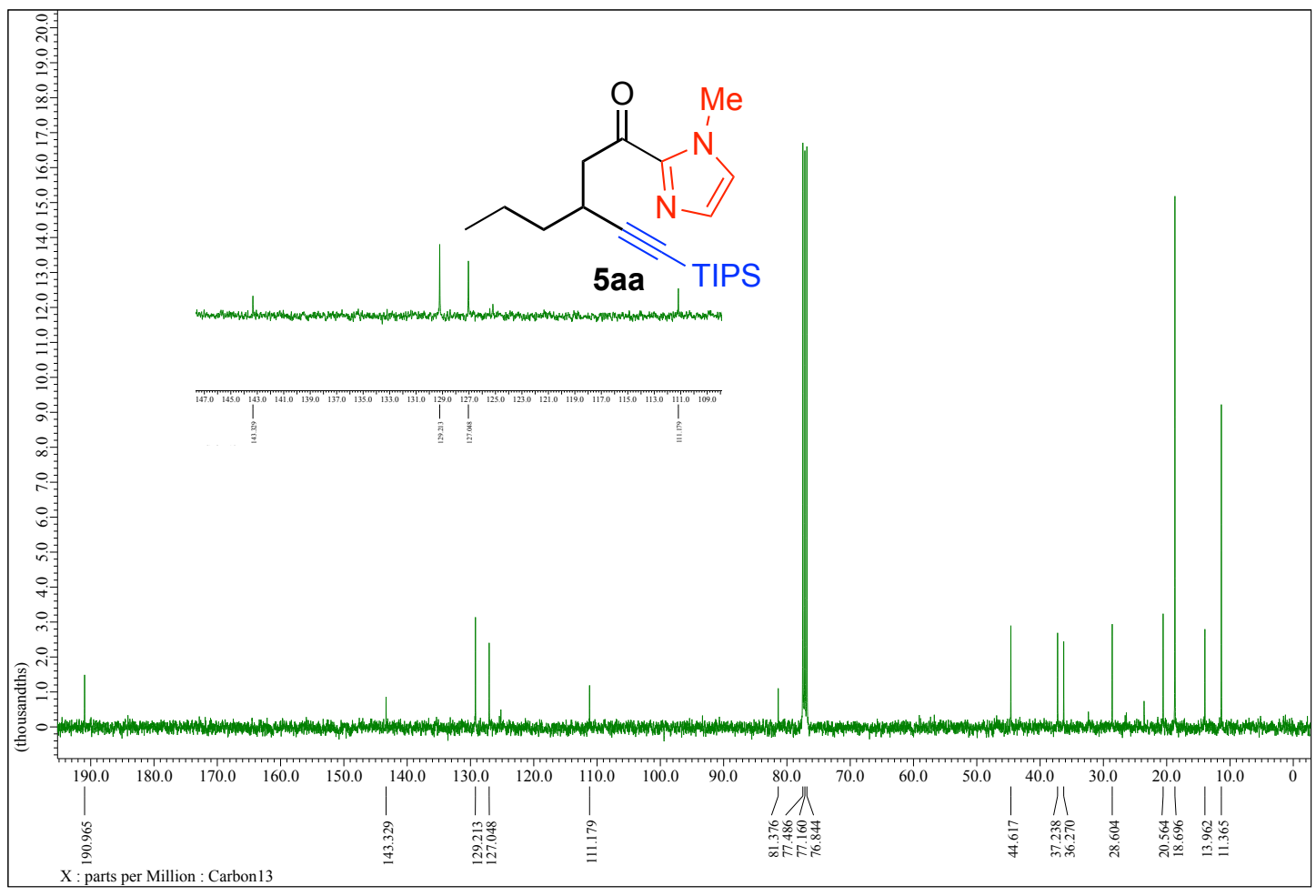

Figure S133: ${ }^{13} \mathrm{C}$ NMR spectrum of compound 5aa $\left(100 \mathrm{MHz}, \mathrm{CDCl}_{3}\right)$. 


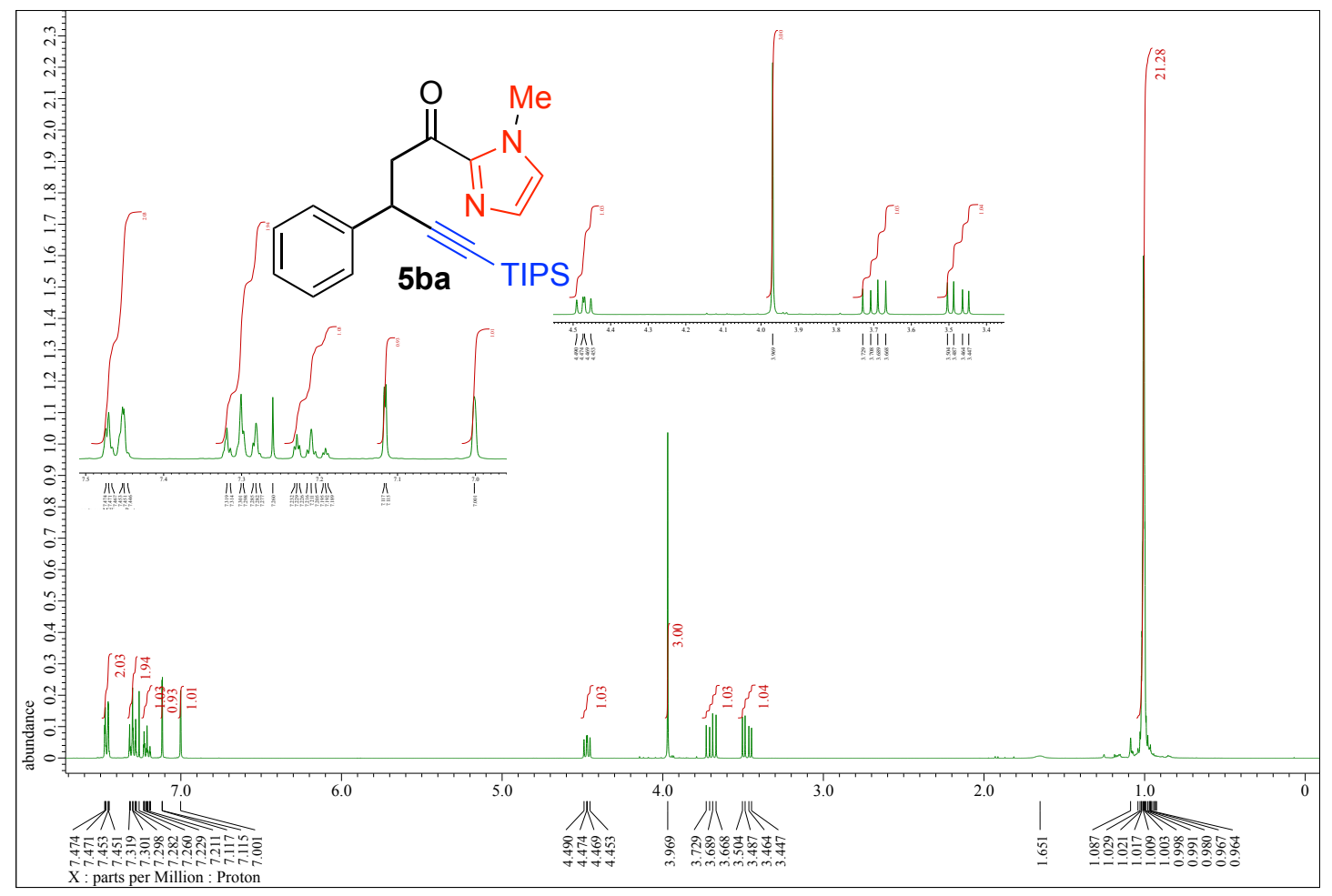

Figure S134: ${ }^{1} \mathrm{H}$ NMR spectrum of compound $5 \mathbf{b a}\left(400 \mathrm{MHz}, \mathrm{CDCl}_{3}\right)$.

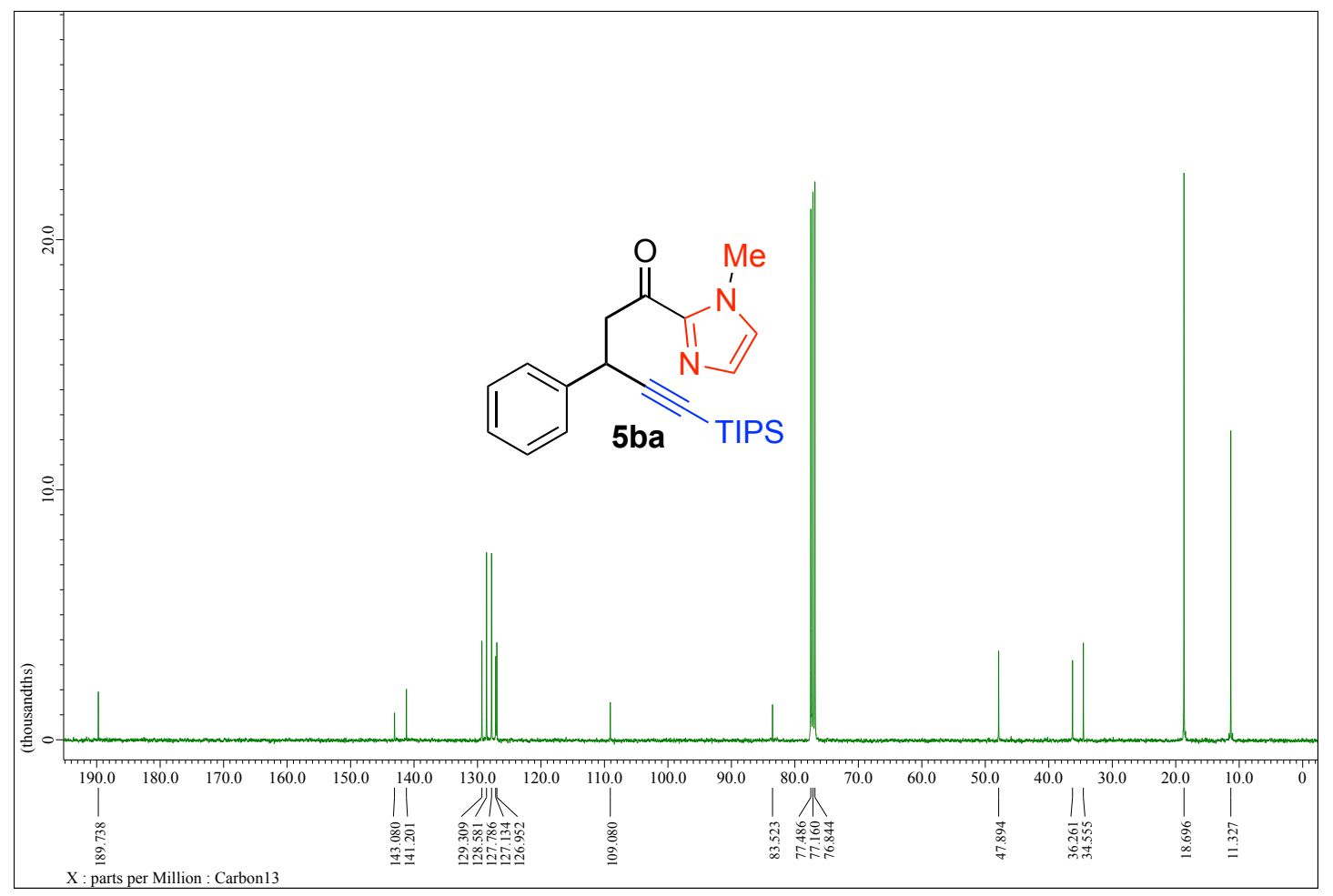

Figure S135: ${ }^{13} \mathrm{C}$ NMR spectrum of compound $5 \mathbf{b a}\left(100 \mathrm{MHz}, \mathrm{CDCl}_{3}\right)$. 


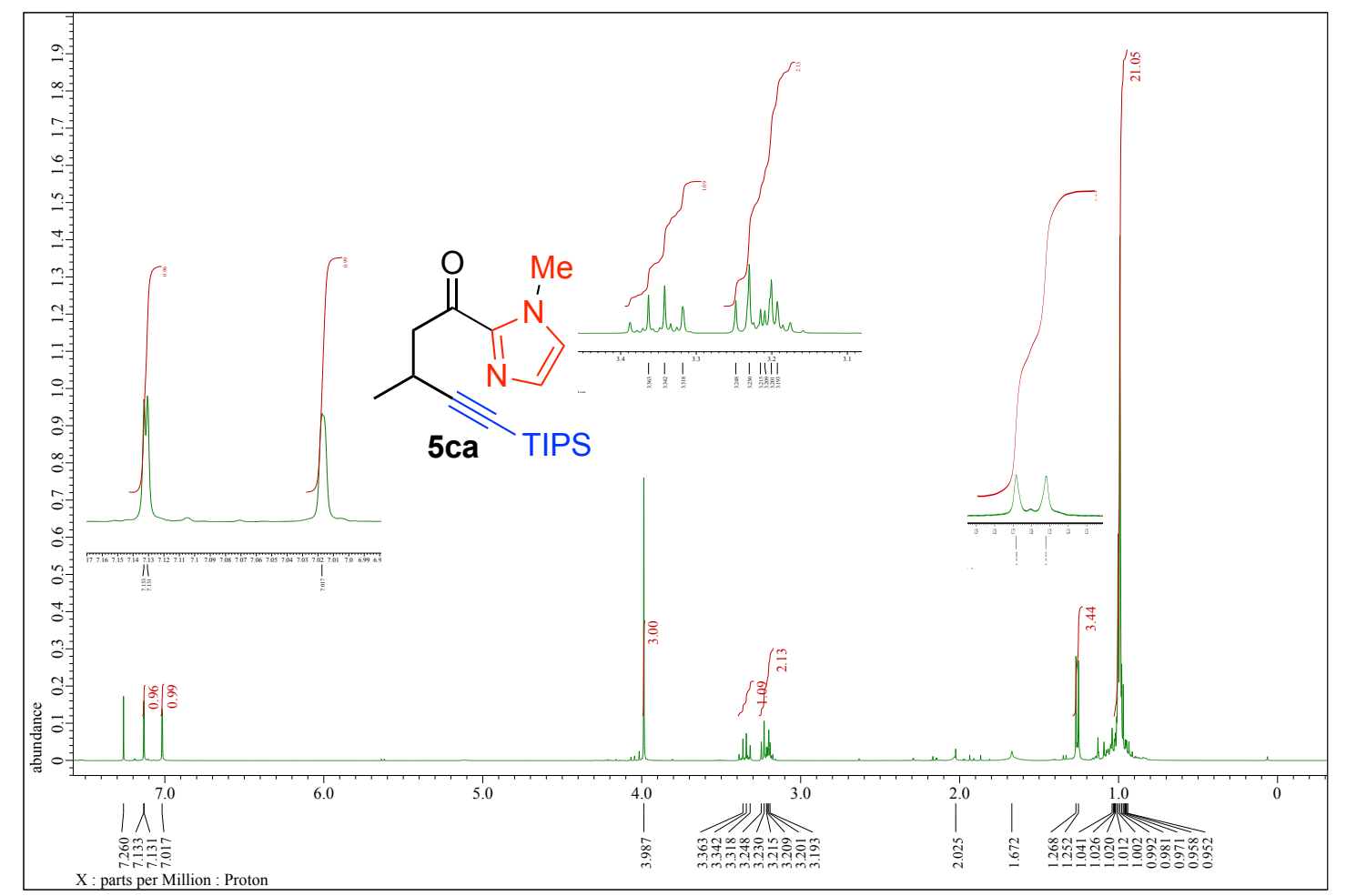

Figure S136: ${ }^{1} \mathrm{H}$ NMR spectrum of compound $\mathbf{5 c a}\left(400 \mathrm{MHz}, \mathrm{CDCl}_{3}\right)$.

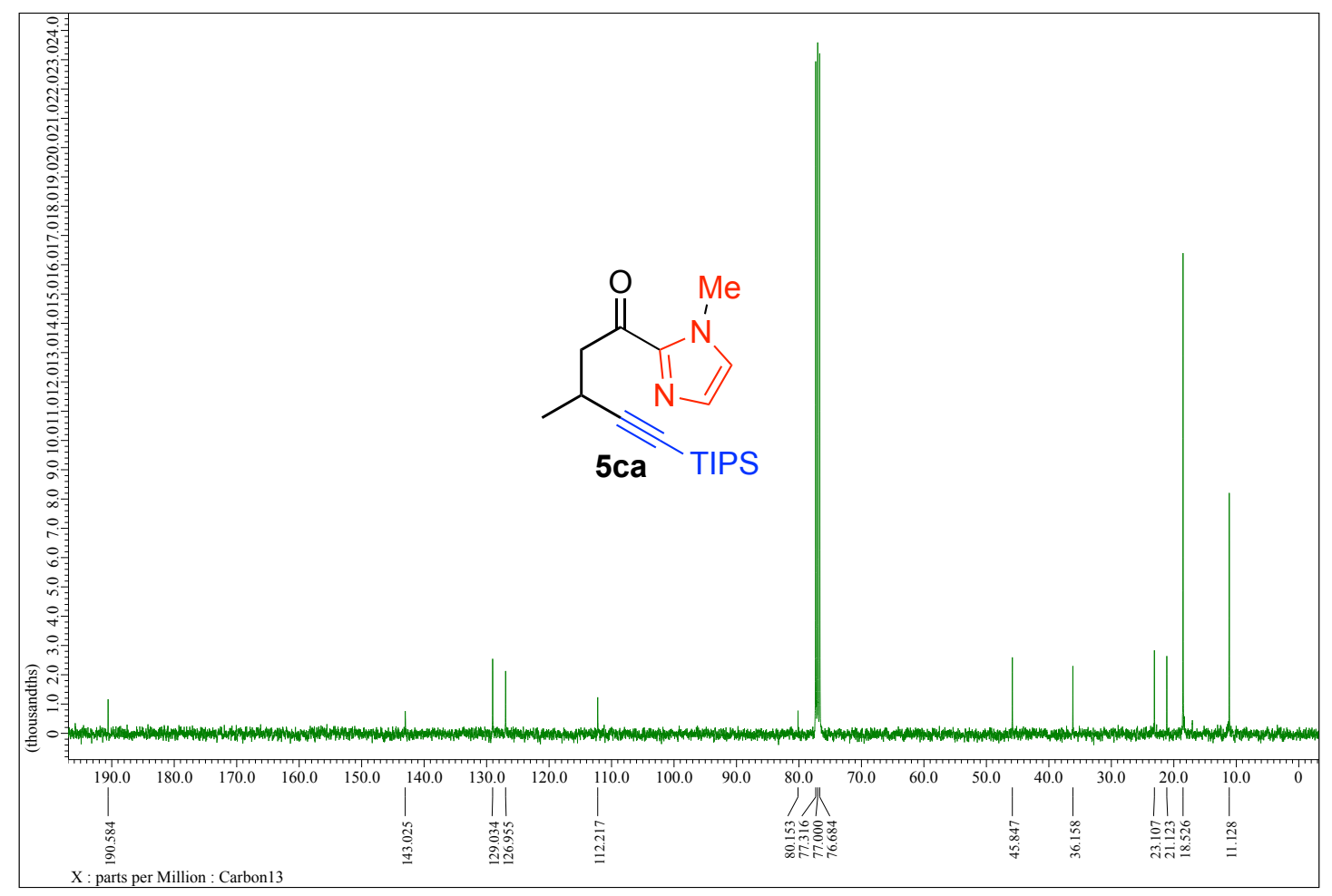

Figure S137: ${ }^{13} \mathrm{C}$ NMR spectrum of compound 5ca $\left(100 \mathrm{MHz}, \mathrm{CDCl}_{3}\right)$. 


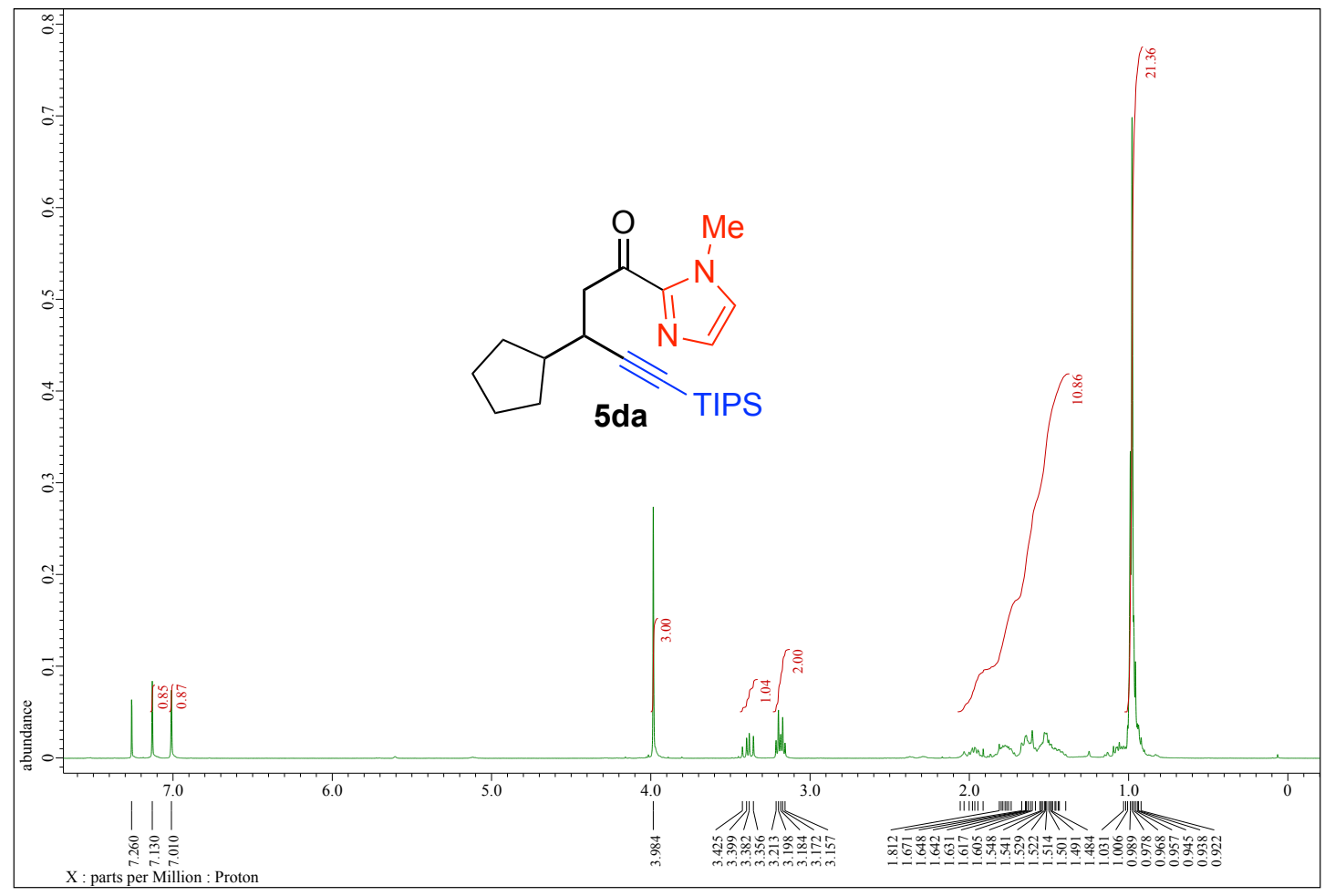

Figure S138: ${ }^{1} \mathrm{H}$ NMR spectrum of compound 5da (400 MHz, $\left.\mathrm{CDCl}_{3}\right)$.

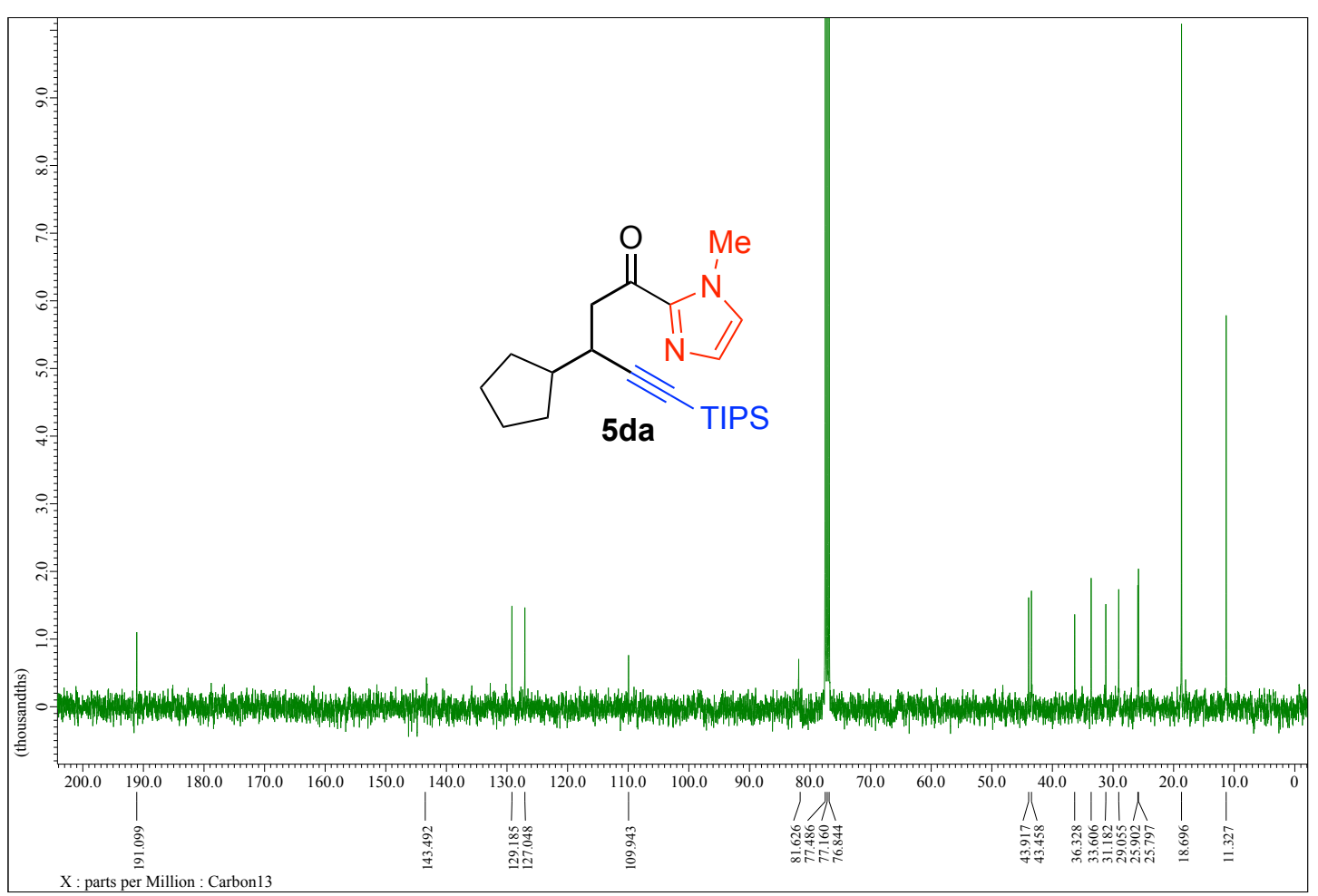

Figure S139: ${ }^{13} \mathrm{C}$ NMR spectrum of compound 5da $\left(100 \mathrm{MHz}, \mathrm{CDCl}_{3}\right)$. 


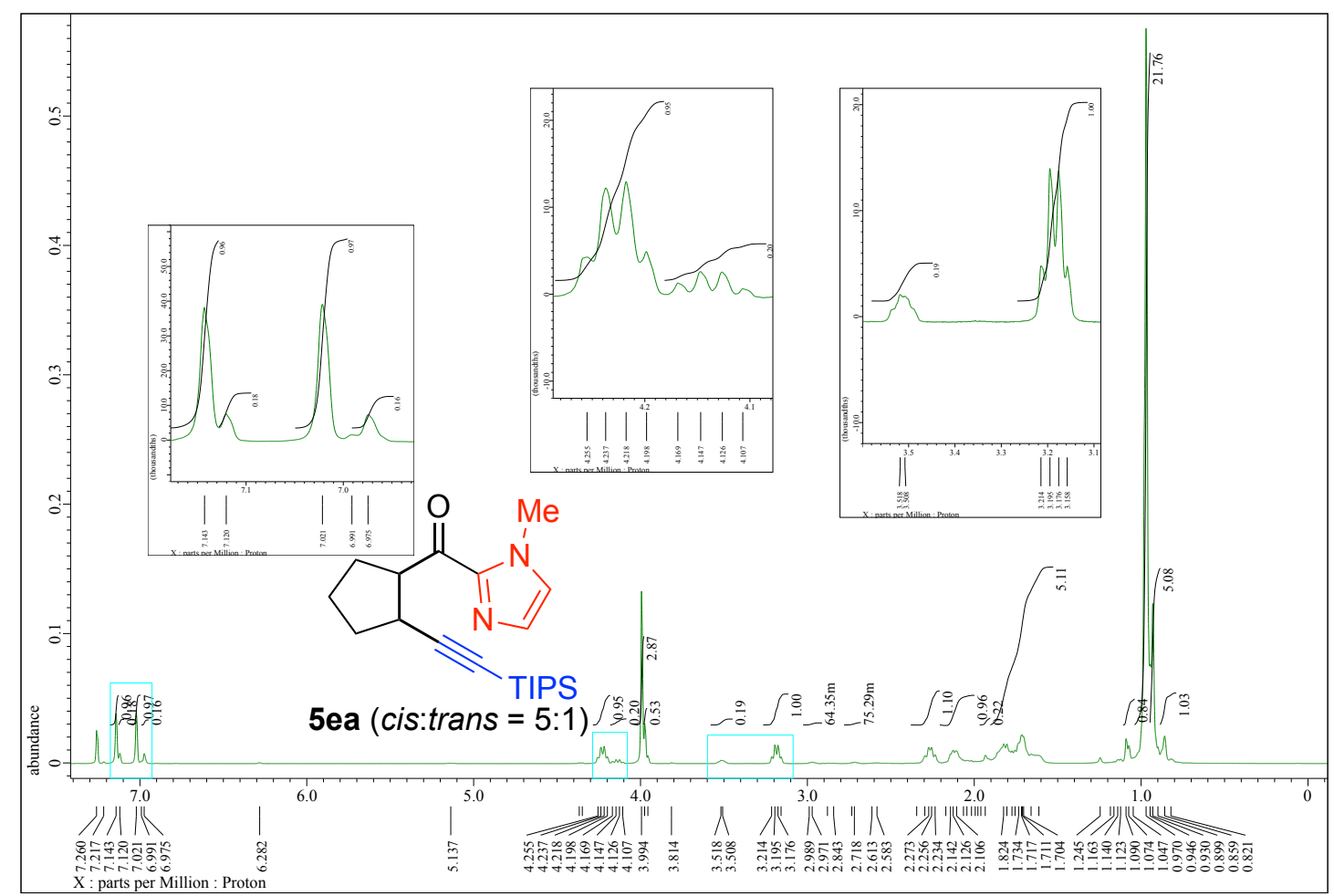

Figure 140: ${ }^{1} \mathrm{H}$ NMR spectrum of compound 5ea (cis:trans $\left.=5: 1\right)\left(400 \mathrm{MHz}, \mathrm{CDCl}_{3}\right)$.

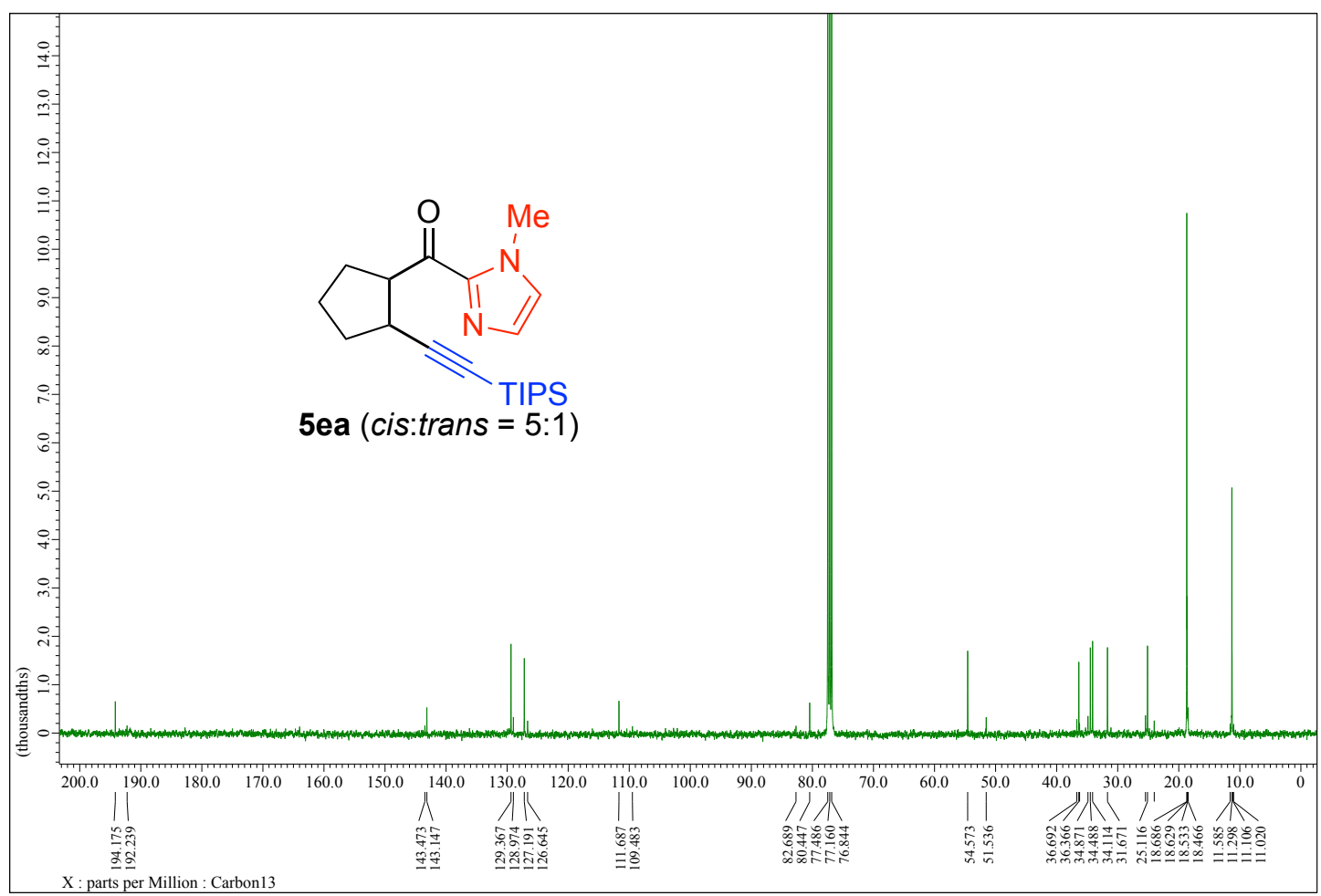

Figure S141: ${ }^{13} \mathrm{C}$ NMR spectrum of compound 5ea (cis:trans $\left.=5: 1\right)\left(100 \mathrm{MHz}, \mathrm{CDCl}_{3}\right)$. 


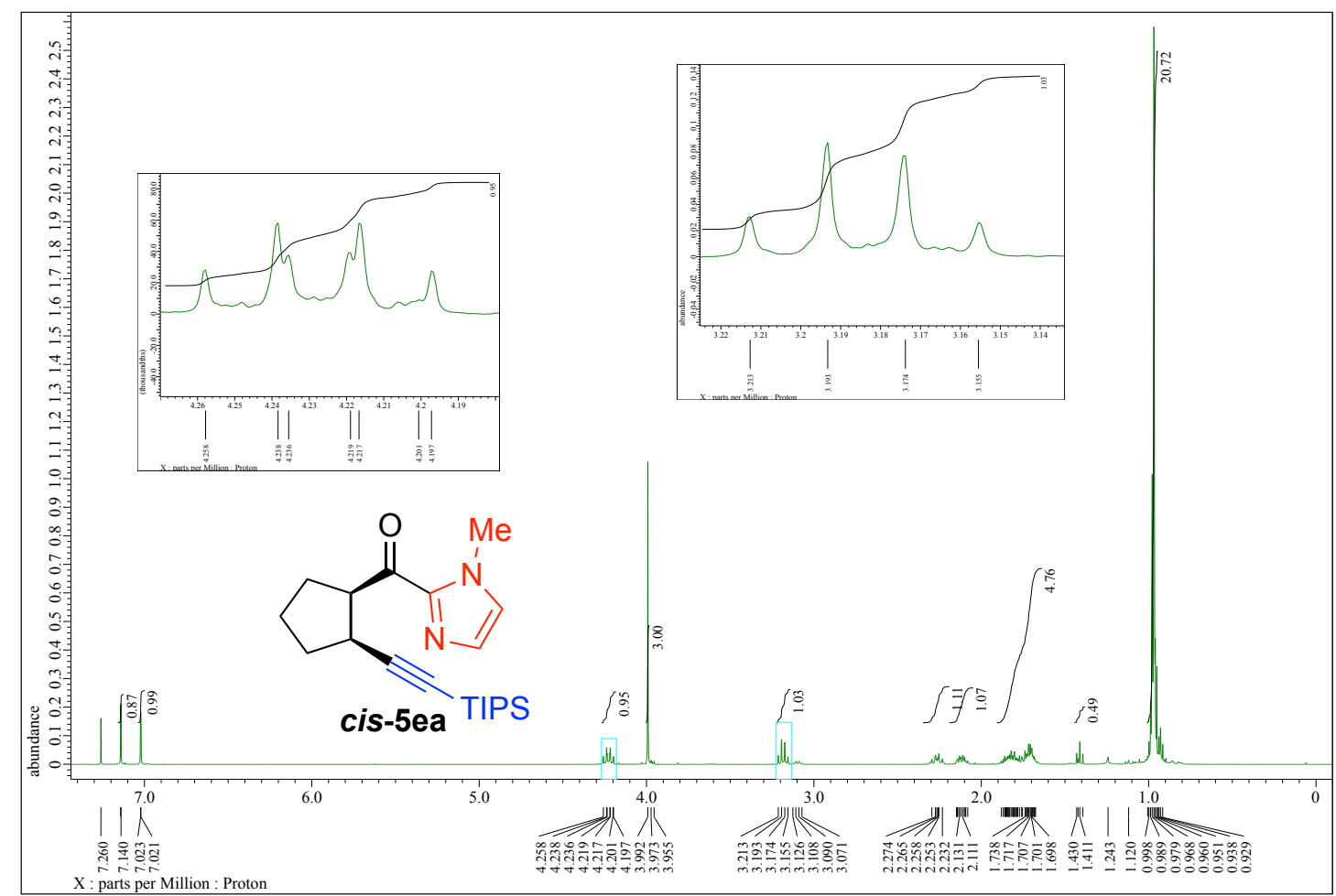

Figure S142: ${ }^{1} \mathrm{H}$ NMR spectrum of compound cis-5ea (400 MHz, $\left.\mathrm{CDCl}_{3}\right)$.

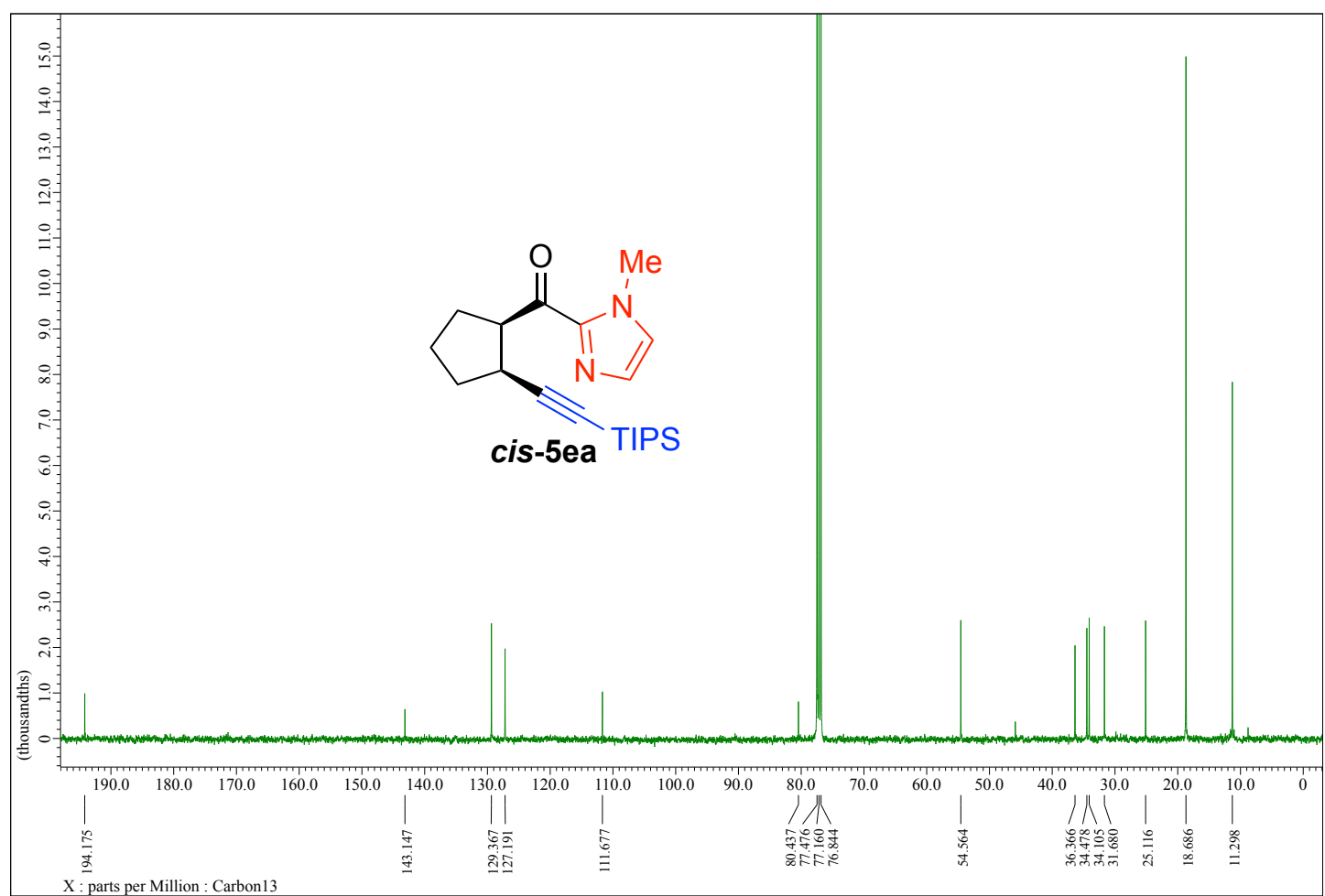

Figure S143: ${ }^{13} \mathrm{C}$ NMR spectrum of compound cis-5ea $\left(100 \mathrm{MHz}, \mathrm{CDCl}_{3}\right)$. 


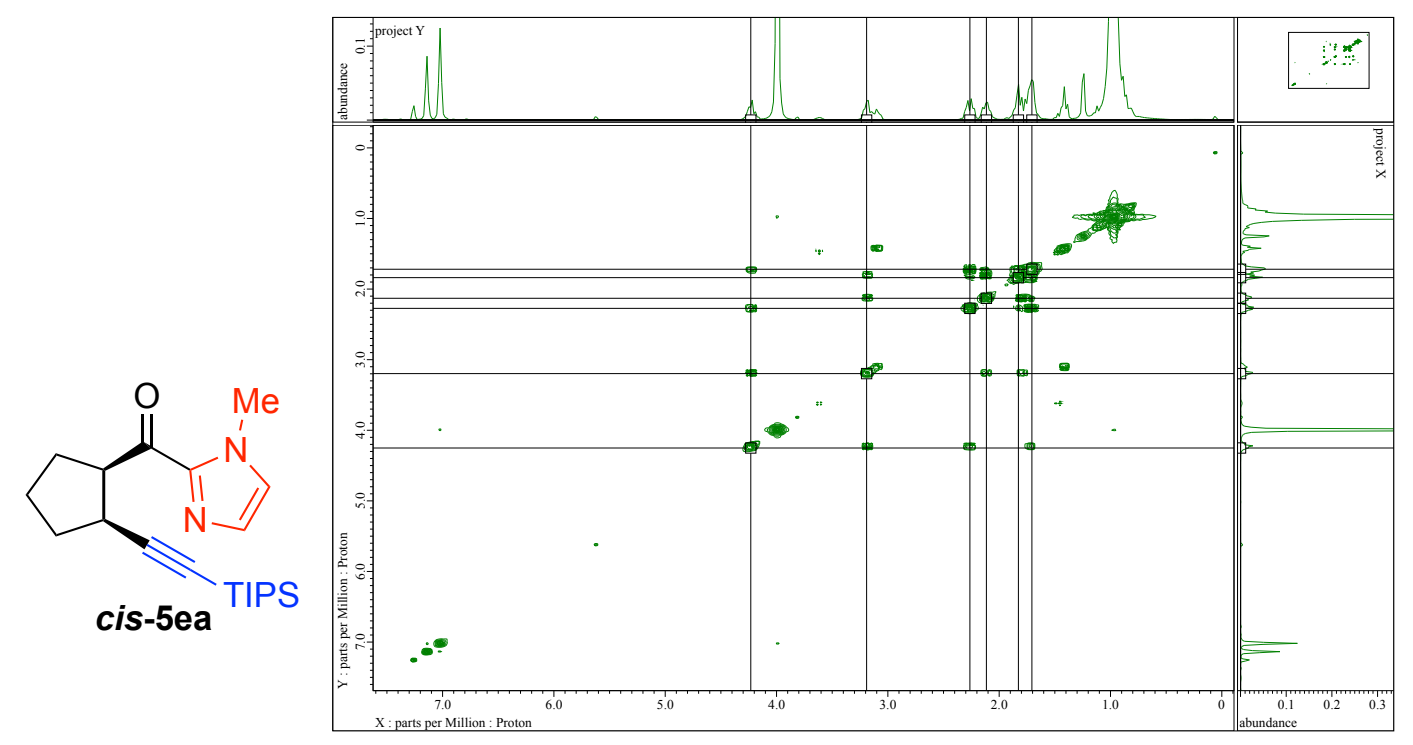

Figure S144: COSY spectrum of compound cis-5ea (400 MHz, $\left.\mathrm{CDCl}_{3}\right)$.

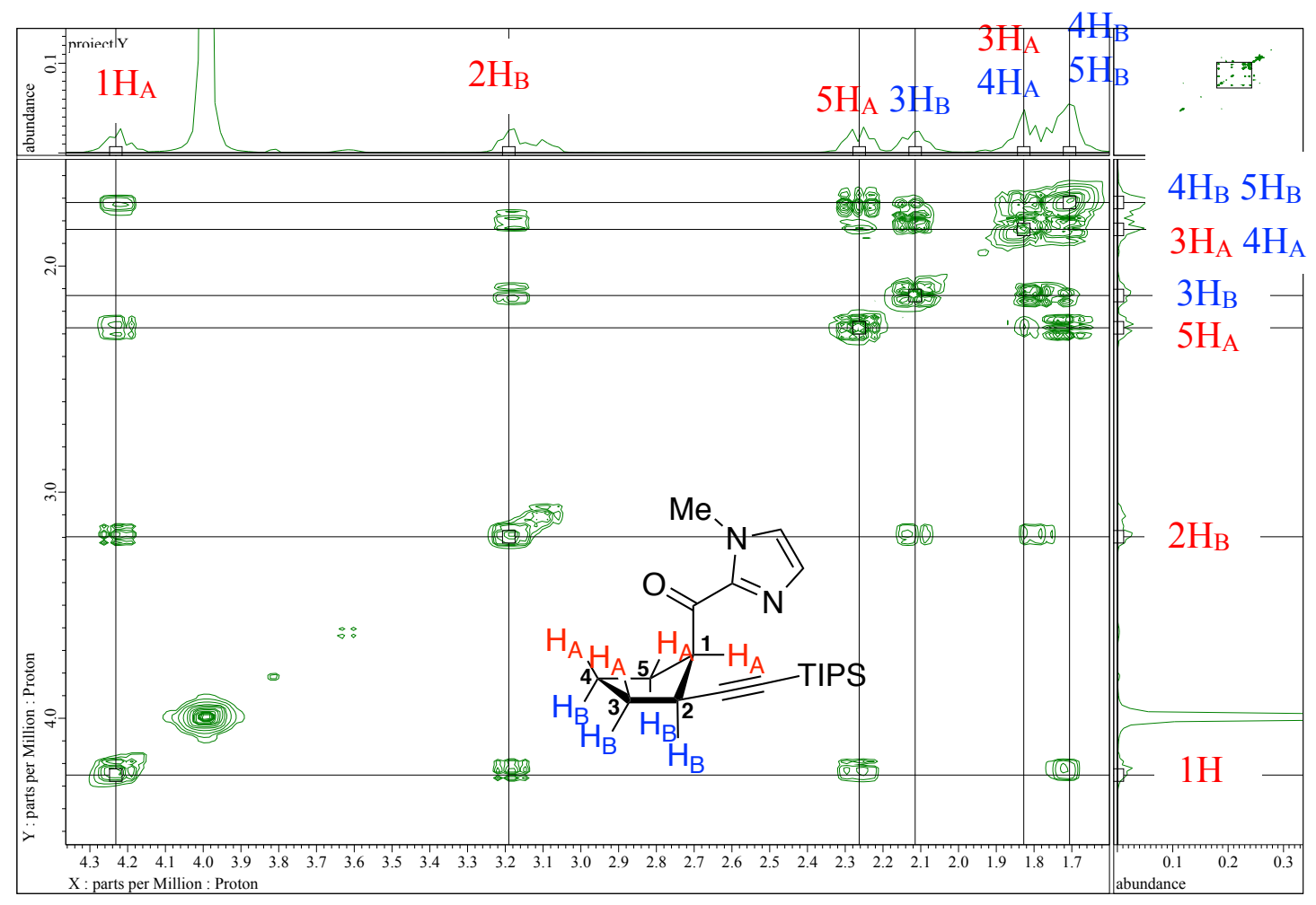

Figure S145: COSY spectrum (expanded) of compound cis-5ea (400 MHz, $\left.\mathrm{CDCl}_{3}\right)$. 


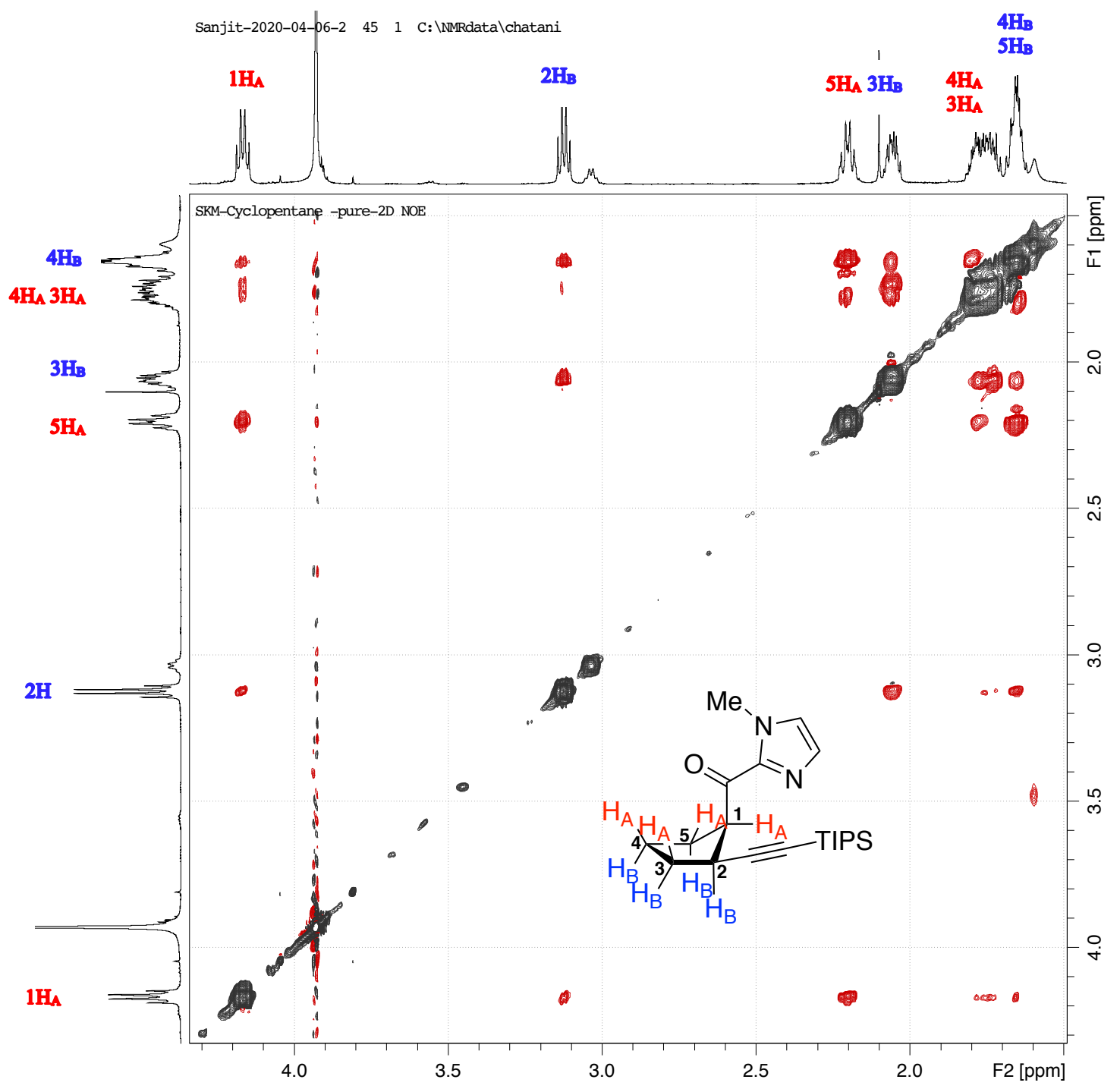

Figure S146: NOESY spectrum for cis-5ea (Bruker; $600 \mathrm{MHz}, \mathrm{CDCl}_{3}$ ). 


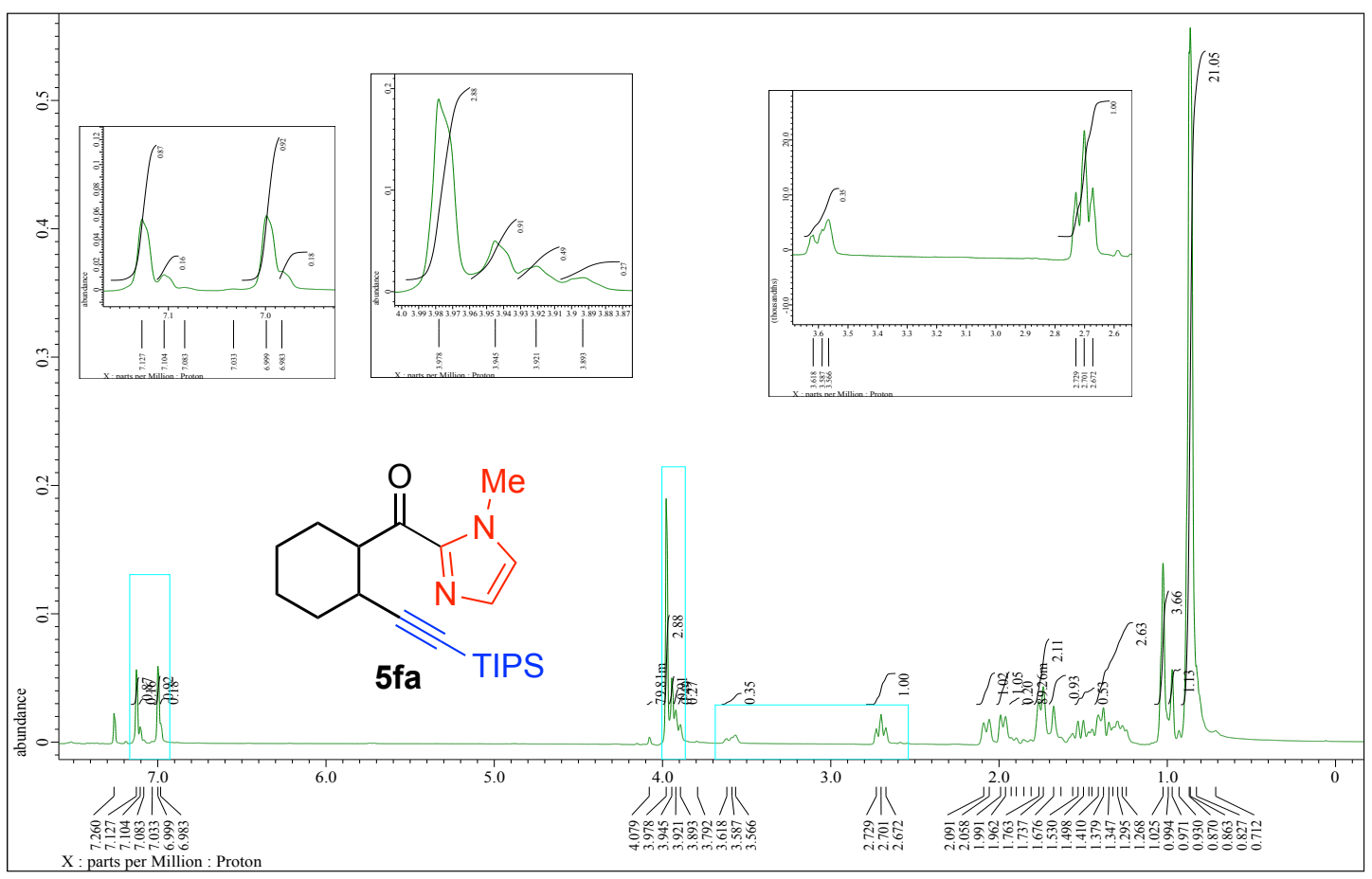

Figure 147: ${ }^{1} \mathrm{H}$ NMR spectrum of compound $\mathbf{5 f a}$ (cis:trans $\left.=10: 3.5\right)\left(400 \mathrm{MHz}, \mathrm{CDCl}_{3}\right)$.

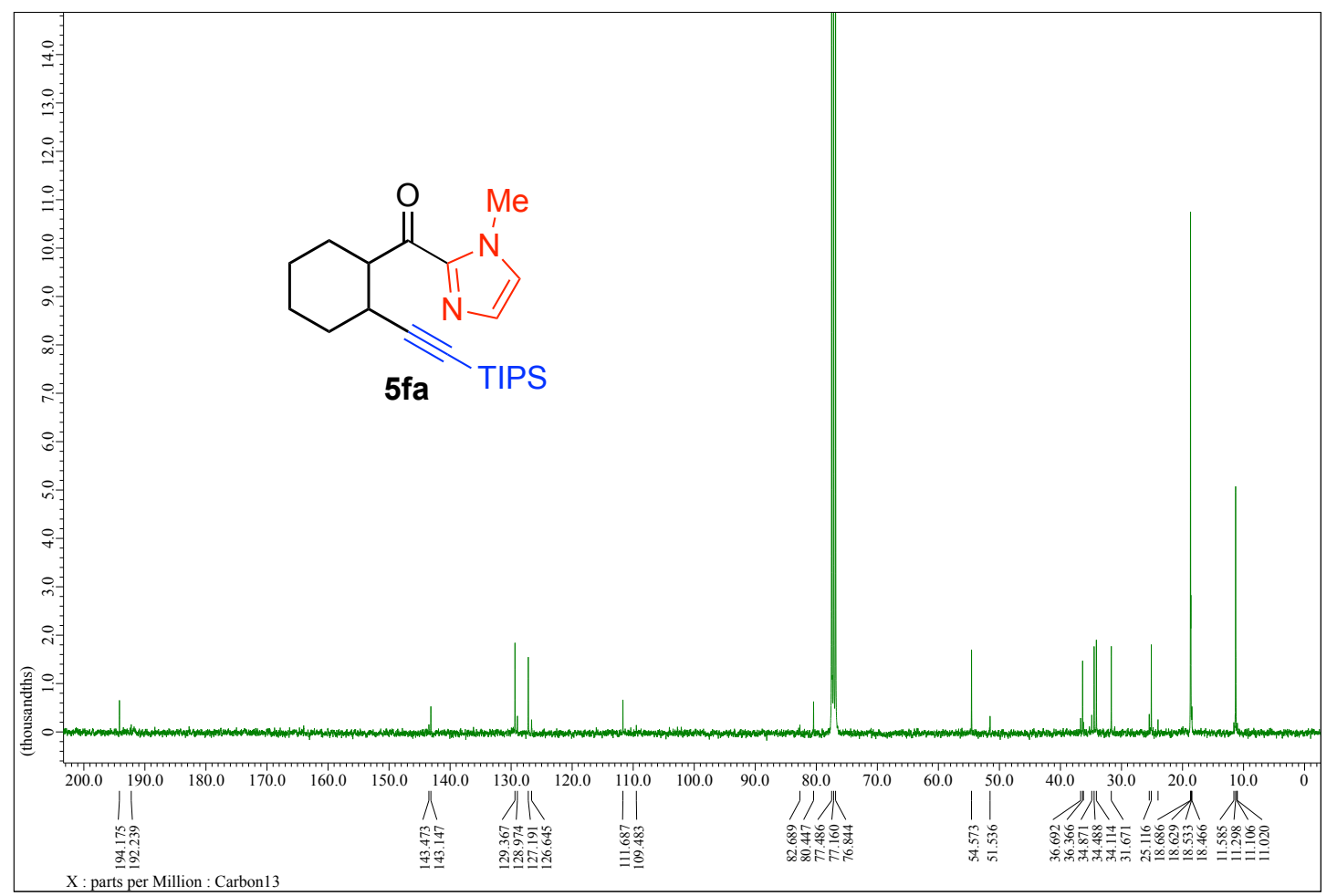

Figure 148: ${ }^{13} \mathrm{C}$ NMR spectrum of compound $\mathbf{5 f a}($ cis:trans $=10: 3.5)\left(100 \mathrm{MHz}, \mathrm{CDCl}_{3}\right)$. 


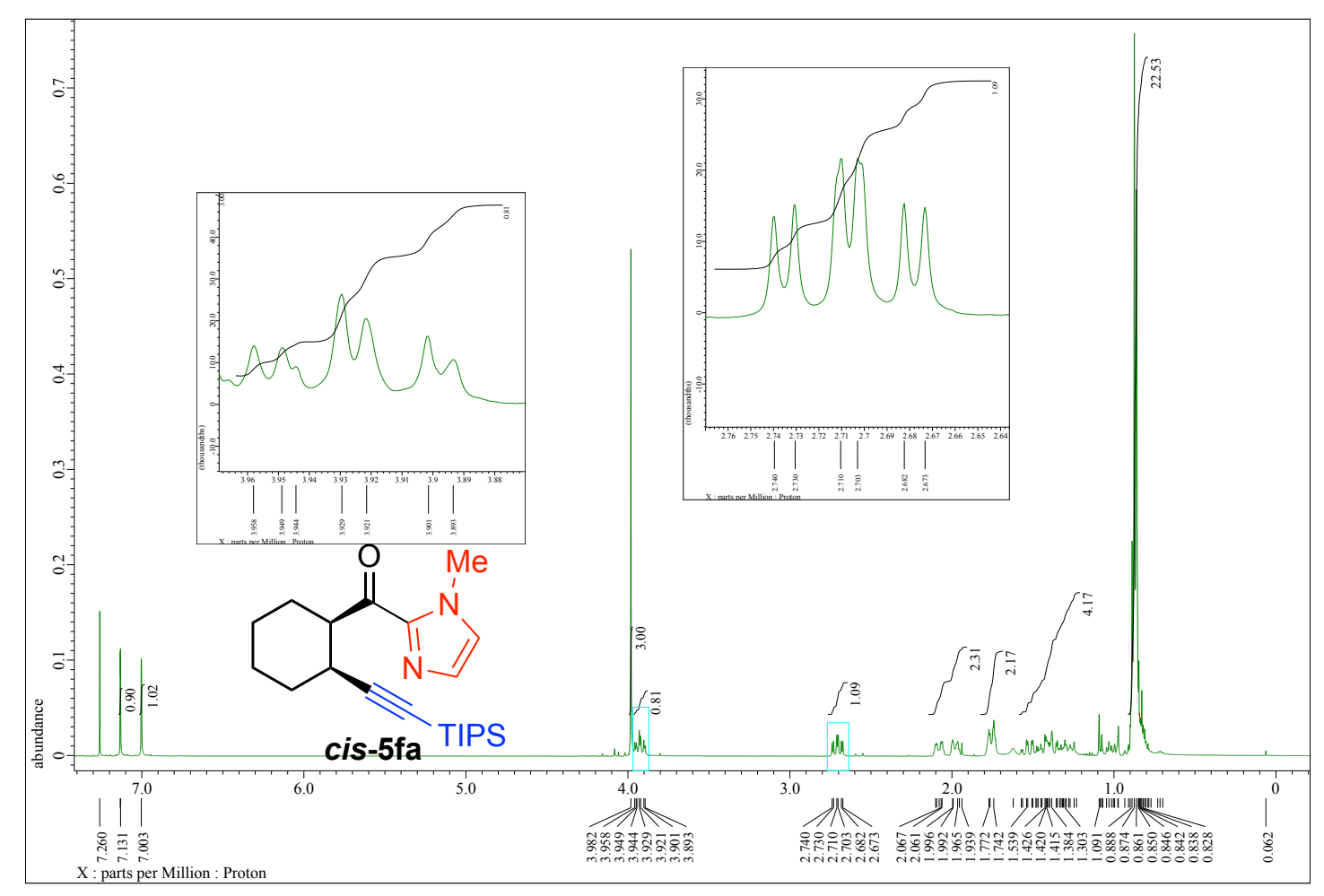

Figure S149: ${ }^{1} \mathrm{H}$ NMR spectrum of compound cis-5fa $\left(400 \mathrm{MHz}, \mathrm{CDCl}_{3}\right)$.

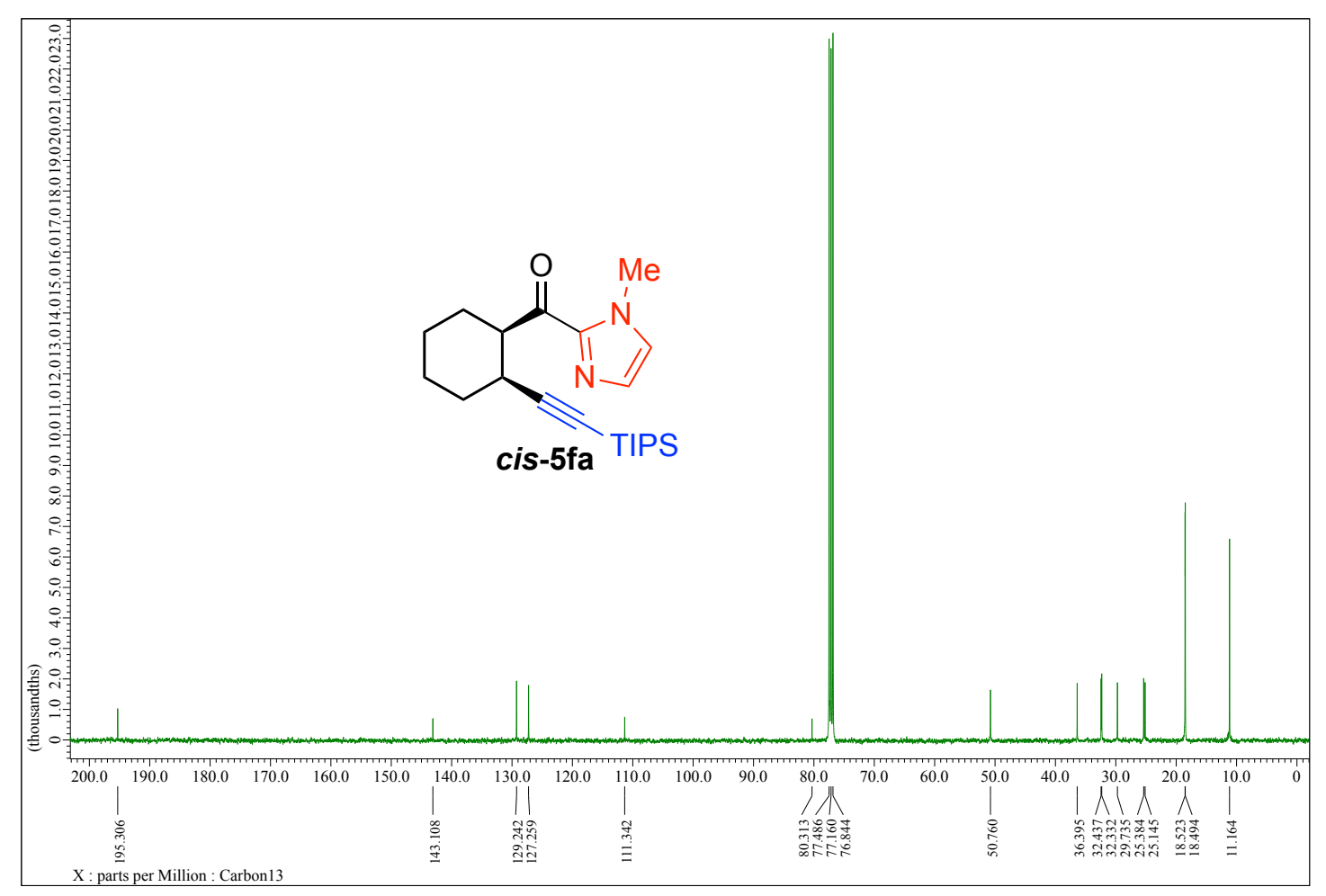

Figure S150: ${ }^{13} \mathrm{C}$ NMR spectrum of compound cis-5fa $\left(100 \mathrm{MHz}, \mathrm{CDCl}_{3}\right)$. 


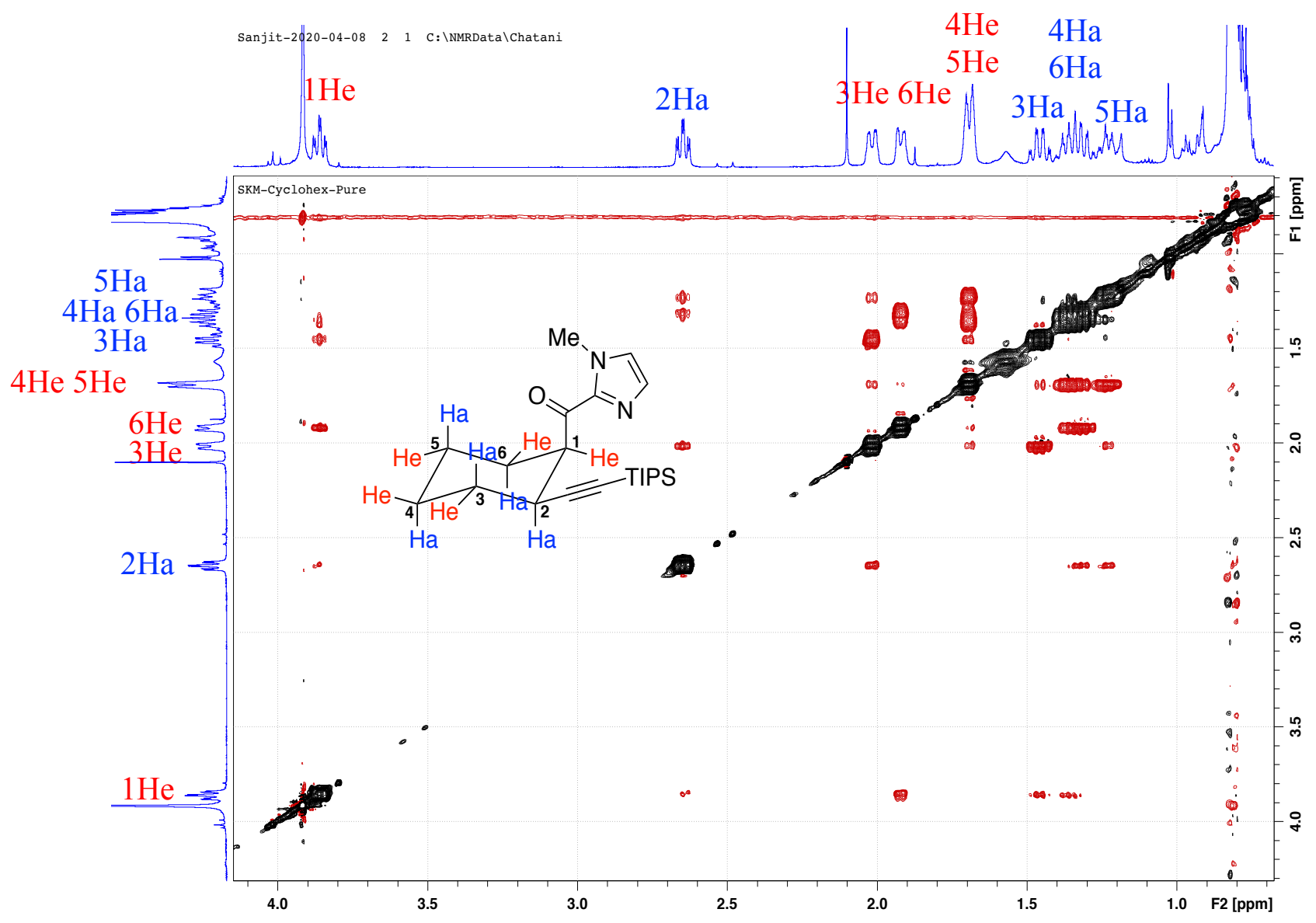

Figure S151: NOESY spectrum for cis-5fa (Bruker; $600 \mathrm{MHz}, \mathrm{CDCl}_{3}$ ). 


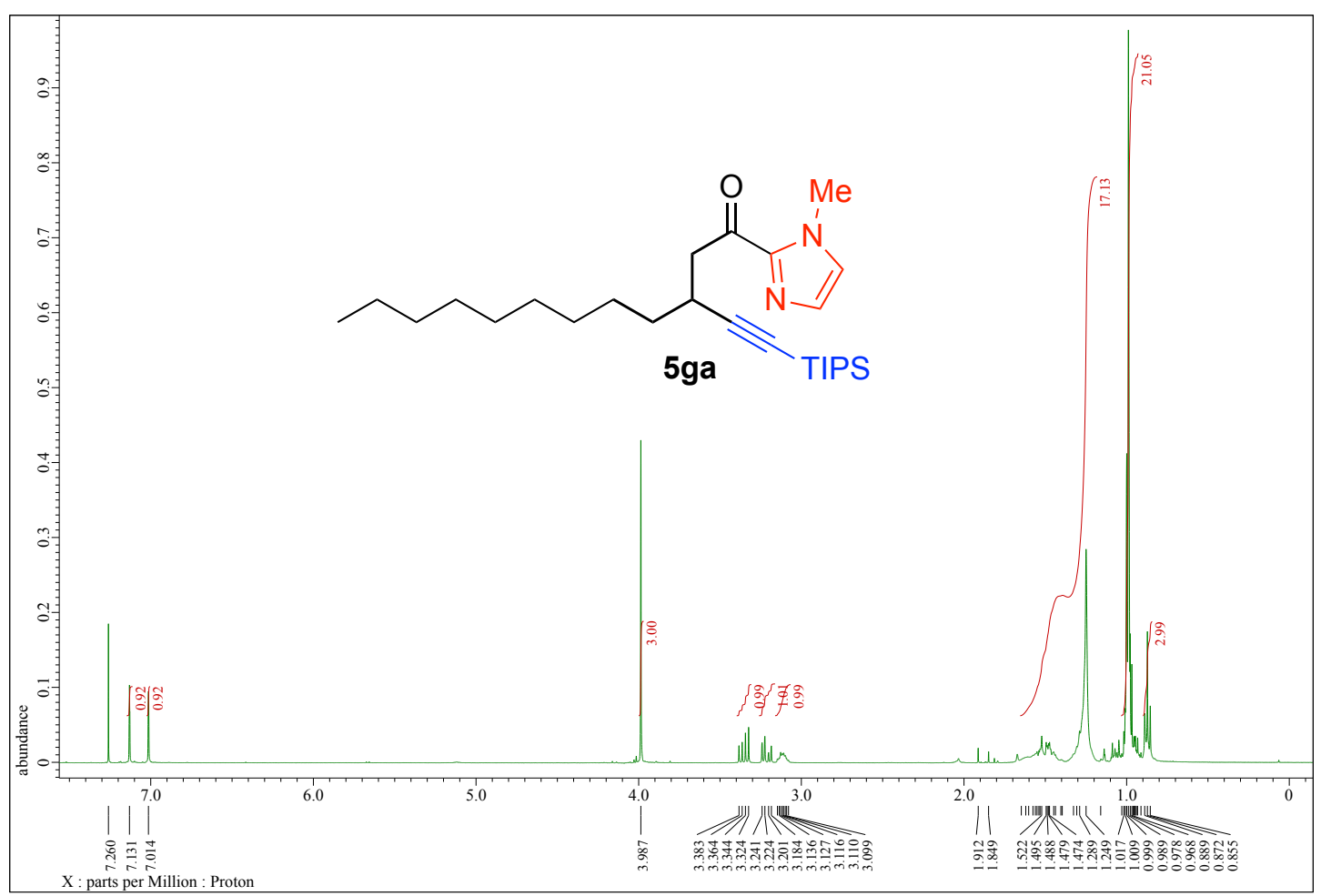

Figure S152: ${ }^{1} \mathrm{H}$ NMR spectrum of compound 5 ga (400 $\left.\mathrm{MHz}, \mathrm{CDCl}_{3}\right)$.

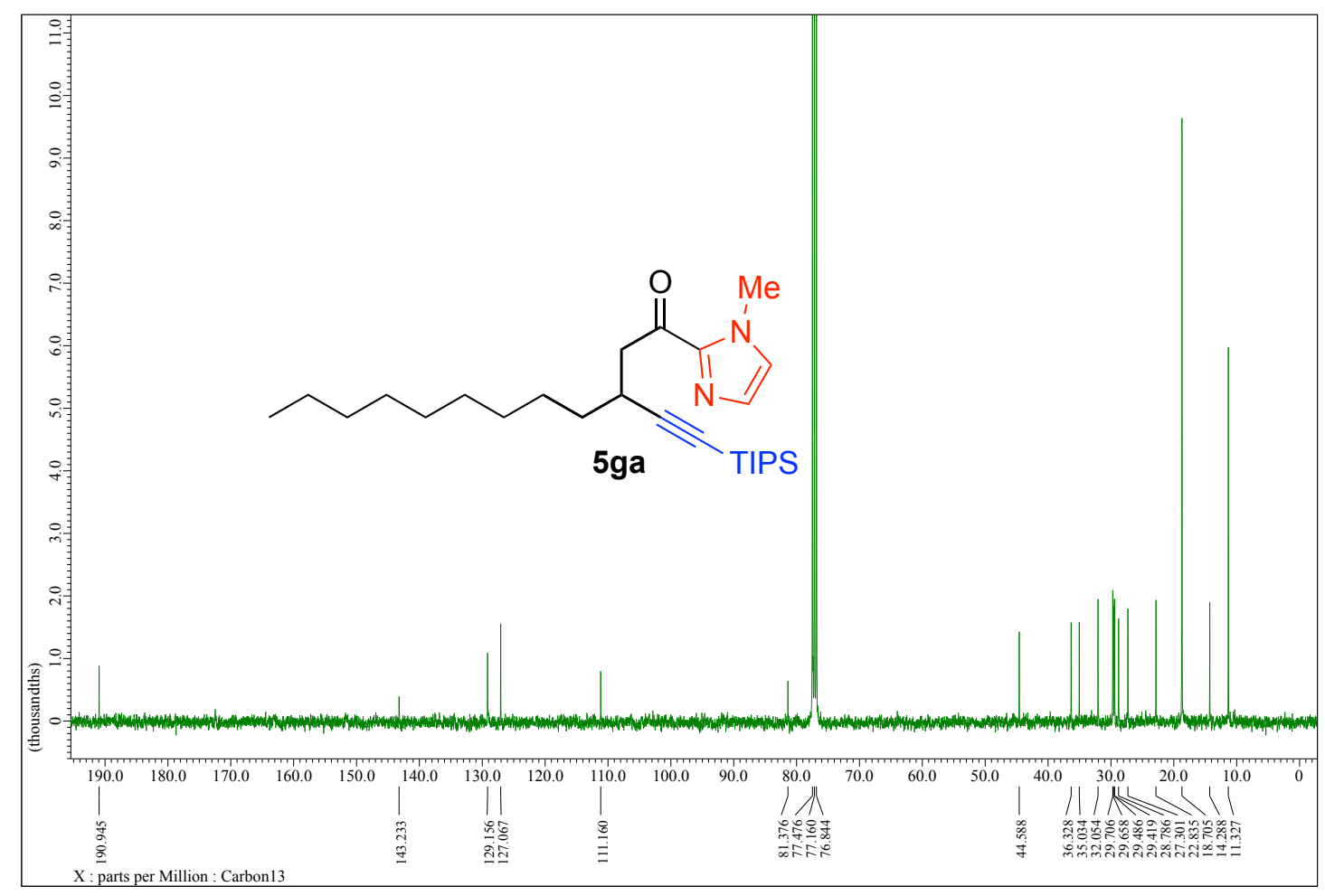

Figure S153: ${ }^{13} \mathrm{C}$ NMR spectrum of compound 5ga (100 $\left.\mathrm{MHz}, \mathrm{CDCl}_{3}\right)$. 


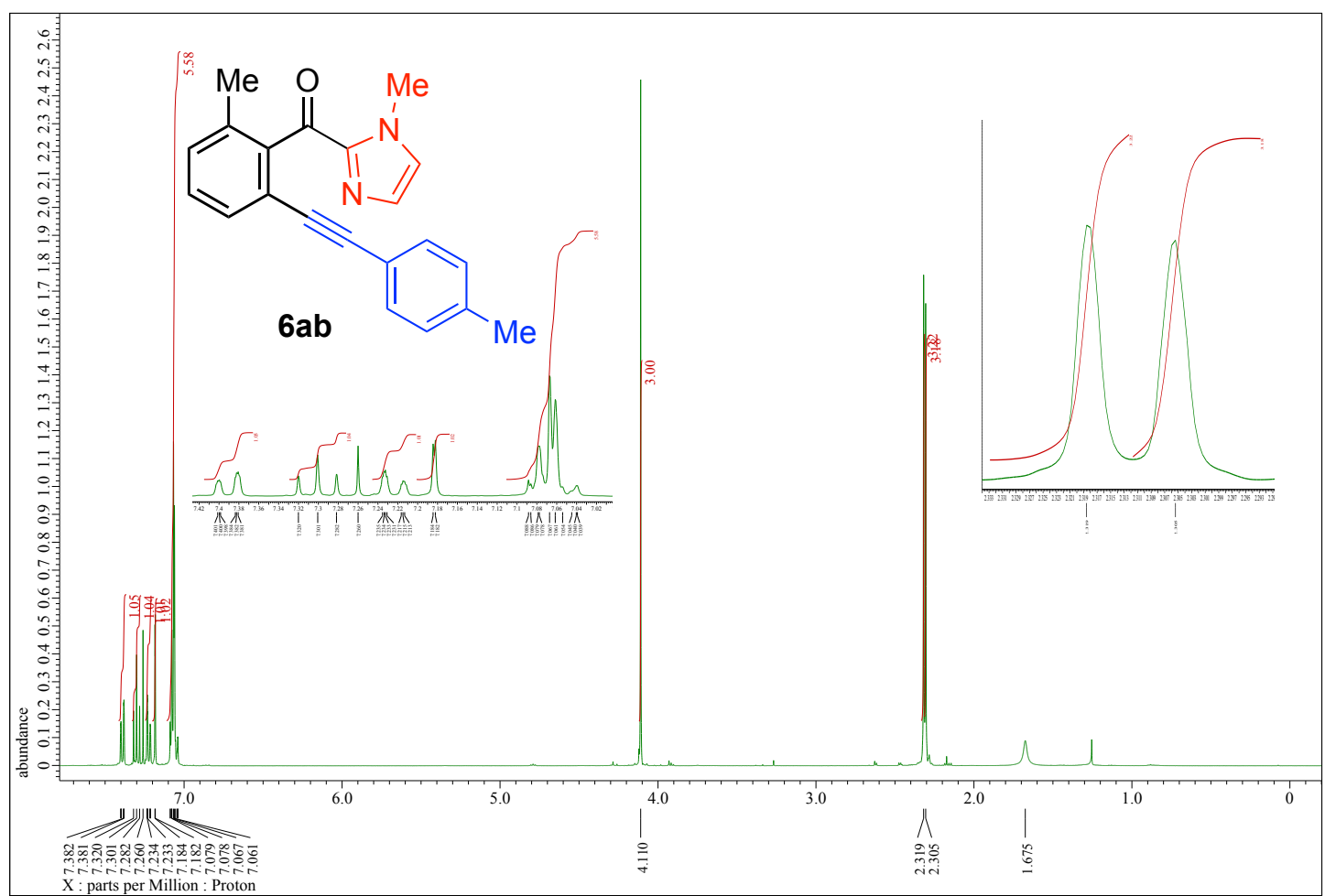

Figure S154: ${ }^{1} \mathrm{H}$ NMR spectrum of compound $\mathbf{6 a b}\left(400 \mathrm{MHz}, \mathrm{CDCl}_{3}\right)$.

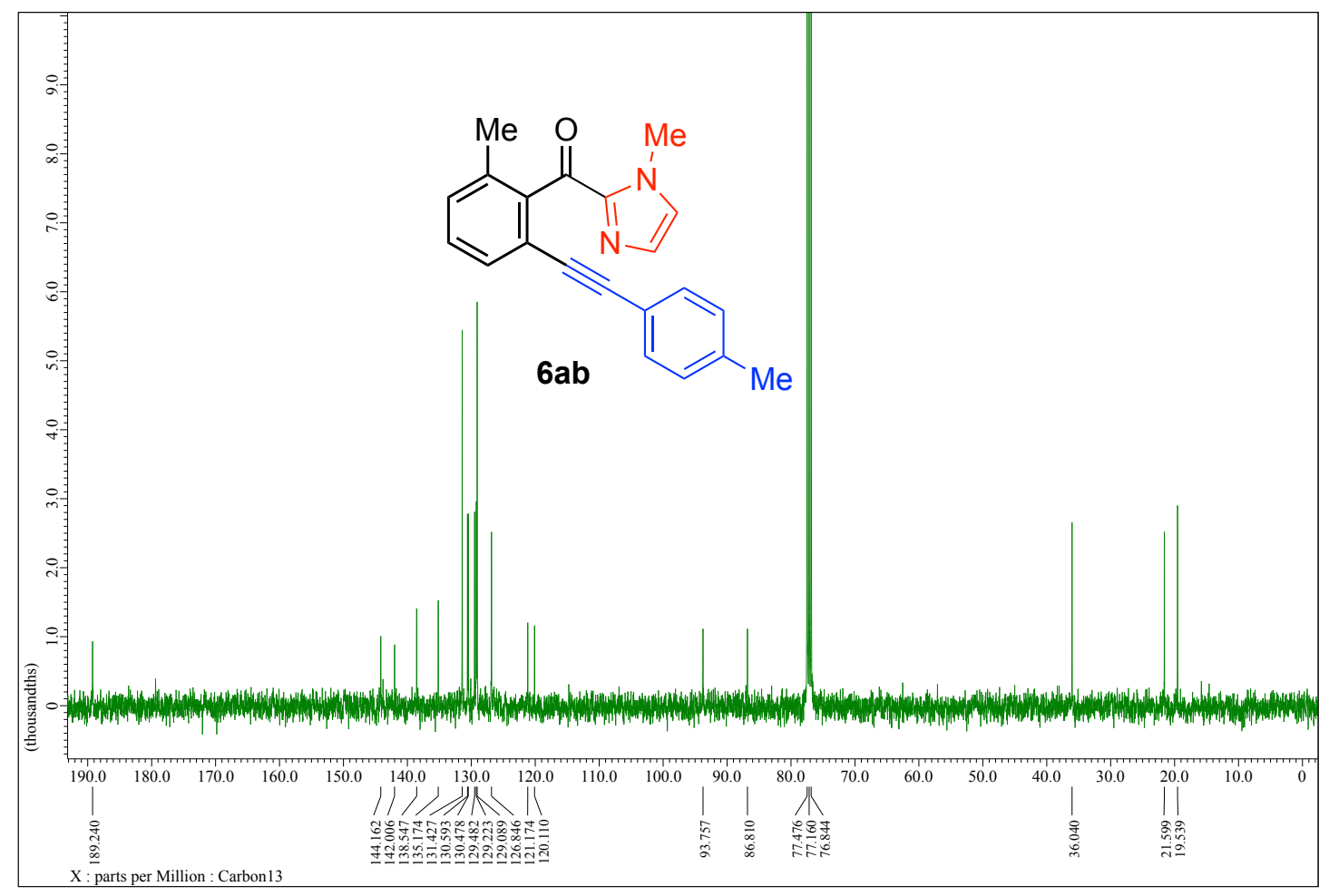

Figure S155: ${ }^{13} \mathrm{C}$ NMR spectrum of compound $\mathbf{6 a b}\left(100 \mathrm{MHz}, \mathrm{CDCl}_{3}\right)$. 


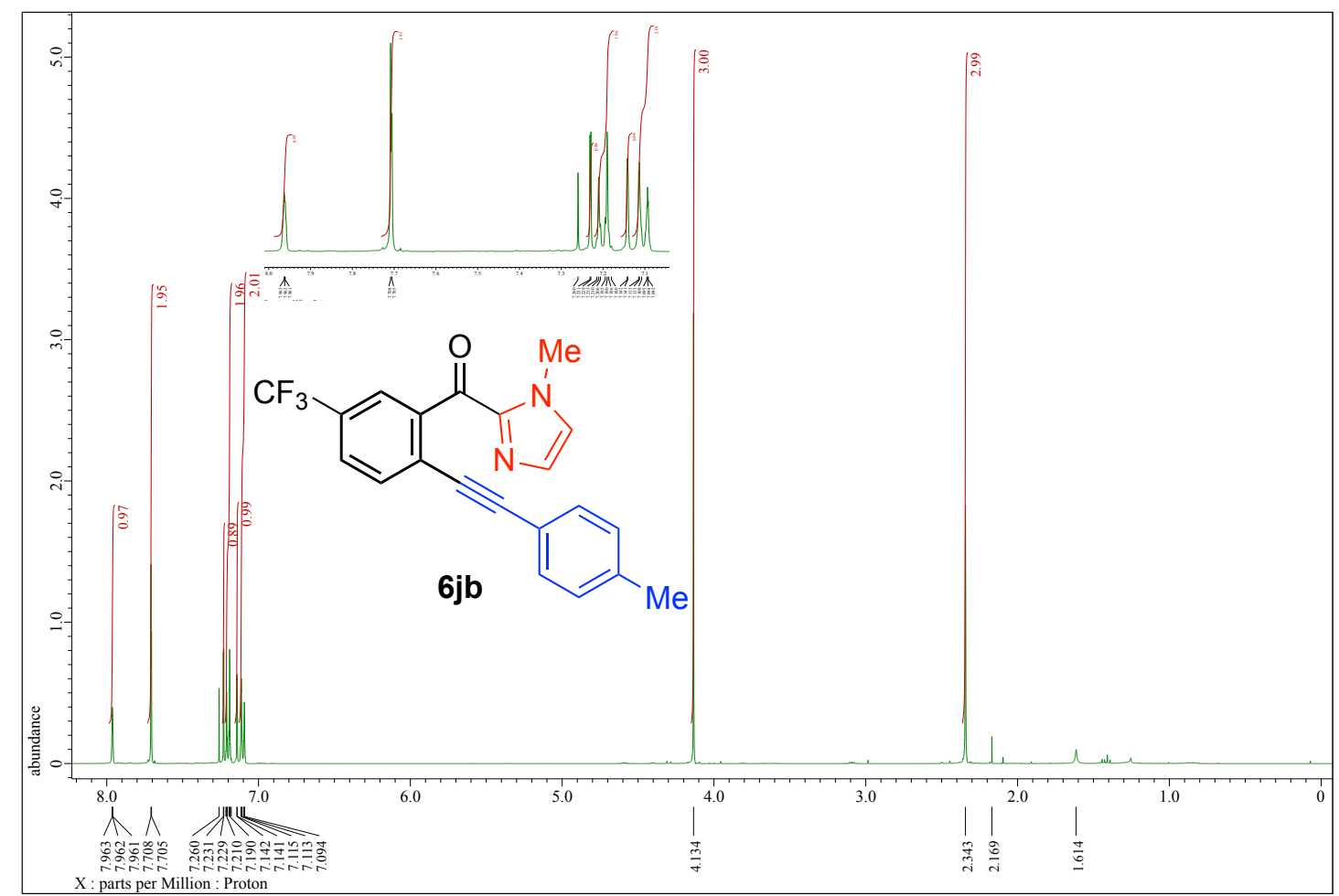

Figure S156: ${ }^{1} \mathrm{H}$ NMR spectrum of compound $\mathbf{6 j b}\left(400 \mathrm{MHz}, \mathrm{CDCl}_{3}\right)$.

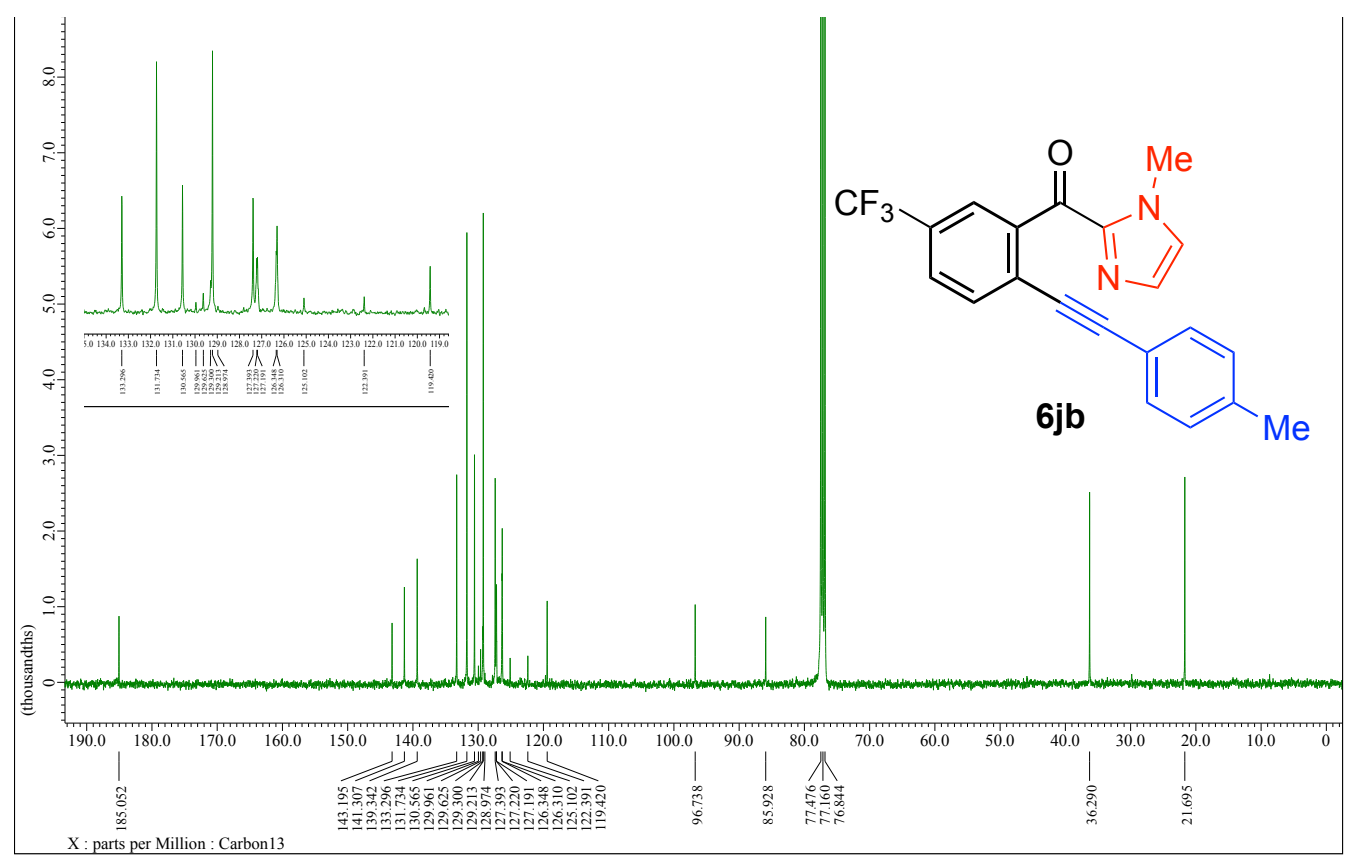

Figure S157: ${ }^{13} \mathrm{C}$ NMR spectrum of compound $\mathbf{6 j b}\left(100 \mathrm{MHz}, \mathrm{CDCl}_{3}\right)$. 


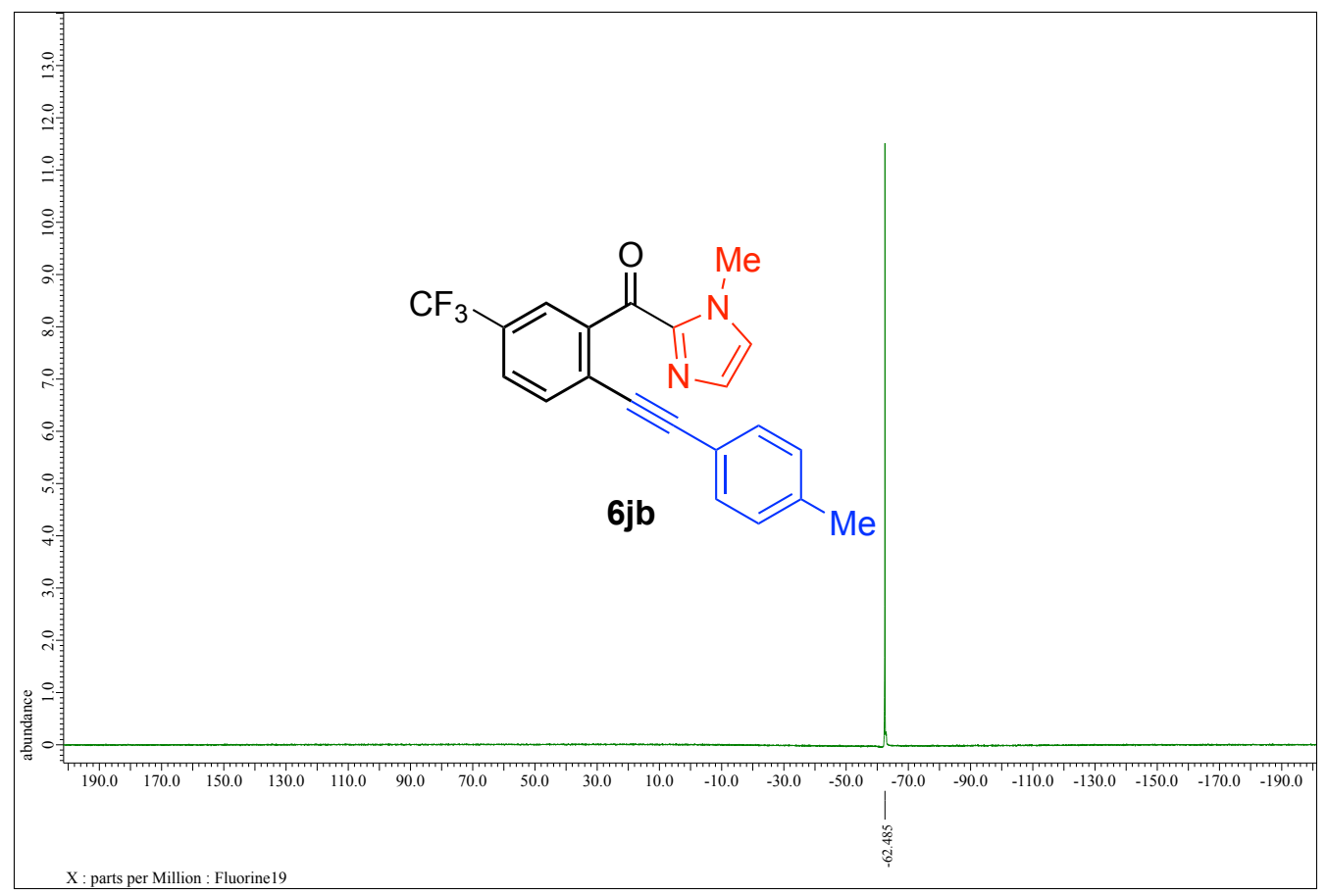

Figure S158: ${ }^{19} \mathrm{~F}$ NMR spectrum of compound $\mathbf{6 j b}\left(376 \mathrm{MHz}, \mathrm{CDCl}_{3}\right)$.

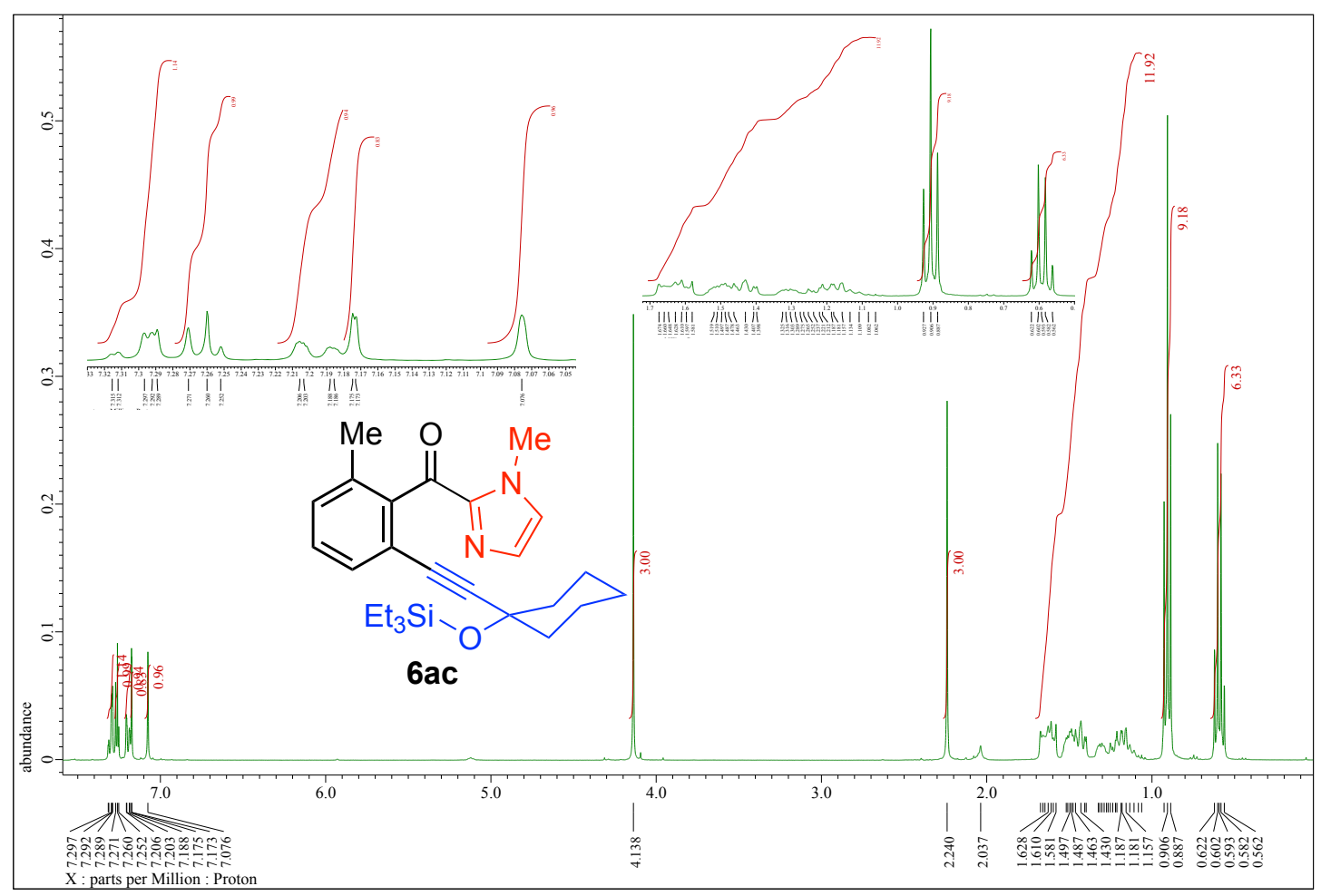

Figure S159: ${ }^{1} \mathrm{H}$ NMR spectrum of compound 6ac $\left(400 \mathrm{MHz}, \mathrm{CDCl}_{3}\right)$. 


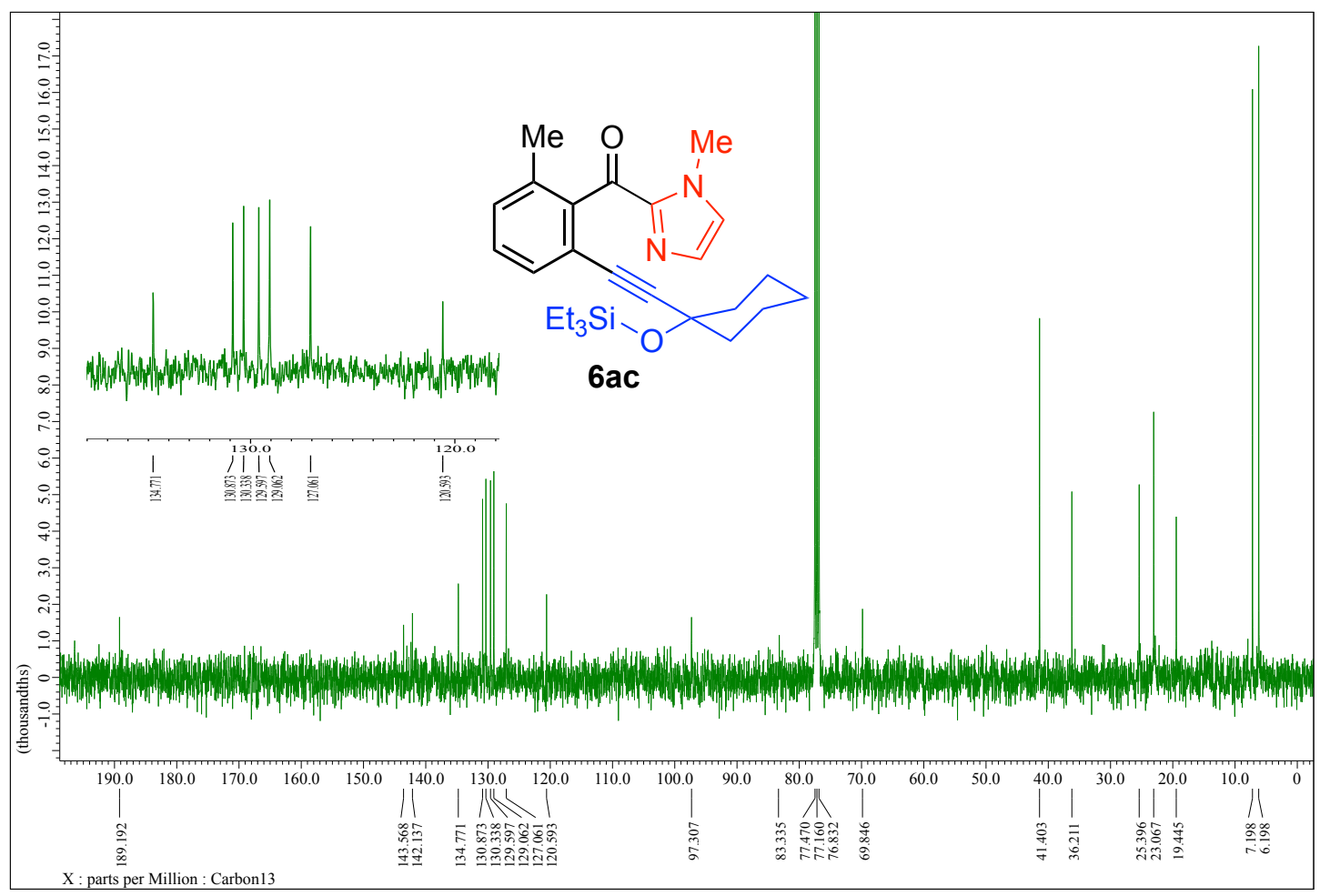

Figure S160: ${ }^{13} \mathrm{C}$ NMR spectrum of compound 6ac $\left(100 \mathrm{MHz}, \mathrm{CDCl}_{3}\right)$.

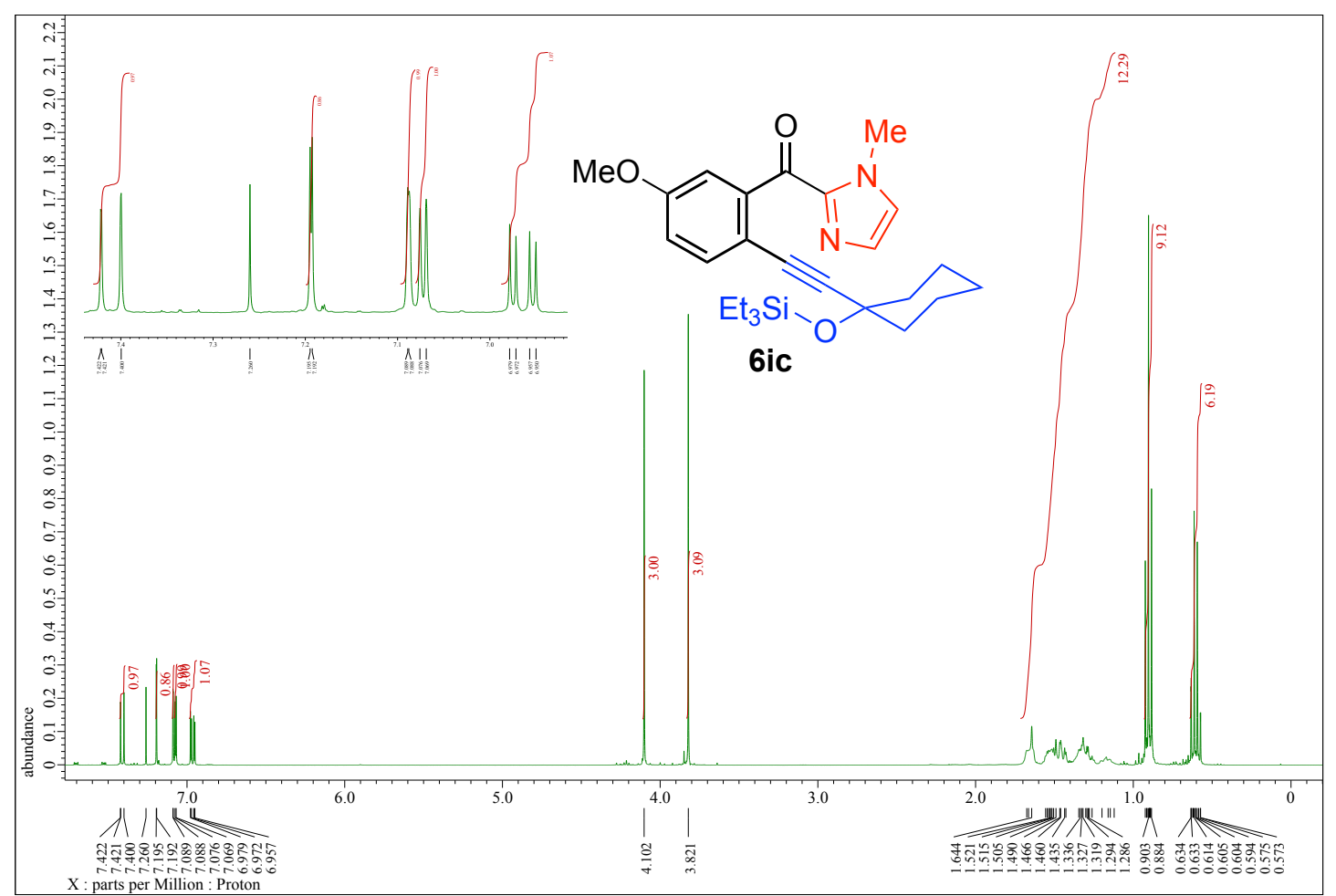

Figure S161: ${ }^{1} \mathrm{H}$ NMR spectrum of compound 6ic $\left(400 \mathrm{MHz}, \mathrm{CDCl}_{3}\right)$. 


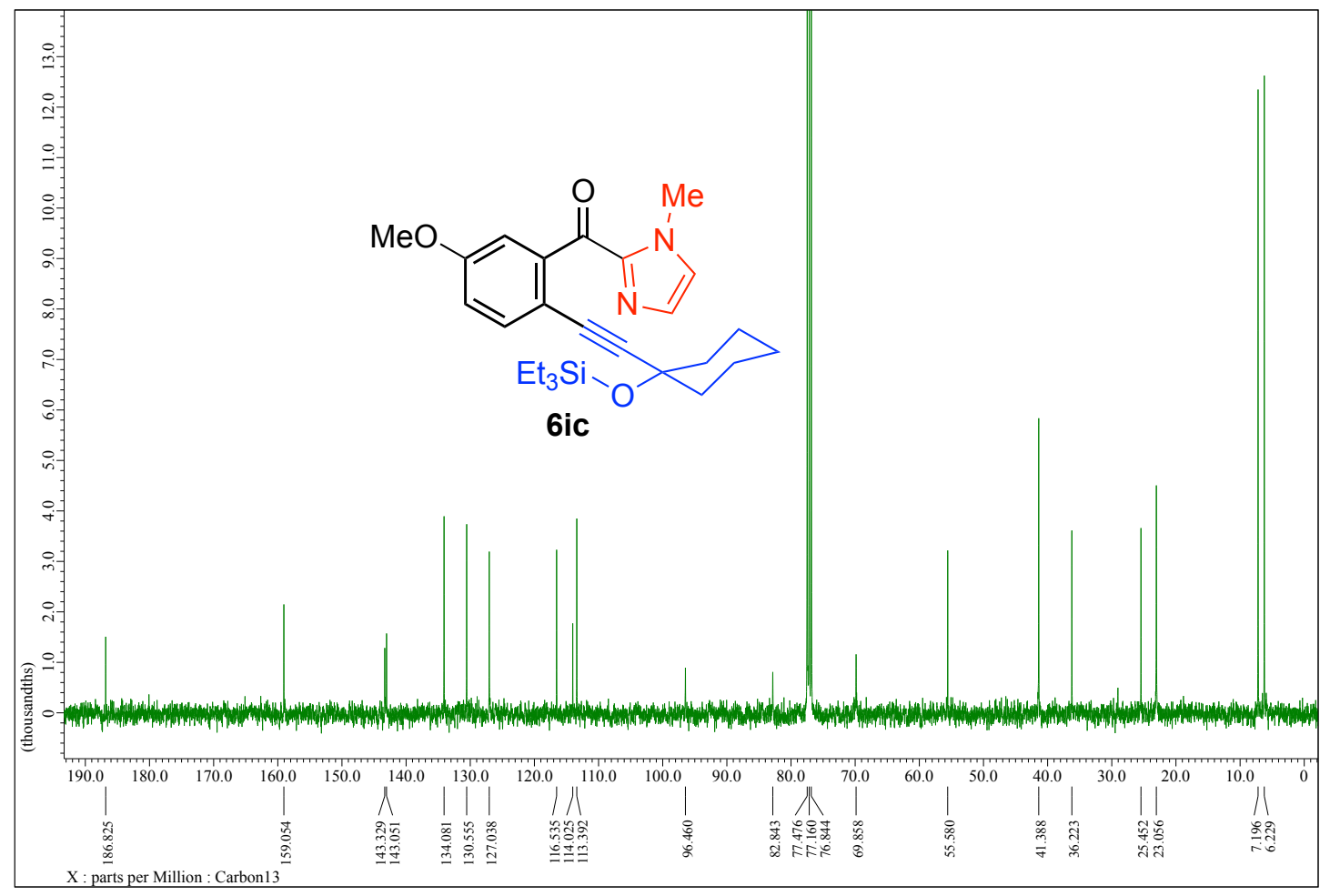

Figure S162: ${ }^{13} \mathrm{C}$ NMR spectrum of compound 6ic $\left(100 \mathrm{MHz}, \mathrm{CDCl}_{3}\right)$.

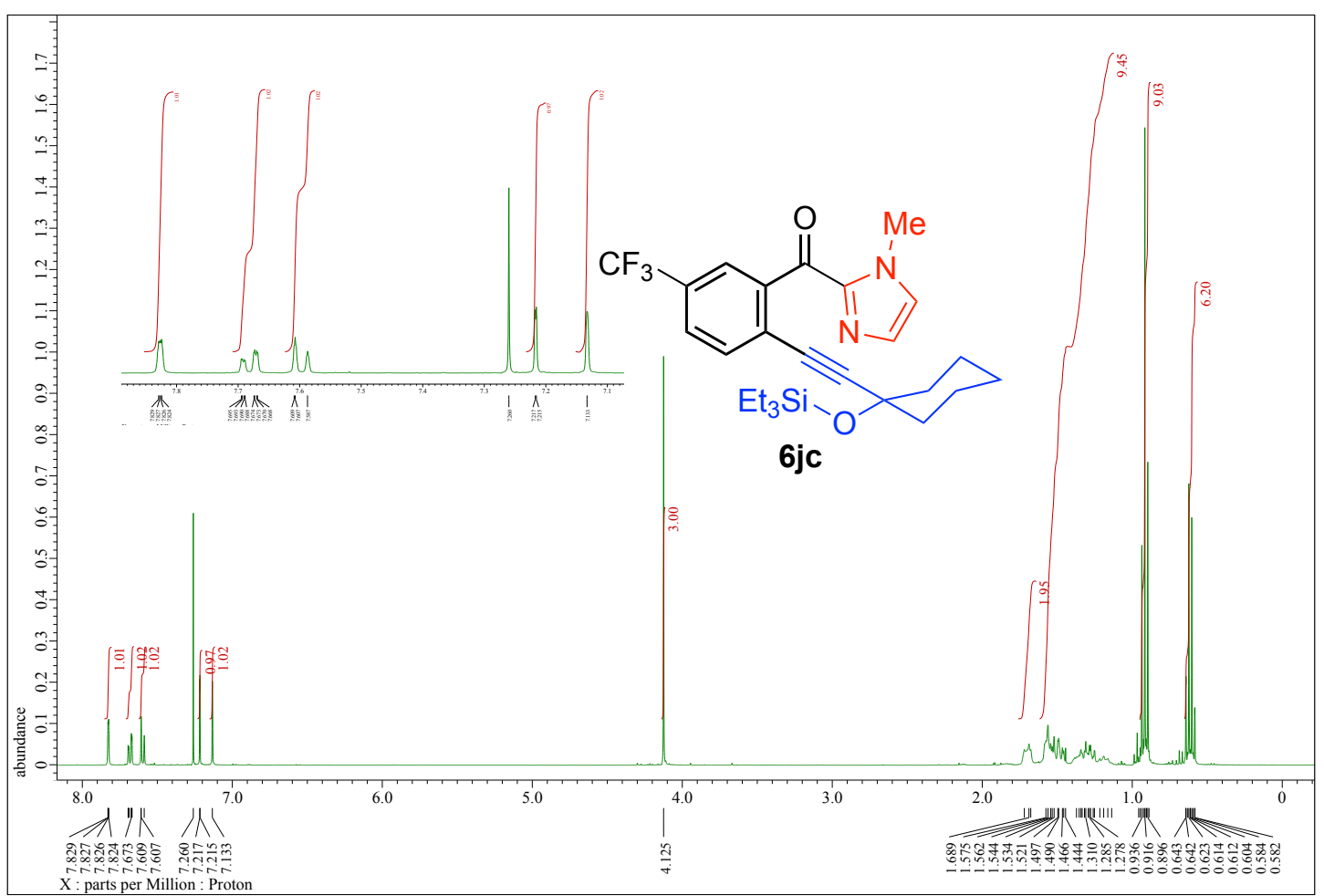

Figure S163: ${ }^{1} \mathrm{H}$ NMR spectrum of compound $\mathbf{6 j c}\left(400 \mathrm{MHz}, \mathrm{CDCl}_{3}\right)$. 


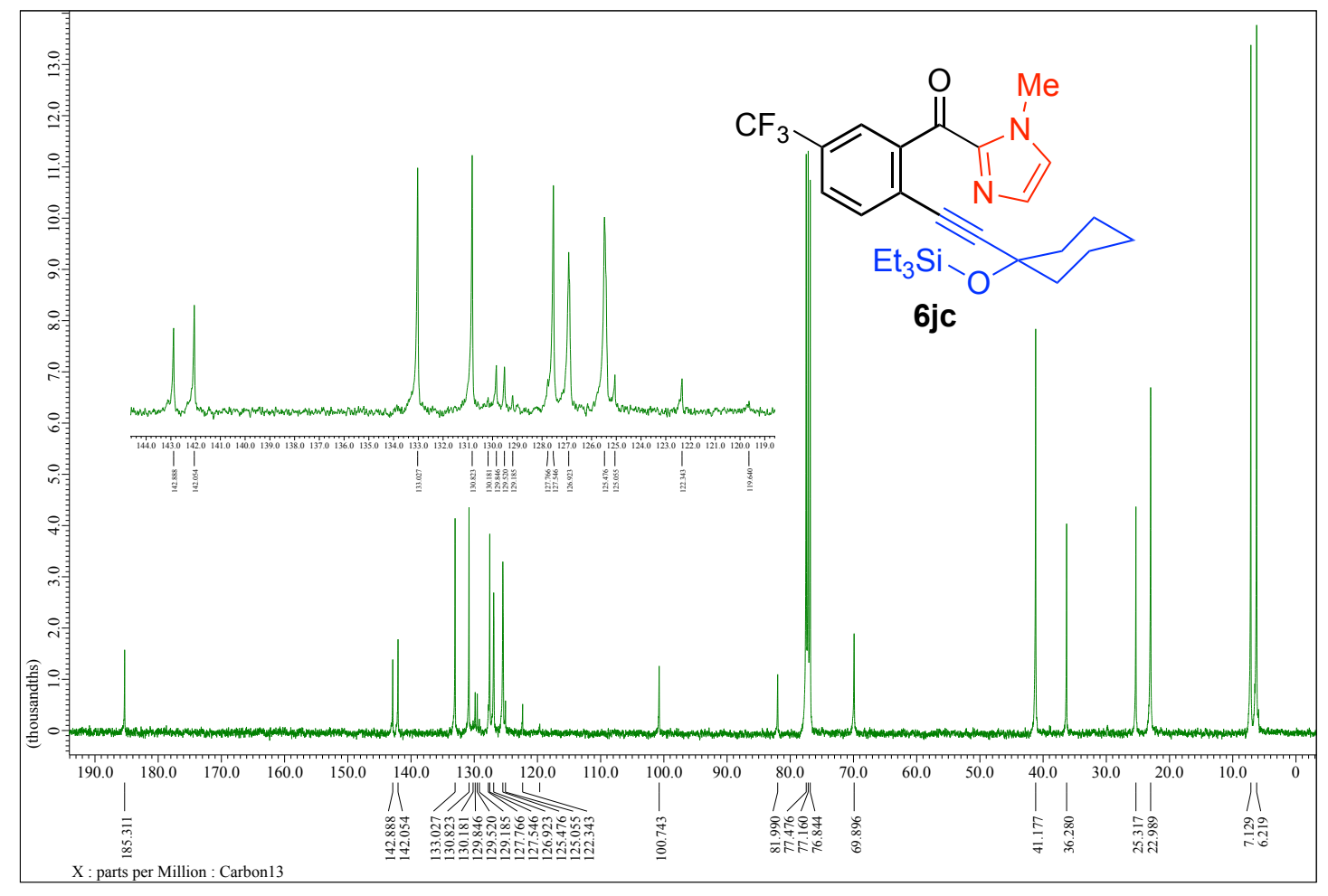

Figure S164: ${ }^{13} \mathrm{C}$ NMR spectrum of compound 6je (100 MHz, $\left.\mathrm{CDCl}_{3}\right)$.

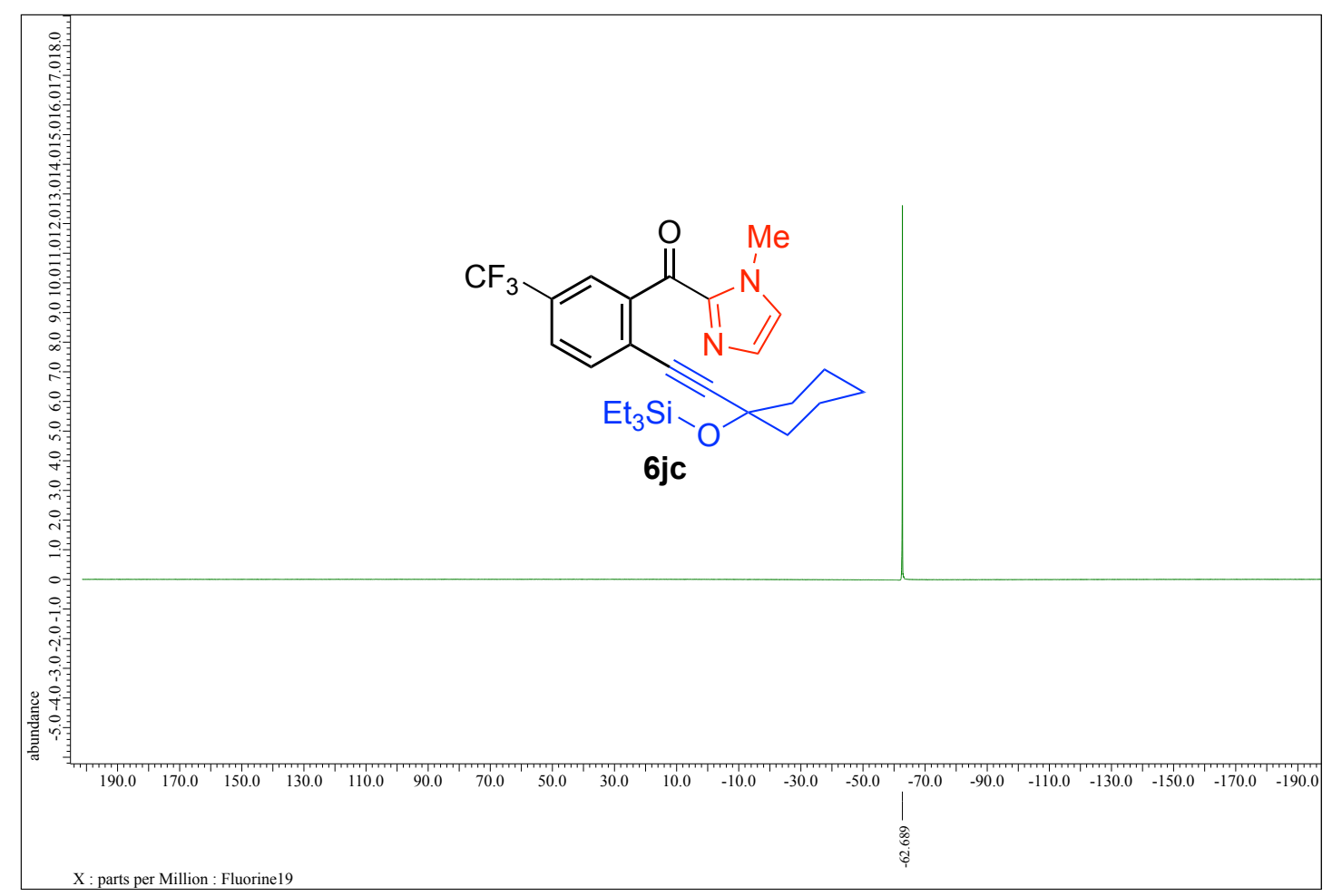

Figure S165: ${ }^{19} \mathrm{~F}$ NMR spectrum of compound 6je (376 MHz, $\mathrm{CDCl}_{3}$ ). 


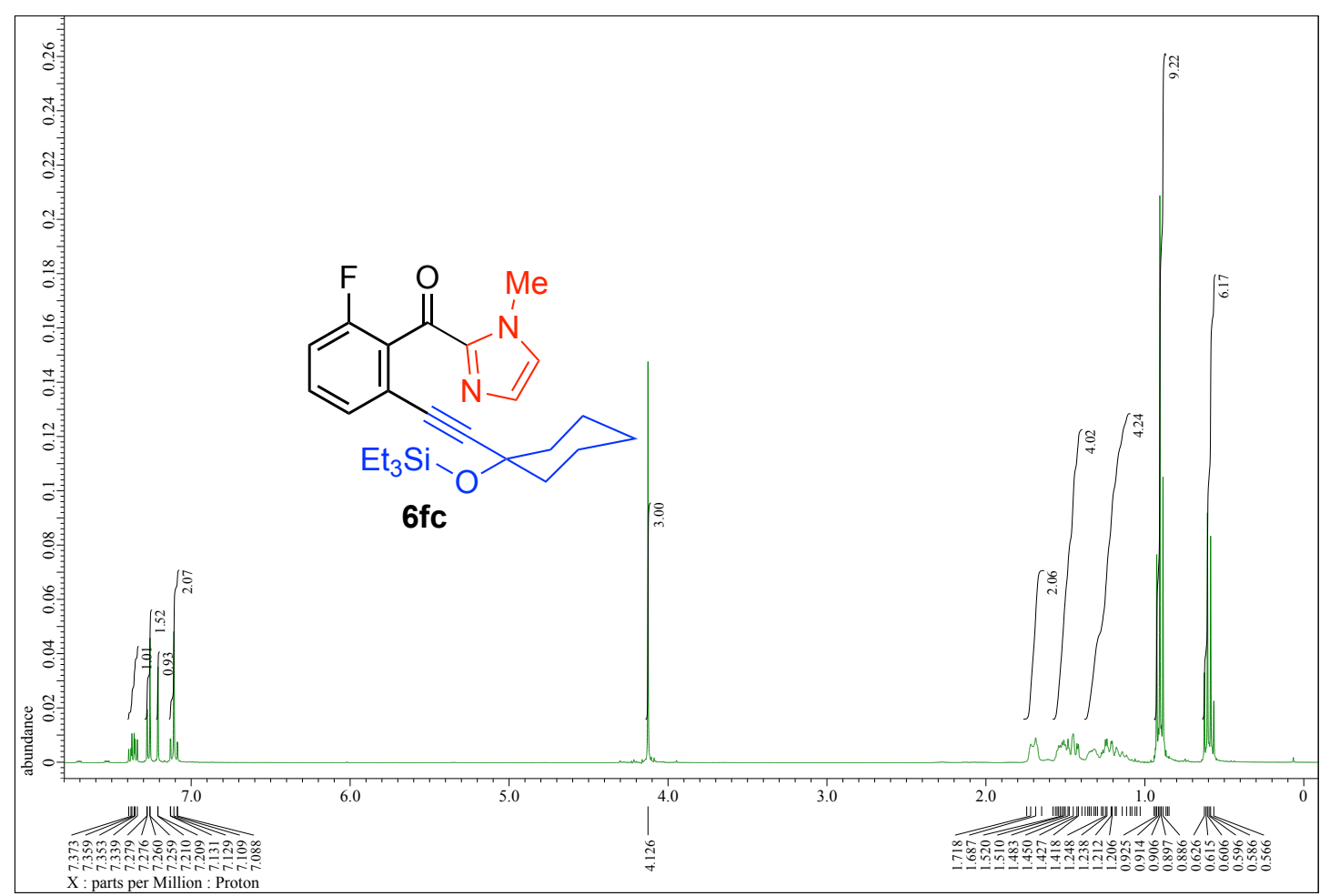

Figure S166: ${ }^{1} \mathrm{H}$ NMR spectrum of compound $6 \mathbf{f c}\left(400 \mathrm{MHz}, \mathrm{CDCl}_{3}\right)$.

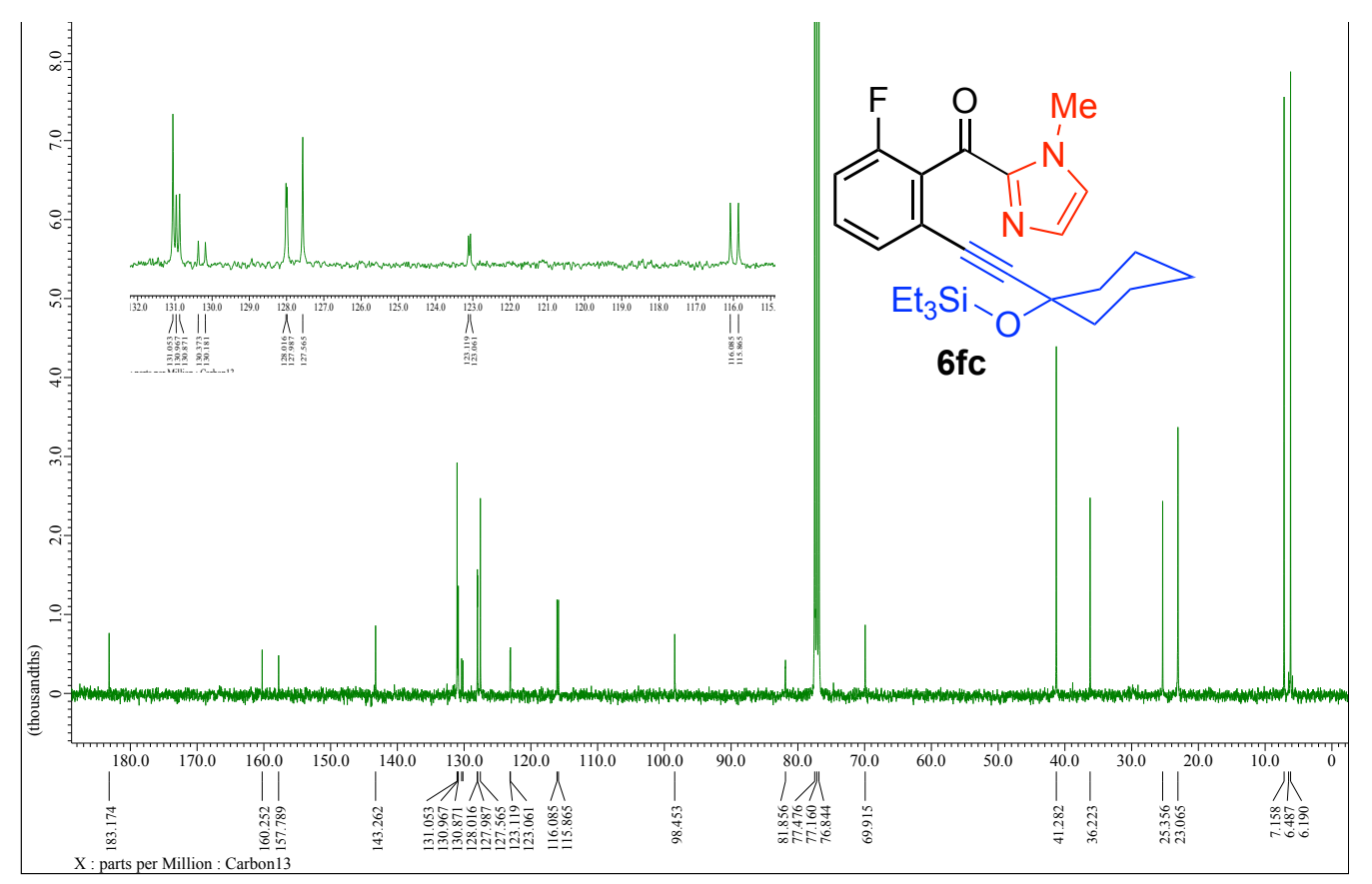

Figure S167: ${ }^{13} \mathrm{C}$ NMR spectrum of compound $6 \mathbf{f c}\left(100 \mathrm{MHz}, \mathrm{CDCl}_{3}\right)$. 


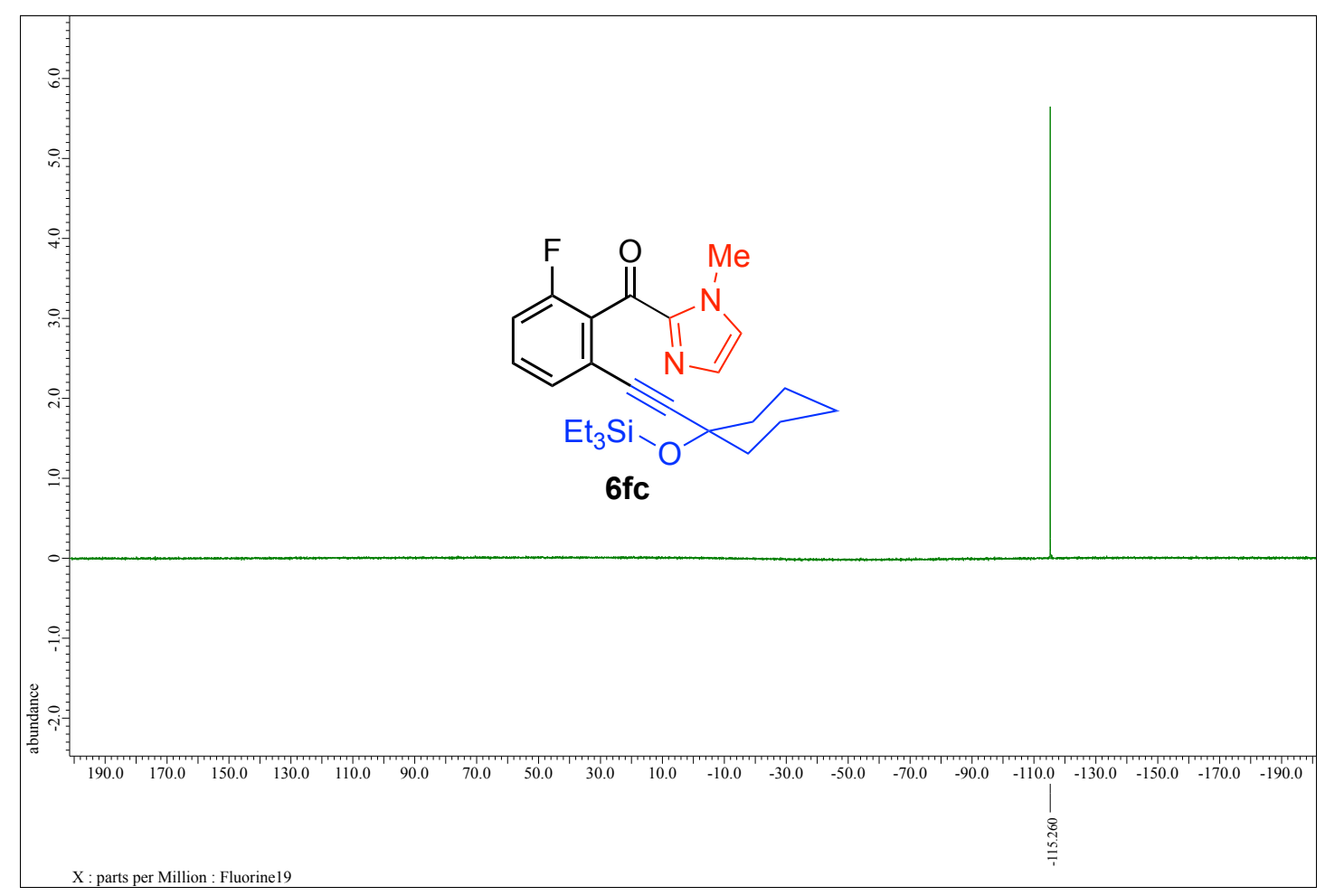

Figure S168: ${ }^{19} \mathrm{~F}$ NMR spectrum of compound 6 fe $\left(376 \mathrm{MHz}, \mathrm{CDCl}_{3}\right)$.

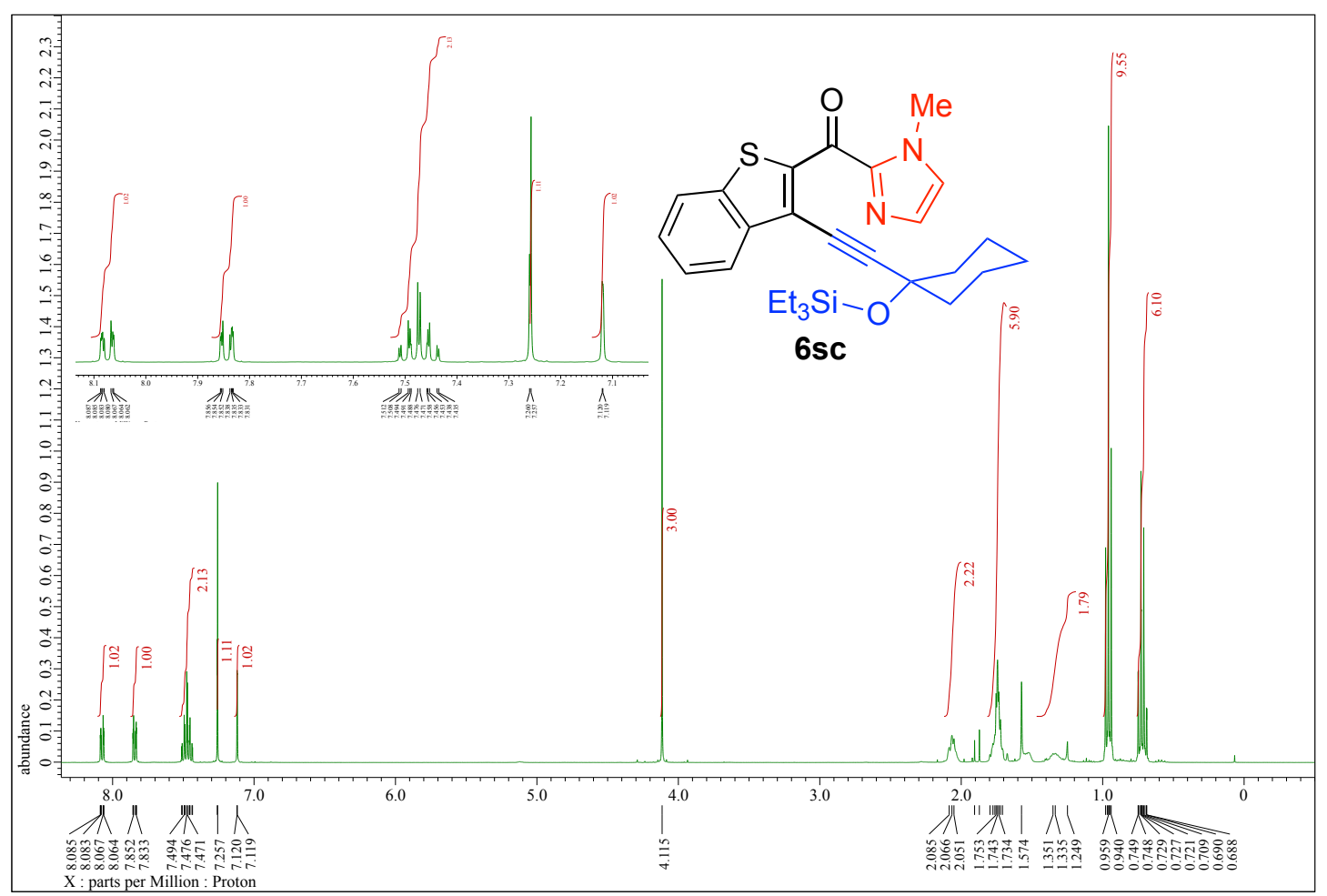

Figure S169: ${ }^{1} \mathrm{H}$ NMR spectrum of compound 6sc (400 MHz, $\left.\mathrm{CDCl}_{3}\right)$. 


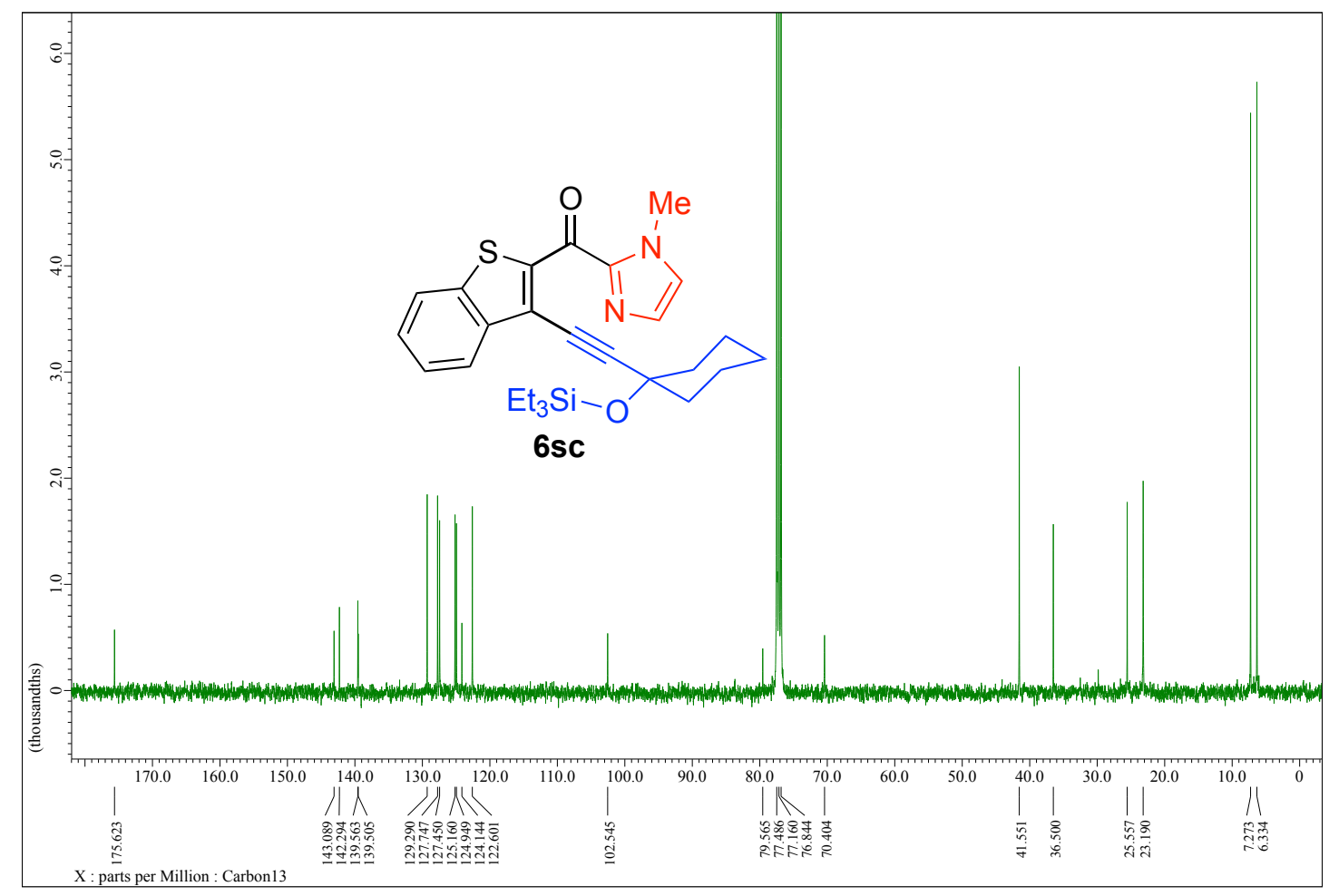

Figure S170: ${ }^{13} \mathrm{C}$ NMR spectrum of compound 6sc $\left(100 \mathrm{MHz}, \mathrm{CDCl}_{3}\right)$.

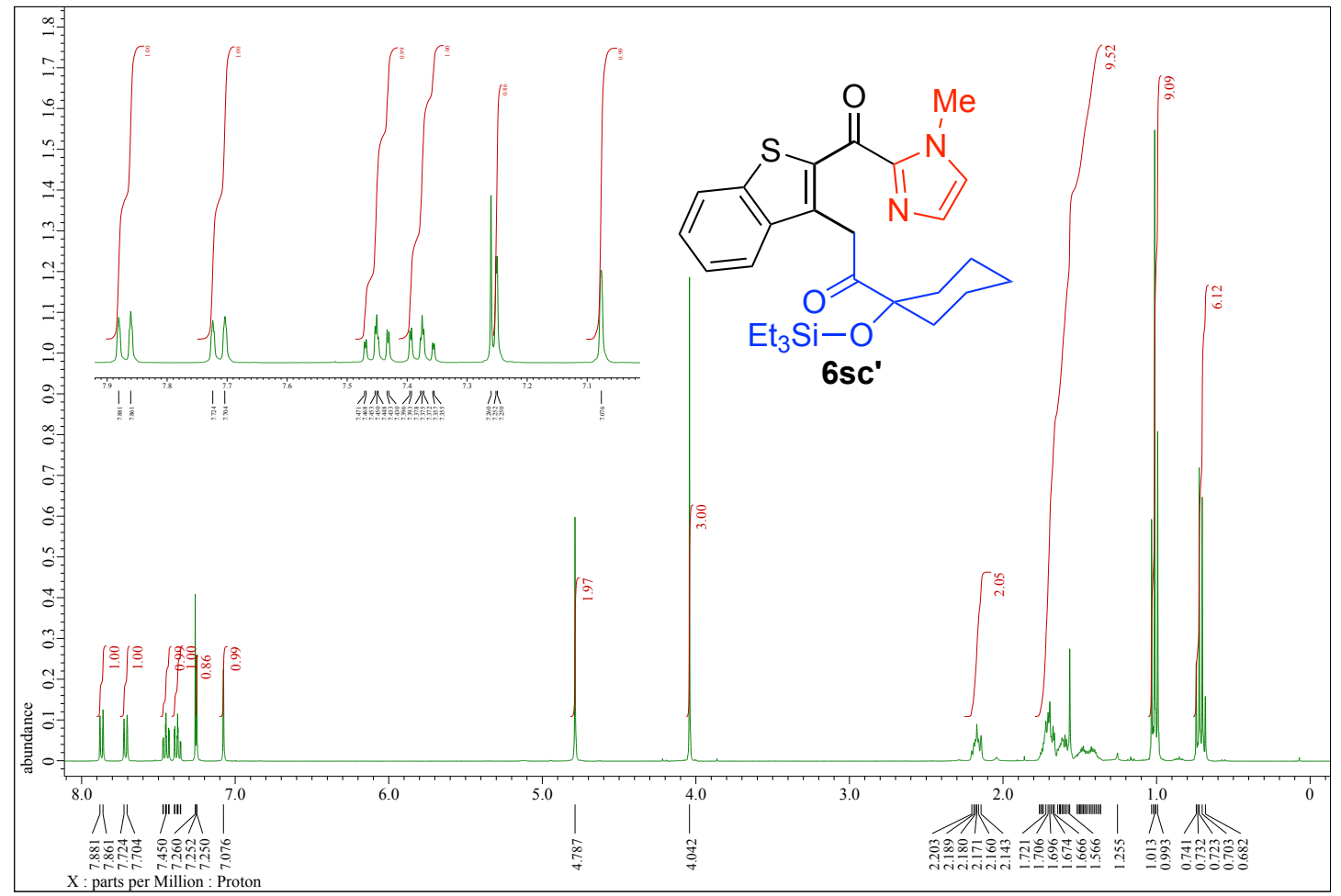

Figure S171: ${ }^{1} \mathrm{H}$ NMR spectrum of compound $\mathbf{6 s c}$ ' $\left(400 \mathrm{MHz}, \mathrm{CDCl}_{3}\right)$. 


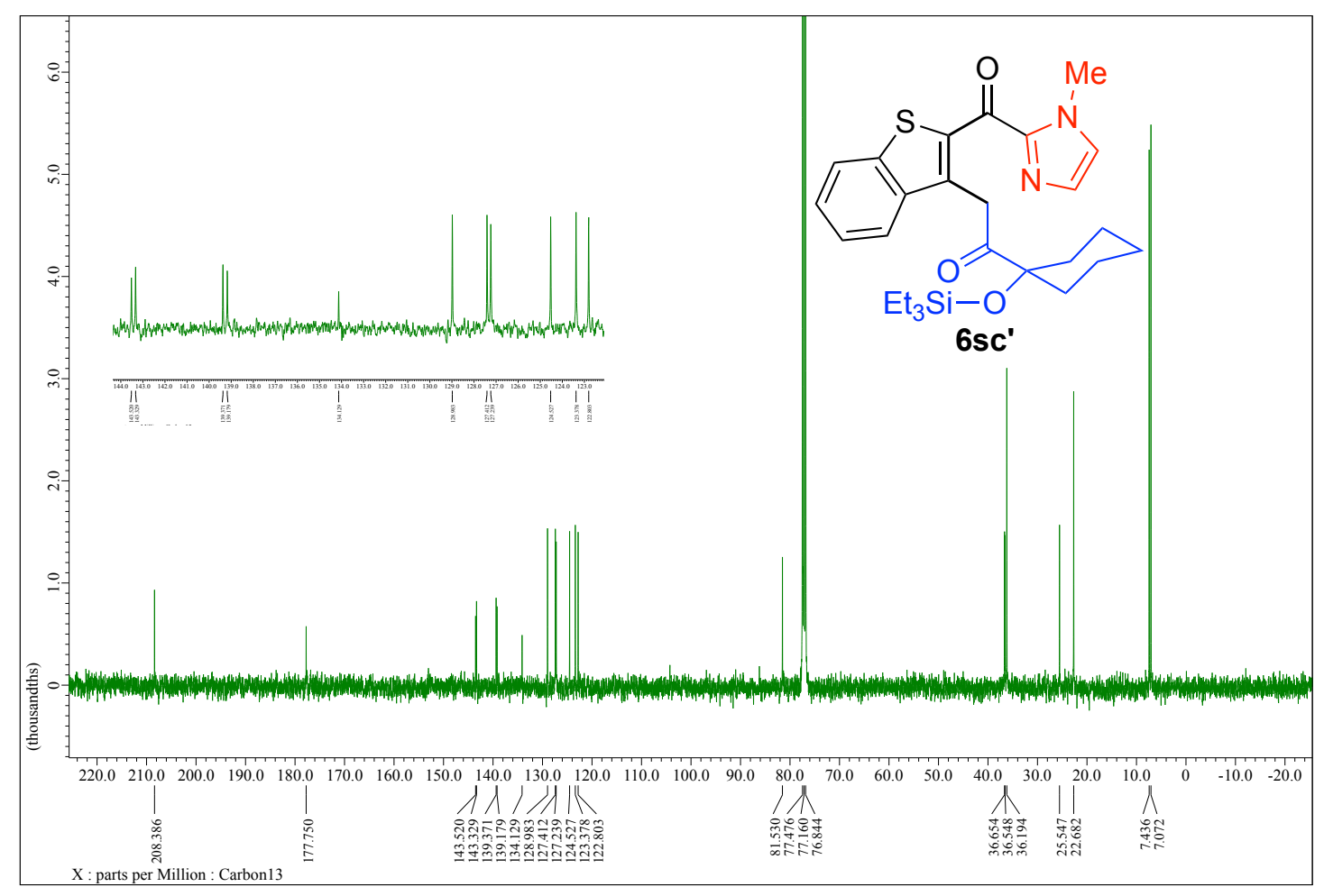

Figure S172: ${ }^{13} \mathrm{C}$ NMR spectrum of compound 6sc' $\left(100 \mathrm{MHz}, \mathrm{CDCl}_{3}\right)$.

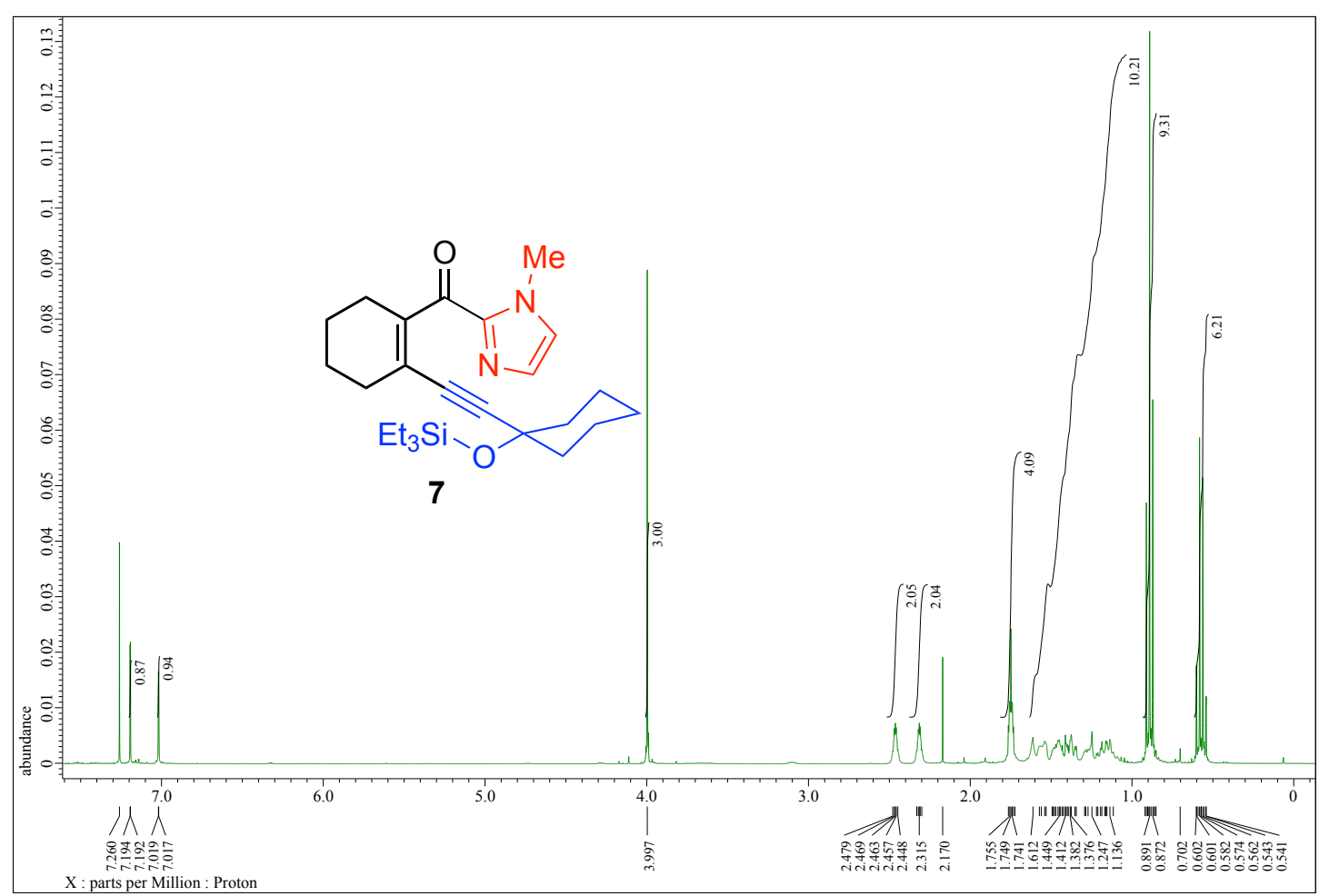

Figure S173: ${ }^{1} \mathrm{H}$ NMR spectrum of compound $7\left(400 \mathrm{MHz}, \mathrm{CDCl}_{3}\right)$. 


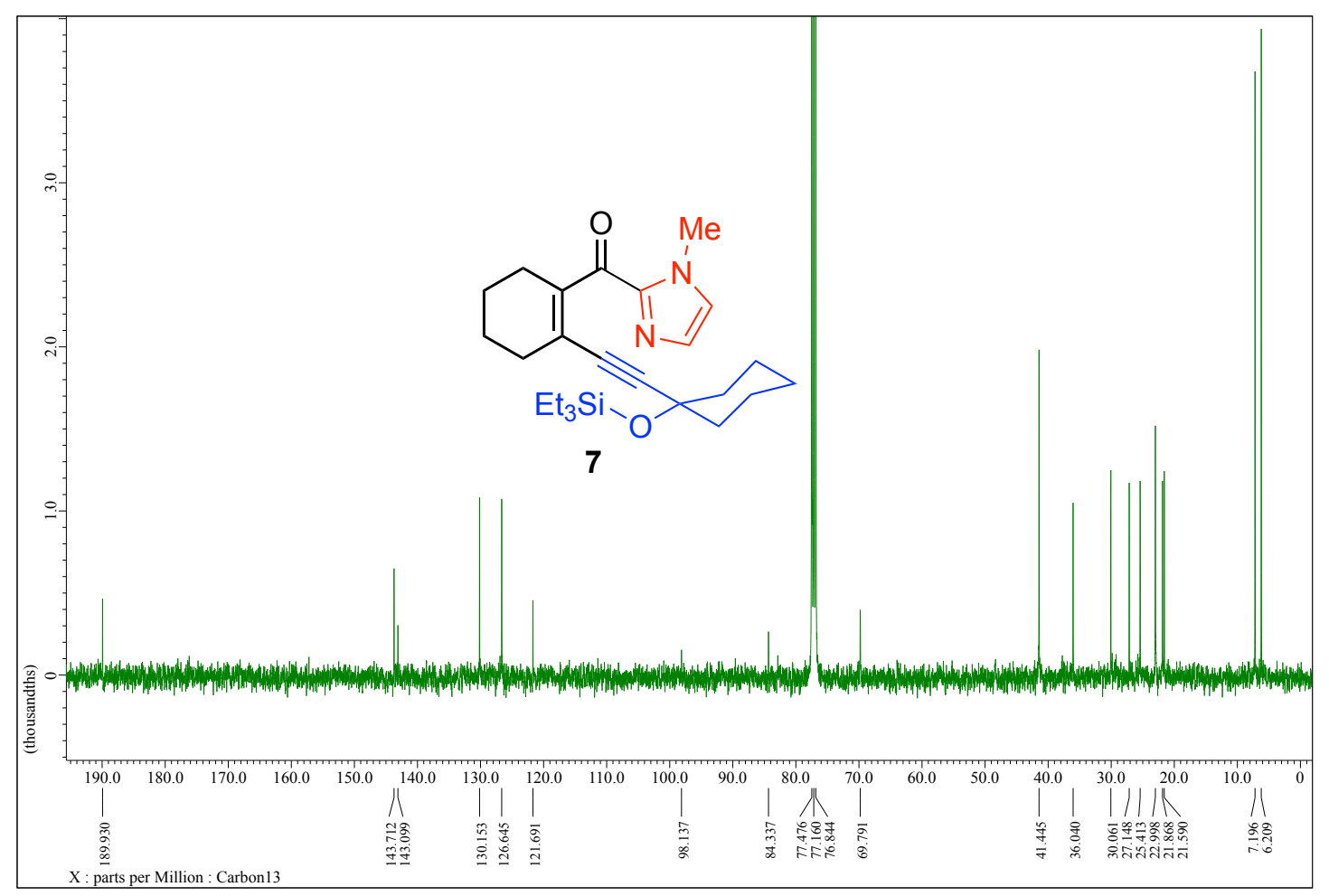

Figure S174: ${ }^{13} \mathrm{C}$ NMR spectrum of compound $7\left(100 \mathrm{MHz}, \mathrm{CDCl}_{3}\right)$.

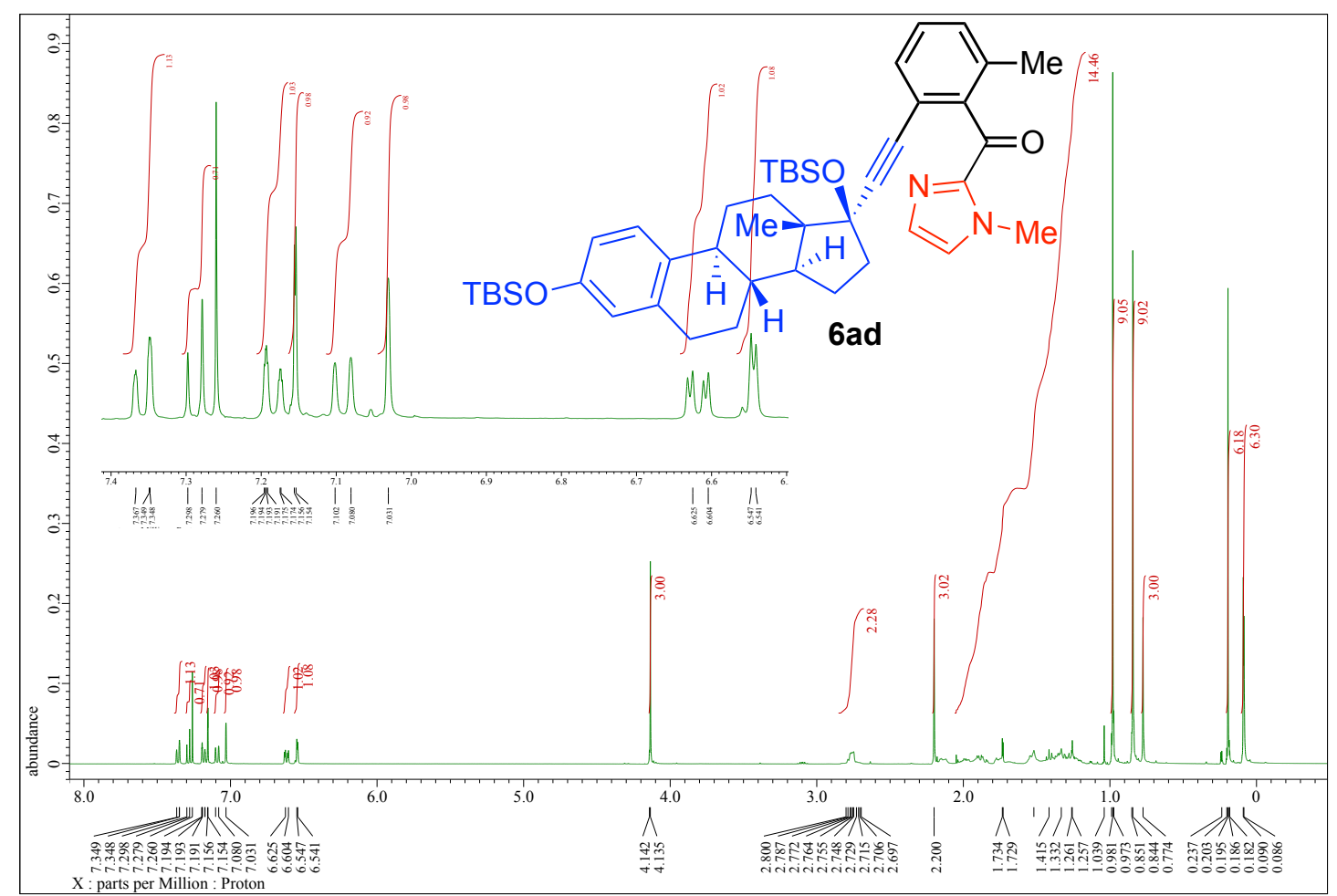

Figure S175: ${ }^{1} \mathrm{H}$ NMR spectrum of compound 6 ad $\left(400 \mathrm{MHz}, \mathrm{CDCl}_{3}\right)$. 


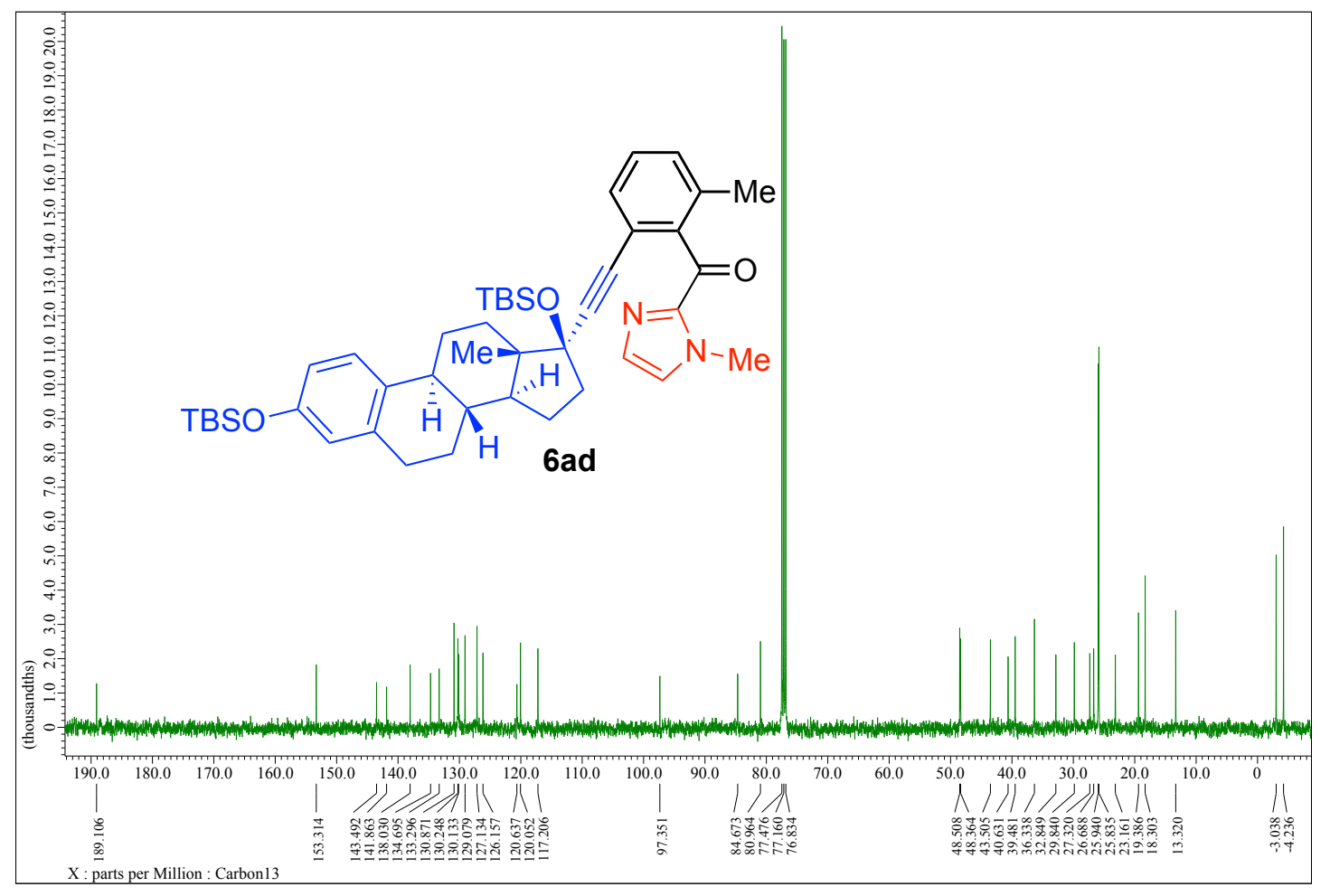

Figure S176: ${ }^{13} \mathrm{C}$ NMR spectrum of compound $\mathbf{6 a d}\left(100 \mathrm{MHz}, \mathrm{CDCl}_{3}\right)$.

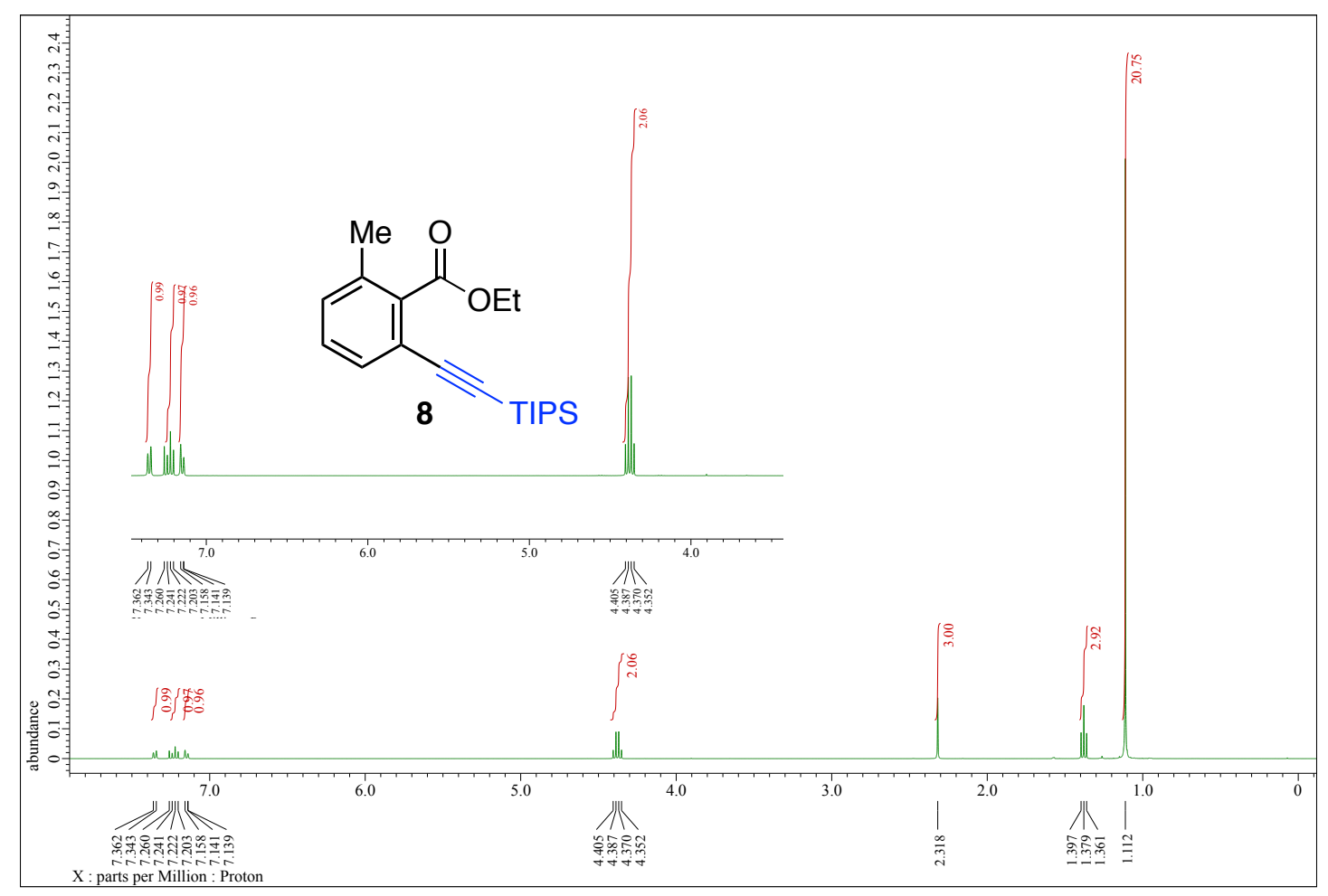

Figure S177: ${ }^{1} \mathrm{H}$ NMR spectrum of compound $8\left(400 \mathrm{MHz}, \mathrm{CDCl}_{3}\right)$. 


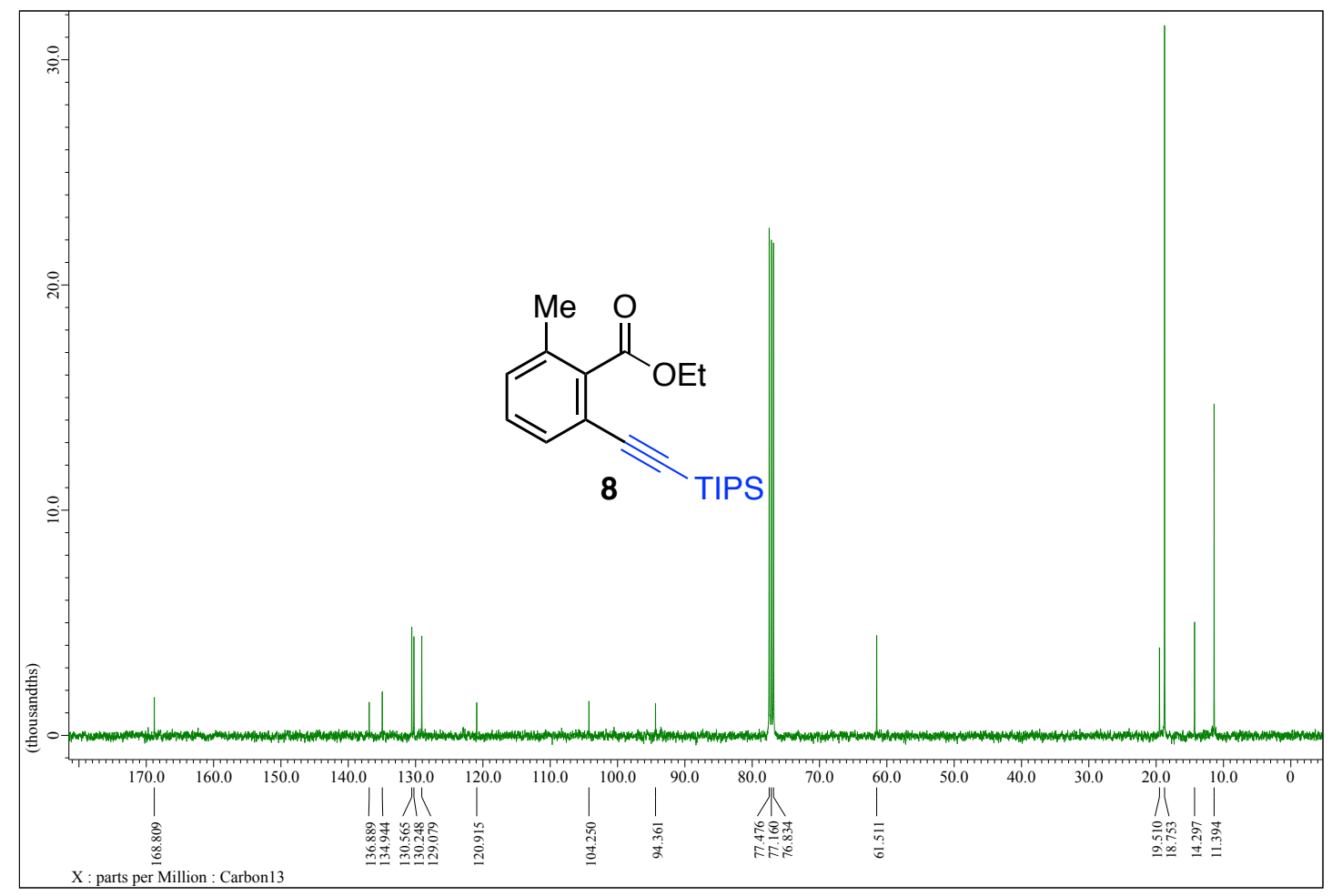

Figure S178: ${ }^{13} \mathrm{C}$ NMR spectrum of compound $8\left(100 \mathrm{MHz}, \mathrm{CDCl}_{3}\right)$.

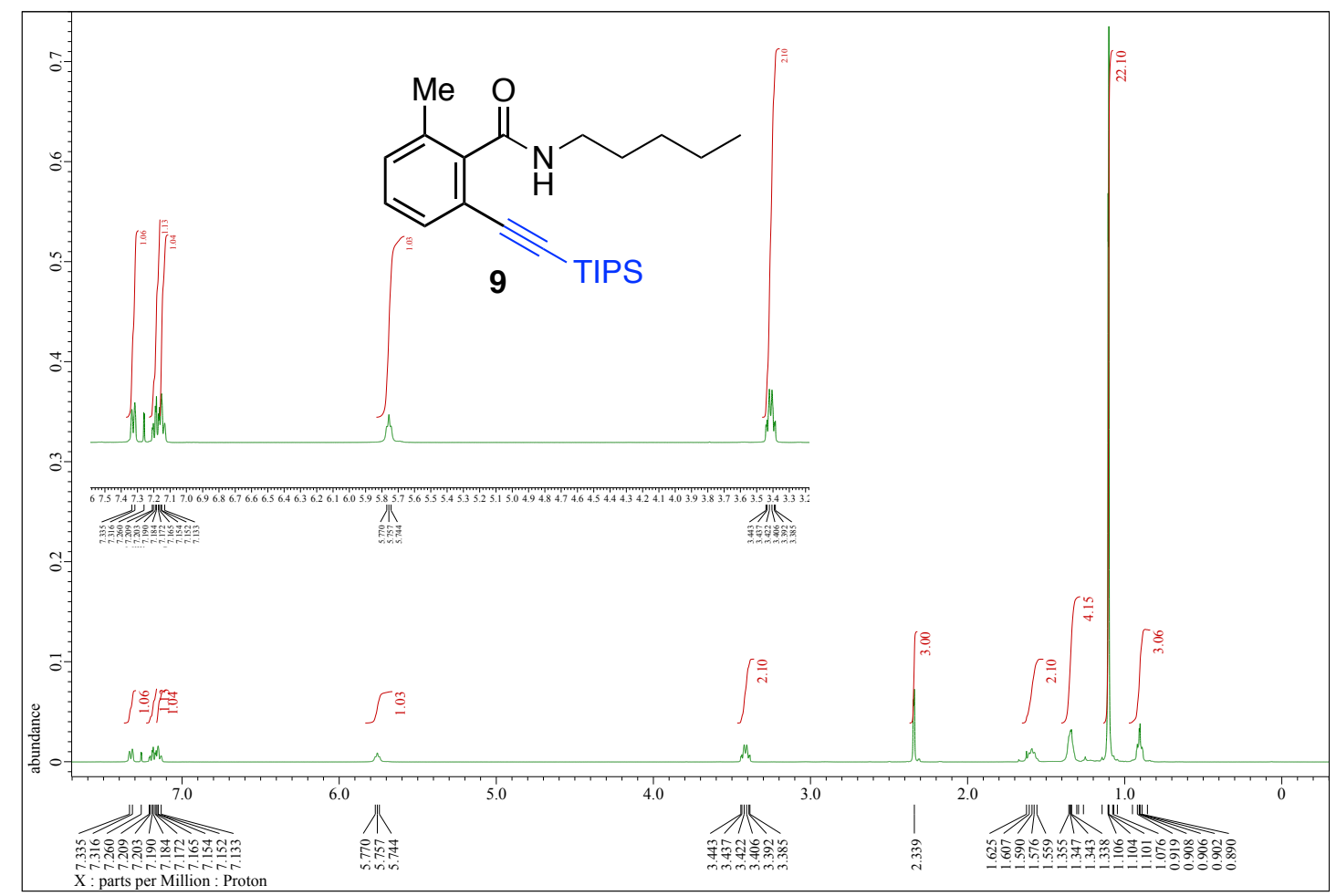

Figure S179: ${ }^{1} \mathrm{H}$ NMR spectrum of compound $9\left(400 \mathrm{MHz}, \mathrm{CDCl}_{3}\right)$. 


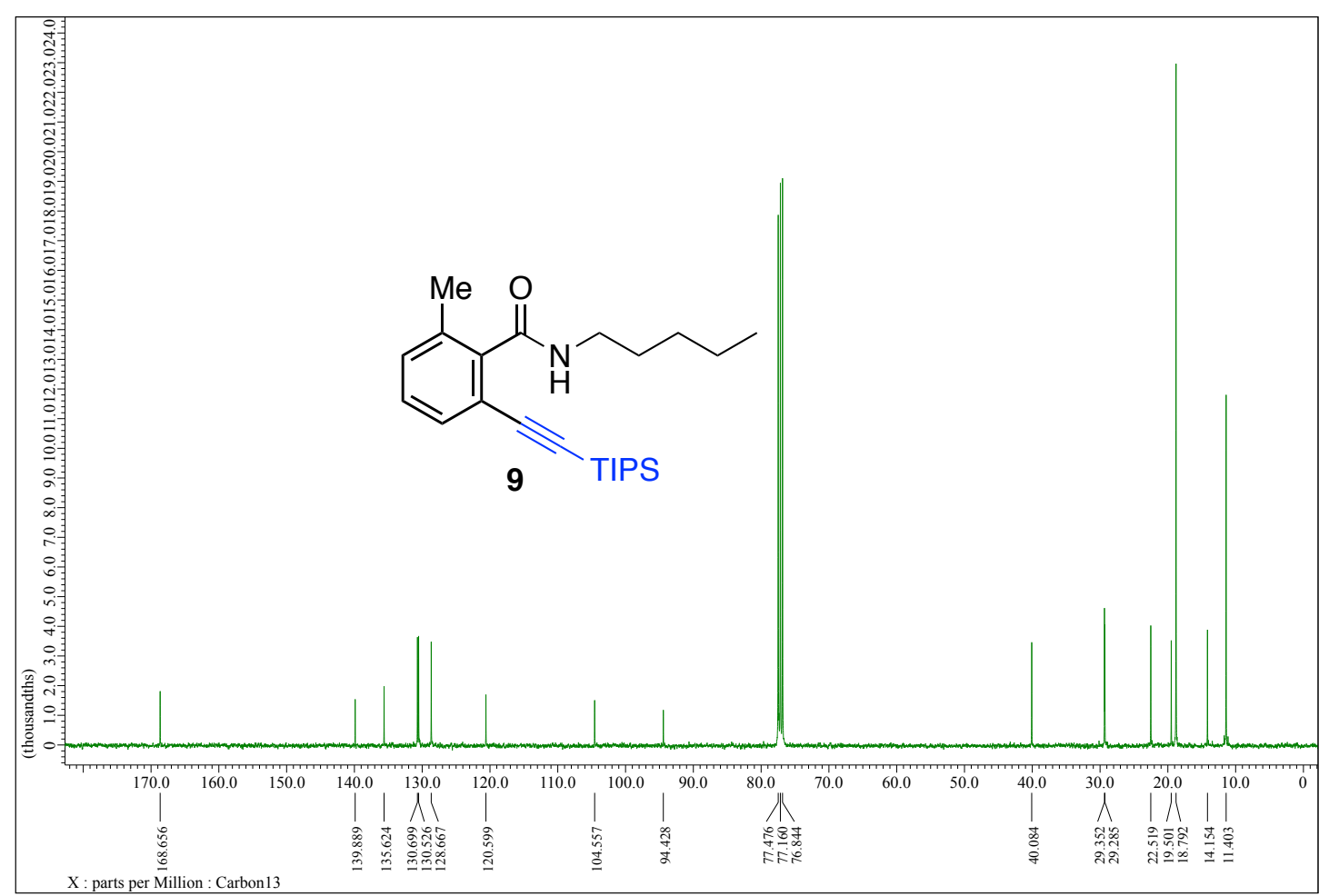

Figure S180: ${ }^{13} \mathrm{C}$ NMR spectrum of compound $9\left(100 \mathrm{MHz}, \mathrm{CDCl}_{3}\right)$.

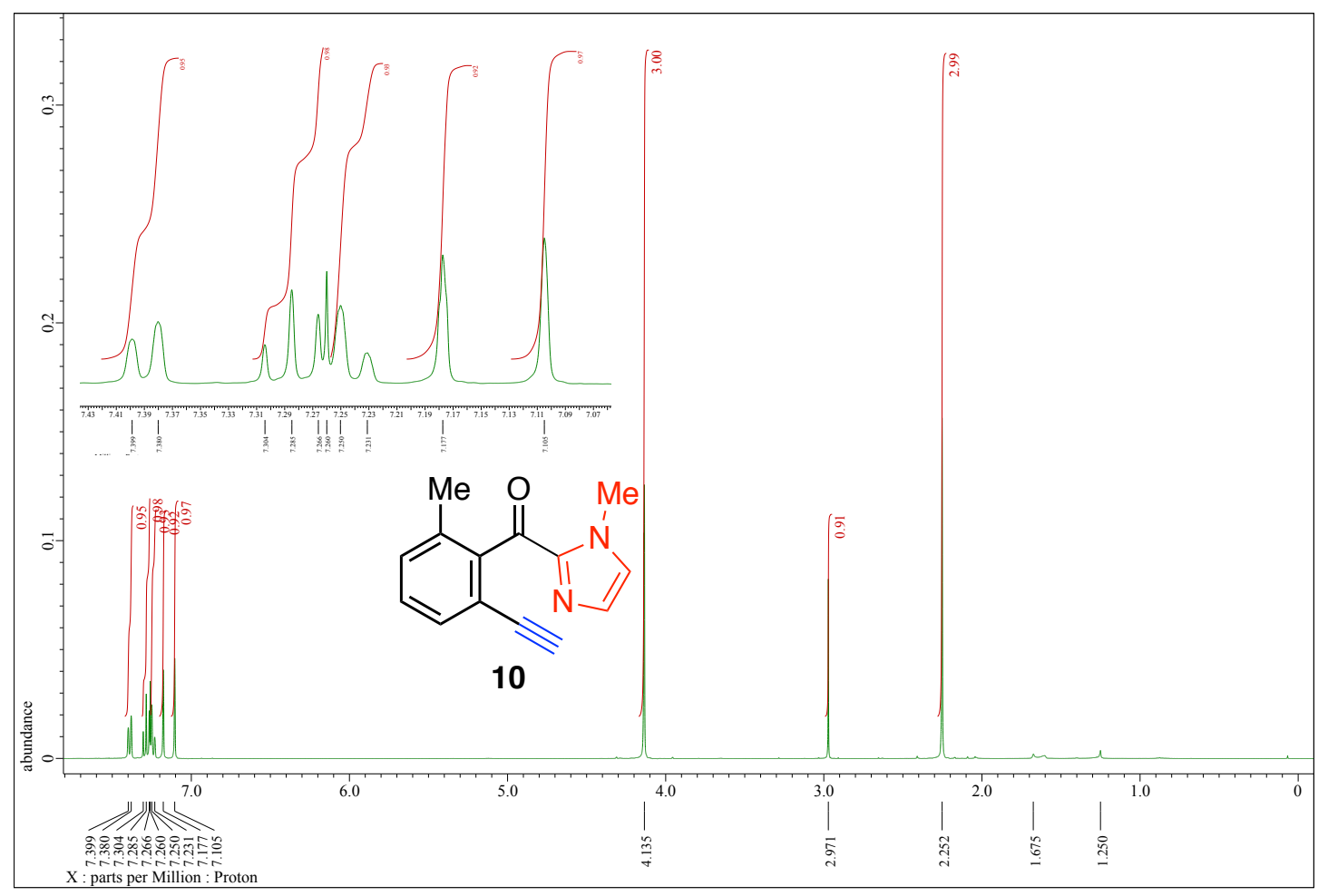

Figure S181: ${ }^{1} \mathrm{H}$ NMR spectrum of compound $10\left(400 \mathrm{MHz}, \mathrm{CDCl}_{3}\right)$. 


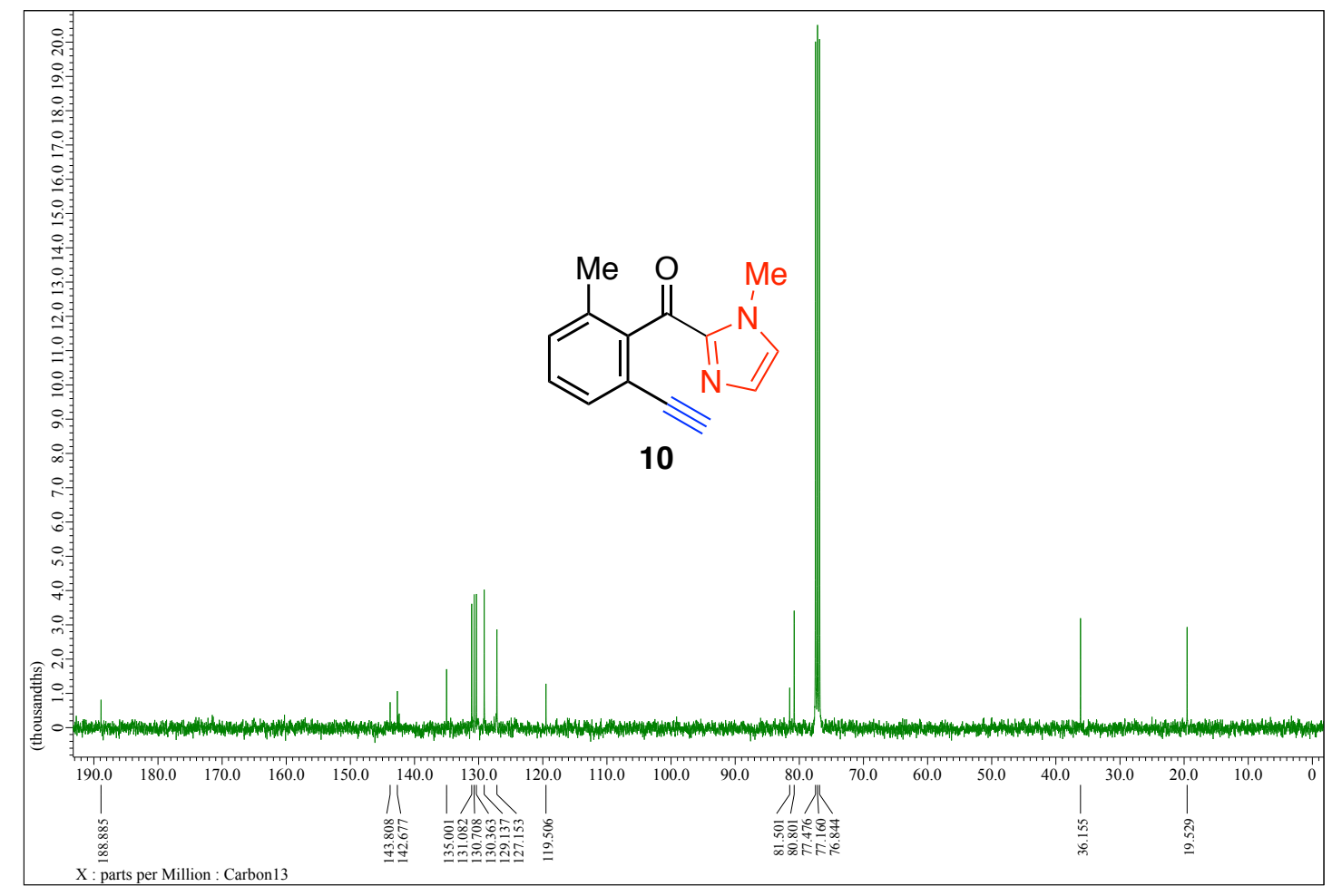

Figure S182: ${ }^{13} \mathrm{C}$ NMR spectrum of compound $10\left(100 \mathrm{MHz}, \mathrm{CDCl}_{3}\right)$. 